DEBORA FROMMELD, ULRIKE SCORNA, SONJA HAUG, KARSTEN WEBER (HG.)

\title{
GUTE TECHNIK \\ FÜR EIN GUTES \\ LEBEN IM ALTER?
}

Akzeptanz, Chancen und Herausforderungen altersgerechter

Assistenzsysteme
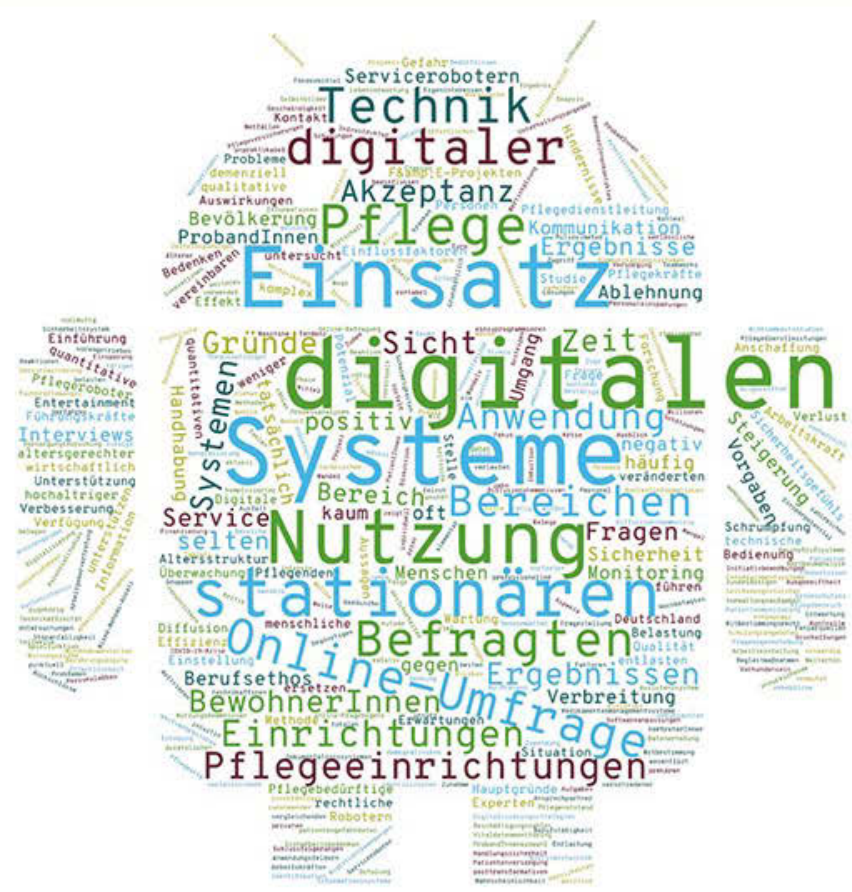

[transcript] ALTER - KULTUR - GESELLSCHAFT 
Debora Frommeld, Ulrike Scorna, Sonja Haug, Karsten Weber (Hg.) Gute Technik für ein gutes Leben im Alter? 
Debora Frommeld (Dr. phil.) ist Soziologin am Institut für Sozialforschung und Technikfolgenabschätzung (IST) der Ostbayerischen Technischen Hochschule (OTH) Regensburg und forscht zu (ästhetischen) Alltagspraktiken an der Schnittstelle von Technik, Digitalisierung, Gesundheit und Körper.

Ulrike Scorna (M.A.) ist Sozialwissenschaftlerin am Institut für Sozialforschung und Technikfolgenabschätzung (IST) an der Ostbayerischen Technischen Hochschule (OTH) Regensburg. Ihre Forschungsschwerpunkte liegen im Bereich Digitaler Assistenztechnik und Medizinsoziologie.

Sonja Haug (Prof. Dr. phil. habil.) ist Soziologin und Professorin für Empirische Sozialforschung an der Ostbayerischen Technischen Hochschule (OTH) Regensburg. Sie leitet gemeinsam mit Karsten Weber das Institut für Sozialforschung und Technikfolgenabschätzung (IST) und forscht unter anderem zu Demografie und sozialen Aspekten von Technik im Bereich Gesundheit.

Karsten Weber (Prof. Dr. phil. habil.), geb. 1967, ist Philosoph und leitet gemeinsam mit Sonja Haug das Institut für Sozialforschung und Technikfolgenabschätzung (IST) der Ostbayerischen Technischen Hochschule (OTH) Regensburg. Er beschäftigt sich mit den sozialen und normativen Auswirkungen der Digitalisierung u.a. im Gesundheitswesen. 
Debora Frommeld, Ulrike Scorna, Sonja Haug, Karsten Weber (Hg.)

\section{Gute Technik für ein gutes Leben im Alter?}

Akzeptanz, Chancen und Herausforderungen altersgerechter Assistenzsysteme

Unter Mitarbeit von Laura Cerullo und Monika Friedl 
DAAS-KIN wurde durch das Bundesministerium für Bildung und Forschung (BMBF) unter dem Förderkennzeichen 16ITA217 unterstützt.

GEFÖRDERT VOM

Bundesministerium

für Bildung

und Forschung

\section{Bibliografische Information der Deutschen Nationalbibliothek}

Die Deutsche Nationalbibliothek verzeichnet diese Publikation in der Deutschen Nationalbibliografie; detaillierte bibliografische Daten sind im Internet über http://dnb.d-nb.de abrufbar.

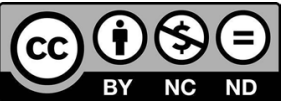

Dieses Werk ist lizenziert unter der Creative Commons Attribution-NonCommercial-NoDerivs 4.0 Lizenz (BY-NC-ND). Diese Lizenz erlaubt die private Nutzung, gestattet aber keine Bearbeitung und keine kommerzielle Nutzung. Weitere Informationen finden Sie unter

https://creativecommons.org/licenses/by-nc-nd/4.o/deed.de

Um Genehmigungen für Adaptionen, Übersetzungen, Derivate oder Wiederverwendung zu kommerziellen Zwecken einzuholen, wenden Sie sich bitte an rights@transcript-publishing.com

Die Bedingungen der Creative-Commons-Lizenz gelten nur für Originalmaterial. Die Wiederverwendung von Material aus anderen Quellen (gekennzeichnet mit Quellenangabe) wie z.B. Schaubilder, Abbildungen, Fotos und Textauszüge erfordert ggf. weitere Nutzungsgenehmigungen durch den jeweiligen Rechteinhaber.

\section{() 2021 transcript Verlag, Bielefeld}

Umschlaggestaltung: Maria Arndt, Bielefeld

Umschlagabbildung: DAAS-KIN 2020

Satz: Laura Cerullo

Druck: Majuskel Medienproduktion GmbH, Wetzlar

Print-ISBN 978-3-8376--1

PDF-ISBN 978-3-8394-5469-5

https://doi.org/10.14361/9783839454695

Buchreihen-ISSN: 2569-2615

Buchreihen-eISSN: 2703-0318

Gedruckt auf alterungsbeständigem Papier mit chlorfrei gebleichtem Zellstoff. Besuchen Sie uns im Internet: https://www.transcript-verlag.de Unsere aktuelle Vorschau finden Sie unter www.transcript-verlag.de/vorschau-download 


\section{Inhalt}

\section{Einführung}

Gute Technik für ein gutes Leben?!

Karsten Weber

Altersgerechte Assistenzsysteme: Ein Überblick

Karsten Weber

\section{Technik und Pflege im professionellen Kontext und (privaten) Alltag}

Die Nutzung von digitalen Kommunikationstechnologien in ambulanten und stationären Pflegeeinrichtungen während der COVID-19-Pandemie

Dominik Domhoff, Kathrin Seibert, Heinz Rothgang, Karin Wolf-Ostermann

Arbeit und Geschlecht.

Strukturelle und normative Grundlagen

von Technisierungsprozessen in der Pflege

Clarissa Rudolph

87

Datenschutz und digitale Ethik

Grundlage guter Technik

Walter Swoboda, Marina Fotteler, Michael Örtl, Felix Holl, Martin Schmieder, Elmar Buchner.. 109

Altern und Verletzlichkeit: Gero-Technologien als Bestandteil einer therapeutisch-rehabilitativen Dyade? 


\section{Altersgerechte Assistenzsysteme und Nutzungshemmnisse}

Die tatsächliche Nutzung digitaler Assistenzsysteme in der Altenpflege Ein Scoping Review

Miriam Vetter und Laura Cerullo 161

Nutzung, Planung und Bewertung

digitaler Assistenzsysteme in der Pflege

Ergebnisse einer Befragung von Führungskräften

in ambulanten und stationären Einrichtungen

Sonja Haug 185

Digitale Technik in der ambulanten und stationären Pflege

Eine Interviewstudie zum Einfluss des Pflegepersonals

auf die Einführung digitaler Assistenzsysteme

Ulrike Scorna

Vertrauen, Wissen, Innovation und Wohltun

als (neue) Herausforderungen im Kontext digitaler Assistenzsysteme

Ergebnisse einer Diskurs- und Wertbaumanalyse

Debora Frommeld

\section{Implementierung und Evaluation von (digitaler) Pflegetechnik}

Möglichkeiten des Bewegungsmonitorings durch Fitnesstracker nach einer Hüftfraktur

Amelie Altenbuchner 265

Digitalisierte Bewegungsentwicklung geriatrischer Traumapatient*innen Konzept und Evaluation eines Bewegungsfeedbacks Laura Cerullo 285

Zum Potenzial grundlagenwissenschaftlicher Technikforschung für ein "gutes Leben im Alter"

Ein Plädoyer für konsequente partizipative Technikgestaltung Jannis Hergesell, Arne Maibaum, Andreas Bischof, Benjamin Lipp.... 
Anforderungen an die Evaluation

von altersgerechten Assistenztechnologien

aus gesundheitsökonomischer Sicht

Mareike Mähs

Technikimplementierung in der Pflege: die Bedeutung

der soziotechnischen Innovationsbewertung

aus gesundheitsökonomischer Sicht

Jürgen Zerth

Autor*innen 

Einführung 



\section{Gute Technik für ein gutes Leben?!}

Karsten Weber

\section{Einleitung}

Mit dem Erscheinen des vorliegenden Sammelbands findet das zweijährige Projekt mit dem etwas sperrigen Titel »Diffusion altersgerechter Assistenzsysteme - Kennzahlenerhebung und Identifikation von Nutzungshemmnissen" (DAAS-KIN) seinen sichtbaren Abschluss. In den zwei Jahren des vom Bundesministerium für Bildung und Forschung (BMBF) geförderten Projekts haben die Herausgeber*innen des vorliegenden Bands untersucht, warum trotz der vielen Mittel, die in die Forschung an und Entwicklung von altersgerechten Assistenzsystemen investiert wurden, vergleichsweise wenig davon in der Praxis angekommen ist. Diese Feststellung ist - manche mögen den damit beschriebenen Sachverhalt für bedauerlich, andere für einen Glücksfall halten $-\mathrm{zu}$ einem Allgemeinplatz geworden und sie wird von Schmidt/Wahl (2019: 546) trefflich auf den Punkt gebracht:

»Wenn man sich auf der einen Seite die sehr hohe Zahl an bislang in Deutschland (und Europa) geförderten Gero-Technologie-Forschungsprojekten (samt den Dutzenden Millionen an Fördergeldern) vor Augen führt und sich auf der anderen Seite die Frage stellt, wo zwischenzeitlich vielversprechende Techniklösungen nachhaltig Einzug in den Alltag älterer Menschen gehalten haben, dann fällt die Bilanz noch verbesserungsbedürftig aus. Was ist wirklich bislang dort sangekommen<?

Man kann hinzufügen: Es sind eben nicht nur »Dutzende [...] Millionen an Fördergeldern « in die Entwicklung altersgerechter Assistenzsysteme investiert worden, sondern Hunderte Millionen - allein in Deutschland und auf Bundesebene.

Anlass des Projekts DAAS-KIN war unter anderem die Frage, die im letzten Satz des obigen Zitats gestellt wird: Was ist denn tatsächlich in der Praxis angekommen? Dazu gibt es erstaunlicherweise wenig empirische Erkenntnisse. Wir hoffen, mit der Präsentation der Projektergebnisse zumindest ein paar Antworten auf diese Frage geben zu können, doch mussten wir im Verlauf des Projekts feststellen, dass eine Bestandsaufnahme auf einige Schwierigkeiten trifft. Mit DAAS-KIN wollten 
wir aber nicht nur das Ziel einer Bestandsaufnahme verfolgen, sondern wir wollten genauer wissen, warum in den einschlägigen wissenschaftlichen und praxisorientierten Debatten stets das Potenzial altersgerechter Assistenzsysteme betont (zumindest, sofern die Debattierenden der Technik im Grundsatz positiv gegenüberstehen), aber mit derselben Regelmäßigkeit und mit Bedauern (oder mit einer gewissen Befriedigung, wenn die Haltung der betreffenden Person der Technik gegenüber eher negativ ausfällt) die Feststellung getroffen wird, dass dieses Potenzial bis heute eben nicht ausgeschöpft wurde. Dieses heute markiert dabei kein festes Datum, sondern fungiert als Platzhalter, der mit dem jeweiligen Datum gefüllt werden kann oder muss. Die Proponenten altersgerechter Assistenzsysteme (zumindest die noch nicht endgültig entmutigten) äußern dann meist die Hoffnung, dass nur noch wenige Jahre (und viele Millionen Euro weitere Fördermittel) benötigt werden würden, um den endgültigen Durchbruch der Technik zu erreichen. Für jene, die ein wenig über Technikgeschichte des 20 . Jahrhunderts Bescheid wissen, drängt sich da womöglich der Vergleich mit der Kernfusion auf - seit Zündung der ersten thermonuklearen Bombe in den frühen 1950er Jahren wird mit der Regelmäßigkeit eines Metronoms angekündigt, dass die zivile Nutzung der Kernfusion in x Jahren erreicht werden wird (man kann für x nun 20, 30 oder auch 50 Jahre einsetzen). Dummerweise werden solche Prognosen bis heute in gleicher Weise ausgesprochen, sodass diese Technik der zivilen Nutzung der Kernfusion stets 20 , 30 oder 50 Jahre entfernt zu sein scheint.

Ganz so düster sieht es im Fall altersgerechter Assistenzsysteme nicht aus. Tatsächlich kann man hoffen, dass die Erfahrungen der Corona-Pandemie mit dazu beitragen werden, dass einige der Diffusionshemmnisse der Einführung dieser Technik in die praktische Anwendung wegfallen könnten. Angesichts der vielen Toten, die der Pandemie zum Opfer gefallen sind, der wahrscheinlich noch zahlreicheren Menschen, die mit den langfristigen Auswirkungen einer Erkrankung zu kämpfen haben, und der durch die ökonomischen Verwerfungen beschädigten oder gar zerstörten Biografien mag es ein wenig zynisch klingen, von positiven Auswirkungen zu sprechen. Es wäre in der Tat unvergleichlich besser, wenn gesellschaftliches Lernen ohne diesen Preis zu erreichen wäre, doch wie es scheint, lassen sich Gesellschaften nur dann auf weitreichende Veränderungen und Umbrüche ein, wenn letztlich keine Alternative mehr gegeben ist.

Schaut man sich die Ergebnisse des Projekts DAAS-KIN an, so wird deutlich, dass es in der Praxis keine grundsätzliche Ablehnung der Technik im Allgemeinen (mehr) gibt; die Einsatzhemmnisse, die wir erheben konnten, sind in der Regel sehr konkret und könnten mit entsprechenden passgenauen Maßnahmen, die nur zum Teil technischer Natur wären, adressiert werden. Allerdings werden bestimmte Technologien sehr skeptisch bewertet - dabei schneiden Roboter für den Einsatz in der Pflege besonders schlecht ab. 
Doch das ist bereits ein Vorgriff auf die Vorstellung der einzelnen Beiträge. Wir haben uns die Freiheit genommen, die Texte nicht nur vorzustellen, sondern sie an der einen oder anderen Stelle auch zu kommentieren und natürlich zueinander in Beziehung zu setzen.

\section{Die Beiträge}

Der vorliegende Sammelband ist in mehrere Abschnitte unterteilt: Einführung, Technik und Pflege im professionellen Kontext und (privaten) Alltag, altersgerechte Assistenzsysteme und Nutzungshemmnisse sowie Implementierung und Evaluation (digitaler) Pflegetechnik. Diese Einteilung soll Orientierung schaffen und als erster Hinweis auf die Themen verstanden werden, die die Autor*innen in den einzelnen Beiträgen verhandeln.

\section{Einleitung}

Die Einleitung umfasst nur zwei Texte - diesen Text sowie einen Überblick zum Themenkomplex. Karsten Weber beschreibt dort in aller Kürze die Genese der Idee altersgerechter Assistenzsysteme, deren Abkunft von Konzepten wie Ubiquitous Computing oder Ambient Intelligence, deren förderpolitisches Auftauchen (inklusive eines Anhangs mit einer Liste der durch das BMBF seit 2008 geförderten Projekte) sowie die demografischen und gesundheitsökonomischen Rahmenbedingungen, die überhaupt zur massiven Förderung dieser Technologie geführt haben. Im Grunde kann man die bisherige Förderung durch die öffentliche Hand als Versuch ansehen, ein Marktversagen zu beheben: Zwar gibt es Nachfrage nach Lösungen für den Pflegenotstand, die auch den Einsatz von Technik umfassen könnten, aber aufgrund der hohen Entwicklungskosten und der ungeklärten Frage, wie die Anschaffung und der Einsatz der Systeme finanziert werden könnten, wird kein Angebot entwickelt. Allerdings zielte die bisherige Förderpolitik im Wesentlichen auf die Anschubfinanzierung der technischen Entwicklung - die anderen offenen Probleme werden davon kaum berührt. Wie sich aber in den folgenden Beiträgen zeigen wird, fehlt es in der Praxis oft schon an den grundlegendsten Voraussetzungen, was ein Hinweis darauf sein könnte, dass die (Nicht-)Verbreitung altersgerechter Assistenzsysteme in allererster Linie kein technisch zu lösendes Problem darstellt. Dazu kommt, wie Weber es beschreibt, dass das Festhalten an überkommenen Leitbildern, Professionsverständnissen und möglicherweise auch Idealvorstellungen der Pflege - so wünschenswert deren Umsetzungen auch wären - verhindert, dass Second-Best-Lösungen zum Einsatz kommen. Anders formuliert: Die allgemeine Verbreitung altersgerechter Assistenzsysteme scheitert derzeit mindestens so sehr an bestimmten Ideologien (im wissenschaftssoziolo- 
gischen Sinne verstanden) wie an noch nicht ausgereifter Technik oder fehlenden Geschäftsmodellen und Finanzierungsmöglichkeiten. Ideologien sind allerdings mit Forschungsförderungspolitik kaum adressierbar.

\section{Technik und Pflege im professionellen Kontext und (privaten) Alltag}

Damit ist die Bühne bereitet; die Konfliktlinien sind grob markiert. Doch um besser verstehen zu können, warum altersgerechte Assistenzsysteme so zögerlich zum Einsatz kommen, obwohl deren Nutzung nun seit mehr als einem Jahrzehnt vorangetrieben wird, müssen die Bedingungen dieses Einsatzes weitaus genauer untersucht werden - sowohl in theoretischer wie praktischer Hinsicht. Hierzu sollen die Texte, die im zweiten Abschnitt des Bandes versammelt sind, einen Beitrag leisten.

Zunächst stellen Dominik Domhoff, Kathrin Seibert, Heinz Rothgang und Karin Wolf-Ostermann mit ihrem Beitrag »Die Nutzung von digitalen Kommunikationstechnologien in ambulanten und stationären Pflegeeinrichtungen während der COVID-19-Pandemie « eine empirische Erhebung vor. Ihr Ausgangspunkt ist dabei, dass die Kontaktbeschränkungen zur Eindämmung der Pandemie den Einsatz insbesondere digitaler Kommunikationstechnologien forcieren und motivieren; Technologie kann also dazu genutzt werden, Kommunikation trotz erschwerter Bedingungen aufrechtzuerhalten. Doch gleichzeitig bringt diese Form der Nutzung digitaler Technik mit sich, dass sich Pflegeeinrichtungen neuen Herausforderungen ausgesetzt sehen, denn sie müssen nun »vulnerable sowie physisch und kognitiv beeinträchtige Menschen in Pflegeeinrichtungen in der Nutzung von Kommunikationstechnologien [...] unterstützen«. Wie sich dieser Gebrauch digitaler Technik unter den Bedingungen der Pandemie tatsächlich gestaltet, haben die Autor*innen durch eine Umfrage eruiert. Sie schließen aus den gewonnenen Ergebnissen, dass »[d]ie COVID-19-Pandemie [...] auch in Pflegeeinrichtungen der Digitalisierung Vorschub geleistet [hat; Erg. KW] «, aber dass die Bedingungen, unter denen dies geschieht, alles andere als optimal waren und sind. Die Autor*innen kommen damit, zwar von einem anderen Ausgangspunkt, zu ähnlichen Ergebnissen wie das Projekt DAAS-KIN. So berichten sie von einer grundsätzlichen Aufgeschlossenheit der Technik gegenüber, aber eben auch von den fehlenden Voraussetzungen. Es wäre zu hoffen, dass der Digitalisierungsschub, den die Pandemie ausgelöst hat, auch über den Tag hinweg anhält.

Dazu müsste aber eine Reihe von Voraussetzungen erfüllt sein, die zum Teil recht grundlegender Natur sind. So beschreibt Clarissa Rudolph in ihrem Beitrag »Arbeit und Geschlecht. Strukturelle und normative Grundlagen von Technisierungsprozessen in der Pflege « sehr eindrücklich »die Arbeitsbedingungen in der bezahlten Pflege und führt diese Arbeitsbedingungen in einer historischen und systematischen Rückschau auf die Vergeschlechtlichung des Pflegeberufs zurück. Diese Rückschau lässt erkennen, dass ein erheblicher Teil der strukturellen Proble- 
me der professionellen Pflege einen zeitlich recht lang zurückliegenden Ursprung hat. Denn Sorge und Pflege wurden schon recht früh als spezifisch weibliche Tätigkeiten institutionalisiert - ökonomische und gesellschaftliche Überlegungen gingen dabei Hand in Hand. Maßnahmen der Kostendämpfung und die Ökonomisierung der Pflege haben wesentlich zur Verschlechterung der Arbeitsbedingungen beigetragen; das allein aber hätte nicht zur weiteren Vergeschlechtlichung der Pflege führen müssen. Doch, wie Rudolph schreibt,

»[u]m trotzdem die Pflege der alten und kranken Menschen weiterhin zu gewährleisten, wurden die Kollektivierungsprozesse durch Re-Familialisierungs- und Vergeschlechtlichungsprozesse begleitet. Insbesondere in der stark wettbewerbsorientierten Altenpflege gilt weiterhin das Motto sambulant vor stationärı, was impliziert, dass die familiäre Pflege überwiegend von Frauen durchgeführt wird, die von ambulanten, wiederum vorwiegend weiblichen, Pflegekräften unterstützt werden.«

Hieran zeigt sich eine der vielen Konfliktlinien in der Pflege wie unter einem Brennglas: Die meisten Menschen wollen tatsächlich zu Hause gepflegt werden, außerdem aber soll die Pflege nicht zu viel kosten - die (vermeintliche) Lösung geht zulasten eines geschlechtlich definierten Teils der Gesellschaft. Dabei scheinen tradierte Leitbilder der Pflege ein wichtiger Hebel zu sein, der auch dann bestehen bleibt, wenn der Einsatz altersgerechter Assistenzsysteme nicht flankiert wird durch eine Änderung dieser Leitbilder - solange Technik männlich konnotiert bleibt und solange die Techniknutzung geschlechtlich zugunsten der Männer aufgeladen wird, so kann man Rudolph verstehen, werden altersgerechte Assistenzsysteme Teil des Problems und nicht der Lösung sein.

Einen Dauerbrenner in der Diskussion um den Einsatz altersgerechter Assistenzsysteme behandeln Walter Swoboda, Marina Fotteler, Michael Örtl, Felix Holl, Martin Schmieder und Elmar Buchner mit ihrem Text über "Datenschutz und digitale Ethik. Grundlage guter Technik«. Das zentrale Ergebnis ihrer breit angelegten Quellenstudie drücken sie selbst am besten aus: »Gute Technik im Alter erfordert die Beachtung der Grundsätze von Datenschutz und digitaler Ethik, da ansonsten die Verbesserung der Lebensbedingungen durch schwerwiegende Nachteile, wie Verletzung des Persönlichkeitsrechts, Verlust der Selbstbestimmung und so weiter erkauft wird.« Solche Nachteile bleiben den potenziellen Nutzer*innen altersgerechter Assistenzsysteme natürlich nicht verborgen - die Folge ist fehlende Akzeptanz und damit letztlich ein massives Diffusionshemmnis. Es muss eigentlich verblüffen, dass Swoboda et al. im Jahr 2021 immer noch zu diesem Fazit gezwungen sind, wenn man bedenkt, dass das Unabhängige Landeszentrum für Datensicherheit Schleswig-Holstein (ULD 2010) schon vor mehr als einem Jahrzehnt auf diese Problematik hingewiesen hatte. Man sollte eigentlich erwarten dürfen, dass die 
Lernkurven der Stakeholder*innen im Umfeld der Pflege hier steiler sein müssten. Allerdings muss man bedenken, dass seit 2010 Social Media, aber auch die Nutzung von Clouds zur Speicherung auch von sensiblen Daten erst richtig Fahrt aufgenommen haben - gerade die Nutzung kommerzieller Clouds wird als kostensparende Variante gegenüber dem Aufbau eigener IT-Infrastrukturen angesehen. So wird hier eine weitere Konfliktlinie sichtbar: Datenschutz auf der einen Seite, Kostenargumente auf der anderen. Wenn man dann zudem aus erster Hand berichtet bekommt, dass lokale Einrichtungen großer Pflegeanbieter*innen beispielsweise die Übergabe zwischen Pflegekräften per WhatsApp organisieren, können die Schlussfolgerungen von Swoboda et al. nicht mehr wirklich verwundern. Hier mischen sich in der Praxis Unwissenheit, fehlende technische und organisatorische Voraussetzungen sowie vermutlich auch eine gewisse Gleichgültigkeit - Faktoren, die in der Summe dann zu schlechter Technik, zumindest aber zu einem schlechten Einsatz von Technik führen.

Neben anderen Überlegungen tragen solche Faktoren sicherlich dazu bei, dass Hartmut Remmers eine ausgesprochen kritische Haltung gegenüber altersgerechten Assistenzsystemen einnimmt. Seinem Beitrag "Altern und Verletzlichkeit: GeroTechnologien als Bestandteil einer therapeutisch-rehabilitativen Dyade? « hat er sicherlich nicht zufällig einen Titel gegeben, der mit einem Fragezeichen endet, denn er sieht den Einsatzmöglichkeiten altersgerechter Assistenzsysteme klare Grenzen gesetzt. Wie andere Autor*innen des vorliegenden Sammelbandes geht Remmers zunächst auf die Genese dieser Technik ein und auf die Rahmenbedingungen, insbesondere bezüglich des Fachkräftemangels, unter denen sie eingesetzt werden soll. Die Zahlen, die Remmers nennt, sind dramatisch, denn er nennt »einen Gesamtbedarf an beruflichen Pflegepersonen für 2030 in Höhe von 1,2 Millionen VZÄ [Vollzeitäquivalente; Erg. KW], der bei fortgesetztem Trend ohne politisch wirksame Eingriffe eine Lücke an beruflichen Pflegepersonen von 44 Prozent oder absolut 517.000 VZÄ aufweisen wird.«Zusammen mit einer eher positiven Beschreibung von Alterungsprozessen in dem Sinne, dass Alter und Altern nicht ausschließlich als Defizitentwicklung, sondern auch als Kompetenzgewinn beschrieben werden müssen, läge es nahe, wenn Remmers der Nutzung altersgerechter Assistenzsysteme, bei aller Vorsicht, positiv gegenüberstünde. Durch seine Sicht auf Altern und Verletzlichkeit schließt Remmers aber auf einen erhöhten Bedarf an sozialer Teilhabe im Alter, um die Resilienz der alternden Menschen zu stärken. Dies wiederum kann, so Remmers, nur in der »therapeutisch-rehabilitativen Dyade« von pflegender und zu pflegender Person erreicht werden - herausgearbeitet wird dies am Beispiel eines im Rahmen des Projekts REHATHESE entwickelten robotischen Systems. In der Tat zeigen die Ergebnisse des Projekts DAAS-KIN, dass insbesondere robotische Systeme auf große Skepsis in der Pflege treffen. Allerdings steht durchaus infrage, ob sich die allgemeine Skepsis, die Remmers ins Feld führt, durch den Verweis auf diese konkrete Technologie rechtfertigen lässt. Die von Remmers 
betonte »therapeutisch-rehabilitative Dyade« sollte aber in jedem Fall bei der Gestaltung >guter Technik für ein >gutes Leben berücksichtigt werden.

\section{Altersgerechte Assistenzsysteme und Nutzungshemmnisse}

Dieser Abschnitt des vorliegenden Sammelbands umfasst ausschließlich Beiträge, die im Rahmen der Arbeit an dem Projekt DAAS-KIN entstanden sind. In DAASKIN wurde ein Mix aus quantitativen, qualitativen und interpretativen Methoden benutzt: eine Umfrage (Haug), leitfadengestützte Interviews (Scorna) sowie eine kombinierte Diskurs- und Wertbaumanalyse (Frommeld). Darüber hinaus wurden die Ergebnisse einer systematischen Literaturanalyse (Vetter/Cerullo) als Beitrag in den Sammelband aufgenommen.

Den Anfang markieren Miriam Vetter und Laura Cerullo, die in ihrem Beitrag »Die tatsächliche Nutzung digitaler Assistenzsysteme in der Altenpflege. Ein Scoping Review « mithilfe der Auswertung zahlreicher wissenschaftlicher Quellen eruieren, inwieweit altersgerechte Assistenzsysteme bereits in die Praxis diffundiert sind. Ihre Vorgehensweise beschreiben die Autorinnen selbst: »Im Rahmen des Reviews wird zunächst ein Überblick zum Themenfeld digitaler Pflegetechnik gegeben. Anschließend werden mögliche Typisierungen der Technik präsentiert. Dem folgt die Darstellung des methodischen Vorgehens und die Präsentation der Ergebnisse, die sich vor allem auf die Verbreitung des Technikeinsatzes in der ambulanten und stationären Altenpflege konzentrieren. "Mit dem ersten Schritt sprechen sie eine nicht zu unterschätzende Problematik an - es gibt keine allgemein akzeptierte Definition altersgerechter Assistenzsysteme. Ungeachtet dessen lassen sich die Kernaussagen des Textes so zusammenfassen: Alle berücksichtigten Studien verwenden jeweils eine eigene Kategorisierung und betrachten unterschiedliche Technologien, die quantitativen Aussagen zur Nutzung der Technik variieren sehr stark, »die elektronische Pflegedokumentation und/oder assistive Technologien wie Hebehilfen [werden; Erg. KW] trotz unterschiedlicher Kennzahlen aktuell am häufigsten in der Altenpflege eingesetzt« und dass die vorliegenden Studien selbst nur selektiv auf Pflegetechnik schauen. Es fehlt, kurzum, an belastbarem Wissen, was angesichts der großen Summen, die beispielsweise durch die öffentliche Hand in die Forschung an und die Entwicklung von altersgerechten Assistenzsystemen investiert wurden, bedenklich stimmen muss. Denn ohne belastbare Zahlen können weder Bedarfe der Praxis noch (Miss-)Erfolge der Förderung verlässlich bestimmt werden.

Der quantitative Teil des Projekts DAAS-KIN wird in dem Beitrag »Nutzung, Planung und Bewertung digitaler Assistenzsysteme in der Pflege. Ergebnisse einer Befragung von Führungskräften in ambulanten und stationären Einrichtungen« von Sonja Haug vorgestellt. Ausgangspunkt dieser Teilstudie ist die Frage in dem weiter oben aufgeführten Zitat von Schmidt/Wahl (2019) - welche Technik ist 
welchem Maße denn nun in der Praxis tatsächlich angekommen? Existierende Potenzialstudien können diese Frage aus zwei Gründen nicht beantworten: Erstens stellen sie Abschätzungen einer möglichen Zukunft dar und zweitens wurden die vorliegenden Studien schon vor geraumer Zeit verfasst; sie können also selbst in Bezug auf das Potenzial nur Aussagen über eine nicht mehr wirklich relevante Vergangenheit enthalten. Den Ergebnissen, die Haug in ihrem Beitrag vorstellt, soll an dieser Stelle zwar nicht vorgegriffen werden, doch so viel sei hier schon gesagt, weil es bereits mehrfach angemerkt wurde: Im Unterschied $\mathrm{zu}$ anderen digitalen Assistenzsystemen haben insbesondere robotische Systeme bislang praktisch keine Verbreitung in der Pflege gefunden und ihr Einsatz wird ausgesprochen skeptisch bewertet. Man kann daraus unterschiedliche und möglicherweise auch nicht miteinander vereinbare Schlussfolgerungen ziehen. Die Projektbeteiligten von DAASKIN tendieren zu der Empfehlung, die öffentliche Förderung der Forschung an und Entwicklung von Robotern für die Pflege auf den Prüfstand zu stellen - auch aus der Erfahrung heraus, dass kommerziell bereits erhältliche Systeme teilweise mehr leisten können als Roboter, die in Deutschland seit vielen Jahren entwickelt werden, aber bisher nicht zur Marktreife gebracht werden konnten. Im Rahmen des ITAForums 2020, bei dem Weber den Zwischenbericht für DAAS-KIN vorstellte, wurde von einem Zuhörer jedoch die genau umgekehrte Schlussfolgerung gezogen - es müsse mehr Forschung und Entwicklung betrieben werden, um bessere Roboter zu bekommen, die dann auch auf mehr Akzeptanz stoßen würden. Tatsächlich zeigt sich hier eine Konfliktlinie, die weit über altersgerechte Assistenzsysteme hinausweist, denn die am Markt erhältlichen Roboter werden (vermutlich ohne Ausnahme) außerhalb Deutschlands und Europas entwickelt und hergestellt - damit gehen potenziell weitreichende Abhängigkeiten einher. Zudem hat eine Evaluation im Rahmen eines anderen Projekts gezeigt, dass die überwiegende Zahl der betrachteten robotischen Systeme (in diesem Fall sogenannte >Telepräsenzroboter`) nicht datenschutzkonform betrieben werden könnten. Hier werden also industriepolitische wie rechtliche Fragen aufgeworfen, deren Antworten zumindest potenziell weitere Diffusionshemmnisse (auch) für altersgerechte Assistenzsysteme mit sich bringen könnten (vgl. Popp/Middel/Raptis 2020).

Ulrike Scorna stellt mit ihrem Beitrag »Digitale Technik in der ambulanten und stationären Pflege. Eine Interviewstudie zum Einfluss des Pflegepersonals auf die Einführung digitaler Assistenzsysteme« den qualitativen Teil des Projekts DAASKIN vor. Ziel der durchgeführten Teilstudie mit leitfadengestützten Interviews war, die Ergebnisse der quantitativen Studie durch >Triangulation $<$ zu validieren. Es wurden insgesamt zwölf Interviews "mit Vertreter*innen der ambulanten und stationären Pflegeeinrichtungen (Führungskräfte und Pflegepersonal) und [...] mit Vertreter*innen aus der Forschung und Wissenschaft « geführt, um deren spezifische Perspektive auf die Nutzung altersgerechter Assistenzsysteme zu eruieren und auf diese Weise weitere Rückschlüsse auf Diffusionshemmnisse für 
diese Technik zu gewinnen. Auch hier soll der Vorstellung der Ergebnisse nicht vorgegriffen werden, zumal vieles, was gerade in Bezug auf die quantitative Studie formuliert wurde, hier wiederholt werden könnte. Vielleicht nur dieses: Insbesondere die Vertreter*innen der ambulanten und stationären Pflegeeinrichtungen hoben sehr deutlich auf die Bedeutung des Verhältnisses zwischen pflegender und gepflegter Person ab-Technik, die dieses Verhältnis berührt, wird mit Vorsicht betrachtet. Auch wenn die Befragten dies nicht mit den Begriffen formulierten, die Hartmut Remmers nutzt, verwiesen sie damit auf die praktische Relevanz der stherapeutisch-rehabilitativen Dyade`. Aber es ist auch zu betonen, dass die Verallgemeinerung, die Remmers trifft, in dieser Form nicht von den von uns befragten Personen geteilt wird; mit Ausnahme von Robotern stoßen altersgerechte Assistenzsysteme auf großes Interesse und deren Nutzen wird durchaus gesehen. Allerdings weisen die Befragten aus der Praxis auch sehr deutlich auf die Defizite der Technik hin - alle Stakeholder*innen, die im Bereich der Gestaltung solcher Systeme arbeiten, sollten diese Aussagen sorgfältig lesen. Eines allerdings muss ernüchtern (Domhoff et al. nennen diesen Punkt ebenfalls): Die Nutzung altersgerechter Assistenzsysteme scheitert offenbar nicht selten schon am Fehlen grundlegender Voraussetzungen wie der Verfügbarkeit eines WLANs. Angesichts des Selbstverständnisses Deutschlands als >Hightech-Standort<, des gesellschaftlichen Reichtums in Deutschland und der Dringlichkeit des Pflegenotstands stellt diese Aussage ein Armutszeugnis dar.

Obwohl Diskursanalysen durchaus als qualitative Methoden gezählt werden, soll hier die Bezeichnung als interpretative Methode genutzt werden, um den Unterschied zu den beiden vorhergehenden Texten und den dafür benutzten Methoden zu markieren - dort wurden empirische Daten erhoben. Für ihren Beitrag »Vertrauen, Wissen, Innovation und Wohltun als (neue) Herausforderungen im Kontext digitaler Assistenzsysteme. Ergebnisse einer Diskurs- und Wertbaumanalyse« hat Debora Frommeld einen diskursanalytischen Zugang gewählt, um im Zuge dieses interpretativen Verfahrens einen Wertbaum zu erstellen. Dazu wurde eine große Zahl wissenschaftlicher Veröffentlichungen zum Thema der altersgerechten Assistenzsysteme untersucht und aus einer Auswahl dieser Texte die dort präsentierten zentralen Normen und Werte extrahiert. Ziel dieser dritten Teilstudie in DAAS-KIN war zu überprüfen, ob es wiederkehrende Muster von Werten und Normen in der einschlägigen Fachliteratur gibt, die - sofern sie nicht angemessen adressiert werden - Hemmnisse bei der Diffusion altersgerechter Assistenzsysteme darstellen können. Da die beiden anderen empirischen Teilstudien eine besonders ausgeprägte Skepsis bezüglich von Robotern in der Pflege ausweisen, hat Debora Frommeld bei ihrer Analyse einen Schwerpunkt auf s Socially Assistive Robots gelegt, die in der Pflege zum Einsatz kommen sollen. Ein weiteres Ziel dieser interpretativen Teilstudie ist die Weiterentwicklung des ethischen Evaluierungswerkezeugs MEESTAR (Weber 2015). Das Modell zur ethischen Evaluation 
sozio-technischer Arrangements wird seit Jahren dazu verwendet, insbesondere altersgerechte Assistenzsysteme während der Entwicklung oder vor deren Implementierung durch möglichst alle beteiligten Stakeholder*innen aus normativer Sicht $\mathrm{zu}$ bewerten. Obwohl sich das Werkzeug hierfür durchaus bewährt hat, enthält es einige Schwächen, die mit den verwendeten Bewertungsdimensionen verbunden sind. Mit der Erstellung eines Wertbaums mithilfe der Diskursanalyse sollten die bestehenden Bewertungsdimensionen in MEESTAR ergänzt beziehungsweise überarbeitet werden. Auch in diesem Fall soll der Darstellung der Ergebnisse hier nicht vorgegriffen werden, doch ein prägnanter Punkt kann bereits jetzt genannt werden: Es zeigt sich, dass Vertrauen beziehungsweise dessen Abwesenheit ein entscheidendes Hemmnis für die Diffusion altersgerechter Assistenzsysteme darstellen kann. Vertrauen liegt vor, wenn bereits bekannte und neu gefundene Dimensionen in MEESTAR positiv bewertet werden. Auch dieses Ergebnis legt nahe, dass für den Einsatz altersgerechter Assistenzsysteme auf der sozialen und womöglich emotionalen Ebene geworben werden muss - allerdings nicht im Sinne einer Produktwerbung, sondern durch eine vertrauenswürdige Gestaltung von Technik.

\section{Implementierung und Evaluation von (digitaler) Pflegetechnik}

Im vierten und letzten Abschnitt des Sammelbands werden Texte präsentiert, die entweder aufzeigen, wie altersgerechte Assistenzsysteme ganz konkret eingesetzt werden können (Altenbuchner und Cerullo), oder eine Bewertung dieser Technologie aus einer gesellschaftlichen und gesundheitsökonomischen Perspektive vornehmen (Hergesell et al., Mähs und Zerth).

Wie in vielen der vorliegenden Beiträge, aber auch in dieser Einführung schon angesprochen wurde, umfassen altersgerechte Assistenzsysteme ein sehr breites Spektrum von Geräten und Anwendungen. Eine innovative Verwendung handelsüblicher Fitnesstracker als therapeutisches beziehungsweise rehabilitatives Assistenzsystem stellt Amelie Altenbuchner in ihrem Bericht über die »Möglichkeiten des Bewegungsmonitorings durch Fitnesstracker nach einer Hüftfraktur« vor. Diese Geräte sind vergleichsweise kostengünstig und leicht zu nutzen. Sie ermöglichen eine sehr viel genauere und kontinuierlichere Erhebung des Bewegungsumfangs der Nutzer*innen, was für die passgenaue Definition therapeutischer beziehungsweise rehabilitativer Maßnahmen genutzt werden kann. In einem umfangreichen Feldversuch hat Altenbuchner das Potenzial der Verwendung von Fitnesstrackern für das Bewegungsmonitoring bei geriatrischen Traumapatient"innen untersucht. In ihrem Text beschreibt sie in erster Linie die Vorgehensweise sowie die Auswahlkriterien für die verwendeten Geräte und trifft erste Aussagen über deren Nutzen. Sie kann dabei eindrücklich zeigen, dass Technik stets als soziotechnisches System verstanden werden muss, denn entscheidend für die sinnvolle Ver- 
wendung der Fitnesstracker ist die Berücksichtigung der Einsatzbedingungen und der Eigenschaften der Nutzer*innengruppe. >One size fits alk funktioniert hierbei nicht - Fitnesstracker für geriatrische Traumapatient*innen müssen Kriterien erfüllen, die für andere Nutzer*innengruppen möglicherweise irrelevant sind. Aus Hersteller*innensicht eröffnet sich mit dieser Feststellung ein erhebliches Dilemma, denn erst große Stückzahlen erlauben günstige Preise, die am Markt angenommen werden, doch große Stückzahlen bedeuten eben auch, dass keine nutzer*innenspezifische Gestaltung der Geräte vorgenommen wird. Dieses Dilemma ist beispielsweise durch >Universal Design « und Anpassbarkeit der Geräte an die Bedürfnisse der jeweiligen Nutzer*innengruppe lösbar, doch bedeutet dies Aufwand und erfordert den Willen zu einem sguten $<$ Design. Anders formuliert: Gute Technik umfasst mehr als bloße Technik.

Diese Einsicht wird durch den Beitrag »Digitalisierte Bewegungsentwicklung geriatrischer Traumapatient*innen. Konzept und Evaluation eines Bewegungsfeedbacks« von Laura Cerullo noch einmal ausdrücklich hervorgehoben. Cerullo hat an dem eben beschriebenen Feldversuch mitgewirkt, die Proband*innen betreut und ihnen ein individuelles Bewegungsfeedback gegeben. Im Rahmen einer Begleitstudie hat sie die existierende wissenschaftliche Literatur zu dieser spezifischen Form der Begleitung und die Reaktionen der Proband*innen untersucht und dokumentiert. Der Darstellung der gewonnenen Ergebnisse soll hier nicht vorgegriffen werden, doch auch an diesem Text wird deutlich, dass Technik-zumal altersgerechte Assistenzsysteme - stets in einen sozialen und auch emotionalen Kontext eingebettet ist. Im Grunde muss man diese Aussage sogar verschärfen und formulieren, dass Technik nur als Bestandteil eines solchen Kontexts zu verstehen ist. Viele Erkenntnisse, die in den vorliegenden Beiträgen präsentiert werden, aber auch in der einschlägigen Literatur zu finden sind, legen den Schluss nahe, dass diese Einsicht in vielen Forschungs- und Entwicklungsprojekten für altersgerechte Assistenzsysteme nicht in einem ausreichenden Maße bedacht wurde. Das mag auch daran liegen, dass ELSA-Begleitforschung, das heißt die Betrachtung ethischer, rechtlicher und sozialer Aspekte, oftmals noch immer als zusätzliche und nicht als integrale Aktivität in entsprechenden Projekten verstanden wird.

Damit steht das Stichwort >Partizipation bereits im (diskursiven) Raum; Jannis Hergesell, Arne Maibaum, Andreas Bischof und Benjamin Lipp nehmen es in ihrem gemeinsamen Beitrag »Zum Potenzial grundlagenwissenschaftlicher Technikforschung für ein sgutes Leben im Alter . Ein Plädoyer für konsequente partizipative Technikgestaltung « auf. Zunächst rekonstruieren sie auf EU-Ebene die Genese der förderpolitischen Entscheidungen zur Unterstützung von Forschung an und Entwicklung von altersgerechten Assistenzsystemen. Damit wird das, was bei Weber nur skizziert wird, noch sehr viel deutlicher herausgearbeitet: Die Arbeit an altersgerechten Assistenzsystemen ist eingebettet in sehr unterschiedliche Kontexte mit 
entsprechenden Eigenlogiken. Es finden sich industrie- und innovationspolitische Überlegungen ebenso wie bevölkerungs- und gesundheitspolitische Erwägungen. Damit wird einmal mehr sichtbar, dass das `So-Sein`von Technik durch zahlreiche Faktoren bestimmt wird, die in der Regel mit Technik im engeren Sinne gar nichts zu tun haben - wir betrachten also nie Technik allein, sondern stets soziotechnische Systeme (oder Arrangements, wie es in MEESTAR heißt). Grundsätzlich ist diese Situation auch nicht notwendigerweise negativ, da man argumentieren kann, dass es eben viele Stakeholder*innen gibt, die eine Stimme bei der Forschung an und Entwicklung von Technik im Allgemeinen und altersgerechten Assistenzsystemen im Speziellen haben sollten. Problematisch wird es jedoch, wenn Stakeholder*innen, die am unmittelbarsten vom Einsatz der Technik betroffen sind, gerade keine Stimme haben: die Gepflegten und die Pflegenden. Hieraus leiten Hergesell et al. ein vehementes Plädoyer für eine strikt partizipativ ausgerichtete Technikentwicklung $\mathrm{ab}$ (die, darauf sollte man hinweisen, ihre ganz eigenen Herausforderungen mit sich bringt), bei der alle Stakeholder*innen von Beginn der Entwicklung an eine Stimme haben, die auch gehört wird - diese Forderung kann ELSABegleitforschung nach wie vor nicht immer erfüllen, weil sie, wie schon mehrfach formuliert, eben nicht immer als integraler Bestandteil der Technikentwicklung begriffen wird. Allerdings muss deutlich betont werden, dass sich in dieser Hinsicht in den letzten zehn Jahren sehr viel zum Besseren hin geändert hat, was sich unter anderem an den Förderbedingungen (nicht nur) des BMBF ablesen lässt. Außerdem haben Partizipation, Folgenabschätzung und Vorausschau in den letzten Jahren gerade im BMBF an Bedeutung gewonnen.

Nicht erst seit Goethe wissen wir: »Nach Golde drängt, am Golde hängt doch alles.«Zwar bezahlen wir heute nicht mehr mit Golddukaten, aber das Prinzip bleibt gleich: Mit der Verfügbarkeit finanzieller Ressourcen entscheidet sich in der Pflege, wie in allen anderen Lebensbereichen auch, was getan werden kann und was nicht. Diese Ressourcen sind grundsätzlich begrenzt; ihr Umfang konkurriert mit den Ausgaben für andere gesellschaftlich relevante Aufgaben. Da die Akteur*innen der Pflege in einem marktwirtschaftlichen Umfeld operieren und zudem teilweise privatwirtschaftlich organisiert sind, aber in jedem Fall wirtschaftlich agieren müssen, ist es unvermeidbar, dass altersgerechte Assistenzsysteme unter (gesundheits-)ökonomischen Gesichtspunkten betrachtet werden müssen. Mareike Mähs zeigt nun in ihrem Beitrag über die "Anforderungen an die Evaluation von altersgerechten Assistenztechnologien aus gesundheitsökonomischer Sicht« auf, dass eine solche Betrachtungsweise alles andere als eine leichte Übung darstellt. Denn Kosten und Nutzen solcher Systeme hängen von zahlreichen voneinander abhängigen und aufeinander wirkenden Faktoren ab, wobei diese Wechselwirkungen nicht immer klar erkennbar beziehungsweise feststellbar sind: Setzt man beispielsweise ein bestimmtes System ein, so kann man womöglich dessen Kosten und Nutzen in der spezifischen Einsatzsituation erheben, aber ob diese höher oder niedriger ausfal- 
len als Kosten und Nutzen der Alternativen, lässt sich in aller Regel nur plausibel abschätzen, aber nicht sicher sagen. Altersgerechte Assistenzsysteme bestehen zudem meist nicht nur aus einem Gerät oder aus Geräten des gleichen Typs, sondern sind eingebettet in ein technisches `Ökosystem` mit möglicherweise großer Komplexität - dies kann eine klare Kosten- und Nutzenzuordnung erheblich erschweren. Nicht zuletzt entscheidet der gewählte Zeithorizont in nicht unerheblichem Maße mit darüber, welche Ergebnisse gesundheitsökonomische Evaluationen zeitigen; allerdings nimmt mit einem weiten Zeithorizont die Genauigkeit von Abschätzungen eher $a b$. Auch wenn die von uns Befragten (siehe die Beiträge von Haug und Scorna) es anders formuliert haben: Hier verbergen sich massive Diffusionshemmnisse, denn aus Sicht der Pflegedienstleister*innen ist der Einsatz altersgerechter Assistenzsysteme zu oft mit einem zu großen und schwer kalkulierbaren wirtschaftlichen Risiko verbunden (ähnliche Ergebnisse haben Vetter/Cerullo durch ihr Scoping Review herausgearbeitet).

Jürgen Zerth untersucht den Einsatz von Technik in der Pflege noch detaillierter. In seinem Beitrag zur »Technikimplementierung in der Pflege: die Bedeutung der soziotechnischen Innovationsbewertung aus gesundheitsökonomischer Sicht « arbeitet Zerth heraus, dass Kosten und Nutzen zwar letztlich in monetären Größen berechnet werden müssen, dass dabei aber verbrauchte oder gewonnene Ressourcen auch in nicht monetärer Gestalt auftreten können - beispielsweise in Form qualitativ hochwertigerer Informationen über den physischen Zustand einer gepflegten Person, die es erlauben passgenauere pflegerische Maßnahmen zu ergreifen und somit zum Beispiel die Ressource Arbeitszeit oder auch Materialeinsatz effizienter bewirtschaften zu können. Doch wie Zerth aufzeigt, sind Überlegungen und Schritte hin zu Effizienzgewinnen noch weitaus komplexer, denn über den Erfolg oder Misserfolg der Implementierung von Technik und deren richtiger Auswahl entscheiden unter anderem Bedingungen, die aus Sicht der Auswahl und Implementierung in der Vergangenheit liegen; dies betrifft insbesondere die Adoptionsneigung der Pflegekräfte als Ausdruck von deren Technikbereitschaft und deren Erwartungen an die Technik. Die im Rahmen von DAAS-KIN befragten Personen (siehe Beitrag Scorna) äußerten sich, wenn auch mit anderen Worten, in ähnlicher Weise; es wurde mehrfach darauf hingewiesen, dass sich die Pflegenden nicht in der Lage sehen, altersgerechte Assistenzsysteme adäquat zu nutzen, und gleichzeitig sind ihre Erwartungen oftmals enttäuscht worden; Konsequenz ist dann meist die Nichtnutzung teurer Technik (ähnliche Ergebnisse finden sich bei Frommeld und bei Vetter/Cerullo). Wollen Pflegedienstleistungsunternehmen also altersgerechte Assistenzsysteme einführen, dann - so kann man einerseits Zerth und andererseits empirische Erkenntnisse interpretieren - steht die Implementierung der Technik nicht am Anfang, sondern stellt einen der letzten Schritte eines langen Weges dar. Bedenkt man, dass die in DAAS-KIN durchgeführte Umfrage (siehe Beitrag Haug) zeigt, dass die Mehrzahl der Pflegedienstleistungsunterneh- 
men keine Digitalisierungsstrategie erstellt haben, besteht in Hinblick auf eine strategische Planung noch sehr viel Aufklärungsbedarf.

\section{Schlussbemerkungen}

Es lohnt sich, die hier nur skizzenhaft vorgestellten Beiträge des Sammelbands im Detail zu lesen. Das gilt nach unserer Ansicht sowohl für Stakeholder*innen aus dem Bereich der Technik und Technikentwicklung wie aus dem Bereich der Pflege - seien es Pflegekräfte, Personen mit Leitungsaufgaben oder jene mit Verwaltungs- und/oder Budgetverantwortung. Es gilt aber auch für Stakeholder*innen der Politik und zumal der Förderinstitutionen, denn wir denken, dass wir hier Erkenntnisse präsentieren, die Anlass zum Überdenken von Förderstrategien geben.

Wie bei jedem anderen Projekt bleibt auch in DAAS-KIN eine Leerstelle: Wir haben die Stakeholder*innengruppe der gepflegten Personen und auch der Angehörigen nicht gleichberechtigt einbeziehen können. Tatsächlich gehörte dies auch nicht zu unserem Auftrag, doch wird deutlich erkennbar, dass diese Leerstelle gefüllt werden sollte, wenn man beispielsweise Partizipation wirklich ernstnehmen möchte. Allerdings dürfen die methodischen Herausforderungen, die damit einhergehen, insbesondere in Bezug auf die gepflegten Personen, nicht unterschätzt werden; zu nennen sind hierbei unter anderem der zeitliche Aufwand und die moralischen Barrieren der Beforschung vulnerabler Personen wie beispielsweise dementer Menschen. Es bleibt zu hoffen, dass diese Herausforderungen an anderer Stelle aufgenommen werden.

Wir haben diesem einleitenden Text den Titel »Gute Technik für ein gutes Leben?! « gegeben und dabei ganz bewusst ein Frage- und ein Ausrufezeichen verwendet. Entsprechend gestaltete altersgerechte Assistenzsysteme können einen Beitrag dazu leisten, dass Menschen aller Altersgruppen, die pflegebedürftig sind, ein >gutes Leben führen können - deshalb das Ausrufezeichen. Die Beiträge des vorliegenden Sammelbands zeigen allerdings gleichzeitig auf, dass die Gestaltung sguter Technik eine erhebliche Herausforderung darstellt, die bisher beileibe nicht immer gemeistert wurde - und daher das Fragezeichen.

In den folgenden Beiträgen kommen Wissenschaftler*innen $\mathrm{zu}$ Wort, die skeptische wie positive Haltungen in Hinblick auf die Nutzung altersgerechter Assistenzsysteme einnehmen. Ohne die unterschiedlichen Bewertungen nivellieren oder ignorieren $\mathrm{zu}$ wollen, kann doch formuliert werden, dass es allen Beitragenden darum geht, die Interessen und berechtigten Ansprüche der beteiligten Stakeholder*innen zur Geltung zu bringen. Damit werden sie zwei Prinzipien gerecht, die zwar meist im Zusammenhang mit medizinethischen Überlegungen geäußert werden, aber insbesondere in der Pflege ebenso Geltung besitzen und 
im Grunde jedes (professionelle) Handeln leiten sollten: das Prinzip des Wohltuns und des Nichtschadens. Nur wenn offensichtlich ist, dass die Gestaltung und der Einsatz altersgerechter Assistenzsysteme diesen Prinzipien gerecht werden, kann Vertrauen in die Technik als Grundlage der Akzeptanz der Technik entstehen.

Wer mehr Technik in der Pflege einsetzen will, muss dafür mit guten Argumenten werben; wer mehr Technik in der Pflege einsetzen will, muss insbesondere ELSA-Begleitung nicht nur als >Sideshow < begreifen, sondern als integralen und der technischen Entwicklung gleichwertigen Teil der Gestaltung und Implementierung der Technik; wer mehr Technik in der Pflege einsetzen will, muss nicht zuletzt die Gestaltung und Implementierung altersgerechter Assistenzsysteme als Bottom-Up-Prozess organisieren; es muss eine Abkehr vom >Technology Push $\prec$ hin zum >Demand Pulk geben. Diese Forderungen sind alles andere als originell, denn sie werden schon seit geraumer Zeit immer wieder erhoben. Zweifelsohne hat sich in den letzten Jahren in dieser Hinsicht vieles zum Besseren entwickelt, aber weitere Verbesserungen sind nicht nur möglich, sondern nach wie vor auch dringend notwendig.

\section{Danksagung}

Der vorliegende Sammelband entstand im Rahmen des durch das Bundesministerium für Bildung und Forschung (BMBF) geförderten Projekts »Diffusion altersgerechter Assistenzsysteme - Kennzahlenerhebung und Identifikation von Nutzungshemmnissen « (DAAS-KIN); die Herausgeber*innen möchten dem Ministerium und allen Verantwortlichen für deren Unterstützung danken. Die Beiträge von Karsten Weber, Sonja Haug, Debora Frommeld, Ulrike Scorna, Laura Cerullo und Miriam Vetter sind in diesem Rahmen entstanden.

Projekte wie dieses funktionieren nur, weil es zahlreiche Personen gibt, die ungeachtet der Frage, ob sie eine offizielle Rolle dabei spielen, Hilfe und Unterstützung leisten - dies gilt natürlich auch für DAAS-KIN. Als Herausgeber*innen des Sammelbands möchten wir daher folgenden Personen ganz herzlich danken (in alphabetischer Reihenfolge): Laura Cerullos Beitrag bei der editorischen Bearbeitung der Beiträge und des ganzen Sammelbands ist kaum zu überschätzen; Edda Currle hat drei Aufsätze Korrektur gelesen und darüber hinaus weitere sehr hilfreiche inhaltliche Hinweise gegeben; Monika Friedl hat ebenfalls zentrale Editions- und Rechercheaufgaben übernommen; Nicla Kaufner hat wichtige Rechercheaufgaben für die Beiträge von Debora Frommeld und Karsten Weber erledigt sowie bei der Gestaltung von Abbildungen in Debora Frommelds Text geholfen; Miriam Vetter hat die Erstellung von Informationen für die Autor*innen des Sammelbands erledigt.

In unserer Eigenschaft als projektdurchführende Personen möchten wir uns bei Sebastian Belda, noch einmal Monika Friedl und Vanessa Mücke für die unver- 
zichtbare Unterstützung bedanken. Der DAAS-KIN-Workshop, der zu Beginn des Jahres 2020 in den Räumen des Regensburg Centers of Health Sciences and Technology (RCHST) stattfand und die Basis des vorliegenden Sammelbands darstellt, wäre ohne die Hilfe Simone Böttgers, Alexander Leis' und Annabell Mehnes nicht so reibungslos verlaufen (Monika Friedl und Miriam Vetter haben hier ebenfalls ihren Beitrag geleistet). Ihnen gilt ebenso unser ausdrücklicher Dank.

Das schon genannte RCHST hat die Durchführung der Tagung zudem finanziell und organisatorisch unterstützt; die vielen Mitarbeiter*innen in der Verwaltung der Ostbayerischen Technischen Hochschule Regensburg ermöglichen durch ihre Arbeit nicht nur DAAS-KIN, sondern viele andere Forschungs- und Entwicklungsprojekte. Ihnen allen schulden wir ein großes »Dankeschön«.

Schließlich gilt, dass ein empirisch ausgerichtetes Projekt wie DAAS-KIN damit steht und fällt, ob es Menschen gibt, die bereit sind an der Forschung teilzunehmen. Wir möchten uns bei allen Personen, die an der Online-Umfrage teilnahmen oder die uns als Befragte in den Interviews zur Verfügung standen, für deren Hilfe und die gewährten Einsichten bedanken.

Wir widmen diesen Band all jenen Personen und Institutionen, die jeden Tag unter teilweise sehr widrigen Umständen dazu beitragen, dass pflegebedürftige Menschen ein gutes Leben führen können.

Regensburg, Februar 2021

\section{Literatur}

Popp, Christof/Middel, Luise/Raptis, Georgios (2020): »Arbeitspapier: Auswahlverfahren für Telepräsenzroboter für die Unterstützung von Schlaganfallpatient"innen, Version 1«, Regensburg. https://www.deinhaus40.de/wp-content/u ploads/2021/01/Marktanalyse_Paper-v1.pdf (Abfrage: 24.02.2021).

Schmidt, Laura/Wahl, Hans Werner (2019): »Alter und Technik«, in: Hank, Karsten/Schulz-Nieswandt, Frank/Wagner, Michael/Zank, Susanne (Hg.), Alternsforschung. Handbuch für Wissenschaft und Praxis, Baden-Baden: Nomos, S. 537-555.

ULD (2010): "Juristische Fragen im Bereich altersgerechter Assistenzsysteme«, Kiel. https://www.datenschutzzentrum.de/uploads/projekte/aal/2011-ULD-Jur istischeFragenAltersgerechteAssistenzsysteme.pdf (Abfrage: 24.02.2021).

Weber, Karsten (2015): »MEESTAR: Ein Modell zur ethischen Evaluierung soziotechnischer Arrangements in der Pflege- und Gesundheitsversorgung «, in: Weber, Karsten/Frommeld, Debora/Manzeschke, Arne/Fangerau, Heiner (Hg.), Technisierung des Alltags - Beitrag für ein gutes Leben?, Stuttgart: Steiner, S. 247-262. 


\section{Altersgerechte Assistenzsysteme: Ein Überblick}

Karsten Weber

\section{Einleitung}

Das Jahr 2020 wird vielen Menschen wohl dauerhaft im Gedächtnis bleiben; COVID-19 hat auf individueller wie gesellschaftlicher Ebene tiefe Spuren im Alltag hinterlassen: Schule, Ausbildung, Beruf - kein Lebensbereich ist von den Auswirkungen der Pandemie verschont geblieben. Die traurige Bilanz zu Beginn des Jahres 2021 (Stand 13.01.2021) in Deutschland beläuft sich auf 1.964 .533 infizierte Personen und 43.559 Tote. COVID-19 hat weltweit nicht nur großes Leid mit sich gebracht, sondern gerade in vielen industrialisierten Ländern erhebliche Schwächen des jeweiligen Gesundheitssystems offengelegt. Nicht nur in Deutschland droht die intensivmedizinische Versorgung der Schwersterkrankten zu kollabieren, die (Über-)Belastung in den Krankenhäusern zwingt dazu, alle nicht unmittelbar notwendigen Operationen $\mathrm{zu}$ verschieben - das deutsche Gesundheitssystem scheint am Abgrund zu stehen. Ähnliches kann für die Pflege älterer und hochbetagter Menschen formuliert werden (vgl. Bauer/Eglseer/Hödl 2020; Bohlken et al. 2020; Hower/Pfaff/Pförtner 2020; Wolf-Ostermann et al. 2020): Um diese durch das Corona-Virus besonders gefährdete Personengruppe zu schützen, werden die Bewohner*innen von Alters- und Pflegeheimen isoliert und Besuchsverbote verhängt; trotzdem scheinen sich solche Einrichtungen $\mathrm{zu}$ Hotspots der Ansteckung zu entwickeln. Das Personal sowohl in Krankenhäusern wie in Pflegeeinrichtungen berichtet von extremen Belastungen, obwohl bereits vor Ausbruch der Pandemie die Situation für diese Berufsgruppe alles andere als rosig beschrieben wurde (vgl. Lübbers 2017; Sahmel 2018; außerdem Domhoff et al. in diesem Band).

So oder so ähnlich kann man die Berichte der letzten Monate, die in den Massenmedien erschienen, zusammenfassen. Folgt man diesen Berichten, steht das bundesdeutsche Gesundheitssystem - und die Pflege wird hierbei mitgedacht - vor der Implosion. Es wird nach mehr Geld, vor allem aber nach mehr Personal gerufen. COVID-19 vergrößert den Pflegenotstand beziehungsweise lässt diesen noch deutlicher sichtbar werden. Doch selbst wenn die notwendigen finanziellen Ressourcen zur Verfügung stünden, scheint die Gewinnung zusätzlichen 
Personals schwierig bis unmöglich. Denn warum sollte unter diesen erschwerten Bedingungen gelingen, was vor der Pandemie schon nicht gelang?

Dieses ungelöste Problem wird zu den Dingen gehören, die insbesondere jene im Gedächtnis behalten werden, die im Gesundheits- und Pflegebereich arbeiten und daher unmittelbar von der Pandemie betroffen sind. Für jene, die sich mit der Ressourcenausstattung dieses Bereichs beschäftigen - beispielsweise aus Perspektive der Pflegewissenschaft, der Versorgungsforschung oder der Technikfolgenabschätzung - stellen sich jedoch noch weitere Fragen.

Eine dieser Fragen soll im Folgenden behandelt werden - beantwortet werden kann sie aber vermutlich nicht endgültig: Welchen Stellenwert nehmen technische Ansätze zur Unterstützung der Pflege in dieser Diskussion ein? Im Grunde ist der gesamte vorliegende Sammelband der Beantwortung dieser Frage gewidmet; im vorliegenden Text soll in erster Linie auf einige Rahmenbedingungen der Entwicklung und des Einsatzes altersgerechter Assistenzsysteme sowie auf die Förderpolitik insbesondere des Bundesministeriums für Bildung und Forschung (BMBF) eingegangen werden, soweit diese öffentlich zugänglich ist.

\section{Wie wichtig sind altersgerechte Assistenzsysteme?}

Die Relevanz eines Themas lässt sich ohne Zweifel auf sehr unterschiedliche Weise messen, belegen oder auch nur behaupten. Gesellschaftliche Diskurse oder politische Debatten gehorchen dabei sicher anderen Regeln, als dies für die Wissenschaft gilt. Für die Wissenschaft gibt es im Kontext der Szientometrie und Bibliometrie quantitative Indikatoren für Relevanz, die zwar selbst im Detail und in Bezug auf ihre Aussagekraft beispielsweise bezüglich der Qualität wissenschaftlicher Arbeiten umstritten sind, aber im Grundsatz durchaus Orientierung geben können. Für die Relevanz gesellschaftlicher Diskurse und/oder politischer Debatten lassen sich sicher auch quantitative Indikatoren angeben, beispielsweise die Zahl der SocialMedia-Follower politischer Akteur*innen oder die Zahl von Leitartikeln in Zeitungen oder Nachrichtensendungen zu einem Thema.

Schwieriger wird es, wenn ein Thema nicht nur zu einer gesellschaftlichen Domäne gehört, sondern gleich in mehreren verortet ist. In solchen Fällen gibt es womöglich eine Konkurrenz der Indikatoren für die Relevanz eines Themas, die sich unter Umständen wechselseitig stärken, aber auch widersprechen können. Für das in diesem Text und in diesem Sammelband behandelte Thema lässt sich zumindest so viel feststellen: Altersgerechte Assistenzsysteme sind verbunden mit gesellschaftlichen Diskursen, politischen Debatten und gesundheitsökonomischen Überlegungen - und möglicherweise weiteren relevanten Handlungsdomänen -, aber sie spielen dort sehr unterschiedliche Rollen, ihre Relevanz ist je verschieden und die Kriterien der Relevanz lassen sich oft nur schwer in Übereinstimmung 
bringen. Dies genau zu untersuchen wäre allerdings die Aufgabe für eine anders angelegte Studie.

Je mehr und je länger man sich mit altersgerechten Assistenzsystemen beschäftigt - zumindest geht es dem hier Schreibenden so - desto unklarer und unschärfer scheint das Thema zu werden; das betrifft die Relevanz des Themas in unterschiedlichen gesellschaftlichen Domänen, aber auch dessen Inhalt. Das beginnt schon damit, dass es keine wirklich gute und allgemein akzeptierte Definition für altersgerechte Assistenzsysteme gibt. Der Ausdruck selbst ist nicht unumstritten: Als in Deutschland das Thema förderpolitisch entdeckt wurde, nutzte man zunächst den Anglizismus Ambient Assisted Living - eine wörtliche Übersetzung ins Deutsche will nicht so recht gelingen: >umgebungsunterstütztes Leben beziehungsweise >umgebungsunterstütztes Wohnen oder einfach >betreutes Wohnen bieten die inzwischen durchaus leistungsfähigen Übersetzungssysteme im Internet an. Ohne Zweifel wird damit ein Aspekt von $A A L$ - so die übliche Abkürzung - getroffen, doch es wird mit diesen Übersetzungen gerade nicht der Aspekt eingefangen, der am Wichtigsten ist: Dass es hier um Technik für betreutes oder umgebungsunterstütztes Leben beziehungsweise Wohnen geht. Selbst das ist zu kurz gegriffen, denn ein erheblicher Teil der Debatten um AAL dreht sich um die Frage der Ersetzung menschlicher Pflegeleistungen mithilfe und durch Technik. Denkt man dies zu Ende, so geht es bei der Diskussion um AAL um nichts weniger als die Art und Weise, wie in Zukunft Pflegeleistungen erbracht werden.

Eine wörtliche Übersetzung von AAL hilft also nicht sonderlich weiter. Das mag mit ein Grund dafür gewesen sein, dass das BMBF auf der Webseite www.aaldeutschland.de (Stand 03.11.2020) mit folgender Definition oder besser Erklärung zitiert wird, was AAL sein könnte:

»Ambient Assisted Living ( $(A A L)$ steht für Konzepte, Produkte und Dienstleistungen, die neue Technologien in den Alltag einführen [sic!] um die Lebensqualität für Menschen in allen Lebensphasen, vor allem im Alter, zu erhöhen. Ins deutsche [sic!] übersetzt steht AAL für Altersgerechte Assistenzsysteme für ein gesundes und unabhängiges Leben.«

Mit diesem Zitat kommt der Technikaspekt sehr viel deutlicher zur Geltung. Es wäre nun durchaus interessant gewesen, die unterschiedlichen Stände der genannten Webseite über die Jahre hinweg zu speichern und damit zu dokumentieren, denn - und das müssen die Leser*innen jetzt einfach glauben - seit diese Webseite eingerichtet wurde, hat sich der Tenor der Aussagen sehr deutlich, ja fast dramatisch verändert. Als das BMBF im Jahr 2008 mit einer ersten AAL-Förderlinie die Entwicklung altersgerechter Assistenzsysteme massiv zu unterstützen begann, war das Thema ausnehmend positiv besetzt, es herrschte Optimismus. Heute jedoch kann man auf der Seite lesen: 
»Während neue Technologien im Alltag fast aller Menschen bereits eine nicht mehr wegzudenkende Rolle spielen, gibt es kaum erfolgreiche Beispiele für den Einsatz neuer Technologien zur Steigerung der Lebensqualität älterer Menschen. Dabei sind die benötigten Technologien bereits vorhanden und könnten relativ einfach angewendet werden. Warum finden vorhandene Konzepte und Technologien also nicht Einzug in den Alltag deutscher Senioren- und Pflegehaushalte?«

Zwölf Jahre, nachdem das BMBF mit der Förderung der Forschung an und Entwicklung von AAL begann (und andere Förderinstitutionen auf EU-, Bundes- und Länderebene schlossen sich an beziehungsweise hatten bereits vorher mit der Förderung begonnen), wird mit diesen wenigen Sätzen doch ein sehr ernüchterndes Fazit gezogen. Obwohl der Vergleich in vieler Hinsicht hinkt und daher mit Vorsicht genossen werden sollte: 2006 brachte Apple das erste iPhone und damit das erste echte Smartphone auf den Markt - inzwischen werden jedes Jahr mehr als eine Milliarde Smartphones unterschiedlicher Hersteller*innen weltweit verkauft und der weltweite Jahresumsatz mit diesen Geräten übersteigt 500 Milliarden Euro (Destatis 2019b). In einem vergleichbar langen (bzw. eher kurzen) Zeitraum hat sich ein digitales Produkt rasant verbreitet, einen gigantischen Markt geöffnet und ein riesiges technologisches Ökosystem geschaffen, das selbst noch viel größer und umsatzstärker ist als der Markt für Smartphones selbst - Facebook, Twitter, Instagram, WhatsApp oder TikTok sind nur wenige Beispiele für Social-MediaPlattformen, die im Wesentlichen mit Smartphones benutzt werden. Gleichzeitig, und darin steckt eine gehörige Portion Ironie, entwickeln sich Smartphones und die mit ihnen ermöglichten Produkte und Dienstleistungen zu einer Plattform, mit der viele der Ziele und Aufgaben, die mit AAL verbunden waren und sind, erreicht und übernommen werden können - hierzu gehören beispielsweise Sprachassistenten unterschiedlicher Hersteller*innen, die neue Formen der Bedienung ermöglichen. Geräte, die ausdrücklich AAL realisieren sollen, fristen hingegen ein Schattendasein.

Für die Frage, warum sich AAL beziehungsweise altersgerechte Assistenzsysteme so und nicht anders entwickelt haben, lässt sich vermutlich keine letztgültige Antwort geben, da diese unter anderem abhängig sein wird von der antwortenden Instanz. Vor allem wäre eine genaue Analyse sehr aufwändig, weil dazu die verschiedenen und zahlreichen Akteur* innen befragt werden müssten und sicherlich auch Archivarbeit geleistet werden müsste - aber der vorliegende Text kann eine solche techniksoziologische und -historische Untersuchung mit Sicherheit nicht leisten. Stattdessen sollen einige Vermutungen und Rahmenbedingungen aufgezeigt werden, die die AAL-Entwicklung beeinflusst haben mögen, und um einige tentative Schlussfolgerungen ergänzt werden. Vorher soll aber versucht werden das Themenumfeld etwas genauer $\mathrm{zu}$ bestimmen. 


\section{Altersgerechte Assistenzsysteme - Begriffsbestimmung, Abgrenzung und Phänomenbeschreibung}

Im deutschsprachigen Raum hat sich für die Beschreibung assistiver Technologien, die in erster Linie den alten Menschen adressieren, zunächst der Ausdruck AAL durchgesetzt. Dieser Ausdruck wird auch in englischsprachigen Publikationen verwendet; so finden sich beispielsweise in den digitalen Bibliotheken der Association for Computing Machinery (ACM) und der Institute of Electrical and Electronics Engineers (IEEE) mehrere Hundert Einträge zu Aufsätzen, Proceedingsbeiträgen und Ähnlichem, die AAL thematisieren (ACM=1184, IEEE=764, Stand: 29.01.2021). Mit dem Ausdruck Ambient Assisted Living oder AAL ist somit eine umfangreiche wissenschaftliche Diskussion verbunden. Allerdings erweist ein etwas genauerer Blick, dass eine Reihe inhaltlich ähnlich definierter Ausdrücke existiert; für AAL gibt es definitorisch keine völlig klare Abgrenzung. Dies erschwert eine wissenschaftliche Auseinandersetzung mit dem Thema erheblich und hat vermutlich mit dazu geführt, dass in der Förderpolitik des BMBF der Begriff zunächst durch die Rede von den saltersgerechten Assistenzsystemen oder ähnlichen Formulierungen ersetzt wurde und inzwischen sehr häufig sehr viel allgemeiner von Mensch-Maschine- beziehungsweise Mensch-Technik-Interaktion (MTI) gesprochen wird.

\section{Definitorische Abgrenzung}

Es gibt eine Reihe mit AAL verbundener Konzepte: Ambient Intelligence, Ubiquitous Computing und Pervasive Computing. Sie stehen beispielsweise für Technologien, die informations- und kommunikationsorientierte Dienstleistungen erbringen sollen, ohne dass die entsprechenden Geräte als technische Artefakte erkennbar wären (vgl. Beigl/Gellersen/Schmidt 2001) ${ }^{1}$. Die entsprechenden Funktionen sollen nicht von klar identifizierbaren und eng lokalisierten einzelnen Geräten ausgeführt werden, sondern die jeweilige Umgebung selbst soll mit technischer Funktionalität aufgerüstet sein; unzählige sehr kleine und meist auch einfache Geräte sollen sich je nach Anforderung spontan und ohne menschliche Eingriffe vernetzen und interagieren, um Benutzer*innenwünsche zu erfüllen. Marc Weiser hatte diese Idee in seinem Aufsatz »The computer for the $21_{\text {st }}$ century« bereits im Jahr 1991 entwickelt und dabei eine Technologie skizziert, die in Bezug auf ihre Funktionalität einen aufzuweisen, wann die entsprechenden Diskussionen ungefähr begonnen haben. Das soll u.a. verdeutlichen, dass über altersgerechte Assistenzsysteme, wenn auch nicht mit dem entsprechenden Ausdruck, aber doch sinngemäß, schon recht lange gesprochen, darüber geforscht und daran entwickelt wird. Dies wirft umso mehr die Frage auf, warum die öffentliche Debatte um den Einsatz dieser Technik nicht umfangreicher ist und größere Aufmerksamkeit auf sich ziehen konnte. 
hohen Grad an Autonomie besäße und gleichzeitig nicht mehr als Technik erkennbar wäre, sondern unsichtbar in der Umwelt aufginge. Weiser (1991: 94) schreibt dazu:

»The most profound technologies are those that disappear. They weave themselves into the fabric of everyday life until they are indistinguishable from it."

Ungeachtet der Frage, ob Weisers Vision bereits in tatsächlich existierenden Geräten umgesetzt wurde (nimmt man ihn beim Wort, dann fällt die Antwort eher negativ aus), findet sich für diese Technologie inzwischen eine große Zahl von Bezeichnungen: Ambient Intelligence, Ubiquitous Computing oder Pervasive Computing sind beileibe nicht die einzigen. Kurzzeitig tauchte die Bezeichnung Anytime Anywhere Communication and Computing (AACC) (vgl. Neitzke et al. 2008) auf; außerdem wird oft vom Internet der Dinge beziehungsweise Internet of Things gesprochen (vgl. die Beiträge in Fleisch/Mattern 2005). Auch die inzwischen in den Massenmedien angekommene Rede vom Smarthome gehört in dieses Umfeld (vgl. Park et al. 2003). Die verschiedenen Bezeichnungen verweisen zum Teil auf verschiedene Phänomene und Verwendungsweisen, besitzen aber gleichzeitig große Überschneidungen - als Bedeutungskern kann man die Idee Marc Weisers identifizieren, dass Sensoren, Aktoren, Computer- und digitale Medientechnologie in die Umgebung eingebettet werden sollen.

Die Bezeichnung Ubiquitous Media taucht seltener in der Literatur auf. Der Unterschied zu den bereits genannten Technologien ist vor allem darin zu sehen, dass im Fall von Ubiquitous Media viel stärker auf mediale Aspekte verwiesen wird und nicht so sehr auf Dienstleistungen; meist wird von Ubiquitous Media im Sinne eines Mediums zur Konstruktion und Vermittlung von Wirklichkeit gesprochen (vgl. Weber et al. 2009). Andere Bezeichnungen, die diesen medialen Aspekt stark hervorheben, sind Mixed beziehungsweise Augmented Reality (Kabisch 2008: 227). Neu sind diese Ideen nicht: Obwohl in den späten 1990er Jahren weder die Bezeichnung Ubiquitous Media genutzt wurde noch die notwendige Technologie zur Umsetzung der dahinterstehenden Ideen zur Verfügung stand, gab es bereits zu dieser Zeit umfangreiche Forschungsprojekte in diesem Bereich (vgl. Mann 1997). Ebenfalls in den 1990er Jahren wurde begonnen, an Virtual-Reality-Anwendungen in der Medizin $\mathrm{zu}$ arbeiten (vgl. Satava 1995); inzwischen sind entsprechende Überlegungen auch in den Gesundheitswissenschaften angekommen (vgl. Lee 2019).

Konzepte der Mixed beziehungsweise Augmented Reality heben nun stärker auf Dezentralisierung und Benutzer*innenzentrierung ab: Konzeptionen beispielsweise von Steve Mann (2004) laufen darauf hinaus, dass Menschen mithilfe von Sensoren und tragbaren Computern die Chance bekommen ihre Sicht auf die Welt zu ergänzen und zu erweitern (engl.: to augment); entsprechende Technologien werden daher oft unter der Bezeichnung Computer Mediated Reality (vgl. 
Rekimoto/Ayatsuka 2000) diskutiert. Informations- und Kommunikationstechnologien sollen dazu genutzt werden, die menschlichen Sinne und deren Fähigkeiten $\mathrm{zu}$ ergänzen, $\mathrm{zu}$ erweitern oder-beispielsweise im Falle gehandicapter Menschen - auch zu ersetzen.

Die Forschung rund um informationstechnische Implantate zur Steigerung kognitiver, sensorischer und motorischer Fähigkeiten (vgl. Benford/Malartre 2008; Warwick 2003) schließlich markiert einen weiteren Bereich, der mit AAL (eher kleine) Überschneidungen aufweist. Vor allem die Erweiterung (engl.: enhancement) menschlicher Fähigkeiten wird in diesem Kontext nicht nur wissenschaftlich-technisch untersucht, sondern es haben sich Gruppierungen wie die Transhumanisten oder die Extropianer (vgl. Bostrom 2005) gebildet, die in der gezielten technischen Modifikation des Menschen einen notwendigen und auch wünschenswerten evolutionären Schritt sehen. Wie schon bemerkt haben diese Ideen mit AAL nicht mehr viel gemein, fließen aber zuweilen in die Diskussionen über AAL mit ein, insbesondere im Kontext der Quantified Self-Bewegung (vgl. Catlaw/Sandberg 2018; Danter/Reichardt/Schober 2016).

Die für die meisten Menschen nach wie vor geläufigste Mensch-MaschineInteraktion basiert auf der Ausgabe von Informationen durch Visualisierung auf einem Bildschirm und der Eingabe über Tastaturen; diese Form der MenschMaschine-Interaktion (oft wird auch der Ausdruck Mensch-Technik-Interaktion genutzt) schließt jedoch visuell oder haptisch eingeschränkte Menschen von der Benutzung solcher Computer weitgehend oder vollständig aus. So kann es kaum überraschen, dass beispielsweise wissenschaftliche Arbeiten, die haptische Benutzer*innenschnittstellen thematisieren, als Zielgruppe Menschen mit einem visuellen Handicap benennen (vgl. Weber 2015). Wall/Brewster (2005: 2140) schreiben hierzu:

»The explosion of software applications, digitally stored data and the subsequent growth in on-line communities, has frequently been denied to visually impaired and blind computer users due to the visual-centric nature of presentation methods employed.«

In jüngerer Zeit bricht sich zunehmend die Idee Bahn, dass alternative Benutzer*innenschnittstellen, die mehr als den visuellen Kanal ansprechen, in vielen Situationen nicht nur für gehandicapte Menschen mit erheblichen Vorteilen verbunden sein können (vgl. Cheng et al. 2010; McGookin/Robertson/Brewster 2010; Shaer et al. 2010). In diesen Bereich gehört die Forschung zu Tangible Media beziehungsweise Tangible Interfaces (vgl. Brereton 2001; Shaer et al. 2004). 


\section{Definitorische Abgrenzung zu anderen medizinischen Anwendungsbereichen}

Die obigen Anmerkungen verweisen darauf, dass im Kontext der Entwicklung neuer Informations- und Kommunikationstechnologien zahlreiche Konzepte und Begriffe entstehen, die letztlich bestimmte grundlegende Einsatzszenarien und Gestaltungsrichtlinien beziehungsweise -ideen umfassen, aber kaum spezielle Anwendungen vorgeben oder andere ausschließen. Es wurde ebenfalls deutlich, dass die Idee der Kompensation physischer und möglicherweise psychischer Handicaps ein Bestandteil des Konzepts allgegenwärtiger Datenverarbeitung ist. Zentral ist, dass Computer(-dienst-)leistungen nicht an einen bestimmten Ort und an eine bestimmte Zeit gebunden sein sollen.

Gerade dieser Aspekt spielt für medizinische Anwendungen der Informationsund Kommunikationstechnologie eine erhebliche Rolle, denn in vielen medizinisch relevanten Situationen kann es passieren, dass kein entsprechend ausgebildetes Personal vor Ort ist (vgl. Flesche/Jalowy/Inselmann 2004). Daher wird schon seit geraumer Zeit die Forschung und Entwicklung an und von computergestützten Systemen der Telemedizin gefördert. Die Anwendungen sind durchaus heterogen und bewegen sich im Umfeld der Beratung jener Patient*innen, die vor Ort keinen Zugang zu medizinischem Personal haben, bis hin zur computer- und robotergestützten Operation, bei denen Ärzt*innen und Patient*innen durch große Distanzen getrennt sind (vgl. Merrell 2005; Satava 2005). Darüber hinaus nehmen medizinische Notfallmaßnahmen (vgl. Skorning et al. 2011) ebenso wie die Prävention von Unfällen im Zusammenhang mit Telemedizin einen erheblichen Raum ein (vgl. Leis 2008).

In vielen dieser Fälle ist die Abgrenzung zum Telemonitoring nur schwer zu ziehen (vgl. Meystre 2005): »Telemonitoring [...] is defined as the use of information technology to monitor patients at a distance« (ebd.: 63). Dieser Aspekt der Überwachung von Vitaldaten taucht sowohl in der Telemedizin als auch im Kontext von eHealth auf. Im Zusammenhang dazu bemerkt Meystre zudem, dass auch Telemonitoring begrifflich noch in Telediagnose und Telekonsultation aufgeteilt werden könnte - auch hier liegt also eine begriffliche Vielfalt vor, die anzeigt, dass es sehr verschiedene Einsatzfelder der jeweiligen Technologie gibt.

eHealth kann nun als überwölbender Begriff für die vorgenannten Anwendungsund Technologiefelder angesehen werden, der darüber hinaus viele andere Bedeutungen beinhaltet (vgl. Oh et al. 2005). In Deutschland wurde eHealth sehr häufig im Zusammenhang mit der elektronischen Gesundheitskarte (eGk) verwendet, ist aber nicht auf diesen Bezug beschränkt. Allerdings ist auch die Vielfalt der genutzten Begriffe groß, denn neben eHealth findet sich beispielsweise auch der Ausdruck $m$ Health für mobile Gesundheitsanwendungen (vgl. Akter/Ray2010; Olla/Shimskey 2015; Rossmann/Krömer 2016). 


\section{AAL und altersgerechte Assistenzsysteme: Eine Arbeitsdefinition}

Die Fülle von Bezeichnungen, Konzepten, Ideen, Anwendungen und dahinterstehenden Technologien ist zunächst verwirrend und erschwert jede Form der Begleitforschung zu AAL beziehungsweise altersgerechten Assistenzsystemen. Allerdings lässt sich die Komplexität, die mit den obigen Bemerkungen verbunden ist, reduzieren. Dazu kann man zunächst die Definition des BMBF heranziehen, wie sie ursprünglich (ab 2008) auf der Webseite www.aal-deutschland.de zu finden war:

»Unter >Ambient Assisted Living`(AAL) werden Konzepte, Produkte und Dienstleistungen verstanden, die neue Technologien und soziales Umfeld miteinander verbinden und verbessern mit dem Ziel, die Lebensqualität für Menschen in allen Lebensabschnitten, vor allem im Alter, zu erhöhen. Übersetzen könnte man AAL am besten mit >Altersgerechte Assistenzsysteme für ein gesundes und unabhängiges Leben<. Damit wird auch schon skizziert, dass AAL in erster Linie etwas mit dem Individuum in seiner direkten Umwelt zu tun hat.«

Die im Folgenden zu betrachtenden Systeme sind damit nicht als rein technische $\mathrm{zu}$ verstehen, sondern als soziotechnische Systeme mit potenziell weitreichenden psychosozialen Auswirkungen. Das Verständnis als soziotechnische Systeme ergibt sich daraus, dass altersgerechte Assistenzsysteme in erster Linie als Unterstützung für Menschen gesehen werden und dies in doppelter Hinsicht: Zum einen sollen beispielsweise ältere und hochbetagte Personen diese Systeme selbst nutzen, um auf diese Weise eine hohe Lebensqualität zu erfahren, zum anderen aber sollen diese Systeme Angehörige ebenso wie das Gesundheits- und Pflegepersonal im sorgenden Umgang beispielsweise mit hilfe- und pflegebedürftigen Menschen unterstützen.

Dabei wird vorausgesetzt, dass jede Lebensphase eines Menschen mit bestimmten Fähigkeiten, aber auch Handicaps verbunden ist, die durch altersgerechte Assistenzsysteme unterstützt beziehungsweise kompensiert werden sollen. Dies gilt für die 25-jährige Frau, die nach einem Motorradunfall querschnittgelähmt ist, ebenso wie für den 80 -jährigen Mann, der altersbedingt einen Teil seiner Bewegungsfähigkeit eingebüßt hat - in beiden Fällen sollen Assistenzsysteme altersgerechte und natürlich auch situationsgerechte Unterstützung bieten. In solchen und vielen anderen Fällen zeigen die entsprechenden Systeme unter anderem Wirkung auf zwischenmenschliche Beziehungen - dies sollte oben mit dem Wort >psychosoziak angezeigt werden.

In vielen Fällen - deshalb auch die umfangreiche Einführung bezüglich Ubiquitous Computing - basieren altersgerechte Assistenzsysteme auf der Idee der unsichtbaren, aber dabei allgegenwärtigen Datenverarbeitung. Für die Nutzer*innen soll es nicht notwendig sein, ein bestimmtes Gerät, beispielsweise einen PC, 
$\mathrm{zu}$ bedienen, um eine Dienstleistung auszulösen. Stattdessen sollen die für die jeweiligen Personen wichtigen Funktionen gleichsam von der Umwelt durch möglichst natürliche Interaktionsformen erbracht werden, wobei beachtet werden soll, dass die Nutzer*innen möglicherweise in ihren sensomotorischen Möglichkeiten eingeschränkt sind. Die Mensch-Maschine-Interaktion soll also nicht nur auf dem klassischen Paradigma von Bildschirm und Tastatur beschränkt sein, sondern multimodale Kanäle bieten. Allerdings war diese Idee der ubiquitären AAL-Technologie vor allem eine Idee: Schaut man sich tatsächlich im Einsatz befindliche Geräte an, so basieren diese in aller Regel auf klar zu identifizierenden Geräten wie Smartphones, Tablet-PCs oder Fitnesstracker in Gestalt sogenannter Smartwatches. Tatsächlich ist AAL gar nicht so sehr bei den gepflegten Personen selbst angekommen, sondern wird derzeit vor allem an die Pflegekräfte adressiert - die Beiträge im vorliegenden Sammelband zeigen dies recht deutlich.

Abbildung 1: Einsatzfelder altersgerechter Assistenzsysteme

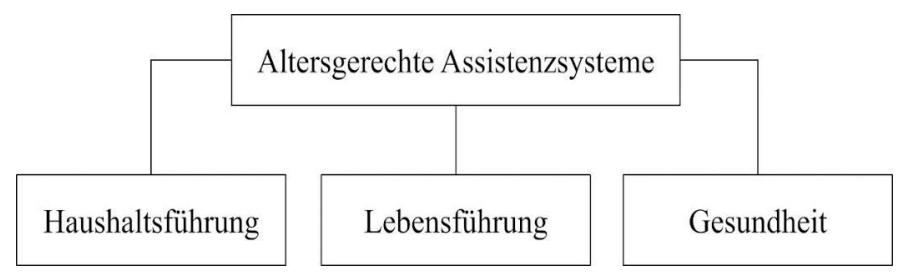

Quelle: Eigene Darstellung

Da altersgerechte Assistenzsysteme zudem dazu beitragen sollen, gerade älteren und hochbetagten Menschen ein längeres Verbleiben im eigenen Haus oder der eigenen Wohnung und dabei ein selbstbestimmtes Leben bei gleichzeitiger sozialer Teilhabe zu ermöglichen, muss berücksichtigt werden, dass Angehörige der genannten Zielgruppe oftmals einen erheblichen Pflegebedarf haben, da sie an (oftmals mehreren) altersbedingten Krankheiten beziehungsweise auch Defiziten leiden. Da zu erwarten ist, dass dieser Pflegebedarf aus verschiedenen Gründen nicht mehr nur durch Pflegekräfte zu erbringen sein wird, sollen altersgerechte Assistenzsysteme beispielsweise Funktionen des Telemonitorings beziehungsweise der Telediagnose und Telekonsultation anbieten; dies gilt insbesondere für dünn besiedelte Regionen mit einer nicht ausreichenden Gesundheits- und Pflegeinfrastruktur.

Zusammenfassend betreffen altersgerechte Assistenzsysteme - wie in Abbildung 1 gezeigt - die selbstbestimmte Haushaltsführung im eigenen Heim, die Lebensführung mit der Frage nach der Teilhabe am sozialen Leben sowie die Versorgung mit Gesundheits- und Pflegeleistungen. Dies kann auf jede Lebensphase und 
jedes Alter bezogen werden. Wie gerade aber schon bemerkt, werden altersgerechte Assistenzsysteme gar nicht so häufig im eigenen Heim von pflegebedürftigen Menschen genutzt, sondern, sofern diese Systeme überhaupt in den alltäglichen Gebrauch diffundiert sind, findet man sie vor allem in der stationären Pflege, wo sie in erster Linie die Arbeit der Pflegekräfte unterstützen sollen.

AAL ist weder auf den Bereich der Pflege und Gesundheit noch auf eine bestimmte Phase des Lebens beschränkt; hierin fallen eben auch alle Anwendungen, die beispielsweise für die Freizeitgestaltung genutzt werden können und sollen. Die Nutzung des Ausdrucks saltersgerechte Assistenzsysteme hingegen soll anzeigen, dass in erster Linie "Konzepte, Produkte und Dienstleistungen« - wie ursprünglich auf der Webseite www.aal-deutschland.de formuliert - in den Blick genommen werden, die eben einen starken Bezug zur Pflege- und Gesundheitsversorgung insbesondere von älteren und hochbetagten Personen aufweisen.

\section{Demografische Rahmenbedingungen}

Die Bundesrepublik Deutschland beziehungsweise ihre Bevölkerungsstruktur und -entwicklung durchlaufen, nicht erst seit heute, eine "posttransformative« Phase (Schulz 2000): Unter der Voraussetzung, dass sich am derzeitigen Reproduktionsverhalten der in Deutschland lebenden Menschen nichts Wesentliches ändert und das Migrationsgeschehen der Jahre 2015/2016 eine Ausnahme bleibt, wird die Population langfristig schrumpfen. Drei quantitative Veränderungen, die für die Gestaltung der Pflege in Deutschland (und zu betonen ist: nicht nur in Deutschland, sondern in vielen industrialisierten Ländern) relevant sind, charakterisieren den demografischen Wandel: Die durchschnittliche Lebenserwartung steigt kontinuierlich, wie den Daten des Statistischen Bundesamtes entnommen werden kann (vgl. Destatis 2020a), der Anteil der älteren und hochbetagten Menschen an der Gesamtbevölkerung wächst, während der Anteil der jüngeren Generationen schrumpft. Für eine wesentlich anders verlaufende Entwicklung spricht wenig; die Migration nach Deutschland wird diesen Schrumpfungs- und Veralterungsprozess kaum aufhalten, sondern allenfalls verlangsamen können. Man kann und muss ergänzen: Die Hoffnung der Entlastung der Solidarsysteme und der öffentlichen Haushalte durch Migration junger Arbeitskräfte ist eher skeptisch zu betrachten (Bonin 2014). Der demografische Wandel stellt damit eine gesellschaftliche Herausforderung in mehreren Dimensionen dar.

Was die öffentliche Debatte über dieses Thema betrifft, kann man zuweilen den Eindruck bekommen, dass der demografische Wandel ein Phänomen sei, das erst kürzlich entdeckt worden wäre. Doch das ist falsch, denn wer (wie der Autor) beispielsweise vor circa 30 Jahren familien- und/oder bevölkerungssoziologische Lehrveranstaltungen besuchte oder die verfügbaren Lehrbücher las, die in den 1980er 
Jahren geschrieben worden waren und Daten und Hochrechnungen der 1970er Jahre wiedergaben, lernte den demografischen Wandel schon damals kennen. Die sexuelle Revolution und die Möglichkeit der effektiven Schwangerschaftsverhütung, die (noch nicht vollständige) Emanzipation der Frauen sowie neue gesellschaftlich wirkmächtige Ideen über Ehe und Familie und nicht zuletzt die deutsche Wiedervereinigung haben zu einem massiven Rückgang der Geburtenrate beigetragen. Es war schon vor mindestens 30 Jahren offensichtlich, dass mit diesen Veränderungen weitreichende gesellschaftliche Herausforderungen verbunden sein würden (vgl. Kaufmann/Leisering 1984; Schmähl 1990; Schulz-Nieswandt 1990; Weizsäcker 1989a \& 1989b \& 1989c). Politisch geschehen ist wenig, um diesen Herausforderungen zu begegnen.

Wie schon bemerkt, ist der demografische Wandel in Deutschland durch mehrere parallel laufende Entwicklungen geprägt; in vieler Hinsicht ist die Schrumpfung des Anteils der jüngeren Generationen der wichtigste Teilaspekt, denn dieser Bevölkerungsteil erwirtschaftet die Ressourcen, die für die Finanzierung einer funktionierenden und angemessenen Gesundheits- und Pflegeversorgung notwendig sind. Es kann dabei gar nicht genug betont werden, was Carol McCarthy (1987: 206) in nüchternen Worten formuliert:

"Ultimately, of course, the people pay all health care costs. Thus, when we say that health care monies come from different sources, we really mean that dollars take different routes on their way from consumers to providers of care.«

Ob Gesundheitsversorgungssysteme durch Steuern (bspw. Großbritannien), solidarische Versicherungssysteme (z.B. Deutschland), direkte Zahlungen und/oder private Versicherungen (in erster Linie USA) finanziert werden, ändert nichts an der Tatsache, die Carol McCarthy zum Ausdruck bringt: »the people pay all health care costs.« Es existieren keine anderen Quellen, aus denen die benötigten Ressourcen sprudeln könnten. Allenfalls kann man unterschiedliche Antworten auf die Frage geben, wer mit "people« gemeint sein könnte. In Deutschland wurde vor einigen Jahren über die Antwort auf diese Frage heftig gestritten, als über Bürger*innenversicherung, allgemeine und einheitliche Versicherungspflicht oder eine Beitragspflicht für Einkommen aus Kapital diskutiert wurde (vgl. Butterwegge 2004; Greß/Lüngen 2017; Langer et al. 2004).

Im Moment gilt aber nach wie vor: Die Finanzierung der Pflege- und Gesundheitssysteme basiert in Deutschland im Prinzip und im Wesentlichen auf Pflichtbeiträgen, die auf Einkommen aus unselbstständiger Arbeit erhoben werden. Steuerfinanzierte Zuschüsse und die Rolle der privaten Kranken- und Pflegeversicherungen sollen hier nicht negiert werden, doch gilt auch für diese, dass sie letztlich durch geleistete Arbeit getragen werden. Wenn es auch eine Vereinfachung darstellt, kann man festhalten, dass die Höhe der Ressourcen, die für Pflege und Ge- 
sundheit zur Verfügung stehen, ganz erheblich von der Zahl der Beschäftigten in Deutschland abhängt. Verringert sich diese Zahl durch den demografischen Wandel, so verringern sich die Ressourcen, die für die Finanzierung der Pflege- und Gesundheitssysteme in Deutschland zur Verfügung stehen, ebenfalls.

Sofern man keine grundsätzlichen und systemverändernden Maßnahmen ergreifen will oder kann (bspw., weil diese politisch vermutlich nicht durchsetzbar wären), eröffnen sich nicht sonderlich viele Handlungsoptionen, um mit dieser Situation umzugehen:

1 Es wäre denkbar, die tatsächlich erbrachte Lebensarbeitszeit zu erhöhen, sodass die Beschäftigten länger zur Finanzierung herangezogen werden (vgl. Höpflinger 2007; Lehr/Kruse 2006). Es erscheint jedoch zweifelhaft, ob dies politisch durchsetzbar wäre: Die immer wieder aufkommenden Debatten über die sogenannte >Work-Life-Balance< deuten eher in die entgegengesetzte Richtung.

2 Die Beiträge für die Renten-, Pflege- und Krankenversicherung könnten erhöht werden, um (annähernd) gleichbleibende Ressourcen für die Versorgung zur Verfügung stehen zu haben. Wiederum steht die Durchsetzbarkeit eher in Zweifel, da die Abgabenlast in Deutschland bereits sehr hoch ist und nicht beliebig weiter erhöht werden kann, weil dadurch unter anderem das Konsumgeschehen negativ beeinflusst werden würde und dies zu erheblichen Steuerausfällen führen könnte.

3 Alternativ könnten die Gesundheits- und Pflegeleistungen, die durch die entsprechenden Versicherungen finanziert werden, reduziert werden auf eine Grundversorgung, die im Bedarfsfall durch zusätzliche Versicherungen oder Eigenzahlungen ergänzt wird. Auch hier kann an der Durchsetzbarkeit gezweifelt werden, wie an den in Deutschland in der Vergangenheit schon geführten Rationierungsdebatten (vgl. Fozouni/Güntert 2000; Mack 2001; Oduncu 2012) ersichtlich wird.

Die Sicherung der Ressourcen für die Finanzierung der Gesundheits- und Pflegeleistungen stößt also an Grenzen, da zu erwarten ist, dass weitere Belastungen der arbeitenden Bevölkerung von dieser kaum hingenommen werden würden. Eine Lebensarbeitszeitverlängerung würde nicht nur, aber eben auch im Bereich der Pflege auf massiven und vermutlich auch berechtigten Widerstand stoßen - Pflegetätigkeiten sind physisch wie psychisch sehr belastend, sodass eine Verlängerung der Lebensarbeitszeit beispielsweise mit einem Renteneintrittsalter von 70 Jahren für viele Beschäftigte in der Pflege kaum durchzustehen wäre und zudem den Arbeitsplatz Pflege noch unattraktiver werden ließe. 
Mit dem bisher Gesagten wurde natürlich nur ein Teil der Rahmenbedingungen angesprochen, der dazu beigetragen hat, dass altersgerechte Assistenzsysteme als mögliche Lösung für einige der Folgen des demografischen Wandels ins Spiel gebracht wurden. Im vorliegenden Sammelband werden zahlreiche weitere Aspekte thematisiert, sodass ein wesentlich vollständigeres Bild entstehen kann, als es hier geboten werden kann. Die mehr als grob skizzierten ökonomischen Bedingungen wurden deshalb hervorgehoben, da die Ergebnisse, die in dem Projekt DAAS-KIN (»Diffusion altersgerechter Assistenzsysteme - Kennzahlenerhebung und Identifikation von Nutzungshemmnissen «) erzielt werden konnten, der Hoffnung auf (womöglich massive) Einsparungen bei den Kosten der Pflege durch den Einsatz von Technik eher widersprechen. Es ist bezeichnend, dass sich dies in den Aussagen auf der schon genannten Webseite www.aal-deutschland.de ebenfalls widerspiegelt:

»Ein weiterer Crund für die mangelnde Durchsetzung vorhandener Technologien ist die fehlende Finanzierung. Senioren- und Pflegehaushalte scheuen die oft hohen Ausgaben.«

Mit anderen Worten: Das (gesundheits-)ökonomische Umfeld erscheint nicht optimal, um technische Innovationen im Bereich altersgerechter Assistenzsysteme $\mathrm{zu}$ fördern und Anreize für den Einsatz solcher Systeme zu bieten. Dadurch entsteht der erste Teufelskreis, der nur schwer zu durchbrechen scheint. Denn da bisher nur eine vergleichsweise geringe Zahl altersgerechter Systeme - sowohl im Sinne typischer Anwendungen als auch im Sinne der im Gebrauch befindlichen Systeme - existiert, verfügen die prospektiven Nutzer*innen sowohl aufseiten der pflegenden wie der gepflegten Personen nur über sehr geringe Erfahrungen im Umgang mit solchen Systemen. Es kann daher kaum überraschen, dass die Haltung dieser Stakeholder*innen nicht auf eigenen Erfahrungen beruht, sondern eher auf Urteilen, die sich zum Beispiel aus dem eigenen Selbstverständnis oder der einschlägigen wissenschaftlichen Literatur speisen. Bedenkt man dann zusätzlich, dass der breite Einsatz von Technologie in anderen Wirtschaftszweigen beziehungsweise Handlungskontexten oft mit Rationalisierungen und damit Arbeitsplatzabbau einherging, dann sollte es wenig verwundern, dass altersgerechte Assistenzsysteme von vielen Stakeholder*innen nicht unbedingt mit offenen Armen empfangen werden (vgl. Frommeld und Scorna in diesem Band) - die Vermutung, dass beispielsweise Serviceroboter dazu genutzt werden könnten, bestimmte derzeit von Menschen erbrachte Dienstleistungen zukünftig durch Maschinen erbringen zu lassen, liegt einfach auf der Hand.

Erschwerend kommt hinzu, dass die fehlenden Erfahrungen in Bezug auf den Einsatz altersgerechter Assistenzsysteme einen zweiten Teufelskreis erzeugen, der ebenfalls schwer zu durchbrechen ist. Es gibt, folgt man beispielsweise Sybille Meyer (2016: 18), schlicht keinen Leistungsnachweis, der hohe Investitionen in AAL- 
Systeme rechtfertigen könnte. Aus den Ergebnissen von Feldversuchen und Modellprojekten würde nicht ausreichend ersichtlich werden, ob solche Systeme tatsächlich die Leistungen erbrächten, die man sich von ihnen in pflegerischer Hinsicht erhofft; die existierenden Bewertungen, so Meyer, beruhten nicht auf empirisch basierten, systematischen Wirksamkeitsstudien, sondern ausschließlich auf Expert*inneneinschätzungen, deren Aussagekraft begrenzt sei:

»Es existieren hohe Erwartungen an die Leistungsfähigkeit assistiver Technologien. Da in die Betreuung und Pflege viele Akteure eingebunden sind, ist neben der direkten Wirkung von Assistenzsystemen beim Anwender ein indirekter Nutzen bei Dritten relevant. Dies können Kosteneinsparungen bei Versicherungsträgern durch vermiedene Krankenhausaufenthalte oder Arztbesuche oder das Vermeiden eines notwendigen Umzugs ins Pflegeheim sein. Auch für diese indirekten Wirkungen ist bisher noch kein Nutzennachweis in der Praxis erbracht. Nicht zuletzt deshalb mangelt es unter anderem noch an der Bereitschaft von Leistungsträgern oder Kommunen, sich an der Finanzierung der meist für den Anwender zu teuren technischen Lösungen zu beteiligen.«

Die wenigen existierenden Potenzialstudien (vgl. Fachinger et al. 2012) und die Überlegungen zu möglichen Geschäftsmodellen (vgl. Beiträge in Gersch/Liesenfeld 2012; Schelisch 2016) in Bezug auf AAL-Systeme sind auch nicht unbedingt geeignet Skepsis gegenüber der Technik abzubauen. Die Potenzialstudien waren in der Regel zu optimistisch, mögliche Geschäftsmodelle konnten angesichts der geringen Verbreitung altersgerechter Assistenzsysteme nicht wirklich auf ihre Tragfähigkeit hin überprüft werden. Kurzum: Es muss erst einmal gezeigt werden können, dass sich AAL-Systeme rechnen (vgl. Zerth in diesem Band).

\section{F\&E-Förderung: Zerschlagung des gordischen Knotens?}

Man könnte die skizzierte Situation der sehr langsamen Diffusion von Technik in die Anwendung als eine Form des Marktversagens ansehen - und damit präsentiert sich die mögliche Lösung gleichsam von selbst: Der Staat beziehungsweise die öffentliche Hand muss eingreifen, um einen Ausweg aus den beiden skizzierten Teufelskreisen zu öffnen. Unter welchen politischen Vorzeichen auch immer (vgl. Hergesell et al. in diesem Band) kann staatliche Förderung von Forschung und Entwicklung (F\&E) dazu beitragen, dass auch risikobehaftete Vorhaben umgesetzt werden, weil die ökonomischen Lasten und Risiken nicht nur auf den Schultern der jeweiligen Unternehmen ruhen, sondern teilweise oder vollständig durch die öffentliche Hand getragen werden. 
Um einen Überblick der in Deutschland durchgeführten F\&E-Projekte, die im Bereich der altersgerechten Assistenzsysteme oder zumindest pflegenaher digitaler Technik durchgeführt wurden und werden, zu schaffen, wurde in dem Projekt DAAS-KIN eine umfangreiche Suche durchgeführt. Dabei wurden ausschließlich durch das BMBF geförderte Projekte berücksichtigt - diese Fokussierung ergibt sich aus den forschungsleitenden Fragen des Projekts DAAS-KIN im Rahmen der BMBF-Förderlinie »Innovations- und Technikanalyse (ITA)«.

Obwohl sich das Team nicht sicher ist, dass die unten im Anhang zu findende Liste wirklich vollständig ist, kann die Zahl der Projekte doch überraschen - es wurden mehr als 100 Projekte gefunden, die vom BMBF gefördert wurden oder aktuell (Stand Herbst 2020) gefördert werden. Soweit es möglich war, wurden bei der Recherche auch Fördersummen erhoben. Hier gibt es ebenfalls gewisse Unsicherheiten, da nicht immer klar ist, ob einzelne Projekte zu größeren Fördervorhaben gehören und somit Projektbudgets womöglich doppelt gezählt wurden. Stellt man dies in Rechnung und rechnet einen gewissen Fehler ein, so beträgt die Gesamtfördersumme der im Anhang dieses Textes aufgeführten Projekte mindestens 200 Millionen und maximal rund 240 Millionen Euro. Zu betonen ist, dass dies die Fördersumme des BMBF für Projekte darstellt, die im Zeitraum von 2009 bis 2023 durchgeführt beziehungsweise zum Recherchezeitraum genehmigt wurden und bereits im Internet zu finden waren. $\mathrm{Zu}$ dieser Summe müssen Projektförderungen hinzugerechnet werden, die beispielsweise vom Bundesgesundheitsministerium (BMG) oder von den Forschungs- und Gesundheitsministerien der Länder finanziert wurden; dazu kommen Mittel aus der europäischen F\&E-Förderung, der Wirtschaftsministerien des Bundes und der Länder, möglicherweise aus dem Innovationsfonds des Gemeinsamen Bundesausschusses (G-BA), von Stiftungen oder rein privatwirtschaftlich finanzierten F\&E-Aktivitäten - vermutlich ist diese Aufzählung ebenfalls nicht vollständig.

Trotz dieser hohen Fördersummen allein durch das BMBF muss der Ertrag daraus als eher ernüchternd eingeschätzt werden. Die Zahlen, die im Rahmen des Projekts DAAS-KIN erhoben wurden (vgl. Haug in diesem Band), deuten darauf hin, dass viele Pflegeeinrichtungen nicht einmal über eine Digitalisierungsstrategie verfügen, also keine systematische Planung bezüglich der Ausstattung mit und des Einsatzes von altersgerechten Assistenzsystemen durchführen. Viele Typen von Systemen werden gar nicht oder extrem selten eingesetzt - dies gilt insbesondere für die Robotik. Somit ist es wenig verwunderlich, wenn Schmidt/Wahl (2019: 546) folgendes Fazit ziehen:

»Wenn man sich auf der einen Seite die sehr hohe Zahl an bislang in Deutschland (und Europa) geförderten Gero-Technologie-Forschungsprojekten (samt den Dutzenden Millionen an Fördergeldern) vor Augen führt und sich auf der anderen Seite die Frage stellt, wo zwischenzeitlich vielversprechende Techniklösungen 
nachhaltig Einzug in den Alltag älterer Menschen gehalten haben, dann fällt die Bilanz noch verbesserungsbedürftig aus. Was ist wirklich bislang dort sangekommen«?

Die Antwort scheint >wenig` zu sein. Die öffentliche Förderung hat daran augenscheinlich wenig geändert. Die Faktoren, die dazu beitragen, dass die Diffusion altersgerechter Assistenzsysteme so zögerlich verläuft, sind vielfältig; die Beiträge im vorliegenden Sammelband weisen hier eine Gemengelage aus ökonomischen, technischen, sozialen und ethischen Aspekten aus. Die Empirie, die im Rahmen des Projekts DAAS-KIN durchgeführt wurde, deutet aber vor allem eines an: Die Probleme in der Praxis sind sehr basal, denn oft scheint die Einführung von AALSystemen bereits am Fehlen von Grundvoraussetzungen wie dem Vorhandensein zuverlässiger Wireless-LAN-Systeme zu scheitern. Die mangelnde Interoperabilität und Kompatibilität der Systeme verschiedener Hersteller*innen und Anwendungsbereiche wird ebenso genannt (vgl. Haug in diesem Band); dies deutet darauf hin, dass es mindestens einen dritten Teufelskreis gibt, der durch fehlende Standardisierung charakterisiert ist. Da aber nur wenige Systeme in Betrieb sind, lohnen sich Standardisierungsprozesse aus Sicht der Hersteller"innen womöglich nicht, sodass sich die Situation nicht verbessert und damit ein weiteres dauerhaftes Diffusionshemmnis existiert.

Die staatliche Förderpolitik konnte den gordischen Knoten der zahlreichen Diffusionshemmnisse also noch nicht oder zumindest nicht vollständig zerschlagen. Man kann das exemplarisch an der Robotik aufzeigen: Als eine Folge der paradoxen Befundlage in der Online-Befragung mit unrealistisch hohen Erwartungen vonseiten der befragten Pflegeführungskräfte an Robotik-Systeme bei gleichzeitiger Ablehnung des Einsatzes solcher Geräte in der Pflegepraxis können berechtigte Zweifel geäußert werden, ob dieser bisher recht umfangreich geförderte Zweig der Forschung und Entwicklung Systeme produzieren wird, die auf Akzeptanz bei ambulanten und stationären Pflegeeinrichtungen stoßen werden. Diese Akzeptanz wäre jedoch notwendig, damit eine flächendeckende Diffusion stattfinden könnte und die oben angesprochenen Teufelskreise in Bezug auf Robotik in der Pflege durchbrochen werden könnten. Die bisherige Förderpolitik in Bezug auf Robotik in der Pflege sollte daher vermutlich überdacht werden - nicht im Sinne eines grundsätzlichen Stopps, sondern einer stärker auf Einsatzfähigkeit in der Praxis gerichteten Forschung und Entwicklung.

Eine weitere Empfehlung könnte aus einem Projekt abgeleitet werden, das womöglich stellvertretend für viele andere ähnlich ausgerichtete Vorhaben stehen kann: Ein Teil des DAAS-KIN-Teams ist in dem durch das bayerische Staatsministerium für Gesundheit und Pflege (StMGP) finanzierte Projekt TePUS, in dem sogenannte Telepräsenzroboter auf Praxistauglichkeit in der ambulanten Pflege getestet werden (siehe https://www.deinhaus40.de/start/). Allerdings wird dabei keine Ent- 
wicklung betrieben, sondern auf am Markt erhältliche Geräte, Software und Apps zurückgegriffen, um eine rasche Umsetzbarkeit in den (Pflege-)Alltag zu erreichen; es geht also nicht um Forschung und Entwicklung im üblichen Sinne, sondern eher um Integration und Evaluation. Erste Rückmeldungen der Praxispartner*innen (u.a. Pflegeeinrichtungen, andere Dienstleistungsunternehmen, herstellende Unternehmen altersgerechter Assistenzsysteme, Krankenkassen) des Projekts lassen vermuten, dass Pflegeeinrichtungen konkrete und möglichst sofort einsatzbare Lösungen erwarten und benötigen; weitere Proof-of-Concept-Projekte werden von diesen Partner*innen hingegen sehr skeptisch betrachtet. Dies gilt im Übrigen nicht nur für Pflegerobotik, sondern generell für altersgerechte Assistenzsysteme. Aus diesen Erfahrungen heraus erscheint es zumindest überlegenswert, ob eine entsprechende (partielle) Neuausrichtung der F\&E-Förderung beispielsweise des BMBF, aber sicherlich auch anderer Förderinstitutionen, einen Beitrag dazu leisten könnte, bestehende Diffusionshemmnisse der Praxis aus dem Weg zu räumen und damit die angesprochenen Teufelskreise zu durchbrechen.

\section{Die Grenzen des Machbaren}

Angesichts der Ergebnisse, die das Projekt DAAS-KIN produziert hat, scheint es nicht übertrieben zu sein, dass dem Einsatz altersgerechter Assistenzsysteme sowohl in der Praxis als auch in akademischen Debatten erhebliche Skepsis entgegengebracht (vgl. Gransche 2017; Selke 2017; außerdem Remmers und Hergesell et al. in diesem Band) und sich dies auch nicht so bald ändern wird. In Hinblick auf die derzeitigen Leistungen und Einsatzmöglichkeiten existierender AAL-Systeme ist zumindest eine zurückhaltende Sichtweise sogar begründbar, denn wenn man die ursprünglichen Visionen altersgerechter Assistenzsysteme, die den Menschen mit Pflegebedarf ein selbstständiges Leben im eigenen Heim ermöglichen sollten, mit den derzeit auf dem Markt verfügbaren Systemen vergleicht, die im Wesentlichen darauf ausgerichtet sind, die Arbeit von Pflegekräften zu erleichtern (was, wenn es funktioniert, auf keinen Fall gering geschätzt werden darf), dann zeigt sich eine erhebliche Diskrepanz. Dies spiegelt sich in eindrücklicher Weise in den Interviews wider, die in DAAS-KIN geführt wurden (vgl. Scorna in diesem Band). Doch es wäre problematisch, den aktuellen technischen Stand als grundsätzliches Argument gegen den Einsatz zukünftiger Technik zu nutzen; denn so zu argumentieren impliziert, die Möglichkeit des technischen und organisatorischen Fortschritts generell zu verneinen. Die Tatsache, dass existierende altersgerechte Assistenzsysteme derzeit (noch) nicht die Erwartungen der verschiedenen Stakeholder*innen (vollständig) erfüllen können, ist für sich allein noch kein schlüssiges Argument gegen die Hoffnung, dass zukünftige Technik hier besser abschneiden könnte. 
Manche der Aussagen in den DAAS-KIN-Interviews können dahingehend gedeutet werden, dass viele Argumente gegen den Einsatz altersgerechter Assistenzsysteme, die mit dem Hinweis auf das Wohl der Gepflegten geäußert werden, eher dem Schutz der jeweiligen Stakeholder*innen-Interessen dienen. Dass beispielsweise Pflegekräfte - hier nur stellvertretend für die verschiedenen Stakeholder*innen-Gruppen - ihre Interessen vertreten, ist völlig legitim; es bedarf eines Interessenausgleichs, der von allen Beteiligten mitgetragen werden kann. Dass sich die Pflege als Profession durch den Einsatz von Technik im Allgemeinen und von AAL-Systemen im Speziellen verändern wird, kann schwerlich bestritten werden. Ob die Einführung einer bestimmten Technologie die Dinge zum Guten oder zum Schlechten verändern wird, ist in der Regel im Vorfeld unklar. Nur sollte man auch nicht mit zweierlei Maß messen: Aus der Sicht der Technikfolgenabschätzung ist beispielsweise schwer verständlich, warum eine technische Hebe- und/oder Wendehilfe oder hochtechnisierte Betten, die helfen einen Dekubitus zu verhindern, anders bewertet werden als beispielsweise altersgerechte Assistenzsysteme, die bestimmte bisher manuell durch Pflegekräfte erbrachte Leistungen erbringen. In beiden Fällen findet eine Veränderung der Pflege und des Verhältnisses zwischen pflegender und gepflegter Person statt. Unterschiede, die zu verschiedenen Bewertungen führen, müssen empirisch belegt werden.

Die Lektüre nicht zuletzt der im vorliegenden Sammelband $\mathrm{zu}$ findenden Beiträge lässt zudem vermuten, dass viele Stakeholder*innen eine implizite, aber nichtsdestoweniger wirkmächtige Annahme treffen: Ökonomisches Denken einerseits und das Festhalten an einem bestimmten Idealbild der Pflege andererseits widersprechen sich (vgl. Frommeld in diesem Band). Vermutlich wäre eine solche Annahme dann richtig, wenn man davon ausginge, dass der Pflege die benötigten Ressourcen vorenthalten werden würden, obwohl diese gesellschaftlich unproblematisch zur Verfügung gestellt werden könnten. Doch so zu denken würde bedeuten eine in der realen Welt geltende Randbedingung zu verkennen, denn die für Gesundheit und Pflege zur Verfügung stehenden Ressourcen sind doppelt begrenzt:

1 Es existiert die prinzipielle Limitierung der verfügbaren Ressourcen, da jede Volkswirtschaft nur eine bestimmte Wertschöpfung erzielen kann und eine unbegrenzte Verschuldung nicht möglich ist (und nebenbei einen massiven intergenerationellen Gerechtigkeitskonflikt heraufbeschwören würde).

2 Es wäre zudem nicht durchführbar, alle verfügbaren Ressourcen ausschließlich für Gesundheit und Pflege zu nutzen, da es weitere gesellschaftliche Aufgaben gibt, die finanziert werden müssen, so beispielsweise Bildung, Justiz, Infrastruktur oder Verteidigung (Childress 1989). Dem Statistischen Bundesamt zufolge wurde 2017 die Schwelle von einer Milliarde Euro pro Tag für Gesundheits- 
und Pflegeausgaben überschritten (Destatis 2019: 150); das entspricht mehr als elf Prozent des Bruttoinlandsproduktes (BIP) beziehungsweise jeder neunte in Deutschland erwirtschaftete Euro wurde 2017 für Pflege und Gesundheit ausgegeben. Der Anteil der öffentlichen Bildungsausgaben am BIP lag in Deutschland 2017 bei 4,2 Prozent (Destatis 2020b), für Verteidigung bei 1,3 Prozent (für das Jahr 2019, Destatis 2020c). Gerade in Hinblick auf Bildung manifestieren sich hier potenzielle intergenerationelle Gerechtigkeitskonflikte.

Daher können Vorstellungen darüber, wie die (zukünftige) Pflege gestaltet werden soll, grundsätzlich nicht von ökonomischen Rahmenbedingungen und von der Frage, welche gesellschaftlichen Bereiche in welcher Höhe an den begrenzten Ressourcen teilhaben sollen, abgekoppelt werden. Anders ausgedrückt: Der Einsatz altersgerechter Assistenzsysteme, ob man das will oder nicht, ist mit grundlegenden Fragen der Gerechtigkeit innerhalb und zwischen den Generationen verbunden, mit Fragen nach der Verteilung von Lasten auf gesellschaftlicher, familiärer und individueller Ebene, mit Fragen der Fairness zwischen den Generationen, Geschlechtern, Einkommensklassen oder Beschäftigtengruppen.

Die aktuelle Pflegesituation, die sich nicht erst seit COVID-19 durch schlechte Bezahlung, psychisch wie physisch belastende Arbeit, ungünstige Arbeitszeiten und Geschlechterungerechtigkeit (vgl. Rudolph in diesem Band) auszeichnet, $\mathrm{zu}$ verbessern, muss ohne jeden Zweifel ein gesellschaftliches Ziel sein. Es muss aber auch klar sein, dass die Schaffung der zusätzlichen Pflegestellen, wie sie derzeit diskutiert und gefordert werden (vgl. Remmers in diesem Band), nicht mit Detailänderungen im Steuer- und Abgabensystem der Bundesrepublik Deutschland $\mathrm{zu}$ erreichen wären, sondern tiefe und systemwechselnde Eingriffe in den Arbeitsmarkt, in Besitzverhältnisse und vermutlich auch in die individuelle Gestaltung von Lebensläufen mit sich brächte. Vorschläge in Richtung einer weiteren deutlichen Erhöhung der Abgabenlast zur Finanzierung angemessener Entgelte in der Pflege oder Ideen wie die Einführung eines verpflichtenden allgemeinen Pflegejahrs sind vermutlich politisch und gesellschaftlich nicht durchsetzbar - nicht zuletzt, weil sie bestehende Probleme und Ungerechtigkeiten nicht lösen, sondern durch Schaffung neuer Belastungen nur auf andere Bevölkerungsgruppen verschieben oder gar neue Unfairness erzeugen würden (vgl. Frommeld in diesem Band). 


\section{Schlussfolgerung}

Ohne Systemwechsel wird die Pflege jedoch den gleichen radikalen Rationalisierungsprozessen unterworfen sein, wie sie alle anderen primären, sekundären und tertiären Wertschöpfungsketten seit der ersten industriellen Revolution durchlaufen haben und immer noch durchlaufen. Es gibt eine erhebliche gesellschaftliche wie individuelle Nachfrage nach Pflege, aber offenkundig weder die Möglichkeit noch Bereitschaft, dafür wesentlich mehr Ressourcen aufzubringen, als dies jetzt schon der Fall ist. Im Rahmen einer (sozialen) Marktwirtschaft werden die Leistungserbringenden daher unweigerlich den Weg der Kostenreduzierung gehen müssen. In diesem Sinne erbringen Pflege- und Gesundheitsversorgung Dienstleistungen beziehungsweise stellen Güter her, deren Produktion mithilfe von Technik im Allgemeinen und altersgerechten Assistenzsystemen im Speziellen effizienter gestaltet werden kann. Man kann diese Sicht mit guten Argumenten beklagen (vgl. Hellige/Meilwes/Seidel 2018), aber muss dann auch bereit und fähig sein, gangbare Alternativen aufzuzeigen, die Probleme nicht nur verschieben-beispielsweise in die Zukunft und/oder zwischen den Generationen - sondern tatsächlich lösen. Pflege- und Gesundheitsversorgung als Dienstleistungsbetrieb $\mathrm{zu}$ verstehen rührt mit Sicherheit nicht nur am Selbstverständnis der beteiligten Professionen, sondern auch an tief verankerten gesellschaftlichen Überzeugungen - man kann aber auch sagen an idealisierten Vorstellungen über Pflege, die schon lange nicht mehr, vielleicht sogar noch nie, der Realität entsprechen und entsprachen.

Man kann das Verständnis der Pflege- und Gesundheitsversorgung als Dienstleistungsbetrieb als neoliberal bezeichnen (vgl. Auth 2012; Krampe 2014), doch das wäre keine sachliche Auseinandersetzung, sondern die Nutzung von Kampfbegriffen. Schaut man sich stattdessen die Interviews, die im Rahmen des Projekts DAAS-KIN geführt wurden (vgl. Scorna in diesem Band), ebenso wie die Auswertung der erhobenen quantitativen Daten (vgl. Haug in diesem Band) sowie die Diskurs- und modifizierte Wertbaumanalyse (vgl. Frommeld in diesem Band) an, so zeigt sich darin ein hoher Gestaltungswille aufseiten der Pflegekräfte und der Pflegeleitungen. Diesen zur Geltung zu bringen, könnte dabei helfen, den Umbruch, den der breite Einsatz altersgerechter Assistenzsysteme in der Pflege mit sich bringen wird, in einer Weise zu gestalten, dass alle Stakeholder*innenInteressen berücksichtigt werden und eine angemessene Pflege $\mathrm{zu}$ angemessenen Bedingungen für alle Beteiligten erreicht werden können. Das Festhalten an überkommenen idealisierten Vorstellungen der Pflege wird das hingegen nicht leisten können. 


\section{Literatur}

Akter, Shahriar/Ray, Pradeep (2010): "mHealth - An ultimate platform to serve the unserved «, in: Yearbook of Medical Informatics, 19, S. 94-100. DOI: 10.1055/s0038-1638697

Auth, Diana (2012): »Ökonomisierung von Pflege in Großbritannien, Schweden und Deutschland «, in: Zeitschrift für Gerontologie und Geriatrie, 45, S. 618-623. DOI: $10.1007 /$ s00391-012-0389-0

Bauer, Silvia/Eglseer, Doris/Hödl, Manuela (2020): »Pflege während der COVID-19 Pandemie: Eine besondere Herausforderung«, in: ProCare, 25, S. 48-53. DOI: 10.1007/s00735-020-1247-8

Beigl, Michael/Gellersen, Hans-W./Schmidt, Albrecht (2001): »Mediacups: Experience with design and use of computer-augmented everyday artefacts", in: Computer Networks, 35, S. 401-409. DOI: 10.1016/S1389-1286(00)00180-8

Benford, Gregory/Malartre, Elisabeth (2008): Beyond human: Living with robots and cyborgs, New York: Tom Doherty Associates.

Bohlken, Jens/Schömig, Friederike/Lemke, Matthias R./Pumberger, Matthias/Riedel-Heller, Steffi G. (2020): »COVID-19 pandemic: Stress experience of healthcare workers - a short current review«, in: Psychiatrische Praxis, 47, S. 190-197. DOI: 10.1055/a-1159-555

Bonin, Holger (2014): Der Beitrag von Ausländern und künftiger Zuwanderung zum deutschen Staatshaushalt, http://ftp.zew.de/pub/zew-docs/gutachten/ZEW_B eitragZuwanderungStaatshaushalt2014.pdf (Abfrage: 07.01.2021).

Bostrom, Nick (2005): »A history of transhumanist thought«, in: Journal of Evolution and Technology, 14, S. 1-25. https://jetpress.org/volume14/bostrom.pdf.

Brereton, Margot (2001): »Drawing lessons in the design of tangible media from a study of interactions with mechanical products «, in: Proceedings of the 2 nd Australasian conference on user interface, Queensland: IEEE Computer Society, S. 124-131. DOI: $10.5555 / 545640.545663$

Butterwegge, Christoph (2004): »Bürgerversicherung - Patentrezept für das Gesundheitswesen?«, in: KrV Kranken- und Pflegeversicherung, 8, S. 206-209. DOI: $10.37307 / \mathrm{j} .2193-5661.2004 .08 .09$

Catlaw, Thomas J./Sandberg, Billie (2018): »The quantified self and the evolution of neoliberal self-government: An exploratory qualitative study", in: Administrative Theory \& Praxis, 40, S. 3-22. DOI: 10.1080/10841806.2017.1420743

Cheng, Kai-Yin/Liang, Rong-Hao/Chen, Bing-Yu/Laing, Rung-Huei/Kuo, Sy-Yen (2010): »iCon. Utilizing everyday objects as additional, auxiliary and instant tabletop controllers «, in: Proceedings of the $28_{\text {th }}$ international conference on human factors in computing systems, Atlanta/Georgia: ACM, S. 1155-1164. DOI: 10.1145/1753326.1753499 
Childress, James F. (1989): »Prioritäten in der Gesundheitsfürsorge«, in: Sass, HansMartin (Hg.), Medizin und Ethik, Stuttgart: Reclam, S. 311-327.

Danter, Stefan/Reichardt, Ulfried/Schober, Regina (2016): »Theorising the quantified self and posthumanist agency. Self-knowledge and posthumanist agency in contemporary US-American literature«, in: Digital Culture and Society, 2, S. 53-67. DOI: $10.14361 / \mathrm{dcs}-2016-0105$

Destatis (2019a): Statistisches Jahrbuch 2019 - Kapitel Gesundheit, https:/www.d estatis.de/DE/Themen/Querschnitt/Jahrbuch/jb-gesundheit.pdf?_blob=publi cationFile (Abfrage: 20.01.2021).

Destatis (2019b): Umsatz mit Smartphones weltweit in den Jahren 2013 bis 2018, h ttps:/de.statista.com/themen/581/smartphones/(Abfrage: 20.01.2021).

Destatis (2020a): Kohortensterbetafeln für Deutschland. Ergebnisse aus den Modellrechnungen für Sterbetafeln nach Geburtsjahrgang, https://www.desta tis.de/DE/Themen/Gesellschaft-Umwelt/Bevoelkerung/Sterbefaelle-Lebenser wartung/Publikationen/Downloads-Sterbefaelle/kohortensterbetafeln-5126101 209004.pdf?_blob=publicationFile (Abfrage: 07.01.2021).

Destatis (2020b): Anteil der Ausgaben der öffentlichen Haushalte in Deutschland für Bildung am Bruttoinlandsprodukt von 1995 bis 2020, https://de.statista.co $\mathrm{m} /$ statistik/daten/studie/161321/umfrage/anteil-der-oeffentlichen-bildungsau sgaben-am-bip/(Abfrage: 20.01.2021).

Destatis (2020c): Anteil der Militärausgaben am jeweiligen Bruttoinlandsprodukt (BIP) der 15 Länder mit den höchsten Militärausgaben im Jahr 2019, https://de.statista.com/statistik/daten/studie/150664/umfrage/anteil-der -militaerausgaben-am-bip-ausgewaehlter-laender/(Abfrage: 21.01.2021).

Fachinger, Uwe/Koch, Hellen/Henke, Klaus-Dirk/Troppens, Sabine/Braeseke, Grit/ Merda, Meiko (2012): Ökonomische Potenziale altersgerechter Assistenzsysteme. Ergebnisse der "Studie zu Ökonomischen Potenzialen und neuartigen Geschäftsmodellen im Bereich Altersgerechte Assistenzsysteme«, https://part ner.vde.com/bmbf-aal/Publikationen/studien/intern/Documents/VDE_PP_A AL_\%C3\%96kon.\%20Potenziale_RZ_oB.pdf (Abfrage: 14.01.2021).

Fleisch, Elgar/Mattern, Friedemann (Hg.) (2005): Das Internet der Dinge - Ubiquitous Computing und RFID in der Praxis, Berlin: Springer. DOI: 10.1007/3-54028299-8

Flesche, Christian W./Jalowy, Andreas/Inselmann, Gerhard (2004): »Telemedizin in der Hochseeschifffahrt? Hightech aus Tradition«, in: Medizinische Klinik, 99, S. 163-168. DOI: 10.1007/s00063-004-1026-5

Fozouni, Behnam/Güntert, Bernhard (2000): »Prioritätensetzung im deutschen Gesundheitswesen - die Triade zwischen Rationierung, Rationalisierung und rationaler Allokation«, in: Das Gesundheitswesen, 62, S. 559-567. DOI: 10.1055/s-2000-13043 
Gersch, Martin/Liesenfeld, Joachim (Hg.) (2012): AAL- und E-Health-Geschäftsmodelle, Wiesbaden: Gabler. DOI: 10.1007/978-3-8349-3521-2

Gransche, Bruno (2017): »Wir assistieren uns zu Tode«, in: Biniok, Peter/Lettkemann, Eric (Hg.), Assistive Gesellschaft, Wiesbaden: Springer, S. 77-98. DOI: 10.1007/978-3-658-13720-5_4

Greß, Stefan/Lüngen, Markus (2017): »Die Einführung einer Bürgerversicherung: Überwindung des ineffizienten Systemwettbewerbs zwischen GKV und $\mathrm{PKV}$ «, in: Gesundheits- und Sozialpolitik, 71, S. 68-74. DOI: 10.5771/1611-5821-2017-34-68

Hellige, Barbara/Meilwes, Michael/Seidel, Sabine (2018): »Digitalisierung und Sorgeverhältnisse - ein unauflöslicher Widerspruch?«, in: Pfannstiel, Mario A./Krammer, Sandra/Swoboda, Walter (Hg.), Digitale Transformation von Dienstleistungen im Gesundheitswesen IV, Wiesbaden: Springer, S. 113-133. DOI: $10.1007 / 978-3-658-13644-4$

Höpflinger, François (2007): »Ausdehnung der Lebensarbeitszeit und die Stellung älterer Arbeitskräfte - Perspektiven aus Sicht einer differenziellen Alternsforschung«, in: Pasero, Ursula/Backes, Gertrud M./Schroeter, Klaus R. (Hg.), Altern in Gesellschaft, Wiesbaden: Springer, S. 307-343. DOI: 10.1007/978-3-53190416-0_14

Hower, Kira Isabel/Pfaff, Holger/Pförtner, Timo-Kolja (2020): »Pflege in Zeiten von COVID-19: Onlinebefragung von Leitungskräften zu Herausforderungen, Belastungen und Bewältigungsstrategien«, in: Pflege, 33, S. 207-218. DOI: 10.1024/1012-5302/a000752

Kabisch, Eric (2008): »Datascape: A synthesis of digital and embodied worlds«, in: Space and Culture, 11, S. 222-238. DOI: 10.1177/1206331208319147

Kaufmann, Franz-Xaver/Leisering, Lutz (1984): »Demographische Veränderungen als Problem für soziale Sicherungssysteme«, in: Internationale Revue für soziale Sicherheit, 37, S. 429-452.

Krampe, Eva-Maria (2014): »Professionalisierung der Pflege im Kontext der Ökonomisierung«, in: Manzei, Alexandra/Schmiede, Rudi (Hg.), 20 Jahre Wettbewerb im Gesundheitswesen, Wiesbaden: Springer Fachmedien, S. 179-197. DOI: 10.1007/978-3-658-02702-5_8

Langer, Bernhard/Mamberer, Florian/Pfaff, Anita B./Pfaff, Martin/Freund, Florian/Rindsfüßer, Christian (2004): »Beitragssatzeffekte bei sofortiger Einführung einer Bürgerversicherung«, in: Gesundheits- und Sozialpolitik, 58, S. 4450. https://www.jstor.org/stable/26890543

Lee, Seung Hak (2019): »Upper extremity rehabilitation for stroke patients using fully immersive virtual reality game: A preliminary study«, in: Archives of Physical Medicine and Rehabilitation, 100, S. e138-e139. DOI: 10.1002/pmrj.12206 
Lehr, Ursula/Kruse, Andreas (2006): »Verlängerung der Lebensarbeitszeit - eine realistische Perspektive? «, in: Zeitschrift für Arbeits- und Organisationspsychologie A\&O, 50, S. 240-247. DOI: 10.1026/0932-4089.50.4.240

Leis, Alexander (2008): »Telemedizin heute«, in: Der Unfallchirurg, 111, S. 146-154. DOI: $10.1007 /$ s00113-008-1416-x

Lübbers, Annette (2017): »Pflegenotstand: Hilft nur noch beten?«, in: Heilberufe, 69, S. 48-50. DOI: $10.1007 /$ s00058-017-2973-6

Mack, Elke (2001): »Rationierung im Gesundheitswesen - ein wirtschafts- und sozialethisches Problem«, in: Ethik in der Medizin, 13, S. 17-32. DOI: 10.1007/s004810100115

Mann, Steve (1997): „Wearable computing: A first step toward personal imaging«, in: Computer, 30, S. 25-32. DOI: 10.1109/2.566147

Mann, Steve (2004): ")Sousveillanceく: Inverse surveillance in multimedia imaging«, in: Proceedings of the 12 th annual ACM international conference on multimedia, New York/New York: ACM, S. 620-627. DOI: 10.1145/1027527.1027673

McCarthy, Carol (1987): »The money we spend and its sources«, in: Brody, Baruch A./Engelhardt Jr., H. Tristam (Hg.), Bioethics: Readings \& cases, Englewood Cliffs/New Jersey: Prentice-Hall, S. 206-213.

McGookin, David/Robertson, Euan/Brewster, Stephen (2010): »Clutching at straws: Using tangible interaction to provide non-visual access to graphs«, in: Proceedings of the $28_{\text {th }}$ international conference on human factors in computing systems, Atlanta/Georgia: ACM, S. 1715-1724. DOI: 10.1145/1753326.1753583

Merrell, Ronald C. (2005): »Telemedicine in surgery«, in: European Surgery, 37, S. 270-273. DOI: 10.1007/s10353-005-0179-7

Meyer, Sibylle (2016): Technische Unterstützung im Alter - was ist möglich, was ist sinnvoll? Expertise zum Siebten Altenbericht der Bundesregierung, http://nbn -resolving.de/urn:nbn:de:0168-ssoar-49980-9 (Abfrage: 14.01.2021).

Meystre, Stephane (2005): »The current state of telemonitoring: A comment on the literature«, in: Telemedicine and e-Health, 11, S.63-69. DOI: 10.1089/tmj.2005.11.63

Neitzke, Peter/Calmbach, Marc/Behrendt, Dieter/Kleinhückelkotten, Silke/Wegner, Elisabeth/Wippermann, Carsten (2008): »Risks of ubiquitous information and communication technologies«, in: GAIA - Ecological Perspectives for Science and Society, 17, S. 362-369. DOI: 10.14512/gaia.17.4.11

Oduncu, Fuat S. (2012): »Verteilungsgerechtigkeit, Rationierung und Priorisierung - das Gesundheitswesen im Spannungsfeld zwischen Medizin, Ökonomie, Ethik und Recht«, in: Medizinrecht, 30, S. 359-367. DOI: 10.1007/s00350012-3162-x

Oh, Hans/Rizo, Carlos/Enkin, Murray/Jadad, Alejandro (2005): „What is eHealth (3): A systematic review of published definitions «, in: Journal of Medical Internet Research, 7, S. 32-40. DOI: 10.2196/jmir.7.1.e1 
Olla, Phillip/Shimskey, Caley (2015): »mHealth taxonomy: A literature survey of mobile health applications«, in: Health and Technology, 4, S. 299-308. DOI: 10.1007/s12553-014-0093-8

Park, Sang Hyun/Won, So Hee/Lee, Jong Bong/Kim, Sung Woo (2003): »Smart home - digitally engineered domestic life«, in: Personal and Ubiquitous Computing, 7, S. 189-196. DOI: 10.1007/s00779-003-0228-9

Rekimoto, Jun/Ayatsuka, Yuji (2000): „CyberCode: Designing augmented reality environments with visual tags", in: Proceedings of DARE 2000 on designing augmented reality environments, Elsinore/Denmark: ACM, S. 1-10. DOI: 10.1145/354666.354667

Rossmann, Constanze/Krömer, Nicola (2016): »mHealth in der medizinischen Versorgung, Prävention und Gesundheitsförderung «, in Fischer, Florian/Krämer, Alexander (Hg.), eHealth in Deutschland, Berlin: Springer, S. 441-456. DOI: 10.1007/978-3-662-49504-9_24

Sahmel, Karl-Heinz (2018): »Pflegenotstand - ist das Ende der Menschlichkeit erreicht? «, in: Pflegezeitschrift, 71, S. 18-22. DOI: 10.1007/s41906-018-0535-4

Satava, Richard M. (1995): »Medical applications of virtual reality«, in: Journal of Medical Systems, 19, S. 275-280. DOI: 10.1007/BFo2257178

Satava, Richard M. (2005): »Telesurgery, robotics, and the future of telemedicine«, in: European Surgery, 37, S. 304-307. DOI: 10.1007/s10353-005-0185-9

Schelisch, Lynn (2016): Technisch unterstütztes Wohnen im Stadtquartier, Wiesbaden: Springer.

Schmähl, Winfried (1990): "Demographic change and social security: Some elements of a complex relationship«, in: Journal of Population Economics, 3, S. 159177. DOI: 10.1007/BFo0163073

Schmidt, Laura/Wahl, Hans Werner (2019): »Alter und Technik«, in: Hank, Karsten/Schulz-Nieswandt, Frank/Wagner, Michael/Zank, Susanne (Hg.), Alternsforschung. Handbuch für Wissenschaft und Praxis, Baden-Baden: Nomos, S. 537-555. DOI: 10.5771/9783845276687-537

Schulz, Reiner (2000): »Die Alterung der Weltbevölkerung«, in: Zeitschrift für Bevölkerungswissenschaft, 25, S. 267-289.

Schulz-Nieswandt, Frank (1990): Stationäre Altenpflege und »Pflegenotstand « in der Bundesrepublik Deutschland. Sozialökonomische Schriften, Bd. 2, Frankfurt a.M.: Peter Lang.

Selke, Stefan (2017): »Assistive Kolonialisierung. Von der >Vita activa $<$ zur >Vita assistiva «, in: Biniok, Peter/Lettkemann, Eric (Hg.), Assistive Gesellschaft, Wiesbaden: Springer, S. 99-119. DOI: 10.1007/978-3-658-13720-5_5

Shaer, Orit/Kol, Guy/Strait, Megan/Fan, Chloe/Grevet, Catherine/Elfenbein, Sarah (2010): »G-nome surfer: A tabletop interface for collaborative exploration of genomic data «, in: Proceedings of the $28_{\text {th }}$ international conference on hu- 
man factors in computing systems, Atlanta/Georgia: ACM, S. 1427-1436. DOI: 10.1145/1753326.1753539

Shaer, Orit/Leland, Nancy/Calvillo-Gamez, Eduardo H./Jacob, Robert J. K. (2004): »The TAC paradigm: Specifying tangible user interfaces«, in: Personal Ubiquitous Computing, 8, S. 359-369. DOI: 10.1007/s00779-004-0298-3

Skorning, Max/Bergrath, Sebastian/Brokmann, Jörg C./Rörtgen, Daniel/Beckers, Stefan K./Rossaint, Rolf (2011): »Stellenwert und Potenzial der Telemedizin im Rettungsdienst «, in: Notfall + Rettungsmedizin, 14, S. 187-191. DOI: 10.1007/s10049-010-1397-5

Wall, Steven/Brewster, Stephen (2005): »Hands-on haptics: exploring non-visual visualization using the sense of touch «, in: CHI'05 extended abstracts on human factors in computing systems, Portland/Oregon: ACM, S. 2140-2141. DOI: 10.1145/1056808.1057127

Warwick, Kevin (2003): „Cyborg morals, cyborg values, cyborg ethics«, in: Ethics and Information Technology, 5, S. 131-137. DOI: 10.1023/B:ETIN.0000006870.65865.cf

Weber, Karsten (2015): »Alternative Benutzerschnittstellen als Möglichkeit der Kompensation sensorischer Handicaps «, in: Kerkmann, Friederike/Lewandowski, Dirk (Hg.), Barrierefreie Informationssysteme, Berlin: de Gruyter, S. 49-70. DOI: 10.1515/9783110337297-004

Weber, Karsten/Nagenborg, Michael/Drüeke, Ricarda/Langewitz, Oliver (2009): »Ubiquitous Media - Ökonomische und technische Rahmung sozialer Handlungsmöglichkeiten«, in: Merz Medien + Erziehung, Zeitschrift für Medienpädagogik, 53, S. 102-110.

Weiser, Mark (1991): »The computer for the 21st century«, in: Scientific American, 265, S. 94-104.

Weizsäcker, Robert K. von (1989a): »Demographischer Wandel und staatliche Einkommenssicherung: Eine Inzidenzanalyse«, in: Jahrbücher für Nationalökonomie und Statistik, 206, S. 181-207. DOI: 10.1038/scientificamericano991-94

Weizsäcker, Robert K. von (1989b): »Demographic change and income distribution«, in: European Economic Review, 33, S. 377-88. DOI: 10.1515/jbnst-19890302

Weizsäcker, Robert K. von (1989c): »Demographischer Wandel, Staatshaushalt und Einkommensverteilung«, in: Schriften des Vereins für Socialpolitik, 187, S. 31-84.

Wolf-Ostermann, Karin/Schmidt, Annika/Preuß, Benedikt/Heinze, Franziska/Seibert, Kathrin/Friedrich, Anna-Carina/Domhoff, Dominik/Stolle, Claudia/Rothgang, Heinz (2020): »Pflege in Zeiten von Corona: Ergebnisse einer deutschlandweiten Querschnittbefragung von ambulanten Pflegediensten und teilstationären Einrichtungen«, in: Pflege, 33, S. 277-288. DOI: 10.1024/1012$5302 / a 000761$ 


\section{Danksagung}

Der Beitrag entstand im Rahmen des durch das Bundesministerium für Bildung und Forschung (BMBF) geförderten Projekts »Diffusion altersgerechter Assistenzsysteme - Kennzahlenerhebung und Identifikation von Nutzungshemmnissen « (DAASKIN). Die Recherche nach den vom BMBF geförderten Entwicklungsprojekten wurde von Frau Nicla Kaufner durchgeführt, die im Projekt DAAS-KIN als studentische Hilfskraft wertvolle Arbeit geleistet hat. Im Namen des gesamten Teams möchte ich mich für die Unterstützung herzlich bedanken. 


\section{Anhang: BMBF-geförderte Projekte zur Entwicklung von AAL}

\begin{tabular}{|c|c|c|}
\hline Projekttitel & Projektleitung/Institution & $\begin{array}{l}\text { Lauf- } \\
\text { zeit }\end{array}$ \\
\hline $\begin{array}{l}\text { Humanzentriertes Assistenzsystem für Sicherheit und } \\
\text { Unabhängigkeit älterer, allein lebender Menschen } \\
\text { (aal@home) }\end{array}$ & $\begin{array}{c}\text { Paritätischer } \\
\text { Niedersachsen e.V., } \\
\text { Sozialzentrum Lüneburg }\end{array}$ & $\begin{array}{l}2009- \\
2012\end{array}$ \\
\hline $\begin{array}{l}\text { Altern lebenswert gestalten: selbstbestimmtes Wohnen } \\
\text { in der Einheit von technischer Lösung und Dienstleis- } \\
\text { tung (AlterLeben) }\end{array}$ & $\begin{array}{l}\text { Verband Sächsischer Woh- } \\
\text { nungsgenossenschaften } \\
\text { e.V. Dresden }\end{array}$ & $\begin{array}{l}2009- \\
2012\end{array}$ \\
\hline $\begin{array}{l}\text { Lange selbstbestimmt zu Hause leben durch situative } \\
\text { Assistenzsysteme und bedarfsgerechte Dienstleistun- } \\
\text { gen für pflegende Angehörige (easyCare) }\end{array}$ & $\begin{array}{l}\text { FZI Forschungszentrum } \\
\text { Informatik, Karlsruhe }\end{array}$ & $\begin{array}{l}2009- \\
2012\end{array}$ \\
\hline $\begin{array}{l}\text { Erhaltung der geistigen und körperlichen Fitness von Se- } \\
\text { nioren durch mikrosystemtechnisch unterstützte Moti- } \\
\text { vation zur körperlichen Aktivität (Motivotion60+) }\end{array}$ & $\begin{array}{l}\text { Vitaphone } \mathrm{CmbH} \text {, } \\
\text { Mannheim }\end{array}$ & $\begin{array}{l}2009- \\
2012\end{array}$ \\
\hline $\begin{array}{l}\text { Entwicklung und Integration von Informations- und } \\
\text { Kommunikationstechnologien zur Unterstützung der } \\
\text { häuslichen Versorgung von Menschen in ihrem letzten } \\
\text { Lebensjahr (PAALIATIV) }\end{array}$ & $\begin{array}{l}\text { Johanniter-Unfall-Hilfe } \\
\text { e.V., Regionalverband } \\
\text { Weser-Ems, Berne }\end{array}$ & $\begin{array}{l}2009- \\
2012\end{array}$ \\
\hline $\begin{array}{l}\text { Selbstbestimmt leben im Alter mit Mikrosystemtechnik } \\
\text { (SELBST) }\end{array}$ & $\begin{array}{l}\text { pme Familienservice } \\
\mathrm{CmbH} \text {, Berlin }\end{array}$ & $\begin{array}{l}2009- \\
2012\end{array}$ \\
\hline Sensorbetreutes Wohnen (sens@home) & $\begin{array}{l}\text { Bruderhaus Diakonie, } \\
\text { Reutlingen }\end{array}$ & $\begin{array}{l}2009- \\
2012\end{array}$ \\
\hline $\begin{array}{l}\text { Sensitiver Bodenbelag zur Unterstützung selbstständi- } \\
\text { gen Lebens im Alter (SensFloor) }\end{array}$ & $\begin{array}{l}\text { Future-Shape } \mathrm{GmbH} \text {, } \\
\text { Höhenkirchen- } \\
\text { Siegertsbrunn }\end{array}$ & $\begin{array}{l}2009- \\
2012\end{array}$ \\
\hline $\begin{array}{l}\text { Plattform zur Unterstützung von sozialen und gesund- } \\
\text { heitlichen Aspekten bei der Cestaltung eines altersge- } \\
\text { rechten autonomen Lebens (SmartAssist) }\end{array}$ & $\begin{array}{l}\text { Lübecker } \\
\text { Wachunternehmen, } \\
\text { Lübeck }\end{array}$ & $\begin{array}{l}2009- \\
2012\end{array}$ \\
\hline $\begin{array}{l}\text { Selbstständig, sicher, gesund und mobil im Alter (Smart- } \\
\text { Senior) }\end{array}$ & $\begin{array}{l}\text { AIS Automations- und } \\
\text { Informationssysteme } \\
\text { CmbH, Kassel }\end{array}$ & $\begin{array}{l}2009- \\
2012\end{array}$ \\
\hline $\begin{array}{l}\text { Versorgungseffizienz durch assistive, modulare Techno- } \\
\text { logien in bedarfsorientierten Szenarien (VAMOS) }\end{array}$ & $\begin{array}{l}\text { SOPHIA Living } \mathrm{CmbH} \text {, } \\
\text { Bamberg }\end{array}$ & $\begin{array}{l}2009- \\
2012\end{array}$ \\
\hline $\begin{array}{l}\text { Sensorbasiertes adaptives Monitoringsystem für die } \\
\text { Verhaltensanalyse von Senioren (SAMDY) }\end{array}$ & $\begin{array}{l}\text { Sozialwerk St. Ceorg e.V., } \\
\text { Celsenkirchen }\end{array}$ & $\begin{array}{c}2009- \\
2013\end{array}$ \\
\hline $\begin{array}{l}\text { Cesund und länger zu Hause leben durch systemüber- } \\
\text { greifende Vernetzung und altersgerechte Assistenzen } \\
\text { (Wohnselbst) }\end{array}$ & ECW mbH, Wiesbaden & $\begin{array}{c}2009- \\
2013\end{array}$ \\
\hline
\end{tabular}




\begin{tabular}{|c|c|c|}
\hline Projekttitel & Projektleitung/Institution & $\begin{array}{l}\text { Lauf- } \\
\text { zeit }\end{array}$ \\
\hline $\begin{array}{l}\text { Automatisierte Assistenz in Gefahrensituationen } \\
\text { (AUTAGEF) }\end{array}$ & ennovatis $\mathrm{GmbH}$, Großpösna & $\begin{array}{l}2010- \\
2013\end{array}$ \\
\hline $\begin{array}{l}\text { Assistenzsystem für Pflegenetzwerke zur Erfassung } \\
\text { von Aktivitäten und existenziellen Erfahrungen des } \\
\text { täglichen Lebens (Daily Care Journal) }\end{array}$ & euregon AG, Augsburg & $\begin{array}{l}2010- \\
2013\end{array}$ \\
\hline Gesund Wohnen mit Stil (GEWOS) & $\begin{array}{l}\text { ISA Informationssysteme für } \\
\text { computerintegrierte } \\
\text { Automatisierung } \mathrm{GmbH} \text {, } \\
\text { Stuttgart }\end{array}$ & $\begin{array}{l}2010- \\
2013\end{array}$ \\
\hline $\begin{array}{l}\text { Technisch-soziales Assistenzsystem für Komfort, Si- } \\
\text { cherheit, Gesundheit und Kommunikation im in- } \\
\text { nerstädtischen Quartier (TSA) }\end{array}$ & $\begin{array}{l}\text { Cemeinnützige } \\
\text { Baugenossenschaft Speyer, } \\
\text { Speyer }\end{array}$ & $\begin{array}{l}2010- \\
2013\end{array}$ \\
\hline $\begin{array}{l}\text { Webbasierte Dienste für ältere Menschen und An- } \\
\text { gehörige (WebDA) }\end{array}$ & $\begin{array}{l}\text { Phoenix Software } \mathrm{GmbH} \text {, } \\
\text { Bonn }\end{array}$ & $\begin{array}{l}2010- \\
2013\end{array}$ \\
\hline $\begin{array}{l}\text { Mobilitätsassistent zur Unterstützung bewegungs- } \\
\text { eingeschränkter Personen (MAID) }\end{array}$ & $\begin{array}{l}\text { KUKA Deutschland } \mathrm{GmbH} \text {, } \\
\text { Augsburg }\end{array}$ & $\begin{array}{r}2012- \\
2015\end{array}$ \\
\hline $\begin{array}{l}\text { Anwendung von akustischen und lautbasierten Er- } \\
\text { kennertechnologien zur Unterstützung pflegender } \\
\text { Dienstleister (AALADIN) }\end{array}$ & $\begin{array}{l}\text { Bosch Sicherheitssysteme } \\
\text { GmbH, Grasbrunn }\end{array}$ & $\begin{array}{r}2012- \\
2015\end{array}$ \\
\hline $\begin{array}{l}\text { Personalisiertes Assistenzsystem mit bedarfsopti- } \\
\text { miertem Akteurs-Netzwerk zur häuslichen Unter- } \\
\text { stützung und automatischen Situationserkennung } \\
\text { (PATRONUS) }\end{array}$ & $\begin{array}{l}\text { Bruderhaus } \\
\text { Diakonie - Stiftung Gustav } \\
\text { Werner und Haus am Berg, } \\
\text { Reutlingen }\end{array}$ & $\begin{array}{r}2012- \\
2015\end{array}$ \\
\hline $\begin{array}{l}\text { Senioren-Technik-Botschafter: Wissensvermittlung } \\
\text { von Älteren für Ältere zu neuen Informations- } \\
\text { und Kommunikationstechnologien (Förderlinie mit } \\
\text { mehreren geförderten Projekten) }\end{array}$ & & $\begin{array}{l}2013- \\
2014\end{array}$ \\
\hline $\begin{array}{l}\text { Assistenzsystem zur Unterstützung adäquater Flüs- } \\
\text { sigkeitsaufnahme in der Pflege (TrinkTracker) }\end{array}$ & INTRATEC GmbH, Ellerau & $\begin{array}{r}2013- \\
2015\end{array}$ \\
\hline $\begin{array}{l}\text { Sprachgesteuerte Haussteuerung mit persönlicher } \\
\text { Assistenz (Dialog+) }\end{array}$ & $\begin{array}{l}\text { Tellur Cesellschaft für } \\
\text { Telekommunikation mbH, } \\
\text { Stuttgart }\end{array}$ & $\begin{array}{l}2013- \\
2015\end{array}$ \\
\hline $\begin{array}{l}\text { Erfolgreiche Technikvermittlung und Technikhand- } \\
\text { habung von Älteren an Ältere (FUTA) }\end{array}$ & Universität Heidelberg & $\begin{array}{l}2013- \\
2015\end{array}$ \\
\hline $\begin{array}{l}\text { Interaktiver RObotischer REha-ASsistent für das } \\
\text { Lauf- und Orientierungstraining von Patienten } \\
\text { nach Schlaganfällen (ROREAS) }\end{array}$ & $\begin{array}{l}\text { MetraLabs } \mathrm{CmbH} \text { Neue } \\
\text { Technologien und Systeme } \\
\mathrm{CmbH}, \text { Ilmenau }\end{array}$ & $\begin{array}{l}2013- \\
2016\end{array}$ \\
\hline
\end{tabular}




\begin{tabular}{|c|c|c|}
\hline Projekttitel & Projektleitung/Institution & $\begin{array}{c}\text { Lauf- } \\
\text { zeit }\end{array}$ \\
\hline $\begin{array}{l}\text { Canzheitliches Konzept zur Unterstützung und Entlas- } \\
\text { tung von ambulanten Pflegekräften und Angehörigen } \\
\text { bei der Betreuung von kognitiv beeinträchtigten Se- } \\
\text { nioren (NeuroCare) }\end{array}$ & $\begin{array}{l}\text { Wohlfahrtswerk für } \\
\text { Baden-Württemberg, } \\
\text { Stuttgart }\end{array}$ & $\begin{array}{l}2013- \\
2016\end{array}$ \\
\hline $\begin{array}{l}\text { Integrierte pflegerische Versorgung im ländlichen } \\
\text { Raum durch technikgestützte Assistenz und Koordi- } \\
\text { nation (Selbstbestimmt und sicher) }\end{array}$ & $\begin{array}{l}\text { Gesundes Kinzigtal } \mathrm{CmbH} \text {, } \\
\text { Haslach }\end{array}$ & $\begin{array}{l}2013- \\
2016\end{array}$ \\
\hline $\begin{array}{l}\text { Unterstützung von Pflege, diskretem Leben und sozia- } \\
\text { ler Teilhabe durch MEMS-Ceruchssensorik (SensOdor) }\end{array}$ & $\begin{array}{l}\text { Binder Elektronik } \mathrm{GmbH} \text {, } \\
\text { Sinsheim }\end{array}$ & $\begin{array}{l}2013- \\
2016\end{array}$ \\
\hline $\begin{array}{l}\text { Unterstützung lokaler kooperativer Sozialmodelle im } \\
\text { ländlichen Raum durch pflegerisch-technische Assis- } \\
\text { tenzsysteme (KoopAS) }\end{array}$ & $\begin{array}{l}\text { Stiftung Liebenau Leben im } \\
\text { Alter gCmbH }\end{array}$ & $\begin{array}{l}2013- \\
2016\end{array}$ \\
\hline $\begin{array}{l}\text { Optimaler Einsatz von Smart-Items-Technologien in } \\
\text { der stationären Pflege (OpSIT) }\end{array}$ & $\begin{array}{c}\text { EnOcean } \mathrm{GmbH} \\
\text { Oberhaching }\end{array}$ & $\begin{array}{l}2013- \\
2016\end{array}$ \\
\hline $\begin{array}{l}\text { Intelligentes Pflegesystem für die Prävention und Be- } \\
\text { handlung von Dekubitus (INSYDE) }\end{array}$ & $\begin{array}{l}\text { Ergo-Tec } \mathrm{CmbH} \\
\text { Wilhelmsdorf }\end{array}$ & $\begin{array}{l}2013- \\
2016\end{array}$ \\
\hline $\begin{array}{l}\text { Kleine autonome Transport- und Handhabungshilfe } \\
\text { (KLARA) }\end{array}$ & Götting KC, Lehrte & $\begin{array}{l}2013- \\
2016\end{array}$ \\
\hline $\begin{array}{l}\text { Mit 60+ mitten im Arbeitsleben: Assistierte Arbeits- } \\
\text { plätze im demografischen Wandel (Förderlinie mit } \\
\text { mehreren geförderten Projekten) }\end{array}$ & & $\begin{array}{l}2013- \\
2016\end{array}$ \\
\hline $\begin{array}{l}\text { Eine multimodale App zur gegenseitigen Nachbar- } \\
\text { schaftshilfe für Senioren (WirlmKiez) }\end{array}$ & Cocomore AC, Frankfurt & $\begin{array}{l}2014- \\
2015\end{array}$ \\
\hline $\begin{array}{l}\text { Integriertes Pflegesystem für Menschen mit Demenz- } \\
\text { erkrankung (SAKI) }\end{array}$ & & $\begin{array}{l}2014- \\
2015\end{array}$ \\
\hline $\begin{array}{l}\text { Lautbasierte Steuerung von Gebäudetechnik, Sicher- } \\
\text { heitstechnik und Multimedia für Menschen in Pflege- } \\
\text { umgebungen (PareSense) }\end{array}$ & $\begin{array}{l}\text { Ilper Elektronik CmbH \& Co. } \\
\text { KC, Tarmstedt }\end{array}$ & $\begin{array}{l}2014- \\
2015\end{array}$ \\
\hline $\begin{array}{l}\text { Erweiterte Interaktionsformen mit digitalen Ceräten } \\
\text { im Alter (Closer) }\end{array}$ & $\begin{array}{l}\text { Universität der Künste } \\
\text { (Berlin) }\end{array}$ & $\begin{array}{l}2014- \\
2015\end{array}$ \\
\hline $\begin{array}{l}\text { Schlafüberwachung im Pflege- und Heimbereich } \\
\text { durch Remotesensorik (SPHERE) }\end{array}$ & $\begin{array}{l}\text { Videmo } \mathrm{GmbH} \& \text { Co. KC, } \\
\text { Karlsruhe }\end{array}$ & $\begin{array}{l}2014- \\
2016\end{array}$ \\
\hline $\begin{array}{l}\text { Aufbau eines Mentoring-Netzwerkes zur Unterstüt- } \\
\text { zung und Koordination der Betreuung pflegebedürfti- } \\
\text { ger Personen (Mentor+) }\end{array}$ & $\begin{array}{l}\text { CIBEK technology + trading } \\
\text { CmbH, Limburgerhof }\end{array}$ & $\begin{array}{l}2014- \\
2016\end{array}$ \\
\hline $\begin{array}{l}\text { Interaktives Sicherheits- und Assistenzsystem unter- } \\
\text { stützt Demenzkranke (InPreS) }\end{array}$ & $\begin{array}{l}\text { KUNDO xT GmbH, St. } \\
\text { Ceorgen }\end{array}$ & $\begin{array}{l}2014- \\
2016\end{array}$ \\
\hline
\end{tabular}




\begin{tabular}{|c|c|c|}
\hline Projekttitel & Projektleitung/Institution & $\begin{array}{c}\text { Lauf- } \\
\text { zeit }\end{array}$ \\
\hline $\begin{array}{l}\text { Bedienkonzepte und Schnittstellen im realen Wohn- } \\
\text { umfeld hochbetagter Nutzer (Bundschuh) }\end{array}$ & $\begin{array}{l}\text { Technische Universität } \\
\text { Ilmenau }\end{array}$ & $\begin{array}{l}2014- \\
2016\end{array}$ \\
\hline Sensoren für eine verbesserte Lebensqualität (SeLe) & $\begin{array}{l}\text { Infineon Technologies AG, } \\
\text { Neubiberg }\end{array}$ & $\begin{array}{l}2014- \\
2017\end{array}$ \\
\hline $\begin{array}{l}\text { Feedback-und Assistenzsystem zur alltagsintegrierten } \\
\text { Reha und aktivierenden Pflege (SiRIA) }\end{array}$ & Saint Elmo's GmbH, Berlin & $\begin{array}{l}2014- \\
2017\end{array}$ \\
\hline $\begin{array}{l}\text { Neue Hilfsmittel für die Bewegungstherapie zu Hause } \\
\text { (BeMobil) }\end{array}$ & $\begin{array}{l}\text { Technische Universität } \\
\text { Berlin }\end{array}$ & $\begin{array}{l}2014- \\
2017\end{array}$ \\
\hline $\begin{array}{l}\text { Lernende Systeme für längere Selbständigkeit zu Hau- } \\
\text { se (KogniHome) }\end{array}$ & $\begin{array}{c}\text { Universität } \\
\text { Bielefeld-Exzellenzcluster } \\
\text { CITEC }\end{array}$ & $\begin{array}{l}2014- \\
2017\end{array}$ \\
\hline $\begin{array}{l}\text { Alters- und alternsgerechte Mensch-Technik- } \\
\text { Interaktion mit telemedizinischen Systemen und } \\
\text { Dienstleistungen (TECH4ACE) }\end{array}$ & $\begin{array}{l}\text { Rheinisch-Westfälische } \\
\text { Technische Hochschule } \\
\text { Aachen }\end{array}$ & $\begin{array}{l}2014- \\
2022\end{array}$ \\
\hline $\begin{array}{l}\text { Fußgänger-Assistenzsystem für ältere Nutzerinnen } \\
\text { und Nutzer im Straßenverkehr (FANS) }\end{array}$ & $\begin{array}{l}\text { Technische Universität } \\
\text { Berlin }\end{array}$ & $\begin{array}{l}2014- \\
2022\end{array}$ \\
\hline $\begin{array}{l}\text { Telepräsenz-Roboter im häuslichen Lebens- und Pfle- } \\
\text { gearrangement von Personen mit Demenz im ländli- } \\
\text { chen Raum (RoboLand) }\end{array}$ & $\begin{array}{l}\text { Hochschule Fulda, } \\
\text { Hochschule Bonn-Rhein } \\
\text { Sieg }\end{array}$ & $\begin{array}{l}2015- \\
2016\end{array}$ \\
\hline $\begin{array}{l}\text { Virtueller Assistent zur Unterstützung von kognitiv be- } \\
\text { einträchtigten Personen auf Basis von Datenbrillen } \\
\text { (CLASSISTANT) }\end{array}$ & Ascora $\mathrm{CmbH}$, Ganderkesee & $\begin{array}{l}2015- \\
2017\end{array}$ \\
\hline $\begin{array}{l}\text { Modelle der Unterstützung der Teilhabe von Menschen } \\
\text { mit geistiger Behinderung im Alter innovativ gestalten } \\
\text { (MUTIC) }\end{array}$ & $\begin{array}{l}\text { Katholische Hochschule } \\
\text { Nordrhein-Westfalen } \\
\text { (KatHO NRW) in Münster }\end{array}$ & $\begin{array}{l}2015- \\
2018\end{array}$ \\
\hline $\begin{array}{l}\text { Interaktives Beleuchtungssystem zur Unterstützung } \\
\text { des Schlaf-Wach-Rhythmus speziell für Menschen mit } \\
\text { Demenzerkrankung (Silverlighting) }\end{array}$ & $\begin{array}{l}\text { licht raum stadt planung } \\
\qquad \mathrm{GmbH} \text {, Wuppertal }\end{array}$ & $\begin{array}{l}2015- \\
2018\end{array}$ \\
\hline $\begin{array}{l}\text { Individualisierte Spracherkennung in der Rehabilitati- } \\
\text { on für Menschen mit Beeinträchtigung in der Sprech- } \\
\text { verständlichkeit (Isi-Speech) }\end{array}$ & $\begin{array}{l}\text { SpeechCare } \mathrm{CmbH} \text {, } \\
\text { Leverkusen }\end{array}$ & $\begin{array}{l}2015- \\
2018\end{array}$ \\
\hline $\begin{array}{l}\text { Entwicklung eines Roboterassistenten für das häusli- } \\
\text { che Umfeld speziell zur Unterstützung älterer Men- } \\
\text { schen (SYMPARTNER) }\end{array}$ & $\begin{array}{l}\text { MetraLabs } \mathrm{CmbH} \text { Neue } \\
\text { Technologien und Systeme, } \\
\text { Ilmenau }\end{array}$ & $\begin{array}{l}2015- \\
2018\end{array}$ \\
\hline $\begin{array}{l}\text { Entwicklung eines selbstlernenden Hausautomations- } \\
\text { systems (LivingCare) }\end{array}$ & $\begin{array}{l}\text { contronics } \mathrm{CmbH} \\
\text { Merzenich }\end{array}$ & $\begin{array}{l}2015- \\
2018\end{array}$ \\
\hline
\end{tabular}




\begin{tabular}{|c|c|c|}
\hline Projekttitel & Projektleitung/Institution & $\begin{array}{l}\text { Lauf- } \\
\text { zeit }\end{array}$ \\
\hline $\begin{array}{l}\text { Intelligente vernetzte Brille als System zur Unter- } \\
\text { stützung im Alltag (CogAge) }\end{array}$ & Universität Siegen, Siegen & $\begin{array}{l}2015- \\
2018\end{array}$ \\
\hline $\begin{array}{l}\text { Umsetzung einer technischen Plattform zur Un- } \\
\text { terstützung älterer Menschen mit eingeschränk- } \\
\text { ten kognitiven oder körperlichen Leistungen (DA- } \\
\text { AN) }\end{array}$ & $\begin{array}{l}\text { Deutsches Forschungszentrum } \\
\text { für Künstliche Intelligenz } \mathrm{GmbH} \text {, } \\
\text { Saarbrücken }\end{array}$ & $\begin{array}{l}2015- \\
2018\end{array}$ \\
\hline $\begin{array}{l}\text { Virtuelle Assistenten als Begleiter für Menschen } \\
\text { mit Unterstützungsbedarf (KOMPASS) }\end{array}$ & Universität Bielefeld & $\begin{array}{l}2015- \\
2018\end{array}$ \\
\hline $\begin{array}{l}\text { Interaktive Biografiearbeit und Erinnerungspflege } \\
\text { (InterMem) }\end{array}$ & $\begin{array}{l}\text { Demenz-Support Stuttgart } \\
\text { gGmbH Zentrum für } \\
\text { Informationstransfer }\end{array}$ & $\begin{array}{l}2015- \\
2018\end{array}$ \\
\hline $\begin{array}{l}\text { Menschen mit Demenz verstehen durch tech- } \\
\text { nisch unterstützte Diagnose- und Entscheidungs- } \\
\text { prozesse (INSIDE-DEM) }\end{array}$ & $\begin{array}{l}\text { Deutsches Zentrum für } \\
\text { Neurodegenerative } \\
\text { Erkrankungen e. V., Standort } \\
\text { Witten und Rostock }\end{array}$ & $\begin{array}{l}2015- \\
2018\end{array}$ \\
\hline $\begin{array}{l}\text { Ein innovatives Musiksystem für musiktherapeuti- } \\
\text { sche Angebote zu Hause (NurMut) }\end{array}$ & $\begin{array}{l}\text { Wohlfahrtswerk für } \\
\text { Baden-Württemberg, Stuttgart }\end{array}$ & $\begin{array}{l}2015- \\
2018\end{array}$ \\
\hline $\begin{array}{l}\text { Ein interaktiver elektronischer Coach, der techni- } \\
\text { sche, medizinische und organisatorische Lösun- } \\
\text { gen miteinander verbindet (PflegeCoDe) }\end{array}$ & Bruderhaus Diakonie Reutlingen & $\begin{array}{l}2015- \\
2018\end{array}$ \\
\hline $\begin{array}{l}\text { Trainingssystem zur Förderung der körperlichen } \\
\text { Leistungsfähigkeit und Mobilität von Demenzpa- } \\
\text { tienten (MobiAssist) }\end{array}$ & $\begin{array}{l}\text { Ambulante Diakonische Dienste } \\
\qquad \mathrm{gCmbH} \text {, Siegen }\end{array}$ & $\begin{array}{l}2015- \\
2018\end{array}$ \\
\hline $\begin{array}{l}\text { Individuelle Aktivierung von Menschen mit De- } \\
\text { menz (I-CARE) }\end{array}$ & $\begin{array}{l}\text { AWO Karlsruhe gemeinnützige } \\
\qquad \mathrm{GmbH}, \text { Karlsruhe }\end{array}$ & $\begin{array}{l}2015- \\
2018\end{array}$ \\
\hline $\begin{array}{l}\text { Ein Projekt zur Verbesserung von Medienkompe- } \\
\text { tenzen bei Seniorinnen und Senioren (KommmiT) }\end{array}$ & $\begin{array}{l}\text { Wohlfahrtswerk für } \\
\text { Baden-Württemberg, Stuttgart }\end{array}$ & $\begin{array}{l}2015- \\
2020\end{array}$ \\
\hline $\begin{array}{l}\text { Bedarfsgerechte Unterstützung für Seniorinnen } \\
\text { und Senioren in der Stadt (UrbanLife+) }\end{array}$ & $\begin{array}{l}\text { Universität Hohenheim, } \\
\text { Stuttgart }\end{array}$ & $\begin{array}{l}2015- \\
2020\end{array}$ \\
\hline $\begin{array}{l}\text { Ein Projekt zur gesundheitsbezogenen Versor- } \\
\text { gung in ländlichen Gegenden (Dorf 2.0) }\end{array}$ & $\begin{array}{l}\text { Gesundheitsregion EUREGIO e. } \\
\qquad \text { V., Nordhorn }\end{array}$ & $\begin{array}{l}2015- \\
2021\end{array}$ \\
\hline $\begin{array}{l}\text { Ein Projekt zum öffentlichen Personennahverkehr } \\
\text { (MobiSaar) }\end{array}$ & $\begin{array}{c}\text { Saarländische-Nahverkehrs- } \\
\text { Service } \mathrm{GmbH}, \\
\text { Völklingen }\end{array}$ & $\begin{array}{l}2015- \\
2021\end{array}$ \\
\hline $\begin{array}{l}\text { Gemeinsam Pflegen in der mobilen Rehabilitation } \\
\text { (MORECARE) }\end{array}$ & $\begin{array}{l}\text { Evangelisches Krankenhaus } \\
\text { »Gottesfriede«in Woltersdorf } \\
\text { GmbH, Woltersdorf }\end{array}$ & $\begin{array}{l}2016- \\
2018\end{array}$ \\
\hline
\end{tabular}




\begin{tabular}{|c|c|c|}
\hline Projekttitel & Projektleitung/Institution & $\begin{array}{c}\text { Lauf- } \\
\text { zeit }\end{array}$ \\
\hline $\begin{array}{l}\text { Pflegeunterstützung mit einer interaktiven } \\
\text { Puppe für informell Pflegende (OurPuppet) }\end{array}$ & Anasoft Technology AC & $\begin{array}{r}2016- \\
2019\end{array}$ \\
\hline $\begin{array}{l}\text { Verlässliche mobile Begleitung für pflegende } \\
\text { Angehörige (MoCaB) }\end{array}$ & Johanniter-Unfall-Hilfe e. V., Berlin & $\begin{array}{l}2016- \\
2019\end{array}$ \\
\hline $\begin{array}{l}\text { Entwicklung eines optoelektronischen } \\
\text { Messsystems zur Steuerung interaktiver } \\
\text { logopädischer Übungen in der } \\
\text { Schlaganfalltherapie (OSLO) }\end{array}$ & linguwerk $\mathrm{CmbH}$, Dresden & $\begin{array}{l}2017- \\
2020\end{array}$ \\
\hline $\begin{array}{l}\text { Robotische Systeme zur Unterstützung } \\
\text { hochgradig motorisch eingeschränkter } \\
\text { Pflegebedürftiger (ROBINA) }\end{array}$ & Pflegewerk Berlin $\mathrm{CmbH}$ & $\begin{array}{l}2017- \\
2020\end{array}$ \\
\hline $\begin{array}{l}\text { Aufmerksamkeitssensitiver Assistenzroboter } \\
\text { (ASARob) }\end{array}$ & $\begin{array}{l}\text { Fraunhofer-Institut für Optronik, } \\
\text { Systemtechnik und } \\
\text { Bildauswertung IOSB, Karlsruhe }\end{array}$ & $\begin{array}{l}2017- \\
2020\end{array}$ \\
\hline $\begin{array}{l}\text { Soziale Angemessenheit für Assistenzsysteme } \\
\text { (poliTE) }\end{array}$ & $\begin{array}{l}\text { Forschungskolleg »Zukunft } \\
\text { menschlich gestalten«(FoKoS) der } \\
\text { Universität Siegen }\end{array}$ & $\begin{array}{l}2017- \\
2020\end{array}$ \\
\hline $\begin{array}{l}\text { Robotisches Reha-System zur Unterstützung } \\
\text { von Diagnose und Therapie (RobExReha) }\end{array}$ & BEC CmbH, Pfullingen & $\begin{array}{l}2017- \\
2020\end{array}$ \\
\hline $\begin{array}{l}\text { Rollatormodul zur Haltungserkennung und } \\
\text { Sturzprävention (ModESt) }\end{array}$ & $\begin{array}{l}\text { Budelmann Elektronik } \mathrm{CmbH} \text {, } \\
\text { Münster }\end{array}$ & $\begin{array}{l}2017- \\
2020\end{array}$ \\
\hline $\begin{array}{l}\text { Verbesserung der Pflege und Betreuung von } \\
\text { älteren Menschen mit chronischen Wunden } \\
\text { (vulnusMON) }\end{array}$ & ifm software $\mathrm{CmbH}$, Zwickau & $\begin{array}{l}2017- \\
2020\end{array}$ \\
\hline $\begin{array}{l}\text { Robuste Wahrnehmungsfähigkeiten für } \\
\text { Roboter zur Unterstützung älterer Nutzer im } \\
\text { häuslichen Umfeld (RoPHa) }\end{array}$ & $\begin{array}{l}\text { Fraunhofer-Institut für } \\
\text { Produktionstechnik und } \\
\text { Automatisierung (IPA), Stuttgart }\end{array}$ & $\begin{array}{l}2017- \\
2020\end{array}$ \\
\hline $\begin{array}{l}\text { Physische Mensch-Roboter-Interaktion für ein } \\
\text { selbstbestimmtes Leben (MobILe) }\end{array}$ & $\begin{array}{l}\text { Westfälische Hochschule } \\
\text { Celsenkirchen Bocholt } \\
\text { Recklinghausen, Celsenkirchen }\end{array}$ & $\begin{array}{l}2017- \\
2020\end{array}$ \\
\hline $\begin{array}{l}\text { Etablierung eines Pflegeinnovationszentrums } \\
\text { (PIZ) }\end{array}$ & OFFIS e.V., Oldenburg & $\begin{array}{l}2017- \\
2022\end{array}$ \\
\hline $\begin{array}{l}\text { Cestengesteuerte Fernbedienung: } \\
\text { nutzerzentrierter, universeller, intuitiver } \\
\text { (SmartPointer) }\end{array}$ & $\begin{array}{l}\text { Laser Components Cesellschaft für } \\
\text { den Vertrieb und die Fertigung von } \\
\text { Lasern und optoelektronischen } \\
\text { Komponenten mbH, Olching bei } \\
\text { München }\end{array}$ & $\begin{array}{l}2018- \\
2020\end{array}$ \\
\hline $\begin{array}{l}\text { Virtual Reality in der physiotherapeutischen } \\
\text { und psychotherapeutischen Schmerztherapie } \\
\text { (ViRST) }\end{array}$ & Cocomore AC, Frankfurt a.M. & $\begin{array}{l}2018- \\
2020\end{array}$ \\
\hline
\end{tabular}




\begin{tabular}{|c|c|c|}
\hline Projekttitel & Projektleitung/Institution & $\begin{array}{l}\text { Lauf- } \\
\text { zeit }\end{array}$ \\
\hline $\begin{array}{l}\text { VR-Trainingssystem für autonome Neurorehabili- } \\
\text { tation (VREHAgo) }\end{array}$ & $\begin{array}{l}\text { Hochschule Reutlingen-Center } \\
\text { for Entrepreneurship }\end{array}$ & $\begin{array}{l}2018- \\
2020\end{array}$ \\
\hline $\begin{array}{l}\text { Interaktionsstrategien für eine robotische Ein- } \\
\text { kaufsassistenz (I-RobEka) }\end{array}$ & Technische Universität Chemnitz & $\begin{array}{r}2018- \\
2021\end{array}$ \\
\hline $\begin{array}{l}\text { Vertrauen und Sympathie schaffender »lebendi- } \\
\text { ger«sozialer Roboter (VIVA) }\end{array}$ & navel robotics $\mathrm{GmbH}$, München & $\begin{array}{r}2018- \\
2021\end{array}$ \\
\hline $\begin{array}{l}\text { Prävention degenerativer Demenz durch menta- } \\
\text { le, interaktive, individualisierte Stimulation (Pro- } \\
\text { demiis) }\end{array}$ & $\begin{array}{l}\text { SilverCreations Software AG, } \\
\text { Hoppstädten-Weiersbach }\end{array}$ & $\begin{array}{r}2018- \\
2021\end{array}$ \\
\hline $\begin{array}{l}\text { Mit Virtueller Realität interaktiv gegen Demenz } \\
\text { und Parkinson (EXGAVINE) }\end{array}$ & Universität Hamburg & $\begin{array}{l}2018- \\
2021\end{array}$ \\
\hline $\begin{array}{l}\text { Sensorgestütztes Bewegungstraining für Senio- } \\
\text { ren in einem intelligenten Augmented Reality } \\
\text { System (BewARe) }\end{array}$ & Ascora $\mathrm{GmbH}$, Ganderkesee & $\begin{array}{r}2018- \\
2021\end{array}$ \\
\hline $\begin{array}{l}\text { Integriertes Diagnose- und e-Assistenzsystem } \\
\text { für Patienten mit altersbedingter Makula- } \\
\text { Degeneration (IDeA) }\end{array}$ & $\begin{array}{l}\text { Ludwig-Maximilians-Universität } \\
\text { München, Human-Centered } \\
\text { Ubiquitous Media }\end{array}$ & $\begin{array}{r}2018- \\
2021\end{array}$ \\
\hline Cluster »Zukunft der Pflege« & & $\begin{array}{l}2018- \\
2023\end{array}$ \\
\hline $\begin{array}{l}\text { Pflegepraxiszentrum für geriatrische digitale As- } \\
\text { sistenz (PPZ-Berlin) }\end{array}$ & $\begin{array}{l}\text { Ev. Johannesstift Altenhilfe } \\
\text { gGmbH, Berlin }\end{array}$ & $\begin{array}{l}2018- \\
2023\end{array}$ \\
\hline $\begin{array}{l}\text { Qualität und Sicherheit für die Pflegepraxis mit } \\
\text { Augmented Reality (Pflegebrille } 2.0 \text { ) }\end{array}$ & TU Clausthal & $\begin{array}{r}2019- \\
2021\end{array}$ \\
\hline $\begin{array}{l}\text { Demenz-Bessere Betreuung und individuelle } \\
\text { Ansprache durch Entwicklung eines interaktiven } \\
\text { Tisches (DAYSI) }\end{array}$ & Böhm GmbH \& Co. KG, Potsdam & $\begin{array}{r}2019- \\
2021\end{array}$ \\
\hline $\begin{array}{l}\text { Textile Oberflächen an den Wänden sorgen für } \\
\text { mehr Komfort im Smart Home (ConText) }\end{array}$ & $\begin{array}{l}\text { Deutsches Forschungszentrum } \\
\text { für Künstliche Intelligenz } \mathrm{GmbH} \text {, } \\
\text { Standorte Bremen und Berlin }\end{array}$ & $\begin{array}{l}2019- \\
2022\end{array}$ \\
\hline $\begin{array}{l}\text { Mobiles Monitoringsystem zum individuellen } \\
\text { Behandlungs- und Medikamentenmanagement } \\
\text { für Pflegebedürftige (KOMMUNIKAP) }\end{array}$ & $\begin{array}{l}\text { PAR Medizintechnik GmbH \& Co. } \\
\text { Kommanditgesellschaft, Berlin }\end{array}$ & $\begin{array}{l}2019- \\
2022\end{array}$ \\
\hline $\begin{array}{l}\text { Interaktive Neuro-Orthetik für die Handfunktion } \\
\text { nach einem Schlaganfall (INERLINC) }\end{array}$ & neuroConn $\mathrm{GmbH}$, Ilmenau & $\begin{array}{l}2019- \\
2022\end{array}$ \\
\hline
\end{tabular}




\begin{tabular}{|c|c|c|}
\hline Projekttitel & Projektleitung/Institution & $\begin{array}{l}\text { Lauf- } \\
\text { zeit }\end{array}$ \\
\hline $\begin{array}{l}\text { Frühdiagnose von Alzheimer und digitaler } \\
\text { Assistent zur Therapie (IASON) }\end{array}$ & $\begin{array}{l}\text { Tokeya Deep Data Dive CmbH \& Co. } \\
\text { KC, Würzburg }\end{array}$ & $\begin{array}{l}2019- \\
2022\end{array}$ \\
\hline $\begin{array}{l}\text { Soziale Interaktion durch Klang-Feedback } \\
\text { (Sentire) }\end{array}$ & Humboldt-Universität zu Berlin & $\begin{array}{l}2019- \\
2022\end{array}$ \\
\hline $\begin{array}{l}\text { Begründungs- und Bewertungsmaßstäbe } \\
\text { von Robotik für die Pflege (BeBeRobot) }\end{array}$ & Universität Osnabrück & $\begin{array}{l}2019- \\
2022\end{array}$ \\
\hline $\begin{array}{l}\text { Simulation von Nudging-Methoden zurStär- } \\
\text { kung der Selbstständigkeit Pflegebedürfti- } \\
\text { ger (SiNuS) }\end{array}$ & Universität Trier & $\begin{array}{c}2020- \\
2021\end{array}$ \\
\hline $\begin{array}{l}\text { Unterstützung der häuslichen Pflege von } \\
\text { Menschen mit Demenz durch eine persona- } \\
\text { lisierte Mensch-Roboter-Interaktion (RUBY- } \\
\text { Demenz) }\end{array}$ & Anasoft Technology AG, Bochum & $\begin{array}{c}2020- \\
2023\end{array}$ \\
\hline $\begin{array}{l}\text { Roboterunterstützung bei Routineaufgaben } \\
\text { zur Stärkung des Miteinanders in Pflegeein- } \\
\text { richtungen (RoMi) }\end{array}$ & pi4 robotics $\mathrm{CmbH}$, Berlin & $\begin{array}{c}2020- \\
2023\end{array}$ \\
\hline $\begin{array}{l}\text { Multifunktionaler Serviceroboter zur Unter- } \\
\text { stützung professioneller Pflege in Kranken- } \\
\text { häusern (HoLLiECares) }\end{array}$ & $\begin{array}{l}\text { Deutsches Institut für angewandte } \\
\text { Pflegeforschung e.V. (DIP), Köln }\end{array}$ & $\begin{array}{c}2020- \\
2023\end{array}$ \\
\hline $\begin{array}{l}\text { Ein adaptives Mehrkomponenten- } \\
\text { Robotersystem für die Pflege (AdaMekoR) }\end{array}$ & $\begin{array}{l}\text { Johanniter-Unfall-Hilfe e. } \\
\text { V.-Landesverband } \\
\text { Niedersachsen/Bremen, Fachbereich } \\
\text { Forschung und Entwicklung, Elsfleth }\end{array}$ & $\begin{array}{c}2020- \\
2023\end{array}$ \\
\hline $\begin{array}{l}\text { Digitale Souveränität im Spiegel der elektro- } \\
\text { nischen Patientenakte (ePA-Coach) }\end{array}$ & Charité-Universitätsmedizin Berlin & $\begin{array}{c}2020- \\
2023\end{array}$ \\
\hline $\begin{array}{l}\text { Mobile Leseförderung: Validierung einer evi- } \\
\text { denzbasierten Lese-App (MobiLe }\end{array}$ & & $\begin{array}{c}2020- \\
2023\end{array}$ \\
\hline
\end{tabular}


Technik und Pflege im professionellen Kontext und (privaten) Alltag 



\section{Die Nutzung von digitalen Kommunikationstechnologien in ambulanten und stationären Pflegeeinrichtungen während der COVID-19-Pandemie}

Dominik Domhoff, Kathrin Seibert, Heinz Rothgang, Karin Wolf-Ostermann

\section{Einleitung}

Innovative digitale Technologien für die Unterstützung und Anwendung in der professionellen pflegerischen Versorgung erfahren seit einigen Jahren zunehmende Aufmerksamkeit in der Forschungsförderung und Entwicklung, um den soziokulturellen und demografischen Herausforderungen der Gesellschaft und einem an Komplexität gewinnenden Versorgungsalltag pflegebedürftiger Menschen zu begegnen (vgl. Fehling 2019; Hielscher/Nock/Kirchen-Peters 2015; Hülsken-Giesler 2010; Krick et al. 2019). Dabei scheint sich das Verhältnis von Pflege und Technik in einem ambivalenten Beziehungsgeflecht $\mathrm{zu}$ bewegen: $\mathrm{Ob}-$ wohl (inter-)national seit langem technische Assistenzsysteme für den Einsatz in der Pflege verfügbar sind, gelingt deren Einbindung in den Versorgungsalltag eher schleppend und kaum flächendeckend (Bräutigam et al. 2017; Fehling 2019; Fehling/Dassen 2017; Merda/Schmidt/Kähler 2017). Auch weisen nationale Studien für Pflegefachpersonen und Entscheidungsträger*innen einerseits Neugierde, positive Einstellungen und ein Verständnis von neuen Technologien als Chance aus, dem gegenüber stehen jedoch auch Skepsis, Vorbehalte und Ablehnung durch die betroffenen Akteur*innen (Braeseke et al. 2017; Bräutigam et al. 2017; Claßen et al. 2010; Roland Berger $\mathrm{GmbH}$ /Deutsches Institut für angewandte Pflegeforschung e.V./Philosophisch-Theologische Hochschule Vallendar 2017; ZQP 2019). Obwohl Entwickler*innen durchaus passgenaue Lösungen präsentieren, wird die Entwicklung von Technologien als an der praktischen Arbeitsrealität der Pflege vorbeigehend empfunden beschrieben (vgl. Merda/Schmidt/Kähler 2017). Erfolgsfaktoren einer gelingenden und nachhaltigen Nutzung, wie etwa entscheidende infrastrukturelle, organisatorische und persönliche Voraussetzungen, sind durchaus bekannt (Ammenwerth/Iller/Mahler 2006; Greenhalgh/Abimbola 2019) - trotzdem ist eine ausbleibende Verstetigung bei hemmenden Kontextfaktoren und fehlen- 
den Gesamtkonzepten zur Entwicklung und Implementierung von Technologien in der Pflegepraxis zu beobachten (Fehling 2019; Hielscher/Nock/Kirchen-Peters 2015).

In einer bundesweiten Studie der Universität Bremen wurden im Jahr 2019 Pflegefachpersonen zu ihren Erfahrungen mit digitalen Technologien befragt. Dabei gaben knapp 95 Prozent von 1.074 Befragten an, praktische Erfahrungen mit digitalen Technologien im Arbeitsalltag zu haben. Sowohl für die ambulante und stationäre Langzeitpflege als auch für Krankenhäuser lagen am häufigsten Erfahrungen mit Informations- und Kommunikationstechnik (IKT) vor, die bei differenzierter Betrachtung vor allem elektronische Pflege- oder Patient*innendokumentation sowie elektronische Prozessplanung (wie Touren-/Dienstpläne) umfassten. Jeweils mehr als 70 Prozent der Befragten hatten mit diesen Arten der IKT bereits Erfahrungen im Arbeitsalltag gesammelt und diese Technologien mehrheitlich als die Kommunikation im Team und mit anderen Gesundheitsdienstleister*innen verbessernd beschrieben (Seibert et al. 2020). Die Teilnehmenden wiesen jedoch in Freitextantworten sowie in den sich der Befragung anschließenden Fokusgruppendiskussionen auf einen allgemein mangelhaften Digitalisierungsgrad in Pflegeeinrichtungen hin, welcher den Einsatz digitaler Technologien, insbesondere auch zur schnittstellenübergreifenden Kommunikation zwischen Leistungserbringenden, verhindere (ebd.). Dieser Befund steht unter anderem im Einklang mit den Ergebnissen der \#SmartHealthSystems-Studie, die Digitalisierungsstrategien einzelner Gesundheitssysteme im internationalen Vergleich betrachtet und Deutschland auf dem vorletzten Platz der siebzehn untersuchten Länder einordnet (Thiel et al. 2018).

Durch den im Gesetz zur Stärkung des Pflegepersonals (PflegepersonalStärkungsgesetz - PpSG) für die Jahre 2019 bis 2021 verankerten Zuschuss für ambulante und stationäre Pflegeeinrichtungen für die Anschaffung von digitaler oder technischer Ausrüstung sowie damit verbundene Schulungen als auch durch die mit der COVID-19-Pandemie einhergehenden Herausforderungen hat der Ausbau der digitalen Infrastruktur und damit auch die Steigerung des Potenzials für den Einsatz von digitalen Technologien in Pflegeeinrichtungen an Bedeutung gewonnen.

Vor allem die auf Besuchs- und Zutrittsbegrenzung abzielenden Maßnahmen der Infektionskontrolle (RKI 2020) begründen einen steigenden Bedarf an Kommunikationstechnologien, die neben den Kontakten zu externen Leistungserbringenden vor allem dabei unterstützen sollen, die sozialen Kontakte pflegebedürftiger Menschen $\mathrm{zu}$ stabilisieren und aufrecht $\mathrm{zu}$ erhalten. Jedoch bringt es neuerliche Herausforderungen mit sich, vulnerable sowie physisch und kognitiv beeinträchtige Menschen in Pflegeeinrichtungen in der Nutzung von Kommunikationstechnologien zu unterstützen (Seifert/Cotten/Xie 2020). Zur Nutzung von technischen Lösungen für die Aufrechterhaltung von sozialen Kontakten liegen bisher auch in- 
ternational kaum Erkenntnisse vor und für Deutschland werden äußerst geringe Nutzungsraten von mit dem Internet verbundenen Kommunikationstechnologien durch Pflegebedürftige in Pflegeheimen berichtet (Schlomann et al. 2020; Seifert/Cotten/Xie 2020). Vor diesem Hintergrund untersuchten das Institut für Public Health und Pflegeforschung (IPP) und das Forschungszentrum Ungleichheit und Sozialpolitik (SOCIUM) der Universität Bremen im Frühling 2020 unter anderem, wie Pflegeeinrichtungen digitale Kommunikationstechnologien während der COVID19-Pandemie nutzen (Rothgang et al. 2020; Wolf-Ostermann et al. 2020b). Der vorliegende Beitrag reichert wesentliche Erkenntnisse der Erhebung durch weiterführende Analysen an, für die folgende Fragestellungen untersuchungsleitend waren:

1 Welche digitalen Kommunikationstechnologien wurden von ambulanten und stationären Pflegeeinrichtungen während der COVID-19-Pandemie vermehrt genutzt?

2 Welche Merkmale beeinflussten eine vermehrte Nutzung von digitalen Kommunikationstechnologien während der COVID-19-Pandemie?

3 Welche weiteren technologie- und digitalisierungsbezogenen Unterstützungsmaßnahmen erachteten die Einrichtungen im Kontext der COVID-19-Pandemie als sinnvoll?

\section{Methoden}

Die nachfolgend präsentierten Ergebnisse basieren auf einer Datenerhebung, die im Rahmen der Studie »Zur Situation der Langzeitpflege in Deutschland während der Corona-Pandemie« (Wolf-Ostermann et al. 2020a) durchgeführt wurde. Zweck dieser Studie war es insbesondere, die Situationen von ambulanten, vollstationären und teilstationären Pflegeeinrichtungen während der COVID-19-Pandemie zu verstehen und Unterstützungsbedarfe zu identifizieren.

\section{Datenerhebung}

Die Datenerhebung erfolgte in der Zeit vom 28. April bis zum 12. Mai 2020. Der Zugang zur Befragung wurde per E-Mail an eine Gelegenheitsstichprobe von 7.723 stationären Pflegeeinrichtungen, 9.547 ambulanten Pflegediensten und 2.731 teilstationären Einrichtungen versandt. Darüber hinaus wurden Interessensvertretungen und Anbieter*innenverbände gebeten, die Befragung unter den ihnen angeschlossenen Einrichtungen bekannt zu machen.

Die Onlinebefragung erfolgte mittels EFS-Survey, Version Fall 2019 (Questback $\mathrm{GmbH}$ 2019). Die Teilnehmenden erhielten vorab mit der Einladung zur Befragung 
Erläuterungen zum Forschungsprojekt und stimmten dem, mit der Datenschutzbeauftragten der Universität abgestimmten, Datenschutzkonzept zu.

Der Fragebogen beinhaltete Fragen zu Art und strukturellen Merkmalen der Pflegeeinrichtungen, zu COVID-19-Fällen oder dem Verdacht auf COVID-19 bei Mitarbeitenden oder Pflegebedürftigen ${ }^{1}$, zu personellen und materiellen Ressourcen, zu getroffenen Maßnahmen, zum Einsatz von Kommunikationstechnik sowie erfahrener und als notwendig erachteter Unterstützung durch Institutionen.

Mit Hinblick auf digitale Kommunikationstechnologien wurden die Teilnehmenden gefragt, welche Technologien sie seit Beginn der Pandemie zur Kommunikation mit welchen Akteur*innengruppen eingesetzt haben. Zudem wurde erhoben, ob sie diese Technologien auch bereits vor der Pandemie eingesetzt hatten und ob der Einsatz seltener, gleichermaßen oder häufiger erfolgt ist, als vor Beginn der Pandemie. Hierbei wurden als mögliche Kommunikationstechnologien E-Mail, Messengerdienste (wie WhatsApp), Videokonferenz sowie spezielle Telecare-Technologien (z.B. Hausnotruf mit Bild, Monitoring-Technologien) als Antwortmöglichkeiten vorgegeben. Darüber hinaus war eine Frage enthalten, ob in der Einrichtung neue digitale Kommunikationstechnologien für die Pflegebedürftigen zur Nutzung für soziale Kontakte geschaffen wurden oder ob dies in Planung sei. Hierbei konnten auch im Freitext die gewählten technischen Lösungen beschrieben werden. Abschließend konnten die Befragten noch weitere Maßnahmen zur Unterstützung von Pflegeeinrichtungen beschreiben, die sie während der Pandemie als sinnvoll erachteten.

Die Befragung wurde so angelegt, dass auch beim Auslassen von Fragen die Folgefragen angezeigt wurden.

\section{Datenauswertung}

Für die folgenden Auswertungen wurden von insgesamt 1.621 Fragebögen lediglich die der vollstationären Pflegeeinrichtungen $(n=824)$ und ambulanten Pflegedienste ( $n=701$ ) einbezogen; die der teilstationären Einrichtungen $(n=96)$ wurden für die Analysen ausgeschlossen, da ein Großteil dieser Einrichtungen zum Befragungszeitpunkt geschlossen war. Ebenso wurden jene Fragebögen der vollstationären und ambulanten Pflegeeinrichtungen ausgeschlossen, welche die Bearbeitung des Fragebogens vor den Fragen zu digitalen Kommunikationstechnologien abgebrochen hatten $(\mathrm{n}=198)$.

Basierend auf den Antworten zur Nutzung von Kommunikationstechnologien seit Beginn der Pandemie und deren Nutzung zuvor wurden die Einrichtungen einem von fünf Nutzungstypen zugeordnet: (1) Neunutzer*innen begannen mit der

1 Dieser Beitrag verwendet im Folgenden den Begriff Pflegebedürftige für von durch Pflegedienste versorgte und in Pflegeheimen lebende Personen gleichermaßen. 
Nutzung einer Technologie erst nach Beginn der Pandemie, (2) häufiger Nutzende setzten eine Technologie bereits vor dem Beginn der Pandemie ein, haben die Nutzungshäufigkeit jedoch seitdem erhöht, (3) konstant Nutzende gaben an, eine Technologie während der Pandemie in der gleichen Häufigkeit zu nutzen wie zuvor, (4) seltener Nutzende gaben an, eine Technologie vor Beginn der Pandemie häufiger zu nutzen als nach deren Beginn und (5) Nichtnutzende setzten eine Technologie zu keinem Zeitpunkt ein.

Alle benannten Fragen wurden deskriptiv ausgewertet und nach ambulanten und stationären Pflegeeinrichtungen unterteilt. Zudem wurde die Häufigkeit der fünf Nutzungstypen zusätzlich danach unterschieden, ob die Einrichtungen COVID-19-Fälle haben oder nicht. Diese Unterscheidung zielt dabei darauf ab, ob in den Pflegeeinrichtungen bis zum Zeitpunkt der Befragung mindestens eine COVID-19-Infektion bei den pflegebedürftigen Personen beziehungsweise den Mitarbeitenden bekannt war.

Um die Faktoren zu identifizieren, die die Wahrscheinlichkeit dafür erhöhen, dass eine Technologie häufiger als vor der Pandemie eingesetzt wird, wurden logistische Regressionsanalysen eingesetzt. Die Zielvariable stellte hierbei eine dichotomisierte Variante des Nutzungstyps dar: Neue beziehungsweise häufigere Nutzung einer Technologie als Zielereignis und keine, seltenere oder konstante Nutzung einer Technologie als Referenzgruppe.

Als mögliche Faktoren, die die Nutzung und Nutzungshäufigkeit von Technologien beeinflussen, wurde die Art der Einrichtung, die Trägerschaft der Einrichtung, die Anzahl der versorgten Pflegebedürftigen, die Anzahl der Mitarbeitenden, der Anteil der COVID-19-Fälle unter den Pflegebedürftigen und der Anteil der COVID-19-Fälle unter den Mitarbeitenden berücksichtigt.

Wenn zu einzelnen Fragen keine Angaben vorlagen, wurden diese von der jeweiligen Auswertung ausgeschlossen, wodurch sich die zu berücksichtigende Fallzahl reduzierte.

\section{Ergebnisse}

In die Analysen wurden Daten von insgesamt 1.327 Pflegeeinrichtungen, davon 599 Pflegedienste und 728 Pflegeheime, eingeschlossen. Tabelle 1 stellt die wesentlichen Merkmale der Einrichtungen dar. Die Mehrzahl der Pflegedienste befand sich dabei in privater Trägerschaft, während sich die Pflegeheime überwiegend in freigemeinnütziger Trägerschaft befanden. Die Pflegedienste verfügten über durchschnittlich 51,9 Mitarbeitende und versorgten im Durchschnitt 157,3 Pflegebedürftige. Die Pflegeheime versorgten im Durchschnitt mit 100,0 Mitarbeitenden 84,8 Pflegebedürftige. 


\section{Vorkommen von COVID-19 Fällen in den Pflegeeinrichtungen}

Bei 9,4 Prozent der befragten Pflegedienste waren zum Zeitpunkt der Befragung COVID-19-Fälle bei den Mitarbeitenden bekannt; bei 17,2 Prozent der Pflegedienste war mindestens ein Fall von COVID-19 bei den Pflegebedürftigen bekannt. Sofern in einem Pflegedienst Fälle auftraten, waren im Durchschnitt 1,8 Mitarbeitende betroffen. Wenn ein Pflegedienst von COVID-19-Fällen bei Pflegebedürftigen berichtete, so waren zum Zeitpunkt der Befragung durchschnittlich Infektionen bei 2,3 Personen bekannt.

18,5 Prozent der Pflegeheime waren von mindestens einem an COVID-19-Fall bei Mitarbeitenden betroffen; 11,8 Prozent der Pflegeheime waren von mindestens einem COVID-19-Fall bei Pflegebedürftigen betroffen. Sofern ein Fall bei Mitarbeitenden vorlag, waren im Durchschnitt 5,5 Infektionen bei den Mitarbeitenden bekannt. Sofern ein Fall bei Bewohnenden vorlag, waren bei durchschnittlich 9,4 Bewohnenden der Einrichtung Infektionen bekannt. Damit waren die teilnehmenden Pflegeheime im Vergleich zu den Pflegediensten häufiger und stärker unmittelbar von COVID-19 betroffen.

Tabelle 1: Beschreibung der Stichprobe ambulanter und stationärer Pflegeeinrichtungen ( $n=1.327 ; \mathrm{zu} 100 \%$ fehlende Werte in einzelnen Antwortoptionen ergeben sich durch fehlende Angaben; SD: Standardabweichung)

\begin{tabular}{|l|l|l|}
\hline & ambulant (N=599) & stationär (N=728) \\
\hline Träger“innen: freigemeinnützig & $193(32,2 \%)$ & $387(53,2 \%)$ \\
\hline Träger“innen: öffentlich & $27(4,5 \%)$ & $77(10,6 \%)$ \\
\hline Träger“innen: privat & $357(59,6 \%)$ & $250(34,3 \%)$ \\
\hline Anzahl Mitarbeitende (SD) & $51,9(211,4)$ & $100,0(322,2)$ \\
\hline $\begin{array}{l}\text { Anzahl versorgter } \\
\text { Pflegebedürftiger (SD) }\end{array}$ & $157,3(191,3)$ & $84,8(43,4)$ \\
\hline $\begin{array}{l}\text { Einrichtungen mit COVID-19- } \\
\text { Fällen bei Mitarbeitenden }\end{array}$ & $56(9,4 \%)$ & $132(18,5 \%)$ \\
\hline $\begin{array}{l}\text { Einrichtungen mit CovID-19- } \\
\text { Fällen bei Pflegebedürftigen }\end{array}$ & $103(17,2 \%)$ & $84(11,8 \%)$ \\
\hline $\begin{array}{l}\text { durchschnittliche Anzahl infizierter Mit- } \\
\text { arbeitender in betroffenen Einrichtungen } \\
\text { (SD) }\end{array}$ & $1,8(1,8)$ & $5,5(7,2)$ \\
\hline $\begin{array}{l}\text { durchschnittliche Anzahl infizierter Pfle- } \\
\text { gebedürftiger in betroffenen Einrichtun- } \\
\text { gen (SD) }\end{array}$ & $2,3(2,0)$ & $9,4(12,1)$ \\
\hline
\end{tabular}

Quelle: Eigene Darstellung 


\section{Arten der verwendeten Kommunikationstechnologie}

Die Nutzung von digitalen Kommunikationstechnologien während der COVID-19Pandemie durch Pflegedienste und -heime unter Berücksichtigung der Kommunikationspartner*innen ist in Tabelle 2 und Tabelle 3 dargestellt. Es zeigt sich, dass E-Mail die vorrangige Art der digitalen Kommunikation darstellt, unabhängig von den Akteur*innengruppen, mit denen die Kommunikation stattfindet. Jeweils fast ein Drittel der Pflegedienste nutzt E-Mail, um mit Angehörigen, externen Dienstleistenden oder externen Leistungserbringenden zu kommunizieren. Pflegeheime nutzen diese Technologie häufiger. So gaben 77,9 Prozent der Pflegeheime an, per E-Mail mit Angehörigen zu kommunizieren. Auch die Kommunikation mit den Pflegebedürftigen selbst erfolge per E-Mail: in Pflegediensten mit 30,9 Prozent häufiger als in Pflegeheimen mit 19,2 Prozent.

Messengerdienste, wie beispielsweise WhatsApp, werden von 46, o Prozent der stationären und 62,6 Prozent der ambulanten Pflegeeinrichtungen zur Kommunikation mit dem Personal genutzt. Auch für die Kommunikation mit Angehörigen sind Messengerdienste relevant, sodass 44,0 Prozent der Pflegeheime diese einsetzen, gegenüber 23,0 Prozent der Pflegedienste. Zudem gab etwa ein Viertel der stationären Pflegeeinrichtungen an, Messenger zu nutzen, um mit Pflegebedürftigen $\mathrm{zu}$ kommunizieren.

Insbesondere Pflegeheime nutzten während der Pandemie Videokonferenzen zur digitalen Kommunikation - 40,1 Prozent der Einrichtungen für die Kommunikation mit Angehörigen; 30,4 Prozent für die Kommunikation mit dem Personal. Ein Viertel der Pflegeheime gab an, mit den Pflegebedürftigen selbst per Videokonferenz zu kommunizieren und 13,3 Prozent nutzten diese Technologie mit externen Leistungserbringenden. Pflegedienste nutzten Videokonferenzen insbesondere zur Kommunikation mit dem Personal, dies erfolgte jedoch nur in 23,9 Prozent der teilnehmenden Einrichtungen und deutlich weniger mit den übrigen Akteur*innengruppen.

Spezielle Telecare-Technologien spielten während der COVID-19-Pandemie nur eine untergeordnete Rolle. Lediglich 4,8 Prozent der Pflegeheime und 1,5 Prozent der Pflegedienste nutzten diese Technologie zur Kommunikation mit Angehörigen oder Pflegebedürftigen. Zur Kommunikation mit dem Personal nutzten darüber hinaus eine solche Art von Technologie noch 2,7 Prozent beziehungsweise 2,2 Prozent der ambulanten beziehungsweise stationären Einrichtungen. 
Tabelle 2: Nutzung von Kommunikationstechnologien durch ambulante Pflegeeinrichtungen während der COVID-19-Pandemie ( $N=599)$

\begin{tabular}{|l|l|l|l|l|}
\hline $\begin{array}{l}\text { Nutzung von ... zur } \\
\text { Kommunikation mit }\end{array}$ & E-Mail & $\begin{array}{l}\text { Messenger- } \\
\text { dienste }\end{array}$ & Videokonferenz & $\begin{array}{l}\text { Telecare- } \\
\text { Technologien }\end{array}$ \\
\hline Angehörige & $69,9 \%$ & $23,0 \%$ & $5,2 \%$ & $1,0 \%$ \\
\hline externe Dienstleister & $62,3 \%$ & $13,0 \%$ & $7,2 \%$ & $0,8 \%$ \\
\hline $\begin{array}{l}\text { externe Leistungser- } \\
\text { bringer }\end{array}$ & $63,9 \%$ & $11,7 \%$ & $6,0 \%$ & $0,8 \%$ \\
\hline Personal & $53,6 \%$ & $62,6 \%$ & $23,9 \%$ & $2,7 \%$ \\
\hline Pflegebedürftige & $30,9 \%$ & $13,7 \%$ & $3,3 \%$ & $1,5 \%$ \\
\hline
\end{tabular}

Quelle: Eigene Darstellung

Tabelle 3: Nutzung von Kommunikationstechnologien durch stationäre Pflegeeinrichtungen während der COVID-19-Pandemie ( $N=728)$

\begin{tabular}{|l|l|l|l|l|}
\hline $\begin{array}{l}\text { Nutzung von ... zur } \\
\text { Kommunikation mit }\end{array}$ & E-Mail & $\begin{array}{l}\text { Messenger- } \\
\text { dienste }\end{array}$ & Videokonferenz & $\begin{array}{l}\text { Telecare- } \\
\text { Technologien }\end{array}$ \\
\hline Angehörige & $77,9 \%$ & $44,0 \%$ & $40,1 \%$ & $4,3 \%$ \\
\hline externe Dienstleister & $69,8 \%$ & $8,2 \%$ & $8,0 \%$ & $0,7 \%$ \\
\hline $\begin{array}{l}\text { externe Leistungser- } \\
\text { bringer }\end{array}$ & $73,8 \%$ & $10,0 \%$ & $13,3 \%$ & $1,2 \%$ \\
\hline Personal & $69,4 \%$ & $46,0 \%$ & $30,4 \%$ & $2,2 \%$ \\
\hline Pflegebedürftige & $19,2 \%$ & $24,6 \%$ & $25,0 \%$ & $4,8 \%$ \\
\hline
\end{tabular}

Quelle: Eigene Darstellung

\section{Vergleich der Nutzungshäufigkeiten vor und während der COVID-19-Pandemie}

Die Nutzung der einzelnen Technologien vor Beginn der COVID-19-Pandemie für Einrichtungen, die die entsprechenden Lösungen während der COVID-19Pandemie einsetzten, kann Abbildung 1 entnommen werden. Unabhängig von der Art der Kommunikationstechnologie zeigt sich, dass etwa ein Viertel bis ein Drittel der Nutzenden die entsprechende technische Lösung vor dem Beginn der Pandemie seltener als zum Zeitpunkt der Befragung einsetzte. Insbesondere bei E-Mail und Messengerdiensten zeigt sich, dass die Hälfte der Einrichtungen diese Technologien bereits vor der Pandemie nutzte. E-Mail wurde dabei von den Einrichtungen häufiger genutzt, Messengerdienste wurden in etwa einem Drittel der Einrichtungen neu eingeführt. Für die übrigen Technologien besteht die 
Mehrheit aus neuen Nutzenden. So nutzten 69,4 Prozent der Einrichtungen, die während der Pandemie Videokonferenzen durchführten, diese Technologie zuvor noch nicht. Gleiches betrifft die lediglich 68 Einrichtungen, die spezielle TelecareLösungen einsetzten. Auch diese wurden von 57,4 Prozent dieser Einrichtungen zuvor nicht genutzt. Daneben berichteten auch Einrichtungen für alle digitalen Kommunikationstechnologien eine verringerte Nutzung, dies sind 5,8 Prozent der Einrichtungen, die eine geringere Nutzung von E-Mails nach Beginn der COVID-19-Pandemie im Vergleich zur vorherigen Situation benannten.

Abbildung 1: Veränderung der Nutzung von Kommunikationstechnologien durch Pflegeeinrichtungen während der Pandemie (N=1.239; ohne Nichtnutzende)

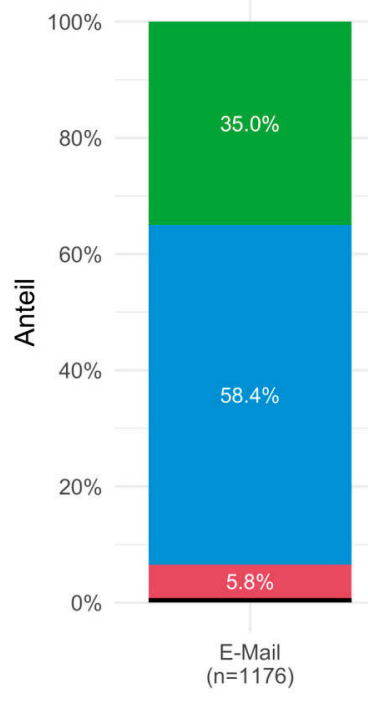

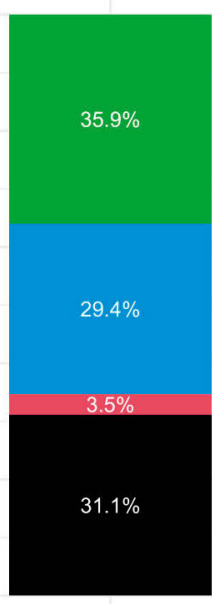

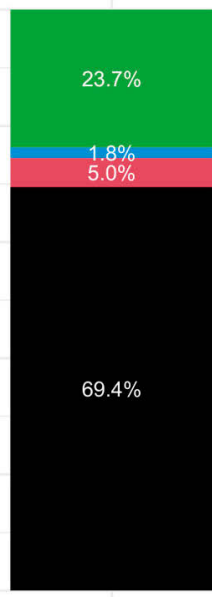

Messenger $(n=793)$

Kommunikationstechnologie

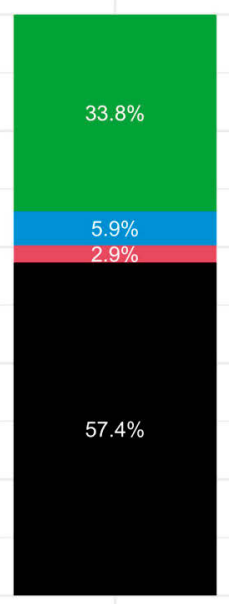

Telecare $(n=68)$ \begin{tabular}{l|l} 
Nutzung vor der Pandemie & ja, aber seltener als jetzt \\
ja, genauso häufig wie jetzt &
\end{tabular}

Quelle: Eigene Darstellung

Abbildung 2 und Abbildung 3 zeigen die Nutzungstypen der Kommunikationstechnologien differenziert nach Setting und der direkten Betroffenheit von COVID19 durch bekannte Infektionen bei Mitarbeitenden oder Pflegebedürftigen für alle Einrichtungen. Es zeigt sich, dass die überwiegende Mehrheit von stationären und ambulanten Pflegeeinrichtungen E-Mail nutzte und diese Nutzung fortgesetzt oder intensiviert hat. Jedoch nutzen 15,3 Prozent der nicht unmittelbar von COVID- 
19 betroffenen und 10,3 Prozent der betroffenen Pflegedienste keine E-Mail. Bei den stationären Einrichtungen betragen die Werte 8,0 Prozent und 3,9 Prozent respektive.

Messenger wurden insbesondere von stationären Einrichtungen während der Pandemie neu eingesetzt, von betroffenen Einrichtungen mit 26,1 Prozent dabei seltener als bei nicht-betroffenen Einrichtungen mit 28,4 Prozent. Bei Pflegediensten lag der Anteil der Nichtnutzenden unabhängig von Setting und Betroffenheit der Einrichtungen durch COVID-19 zwischen 38,3 Prozent und 42,9 Prozent. In beiden Settings war der Anteil der Neunutzenden dabei bei direkt betroffenen Einrichtungen geringer als bei nicht-betroffenen Einrichtungen.

Videokonferenzen hielten während der Pandemie insbesondere in stationären Einrichtungen Einzug. So schafften 48,4 Prozent der von COVID-19 betroffenen und 42,4 Prozent der nicht-betroffenen Einrichtungen neue Möglichkeiten zur Nutzung dieser Technologie. Bei den ambulanten Pflegeeinrichtungen bleiben 73,9 Prozent der nicht-betroffenen Einrichtungen und 62,7 Prozent der von COVID-19 betroffenen Einrichtungen Nichtnutzende.

Spezielle Telecare-Lösungen zeigen sich weder in den stationären noch in den ambulanten Pflegeeinrichtungen der Teilnehmenden als verbreitet. Lediglich 3 bis 7 Prozent der Einrichtungen hatten eine solche Technologie jemals im Einsatz. Unterschiede zwischen betroffenen und nicht-betroffenen Einrichtungen finden sich hierbei nicht.

Insgesamt zeigt sich, dass von COVID-19-Fällen direkt betroffene Einrichtungen etwas häufiger digitale Kommunikationstechnologien einsetzten als nicht-betroffene Einrichtungen der gleichen Einrichtungsart. Stationäre Pflegeinrichtungen haben in deutlich höherem Umfang als ambulante Pflegeeinrichtungen die Nutzung von Videokonferenzen zur Kommunikation aufgenommen.

\section{Merkmale, die eine vermehrte Nutzung digitaler Kommunikationstechnologien beeinflussen}

Zur Untersuchung, welche Merkmale der befragten Einrichtungen zu einer häufigeren oder neu aufgenommenen Nutzung von Technologien führten, wurden multivariate logistische Regressionsanalysen durchgeführt. Tabelle 4 zeigt die Ergebnisse für die vier berechneten Modelle. Die abhängige Variable stellte in der Analyse dichotom die neue oder vermehrte Nutzung der jeweiligen Kommunikationstechnologie dar, im Vergleich zu konstanter, geringerer oder gar keiner Nutzung als Referenz.

Die Ergebnisse zeigen, dass stationäre Pflegeeinrichtungen während der COVID-19-Pandemie deutlich häufiger digitale Kommunikationstechnologien neu einführten oder verstärkt nutzten als ambulante Pflegeeinrichtungen. Dies 
Abbildung 2: Nutzungstypen digitaler Kommunikationstechnologien differenziert nach COVID-19-Betroffenheit, ambulante Pflegeeinrichtungen $(\mathrm{N}=598)$

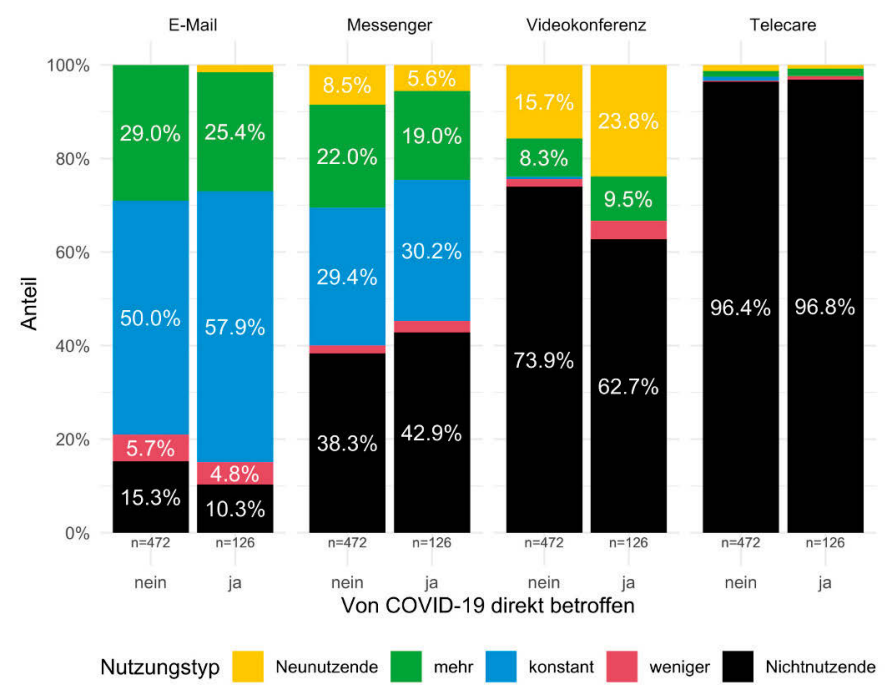

Quelle: Eigene Darstellung

spiegelt sich in Odds Ratios für dieses Merkmal zwischen 2,59 für Messengerdienste und 4,25 für Videokonferenz wider. Darüber hinaus zeigen die übrigen untersuchten Merkmale mit der Anzahl der Mitarbeitenden der Einrichtung, der Anzahl der Pflegebedürftigen, der Trägerschaft und den Anteilen von an COVID-19-Fällen bei den Mitarbeitenden und Pflegebedürftigen keinen offenkundigen Zusammenhang, der mit einer Neueinführung oder stärkeren Nutzung der genannten Kommunikationstechnologien assoziiert ist. Lediglich bei Videokonferenzen zeigt sich für die Anzahl der Pflegebedürftigen, die durch eine Einrichtung versorgt werden, ein statistisch signifikantes positives Odds Ratio, welches jedoch nur geringfügig über eins liegt und damit eine zu vernachlässigende Effektstärke aufweist. Ebenso legen die Ergebnisse nahe, dass Einrichtungen in öffentlicher Trägerschaft deutlich seltener Telecare-Technologien einsetzen. Bei der Bewertung dieses Ergebnisses ist zu beachten, dass nur eine geringe Anzahl von Einrichtungen, die Telecare einsetzten, und nur wenig öffentlich getragene Pflegeeinrichtungen befragt wurden. 
Abbildung 3: Nutzungstypen digitaler Kommunikationstechnologien differenziert nach COVID-19-Betroffenheit, stationäre Pflegeeinrichtungen $(N=712)$

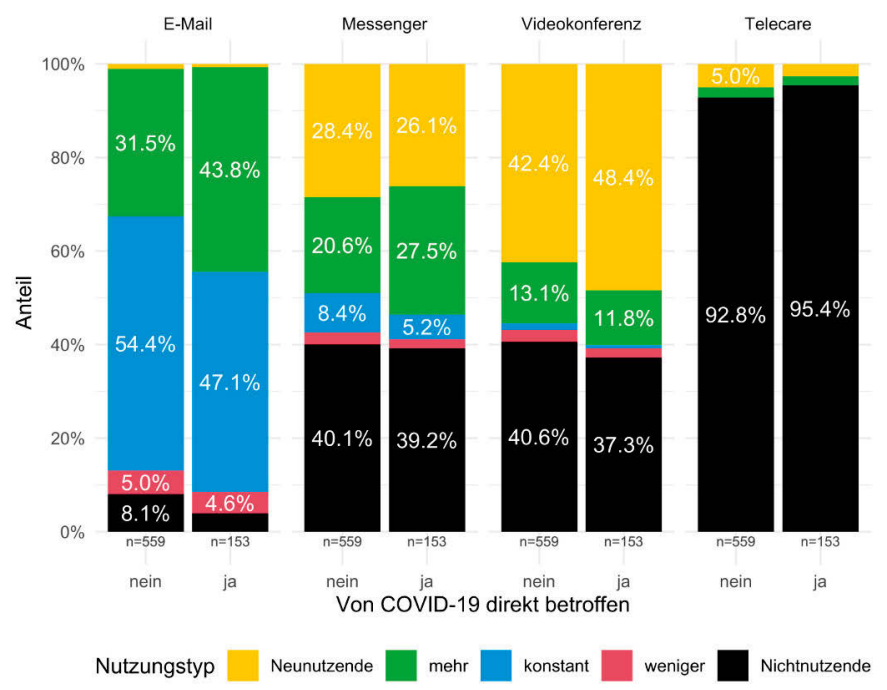

Quelle: Eigene Darstellung

Tabelle 4: Logistische Regressionsanalysen zu Faktoren der neuen oder vermehrten Nutzung von Kommunikationstechnologien ( $N=1.231)$

\begin{tabular}{|l|l|l|l|l|}
\hline & E-Mail & Messenger & Videokonferenz & Telecare \\
\hline Merkmal & $\begin{array}{l}\text { OR } \\
{[95 \%-K I]}\end{array}$ & $\begin{array}{l}\text { OR } \\
{[95 \%-K I]}\end{array}$ & $\begin{array}{l}\text { OR } \\
{[95 \%-K I]}\end{array}$ & $\begin{array}{l}\text { OR } \\
{[95 \%-K I]}\end{array}$ \\
\hline $\begin{array}{l}\text { Einrichtung: } \\
\text { ambulant }\end{array}$ & $\begin{array}{l}1,00 \\
\text { (Ref.) }\end{array}$ & $\begin{array}{l}1,00 \\
\text { (Ref.) }\end{array}$ & $\begin{array}{l}1,00 \\
\text { (Ref.) }\end{array}$ & $\begin{array}{l}1,00 \\
\text { (Ref.) }\end{array}$ \\
\hline $\begin{array}{l}\text { Einrichtung: } \\
\text { stationär }\end{array}$ & $\begin{array}{l}1,21 \\
{[0,92-1,65]}\end{array}$ & $\begin{array}{l}2,59^{*} \\
{[1,99-3,38]}\end{array}$ & $\begin{array}{l}4,25^{*} \\
{[3,25-5,59]}\end{array}$ & $\begin{array}{l}3,16^{*} \\
{[1,63-6,87]}\end{array}$ \\
\hline $\begin{array}{l}\text { Anzahl der } \\
\text { Mitarbeitenden }\end{array}$ & 1,00 & 1,00 & 1,00 & 1,00 \\
\hline$[1,00-1,00]$ & {$[1,00-1,00]$} & {$[1,00-1,00]$} & {$[1,00-1,00]$} \\
\hline $\begin{array}{l}\text { Anzahl der Pflege- } \\
\text { bedürftigen }\end{array}$ & $\begin{array}{l}1,00 \\
{[1,00-1,00]}\end{array}$ & $\begin{array}{l}1,00 \\
{[1,00-1,00]}\end{array}$ & $\begin{array}{l}1,00^{*} \\
{[1,00-1,00]}\end{array}$ & $\begin{array}{l}1,00 \\
{[1,00-1,00]}\end{array}$ \\
\hline $\begin{array}{l}\text { Träger"innen: } \\
\text { freigemeinnützig }\end{array}$ & 1,00 (Ref.) & 1,00 (Ref.) & 1,00 (Ref.) & 1,00 (Ref.) \\
\hline
\end{tabular}




\begin{tabular}{|l|l|l|l|l|}
\hline & E-Mail & Massenger & Videokonferenz & Telecare \\
\hline $\begin{array}{l}\text { Träger*innen: } \\
\text { öffentlich }\end{array}$ & $\begin{array}{l}0,7 \\
{[0,43-1,11]}\end{array}$ & $\begin{array}{l}0,78 \\
{[0,49-1,22]}\end{array}$ & $\begin{array}{l}0,99 \\
{[0,63-1,55]}\end{array}$ & $\begin{array}{l}0,17^{*} \\
{[0,01-0,81]}\end{array}$ \\
\hline $\begin{array}{l}\text { Träger*innen: } \\
\text { privat }\end{array}$ & $\begin{array}{l}0,79 \\
{[0,61-1,03]}\end{array}$ & $\begin{array}{l}1,07 \\
{[0,83-1,38]}\end{array}$ & $\begin{array}{l}0,91 \\
{[0,70-1,18]}\end{array}$ & $\begin{array}{l}0,99 \\
{[0,56-1,74]}\end{array}$ \\
\hline $\begin{array}{l}\text { Anteil COVID-19- } \\
\text { Fälle bei Mitarbei- } \\
\text { tenden (\%) }\end{array}$ & $\begin{array}{l}1,06 \\
{[0,98-1,15]}\end{array}$ & $\begin{array}{l}1,01 \\
{[0,93-1,1]}\end{array}$ & $\begin{array}{l}1,03 \\
{[0,95-1,11]}\end{array}$ & $\begin{array}{l}1,03 \\
{[0,78-1,24]}\end{array}$ \\
\hline $\begin{array}{l}\text { Anteil COVID-19- } \\
\text { Fälle bei Pflegebe- } \\
\text { dürftigen (\%) }\end{array}$ & $\begin{array}{l}1,00 \\
{[0,95-1,05]}\end{array}$ & $\begin{array}{l}1,01 \\
{[0,97-1,06]}\end{array}$ & $\begin{array}{l}0,98 \\
{[0,94-1,03]}\end{array}$ & $\begin{array}{l}0,93 \\
{[0,73-1,08]}\end{array}$ \\
\hline
\end{tabular}

Quelle: Eigene Darstellung; OR: Odds Ration; * p < 0,05; 95 \%-KI: 95 \%-Konfidenzintervall

Im Hinblick auf die Unterstützung sozialer Kontakte der Pflegebedürftigen wurden von den Einrichtungen ebenfalls Möglichkeiten geschaffen, diese mittels digitaler Kommunikation zu erreichen (siehe Abbildung 4). So gaben 75,9 Prozent der stationären Einrichtungen an, dass dies zum Befragungszeitpunkt bereits geschehen ist, weitere 12,7 Prozent gaben an, dass sich dieses in Planung befindet. Bei den ambulanten Pflegeeinrichtungen gaben 14,9 Prozent an, dass bereits entsprechende Möglichkeiten geschaffen wurden, während sich 5,8 Prozent noch in der Planung befinden.

In 537 auswertbaren Freitextantworten benannten die Befragten aus 63 Pflegediensten und 474 Pflegeheimen die Art dieser zusätzlich geschaffenen Kommunikationsmöglichkeiten. Dabei dominierten kostenlose Videokonferenzsysteme wie Skype oder Microsoft Teams in jeweils über 70 Prozent der formulierten Antworten die Beschreibungen, gefolgt von Messengerdiensten wie WhatsApp, die in jedem vierten Textbeitrag aus ambulanten Pflegediensten und in knapp 16 Prozent der Texte aus Pflegeheimen benannt wurden. Gleichsam wurden in den Antworten jedoch auch Hürden für den Einsatz von digitalen Kommunikationstechnologien beschrieben, etwa, wenn Pflegebedürftige diese nicht nutzen möchten oder seitens der Angehörigen entsprechende technische Voraussetzungen und Kompetenzen fehlen. E-Mail sowie Telecare-Technologien spielen für die Unterstützung sozialer Kontakte der Pflegebedürftigen kaum eine Rolle und wurden insgesamt in unter 2 Prozent der Freitextantworten genannt (genannt wurden hier Medifox Connect, CGM ELVI, myo App und die Patientus Video Sprechstunde). Acht ambulante Pflegedienste und 155 Pflegeheime beschrieben jedoch auch die Anschaffung von entsprechender Hardware, darunter vor allem Tablets, Laptops sowie Smartphones, die in einigen Pflegeheimen durch eine von der Telekom initiierte Spendenaktion bereitgestellt wurden. Nur vereinzelt wiesen die Antwortenden darauf hin, dass Ange- 
hörige angehalten waren, entsprechende Hardware zu kaufen oder bereitzustellen. Wenige weitere Antworten beschrieben den Ausbau der digitalen Infrastruktur in Bezug auf (W)LAN, die Befreiung von Telefongebühren für Bewohner*innen von Pflegeheimen oder die verstärkte Nutzung und Pflege der eigenen Webseite für die Außenkommunikation und Information von Angehörigen.

Abbildung 4: Zusätzliche digitale Kommunikationsmöglichkeiten für Pflegebedürftige $(N=1.200)$

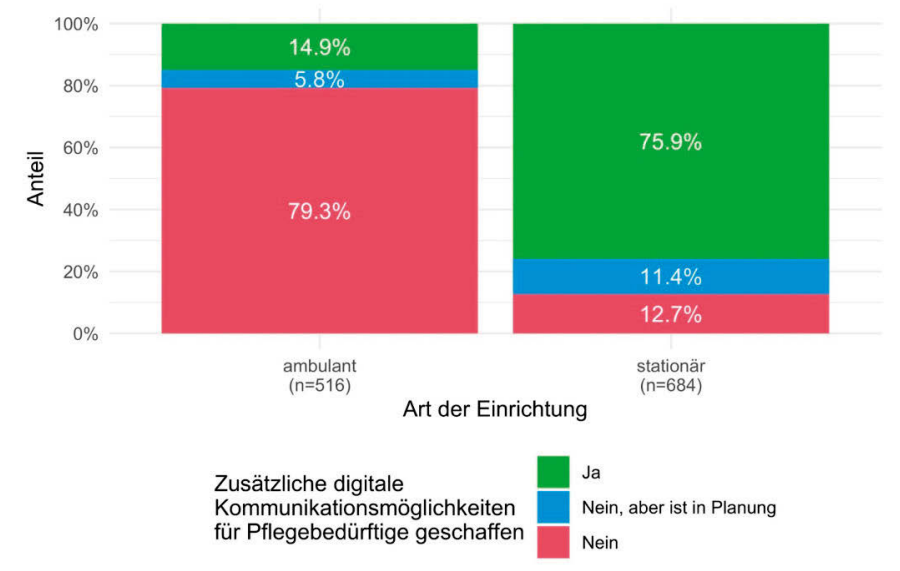

Quelle: Eigene Darstellung

In weiteren 490 auswertbaren Freitextantworten äußerten sich die Befragten $\mathrm{zu}$ aus ihrer Sicht sinnvollen Maßnahmen, um Pflegeeinrichtungen während der Pandemie zu unterstützen. Als Nebenbefund ließen sich hier neunzehn Beiträge (elf Pflegedienste, acht Pflegeheime) identifizieren, die das Thema der digitalen Kommunikation oder Digitalisierung von Pflegeeinrichtungen aufgriffen und sich dafür aussprachen, Digitalisierung verstärkt voranzutreiben und finanziell $\mathrm{zu}$ unterstützen. Insbesondere für die schnittstellenübergreifende Kommunikation mit Leistungserbringenden der Primärversorgung sowie zur Etablierung von E-Learning Angeboten für Schulung und Fortbildungen der Mitarbeitenden, aber auch zur teaminternen Kommunikation erschien den Befragten dabei der Einsatz von digitalen Lösungen während der COVID-19-Pandemie sinnvoll. 


\section{Diskussion}

Dieser Beitrag untersucht das Nutzungsverhalten und beeinflussende Faktoren der Nutzung von digitalen Kommunikationstechnologien in ambulanten und stationären Pflegeeinrichtungen in Deutschland während der COVID-19-Pandemie im Frühjahr des Jahres 2020. Wie in anderen Lebensbereichen (Bär et al. 2020; Krcmar/Wintermann 2020) verändert die COVID-19-Pandemie auch in den Pflegeeinrichtungen den Umgang mit digitalen Kommunikationstechnologien.

Die Ergebnisse zeigen, dass ambulante und insbesondere stationäre Pflegeeinrichtungen im Zuge der COVID-19-Pandemie vermehrt digitale Kommunikationstechnologien zur Kommunikation innerhalb der Institution, aber auch zur Wahrung von sozialen Kontakten der von ihnen versorgten pflegebedürftigen Personen eingesetzt haben. Der Fokus der verwendeten Technologien lag dabei auf frei verfügbaren Lösungen, die nicht spezifisch für die Pflege entwickelt wurden. E-Mail wurde bereits vor dem Beginn der Pandemie von der stark überwiegenden Mehrheit aller Einrichtungen genutzt, wobei sich die Intensität der Nutzung seitdem erhöht hat. Zugleich existiert in beiden Settings eine nennenswerte Anzahl von Einrichtungen, die dieses mittlerweile als geläufig zu bezeichnende Kommunikationsmittel bisher nicht umfassend einzusetzen scheinen. Die Nutzung von Messengerdiensten und Videokonferenzen hat deutlich zugenommen. Spezielle Telecare-Systeme, also spezifisch für die Pflege beziehungsweise für die Anwendung in der gesundheitlichen Versorgung entwickelte Kommunikationslösungen, wurden hingegen kaum eingesetzt.

Unabhängig von der Art der Technologie zeigt sich, dass stationäre Einrichtungen insbesondere die Nutzung von digitalen Technologien deutlich verstärkten - sowohl für die interne Kommunikation, beispielsweise mit den Mitarbeitenden, als auch für die Unterstützung der sozialen Kontakte der versorgten Personen. Eine generell höhere Nutzung digitaler Kommunikationstechnologien in stationären gegenüber ambulanten Einrichtungen zeigt sich auch in den Ergebnissen einer Befragung ambulanter und stationärer Pflegeeinrichtungen im Jahr 2019 (vgl. Haug in diesem Band). Weitere Faktoren der Einrichtungen, wie deren Größe oder die Trägerschaft, zeigten ebenso keinen Zusammenhang mit der Technologienutzung wie die direkte Betroffenheit der Einrichtungen durch COVID-19-Fälle bei Mitarbeitenden oder Pflegebedürftigen.

Allgemein sahen Teilnehmende beider Versorgungssettings den Ausbau der digitalen Infrastruktur von Pflegeeinrichtungen und die Steigerung des Digitalisierungsgrades auch im Kontext der COVID-19-Pandemie als sinnvoll an. Vor allem die schnittstellenübergreifende Kommunikation mit externen Leistungserbringenden sowie die Möglichkeiten von digitlen Schulungs- und Fortbildungsangeboten für die Mitarbeitenden wurden als Entwicklungs- und Anwendungsfelder für di- 
gitale Lösungen beschrieben, wobei verstärkte finanzielle Unterstützung hierfür gefordert wurde.

Die von den Befragten in den Einrichtungen genannten Lösungen, vorrangig Skype und WhatsApp, werden insbesondere von Privatpersonen genutzt. Dies lässt vermuten, dass vor allem solche Kommunikationstechnologien auf das eigene Arbeitssetting übertragen wurden, die den Befragten aus anderen (privaten) Anwendungskontexten bereits bekannt waren. Die Nutzung von frei verfügbaren Videokonferenzlösungen und Messengerdiensten stützt die bereits in anderen Forschungsprojekten zum Thema >Technik und Pflege`, wie dem Pflegeinnovationszentrum (IPP 2020), formulierte Forderung, bestehende digitale Lösungen hinsichtlich ihrer Übertragbarkeit und Eignung für die Anwendung in pflegerischen Arbeitssettings zu prüfen (Seibert et al. 2020).

Für Kommunikationstechnologien, die pflegespezifische Inhalte oder Anforderungen aufgreifen, scheint hingegen Implementierungs- und eventuell auch Entwicklungspotenzial zu bestehen. In der Studie zur Bedarfsermittlung von innovativen Technologien in der Pflege (Seibert et al. 2020) wurde auch die Entwicklung von pflegespezifischen Technologien von teilnehmenden Pflegefachpersonen hervorgehoben, welche die Besonderheiten des Pflegeprozesses und der Organisation von Pflege berücksichtigten. In der hier gegenständlichen Befragung wurde die Nutzung von speziellen Telecare-Technologien hingegen nur von einem äußerst geringen Anteil der Befragten beschrieben. Neben der Kenntnis über verfügbare Telecare-Technologien benötigt deren Anwendung eventuell ein höheres Ausmaß an Implementationsbemühungen als das Bereitstellen eines Tablets mit der Möglichkeit für Videokonferenzen. Zudem beklagte eine Vielzahl von Einrichtungen auch eine unzureichende Information oder Unterstützung während der COVID-19Pandemie durch externe, beratende und prüfende Institutionen (Wolf-Ostermann et al. 2020a). Beides kann zu einem Zeitpunkt, an dem Pflegeeinrichtungen und deren Mitarbeitende mit vielfältigen und neuen Handlungsrichtlinien während des Pandemiegeschehens konfrontiert wurden und eine erhöhte Belastung aufwiesen (Hower/Pfaff/Pförtner 2020; Wildgruber et al. 2020), eine zusätzliche Implementierungshürde für Telecare-Technologien darstellen.

In Ermangelung einer allgemeingültigen Definition von >Telecare (Turner 2015) könnten auch außerhalb der Pflege entwickelte und eingesetzte Technologien als pflegespezifisch betrachtet werden, wenn sie für die Gestaltung des Pflegeprozesses eingesetzt werden. Jedoch sind derartige Technologien primär durch das Fehlen pflegespezifischer Inhalte oder Nutzungskonzepte gekennzeichnet. Hier kommt der Kreativität der Anwender*innen für die Gestaltung der Nutzung in diesen Fällen eine besondere Rolle $\mathrm{zu}$, während diese bei der Anwendung von konkret für den Einsatz in Pflegeeinrichtungen entwickelten Technologien durch den vom herstellenden Unternehmen definierten Anwendungsbezug vorgegeben wird. Offen bleibt an dieser Stelle, ob und, wenn ja, welche pflegespezifischen Anforderungen 
an Kommunikationstechnologien zu stellen und gegebenenfalls auf verfügbare Lösungen in anderen Bereichen anzuwenden sind.

Auffallend ist, dass jede vierte befragte Person aus Pflegeheimen angab, Videokonferenzen und Messengerdienste für die Kommunikation mit den Pflegebedürftigen zu nutzen. In Zusammenhang mit den in den Freitexten benannten hohen Anteilen von Videokonferenzen für die Unterstützung sozialer Kontakte der Pflegebedürftigen ist dies möglicherweise ein Hinweis darauf, dass die Formulierung der Frage nach der Art der Technologie für die Kommunikation mit verschiedenen Akteur*innengruppen nicht von allen Befragten eindeutig im Sinne des Items verstanden und beantwortet wurde. Vielmehr scheinen hier auch die Erfahrungen in der Begleitung und dem Angebot der Kommunikation anhand von Videokonferenzen und Messengerdiensten zwischen den Bewohner*innen und deren Angehörigen abgebildet worden zu sein. Dies wäre insofern zu begrüßen, als dass es neben einem Hinweis auf vermehrte Aktivitäten zur digitalen Teilhabe pflegbedürftiger Menschen auch aufzeigt, dass mit der Wahl von bildgestützter Kommunikation ein für ältere Menschen eventuell eher geeignetes Medium als Textkonversation gewählt wurde (Seifert/Cotten/Xie 2020). Vor allem in Hinblick auf die während der COVID-19-Pandemie für ältere und vulnerable Personengruppen in Pflegeeinrichtungen verstärkte Ausgrenzung von sozialer Interaktion und der für diese Personengruppe beschriebenen digitalen Kluft (engl. digital divide) (Seifert/Cotten/Xie 2020) scheinen ungenutzte Einsatzpotenziale für Kommunikationstechnologien zu bestehen, die sich in dieser Befragung in der generellen Nutzungsbereitschaft der Teilnehmenden ausdrücken. In diesem Kontext ist auch der Zeitpunkt der Befragung zu betrachten, welcher mit etwa sechs bis acht Wochen nach der Etablierung von kontaktreduzierenden Maßnahmen als seher am Anfang، der COVID-19-Pandemie in Deutschland zu verorten ist. Aufgrund einer dynamischen Entwicklung und in der Erwartung einer >zweiten Welle ist davon auszugehen, dass entsprechende Kommunikationstechnologien seitdem noch ausgeweitet wurden beziehungsweise durch andere Lösungen ersetzt wurden. Die von den Teilnehmenden benannte Notwendigkeit einer weitergehenden Digitalisierung in Pflegeeinrichtungen könnte hierbei Lösungen hervorbringen, die neben pflegespezifischen Anwendungsfällen auch die Anforderungen an den Datenschutz beinhalten, sodass eine weitergehende Nutzung, etwa beim Austausch von pflegerischen und medizischen Daten, sicher ermöglicht wird.

Für die Generalisierbarkeit der berichteten Ergebnisse ist einerseits das gewählte Vorgehen der Stichprobenbildung zu beachten. Im Vergleich zu den Daten der Pflegestatistik (Destatis 2018) fällt auf, dass sich tendenziell eher Personen aus größeren Einrichtungen beteiligten. Die Nutzungserfahrungen digitaler Kommunikationstechnologien vor allem kleinerer Einrichtungen sind in den Ergebnissen eventuell eingeschränkt abgebildet. Da die Rekrutierung sowie die Beantwortung des Fragebogens ebenfalls online erfolgten, ist es andererseits möglich, dass die 
Befragten in grundsätzlich eher technikaffinen Pflegeeinrichtungen tätig sind und hier eventuell die Nutzungserfahrungen aus eher technikaversen Einrichtungen unterrepräsentiert sind. Zugleich finden sich unter den Teilnehmenden der Befragung jedoch auch Einrichtungen, die für die Kommunikation mit den in der Befragung erhobenen Akteur*innengruppen keine E-Mail nutzten. Dies weist darauf hin, dass auch Einrichtungen teilnahmen, die Ausweitungspotenzial in Bezug auf digitale Kommunikation aufweisen.

Dies weist auf weitere Limitationen der Studie hin, die für die Interpretation der berichteten Ergebnisse zu diskutieren sind. Darunter zuvorderst das Verständnis und die Trennschärfe der gewählten Kategorien der Items. Besonders für Videokonferenzen und Messengerdienste, aber ebenso für Telecare-Technologien, ist nicht auszuschließen, dass die Teilnehmenden diese in allen Fällen, trotz beispielhafter Nennung einzelner Anwendungen im Fragebogen, nicht eindeutig voneinander abgrenzen konnten. Eine solche Abgrenzung wird nicht zuletzt durch die Multifunktionalität einzelner Anwendungen erschwert, die bei der Betrachtung der Ergebnisse zu berücksichtigen ist.

Die Freitextantworten lassen generell darauf schließen, dass die Teilnehmenden ein hohes Interesse an der Thematik der zugrunde liegenden Befragung aufwiesen. Obwohl Digitalisierung und die Anwendung digitaler Technologien in der Pflege nicht explizit abgefragt beziehungsweise nur in wenigen Items des Fragebogens inhaltlich aufgegriffen wurden, fällt auf, dass diese Themen dennoch von den Teilnehmenden im Kontext von sinnvollen Unterstützungsmaßnahmen aufgegriffen wurden. Dies unterstreicht die an anderer Stelle mehrfach beschriebene Bedeutung und das Optimierungspotenzial des Einsatzes digitaler Technologien in der Pflegepraxis, besonders für die Gestaltung von schnittstellenübergreifender Information und Kommunikation (Bündnis Digitalisierung in der Pflege 2020; Roland Berger $\mathrm{GmbH} /$ Deutsches Institut für angewandte Pflegeforschung e.V./Philosophisch-Theologische Hochschule Vallendar 2017). Dabei ist jedoch zu beachten, dass eine vermehrte Nutzung von digitalen Technologien in der Pflege neben infrastrukturellen Anpassungen auch Veränderungen von Kompetenzprofilen der Anwendenden (Seifert/Cotten/Xie 2020) und eine Neugestaltung von Arbeits- und Organisationsprozessen in Pflegeeinrichtungen erforderlich macht.

\section{Schlussfolgerung und Ausblick}

Die COVID-19-Pandemie hat auch in Pflegeeinrichtungen der Digitalisierung Vorschub geleistet. Unter Berücksichtigung der vorliegenden Evidenz sowie vorangegangenen Untersuchungen zu Technik und Digitalisierung in der Pflege ist davon auszugehen, dass Pflegeeinrichtungen für eine kurzfristige Umsetzung umfassender digitaler Kommunikationsmöglichkeiten eine ungünstige Ausgangspositi- 
on aufwiesen. Ein Ausbau der technischen Infrastruktur wird gemeinhin als notwendig und überfällig erachtet, als Hemmnis werden hierfür insbesondere unzureichende Finanzierungsmöglichkeiten für Anschaffungen, Betrieb und Beratung benannt. Zugleich wird jedoch auch die fachliche Begleitung der Auswahl, der Implementation und auch der Entwicklung von technischen Lösungen in der Pflege gefordert. Dies tritt noch einmal mehr in den Vordergrund, wenn etwa digitale Kommunikationstechnologien die gestaltende und vermittelnde Rolle der Pflegefachpersonen in der Anwendung mit pflegebedürftigen Menschen verstärkt erforderlich machen, etwa bei der Unterstützung im Umgang mit Videotelefonaten. Neben der Entwicklung von Implementierungs-, Schulungs- und Evaluationskonzepten werden somit zukünftig auch die Vermittlung digitaler Kompetenzen in Ausbildung und Studium für Pflegefachpersonen und die Untersuchung der Wirkungen des Technikeinsatzes auf Pflegebedürftige und deren Angehörige, aber auch auf das Berufsfeld Pflege und die Organisationsprozesse von Pflegeeinrichtungen für die Pflegeforschung an Bedeutung gewinnen. Einer qualifizierten externen Beratung und Begleitung von Einrichtungen kann hier derzeit höchstens punktuell nachgekommen werden. Interdisziplinäre, unabhängige Kompetenzzentren, die Pflegeeinrichtungen im Auswahl-, Einführungs- und Anwendungsprozess von digitalen Technologien begleiten, aber auch Forschung und Entwicklung im Designprozess bedarfsgerechter, pflegespezifischer Lösungen beraten, gewinnen daher zunehmend an Bedeutung.

Neben den bestehenden Lücken zeigt sich jedoch auch, dass Lösungen für vielfältige Einsatzbereiche existieren und von den Pflegefachpersonen und Pflegeeinrichtungen kurzfristig eingesetzt werden können. Infrastrukturelle und personelle Ressourcen bleiben jedoch notwendig, um diesen Technologieeinsatz qualitativ und quantitativ zu befördern. Nicht zuletzt bleiben die Aus- und Wechselwirkungen des Technologieeinsatzes auf die individuellen pflegebedürftigen Menschen und für sie relevante Endpunkte, wie die Qualität der Versorgung, ihre soziale Teilhabe oder Lebensqualität, sowie die Akteur*innen der Pflegeeinrichtungen und deren Prozesse und Organisationsformen zu untersuchen, um den Beitrag digitaler (Kommunikations-)Technologien zur pflegerischen Versorgung der Gesellschaft abzubilden.

\section{Dank und Anerkennung}

Wir bedanken uns bei allen teilnehmenden Personen aus den Pflegeeinrichtungen für die Mitwirkung an der Studie und bei Anna-Carina Friedrich, Franziska Heinze, Benedikt Preuss, Annika Schmidt und Claudia Stolle für die Mitarbeit im zugrundeliegenden Forschungsprojekt. 


\section{Literatur}

Ammenwerth, Elske/Iller, Carola/Mahler, Cornelia (2006): »IT-adoption and the interaction of task, technology and individuals: A fit framework and a case study«, in: BMC Medical Informatics and Decision Making 6, S. 3.

Bär, Dorothee/Bentkämper, Petra/Berg, Achim/Sager, Reinhardt (2020): Digitaltag 2020: Gemeinsam digitale Teilhabe fördern. Berlin 16. Juni 2020, https:/digit altag.eu/sites/default/files/2020-06/Pr\%C3\%A4sentation\%20Digitaltag-Auftak t-PK\%2016\%2006\%202020_final.pdf vom 04.05.2021.

Braeseke, Grit/Meyer-Rötz, Sinja H./Pflug, Claudia/Haaß, Friederike (2017): Digitalisierung in der ambulanten Pflege - Chancen und Hemmnisse. Berlin: IGES.

Bräutigam, Christoph/Enste, Peter/Evans, Michaela/Hilbert, Josef/Merkel, Josef/Öz, Fikret (2017): Digitalisierung im Krankenhaus. Mehr Technik - bessere Arbeit? Forschungsförderung. Düsseldorf: Hans-Böckler-Stiftung.

Bündnis Digitalisierung in der Pflege (2020): Digitalisierung in der Pflege: Eckpunkte einer nationalen Strategie. Bündnis Digitalisierung in der Pflege, 4. August 2020, https://deutscher-pflegerat.de/wp-content/uploads/2020/09/ 2020-09-01_Positionspapier_Verb\%C3\%A4ndeb\%C3\%BCndnis_Digitalisierung _Pflege.pdf vom 04.05.2021.

Claßen, Katrin/Oswald, Frank/Wahl, Hans-Werner/Heusel, Christof/Antfang, Peter/Becker, Clemens (2010): »Bewertung neuerer Technologien durch Bewohner und Pflegemitarbeiter im institutionellen Kontext. Befunde des Projekts BETAGT«, in: Zeitschrift für Gerontologie und Geriatrie 43, S. 210-218.

Destatis (Statistisches Bundesamt) (2018): Pflegestatistik 2017. Pflege im Rahmen der Pflegeversicherung Deutschlandergebnisse. Wiesbaden: Statistisches Bundesamt.

Fehling, Patrick (2019): »Entwicklungsstand der gegenwärtigen und künftigen technischen Assistenzsysteme«, in: Pflege \& Gesellschaft 24, S. 197-205.

Fehling, Patrick/Dassen, Theo (2017): »Motive und Hürden bei der Etablierung technischer Assistenzsysteme in Pflegeheimen. Eine qualitative Studie«, in: Klinische Pflegeforschung 3, S. 61-71.

Greenhalgh, Trisha/Abimbola, Seye (2019): »The NASSS Framework - A Synthesis of Multiple Theories of Technology Implementation «, in: Studies in Health Technology and Informatics 263, S. 193-204.

Hielscher, Volker/Nock, Lukas/Kirchen-Peters, Sabine (2015): Technikeinsatz in der Altenpflege. Potenziale und Probleme in empirischer Perspektive. BadenBaden: Nomos.

Hower, Kira Isabel/Pfaff, Holger/Pförtner, Timo-Kolja (2020): »Pflege in Zeiten von COVID-19: Onlinebefragung von Leitungskräften zu Herausforderungen, Belastungen und Bewältigungsstrategien«, in: Pflege 33, S. 207-218. 
Hülsken-Giesler, Manfred (2010): »Technikkompetenzen in der Pflege«, in: Pflege \& Gesellschaft 15, S. 330-352.

IPP (Institut für Public Health und Pflegeforschung) (2020): Pflegeinnovationszentrum (PIZ), https://www.ipp.uni-bremen.de/abteilungen/pflegewissenschaftli che-versorgungsforschung/projekte/laufende-projekte/?proj=719\&page=1 vom 24.09.2020.

Krcmar, Helmut/Wintermann, Ole (2020): Studie $\mathrm{zu}$ den Auswirkungen der Corona-Pandemie in gesellschaftlicher, wirtschaftlicher und technologischer Hinsicht. Gütersloh, München, Heilbronn: Bertelsmann Stiftung, Münchner Kreis e.V., TUM Campus Heilbronn.

Krick, Tobias/Huter, Kai/Domhoff, Dominik/Schmidt, Annika/Rothgang, Heinz/Wolf-Ostermann, Karin (2019): "Digital technology and nursing care: A scoping review on acceptance, effectiveness and efficiency studies of informal and formal care technologies«, in: BMC Health Services Research 19, S. 400 .

Merda, Meiko/Schmidt, Kristina/Kähler, Bjørn (2017): Pflege 4.0 - Einsatz moderner Technologien aus der Sicht professionell Pflegender. Forschungsbericht. Hamburg: Berufsgenossenschaft für Gesundheitsdienst und Wohlfahrtspflege (BGW).

Questback GmbH (2019): EFS Survey, Version Fall 2019. Köln: Questback GmbH.

RKI (2020): Prävention und Management von COVID-19 in Alten- und Pflegeeinrichtungen und Einrichtungen für Menschen mit Beeinträchtigungen und Behinderungen. Empfehlungen des Robert Koch-Instituts für Alten- und Pflegeeinrichtungen und Einrichtungen für Menschen mit Beeinträchtigungen und Behinderungen und für den öffentlichen Gesundheitsdienst. V.o6. Berlin: Robert Koch-Institut.

Roland Berger $\mathrm{GmbH} /$ Deutsches Institut für angewandte Pflegeforschung e.V./Philosophisch-Theologische Hochschule Vallendar (2017): ePflege. Informations- und Kommunikationstechnologie für die Pflege. Stude im Auftrag des Bundesministeriums für Gesundheit. Berlin, Vallendar, Köln: Roland Berger $\mathrm{GmbH}$, Deutsches Institut für angewandte Pflegeforschung e.V., Philosophisch-Theologische Hochschule Vallendar.

Rothgang, Heinz/Domhoff, Dominik/Friedrich, Anna-Carina/Heinze, Franziska/Preuss, Benedikt/Schmidt, Annika/Seibert, Kathrin/Stolle, Claudia/WolfOstermann, Karin (2020): »Pflege in Zeiten von Corona: Zentrale Ergebnisse einer deutschlandweiten Querschnittsbefragung vollstationärer Pflegeheime«, in: Pflege 33, S. 265-275.

Schlomann, Anna/Seifert, Alexander/Zank, Susanne/Rietz, Christian (2020): »Assistive Technology and Mobile ICT Usage Among Oldest-Old Cohorts: Comparison of the Oldest-Old in Private Homes and in Long-Term Care Facilities«, in: Research on Aging 42, S. 163-173. 
Seibert, Kathrin/Domhoff, Dominik/Huter, Kai/Krick, Tobias/Rothgang, Heinz/ Wolf-Ostermann, Katrin (2020): »Application of digital technologies in nursing practice: Results of a mixed methods study on nurses' experiences, needs and perspectives«, in: Zeitschrift für Evidenz, Fortbildung und Qualität im Gesundheitswesen 158, S. 94-106.

Seifert, Alexander/Cotten, Shelia R./Xie, Bo (2020): »A Double Burden of Exclusion? Digital and Social Exclusion of Older Adults in Times of COVID-19«, in: The Journals of Gerontology: Series B 76, S. e99-e103

Thiel, Rainer/Deimel, Lucas/Schmidtmann, Daniel/Piesche, Klaus/Hüsing, Tobias/Rennoch, Jonas/Stroetmann, Veli/Stroetmann, Karl (2018): \#SmartHealthSystems. Digitalisierungsstrategien im internationalen Vergleich. Gütersloh: Bertelsmann Stiftung.

Turner, Kenneth J. (2015): »The Ecology of Home Sensor Networks for Telecare«. In: Saito, N., Menga, D. (Hg.): Ecological Design of Smart Home Networks: Technologies, Social Impact and Sustainability. Swaston: Woodhead Publishing.

Wildgruber, Domenika/Frey, Jana/Seer, Max/Pinther, Kristina/Koob, Clemens/Reuschenbach, Bernd (2020): »Arbeitsengagement und Belastungserleben von Health Professionals in Zeiten der Corona-Pandemie«, in: Pflege 33, S. 299-307. Wolf-Ostermann, Karin/Rothgang, Heinz/Domhoff, Dominik/Friedrich, AnnaCarina/Heinze, Franziska/Preuß, Benedikt/Schmidt, Annika/Seibert, Kathrin/Stolle, Claudia (2020a): Zur Situation der Langzeitpflege in Deutschland während der Corona-Pandemie. Ergebnisse einer Online-Befragung in Einrichtungen der (teil)stationären und ambulanten Langzeitpflege. Bremen: IPP, SOCIUM.

Wolf-Ostermann, Karin/Schmidt, Annika/Preuß, Benedikt/Heinze, Franziska/Seibert, Kathrin/Friedrich, Anna-Carina/Domhoff, Dominik/Stolle, Claudia/Rothgang, Heinz (2020b): »Pflege in Zeiten von Corona: Ergebnisse einer deutschlandweiten Querschnittbefragung von ambulanten Pflegediensten und teilstationären Einrichtungen«, in: Pflege 33, S. 277-288.

ZQP (Zentrum für Qualität in der Pflege) (2019): ZQP-Report Pflege und Digitale Technik. Berlin: Zentrum für Qualität in der Pflege. 


\section{Arbeit und Geschlecht. Strukturelle und normative Grundlagen von Technisierungsprozessen in der Pflege}

Clarissa Rudolph

\section{Klatschen für die Pflege?}

2020 war das »Internationale Jahr der Pflegenden und Hebammen« und 2020 wurde über die Arbeitsbedingungen von Pflegekräften in Deutschland tatsächlich viel gesprochen. Im Zuge der Corona-Pandemie wurde dieses Thema in einem Ausmaß öffentlich, wie es sich viele Akteur*innen lange Zeit gewünscht hätten (Rudolph/Schmidt 2019a; Schmidt 2019): Die `Systemrelevanz`der Pflegekräfte wurde ausgiebig gelobt und es wurde beklagt, dass das Einkommen niedrig sei, die Arbeitsumstände schwierig und anstrengend und die Anerkennung gering (vgl. Koebe et al. 2020). Die Reaktionen darauf bezogen sich dann insbesondere auf die Anerkennung: Das Bild der klatschenden Bevölkerung als Dank für die aufopfernde Arbeit der Pflegekräfte ist eines der Sinnbilder der Krise - und es ist eines der Sinnbilder für den gesellschaftlichen Blick auf die Arbeit und die Arbeitsbedingungen in der Pflege. Pflegearbeit umgibt die Aura der Aufopferung und man kann den Pflegekräften gar nicht genug danken dafür, dass sie sich um eine*n selbst, die Mutter oder die Kinder kümmern, wenn Pflegebedürftigkeit herrscht - und weil man gar nicht genug danken kann, probieren wir es mit einer Schachtel Pralinen oder dem Beifall auf dem Balkon.

Dieser gesellschaftliche Blick auf Pflege und Pflegearbeit ist eng verwoben mit den Geschlechterverhältnissen und Entstehungskontexten bezahlter Pflegearbeit. Daraus resultieren vergeschlechtlichte Arbeitsbedingungen und Einkommensverhältnisse, die sich durch die Ökonomisierungsprozesse im deutschen Gesundheitswesen weiter manifestiert haben. Gleichzeitig führt dieses Verständnis von Pflege auch dazu, dass sich Pflege beziehungsweise Pflegende selbst insbesondere durch ihre personenbezogenen Tätigkeiten charakterisieren und auszeichnen, mit der Folge - so meine These - dass die Technisierung der Pflege als Angriff auf dieses Spezifische der Tätigkeiten und auf ihr Selbstverständnis verstanden wird. Auch 
daraus resultiert unter anderem eine sichtbare Distanz zur Technisierung und Digitalisierung in der Pflege.

Zur weiteren Erläuterung dieser These werde ich im Folgenden zunächst die Arbeitsbedingungen in der bezahlten Pflege skizzieren. Um die Bedeutung der Vergeschlechtlichung für den Pflegeberuf sichtbar zu machen, werde ich dann die Verankerung der Pflege als Teil des Care-Systems und die spezifischen Entstehungsbedingungen dieses >Frauenberufs aufzeigen, die in einer normativen Leitidee von >guter Pflege münden. Inwiefern sich dieses Leitbild auf das Verhältnis zu Technisierungsprozessen auswirkt, diskutiere ich anschließend. Ich ende mit einem Ausblick, der sich mit der Frage beschäftigt, inwieweit die Corona-Krise das Verhältnis von Pflegekräften zur Technik ändert/ändern könnte ${ }^{1}$.

\section{Arbeitsbedingungen von Pflegekräften in der bezahlten Pflegearbeit}

Die Pflegearbeit findet in drei unterschiedlichen Settings statt: Stationär, dies umfasst vor allem die Pflege im Krankenhaus und in Alten-Pflegeheimen; ambulant, hier geht es um ambulante Pflegedienste, die Pflegebedürftige in ihrer häuslichen Umgebung versorgen, und schließlich die häusliche Pflege, in der meist migrantische, sogenannte 24-Stunden-Kräfte die Pflegebedürftigen und ihre Angehörigen zu Hause unterstützen. Diese Ausdifferenzierung der bezahlten Pflegearbeit setzt sich weiter fort. Der Pflegesektor ist gekennzeichnet durch eine Vielzahl von Träger*innen der Pflegeeinrichtungen (staatlich/kommunal, gemeinnützige und private Träger*innen), durch unterschiedliche beziehungsweise fehlende Tarifverträge ${ }^{2}$ sowie - bisher - durch unterschiedliche Ausbildungsgänge. Gleichwohl eint die Beschäftigten in der Pflege, dass ihre Arbeitsbedingungen komplex, kritisch und prekär sind.

Meine Überlegungen basieren u.a. auf dem Forschungsprojekt »Arbeitsbedingungen und Interessenvertretung von Pflegekräften in Bayern«, das ich von 2015 bis 2019 geleitet und zusammen mit Katja Schmidt durchgeführt habe. Neben der Literatur- und Dokumentenanalyse haben wir 32 Interviews mit Pflegekräften aus der stationären, der ambulanten und der häuslichen bezahlten Pflege durchgeführt; einige Interviewpassagen dienen der Erläuterung einzelner Argumente.

2 Die Beschäftigten in der häuslichen Pflege haben oftmals keine berufsspezifische Ausbildung; sie unterliegen auch keinem Beschäftigungstarif und ihre Arbeitsbedingungen sind weitgehend ungeregelt (ILO 2018; Karakayali 2010). Da die Kontextbedingungen so unterschiedlich sind, werde ich mich im Folgenden auf die Alten- und Krankenpflege konzentrieren. Für die spezifischen Aspekte der migrantischen Pflegearbeit vergleiche hierzu Emunds et al. 2019, Lutz/Palenga-Möllenbeck 2010 sowie Theobald 2017. Es gibt aber auch im ambulanten und stationären Bereich viele Einrichtungen ohne Tarifbindung. 
2018 arbeiteten 1,7 Millionen Personen in der Pflege. 1,1 Millionen von ihnen waren in der Krankenpflege und 0,6 Millionen in der Altenpflege tätig; 80 Prozent (Krankenpflege) beziehungsweise 84 Prozent (Altenpflege) der Pflegekräfte sind weiblich (BA 2020: 6). Fast 60 Prozent der in der Pflege Beschäftigten arbeiten als Teilzeitkraft oder in einer geringfügigen Beschäftigung, wobei auch hier der Geschlechterunterschied deutlich ist: 62 Prozent der Frauen und 37 Prozent der Männer arbeiten Teilzeit (ebd.). In der Altenpflege arbeitete 2019 fast die Hälfte der dort Beschäftigten als Altenpflegehelfer*in (48\%), in der Krankenpflege beträgt der Helfer*innenanteil immerhin auch 16 Prozent (ebd.: 6-7).

Wie in Abbildung 1 sichtbar wird, herrscht zwischen Kranken- und Altenpflege ein deutliches Einkommensgefälle vor. Die Krankenpflege wird deutlich besser bezahlt, mit Ausnahme der Expert*innen (von denen es aber nicht sehr viele gibt). Wenn man darüber hinaus bedenkt, dass der Helfer*innenanteil in der Altenpflege fast 50 Prozent beträgt, dann vergrößern sich dadurch nochmals die Einkommensunterschiede.

Abbildung 1: Bruttolöhne der bezahlten Pflegearbeit im Vergleich. Deutschland, Dezember 2018, Veränderung zum Vorjahr. Medianentgelte sozialversicherungspflichtig Vollzeitbeschäftigter

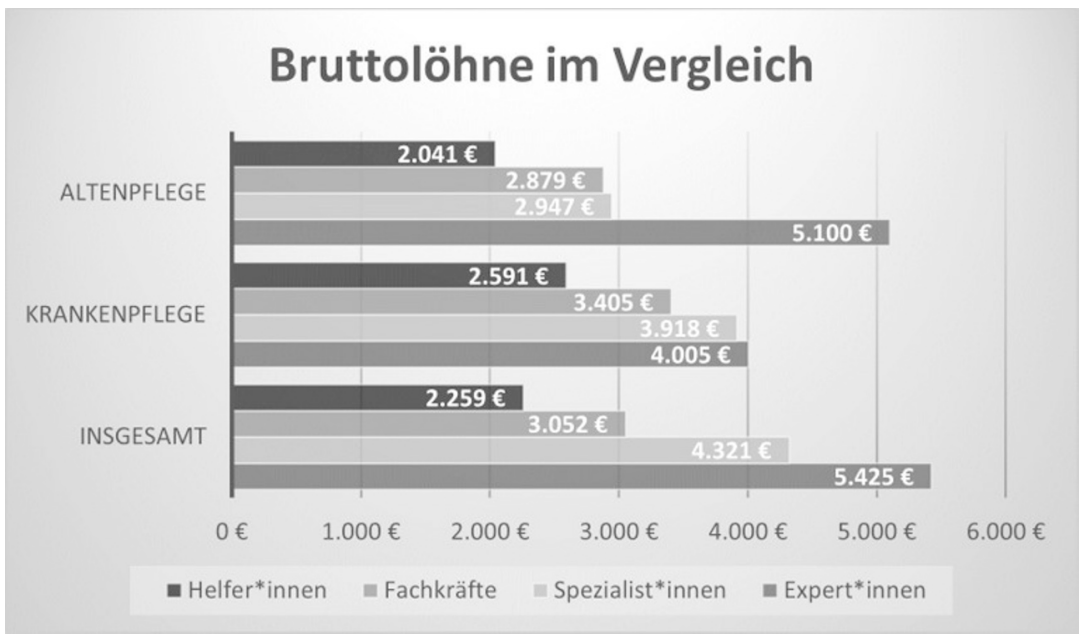

Quelle: Statistik der Bundesagentur für Arbeit (BA 2020: 7)

Im Vergleich mit den Durchschnittseinkommen in Deutschland zeigt sich, dass die Einkommen unter dem Durchschnitt liegen, mit Ausnahme der Krankenpflegefachkräfte. Auch hier gilt es aber zu bedenken, dass der überwiegende Anteil der Pflegekräfte in Teilzeit tätig ist, sodass diese nicht das Durchschnitts- 
einkommen erreichen. Obwohl die Gehälter in der Pflege in den letzten Jahren überdurchschnittlich stark gestiegen sind, reichen sie noch immer nicht an das Brutto-Mediangehalt bei sozialversicherungspflichtigen Vollzeitbeschäftigten heran ${ }^{3}$. Dabei sind die Anforderungen und Bedingungen der Arbeit beschwerlich:

»Also wir sind praktisch eine gewisse Anzahl Pflegekräfte in einer Schicht und haben halt so und so viel Patienten, die teilen wir dann auf. Aber es ist so, in den letzten Jahren sind wir meistens ein bis zwei Leute weniger pro Schicht, vor allem nachts und wenn einer krank wird, wird jetzt auch nicht gesucht, ob man den ersetzen könnte, sondern es bleibt dann einfach so. Und wir haben aber auch insgesamt viel mehr Patienten als vorher. [...] manchmal im Spätdienst kommen sieben neue Patienten, dann bleiben welche für ein paar Stunden und gehen dann wieder und dann kommen schon die nächsten und da weiß einfach der Arzt überhaupt nicht [...] was man da überhaupt alles machen muss, um das neu vorzubereiten und da bleibt dann praktisch der Patient, der da ist, oft auf der Strecke, weil man mit so organisatorischen Sachen beschäftigt ist. Und Schreibkram ist auch viel mehr geworden.«(PKSw3) ${ }^{4}$

Die permanente Schichtarbeit, die zunehmende Arbeitsverdichtung, die bürokratischen Anforderungen an die Dokumentation der Tätigkeiten, die körperlichen Anstrengungen und die große emotionale Belastung in der Auseinandersetzung mit Krankheit, Alter und Tod führen zu einer extrem starken physischen und psychischen Gesundheitsbelastung, die zu hohen Fehlzeiten aufgrund von Krankmeldungen und in der Konsequenz zu einem überdurchschnittlich häufigen Ausstieg aus dem Beruf führen (Bogai 2017; Hackmann/Müller 2012). Bei all denjenigen, die im Beruf bleiben, leidet die Qualität der Pflege unter den prekären Arbeitsbedingungen (Institut DGB-Index Gute Arbeit 2018): 46 Prozent der in der DGB-Studie "Arbeitsbedingungen in der Alten- und Krankenpflege« Befragten gaben an, dass sie sehr häufig oder oft Abstriche bei der Qualität der Arbeit machen müssen, um ihr Arbeitspensum zu schaffen (ebd.: 16). Auf einer 100-Punke-Skala erreicht die durchschnittliche Arbeitsqualität aus Sicht der Beschäftigten lediglich 53 Punkte und liegt damit nur knapp über dem Wert für schlechte Arbeitsbedingungen; bei den Beschäftigten aller Berufe liegt der Durchschnittswert im (fast oberen) Mittelfeld bei 62 Punkten (ebd.: 19-21). gionalen Unterschiede im Entgelt hin: So verdiente 2016 eine Fachkraft für Altenpflege in Sachsen-Anhalt 1.985 Euro und in Baden-Württemberg 2.937 Euro. Pflegekraft, S für den stationären Bereich und $A$ für den ambulanten. Exp steht für Expert*in, $\mathrm{m}=$ männlich und w=weiblich. GD steht für Gruppendiskussion. Die Zitate wurden um der Lesbarkeit willen teilweise sprachlich geglättet. 
Die schon seit langer Zeit geforderte Verbesserung der Arbeitsbedingungen von Pflegekräften würde also nicht nur eine Verbesserung der Existenzsicherung der Beschäftigten darstellen und könnte einen wichtigen Beitrag zur Fachkräftesicherung leisten, sondern ist auch unmittelbar mit der Qualität der Pflege und der Sicherung des Gemeinwohls verknüpft (Schnerring/Verlan 2020; Winker 2015). Die Gründe für die nur langsamen Veränderungen sind unmittelbar in den Vergeschlechtlichungsprozessen von Care-Arbeit und der Ökonomisierung des Gesundheitswesens zu finden.

\section{Vergeschlechtlichung und Ökonomisierung der Pflege}

Die inkrementellen Reformen im Gesundheits- und Pflegesystem standen $a b$ Mitte der 1970er Jahre unter dem dringlichsten Ziel der Kostendämpfung (Gerlinger/Reiter 2017). Wichtige Strategien, die dabei umgesetzt wurden, waren die Wettbewerbsorientierung, Vermarktlichung und Privatisierung bei gleichzeitiger hierarchischer staatlicher Re-Regulierung. Daneben sind aus Sicht der Pflege vor allem die Einführung der Pflegeversicherung 1995 als >Teilkaskoversicherung und der Fallpauschalen 2003 als Vergütungsansatz in Krankenhäusern wesentliche Neuerungen gewesen. In der Folge der Einführung des Fallpauschalensystems (DRGs - Diagnosis Related Groups) sind die Stellen im Pflegedienst deutlich zurückgegangen, während die Anzahl der ärztlichen Stellen gestiegen ist (Braun 2014). Mit der Pflegeversicherung ist (die Absicherung der) Pflege zumindest ansatzweise als kollektive Aufgabe der Gesellschaft implementiert und wohlfahrtsstaatlich abgesichert worden (Auth 2017 \& 2019). Dies hat einerseits $\mathrm{zu}$ einem Professionalisierungsschub der Pflege geführt und andererseits durch die Ökonomisierungsziele die Arbeitsbedingungen verschlechtert (ebd.). Um trotzdem die Pflege der alten und kranken Menschen weiterhin zu gewährleisten, wurden die Kollektivierungsprozesse durch Re-Familialisierungs- und Vergeschlechtlichungsprozesse begleitet. Insbesondere in der stark wettbewerbsorientierten Altenpflege gilt weiterhin das Motto sambulant vor stationär<, was impliziert, dass die familiäre Pflege überwiegend von Frauen durchgeführt wird, die von ambulanten, wiederum vorwiegend weiblichen, Pflegekräften unterstützt werden. Wenngleich immer wieder betont wird, dass ein Verbleib in der eigenen Wohnung auch dem Willen der Pflegebedürftigen entspräche, führt dies in der Konsequenz dazu, dass es zu wenige Pflegeeinrichtungen für alte Menschen gibt, in denen zudem aufgrund des Fachkräftemangels oftmals prekäre Zustände für Bewohner*innen und Pfleger"innen herrschen. Dies entspricht dem konservativen Wohlfahrtsstaatenmodell, das immer wieder auf die Fürsorgearbeit von Frauen zurückgreift und dies mit Geschlechterbildern und einem Familienmodell legitimiert, in denen Frauen die Verantwortung für Care zugesprochen wird, auch wenn Frauen mitt- 
lerweile in einem hohen Maße erwerbstätig sind. Diese Geschlechterbilder finden sich auch im Bild der Pflege wieder:

»Also zum einen ist ja die Pflege eigentlich so ein Frauenberuf, [...] also früher waren das halt so Krankenschwestern, deswegen, das ist ja noch so drinnen. Und ich denke, viele machen das halt gerne, helfen und ein bisschen kümmern, (lacht) sage ich jetzt mal.«(PKSw5)

Die Naturalisierung von Fürsorge (`Frauen machen das halt gerne`) kann in der Historie der Entstehung des Pflegeberufs nachvollzogen werden (MeusslingSentpali 2019) und wurde verknüpft mit der christlichen Idee des >Dienens` und der ökonomischen Notwendigkeit, dass im 19. Jahrhundert Berufsfelder für unverheiratete Frauen gesucht wurden, um die Herkunftsfamilie von ihrer Versorgung zu entlasten. Die Organisierung der Pflege in Diakonissengemeinschaften war an die bürgerlich-patriarchale Familie angelehnt und verband die »väterlich-strenge und bestimmende Rolle des Vorstehers« (Nolte 2020: 122) mit den dienenden Tätigkeiten der Frauen (Schmidt 1998)-damit wurden die Geschlechterrollen reproduziert und versucht, die Ehrbarkeit der arbeitenden Frauen zu sichern. Auch der bürgerliche Flügel der ersten Frauenbewegung betonte die "geistige Mütterlichkeit« von Frauen als natürliche Kompetenz von Frauen:

»Hat in bezug auf den mütterlichen Beruf das Wort Menschheit schon eine Bedeutung für das Weib gewonnen? so, daß die geistige Mütterlichkeit mit ihrer pflegenden Kraft, ihrer wärmenden Liebe sich nicht allein an die eigene Kinderstube, nicht allein an die physische Mütterlichkeit bindet: sondern daß überall, wo Hilfsbedürftige sind an Leib und Seele, die Frau auch außerhalb des Hauses zum mütterlichen Wirken berufen ist, wenn keine eigenen Familienbande sie fesseln oder ihre Zeit genügend ausfüllen können« (Schrader-Breymann o.J., zitiert nach: Jacobi 1990: 211).

Diese Vergeschlechtlichung hält bis heute an, auch wenn sie erweitert worden ist, zum Beispiel durch den Verweis darauf, dass sich Sorge-Berufe oftmals besser vereinbaren lassen mit der familiären Fürsorge - was sich unter anderem in der hohen Teilzeitquote in der Pflege ausdrückt. Im Zuge der Industrialisierung und der Durchsetzung der kapitalistischen Produktionsweise ist aber nicht nur ein vergeschlechtlichter Arbeitsmarkt, sondern auch ein hierarchisierter entstanden: Produzierende Berufe, deren Produkte vermarktlicht werden können, haben eine größere Bedeutung und gesellschaftliche Anerkennung als sogenannte reproduktive Tätigkeiten, bei denen kein verkaufbares Produkt entsteht. Die Folgen der Hierarchisierung zeigen sich beispielsweise in den deutlichen Gehaltsunterschieden zwischen industrieller und Care-Arbeit (vgl. Scheele 2019) beziehungsweise, wie Nancy Fraser (2017: 108) es formuliert: Kapitalistische Gesellschaften »vergüten die 
>reproduktiven Tätigkeiten in der Währung der >Liebe und >Tugend , die >produktive Arbeit hingegen mit Geld«:

»Also die Frauen, die sind es gewohnt, dass sie alles machen. Möglichst für nichts« (PKAw1).

Die Vergeschlechtlichung grenzt nicht nur Lebenswege für Frauen (und Männer) ein, sondern beschränkt die Möglichkeiten der eigenen materiellen Existenzsicherung und reproduziert auch so erneut das modernisierte ErnährerZuverdienerinnen-Modell (Brand/Rudolph 2014). Zudem leiten sich aus der Vergeschlechtlichung und der Charakterisierung der Care-Arbeit die Leitbilder sguter Pflege $a b$, die direkten Einfluss auf das Verhältnis der Pflege zur Technisierung und Digitalisierung haben.

\section{Care-Arbeit als Konzept und das Leitbild sguter Pfleger}

Das Konzept von Care(-Arbeit) erweitert und präzisiert die Debatte über individuelle und gesellschaftliche Für-Sorge und Sorge-Arrangements. Die Frage >Wer sorgt unter welchen Bedingungen für wen?< erscheint dadurch nicht mehr nur als reines >Frauenthema und konturiert »Selbst- und Fürsorge als elementare Bestandteile der modernen Gesellschaft « (Scheele 2019: 25). Es werden familialisierte und erwerbsförmige Care-Tätigkeiten sichtbar, die Menschen ihr ganzes Leben lang begleiten und die jeder Mensch braucht, und die sowohl bezahlt als auch unbezahlt erbracht werden.

»)Care< umfasst den gesamten Bereich weiblich konnotierter, personenbezogener Fürsorge und Pflege, d.h. familialer und institutionalisierter Aufgaben der Versorgung, Erziehung und Betreuung und stellt sowohl eine auf asymmetrische Beziehungen beruhende Praxisform als auch eine ethische Haltung dar« (Brückner 2010: 44).

Zudem gelten Emotionen und soziale Beziehungen als zentrale Bestandteile von Care-Arbeit, was mit den Begriffen der >Emotions- und Gefühlsarbeit ${ }^{5}$ als Teil des beruflichen Handelns erfasst wird (Giesenbauer/Glaser 2006; vgl. HülskenGiesler/Daxberger 2018). Bei Care/Pflege geht es nicht nur um Kompetenzen und Techniken, sondern auch um eine bestimmte Haltung und um personale Beziehungen und Interaktionen. Die Bedeutung, die Gefühle in der Pflege haben, gilt

`Gefühlsarbeit، bezeichnet die Arbeit an den Gefühlen der Patient*innen und Klient*innen, während es bei der >Emotionsarbeit ‘ um den Umgang mit den eigenen Gefühlen geht. Der Einsatz von Gefühlen als Arbeitsmittel findet sich im Konzept des »subjektivierenden Arbeitshandelns« wieder (Giesenbauer/Claser 2006: 61). 
jedoch oft nicht als Qualitätsmerkmal der Arbeit, sondern als Einschränkung der Professionalität:

»]a, also prinzipiell möchte ich ja auch, dass die Pflege als Profession gesehen wird. Dass die Pflege als Beruf gesehen wird und eben nicht mehr als Herzensangelegenheit und Berufung. Trotzdem geht es nicht ganz ohne [Gefühle; Erg. CR].« (PKAm8)

Professionalität bedeutet nämlich »die Kernkompetenz pflegerischen Handelns als systematisch plan- und evaluierbarer Problemlösungsprozess im Zuständigkeitsund Verantwortungsbereich beruflich Pflegender (Mohr et al. 2020: 204). Eine so verstandene Professionalität gilt als Voraussetzung für mehr gesellschaftliche Anerkennung und auch höhere Entlohnung, weil damit sichtbar wird, dass die Voraussetzung für solche Kernkompetenzen nicht die weibliche Natur ist, sondern eine qualifizierte, heute auch teilweise akademisierte Ausbildung. Für die Pflegekräfte führen diese Entwicklungen jedoch zu einem Spannungsverhältnis:

»Aber andererseits wird er auch irgendwie ein bisschen herabgewertet, der Beruf, habe ich das Cefühl. Also es ist eher so, dass die Krankenschwester, mei, die kümmert sich halt um die alten Leute und gibt ihnen Essen und wäscht die, aber aus dem medizinischen und fachlichen Bereich ist es schon viel, viel mehr, also ganz anders. Und das finde ich halt ein bisschen schade, dass das so in der Cesellschaft also irgendwie herabgewertet wird dadurch. Also einerseits ist es schon schmeichelnd, klar, wenn man mir Fürsorglichkeit und Freundlichkeit irgendwie zuschreibt durch meinen Beruf, ist es schon sehr schmeichelnd.«(PKSw2)

Auch aus Sicht der Pflegebedürftigen stellen die emotionalen Komponenten eine zentrale Rolle für ihre Bewertung der Pflege dar. Christel Kumbruck (2010: 202) fasst zusammen, dass "Gefühle als Arbeitsmittel dienen und genutzt werden. [...] Bei der Bewertung der Pflege orientierten sich die Patienten vor allem an der Beziehungsqualität, dann an generellen Pflegeaktivitäten und zuletzt an konkreten Pflegehandlungen« (vgl. Hauer 2017). Es existiert somit ein Zirkelschluss: Emotionen werden von Pflegebedürftigen und Pflegekräften als zentraler Bestandteil einer guten Pflege betrachtet, gleichzeitig dient der emotionale Anteil der Pflege als Grund gesellschaftlicher Abwertung und geringer Anerkennung, zumal Gefühle ja nicht gelernt werden müssen, weil Frauen sie von >Natur aus $<$ haben.

»In Deutschland wurden Pflegeberufe traditionell nicht als Berufe wie andere auch betrachtet. Vielmehr war Pflege (besonders seit dem 19. Jahrhundert) mit der Aura einer besonderen Berufung und Lebensform verbunden, für die sich Frauen aus einem besonderen Geist heraus entschieden hatten. In der Bezeichnung sSchwester finden sich die Vorstellungen eines nicht mit dem 
üblichen Berufsleben vergleichbaren >Liebesdienstes` in verdichteter Gestalt« (Senghaas-Knobloch 2008: 226).

Benedix/Medjedovic (2014), die eine qualitative Befragung von Pflegekräften sowie Expert"innengespräche durchgeführt haben, fassen das Berufsverständnis der befragten Pflegekräfte als "ganzheitliche Pflege« zusammen. Dazu gehört, wie oben schon beschrieben, die Planung des gesamten Pflegeprozesses und die Ausführung aller Aspekte der Pflege, aber auch eine professionelle Beziehungsarbeit (ebd.: 33). Im Kontext der Ökonomisierung sind allerdings die Möglichkeiten einer professionellen Beziehungsarbeit erheblich eingeschränkt worden, weil die Tätigkeiten nicht messbar und quantifizierbar und damit auch nicht abrechenbar sind. Ein System effizienzorientierter Konkurrenz (ambulante Pflege) beziehungsweise pauschalisierter Abrechnungen (DRGs in Krankenhäusern) lässt keinen Raum für fürsorgliche Beziehungsarbeit, insofern sehen 85 Prozent einer Bevölkerungsbefragung durch das Zentrum für Qualität in der Pflege (ZQP) den größten Verbesserungsbedarf bei der persönlichen Zuwendung und Kommunikation (Staudhammer 2018: 97). Mehr als zwei Drittel der Befragten sehen den Grund für die Mängel in der Pflege beim fehlenden Personal und der daraus resultierenden Überlastung.

In einem Zwischenfazit können nunmehr mehrere Dilemmata (in) der Pflege konstatiert werden:

- Die Arbeitsbedingungen in der Pflege sind gleichermaßen komplex und prekär. Die Anforderungen an Pflegekräfte sind umfassend und erfordern eine gute Ausbildung sowie gleichermaßen berufliche und soziale Kompetenzen. Diese hohen Anforderungen und die daran gemessen schlechte Bezahlung führen zu einem Fachkräftemangel in der Pflege.

- Die Vergeschlechtlichung und die Ökonomisierung prägen das Bild und die Strukturbedingungen der Pflege. Effizienzkriterien führen aus Sicht von Träger*innen und Investor*innen zur Notwendigkeit von Einsparungen in der Pflege, also zur Reduzierung von Personal(-kosten). Mithilfe geschlechtlicher Zuweisungen werden die prekären Arbeitsbedingungen legitimiert: Frauen pflegen von Natur aus gerne und sind außerdem Zuverdienerinnen, weshalb eine angemessene Entlohnung entbehrlich ist.

- Das Spannungsverhältnis von Beruf und Berufung, von Professionalisierung und Ganzheitlichkeit verweist auf die komplexen Herausforderungen und Selbstbilder des Pflegeberufs. Gleichwohl verbleibt die Anerkennung für die Bewältigung der Komplexität auf einer symbolischen Ebene, die Interessen der Pflegekräfte werden im System der konkurrierenden Interessen des Pflegeund Gesundheitssystems weiter marginalisiert. 
Im Rahmen zunehmender Technisierung und Digitalisierung scheint auch in diesem gesellschaftlichen Bereich ein vermehrter Technikeinsatz eine Lösungsoption für diese Dilemmata zu sein. Allerdings entspricht Technik nicht unbedingt den bisher dargestellten (Selbst-)Bildern von Pflege.

\section{Technik als Lösung? Pflege und Technik(-einsatz) - Einstellungen und Geschlechterverhältnisse}

Die Technologisierung des Gesundheitswesens wird überwiegend als Fortschritt definiert, weil wir uns dadurch eine bessere Diagnose und Therapie erwarten beziehungsweise hoffen, dass Alterungs- und Sterbeprozesse dadurch hinausgezögert werden können. Dies gilt im Bereich der Pflege nicht unbedingt. Obwohl der Technikeinsatz in der Pflege immer wieder als zukunftsträchtiges Thema im Kontext von Pflegebedarf und Fachkräftemangel angeschoben wird (Zöllick et al. 2020: 212), spielt es in den meisten Studien und Publikationen zur (Verbesserung der) Situation der Pflege eine nachgeordnete oder gar keine Rolle. Auch die Frage nach Anerkennung und Umverteilung von Care-Arbeit entscheidet sich bisher nicht nach dem Ausmaß und der Qualität von Technologien und Unterstützungssystemen in der Pflege. Mit diesen Differenzierungen im Hintergrund möchte ich an dieser Stelle zwei Fragen diskutieren, nämlich inwieweit die Akzeptanz der Technik im Kontext der Care-Debatte einzuschätzen ist und ob Technik(-einsatz) zu einer Aufwertung von Pflegearbeit führen könnte.

\section{Technik in der Pflege - Einstellungen und Geschlechterverhältnisse}

Der Einsatz von Technik in den Pflegealltag trifft immer wieder auf Zurückhaltung auch aufseiten der Pflegekräfte und weckt unterschiedliche Befürchtungen:

»Das [Robotik; Erg. CR] ist eine Ergänzung für die Pflege auf alle Fälle. Ich würde sie nie abschreiben, die würde ich schon annehmen, würde sie nutzen als Ergänzungsmittel, als Ergänzung, aber niemals als Ersatz für eine Pflegekraft. [...] Das kann ich mir beim besten Willen nicht vorstellen und wünsche ich mir in meinem Alter auch nicht, ganz ehrlich in einen Roboter dann reinzuschauen und der mir dann, der erkennt, dass mein Cesicht heute mal nicht so ist, wie es sein sollte und mir das dann erzählt. Ich glaube, ich möchte mit jemandem richtig sprechen können. Ich möchte mit meinem Enkel reden können [...] oder mit jemandem über meine Familie sprechen können. Das kann der Roboter gar nicht. Kann der also nie ersetzen, diese Wärme eines Menschen [...] die Gestik, die ganzen nonverbalen Kommunikationen, die kriegt nie einmal der Roboter hin.« (Expm9) 
Der hier befragte Pfleger und Personalratsvorsitzende spricht in seiner Aussage eine weit verbreitete Befürchtung im Hinblick auf den Einsatz von Technik in der Pflege aus, nämlich dass die Technik die Pflegekräfte ersetzen können soll. Allerdings rekurriert er in der Begründung seiner skeptischen Haltung nicht auf die fachlichen Kompetenzen, die er einem Roboter voraushat, sondern nimmt die Position eines alten pflegebedürftigen Menschen ein, der >mit jemandem richtig sprechen « möchte. Der Altenpfleger verweist auf das oben skizzierte Selbstbild der guten, ganzheitlichen Pflege, das - neben der professionellen Planung und Umsetzung des Pflegeprozesses - insbesondere aus Fürsorglichkeit, Emotionalität und sozialen Kompetenzen besteht. Die Begründung ist also keine arbeitspolitische, sondern eine care-politische; gleichzeitig werden die Erfahrungen der Ökonomisierung sichtbar. So könnte man ja auch argumentieren, dass die durch Technik eingesparte Zeit den Patient*innen und ihren Bedürfnissen zugutekommt; die Pflegekräfte haben aber in den letzten Jahren die Erfahrung gemacht, dass zeitliche Einsparungen direkte ökonomische Folgen haben, also zu einem Abbau von Pflegepersonal führen. Insofern befürchten viele Pflegekräfte von Technikeinsätzen und Digitalisierungsprozessen in der Pflege insbesondere im Bereich der emotionalen und sozialen Zuwendung einen Qualitätsverlust (Zöllick et al. 2020: 216). »Aufseiten der befragten Pflegekräfte herrscht hier Skepsis vor: Der Einsatz dieser Technik steht mehr als andere Anwendungen in Konkurrenz zum Berufsbild der Pflege, das Fürsorge und Mitmenschlichkeit ins Zentrum der Arbeit stellt, eine Arbeit, die Maschinen - so die Position - nicht ersetzen können« (Kuhlmey et al. 2019: 34):

»Wir sind doch Pflegekräfte geworden, weil wir mit Menschen arbeiten wollen und nicht mit technischen Geräten. Sonst wären wir jetzt Techniker« (Evans/Hielscher/Voss 2018: 7).

Eine deutlich höhere Akzeptanz von Technik seitens der Pflegekräfte liegt dann vor, wenn es um körperliche Unterstützung geht (z.B. Hebebühnen) und teilweise auch im Hinblick auf die Digitalisierung der Dokumentation der Pflegeleistungen (Bräutigam et al. 2017). Um die Akzeptanz beispielsweise von Pflegerobotern beziehungsweise Robotern, die den Alltag älterer Menschen unterstützen sollen, zu erhöhen, greifen die herstellenden Unternehmen deshalb auf die Betonung vermeintlich sozialer - menschlicher - Eigenschaften ihrer Maschinen zurück. So bewirbt das Fraunhofer IPA seinen »Personal Roboter «, den Care-O-bot, als elektronischen Butler mit folgenden Eigenschaften: »Er ist freundlich, sympathisch und zugleich unsichtbar, wenn man ihn nicht braucht (IPA 2015; vgl. Jeanrenaud 2016). Ähnlich in der Darstellung von Janowski et al. (2018), nach der das effiziente Funktionieren von "sozial interagierenden Robotern « dann am besten gelingt, wenn der Roboter die Rolle als »persönlicher Begleiter« oder noch besser »Freund« (ebd.: 65) einnimmt und wenn er »als eigenständige Persönlichkeit mit konsistenten Verhal- 
tensweisen wahrgenommen« wird (ebd.: 66). Während die Autor*innen dadurch eine Akzeptanzsteigerung von Pflegerobotern erwarten, verweisen die genannten Studien darauf, dass gerade Fürsorglichkeit den Kern der Pflege ausmacht, der nicht durch Technik ersetzbar ist.

Die sozialen Fähigkeiten stellen den Grundstock der Vergeschlechtlichung der Care-Arbeit dar und reproduzieren das Bild einer nur halbprofessionalisierten, weiblich konnotierten Pflege. Dazu passt, dass die Pflegebereiche, die heute schon technisch stark dominiert sind, wie die Intensivpflege, einen verhältnismäßig höheren Anteil an männlichen Pflegekräften zu verzeichnen haben:

"Cenau, auch weil [im Krankenhaus; Erg. CR] viel mehr Maschinen noch mit im Spiel sind, die Beatmung und Infusion, und ich glaube deswegen arbeiten auch mehr Männer im Krankenhaus als in der Altenpflege, weil es da eben noch eine technische Perspektive gibt. Besonders in der Intensivmedizin arbeiten ja viel mehr Männer als Frauen, da ist es dann oft umgedreht.« (PKAw4)

Der Bereich der Intensivpflege ist einer der besser bezahlten in der stationären Krankenpflege, gleichzeitig genießt er ein vergleichsweise hohes Ansehen innerhalb des Pflegesystems. Damit reproduziert sich entlang des Technikeinsatzes und der Technikanwendung das geschlechtshierarchische System der Arbeitsteilung. In Befragungen werden »tendenziell positive [...] Auswirkungen der Digitalisierung [...] teilweise von Männern stärker gesehen als von Frauen und von Beschäftigten auf Intensivstationen häufiger genannt als von denen auf Normalstationen « (Bräutigam et al. 2017: 52).

»Viele [Männer; Erg. CR] sind zum Beispiel im OP, OP-Pfleger oder so, oder in irgendwelchen Funktionen, eben auf Intensivstationen auch. Also ich glaube, dass die Männer sich eher spezialisieren und diese allgemeine Pflege nicht so wollen. [...] Die sind bei uns auch alle technikvernarrt und - also auch, wenn die noch ganz jung sind, sind die da immer gleich Feuer und Flamme: Also die sind immer total heiß drauf, diese ganz besonders technisch ausgerüsteten Patienten zu übernehmen.«(PKSw3)

Auch im Verhältnis der Pflege zur Technik spiegeln sich Geschlechterverhältnisse und Geschlechterbilder wider. In technikintensiven Arbeitsbereichen sind schon jetzt die männlichen Pflegekräfte überrepräsentiert, und diese Geschlechterverteilung entspricht auch den Erwartungen der Pflegekräfte insgesamt. Hierarchisierungen betreffen also nicht nur die Berufsgruppen (produzierendes Gewerbe vs. soziale Dienstleistungen), sondern werden auch innerhalb einer Berufsgruppe über Techniknähe und Technikdistanz hergestellt. Im Grunde ist es ausgesprochen widersprüchlich: Obwohl die Pflegekräfte (und nicht nur diese) das Besondere der Pflege in der fürsorglichen Praxis sehen (Senghaas-Knobloch 2008), erhalten 
techniknahe Fachkräfte nicht nur höhere Anerkennung, sondern auch mehr Geld. Fürsorglichkeit als persönliche und unbezahlbare Eigenschaft verbleibt als vergeschlechtlichter Teil des Pflegeberufs.

\section{Aufwertung von Pflegearbeit durch Technik?}

Im Rahmen der bisherigen Pflegearbeit führen Technikanwendung und Technikaffinität zumindest teilweise zu mehr Anerkennung und mehr Umverteilung im Sinne gesellschaftlicher Aufwertung, der Übernahme von Care-Arbeit durch mehr Menschen und einem größeren Ausgleich zwischen den Geschlechtern (Fraser 1996). Schreibt man diesen Befund fort, so könnte durch eine zunehmende Technisierung der Fachkräftemangel behoben werden, weil der Pflegeberuf insgesamt und besonders für Männer attraktiver würde. Heilmann (2020) hat ebendiese Frage untersucht, nämlich inwieweit durch Digitalisierung eine Aufwertung der Krankenpflege erreicht werden kann. Digitalisierung ist dabei sehr weit gefasst und beinhaltet Robotik, vernetzte Monitoring- und Logistiktechnologien, digitale Informations- und Verwaltungssysteme sowie Endgeräte zur Nutzung digitaler Technologien (ebd.: 4-5). In der qualitativen Studie, in der Expert"innen der Pflegepraxis und der Pflegeforschung befragt wurden, zeigte sich zunächst, dass in der Umsetzung der Technisierung und der Anwendung von Technik in der Pflege vor allem die elektronische Patient"innenakte, unterschiedliche Endgeräte zur Nutzung digitaler Technik und das Telemonitoring zum Einsatz kommen. Obwohl diese Anwendungen durchaus mit neuen Anforderungen an die Ausbildung und die ausgeübten Tätigkeiten verbunden sind, findet - so Heilmann - keine Aufwertung der Krankenpflege statt. Als Gründe führt er auch die hier schon genannten Rahmenbedingungen der Pflege an: Aufgrund der Krankenhaus- und Pflegefinanzierung im Rahmen der sozialen Sicherungssysteme sind politische Entscheidungen für die Erhöhung der Ausgaben im Gesundheitssystem notwendig. Zudem würden die bisherigen Digitalisierungs- und Technikmaßnahmen im Krankenhaus lediglich als unterstützend und helfend wahrgenommen und fügten sich in die allgemeine Wahrnehmung der Pflegetätigkeit als lediglich halbprofessionelle Arbeit ein. Letztendlich erwarten sich die von Heilmann befragten Expert"innen durch die Digitalisierung auch keine Verbesserung der durch Personalmangel hervorgerufenen Arbeitsbedingungen, weil »sich die für die Krankenpflege zentrale Interaktionsarbeit zwischen Pflegenden und Patient"innen nicht durch den Einsatz digitaler Technologien substituieren lässt« (ebd.: 17). Im Kern ist es also immer wieder die soziale Interaktion, um die sich die Bewertung von Pflege dreht, und gerade die erscheint sowohl in der gesellschaftlichen 
Betrachtung wie auch in tarifpolitischen Bewertungsprozessen als nachrangig beziehungsweise als selbstverständlicher Bestandteil >weiblicher Arbeitく.

Verstärkt wird dieser Befund durch die Befürchtung, dass Technik nicht nur nicht zu einer Aufwertung, sondern vielmehr zu einer De-Professionalisierung der Pflege und damit zu einer Abwertung führt (Becka/Bräutigam/Evans 2020: 4). Hielscher (2014: 11) verweist

»auf die widersprüchlichen Entwicklungen, denen zu Folge die Pflege einerseits durch eine Zunahme der Zahl von Beschäftigten mit Hilfs- und Assistenzfunktion, die vor allem die direkte Pflege am Bett vornehmen, andererseits durch Spezialisierung für bestimmte technische Funktionen gekennzeichnet ist. Diese >coexistence of conditions of >upskilling « and >deskilling in nursing [ [Windsor 2007; Erg. CR] sei mit Blick auf die dominierenden Paradigmen von Wettbewerb und Produktivität politisch zu hinterfragen.«

In der Altenpflege noch mehr als in der Krankenpflege arbeitet ein relevanter Anteil von Hilfskräften (s.o.) und die Befürchtung besteht, dass durch mehr Technik die Pflegefachkräfte durch noch mehr Helfer*innen ersetzt werden, die nur die Maschinen anschalten müssen. Eine solche Entwicklung zeichnet sich schon durch die Einführung des Pflegebudgets und die Debatten zur Personalbemessung ab, mit der Folge, dass zum einen im Krankenhaussektor Pflegefachkräfte die Arbeit von Hilfskräften zusätzlich übernehmen sollen und zum anderen, dass insbesondere in der Langzeitpflege die Fachkraftquote ersetzt wird durch die überproportionale Einstellung von Pflegehilfskräften (Sell 2020a \& 2020b).

Insgesamt, das zeigen verschiedene Studien (vgl. Becka/Bräutigam/Evans 2020; Hielscher 2014) und auch unsere Befragungen, müssen die Einstellungen der in der Pflege Beschäftigten differenziert nach Technik-Typen und -Funktionen betrachtet werden. Hilfe und Unterstützung bei beschwerlichen und Routineaufgaben gelten durchaus als positiv beziehungsweise eröffnen die Option, mehr Zeit für >das Wesentliche`, also die Pflegearbeit an und mit den Patient*innen, zu haben (Evans/Hielscher/Voss 2018: 3).

»Davon heben sich Technologien ab, die den Pflegebedürftigen emotionale Nähe und soziale Unterstützung bieten sollen. Aufseiten der befragten Pflegekräfte herrscht hier Skepsis vor: Der Einsatz dieser Technik steht mehr als andere Anwendungen in Konkurrenz zum Berufsbild der Pflege, das Fürsorge und Mitmenschlichkeit ins Zentrum der Arbeit stellt, eine Arbeit, die Maschinen-so die Position - nicht ersetzen können.« (Kuhlmey et al. 2019: 34; vgl. Hielscher 2014: 14, 33)

Technik als Lösung? Eigentlich ist die Frage falsch gestellt, weil die Probleme (in) der Pflege nicht technischer Natur sind, sondern eine Frage der Anerkennung und Bewertung, der Arbeitsbedingungen und der Zukunftsperspektiven. In den letz- 
ten Jahren haben Pflegekräfte oft die Erfahrung gemacht, dass nicht sie, sondern Arbeitgeber*innen und politische Akteur*innen Entscheidungen getroffen haben, die diese Fragen nicht zufriedenstellend beantwortet haben. Wenn Technik diese Probleme nun lösen soll, dann herrscht aufgrund der bisherigen Erfahrungen eher Skepsis vor. Evans/Hielscher/Voss (2018) weisen infolgedessen darauf hin, dass die Voraussetzung zur Integration von Digitalisierung und Technik in die Pflegearbeit die Partizipation der Pflegekräfte bei den Debatten und bei der Einführung ist - und insgesamt eine Stärkung der Mitbestimmung und Interessenvertretung von Pflegekräften erfolgen muss (vgl. Rudolph/Schmidt 2019a).

\section{Fazit: Ambivalenzen der Fürsorglichkeit}

Die strukturellen Rahmenbedingungen von Pflege, und hier vor allem die Vergeschlechtlichung und die wohlfahrtsstaatliche Ökonomisierung, führen zu prekären Arbeitsbedingungen einer anspruchsvollen und komplexen beruflichen Arbeit. Entsprechend befinden sich die Stellschrauben zur Veränderung insbesondere auf der politischen Ebene, weil dort die Verantwortlichkeiten für die Finanzierung des Gesundheitssystems und für die Ausgestaltung der Pflegeversicherung liegen ( $\mathrm{Ru}$ dolph/Schmidt 2019b). Zudem sind Länder und Kommunen als Träger*innen von Krankenhäusern auch Tarifpartner*innen bei der arbeitspolitischen Aushandlung von Tarifverträgen und Arbeitsbedingungen. Dabei geht es nicht nur um eine bessere Entlohnung, sondern vor allem auch um die Personalausstattung, die Arbeitszeiten und Personaluntergrenzen als Maßstab für die Betreuungsrelation zwischen qualifizierten Pflegekräften und Patient*innen. Die Implementation diesbezüglicher Verbesserungen wäre auch ein gesellschaftliches Signal für eine andere Wahrnehmung und Aufwertung bezahlter Care-Arbeit und könnte die Attraktivität der Pflegeberufe erhöhen. Denn obwohl die intrinsische Motivation der Pflegekräfte für die Ausübung ihres Berufs oft sehr hoch ist (»Ich würde immer wieder, wenn ich einen neuen Beruf erlernen müsste, die Pflege nehmen. Also die Pflege muss man lieben«; PKAw2), wirken die Bedingungen der Arbeit demotivierend.

Schien es zu Beginn der Corona-Krise noch so, als ob sich die gesellschaftliche und politische Wahrnehmung verändern würde, hat sich die Frustration unter den Pflegekräften augenscheinlich erhöht (vgl. DBfK 2020). Der angekündigte >Pflegebonus wird nicht an die Pflegekräfte in den Krankenhäusern gezahlt, sondern nur an die Pfleger*innen in der Altenpflege, und auch hier je nach Bundesland nicht in der angekündigten Höhe von 1.500 Euro. In den bisherigen CoronaKonjunkturpaketen (Stand August 2020) kommt die Pflege nicht vor. Und insgesamt ist in der Corona-Krise ein Rückgriff auf tradierte Muster der Kleinfamilie sichtbar geworden (vgl. Baum 2020), der nicht nur bezahlt und unbezahlt Pflegende an den Rand der Belastung gebracht hat, sondern der insgesamt keine zukunfts- 
orientierte Antwort auf Care-Bedarfe formulieren konnte. Insbesondere auch die Betreuung älterer Menschen, die sonst in Tageszentren oder ähnliche Einrichtungen gehen und die dann geschlossen waren, hat die Betroffenen und ihre Angehörigen vor teilweise traumatische Herausforderungen gestellt.

Die Corona-Krise und das zeitweise umgesetzte Kontaktverbot zwischen alten und kranken Menschen und ihren Angehörigen hat noch einmal deutlich gemacht, wie fundamental soziale Interaktionen und soziale Beziehungen im Pflegekontext sind. Nicht die Chancen, die Technik in der Pflege für einen besseren Infektionsschutz bieten könnte, wurden diskutiert, sondern die Notwendigkeit (und der Mangel) ausreichender Schutzmaterialen, damit eine qualifizierte und professionelle Pflege mit der dazugehörenden Fürsorge aufrechterhalten werden kann (AEM 2020; Weber 2020). Insofern hat auch die Corona-Krise nicht dazu beigetragen, der Technisierung und Digitalisierung in der Pflege einen neuen Schub zu geben, sondern das Paradigma der Fürsorge ins Zentrum der Debatten gestellt; und zumindest anfangs auch die prekären Arbeitsbedingungen der Pflegekräfte, auch wenn dies schnell wieder in Vergessenheit geriet.

Die Ambivalenzen (in) der Pflege sind vielfältig. Man mag die Vergeschlechtlichung und Emotionalisierung der Pflege beklagen, doch gleichzeitig sind Fürsorglichkeit und Empathie die zentralen Anforderungen, die Pflege aus der Sicht der Pflegebedürftigen und der Pflegekräfte zur >guten Pflege die geringe Bereitschaft zur Interessenvertretung seitens der Pflegekräfte irritierend finden; gleichwohl beruht das momentane Funktionieren des Pflegesystems darauf, dass Pflegekräfte bereit sind, sich auf die schwierigen Arbeitsbedingungen einzulassen. Und man mag die Skepsis gegenüber dem Technikeinsatz vieler Pflegenden rückwärtsgewandt finden, doch gleichzeitig zeigen die Analysen und Studien, dass sich Pflegekräfte eine Aufwertung der Pflege und eine Verbesserung der Arbeitsbedingungen wünschen, und dass ein erhöhter Technikeinsatz dabei eine unterstützende Funktion einnehmen könnte, wenn er nicht für Rationalisierungsprozesse genutzt wird. Zudem scheint es entscheidend zu sein, Technikentwicklung und -einsatz partizipativ mit den Pflegekräften zu gestalten und dabei einerseits ihre Selbstbilder zu berücksichtigen und andererseits einen attraktiven Zusammenhang von Technik und Professionalisierung herzustellen, ohne die Bedeutung von Fürsorglichkeit infrage zu stellen. Um dies zu erreichen, bleibt es weiterhin notwendig, die Vergeschlechtlichungsmuster in Pflege- und Gesundheitsberufen offen zu legen und als Analysekategorie für Arbeitsbedingungen und gesellschaftliche wie politische Gestaltungsprozesse heranzuziehen.

Die größte Ambivalenz liegt aber darin, dass wir alle im Laufe unseres Lebens sowohl Care-Taker als auch Care-Receiver sind, von der Wiege bis zur Bahre, um genau zu sein, und dass wir trotzdem dieses essenzielle gesellschaftliche Verhältnis so gering schätzen. 2020 als »Internationales Jahr der Pflegenden und Hebammen« - insgesamt war dies eher kein gutes Jahr für diese Berufsgruppen. 


\section{Literatur}

AEM (Akademie für Ethik in der Medizin) (2020): Diskussionspapier der Akademie für Ethik in der Medizin. Pflegeethische Reflexion der Maßnahmen zur Eindämmung von Covid-19 (https://www.aem-online.de/fileadmin/user_uplo ad/2020_05_12_Pflegeethische_Reflexion_Papier.pdf; Zugriff 18.8.2020).

Auth, Diana (2017): Pflegearbeit in Zeiten der Ökonomisierung. Wandel von CareRegimen in Großbritannien, Schweden und Deutschland, Münster: Westfälisches Dampfboot.

Auth, Diana (2019): Der Wandel der Arbeitsbedingungen in der Pflege im Kontext von Ökonomisierungsprozessen, in: Rudolph, Clarissa/Schmidt, Katja (Hg.): Interessenvertretung und Care, Münster: Westfälisches Dampfboot, S. 54-71.

BA (Bundesagentur für Arbeit - Statistik) (2020): Berichte: Blickpunkt Arbeitsmarkt - Arbeitsmarktsituation im Pflegebereich, Nürnberg: BA.

Baum, Antonia (2020): Hannelore radikalisiert sich (https://www.zeit.de/kultur/2 020-04/kinderbetreuung-berufstaetige-frauen-rollenverteilung-familie-coron a-krise-1onach8; Zugriff 14.8.2020).

Becka, Denise/Bräutigam, Christoph/Evans, Michaela (2020): „Digitale Kompetenz« in der Pflege. Ergebnisse eines internationalen Literaturreviews und Herausforderungen beruflicher Bildung, Forschung Aktuell 8/2020, Gelsenkirchen/Bocholt/Recklingshausen: IAT.

Benedix, Ulf/Medjedovic, Irena (2014): Gute Arbeit und Strukturwandel in der Pflege: Gestaltungsoptionen aus Sicht der Beschäftigten. Reihe Arbeit und Wirtschaft in Bremen 6/2014, Bremen: Institut Arbeit und Wirtschaft (IAW), Universität Bremen und Arbeitnehmerkammer Bremen.

Bogai, Dieter (2017): Der Arbeitsmarkt für Pflegekräfte im Wohlfahrtsstaat, Berlin/Boston: De Gruyter Oldenbourg.

Brand, Ortrun/Rudolph, Clarissa (2014): Auf zu neuen Ufern? Geschlechterleitbilder im Wandel, in: WSI-Mitteilungen 2/2014, S. 89-96.

Braun, Bernard (2014): Auswirkungen der DRGs auf Versorgungsqualität und Arbeitsbedingungen im Krankenhaus, in: Manzei, Alexandra/Schmiede, Rudi (Hg.): 20 Jahre Wettbewerb im Gesundheitswesen, Wiesbaden: Springer VS, S. $91-113$.

Bräutigam, Christoph/Enste, Peter/Evans, Michaela/Hilbert, Josef/Merkel, Sebastian/Öz, Fikret (2017): Digitalisierung im Krankenhaus. Mehr Technik - bessere Arbeit?, Hans-Böckler-Stiftung Study Nr. 364, Düsseldorf: Hans-BöcklerStiftung.

Brückner, Margrit (2010): Entwicklungen der Care Debatte - Wurzeln und Begrifflichkeiten, in: Apitzsch, Ursula/Schmidbaur, Marianne (Hg.): Care und Migration, Opladen: Budrich, S. 43-58. 
DBfK (Deutscher Berufsverband für Pflegeberufe) (2020): Stimme erheben! Für die \#PflegeNachCorona (https://pflegenachcorona.de/; Zugriff 27.11.2020).

Emunds, Bernhard/Hagedorn, Jonas/Leiber, Simone/Rossow, Verena (2019): Hintergrundpapier zum Fachworkshop: Gestaltungsoptionen der sogenannten "24-Stunden-Pflege« (https://www.schader-stiftung.de/fileadmin/content/Hi ntergrundpapier_Die_sogenannte_24-Stunden-Pflege.pdf; Zugriff 20.8.2020).

Evans, Michaela/Hielscher, Volker/Voss, Dorothea (2018): Damit Arbeit 4.0 in der Pflege ankommt, Hans-Böckler-Stiftung Policy Brief 004/2018, Düsseldorf: Hans-Böckler-Stiftung.

Fraser, Nancy (1996): Die Gleichheit der Geschlechter und das Wohlfahrtssystem: Ein postindustrielles Gedankenexperiment, in: Nagl-Docekal, Herta/PauerStuder, Herlinde (Hg.): Politische Theorie. Differenz und Lebensqualität, Frankfurt a.M.: Suhrkamp, S. 469-498.

Fraser, Nancy (2017): Who cares? Die Ausbeutung der Sorgearbeit und ihre Krise, in: Blätter für deutsche und internationale Politik, 4/2017, S. 105-114.

Gerlinger, Thomas/Reiter, Renate (2017): Pflegepolitik, in: Reiter, Renate (Hg.): Sozialpolitik aus politikfeldanalytischer Perspektive, Wiesbaden: Springer VS, S. 275-296.

Giesenbauer, Björn/Glaser, Jürgen (2006): Emotionsarbeit und Gefühlsarbeit in der Pflege - Beeinflussung fremder und eigener Gefühle, in: Böhle, Fritz/Glaser, Jürgen (Hg.): Arbeit in der Interaktion - Interaktion als Arbeit. Arbeitsorganisation und Interaktionsarbeit in der Dienstleistung, Wiesbaden: Springer VS, S. 59-83.

Hackmann, Tobias/Müller, Daniela (2012): Berufsausstieg in der Pflege-Herausforderungen an die betriebliche Praxis, in: Sozialer Fortschritt, 9/2012, S. 227-236.

Hauer, Katharina (2017): Brauche ich das überhaupt?! - Qualität assistiver Technologien aus Sicht von älteren Personen in häuslicher Pflege, in: Pfannstiel, Mario A./Krammer, Sandra/Swoboda, Walter (Hg.): Digitale Transformation von Dienstleistungen im Gesundheitswesen III. Impulse für die Pflegepraxis, Wiesbaden: Springer Gabler, S. 307-325.

Heilmann, Tom (2020): Aufwertung der Krankenpflege. Welchen Beitrag kann die Digitalisierung leisten? IAQ-Report 2/2020, Duisburg: Institut Arbeit und Qualifikation.

Hielscher, Volker (unter Mitarbeit von Niklas Richter) (2014): Technikeinsatz und Arbeit in der Altenpflege. Ergebnisse einer internationalen Literaturrecherche, iso-Report Nr. 1, Saarbrücken: Institut für Sozialforschung und Sozialwirtschaft e. V.

Hülsken-Giesler, Manfred/Daxberger, Sabine (2018): Robotik in der Pflege aus pflegewissenschaftlicher Sicht, in: Bendel, Oliver (Hg.): Pflegeroboter, Wiesbaden: Springer Gabler, S. 125-139. 
ILO (International Labour Organisation) (2018): Care work and care jobs for the future of decent work, Genf: International Labour Office.

Institut DGB-Index Gute Arbeit (2018): Arbeitsbedingungen in der Alten- und Krankenpflege, Berlin: DGB.

IPA (Fraunhofer-Institut für Produktionstechnik und Automatisierung) (2015): Roboter als vielseitiger Gentleman. Pressemitteilung (https://www.care-o-bot .de/content/dam/careobot/de/documents/Pressemitteilungen/2015_01_13_Car e-O-bot_4_final.pdf; Zugriff 13.8.2020).

Jacobi, Juliane (1990): »Geistige Mütterlichkeit«: Bildungstheorie oder strategischer Kampfbegriff gegen Männerdominanz im Mädchenschulwesen?, in: Die Deutsche Schule DDS, Beiheft/1990, S. 209-224.

Janowski, Kathrin/Ritschel, Hannes/Lugrin, Birgit/André, Elisabeth (2018): Sozial interagierende Roboter in der Pflege, in: Bendel, Oliver (Hg.): Pflegeroboter, Wiesbaden: Springer Gabler S. 63-86.

Jeanrenaud, Yves (2016): All this talk of getting old - Ein Kommentar zu Technik und Care (http://blog.soziologie.de/2016/02/all-this-talk-of-getting-old-ein-k ommentar-zu-technik-und-care/; Zugriff 13.8.2020).

Karakayali, Juliane (2010): Transnational Haushalten. Biografische Interviews mit care workers aus Osteuropa, Wiesbaden: Springer VS.

Koebe, Josefine/Samtleben, Claire/Schrenker, Annekatrin/Zucco, Aline (2020): Systemrelevant, aber dennoch kaum anerkannt: Entlohnung unverzichtbarer Berufe in der Corona-Krise unterdurchschnittlich, DIW aktuell 48/2020, Berlin: DIW.

Kuhlmey, Adelheid/Blüher, Stefan/Nordheim, Johanna/Zöllick, Jan (2019): Ressource oder Risiko: Wie professionell Pflegende den Einsatz digitaler Technik in der Pflege sehen, in: Zentrum für Qualität in der Pflege (Hg.): ZQP-Report - Pflege und digitale Technik, Berlin: ZQP, S. 31-35.

Kumbruck, Christel (2010): Menschenwürdige Gestaltung von Pflege als Interaktionsarbeit, in: Becke, Guido/Bleses, Peter/Ritter, Wolfgang/Schmidt, Sandra (Hg.): >Decent Work ‘. Arbeitspolitische Gestaltungsperspektive für eine globalisierte und flexibilisierte Arbeitswelt, Wiesbaden: Springer VS, S. 187-207.

Lutz, Helma/Palenga-Möllenbeck, Ewa (2010): Care-Arbeit, Gender und Migration: Überlegungen zu einer Theorie der transnationalen Migration im Haushaltsarbeitssektor in Europa, in: Apitzsch, Ursula/Schmidbaur, Marianne (Hg.): Care und Migration, Opladen: Budrich, S. 143-162.

Meussling-Sentpali, Annette (2019): Professionalisierung und Geschlechterverhältnisse in der Pflege, in: Rudolph, Clarissa/Schmidt, Katja (Hg.): Interessenvertretung und Care, Münster: Westfälisches Dampfboot, S. 37-53.

Mohr, Jutta/Fischer, Gabriele/Lämmel, Nora/Höß, Tanja/Reiber, Karin (2020): Pflege im Spannungsfeld von Professionalisierung und Ökonomisierung. Oder: Kann der Pflegeberuf wirklich attraktiver werden?, in: Bundeszentrale für po- 
litische Bildung (Hg.): Pflege. Praxis - Geschichte - Politik, Bonn: Bundeszentrale für politische Bildung, S. 203-213.

Nolte, Karen (2020): Sorge für Leib und Seele. Krankenpflege im 19. und 20. Jahrhundert, in: Bundeszentrale für politische Bildung (Hg.): Pflege. Praxis - Geschichte - Politik, Bonn: Bundeszentrale für politische Bildung, S. 120-132.

Rudolph, Clarissa/Schmidt, Katja (Hg.) (2019a): Interessenvertretung und Care. Voraussetzungen, Akteure und Handlungsebenen, Münster: Westfälisches Dampfboot.

Rudolph, Clarissa/Schmidt, Katja (2019b): Politikfeld Pflege - kollektives Handeln und Interessenvertretung von Pflegekräften, in: Rudolph, Clarissa/Schmidt, Katja (Hg.): Interessenvertretung und Care, Münster: Westfälisches Dampfboot, S. 72-92.

Scheele, Alexandra (2019): Abwertung von Care-Arbeit durch Vergeschlechtlichung, in: Rudolph, Clarissa/Schmidt, Katja (Hg.): Interessenvertretung und Care, Münster: Westfälisches Dampfboot, S. 24-36.

Schmidt, Jutta (1998): Beruf: Schwester. Mutterhausdiakonie im 19. Jahrhundert, Frankfurt/New York: Campus.

Schmidt, Katja (2019): Kollektive Auseinandersetzungen um Pflege. Formen und Praxen pflege- und carepolitischer Interessenartikulation, in: Rudolph, Clarissa/Schmidt, Katja (Hg.): Interessenvertretung und Care, Münster: Westfälisches Dampfboot, S. 250-265.

Schnerring, Alm/Verlan, Sascha (2020): Equal Care. Über Fürsorge und Gesellschaft, Bonn: bpb.

Seibert, Holger/Carstensen, Jeanette/Wiethölter, Doris (2018): Entgelte von Pflegekräften - weiterhin große Unterschiede zwischen Berufen und Regionen (h ttps://www.iab-forum.de/entgelte-von-pflegekraeften-weiterhin-grosse-unte rschiede-zwischen-berufen-und-regionen/?pdf=6353; Zugriff 10.1.2020).

Sell, Stefan (2020a): Der Irrsinn mit den Kräften in der Pflege. Oder: Folge dem Geld, dann wird aus dem offensichtlichen Irrsinn ein betriebswirtschaftlich durchaus rationales Vorgehen (https://aktuelle-sozialpolitik.de/2020/03/10/de r-durchaus-rationale-irrsinn-in-der-pflege/; Zugriff 17.8.2020).

Sell, Stefan (2020b): Die Zukunft der stationären Altenpflege zwischen Mindestlohn und wenn, dann mehr Hilfskräften? Kritische Anmerkungen angesichts einer doppelten Absenkung in einem ganz besonderen Arbeitsfeld (https://aktuelle-sozialpolitik.de/2020/02/25/die-zukunft-der-stationaere n-altenpflege-zwischen-mindestlohn-und-hilfskraeften/; Zugriff 17.8.2020).

Senghaas-Knobloch, Eva (2008): Care-Arbeit und das Ethos fürsorglicher Praxis unter neuen Marktbedingungen am Beispiel der Pflegepraxis, in: Berliner Journal für Soziologie, 2/2008, S. 221-243.

Staudhammer, Martina (2018): Prävention von Machtmissbrauch und Gewalt in der Pflege, Berlin: Springer. 
Theobald, Hildegard (2017): Care workers with migration backgrounds in formal care services in Germany: A multi-level intersectional analysis, in: International Journal of Care and Caring, 1/2017, S. 209-226.

Weber, Jenny (2020): Pflege: Der alltägliche Ausnahmezustand, in: Blätter für deutsche und internationale Politik, 6/2020, S. 13-16.

Winker, Gabriele (2015): Care Revolution. Schritte in eine solidarische Gesellschaft, Bielefeld: transcript.

Zöllick, Jan C./Kuhlmey, Adelheid/Suhr, Ralf/Eggert, Simon/Nordheim, Johanna/Blüher, Stefan (2020): Akzeptanz von Technikeinsatz in der Pflege. Zwischenergebnisse einer Befragung unter professionell Pflegenden, in: Jacobs, Klaus/Kuhlmey, Adelheid/Greß, Stefan/Klauber, Jürgen/Schwinger, Antje (Hg.): Pflege-Report 2019. Mehr Personal in der Langzeitpflege - aber woher?, Berlin: Springer, S. 211-218. 



\title{
Datenschutz und digitale Ethik Grundlage guter Technik
}

\author{
Walter Swoboda, Marina Fotteler, Michael Örtl, Felix Holl, Martin Schmieder, \\ Elmar Buchner
}

\section{Was ist Digitalisierung?}

Computer im heutigen Sinne, also programmierbare und digital-elektronisch gesteuerte Automaten, gibt es schon seit der ersten Hälfte des letzten Jahrhunderts, wobei sich die Basistechnologie (aufbauend auf drei einfachen logischen Operationen) nicht grundlegend geändert hat. Die entscheidende Beschleunigung hat die Technologie im Jahre 1974 erfahren: In diesem Jahr begann die Firma Intel mit der Produktion des Mikroprozessors, der Computer nicht nur miniaturisierte, sondern radikal preiswerter machte. In den folgenden 40 Jahren verbreitete sich die Technologie rasch. Heute sprechen wir von der Digitalisierung praktisch aller Lebensbereiche.

Damit ist gemeint, dass es keine menschliche Tätigkeit mehr gibt, in der computergesteuerte Anwendungen nicht zum Einsatz kämen; einige Anwendungen haben sogar Bedürfnisse geschaffen, die es vorher noch gar nicht gab: Social Networking ist hierfür ein gutes Beispiel, aber bei weitem nicht das einzige. Ob die Digitalisierung dabei die zugrunde liegenden Prozesse immer verbessert, oder ob insgesamt durch Digitalisierung eine Verbesserung eintritt, ist durchaus umstritten: Den unumstrittenen Vorteilen (z.B. Wegstreckeneinsparung durch Navigationsgeräte, ubiquitäre Verfügbarkeit von Information durch das Internet, Wettervorhersagen durch massiv parallele Großrechner) stehen nicht wegzudiskutierende Risiken gegenüber. Moderne Computersysteme sind grundsätzlich mit großer Speicherkapazität ausgestattet und miteinander vernetzt, das heißt, es können Daten in einer Menge gespeichert und ausgetauscht werden, wie es noch vor einigen Jahren undenkbar gewesen wäre. Gegenüber herkömmlichen Datenspeichern, wie beispielsweise Aktenordnern oder Mikrofiches, hat sich die potenzielle Quantität erhöht, nicht aber die Qualität des Datenschutzrisikos. Dies führt in der öffentlichen Diskussion manchmal zu voreiligen Schlüssen, wie etwa dem Einwand, dass auch früher Patient*innenakten versehentlich Dritten zur Einsicht gelangten. Das mag richtig sein, aber durch datentechnische Unzulänglichkeiten moderner Informa- 
tionssysteme gelangen eben nicht nur einzelne Datensätze, sondern häufig ganze Datenarchive mit vielen Tausend Datensätzen in die Hände nicht autorisierter Personen.

Durch Einsatz von neuen, teilweise revolutionären Technologien wie Big Data, maschinellem Lernen und eventuell künftig von Quantencomputern können riesige Datenmengen zudem sehr effizient gefiltert und verarbeitet werden, womit auch kommerziell verwertbares Wissen extrahiert werden kann. Plattformen wie Amazon oder Alibaba arbeiten daran, ihre Kund*innendaten derartig effizient auszuwerten, dass es möglich ist, die nächste Bestellung vorzubereiten, bevor die Kund"innen bestellen. Der Anfang ist gemacht und diesbezügliche Versuche laufen bereits.

\section{Warum Digitalisierung für Senior*innen?}

Die menschlichen Lebensgewohnheiten werden bereits heute durch die mit Miniaturisierung und Verbilligung ausgelöste technologische und soziale Revolution der Digitalisierung mehr verändert als durch die drei industriellen Revolutionen der Mechanisierung, Massenproduktion und Automatisierung zuvor. Bisher ist kein Ende in Sicht, im Gegenteil scheint sich die Entwicklung sogar noch weiter $\mathrm{zu}$ beschleunigen. Allerdings nehmen nicht alle Bevölkerungsschichten gleichermaßen an dieser Entwicklung teil. Der vielbesprochene >social divideく, das heißt, der schlechtere Zugang zur Technologie und die daraus resultierende Benachteiligung vor allem von älteren, sozial schwachen und behinderten Menschen, ist Realität und kann auch in jüngerer Zeit bestätigt werden (Hollier 2007), wenngleich das Problem mittlerweile differenzierter betrachtet werden muss (Paul/Stegbauer 2005). Entsprechend wird von staatlichen Institutionen gegengesteuert; einige Maßnahmen sind im 8. Altersbericht des Bundesministeriums für Familie, Senioren, Frauen und Jugend (BMFSFJ) zusammengefasst (BMFSFJ 2020).

\section{Nicht zwingende Gründe}

Besteht hier Nachholbedarf, oder anders ausgedrückt: Sollen wir die Digitalisierung der Lebensumstände von Älteren überhaupt fördern? Brauchen Senior*innen unbedingt Digitalisierung? Viele würden hier sicher argumentieren: Nicht unbedingt. Allerdings entstehen dann durch die sich ändernden gesellschaftlichen Rahmenbedingen Einschränkungen, die nicht ohne Weiteres aus dem Weg geräumt werden können, so zum Beispiel:

- Finanzielle Einschränkungen (z.B. wird eine Banküberweisung künftig für diejenigen deutlich teurer werden, die den herkömmlichen, analogen Weg bevor- 
zugen; Verbraucher"innen, die mittels App und/oder Kund"innenkarte Mitglied in einem digitalen Bonusprogramm sind, bekommen einlösbare Punktegutschriften oder erhalten Rückzahlungen).

- Gesellschaftliche Einschränkungen (z.B. soziale Kontakte finden vermehrt über die digitalen Netzwerke statt, physische Veranstaltungen werden darüber geplant).

Obwohl diese Nachteile zwar messbar sind, werden sie wohl aber nicht als derart gravierend angesehen, die Digitalisierung der Lebensbereiche von Senior*innen zu fördern oder eventuell gar zu fordern: Die Einzelperson mag die Konsequenzen abwägen und individuell entscheiden.

\section{Zwingende Gründe}

Allerdings existiert mindestens ein weiterer Punkt, der sich ganz grundlegend von den angeführten Einschränkungen unterscheidet und eine zumindest teilweise Digitalisierung voraussetzt. Es handelt sich um die Möglichkeit der adäquaten künftigen pflegerischen und medizinischen Versorgung Älterer, beziehungsweise um die Unmöglichkeit dieser, wenn die etablierten Prozesse ohne grundlegende Änderung fortgesetzt werden. Es gibt in Deutschland, wie in anderen Ländern, außerdem einen gravierenden Mangel an Pflegepersonal, der zwar durch äußere Umstände (Bezahlung, Arbeitsbedingungen) beeinflussbar ist, aber ganz grundsätzlich durch zu wenig verfügbare personelle Ressourcen verursacht wird, wobei sich diese Faktoren natürlich gegenseitig beeinflussen (siehe Abbildung 1).

Der dargestellte, sich selbst verstärkende Kreislauf kann nur durchbrochen werden, wenn mindestens einer der Faktoren Arbeitsbelastung, Attraktivität, und/oder Arbeitskräftemangel entscheidend positiv beeinflusst wird.

Dem Mangel an Personal wird mit überschaubarem Erfolg entgegengetreten, indem Pflegekräfte aus dem Ausland gezielt angeworben werden. Auch Versuche, die Höhe der Bezahlung den verantwortungsvollen und risikoreichen Aufgaben anzupassen, haben eher enge Grenzen finanzieller Art. Dagegen lässt sich die Belastung am Arbeitsplatz voraussichtlich deutlich durch Einsatz der Digitalisierung verringern, vor allem wenn man bedenkt, dass mindestens ein Drittel der pflegerischen Arbeitszeit mit eigentlich pflegefremden Tätigkeiten verbracht wird, zum Beispiel Tätigkeiten der Verwaltung oder Dokumentation (Hendrich et al. 2008). Werden Pflegekräfte selbst befragt, so würden sie übrigens selbst bessere Arbeitsbedingungen einer besseren Bezahlung vorziehen (Vereinigung der Bayerischen Wirtschaft 2020).

Zudem, und dieser Punkt wird häufig übersehen, ist davon auszugehen, dass eine moderne und effiziente Arbeitsumgebung dazu beitragen kann, die Attraktivität des Berufs zu steigern. Zeitgerechte Ausstattung hebt durchaus die Motivati- 
Abbildung 1: Abhängigkeitsmodell berufliche Attraktivität Pflege

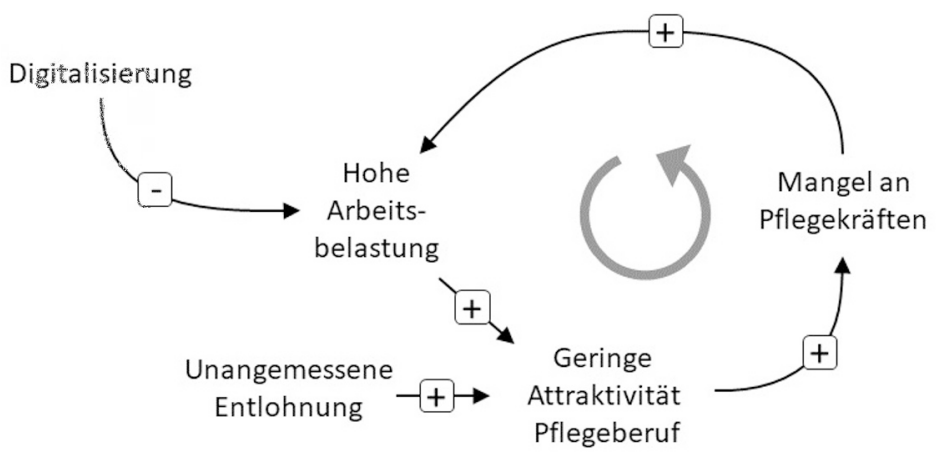

Quelle: Eigene Darstellung

on und gerade in den pflegerischen Berufen besteht hier Nachholbedarf. Während zum Beispiel Automechaniker*innen zu Mechatroniker*innen werden und selbstverständlich nicht nur mit Motoren umgehen, sondern auch programmieren und Software aktualisieren, wird in der Pflege - abgesehen von vereinzelten Inselversuchen mit Pflegeinformationssystemen - zumeist immer noch mit herkömmlichen Methoden, wie beispielsweise papierbasierter Dokumentation, gearbeitet.

Die Sinnhaftigkeit der Digitalisierung von medizinischer und pflegerischer Versorgung von Senior*innen sollte damit außer Frage stehen, allerdings gilt es, vorhandene Risiken hinreichend kritisch zu bewerten und zu berücksichtigen.

\section{Was ist sinnvoll?}

Außer Frage steht, dass es sich bei medizinischer Versorgung um einen sehr arbeitsteiligen Prozess handelt. Das gilt auch für die Pflege und zwar sogar zunehmend. Durch die Bemühungen, die Pflege von Senior*innen verstärkt ambulant zu betreiben, sind häufig unterschiedliche Dienstleister*innen am Prozess beteiligt. Vernetzung und Abstimmung erfolgen dabei nicht immer in ausreichendem $\mathrm{Ma}$ ße (Lavander/Meriläinen/Turkki 2016). Davon ausgehend muss die Frage gestellt werden: Was ist weiterhin sinnvoll? 


\section{Literaturrecherche}

Wir haben eine breit angelegte Literaturrecherche $\mathrm{zu}$ assistiven Technologien für Senior*innen begonnen, die als vorläufige Veröffentlichung vorliegt (Fotteler et al. 2019). Einschlusskriterien waren unter anderem, dass es sich um eine randomisierte kontrollierte Studie (engl. srandomized controlled triak, RCT) mit mindestens einer Kontrollgruppe handelt, die peer-reviewed nach dem 01.01.2009 veröffentlicht wurde und sich mit üblichen Digitalisierungsschwerpunkten der assistiven Versorgung älterer Menschen (Durchschnittsalter $\geq 65$ Jahre) beschäftigt. Ausgeschlossen wurden die Themen Telemedizin, Virtual Reality, Robotik, Lifestyle-Interventionen und weitere Technologien für rehabilitative und therapeutische Ansätze. Die Suche wurde in den Datenbanken Medline, CINAHL und IEEEXplore durchgeführt. Es wurden 11.400 Studien gesichtet; nach eingehender Prüfung blieben neunzehn Studien übrig, die sich durch einen hohen Grad der Heterogenität auszeichneten. Sechs Kategorien wurden in der Auswertung identifiziert: Hören, Medikation, Sehen, mentale Unterstützung, Mobilität und persönliches Krankheitsmanagement. Lediglich Hörgeräte und Anwendungen zum persönlichen Krankheitsmanagement erscheinen den vermeintlich größten Nutzen für ältere Menschen zu haben. Es gibt jedoch noch $\mathrm{zu}$ wenige randomisiert-kontrollierte Studien zur Thematik, zudem kaum RCTs zu den aktuell populären Technologien wie Sturz-Sensorik et cetera, weshalb die Evidenzlage nicht eindeutig bestimmt werden kann.

\section{Konsortialprojekt CARE REGIO}

Das Projektkonsortium CARE REGIO wurde im Raum Bayerisch-Schwaben ins Leben gerufen, um speziell die Möglichkeiten der Digitalisierung der Pflege zu erproben. In der bereits abgelaufenen Vorphase des Projekts wurden mehrere Befragungen durchgeführt, um den Bedarf der Pflegekräfte zu evaluieren (Veröffentlichung in Vorbereitung). Ein ganz eindeutiges Ergebnis ist, dass die Befragten einen erhöhten Bedarf bei der Erleichterung der als sehr arbeitsintensiv empfundenen Dokumentationspflichten und bei der inter-institutionellen Nachrichtenvernetzung sehen. Demzufolge wird CARE REGIO neben dem Einsatz von assistiver robotischer Technologie auch einen besonders aufwendigen Dokumentations- und Kommunikationsprozess (Überleitungsmanagement) und einen zentralen Datenpool für pflegerische Datensätze (`Data Lake`) erproben. 


\section{Wie wird aus Digitalisierung gute Technik?}

In diesem Artikel sollen weniger die technischen Lösungsansätze beleuchtet werden, da diese teilweise vorhanden, wenn auch nicht im gegebenen Umfeld erprobt sind. Vor allem die Einbindung der pflegerischen Prozesse in die deutsche Telematik-Infrastruktur wird eine wesentliche Aufgabe darstellen. Bisher gibt es diesbezüglich kaum etablierte Ansätze, zumal der Fokus der mit der Bereitstellung beauftragten Gesellschaft für Telematikanwendungen der Gesundheitskarte $\mathrm{mbH}$, genannt gematik (Drees 2007), in der Vergangenheit mehr auf die medizinische Versorgung ausgerichtet war. Dies wird nunmehr für die Pflege nachgeholt, entsprechende Module und Modifikationen sind bereits eingeleitet.

Im Folgenden wird detailliert auf Anforderungen von Datenschutz, Datensicherheit und ethische Belange eingegangen, die im sensiblen Umfeld der Digitalisierung von Medizin und Pflege während des gesamten Einführungs- und Betriebsprozesses Vorrang vor technischen und ökonomischen Gegebenheiten haben sollten (siehe Abbildung 2).

Abbildung 2: Einflussfaktoren auf den Einführungs- und Betriebsprozess

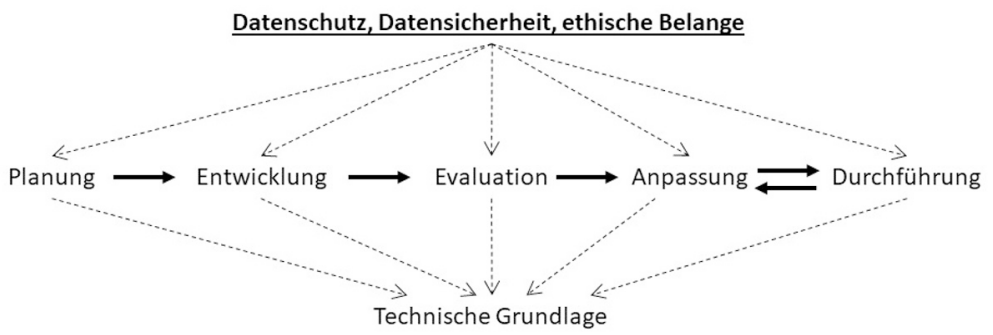

Quelle: Eigene Darstellung

\section{Datenschutz und Datensicherheit}

Insbesondere in Medizin und Pflege stellt der Schutz der patient"innenrelevanten Daten eine besonders wichtige Aufgabe dar. Dabei ist Datenschutz von der Datensicherheit zu unterscheiden: Das Erste meint den Schutz vor Zugriffen unautorisierter Dritter, das Zweite die sichere Speicherung und Übertragung der Datensätze. Hierunter fallen zum Beispiel der Schutz vor Löschung und Veränderung, die Verfügbarkeit und die Nichtabstreitbarkeit von Daten. 


\section{Gesetzliche Grundlagen}

Ganz grundsätzlich ist für jede Maßnahme der Digitalisierung in der (Alten-)Pflege ein >Datenschutzbeauftragter nach Artikel 37 der Datenschutz-Grundverordnung (DSGVO) zu benennen, welcher aufgrund der beruflichen Qualifikation und des Fachwissens gewählt werden muss.

Eine weitere wichtige Voraussetzung ist die Einhaltung der Richtlinien des Bundesdatenschutzgesetzes (Nagel/Kiefer 2008) und der Datenschutz-Grundverordnung der Europäischen Union (Voigt/von dem Bussche 2018). Hier wird vor allem das Persönlichkeitsrecht der Patient*innen geschützt: Unbedingt erforderlich ist die Einholung einer freiwilligen, für den jeweiligen Einsatz zweckgebundenen, schriftlichen Patient*innenzustimmung mit vorgehender vollständiger Aufklärung in einer für die Patient*innen verständlichen Sprache. Selbstverständlich dürfen Erhebung, Verarbeitung und Nutzung der personenbezogenen Daten nur für die medizinisch-pflegerische Behandlung (unter Patient*inneneinwilligung) verwendet werden. Die Erhebung, Verarbeitung und Nutzung von ausschließlich nicht personenbezogenen Daten für eine wissenschaftliche Auswertung ist möglich, muss jedoch in der Patient*inneneinwilligung ausdrücklich vermerkt werden. Ganz grundsätzlich unterliegen alle Datensätze der Erforderlichkeit und Zweckbindung, das heißt, Daten werden nur erhoben, wenn sie für den medizinisch-pflegerischen und eventuell wissenschaftlichen Einsatzzweck erforderlich und zweckmäßig sind.

Einige wichtige neuere Anforderungen ergeben sich durch das Patientendatenschutzgesetz (Fricke 2020). Dieses beinhaltet im Wesentlichen, dass Patient*innen in Deutschland das Recht besitzen, dass ihre medizinisch und auch pflegerisch relevanten Daten in den Datensenken der deutschen Telematik-Infrastruktur (auf Verlangen) eingetragen werden. Unabdingbare Voraussetzung dafür ist, dass Digitalisierungsvorhaben für Senior*innen mittels Konnektoren überhaupt eingebunden werden und die betreuenden Fachkräfte Zugang zur Telematik-Infrastruktur erhalten. Dies scheitert im Moment noch an der Tatsache, dass der dafür notwendige elektronische Heilberufe-Ausweis (eHBA) an Ärzt*innen, jedoch noch nicht an Pflegekräfte ausgegeben wird. Der Grund hierfür liegt in einer uneinheitlichen organisatorischen Verteilerstruktur, die zwar im medizinischen Bereich mit der Bundesärztekammer vorhanden ist (und von den Ärzt*innen finanziert wird); eine entsprechende Einrichtung für die Pflege hingegen jedoch fehlt. Eine Lösung mittels Gruppenzugang ist im Sinne der Nachverfolgbarkeit von Datenzugriff, änderung und -eingabe kein geeigneter Weg.

\section{Verschlüsselung}

Erster und wichtigster Schritt für die Sicherstellung der datenschutzrechtlichen Anforderungen ist die Verschlüsselung von Daten. Je nach verwendetem Verfah- 
ren ist eine Entschlüsselung durch unautorisierte Dritte mehr oder weniger leicht möglich. Im Sinne eines mathematischen Nachweises kann keine der heute verwendeten Verschlüsselungsalgorithmen einen absolut sicheren Schutz bieten. ${ }^{1}$ Gängige Verfahren erhöhen nur den Aufwand, der zur Entschlüsselung betrieben werden muss, auf ein Maß, das sie heute faktisch unmöglich macht. Allerdings geht jede*r Anwender*in das Risiko ein, dass verschlüsselte Daten gespeichert werden und in Zukunft mit neuen Verfahren relativ einfach in Klartext verwandelt werden können. Wesentlichste Unsicherheitsfaktoren sind dabei die Möglichkeit wissenschaftlicher Fortschritte auf verschiedenen Gebieten der Kryptografie, zum Beispiel zeitlich verzögerte >Brute-Force-Attacke oder die Anwendung eventuell entwickelter neuer Möglichkeiten der Primzahlenzerlegung. Auch schlichtes menschliches Versagen trägt dazu bei, dass Daten entschlüsselt werden können.

Herkömmliche Verfahren basieren grundsätzlich auf einem symmetrischen Schlüsselaustausch, bevor der eigentliche Datenaustausch erfolgt. Alle am Verfahren beteiligten Partner*innen nutzen dann (eventuell für einen sehr kurzen Zeitraum) denselben Schlüssel, mit dem die Daten über einen bekannten Prozess behandelt werden. Das inverse Verfahren liefert dann wieder den ursprünglichen Text. Formal betrachtet verhält es sich wie folgt: Sei $C$ eine beliebige Zeichenkette, f ein Verschlüsselungsverfahren, $S$ der zugehörige Schlüssel, $C^{\prime}$ die verschlüsselte Zeichenkette und $\mathrm{f}^{\prime}$ das Umkehrverfahren zu $f$ :

$$
\begin{gathered}
C=\left\{\mathrm{c} \in(\mathrm{a} . \mathrm{z}, \mathrm{A} . \mathrm{Z}, \ldots) \mid \mathrm{c}_{0} \cdot \mathrm{c}_{\mathrm{n}}\right\} \\
\mathrm{f}(\mathrm{S}, \mathrm{C})=\mathrm{C}^{\prime} \\
\mathrm{f}^{\prime}\left(\mathrm{S}, \mathrm{C}^{\prime}\right)=\mathrm{C}
\end{gathered}
$$

Es ist leicht einsehbar, dass eine dritte Person alle Nachrichten entschlüsseln kann, wenn ihr der Schlüssel bekannt ist. Wird dieser über denselben Nachrichtenkanal wie die eigentliche Nachricht ausgetauscht, ist dies sogar besonders einfach (>ManIn-The-Middle-Attack ). Trotzdem wird das Verfahren häufig wegen seiner verhältnismäßigen Einfachheit, des geringen Ressourcenverbrauchs und seiner Perfor-

$1 \quad$ Es gibt zwar >beweisbar sichere Verfahren<, diese fußen aber auf Annahmen, die heute noch nicht bewiesen werden können. Außerdem werden diese Verfahren in der Praxis wegen des damit verbundenen hohen Aufwands kaum eingesetzt. 
mance angewendet, auch bei der Verschlüsselung von WLAN-Übertragungen über das Protokoll Wired Equivalent Privacy (WEP).

Bei der asymmetrischen Verschlüsselung werden zwei Schlüssel verwendet, ein sogenannter privater Schlüssel und ein öffentlicher Schlüssel:

$$
\begin{gathered}
\mathrm{f}\left(\mathrm{S}_{\text {"offentlich }}, \mathrm{Z}\right)=\mathrm{S}^{\prime} \\
\mathrm{f}^{\prime}\left(\mathrm{S}_{\text {privat }}, \mathrm{Z}^{\prime}\right)=\mathrm{S}
\end{gathered}
$$

Der private Schlüssel kann frei verteilt werden; damit behandelte Dateien lassen sich nur dann wieder in den Klartext verwandeln, wenn der geheime Schlüssel zur Verfügung steht. In der Praxis verteilt ein*e potenzielle* $r$ Empfänger*in einer Nachricht seinen*ihren öffentlichen Schlüssel zum Beispiel über eine Web-Page, die jede*r einsehen kann. Ein*e potenzielle*r Nachrichtensender*in verschlüsselt dann seine*ihre Datei mit diesem öffentlichen Schlüssel und entzieht sie damit dem Zugriff (auch durch ihn*sie selbst, d.h., der*die Sender*in kann dann die Nachricht ebenfalls nicht mehr lesen). Kommt die Nachricht bei dem*der Empfänger*in an, so entschlüsselt diese*r die Nachricht mittels seines*ihres geheimen privaten Schlüssels.

Wie kann dies funktionieren? Dieses Verfahren basiert auf der Tatsache, dass bestimmte mathematische Rechnungen auf Computern in einer Richtung sehr schnell, in der anderen Richtung aber extrem langsam ablaufen. So lässt sich jede beliebige ganze Zahl in ein Produkt aus Primzahlen zerlegen, diese Operation kann aber bei großen Zahlen sehr viel Zeit erfordern, bei Zahlen mit 200 Stellen zehntausende Jahre oder mehr bei heutiger Computertechnik. Aus beliebigen ganzen Primzahlen lässt sich aber in Bruchteilen von einer Sekunde ein Produkt bilden, dessen Ergebnis eine Zahl mit den genannten 200 Stellen ergibt.

In der Praxis werden sinnvollerweise beide Verfahren, also symmetrische und asymmetrische Verschlüsselung, kombiniert. Der Algorithmus, der sich hinter >sicheren $<$ Web-Pages befindet, ist Hypertext Transfer Protocol Secure (HTTPS). Das Verfahren bewerkstelligt den Schlüsselaustausch über asymmetrische, die Nachrichtenverschlüsselung über symmetrische Verfahren.

Manchmal wird übersehen, dass die Daten nicht nur während der eigentlichen Übertragung geschützt werden müssen, sondern auch bei der Datenspeicherung in allen beteiligten System-Modulen (siehe Abbildung 3).

Auch jene Daten, die auf dem Server liegen, müssen verschlüsselt werden. Das gilt auch für die Zugriffsmöglichkeiten der Administrator*innen! Beteiligte Perso- 
Abbildung 3: Notwendige Absicherungen von Modulen in einem Beispielsystem (WLAN=Funkverbindung, LAN=Lokales Netzwerk, WAN=Weitverkehrsnetzwerk)

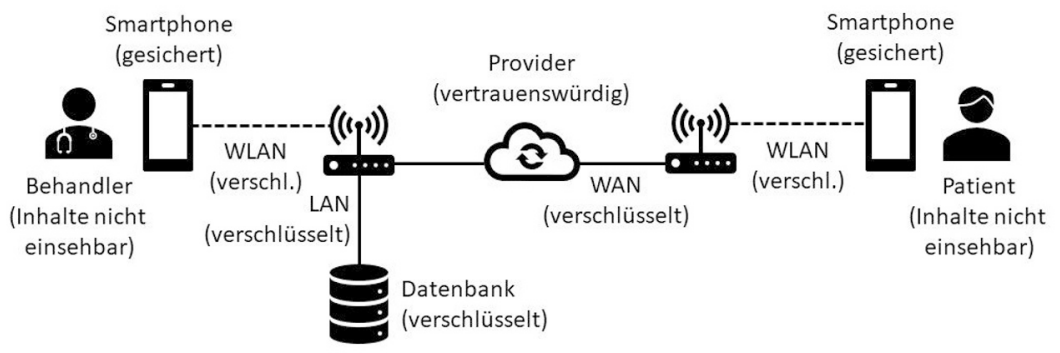

Quelle: Eigene Darstellung

nen müssen dafür Sorge tragen, dass Bildschirminhalte, Töne und so weiter nicht von Dritten eingesehen oder abgehört werden können. In den meisten Fällen wird es nötig sein, eigene Räume für die angemessene physische IT-Sicherheit zur Verfügung zu stellen.

\section{Anonymisierung}

Bei jeglicher Auswertung von Ergebnissen durch nicht am Behandlungsprozess Beteiligte ist es notwendig, dass Daten entfernt werden, die nicht für die Auswertung erforderlich sind, aber eine Identifizierung ermöglichen. Dieser Transfer von patient*innenrelevanten Daten (für die Behandlung) hin zu nicht-patient*innenrelevanten Daten erfolgt grundsätzlich durch die Anonymisierung der Datensätze. Hierbei werden persönliche Daten in einer möglichst räumlich, organisatorisch und personell getrennten Vertrauensstelle vor Weiterleitung entfernt und alle noch vorhandenen Datensätze mittels eines Randomisierung-Algorithmus zwischen den Datensätzen permutiert. Dabei bleiben zwar summarische Auswertungen (z.B. durchschnittlicher zeitlicher Arbeitsaufwand) erhalten, die Daten eignen sich aber nicht mehr für die eingehende wissenschaftliche Untersuchung, insbesondere, wenn verschiedene Gruppen unter Einbeziehung von Kofaktoren evaluiert werden sollen. Deshalb wird bei einer geplanten Untersuchung normalerweise die Methode der Pseudonymisierung angewendet, bei der auf die permutierende Neuverteilung der Daten verzichtet wird. Es ist leicht nachvollziehbar, dass durch auftretende Datenmuster bei Diagnosen und Behandlungen auch nach der Pseudonymisierung eine teilweise sichere Zuordnung zur Persönlichkeit der Patient*innen 
möglich bleiben kann. Deshalb wird das Verfahren als alleinige Maßnahme für den Datenschutz als nicht ausreichend angesehen.

Eine höhere Konformität zum Bundesdatenschutzgesetz bietet die k-Anonymität (Sweeney 2002) kombiniert mit einer Pseudonymisierung. Hierzu werden neben den personenrelevanten Datensätzen (>Identifier<, diese werden entfernt) andere Variablen als quasi-identifizierend (`Quasi-Identifier $)$ definiert. QuasiIdentifikatoren sind solche, die für sich genommen keine Zuordnung erlauben würden, aber in Kombination mit anderen Daten eine Zuordnung ermöglichen. Ein Anwendungsbeispiel: Die Vertrauensstelle gibt einen Datensatz erst dann weiter, wenn mindestens zwei Datensätze mit gleichen quasi-identifizierenden Einträgen vorhanden sind, da erst dann davon ausgegangen werden kann, dass eine sichere Zuordnung nicht mehr möglich ist. Etwas formeller lässt sich der Zusammenhang so darstellen: Gegeben sei eine Gruppe von Patient*innen G, die über die identifizierende Datenmenge Imit ihren Variablen $V_{0 . n}$, einer quasiidentifizierenden Datenmenge $\mathrm{Q}$ mit ihren Variablen $\mathrm{V}_{\mathrm{n}+1}$.m und eine weitere sensible Datenmenge $\mathrm{S}$ mit ihren Variablen $\mathrm{V}_{\mathrm{m}+1 \text {.o }}$ beschrieben wird:

$$
\mathrm{G}=\left\{\mathrm{I}\left(\mathrm{V}_{0 . \mathrm{n}}\right) \cup \mathrm{Q}\left(\mathrm{V}_{\mathrm{n}+1 . \mathrm{m}}\right) \cup \mathrm{S}\left(\mathrm{V}_{\mathrm{m}+1 . \mathrm{o}}\right)\right\}
$$

Der für eine Untersuchung zur Verfügung stehende Datensatz lautet $G^{\prime}$ :

$$
\mathrm{G}^{\prime}=\left\{\mathrm{Q}\left(\mathrm{V}_{\mathrm{n}+1 . \mathrm{m}}\right) \cup \mathrm{S}\left(\mathrm{V}_{\mathrm{m}+1 . \mathrm{o}}\right)\right\}
$$

Dabei gibt es eine Zuordnung f der Vertrauensstelle:

$$
\mathrm{f}: \mathrm{G}^{\prime} \rightarrow \mathrm{G}
$$

T sei eine Tabelle von Werten der Variablen aus Q. Der Datensatz G'ist genau dann $\mathrm{k}$-anonym, wenn jede Teilmenge jeder Zeile $\mathrm{x}$ aus $\mathrm{T}$ mindestens $\mathrm{k}$-mal in $\mathrm{T}$ vorkommt: 


$$
\begin{gathered}
\mathrm{t}(\mathrm{x}) \subset \mathrm{T}(\mathrm{x}) \\
\forall \mathrm{t}(\mathrm{x}): \exists \mathrm{x}_{1 . \mathrm{k}} \text { mit }\left\{1 . \mathrm{k} \in \aleph \mid \mathrm{x}=\mathrm{x}_{1}, \ldots, \mathrm{x}=\mathrm{x}_{\mathrm{k}}\right\}
\end{gathered}
$$

Man spricht bei diesen Einträgen auch von einer `Äquivalenzklasseく. Damit ergibt sich:

$$
\nexists \mathrm{f}^{\prime}: \mathrm{Q} \rightarrow \mathrm{G}
$$

In der praktischen Arbeit ergeben sich trotzdem Möglichkeiten zur Deanonymisierung, die bekannteste davon ist die >Homogenitäts-Attackeく. Wenn alle Einträge einer Äquivalenzklasse in einem sensiblen Datensatz den gleichen Wert haben, ist eine Zuordnung sensibler Daten möglich (Beispiel: Alle 85-jährigen Männer haben eine Prostata-Hyperplasie). Auch lassen sich durch Verknüpfungen mit anderen Datenbanken einzelne Datensätze direkt zuordnen (>Background-KnowledgeAttack $)$.

\section{Schutzziele}

Es gibt eine Reihe weiterer zu realisierenden Maßnahmen, um Schutzziele bei allen Digitalisierungsvorhaben in der Medizin zu verfolgen, insbesondere bei solchen, bei denen keine statistische Auswertung erfolgt.

Zunächst ist hier die Vertraulichkeit von Daten zu nennen. Darunter versteht man, dass Daten nur von den Personen eingesehen oder offengelegt werden dürfen, die dazu auch berechtigt sind, was normalerweise über Verschlüsselungen erreicht wird.

Zweitens ist die Verfügbarkeit von Daten zu nennen. Sind Daten, die in einer Behandlung sinnvoll eingesetzt werden können, digital verfügbar, so müssen sie auch mit vertretbarem Aufwand einsehbar sein. Es wäre nicht vertretbar, wenn ein entsprechender Server gerade gewartet würde und daher nicht auf ihn zugegriffen werden könnte, sei es auch nur für eine begrenzte Zeit. Dieses Schutzziel wird normalerweise durch Einsatz redundanter Systeme erreicht, das heißt, durch mehrere Server, bei denen im Fall eines Einzelausfalls zusätzliche Hard- und Software vorgehalten wird. Um es gar nicht so weit kommen zu lassen, wird auch der 
Einsatz von unterbrechungsfreien Stromversorgungen diskutiert, die aber jeweils für sich wieder eine zusätzliche Fehlerquelle darstellen.

Die Daten-Integrität, also der Schutz vor unerlaubter (und eventuell sogar unentdeckter) Einsicht, Verfälschung oder gefälschtem Herkunftsnachweis, kann durch technische Maßnahmen wirkungsvoll verhindert werden. Verschlüsselung, Hash-Algorithmen und digitale Signaturen sind wirksame technische Methoden bei Speicherung und Kommunikation, die in jedem Fall durch zusätzliche organisatorische Maßnahmen (`Closed Roomく, Zugangsbeschränkung) ergänzt werden müssen. Der Verwendung von asymmetrischen Methoden, bei denen kein vorhergehender Austausch von privaten Schlüsseln notwendig ist (Andalib/Azad 2015), ist dabei zu bevorzugen.

Zusätzlich bestehen folgende ergänzende Schutzziele: Revisionssicherheit (revisionssichere Archivierung), Transparenz (ständige Möglichkeit des Einblicks gespeicherter Datensätze), Nichtverkettbarkeit von Datensätzen (s.o.) und Invertierbarkeit, also die vollständige Kontrolle durch Patient*innenrechte. Hierbei sind zu gewährleisten:

- Recht auf Widerspruch: Patient"innen haben ein Recht auf Widerspruch, dieses Recht gilt auch für Daten, welche zu Forschungszwecken eingesetzt werden unter Nennung von Gründen. Damit wird eine Teilnahmevereinbarung unter sofortiger Wirkung ungültig und alle Daten sind zu löschen.

- Recht auf Widerrufung: Patient*innen haben zu jeder Zeit die Möglichkeit, das Einverständnis zur Verarbeitung der personenbezogenen Daten zurückzuziehen. Im Unterschied zur Widerrufung wird dabei das Zustandekommen und die Gültigkeit einer Teilnahmevereinigung nicht bestritten, aber zu einem späteren Zeitpunkt beendet. Eventuell bereits gespeicherte Daten können damit erhalten bleiben.

- Recht auf Löschung von Daten, auch teilweise.

- Recht auf Auskunft über das Vorhandensein eigener Daten.

- Recht auf Nichtwissen: Patient*innen können sich dazu entscheiden, nicht über eventuelle Erkrankungen und Vorkommnisse aufgeklärt zu werden und Informationen über Ergebnisse nicht zu erfahren.

\section{Weitere Schutzziele}

Es gibt eine Reihe von zusätzlichen Forderungen, die schon in die ethischen Erfordernisse hineinreichen, aber technisch bedingt sind, weshalb sie hier besprochen werden:

- Unaufdringlichkeit: Die eingesetzten digitalen Methoden zwingen aus sich selbst heraus nicht zum Handeln der Patient*innen. 
- Situationsangemessenheit: Die eingesetzten digitalen Methoden reagieren angemessen auf die Bedürfnisse der Nutzer*innen, der Umgebung und der Situation.

- Zulassung von Sensoren und Aktoren: Sämtliche Sensoren, Aktoren und jegliche weitere Hard- und Software unterliegen der Medizingeräteverordnung (Nöthlichs/Weber 2003) und müssen diesbezüglich freigegeben sein.

- Robustheit: Die Robustheit der Systeme muss dem Einsatzzweck angemessen sein.

- Technikfolgenabschätzung: Grundsätzlich sind die Folgen des Einsatzes einer Digitalisierungs-Technologie für Patient*innen, Personal und Gesellschaft vor dem Einsatz geeignet abzuschätzen. Zu dieser Abschätzung gehört die Einbeziehung von medizinisch-pflegerischen, technischen, aber auch soziologischen und wirtschaftlichen Faktoren. Aktuell mangelt es noch an pflegebezogenen Technikfolgenabschätzungen. Sinnvoll erscheint daher analog zum etablierten Health Technology Assessment (HTA) ein `Care Technology Assessmentく. Dazu gehören Nutzenbewertung und Technikfolgeabschätzung. Ein klassisches HTA hat die Aufgabe, die beste Lösung für eine Entscheidung hinsichtlich der Gesundheitsversorgung insgesamt wissenschaftlich zu unterstützen. Dies setzt jedoch eine gründliche vorausgegangene Evaluierung einer Anwendung voraus, das heißt, klinische Studien liegen bereits vor und die Anwendung wird bereits genutzt und erprobt. Ein prospektives HTA, das in früheren Phasen ansetzt, kann Einsatzpotenziale und Schwachstellen einer Innovation aufzeigen und so helfen, Chancen und Risiken frühzeitig zu bewerten und damit als strategisches Entscheidungsinstrument für die Umsetzung dienen.

Tabelle 1 fasst alle oben genannten Maßnahmen zusammen. 
Tabelle 1: Maßnahmen zu Datenschutz und Datensicherheit

\begin{tabular}{|c|l|}
\hline Cesetzliche Anforderungen & $\begin{array}{l}\text { Datenschutzgesetze } \\
\text { Patientendatenschutzgesetz }\end{array}$ \\
\hline Verschlüsselung & Symmetrische Verfahren \\
& Asymmetrische Verfahren \\
& Kombinierte Verfahren \\
\hline Anonymisierung & Klassische Anonymisierung \\
& Pseudonymisierung \\
& k-Anonymisierung \\
\hline Schutzziele & Vertraulichkeit \\
& Verfügbarkeit \\
& Datenintegrität \\
& Transparenz \\
& Nichtverkettbarkeit \\
& Invertierbarkeit \\
\hline Weitere Schutzziele & Unaufdringlichkeit \\
& Angemessenheit \\
& Zulassung \\
& Robustheit \\
& Folgenabschätzung \\
\hline
\end{tabular}

Quelle: Eigene Darstellung

\section{Erfordernisse der digitalen Ethik}

\section{Abgrenzung und Ethikkommission}

Datenschutz und Datensicherheit sind Teil der ethischen Anforderungen, da ihre Nichtbeachtung zu entsprechenden Nachteilen für die Klient"innen führen kann. Weitere ethische Erfordernisse werden im Folgenden genannt. $\mathrm{Zu}$ beachten ist, dass eine formale Aufzählung nicht vollständig sein kann, da sie von den spezifischen Gegebenheiten eines Systems abhängt, womit dann neue Einträge erzwungen werden können. Im Zweifelsfall wird die zuständige Ethikkommission auf einzelne Punkte hinweisen, bevor sie ein Vorhaben positiv bewerten kann.

Ein Votum einer Ethikkommission ist immer dann erforderlich, wenn Forschung an lebenden oder verstorbenen Menschen oder an entnommenen Körpergeweben vorgenommen wird oder epidemiologische Untersuchungen erfolgen, bei denen personenbezogene Daten verarbeitet (nicht unbedingt ausgewertet) werden.

\section{Forderungen}

Zur Orientierung werden hier einige Sachverhalte angesprochen, die in den meisten Projekten für die technische Versorgung Älterer zur Anwendung kommen dürften: 
- Risiken: Die Deklaration von Helsinki (Bundesärztekammer 2013) fordert schon seit 1964 eine Chancen-/Risiko-Abwägung, allerdings nur für medizinisch-pflegerische Forschungsvorhaben: Ganz grundsätzlich dürfen Vorhaben nur dann umgesetzt werden, wenn die positiven Effekte für die Klient*innen überwiegen. Einzelne negative Effekte müssen durch einen Gewinn für die Gesamtheit aller Beteiligten mehr als ausgeglichen werden. Diese Forderung hat erhebliche Bedeutung für alle Digitalisierungsvorhaben und sollte daher auch hier Berücksichtigung finden.

- Selbstbestimmung: Eines der Hauptziele von senior*innengerechten technischen Hilfen ist es, den Klient*innen ein selbstbestimmtes Leben zu ermöglichen. Kein System oder Teilsystem sollte deshalb ausschließlich autonom agieren. In allen Fällen werden unter Einbeziehung der Klient*innen Entscheidungen von Menschen getroffen. Hierzu gehört auch, dass alle Personen das Recht haben, auf ein Hilfsmittel zu verzichten (s.u.).

- Eingeschränkte Selbstbestimmung: Ein Unterstützungsangebot sollte sich ausschließlich an entscheidungsfähige Patient"innen richten, deren kognitive Fähigkeiten weder durch Krankheit noch durch Behinderung soweit eingeschränkt sind, dass obige Voraussetzung nicht mehr gegeben ist. Diese Forderung ist in vielen Fällen nicht $\mathrm{zu}$ erfüllen, vor allem dann, wenn die Empfänger*in der Leistung diese zwar benötigen würde, aber aufgrund beispielsweise mangelnder kognitiver Leistung dazu nicht mehr oder vorübergehend nicht mehr in der Lage ist (Beispiel: Frühmobilisation großer Gelenke nach Schlaganfall mit Unterstützung durch Bewegungsroboter). Hier sollte versucht werden, Angehörige hinzuzuziehen. Ist dies nicht möglich, sollte eine rechtliche Vertretung gefunden werden. ${ }^{2}$ Eine eventuell vorhandene Patient*innenverfügung ist auf alle Fälle bindend.

- Teilhabe: Alle Teilnehmer*innen des Projekts (Personal, Patient*innen, Angehörige) werden in das Projekt einbezogen und befragt, auch zu Verbesserungsmöglichkeiten. Alle Meinungsäußerungen sind gleichwertig zu behandeln.

- Gerechtigkeit und Vermeiden von Diskriminierung: Alle Personen werden ausschließlich durch Anwendung der Ein- und Ausschlusskriterien ohne Ansehen von Geschlecht, Religion oder ethnischer Gruppenzugehörigkeit einbezogen. Die erwähnten Kriterien werden vor der eigentlichen Untersuchung festgelegt und nachträglich nicht verändert, solange sich daraus keine ethischen Konflikte (Risikoerhöhung) ergeben. Um dies auszuschließen, werden regelmäßig Zwischenuntersuchungen anhand der bereits verfügbaren Daten durchgeführt.

2 Das gilt ausschließlich für nicht-invasive Verfahren, andernfalls sind deutlich verschärfte Bedingungen bindend. 
- Privatheit: Daten aus der Privatsphäre aller beteiligten Personen werden nur insoweit gespeichert, als sie der medizinischen Behandlung dienen und für diese unabdingbar sind. Die wissenschaftliche Auswertung findet nur unter der Minimalvoraussetzung der Pseudonymisierung der Datensätze (s.o.) statt.

- Aufklärung: Bei allen Klient*innen findet eine schriftliche Aufklärung statt, die im Falle der Zustimmung zur Projektteilnahme mit Unterschrift der Aufklärenden und der Patient*innen bestätigt wird. Die Aufklärenden werden vor der Durchführung des ersten Aufklärungsgesprächs im Projekt über Risiken und Gefahren geschult. Die Aufklärungsdokumente werden an zentraler Stelle aufbewahrt und vor nachträglicher Änderung geschützt. Siehe hierzu auch die Forderung nach Selbstbestimmung, beziehungsweise eingeschränkter Selbstbestimmung.

- Sicherheit und Haftung: Sicherheit für alle Beteiligten steht an erster Stelle und wird durch Anwendung geprüfter Technologie gewährleistet. Sollten dennoch unerwünschte Wirkungen auftreten, so werden diese dokumentiert und den zuständigen Behörden gemeldet (Bundesinstitut für Arzneimittel und Medizinprodukte, BfArM). Ansonsten tritt die Haftung der betreuenden Einrichtungen in Kraft, die durch eine entsprechende Pflichtversicherung abgedeckt sein muss.

- Anwender*innenfreundlichkeit: Um den Projekterfolg nicht zu gefährden, werden alle digitalen Verfahren anwender*innenfreundlich gestaltet und diesbezüglich optimiert (`Usability<).

- Vertragsabstimmung: Klient*innen haben das Recht, im Projektverlauf aus dem Projekt jederzeit auszusteigen und die Teilnahme zu beenden (s.u.).

- Weiterbildung: Beteiligtes Personal wird vor Beginn einer Maßnahme ausführlich, nachvollziehbar und nachweisbar geschult, um das Risiko einer negativen Behandlungswirkung für alle Beteiligten auszuschließen.

- Verantwortung: Alle Projektmitarbeiter*innen und alle beteiligten Lieferant*innen werden in ihrer Verantwortung gegenüber Bedürfnissen, Wünschen und Lebensprozessen der Patient*innen belehrt; die Einhaltung wird überprüft und dokumentiert.

- Sozialräumliche Strukturen: Bei der Teilnehmer*innenakquise spielt weder die ökonomische Situation, die Bildung, oder Herkunft eine Rolle, außer sie sind Gegenstand der Untersuchung selbst. Alle unterstützungsbezogenen Aufwendungen werden ersetzt.

- Technisierung: Die eingesetzten Methoden bleiben im alltäglichen Hintergrund und tragen nicht mehr als notwendig zur Technisierung des Alltags bei. Insbesondere wird keine Technik verwendet, die aus sich selbst heraus eine Reaktion oder Eingabe der Patient*innen erfordert. 


\section{Zusammenfassung}

Gute Technik im Alter erfordert die Beachtung der Grundsätze von Datenschutz und digitaler Ethik, da ansonsten die Verbesserung der Lebensbedingungen durch schwerwiegende Nachteile, wie Verletzung des Persönlichkeitsrechts, Verlust der Selbstbestimmung und so weiter erkauft wird. Dies hat nicht nur Auswirkungen auf die Anwender*innen, sondern auch auf die Anbietenden: Werden derart grundlegende Werte nicht beachtet, wird vermutlich die Akzeptanz der Produkte sinken. Dabei ist es nicht so, dass Senior*innen gute Technik ablehnen würden; das Gegenteil ist eher der Fall (Demiris et al. 2004). Es herrscht ein gewisses Vertrauen von Senior*innen, dass der Einsatz von Technik das Leben im Alter erleichtert und insbesondere eine längere Selbstständigkeit ermöglichen kann. Es gilt, dieses Vertrauen nicht zu enttäuschen.

Hier wurde der Versuch unternommen, einerseits die Notwendigkeit der Digitalisierung für Ältere darzulegen, andererseits die damit verbundenen datenschutzrechtlichen und ethischen Anforderungen aufzuzeigen. Die Fülle mag überraschen und es wird schwer sein, zu jedem Einzelvorhaben alle aufgeführten Punkte $\mathrm{zu}$ realisieren. Ob und inwieweit dies sinnvoll ist, muss immer die Betrachtung des Einzelfalls zeigen. Zudem ist es wahrscheinlich, dass in der konkreten Durchführung eines Projekts Anforderungen auftreten, die hier nicht vorkommen, deren Einhaltung jedoch wesentlich sein können.

\section{Literatur}

Andalib, Saad/Azad, Saiful (2015): »The RSA Algorithm«, in: Saiful Azad/Al-Sakib Khan Pathan (Hg.), Practical Cryptography: Algorithms and Implementations Using C++, Boca Raton: CRC Press, 135-146.

BMFSFJ Bundesministerium für Familie, Senioren, Frauen und Jugend (2020): »Achter Altersbericht. Ältere Menschen und Digitalisierung«, https://www.bm fsfj.de/blob/159938/3970eecafb3c3c630e359379438c6108/achter-altersbericht-la ngfassung-data.pdf vom 02.12.2020.

Bundesärztekammer (2013): »Deklaration von Helsinki«, https://www.bundesaerz tekammer.de/fileadmin/user_upload/downloads/pdf-Ordner/International/D eklaration_von_Helsinki_2013_20190905.pdf vom 02.12.2020.

Demiris, George/Rantz, Marilyn/Aud, Myra/Marek, Karen/Tyrer, Harry/Skubic, Marjorie/Hussam, Ali (2004): »Older adults' attitudes towards and perceptions of >smart home technologies: A pilot study«, in: Med. Inform. Int. Med. 29, 87-94.

Drees, Dirk (2007): ISSE/SECURE 2007 - Securing Electronic Business Processes, Braunschweig: Vieweg. 
Fotteler Marina/Mühlbauer Viktoria/Holl, Felix/Swoboda, Walter/Dallmeier, Daria/Denkinger, Michael (2019): »The Usefulness of Assistive Technologies for Older Adults: Preliminary Results of a Systematic Literature Review«, in: 14. Jahrestagung der DGEpi, Ulm: HNU.

Fricke, Arno (2020): »Koalition bringt E-Patientenakte Richtung Versorgung«, in: InFo Hämatol. + Onkol. 9, 60-61.

Hendrich, Ann/Chow, Marilyn/Skierczynski, Boguslaw/Lu, Zhenqiang (2008): »A 36-Hospital Time and Motion Study: How Do Medical-Surgical Nurses Spend Their Time? ", in: Perm. J. 12, 25-34.

Hollier, Scott (2007): »The Disability Divide: A Study into the Impact of Computing and Internet-related Technologies on People who are Blind or Vision Impaired«, Doctoral Thesis, Faculty of Media, Society and Culture, Perth: Curtin University.

Lavander, Päivi/Meriläinen, Merja/Turkki, Leena (2016): »Working time use and division of labour among nurses and health-care workers in hospitals - a systematic review«, in: J. Nurs. Manag. 24, 1027-1040.

Nagel, Kurt/Kiefer, Erich (2008): Informationsbroschüre zum Bundesdatenschutzgesetz in der Fassung der Neubekanntmachung vom 14. Januar 2003 mit den Änderungen vom 22. August 2006, Berlin: De Gruyter.

Nöthlichs, Matthias/Weber, Horst Peter (2003): Sicherheitsvorschriften für Medizinprodukte, Berlin: Erich Schmidt.

Paul, Gerd/Stegbauer, Christian (2005): »Is the digital divide between young and elderly people increasing?«, in: First Monday 10.

Sweeney, Latanya (2002): »k-Anonymity: A Model for Protecting Privacy«, in: International Journal of Uncertainty, Fuzziness and Knowlege-Based Systems 5, 557-570.

Vereinigung der Bayerischen Wirtschaft (2020): »Zukunft der Pflege in Bayern«, h ttps://www.wifor.com/de/studien/ vom 02.12.2020.

Voigt, Paul/von dem Bussche, Axel (2018): EU-Datenschutz-Grundverordnung (DSGVO), Berlin, Heidelberg: Springer. 



\section{Altern und Verletzlichkeit: Gero-Technologien als Bestandteil einer therapeutisch-rehabilitativen Dyade?}

Hartmut Remmers

\section{Einleitung}

Aus unterschiedlichen Gründen, beispielsweise Einbußen körperlicher und kognitiver Funktionen infolge akuter oder chronischer Erkrankungen, benötigen ältere Menschen in wachsendem Umfang medizinisch-pflegerische Maßnahmen der Rehabilitation. Die Entwicklung zahlreicher Gero-Technologien zielt auf die Unterstützung dieser Maßnahmen. Es stellt sich die Frage, inwieweit dafür vorgesehene technische Systeme wie etwa Roboter jenseits rein verhaltensbezogener Funktionalitäten den Anforderungen eines als interpersonale Einheit verstandenen therapeutisch-rehabilitativen Geschehens gerecht werden können. Zur Beantwortung dieser Frage soll nach einer Skizzierung einschlägiger Technologieförderpolitik vor dem Hintergrund einer sich zuspitzenden Personalkrise in Pflegeberufen zunächst gezeigt werden, dass Alternsprozesse verschiedene Ausprägungen und Charakteristika aufweisen, die bei speziell auf diese Personengruppe ausgerichteten Technologien stärker berücksichtigt werden müssen. Daran anschließend wird auf einer zweiten Analyse-Ebene gezeigt, welche strukturellen Besonderheiten Care-Arbeit auszeichnet und welche konzeptionellen Zusammenhänge es sowohl mit einer aktivierenden als auch rehabilitativen Pflege gibt. In einem dritten Schritt werden Anforderungen an rehabilitative Maßnahmen im häuslichen Rahmen thematisiert, allerdings zunächst beschränkt auf rehabilitative Trainings, um sodann zu zeigen, dass genau darauf funktionell ausgerichtete robotische Systeme nur begrenzt geeignet sind, im Kern dyadisch strukturierte und naturgemäß eigendynamisch verlaufende und in dieser Weise höchst komplexe rehabilitative Prozesse als Care Work adäquat zu unterstützen. Damit sind der technischen Substituierbarkeit therapeutisch-rehabilitativen Handelns offenbar Grenzen gesetzt. 


\section{Rahmenbedingungen der gesundheitlichen Versorgung älterer Menschen}

\section{Politik der Technikentwicklung}

Mit ihrer Hightech-Initiative verfolgt die deutsche Bundesregierung seit bald zwei Jahrzehnten ein ambitioniertes Programm der Technikförderung, in dem auch die modernen Dienstleistungsberufe, die technisch-gewerblichen ebenso wie die personenbezogenen, eine bedeutende Rolle spielen. Unter der Annahme, dass vor dem Hintergrund eines tiefgreifenden demografischen Wandels mit dem Aufbau technischer Rationalisierungsreserven sich in Zukunft zuspitzende Personalengpässe eher bewältigen lassen werden und auch auf diesem Wege eine sich schon jetzt abzeichnende pflegerische Versorgungkrise gemildert werden könne, sind beträchtliche Finanzmittel in diesen Bereich geflossen. ${ }^{1}$ Bis heute aber blieben dabei zwei Fragen unbeantwortet: Was hat sich bislang in der Technikförderung als zweckdienlich erwiesen und praktisch bewährt?

Diese Fragen stellen sich auch mit Blick auf den ebenso beachtlichen Förderbereich der Gero-Technologien, denen angesichts des demografischen Wandels eine nicht minder große Bedeutung zukommt. Diesbezüglich stellt sich eine weitere Frage sehr grundsätzlicher Natur, die deswegen zu beantworten ist, weil damit die Sinnhaftigkeit der zu entwickelnden technischen Artefakte jenseits vordergründig funktionalistischer Erwägungen thematisiert wird: Von welchen Vorstellungen und Wünschen lassen sich Mitglieder unserer Gesellschaft im Hinblick auf ein gutes Leben im fortgeschrittenen und hohen Alter leiten und welche höchst persönliche Bedeutung hat für sie ein menschenwürdiges Altern (vgl. u.a. die Beiträge in Kruse/Rentsch/Zimmermann 2012). Erst mit Beantwortung dieser Fragen wären Prämissen formulierbar für eine an umfassenden und nicht bloß punktuellen Bedarfen orientierte Technikförderpolitik.

Im Gegensatz dazu verlief die Förderung sogenannter Gero-Technologien eher klassisch >pfadabhängig`: das heißt nach Maßgabe von Systemimperativen ökonomischen Wachstums als Innovationstreiber (Ropohl 2009). Es zeigt sich, dass Forschungs- und Entwicklungsprogramme, die primär der gesundheitlichen Versorgungssicherheit im Alter dienen sollen, mit Förderinteressen von HightechIndustrien in auffälliger Weise verwoben sind. Das wäre kein beklagenswerter

1 Die in hohem Maße auf alters- beziehungsweise pflegebezogene Technikentwicklung ausgerichtete Förderlinie des BMBF firmiert unter dem Titel »Mensch-Technik-Interaktion«. Der Anteil dieser Förderlinie am gesamten BMBF-Haushalt des Jahres 2018 beträgt 1,24 Prozent. Dieser Anteil erscheint auf den ersten Blick eher als marginal, gemessen am Gesamtjahreshaushalt des BMBF von 17,6 Milliarden Euro. Näher besehen ist für diese besondere Förderlinie eine jährliche Soll-Stellung von 80 Millionen Euro vorgesehen. Inzwischen dürfte die Summe aufgrund der Förderung überregionaler Pflegeinnovationszentren angewachsen sein. 
Zustand, wenn nicht diese Interessen in Führung gingen. Das aber ist der Fall, wenn Vertreter*innen der Hightech-Branche routinemäßig an prominenter Stelle in die politische Agenda der European Commission (2010) einbezogen werden, während Vertreter*innen von Interessenorganisationen älterer Menschen oder verschiedener Caring Professions dort nicht anzutreffen sind. Bislang bemaßen sich daher auch Erfolgskriterien technisch unterstützten Handelns (Kornwachs 2013) eher am technisch-gewerblichen Produktions- beziehungsweise am kaufmännischen Distributionssektor als an einem Bewertungssystem, mit dem qualitativen Anforderungen an persönliche Gesundheitsdienstleistungen vor allem unter Bedingungen menschlicher Fragilität und Verletzlichkeit Rechnung zu tragen ist (Remmers 2020a). Technikentwicklung wies bis dahin eine bestimmte Richtung auf, die sich als abhängig von wirtschaftlichen Verwertungsmöglichkeiten neuer technischer Produkte erweist und damit auch als das Resultat beschleunigter Kapitaldynamik zu betrachten ist (Kornwachs 2013 gestützt auf die Systemtheorie der Technik von Ropohl 2009). Mit technologischen Innovationen lassen sich bis dahin verborgene ökonomische Rationalisierungsreserven erschließen (Nemet 2009), indem kostenökonomisch teure Arbeitskraft apparativ - teilweise - ersetzt werden kann, wenn beispielsweise maschinelle Investitionskosten signifikant unterhalb dadurch eingesparter Arbeitskosten liegen.

Dieser Zusammenhang liegt unmittelbar auf der Hand, wenn man sich $\mathrm{Zu}$ kunftsszenarien eines sich fortlaufend verringernden Reservoirs an Pflegefachpersonen deutlich macht, den zu kompensieren das Ziel einer technologischen Rationalisierung pflegerischen Handelns, vor allem damit verbundener Arbeitsabläufe, ist. Und auch die Entwicklung von Gero-Technologien in Verbindung mit Weiterentwicklungen im Umkreis von Smarthome und verwandten Technologien zielt in vielerlei Hinsicht auf eine Substituierbarkeit (professioneller) Betreuungspersonen unter dem plakativen Vorzeichen eines dadurch zugleich zu erreichenden höheren Grads an autonomer Lebensführung. Es wird sich zeigen, dass mit diesen Zielen und ihrer Operationalisierung in bestimmten technischen Entwicklungsprodukten möglicherweise konzeptionelle Verkürzungen verbunden sind: zum einen eine Verkennung dessen, was Alter und Altern in ihren vielfältigen Ausprägungen bedeuten, zum anderen eine Verkennung dessen, was Care Work, speziell pflegerisches Handeln, auszeichnet. Es ist nicht zu erwarten, dass dem zunehmenden Fachkräftemangel mit dem Instrument der Technikentwicklung effektiv begegnet werden kann. Unzureichend erscheint es auch, Anforderungen an die Technikentwicklung am Leitfaden verhaltensbezogener Funktionalitäten erfüllen zu können, weil dabei die Dimension körperlich-leiblichen Erlebens, die in einer pflegerischrehabilitativen Dyade eine bedeutsame Rolle spielt, verkannt wird. 


\section{Fachkräftemangel im Berufsfeld Pflege}

Fortgeschrittenes Alter ist in vielen Fällen mit Pflegebedürftigkeit assoziiert. Die gegenwärtig bekannte Zahl pflegebedürftiger Menschen beläuft sich für das Jahr 2018 auf 3.685.389 (BMG 2019). Dabei handelt es sich um einen Anstieg von 346.210 Personen oder 9,4 Prozent gegenüber 2017. Im Vergleich mit der Zahl von Pflegebedürftigen für 2016 mit 2.749.201 zeigt sich ein bemerkenswerter Anstieg um 25,5 Prozent, der auf die Umstellung auf Pflegegrade mit einem neuen Bemessungssystem zurückzuführen ist. Die zu verzeichnende Zunahme der stationären Versorgung gegenüber 2017 von nur 0,2 Prozent ist sehr gering, während die $\mathrm{Zu}$ nahme der ambulanten Versorgung von etwa 13,4 Prozent gegenüber 2017 bemerkenswert groß ist und auch strukturell erwünscht. Allerdings ist bei dieser Zunahme eine wachsende Zahl ausländischer informeller Pflegekräfte und Haushaltshilfen $\mathrm{zu}$ berücksichtigen. ${ }^{2}$ Dieser Wachstumstrend in der ambulanten Versorgung wird sich wahrscheinlich in Zukunft fortsetzen, wobei aus Gründen sozialstruktureller Veränderungen wie wachsende Mobilität beispielsweise von Angehörigen, Zunahme von Single-Haushalten, zunehmende Berufstätigkeit von Frauen (bspw. als pflegende Töchter/Schwiegertöchter) sich das Potenzial innerfamilial gewährleisteter Pflege verringern wird. Erwartungen, dass sich der absolute Anstieg von Pflegebedürftigkeit in Zukunft aufgrund wachsender Gesundheitspotenziale im Alter verlangsamt (Kompressionsthese), sollten nicht $\mathrm{zu}$ hoch geschraubt werden. ${ }^{3}$

Es handelt sich hier um einen rechtsunsicheren Markt jener ausländischen Betreuungskräfte, die gegenwärtigen Schätzungen nach in etwa 160.000 Haushalten beschäftigt sind. Aufgrund der Pendelbewegungen dürfte die Personenanzahl noch größer sein (Leiber/Rossow/Frerk 2020).

3 Unsere eher skeptische Beurteilung der an die Kompressionsthese von Fries (1980) geknüpften Hoffnungen stützt sich auf die Tatsache, dass bis dato in mancherlei Hinsicht Evidenz fehlt. Einer im Auftrag der Barmer Ersatzkasse erfolgten Studie von Niehaus (2012: 63) zufolge gibt es »empirische Beobachtungen«, die darauf hinzudeuten scheinen, »dass die Menschen bei Zunahme der Lebenserwartung eine längere beschwerdefreie Lebenszeit gewinnen. « Zu einer ähnlich vorsichtigen Einschätzung kommt Ceyer (2015). Ihm zufolge deuten Befunde bei Menschen, die ihre Cesundheit selbst einschätzen können, aber auch bei denen, die unter Multimorbidität mit gut diagnostizierbaren Erkrankungen und sogar kognitiven Beeinträchtigungen leiden, auf eine Morbiditätskompression hin. Allerdings sind aus seiner Sicht Einschränkungen geboten wegen der stark selektiven Einbeziehung bestimmter Erkrankungen und gesundheitlicher Beeinträchtigungen wie z.B. Diabetes Typ 2 und Krebskrankheiten. Zu wenig untersucht wurden bisher nicht nur komplette Krankheitsverläufe, sondern auch viele Fälle, bei denen die Todesursache nicht eindeutig zu klären war. Eine Evidenzrecherche des Departments für Evidenzbasierte Medizin und Klinische Epidemiologie der Donau-Universität Krems (Strobelberger/Grillich/Gartlehner 2012) kommt zu dem Ergebnis, dass ein Zusammenhang zwischen gestiegener Lebenserwartung und Morbiditätskompression aufgrund der Qualität der gefundenen Studien, unterschiedlicher verwendeter Messgrößen und unterschiedlicher regionaler Gegebenheiten nicht eindeutig nachzuwei- 
Die Anzahl pflegebedürftiger Menschen mit unterschiedlichen Graden der Pflegebedürftigkeit gibt Auskunft über den Bedarf an Pflegeleistungen, die familiär durch Angehörige, Freund*innen sowie andere vertraute Personen oder durch professionelle Dienste erbracht werden können. Dem wachsenden Bedarf stehen indessen zwei strukturell bedeutsame Trends entgegen: ein langjähriger, erst jüngst gestoppter Stellenabbau in der krankenhausbasierten Akutpflege sowie wachsende Rekrutierungsprobleme qualifizierter Fachpersonen in der ambulanten und stationären Langzeitpflege. Der sich ausdehnende Fachkräftemangel berechtigt von einer Krise historisch überragend weiblich besetzter Care-Berufe zu sprechen.

Neben sehr ernst zu nehmenden beruflichen Motivationsproblemen erwachsen aus dem zu erwartenden Rückgang des Pflegepersonals aus Gründen der demografischen Alterung der Gesellschaft und des damit zusammenhängenden Ausscheidens derzeit Beschäftigter weitere Rekrutierungsprobleme. Einer von der FriedrichEbert-Stiftung in Auftrag gegebenen Studie zufolge wird auf der Grundlage einer Status-quo-Prognose die Anzahl von Beschäftigten in Pflegeberufen um 59.000 Vollzeitäquivalente (VZÄ) auf 645.000 VZÄ im Jahr 2030 sinken (Ehrentraut et al. 2015: 12). Dabei wird von einer proportional zur altersgleichen Bevölkerung konstanten Ausbildungsquote ausgegangen.

Demgegenüber wird angenommen, dass die demografische Entwicklung sowie Auswirkungen des Pflegestärkungsgesetzes II voraussichtlich einen zusätzlichen Bedarf an Pflegefachpersonen von 406.000 VZÄ für das Jahr 2030 erzeugen könnten. Darüber hinaus könnte neben dem demografischen Wandel die zunehmende Erwerbstätigkeit von Frauen zusätzlichen Ersatzbedarf in der informellen Pflege von 52.000 VZÄ mit sich bringen. Die Studie, die den leicht ansteigenden Anteil von Männern in der Pflege ebenso wie (schwer einschätzbare) Auswirkungen von Migration unberücksichtigt lässt, bilanziert insofern einen Gesamtbedarf an beruflichen Pflegepersonen für 2030 in Höhe von 1,2 Millionen VZÄ, der bei fortgesetztem Trend ohne politisch wirksame Eingriffe eine Lücke an beruflichen

sen ist. Als eines der zentralen Probleme wird die Konstruktvalidität und Messbarkeit einer Kompression von Morbidität erachtet. Ähnlich argumentierten bereits Crimmins/BeltránSánchez (2011). Auf Basis eines breit angelegten Reviews zu Morbiditäts- und Mortalitätstrends in den USA kommen sie zu dem Ergebnis, dass die vorliegenden Daten die These der Kompression nicht unterstützen, wenn Morbidität als Haupterkrankung mit körperlichem Mobilitätsverlust gesehen wird: »Our empirical example showed that in recent years, there has been an expansion of life with disease and mobility functioning loss. Our results are quite different from other analyses of changes in healthy life because our definition of healthy life differs. It is, however, a definition we feel is more in concert with the idea of compression of morbidity. When mortality declines because people survive longer with a disease rather than because people were less likely to get a disease, there will be an expansion of disease morbidity.«(Crimmins/Beltrán-Sánchez 2011: 83) 
Pflegepersonen von 44 Prozent oder absolut 517.000 VZÄ aufweisen wird. Eine etwas ältere Studie von Pohl (2011) kommt zu ähnlichen, quantitativ leicht darunter liegenden Ergebnissen unter Berücksichtigung einiger Produktivitätsfaktoren wie: besserer qualifikationsbezogener Personalmix, besserer Einsatz technischer Hilfsmittel, Verbesserung der Organisation und Abläufe sowie neue Versorgungs- und Wohnformen.

$\mathrm{Zu}$ den Anstrengungen, den zukünftig sich verschärfenden Pflegepersonalmangel - wenigsten teilweise - zu kompensieren, gehören die Entwicklung und Erprobung neuer Assistenztechnologien, mit denen zum einen beruflich hohe physische und nervliche Beanspruchungen reduziert (Gliesche et al. 2020), zum anderen mit Blick auf ältere Menschen ein selbstbestimmtes Leben auch bei eingeschränkter Selbständigkeit und Pflegebedürftigkeit gewährleistet werden soll (BMFSFJ 2020). Nach technischen Lösungen eines sozialen Versorgungsproblems $\mathrm{zu}$ suchen, darf als eine an Pflichten grenzende gesamtgesellschaftliche Aufgabe betrachtet werden, die allerdings auch die Gefahr einer Problemverschiebung in sich birgt angesichts kaum überzeugender Initiativen, den Pflegeberuf (nicht nur monetär) attraktiver zu machen. Daher darf das Potenzial dieser Lösung nicht überschätzt werden (Neven 2015). Als fachlich unstrittig gilt, dass durch moderne Technologien persönliche Zuwendung nicht ersetzt werden kann oder darf (Barnard 2016; Deutscher Ethikrat 2020; Hülsken-Giesler/Remmers 2020; Remmers 2019). Dies gilt insbesondere hinsichtlich der Pflege älterer Menschen und ihrer Charakteristika, die im Folgenden ausführlicher thematisiert werden sollen.

\section{Alternsprozesse}

\section{Vorbemerkungen zur Pathophysiologie des Alterns}

Alternsprozesse bergen eine erhöhte Wahrscheinlichkeit des Auftretens pathologischer Zustände. Vermindert sind die organische Adaptionsfähigkeit und die Aufrechterhaltung innerer Homöostase. Typische Alterskrankheiten sind: Arteriosklerose, Arthrosen, Osteoporose, Lungenemphysem, pathologische Erhöhung des systolischen Blutdrucks, Veränderungen des Immunsystems mit der Folge beispielsweise einer Fehlregulierung der Bildung von Blutzellen, aber auch von Krebserkrankungen. Nicht vergessen werden sollte die erhöhte Wahrscheinlichkeit einer Demenz. Bei zunehmendem Alter mit Nachlassen sensorischer Funktionen häufen sich Stürze mit dem Risiko der Langzeitpflegebedürftigkeit (von Renteln-Kruse/Krause 2004). Bei Multimorbidität kommt es zu einer Überlagerung vielfältiger Symptome, die besonders belastend sind. Auch verstärkt sich im Alter aus physiologischen Gründen die Empfindlichkeit gegenüber Me- 
dikamenten. Schließlich tritt Gebrechlichkeit (Frailty) bei hochgradig reduzierter Reservekapazität und erhöhter Vulnerabilität des alten Menschen auf (Fried et al. 2001). In solchen kritischen Lebenslagen stellen sich entscheidende Fragen des personalen Selbstverständnisses im existenziellen Horizont der biografischen Entwicklung. Der Belastungscharakter solcher Situationen kann möglicherweise zu einer persönlichen Umorientierung zwingen.

Ältere und alte Menschen erleben sich im Zuge fortschreitender Einschränkungen vermehrt als verletzbar und ihr Leben als vergänglich. Eintretende Pflegebedürftigkeit bedeutet für sie Abhängigkeit. Kuhlmey (2009) beschreibt sie als einen Zustand höchster körperlicher, psychischer und sozialer Vulnerabilität. Einschränkungen individueller Selbstständigkeit müssen jedoch nicht zwangsläufig Einschränkungen der Fähigkeit eines selbstbestimmten Lebens zur Folge haben. Allerdings kann die Wahrnehmung und Durchsetzung von Selbstbestimmung durch wachsende Erfordernisse der Rücksichtnahme auf Wünsche, Bedürfnisse und anderweitige Verpflichtungen der Pflegepersonen eingeschränkt sein. Manifeste Einschränkungen erleben pflegebedürftige Menschen im Bereich der Alltagsverrichtungen wie Duschen, Waschen, An- und Ausziehen, Toilettennutzung, Nahrungsaufnahme. Einschränkungen können auch instrumentelle Aktivitäten umfassen wie Einkaufen, Reinigungsarbeiten, Zubereitung von Mahlzeiten oder die Regelung finanzieller Angelegenheiten. Die Betroffenen versuchen über eine lange Zeit mit hohem Aufwand, Autonomie durch selbstständige Bewältigung gesundheits- und altersbedingter Probleme $\mathrm{zu}$ bewahren. Dennoch münden viele Anstrengungen und Verläufe etwa bei Kumulation von Gesundheitsund Krankheitsproblemen in Pflegebedürftigkeit.

\section{Altern und Potenziale}

Nun hat die gerontologische Forschung gezeigt, dass das Alter in unserer Zeit nicht nur durch Einschränkungen, sondern auch durch Entwicklungspotenziale gekennzeichnet ist. Es gibt biologische sowie sozioemotionale Veränderungen im Alter, welche beachtliche Entwicklungsmöglichkeiten enthalten. Festzustellen ist, dass das Alter eigentlich ein relativ junges Phänomen ist angesichts der frühen Sterblichkeit des Menschen vor Einführung der gesetzlichen Rentenversicherung. Alterung ist aber biologisch unvermeidlich.

Freilich sind Menschen in der Lage, die mit dem Alter verbundenen Verluste auszugleichen: zum einen auf sozialer Ebene durch Neuorganisation der Lebensumwelt, zum anderen durch einen im Persönlichkeitssystem des älteren Menschen sich vollziehenden Entwicklungsprozess (Remmers 2020a: 102-103). Ältere Menschen sind in der Lage, Ziele umzustellen oder sich neue Ziele zu setzen, andere Handlungsschemata und Routinen zu entwickeln als jene, die sie in früheren Lebensjahren erworben haben. Sie sind mehr und mehr in der Lage, ihre zunehmend 
biologisch begrenzten Kapazitäten zu kompensieren. Das von Baltes/Baltes (1990) formulierte Entwicklungsmodell lautet: Selektion und Optimierung von Möglichkeiten bei gleichzeitiger Kompensation von Einbußen in anderen Funktionsbereichen.

Diese evolutionäre Selektion wirkt sich auf das Leben im Alter zum Beispiel so aus: Es werden vermehrt Fähigkeiten erworben, anderen kompetent zu helfen. Es wird ein eigener Erfahrungs- und Wissensfundus an Jüngere weitergegeben. Im Vergleich mit instrumentellen Zielen wächst zudem mit zunehmendem Alter die Bedeutung emotionaler und sozialer Ziele. Auch die Zeitperspektive verändert sich beim älteren Menschen: Das Denken ist stärker auf zukünftiges Wohlergehen anderer Menschen ausgerichtet und an Fragen, wie Bleibendes geschaffen werden kann (Tornstam 1989).

Aufgrund ihrer Erfahrungen verfügen ältere Menschen über Fähigkeiten, mit Grenzen sowohl im privaten als auch öffentlichen, beruflichen Alltag umzugehen. Solche Fähigkeiten finden größere Realisierungschancen im Alter, weil kreatives Handeln in diesem Lebensabschnitt (Kruse 2011) nicht mehr mit den für jugendliche Entwicklungsphasen charakteristischen Risiken - etwa berufliches Scheitern behaftet ist. So befähigt die Verarbeitung lebensgeschichtlicher Grenzerfahrungen zu einem gelasseneren Umgang mit Ängsten und zur nüchternen Problemanalyse. Der Bedarf neuer Informationen in Entscheidungssituationen wird kontrollierter abgeschätzt. Auch Verlusterfahrungen und sich einstellende Gefühle der Vergänglichkeit können thematisiert und damit zum Gegenstand einer >reflektierten Auseinandersetzung`gemacht werden. Unter günstigen Bedingungen lassen sich solche im Prozess des Alterns entstandenen Haltungen, dem Prinzip der Generativität gemäß, als Tugenden der Reife weitergeben.

Studien haben gezeigt, dass die Verarbeitung von Erfahrungen zu einer Neubestimmung von Lebenssinn führen kann, welcher dem Anspruch selbstbestimmten Lebens neue Bedeutung verleiht. Staudinger (2005: 753) spricht daher auch von einer >Dynamik des Lebenssinns nes $<$, das wir dann besitzen, sondern Lebenssinn ist dynamisch. Lebenssinn muss in der Auseinandersetzung mit den jeweils gegebenen Lebensumständen immer wieder neu gefunden, besser gesagt, konstruiert werden. « Altern sollte deswegen auch nicht automatisch mit einem Zuwachs reflektierter Erfahrungen, geschweige denn mit einem Zuwachs an Weisheit, gleichgesetzt werden. In den Augen Staudingers muss vielmehr der Prozess des Alterns stets neu mit Sinn erfüllt werden (ebd.).

Freilich erlaubt es die psychologische Perspektive nicht, sozialstrukturelle >Determinanten $<$ des Alterns unbeachtet zu lassen; zum Beispiel den wachsenden Einfluss sozialer Unterschiede im Alter; die zunehmende Auswirkung sozialer Ungleichheiten mit gewissen Kumulationseffekten hinsichtlich Armuts- und Gesundheitsrisiken im Alter (Richter-Kornweitz 2012). Unabhängig davon erweisen sich 
Menschen bis ins hohe Alter in bestimmten Bereichen als entwicklungsfähig. Selbst unter Bedingungen gesundheitlicher Einbußen sind sie häufig zu kreativen Leistungen in der Lage.

Erste Zwischenbemerkung Im Hinblick auf solche Potenziale des Alters stellt sich die Frage, welche Bedeutung ihnen bei der Entwicklung von Gero-Technologien zugemessen wird; inwieweit das Kreativitätsvermögen älterer Menschen bei der Entwicklung von Gero-Technologien entweder technisch-konstruktiv aufgegriffen und genutzt wird oder inwieweit es durch bestimmte konstruktive Eigenschaften technischer Artefakte stimuliert werden soll. Wirft man einen Blick auf den gegenwärtigen Entwicklungsstand, so kann man etwas überpointiert sagen: Durch Eingabe von Tastenbefehlen bei einem Roboter mit Angehörigen, Freund*innen und Anderen elektronisch in Verbindung treten zu können ist keineswegs kreativ oder kreativitätsfördernd.

\section{Altern und Verletzlichkeit}

Wurden bisher die kreativen Entwicklungspotenziale des Alters unterstrichen, so wird man sich ebenso gegenläufige Entwicklungstatsachen klarmachen müssen. Denn im hohen Alter von 80 und mehr Jahren wächst, wie bereits angedeutet, das Risiko von Mehrfacherkrankungen. Es nehmen chronisch-degenerative Erkrankungen einschließlich neurodegenerativer Erkrankungen vom Alzheimer-Typ exponentiell zu. Besonders das hohe Alter ist durch zunehmende Verletzlichkeit mit begrenzter psychischer Widerstandsfähigkeit charakterisiert (Kruse 2017: 167-272, 317-353). Verletzlichkeit ist aber auch ein Bestandteil der Grundverfasstheit des Menschen. Anders lässt sich sorgendes Verhalten gegenüber kranken, hilfe- und pflegebedürftigen Menschen nicht verstehen. Care Work gehört zur menschlichen Kultur. Sie schließt ebenso instrumentelle Praktiken der Kompensation von Einbußen, die bis zu völliger Schutzlosigkeit reichen können, ein. Darauf wird später zurückzukommen sein.

Das Alter ist jene Lebensphase, in welcher zunehmend eine Auseinandersetzung mit körperlichen, kognitiven und sozialen Einbußen stattfindet. Dabei ist der emotionalen Verletzlichkeit besondere Beachtung zu schenken. Unbestritten ist, dass bestimmte Lebensbedingungen unmittelbaren Einfluss auf den Grad und die Formen der Verletzlichkeit haben (Holt-Lunstad/Smith/Layton 2010). Verletzlichkeit kann das Ergebnis einer lebenslangen Entwicklung sein oder das Ergebnis einer aktuell gegebenen Lebenssituation, beispielsweise einer akuten Erkrankung. Dabei sollte bedacht werden, dass Individuen auch im Alter unter günstigen, teilweise sogar unter ungünstigen Lebensumständen, in der Lage sind, bestimmte Formen und Ausprägungen von Verletzlichkeit zu vermeiden, zu lindern oder in 
einer Weise zu kompensieren, dass ein weitgehend selbstbestimmtes Leben unter Bedingungen sozialer Teilhabe möglich wird.

Sozialer Teilhabe kommt damit eine große Bedeutung zu. Es ist unbestritten, dass fehlende Möglichkeiten der Teilhabe am Entwicklungs- und Wohlstandsniveau einer Gesellschaft ein entscheidender Grund für ausgeprägte Vulnerabilität sind. Allerdings reagieren Menschen auf objektiv vergleichbare Belastungen sehr unterschiedlich. Nicht immer müssen Belastungen in hohe emotionale Verletzlichkeit münden. Das zeigen Ergebnisse der Resilienzforschung, das heißt der Untersuchung komplexer Prozesse der Belastungsverarbeitung. Dabei hat sich, etwas verkürzt gesagt, herausgestellt, dass bestimmte Prozesse der Belastungsverarbeitung an die Verfügbarkeit sozialer Netzwerke und die durch sie geleistete Unterstützung gekoppelt sind (Bengel/Strittmatter/Willmann 2001; Kunzler et al. 2018) - ein Ansatzpunkt übrigens für die Entwicklung und den Einsatz technischer Assistenzsysteme.

Stets sollte bedacht werden, dass die Entstehung und Aufrechterhaltung von Resilienz verknüpft ist mit einem Zusammenspiel psychischer, sozialer und institutioneller Faktoren. Vor allem ältere Menschen sind auf ein Umfeld vertrauter beziehungsweise vertrauenswürdiger Personen angewiesen. Bei alledem zeigt sich laut Berliner Altersstudie, dass ältere Menschen auch bei Einbußen zu produktiven Anpassungsleistungen fähig sind (Lindenberger et al. 2010). Resilienz erweist sich somit als eine spezifische Form der Plastizität menschlichen Leistungs- und Organisationsvermögens (Remmers 2020a: 111). Dabei ist jedoch vor Fehlschlüssen zu warnen.

Zweite Zwischenbemerkung Alternsprozesse sind bei Zunahme von Verletzlichkeit sowie bei Abnahme an Ressourcen im hohen Alter nicht beliebig manipulierbar. Der Konstruktion technischer Assistenzsysteme liegen oftmals Fehlannahmen zugrunde. Sie sind häufig zu stark an fragwürdigen sozialen Leitbildern der Selbstoptimierung orientiert (Bröckling 2007; Reckwitz 2017). Anforderungen an technische Assistenzsysteme lauten vielmehr: Sind sie geeignet, Individuen zum Beispiel in bereits angebahnten Präventionsstrategien $\mathrm{zu}$ unterstützen und $\mathrm{zu}$ stabilisieren? Inwieweit können technische Assistenzsysteme einen Beitrag dazu leisten, ältere Menschen in seelisch-geistigen Wachstumsprozessen zu stimulieren? Inwieweit sind sie von ihrer konzeptionellen Anlage und ihrer Funktionalität her geeignet, ältere Menschen bei der Verarbeitung belastender Situationen im Zusammenspiel mit professionellen Akteur*innen zu unterstützen? 


\section{Konzeptionelle Grundlage aktivierender und rehabilitativer Pflege}

Vor dem Hintergrund einer sehr knappen Beschreibung typischer Phänomene des Alters und Alterns sollen im Folgenden strukturelle Besonderheiten von Care Work, insbesondere pflegerischer Arbeitsprozesse, dargestellt werden. Damit sollen gleichzeitig Grundlagen geschaffen werden für Anforderungen an moderne, den alten Menschen und/oder seine gesundheitliche Versorgung unterstützende Technologien.

\section{Struktureigenschaften von Care Work}

Care versteht sich als eine universale Kategorie. Sie umfasst vielfältige und vielgestaltige Tätigkeiten, Eigenschaften und Haltungen. Die konzeptionellen Grundlagen von Care sind entsprechend vielschichtig. Ausgegangen wird dabei von einer anthropologischen Grundannahme im Sinne einer condition humaine (Conradi 2001), welche besagt, dass Menschen biologisch und sozial auf Andere angewiesen sind. Demzufolge handelt es sich bei Care um eine menschliche Praxis, die mit den reproduktiven Funktionen menschlichen Lebens sowie menschlicher Lebenswelten unmittelbar verknüpft ist.

Die konzeptionellen Grundlagen von Care (vor allem hinsichtlich Verantwortlichkeit, Verlässlichkeit, Achtsamkeit, gegenseitiger Wertschätzung) weisen eine starke Affinität auf mit Anliegen und Grundgedanken einer Ethik menschlichen Gedeihens, Wohlergehens und Gelingens, welche ebenso individuelle Rechte auf soziale Grundgüter sowie Ansprüche auf Beseitigung der Voraussetzungen schweren Leidens miteinschließt (Bertolini/Arian 2020; Siep 2004). Care lässt sich auf das berufliche Leistungsspektrum der Pflege nicht einschränken, sondern umschließt alle Tätigkeiten, die auf die Rückgewinnung einer möglichst autonomen Lebenspraxis auch unter Einschränkungen in allen Lebensbereichen ausgerichtet ist. Daraus lassen sich Rechte, aber auch Pflichten zurechnungsfähiger Personen ableiten (Pauer-Studer 2006:353), aber auch Ansprüche an bestimmte Organisationsformen und an damit verbundene Verhaltensstile, welche der Tatsache jener konstitutiven Versehrbarkeit und Hilfsbedürftigkeit des Menschen zu entsprechen haben.

Darüber hinaus bedarf Care Work des Aufbaus geeigneter Organisationsbedingungen und Organisationsformen, das heißt des Auf- und Ausbaus von Care-Systemen durch eine flankierende Care Policy bei gleichzeitig dezidierter gesellschafts- beziehungsweise machtkritischer Einflussnahme professioneller Akteur*innen (Tronto 1993). Indes zeigen moderne, durch industrielle Produktion und große bürokratische Apparate gekennzeichnete Gesellschaften für den spezifischen Arbeits- und Leistungstypus von Care wenig Verständnis. Aufgrund eines stark industriellen Produktions-Apriori lassen diese Gesellschaften bislang 
ein Bewusstsein für einen ganz anderen >Produktions«-Typus, nämlich Care, vermissen.

Worin besteht nun die besondere Arbeitsform von Care? Care versteht sich im Kern als Beziehungsarbeit. Sie schließt eine Vielfalt an Tätigkeiten ein, welche Alltagsverrichtungen sind, die in ihrer Verschiedenheit jedoch miteinander kombiniert werden müssen. Entscheidend ist dabei die zeitliche Synchronisierung verschiedener Tätigkeiten: Es müssen die materiellen und instrumentellen Voraussetzungen von Versorgung organisiert werden. Gleichzeitig bedürfen die zu versorgenden Personen der leiblichen Zuwendung. Immer wieder muss zwischen dem eigenen Verhalten des Dienstleistenden und dem seelischen Befinden des Gegenüber eine affektive Balance hergestellt werden. Dies setzt ein hohes Maß mimetischen Wahrnehmungs- und Ausdrucksvermögens voraus. Care-Beziehungen sind nicht-symmetrisch strukturiert. Es fehlt ihnen die für alltägliches Handeln charakteristische Reziprozität gleichwertiger Erwartungen.

Eine damit verbundene Schwierigkeit besteht darin, dass Beziehungsarbeit an primären Bedürfnissen hilfsbedürftiger Personen orientiert ist. Diese Bedürfnisse kehren zyklisch wieder, können sich jedoch situativ wandeln. Beziehungsarbeit kann daher nicht zeitlich linear strukturiert und organisiert werden. Möglichkeiten rationaler Planung sind wegen überraschender Entwicklungen, Ungewissheiten oder auch sich wandelnder Gemütslagen gering. Care-Tätigkeiten sind nicht repetitiv, nicht jederzeit wiederholbar. Sie lassen sich deswegen auch wenig formalisieren und standardisieren. Vorrang hat das Prinzip der Individualisierung von Hilfeleistungen. Eine besondere Schwierigkeit besteht darin, dass Leistungen gleichzeitig erbracht und konsumiert werden (uno actu-Prinzip). Im Stillen eines Bedürfnisses diffundiert Care. Es fehlt meist die Anschaulichkeit eines Produkts. Hinzu kommt ein weiterer Belastungsfaktor: Care-Arbeit verlangt in vielen Fällen körperliche Nähe, die mit Scham und Peinlichkeit verbunden sein kann. Chancen der Distanzierung und des Rückzugs sind sehr gering.

Pflegebedürftige Menschen als Adressaten von Care sind vor allem jene älteren Menschen, bei denen körperliche, geistige, auch seelische Verlusterfahrungen mehr und mehr in den Vordergrund treten. Dazu zählen die Auswirkungen chronischer Erkrankungen sowie häufiger Multimorbidität. Dabei sollte aber nicht übersehen werden, dass ältere Menschen auch im Pflegeheim noch über Potenziale verfügen. Sie zu aktivieren ist eine Aufgabe von Care. Es gibt jedoch Hinweise darauf, dass einige für Caring Professions wichtige Grundhaltungen und Einstellungen wie beispielsweise Hilfs- und Hingabebereitschaft kontraproduktive Wirkungen erzeugen, wenn sie situativ unangemessen oder mit nicht-adäquaten, an Defiziten orientierten Altersbildern assoziiert sind. So haben Baltes/Wahl (1996) in einer Studie zeigen können, dass altersbezogene Stereotype im Care-Bereich dazu führen, dass Abhängigkeit erzeugt, Hilflosigkeit verfestigt und vorhandene Selbstständigkeitspotenziale ignoriert werden. In einer anderen Studie von Remmers/Ren- 
neke (2012) konnte gezeigt werden, dass Studierende in Pflegestudiengängen mit Praxiserfahrung einen Trend zu einem relativ positiven, teilweise auch idealisierten Altersbild repräsentieren. Alter wird mit persönlicher Entwicklung und persönlichem Gewinn assoziiert, weniger mit sozialen, stärker jedoch mit körperlichen Verlusten wie eingeschränkter Gesundheit oder mangelnder körperlicher Belastbarkeit. Da Selbstbilder sehr häufig auch Altersbilder beeinflussen, wurden die Studierenden auch hinsichtlich ihrer Selbsteinschätzung befragt. Dabei zeigte sich, dass positive Erwartungen der Befragten hinsichtlich der eigenen Zukunft ihre Sichtweise auf die ältere Generation ebenso positiv prägen. Älteren Menschen werden umso mehr Kompetenzen zugesprochen, je älter und beruflich erfahrener die befragten Studierenden der Pflege waren. Im Vergleich mit einer gleichzeitigen Befragung angehender Ärzt*innen zeigte sich, dass diese sich trotz positiv gewandelter Altersbilder in ihren Therapieentscheidungen sehr häufig vom kalendarischen Lebensalter ihrer Patient*innen leiten lassen (Remmers/Walter 2012).

\section{Aktivierende Pflege}

Die zunehmende Verletzlichkeit im Alter ist nicht allein auf physische, auch nicht allein auf kognitive Abbauprozesse zurückzuführen, sondern immer auch Resultat begrenzter psychischer Widerstandsfähigkeit, wie bereits vorstehend deutlich geworden sein dürfte. Mit Inkrafttreten des Zweiten Pflegestärkungsgesetzes hat nun der Ansatz einer darauf bezogenen aktivierenden Pflege eine Aufwertung und Stärkung erfahren. Entsprechende Leistungen der Sozialen Pflegeversicherung können nunmehr bei moderater Einschränkung der Alltagskompetenz (Pflegegrad 1) in Anspruch genommen werden, die für eine zusätzliche Betreuung und Aktivierung in stationären Pflegeeinrichtungen vorgesehen sind ( $\$ 43 \mathrm{~b}$ SGB XI in Verbindung mit $\$ 28$ a SGB XI). Die Einschätzung dieses Bedarfs erfolgt auf der Grundlage von sechs Modulen, mit denen das Vorhandensein beziehungsweise die Beeinträchtigung von Fähigkeiten der Selbständigkeit ermittelt werden. Alle von der Pflegekasse zu finanzierenden Leistungen beziehen sich auf die Aktivierung bestimmter alltagspraktischer Fähigkeiten. Pflegebedürftige in stationären Pflegeeinrichtungen haben damit einen individuellen Rechtsanspruch auf Maßnahmen der aktivierenden Pflege. Inwieweit entsprechende Maßnahmen in der stationären und auch in der ambulanten Altenpflege durchgeführt werden, ist nicht ganz klar. Ihre Dringlichkeit ergibt sich aber aus der Tatsache, dass im Konzept der aktivierenden Pflege zugleich präventive Anteile eine bedeutende Rolle spielen. Häufig wird in der Fachöffentlichkeit auch von saktivierend-rehabilitativer Pflege gesprochen, wobei eine Abgrenzung der jeweiligen Ausrichtung eher von leistungsrechtlicher und weniger von fachlicher Bedeutung ist, da sie vom systematischen Ansatz her vielfach ineinandergreifen, wie später zu sehen sein wird. 
In der Altenpflege wurde das ursprünglich auf Cavan et al. (1949) zurückgehende Konzept der Aktivierung im Gegensatz zu einer lange Jahre vorherrschenden Theorie des Disengagement (Cumming/Henry 1961) eingeführt. Dabei wird davon ausgegangen, dass zwischen Lebenszufriedenheit und der Höhe des Aktivitätsniveaus und der Dauer der Aktivität einer Person im Erwachsenenalter ein Zusammenhang besteht. Dieser Zusammenhang lässt sich aber nicht undifferenziert auf das Alter übertragen. Denn im Alter kommt es zu einem Verlust biografisch eingeübter Rollen und zu einer Reduzierung von Aktivitäten. Damit aber aus eigener Kraft ein aktiver und befriedigender Lebensstil erreicht werden kann, muss eine anregende Umgebung existieren oder geschaffen werden. Häufig anzutreffenden Phänomenen der Unterforderung und Langeweile alter Menschen im Pflegeheim soll daher durch aktivierende Maßnahmen entgegengewirkt werden. Dabei kann unterstellt werden, dass Aktivitäten ihre Wirkung nur dann entfalten können, wenn sie auch als sinnvoll empfunden werden. Berücksichtigt werden sollte, dass es im Leben älterer Menschen Phasen beispielsweise der Trauer, des Abschiednehmens, des intensiven Erinnerns und der Aufarbeitung lebensgeschichtlicher Ereignisse gibt, in denen sie sich berechtigterweise zurückziehen.

Den Ansatz einer aktivierenden Pflege, die vorhandenen Fähigkeiten der Patient*innen zu unterstützen und zu fördern, findet man ebenfalls im Bereich der akutmedizinischen und rehabilitativen Behandlung. Als anzustrebende Ziele gelten hier: der Abbau von Abhängigkeiten, der Rückgewinn von Selbständigkeit, Beweglichkeit und eines positiven Selbstwertgefühls sowie von Kompetenzen der Alltagsbewältigung und der Selbstversorgung. Zumindest die vollständige Übernahme von Pflege durch Andere soll vermieden werden. Nur durch eine ausreichende Bedürfnisanpassung aktivierender Maßnahmen werden Erfolge zu erzielen sein. Um Betroffene effektiv motivieren zu können, bedarf es gezielter Information und vertrauensvoller Beratung. Der für solche Leistungen erforderliche zeitliche Aufwand ist vergleichsweise hoch und stellt Anforderungen auch an die Patient*innen, die jedoch nicht überfordert werden dürfen. Deswegen sind individuelle Pflegepläne mit betroffenen Personen und ihren Angehörigen abzustimmen. Auf jene individuellen Pflegepläne wiederum ist auch die Ablauforganisation einer Stationseinheit einzustellen, um kontraproduktive Effekte einer rein funktionell ausgerichteten Versorgung nach formalisierten Zeitplänen zu vermeiden.

Alle Versuche, Elemente eines aktivierenden Ansatzes von Care apparativ aufzugreifen und technologisch vollständig oder zum Teil zu substituieren, stehen vor der Schwierigkeit, das komplizierte Interaktionsgeschehen nicht zu stören, sondern so unauffällig wie möglich zu unterstützen. 


\section{Rehabilitative Pflege}

Akute Anlässe rehabilitativer Pflege sind vor allem durch zwei Erkrankungsereignisse charakterisiert: einen den Körper in seinen integralen hirnorganischen Funktionen zutiefst beschädigenden akuten Schlaganfall und eine den Körper ebenso in seiner Integrität zutiefst beeinträchtigende onkologische Erkrankung. Für beide im Alter gehäuft auftretenden Erkrankungen gilt, dass sie die biopsychosoziale Einheit menschlichen Lebens und Erlebens im Kern berühren.

Konzentriert man sich nun auf die im Alter gehäuft auftretenden Schlaganfallereignisse, so wird man sagen können, dass die Konzepte der medizinisch-pflegerischen Rehabilitation einen inzwischen klinisch gut evaluierten Entwicklungsstand erreicht haben. Als wegweisend für die Begründung rehabilitativer Interventionskonzepte haben sich Konstrukte der hirnanatomischen Plastizität und - im Umkreis der Gestalttheorie - der Aktualgenese oder Mikrogenese (Goldstein 2014 [1934]) erwiesen, die durch Befunde verschiedener Studien empirisch und methodisch bestätigt werden konnten. Sowohl bei Maßnahmen der stationären als auch der ambulanten Rehabilitation erweist sich das Konstrukt der Plastizität von Nutzen. Auch Menschen im höheren Alter verfügen über Reservekapazität, die bei kognitivem Training und bei Maßnahmen der Förderung von Selbstständigkeit eine wichtige Voraussetzung ist. Methodisch haben sich vor allem am Heidelberger Institut für Gerontologie durchgeführte Mikrolängsschnittstudien als besonders geeignet erwiesen, auch kleinste (und trotzdem stabile) Veränderungen in kognitiven und sensomotorischen, für die Selbstständigkeit älterer Menschen bedeutsamen, Fähigkeiten zu erfassen.

Durch solche Studien auf dem Gebiet der Rehabilitations- und Pflegeforschung (vgl. u.a. Becker et al. 2006; Schneider et al. 2006) konnte der Nachweis erbracht werden, dass die emotionale Befindlichkeit und die damit zusammenhängende Widerstandsfähigkeit eines Menschen einen eindeutig nachweisbaren Einfluss auf den Verlauf der bereits bestehenden chronischen Erkrankung und damit auch auf den Grad der Hilfe- oder Pflegebedürftigkeit ausüben kann. Die Ergebnisse weisen klar darauf hin, dass rehabilitative Interventionen klinisch-psychologische und psychosomatische Dimensionen einschließen müssen. Sie legen daher auch einen ganzheitlichen Ansatz (sense of coherence) des nicht anders als interaktiv vorstellbaren Rehabilitationsgeschehens nahe. Dies gilt auch für jene, der stationären Rehabilitation folgenden, rehabilitativen Versorgungsmaßnahmen im häuslichen Umfeld, die selbst bei verringertem Rehabilitationspotenzial die Aktivierung basaler sensorischer, motorischer und kognitiver Funktionen zum Ziel haben.

Erheblich zum Erfolg der rehabilitativen Pflege trägt bei, wenn diese in der unmittelbaren Nähe zum eigenen Haushalt und in enger Kooperation mit den betreuenden Angehörigen durchgeführt wird. Der Erfolg lässt sich an einer statistisch signifikanten Zunahme der Selbstständigkeit messen, die durch kognitive Maße, 
durch kommunikative Fähigkeiten, durch Aktivitäten des täglichen Lebens und durch spezifische sensomotorische Funktionen bestimmt ist. Selbstständigkeitsund kompetenzförderliche Potenziale können durch die Einbeziehung von Konzepten der Neuropsychologie erhöht werden. Es hat sich gezeigt, dass spezielle »therapeutische Maßnahmen, beispielsweise bei Depressivität, oder unterstützende Interventionen bei Kommunikationseinschränkungen [...] dem individuellen Bedarf der Patienten angepasst werden [müssen; Erg. HR]« (Becker et al. 2006: 369).

Die hier vertretene These lautet, dass ein auf lediglich verhaltensbezogene Funktionalitäten eingeschränktes rehabilitatives Training, wie beispielsweise des Gangs, die in jedem therapeutischen Akt unabweisbare Totalität des rehabilitativen Geschehens verfehlt. Jedes rehabilitative Training muss als ein solches der jeweils betroffenen Person in ihrer Totalität verstanden werden. Allein schon die Bedeutsamkeit psychosomatischer Aspekte der Rehabilitation, das Zusammenspiel geistig-seelischen und physischen Ausdruckserlebens einer Person spricht dafür, das rehabilitative Geschehen als ein zwischenmenschliches Geschehen der Responsivität $\mathrm{zu}$ betrachten. Ihren strukturellen Eigenschaften nach muss auch für eine in dichten, leiblich konstituierten Interaktionszusammenhängen basierte rehabilitative Einheit gelten, was Rosa (2018: 656) als die »soziale Relevanz resonanter [Herv. HR] Weltbeziehungen« akzentuiert. Auch die konzeptionellen Ansätze einer aktivierenden oder rehabilitativen Pflege beruhen letztlich auf einer Reproduktion jener "primären, basalen Bezogenheit« des Menschen, die sich in einem Wechselspiel von »Berührung und Selbstwirksamkeit beziehungsweise Spontaneität« (ebd.: 637-638f.) ausdifferenziert. Auch rehabilitative Prozesse sind strukturell in lebensgeschichtlich aufgebaute, jedoch teilweise extrem beschädigte und reduzierte »Weltbeziehungen « eingebettet. Folgt man der Sichtweise Rosas, so wäre bei allen Versuchen eines Rückgewinns verlorener Kompetenzen zu beachten, dass deren erneuter Aufbau in einem wechselseitigen Wirkungszusammenhang zwischen »leiblichen und emotionalen Regungen und Bewegungen « (ebd.: 640) einerseits und einer räumlichen, gegenständlich gestalteten Umwelt andererseits erfolgt, welche ihrerseits die Qualität einer Resonanzsphäre besitzt. Über diesen komplexen Wirkungszusammenhang lässt sich allerdings nur begrenzt technisch verfügen. In ihm spielen ebenso Sensibilitäten wie auch »Indifferenzneigungen « als habituelle Grundmodi eine disponierende Rolle, weshalb stets die Gefahr von »Entfremdung«, von Verdinglichungseffekten besteht: Es werden »eher Echoeffekte als Resonanzwirkungen erzeugt.« (ebd.: 644-645).

Zwischenergebnis Rehabilitative Prozesse gestalten sich wesentlich in dyadischen Beziehungen. Sie erfolgen in Form einer interpersonalen Einheit. Eine Isolation des rehabilitativen Geschehens etwa auf ein bloß funktionelles Training wäre insuffizient. Demgegenüber wird mit dem Prinzip der Responsivität beziehungsweise der Resonanz die Mehrschichtigkeit rehabilitativer Prozesse in Form eines 
Zusammenspiels körperlich-leiblicher, psychischer und sozial-umweltlicher Wirkungsfaktoren als Manifestation von Plastizität hervorgehoben.

\section{Rehabilitation als Training}

Bisher standen vor allem Struktureigenschaften der rehabilitativen Pflege im Zentrum der Aufmerksamkeit, wobei das besondere Augenmerk auf das rehabilitative Geschehen in Gestalt einer rehabilitativen Dyade gelenkt wurde. Im Folgenden sollen stärker die räumlichen Voraussetzungen einer auf die Häuslichkeit beeinträchtigter Personen zielenden Rehabilitation in den Blick genommen werden. Und es sollen Fragen der Abwägung jeweils als sinnhaft empfundener Installationen assistiver technischer Systeme beantwortet werden einschließlich der Funktionalitäten jener robotischen Systeme, die für Personen mit starken krankheitsbedingten Einschränkungen entwickelt wurden.

\section{Ambulantes Setting und Häuslichkeit}

Im Gegensatz zu Konzepten einer als Interaktionszusammenhang verstandenen Rehabilitation soll nunmehr von einem engeren Verständnis von Rehabilitation als Training ausgegangen werden. Mithilfe eines gezielten Trainings soll durch ein akutes Krankheitsereignis plötzlich oder durch ein chronisches Krankheitsgeschehen oder durch `natürliches` Altern langsam sich einstellenden Verlusten an geistiger und körperlicher Aktivität entgegengewirkt werden, und zwar im Sinne der tertiären Prävention als Verlangsamung durch Aktivierung. Dabei versteht sich rehabilitatives Training als Bestandteil eines Bündels medizinischer, pflegerischer und/oder physiotherapeutischer Maßnahmen zur Wiederherstellung möglichst eines Zustands vor einer akuten Erkrankung, der Wiederbefähigung zur Ausübung von Tätigkeiten des privaten und beruflichen Alltags und damit auch der Wiedereingliederung in das Alltagsleben. Bezogen auf ältere Menschen spielen Wiedereingliederungen in den Beruf eine eher marginale Rolle. Viel entscheidender sind die private, soziale und räumlich-materielle Umgebung, mit denen zum einen $\mathrm{Pa}$ rameter wiedergewonnener Fähigkeiten markiert werden, zum anderen jene konkreten Lebensbedingungen, welche verändert werden müssen, um ein möglichst selbständiges Leben nach erlebten Einbußen von Kompetenzen führen zu können.

Unter der Fragestellung, welche technischen Systeme ein möglichst selbstständiges Leben unterstützen können, spielt die häusliche Lebenssituation, insbesondere die materielle Ausstattung der Wohnung eine wichtige Rolle. Die Möblierung einer Wohnung darf dabei nicht allein unter Gesichtspunkten purer Zweckmäßigkeit, das heißt eines funktionellen Arrangements reiner Gebrauchsgegenstände mit eng umschreibbaren Funktionalitäten, betrachtet werden. Diese lassen 
sich unter Berücksichtigung beispielsweise erforderlicher Mobilitätshilfen schnell umgruppieren und neu funktionalisieren. Eine Kommode beispielsweise hat ihren angestammten Ort. Um etwa eingeschränkte Mobilität besser aufrechterhalten beziehungsweise trainieren zu können, müsste sie eventuell neu platziert oder so umgestellt werden, dass der mit ihr entstehende Eindruck einer Dysfunktionalität aufgehoben wird. Sie könnte umfunktionalisiert werden als sichere Halteposition bei zunehmender Gangunsicherheit, als Gegenstand, bei dem man als Stützhilfe kurz verweilen kann. Spätestens dann aber, wenn diese Kommode wegen eines sichereren Gangtraining in der Wohnung, möglicherweise wegen des zusätzlichen Einsatzes technischer Unterstützungsinstrumente, neu platziert werden muss, stellt sich die Frage nach der symbolischen Funktion, nach dem für die Person inhärenten und insofern funktionell indifferenten Wert eines Möbels. Auch unter eingeschränkten Bedingungen vollzieht sich das Leben einer älteren Person in einem wohnlichen Ensemble materieller Gebrauchsgegenstände, die als solche eher weniger wahrgenommen werden, vielmehr als solche, die einen anschauen, die einen lebensgeschichtlich intrinsischen (Erinnerungs-)Wert besitzen und dergestalt zu einem auratischen Lebensgefühl beitragen (Karol 2016). Die Einbettung technischer Unterstützungs- und Überwachungssysteme in die private räumliche Umgebung des älteren Menschen kann diesen Charakter eines lebensgeschichtlich hochbedeutsamen materiellen Ensembles, das mit stabilisierenden Gefühlen des Vertrautseins und der Kontinuität eines diesen Gegenständen zugeschriebenen lebendigen Erfahrungszusammenhangs assoziiert ist, erheblich stören (Johansson 2013). Die Umwandlung einer Wohnung als Lebensraum in einen durch funktionelle Sicherheitstechnologien >okkupierten Überwachungsraum mag nach Maßgabe eines vitalen Interesses an einem möglichst langen, gesicherten Leben durchaus erwünscht sein. Ob bei solchen Umwandlungen eine Wohnung für eine Person noch als >lebenswert erachtet wird, wird von der weiterhin garantierten Erfüllung ihrer persönlichen Bedürfnisse abhängen; inwieweit diese Wohnung als `barrierefrei gelten kann, von den jeweils installierten Smarthome-Technologien. Die >Adaptivität $<$ der Wohnung wiederum bemisst sich daran, wie leicht >Barrierefreiheit $<$ durch Umwandlungen erreicht werden kann bei gleichzeitigem Erhalt eines möglichst hohen Maßes nicht nur an >Lebensdienlichkeit<, sondern vor allem an >Lebenswertigkeit< (Friedman 2002; Palmer/Ward 2013).

Hier nun sind Übergänge zu einer technischen Ausstattung der Wohnung gegeben, mit der eine Art gegenständlich rehabilitative Umgebung durch Einbau multifunktioneller robotischer Mikrosysteme zur Überwachung und Unterstützung diverser Aktivitäten des täglichen Lebens (ADLs) hergestellt werden kann (Chan et al. 2008). $\mathrm{Zu}$ sprechen wäre hier von einem Ensemble robotischer Systeme, welche zum einen auf der Basis von Telepräsenzplattformen soziale Interaktionen mit der Außenwelt ermöglichen sollen (Cesta et al. 2010); zum anderen durch den Einsatz elektronischer Spiele Rückschlüsse auf gegebenenfalls vorhandene kognitive Ein- 
schränkungen erlauben, um die Teilnehmer*innen gleichzeitig durch Reduzierung kognitiver Anforderungen zu einer Rückkehr zum Spiel zu motivieren (Khaksar et al. 2016). Dabei handelt es sich um ein Programm, dessen Ziel die Reduzierung sozialer Vulnerabilität ist und mit dem ältere Menschen, welche als nur begrenzt emotional belastbar gelten, beispielsweise durch ein spezielles Warnsystem vor dem Hören sernster` Musik gewarnt und ihre betreuenden Personen aufmerksam gemacht werden sollen (Khaksar et al. 2016:449). Zu nennen sind des Weiteren technisch ebenso anspruchsvolle Systeme, welche bestimmte Fortschritte im Rehabilitationsprozess durch Detektion funktioneller Eigenschaften beispielsweise der Mobilität, aber auch weiterer Vitaldaten, in unterschiedlichen körperlichen Positionen messen, beginnend etwa mit einer einfachen eWatch (Cooper et al. 2008).

Auf einer wesentlich höheren Entwicklungsstufe finden sich Roboter, welche den Rückgewinn von Mobilität durch direkte technische Intervention aktiv fördern, beispielsweise durch ein an zeitlich variierende Präferenzen der Nutzer*innen anknüpfendes und auf diese Weise motivierendes System der Instruktion körperlicher, aber auch geistiger Übungen (Gamification) (Chong Li et al. 2014; Fasola/Matarić 2012), durch Unterstützung von Arm- oder Beinbewegungen (Interactive Motion Technologies), welche den Muskelaufbau von Extremitäten stimulieren oder durch einen schienenmontierten Roboterarm, der es ermöglicht, manuell kontrollierte Bewegungen wieder auszuführen (Beasley 2012; Ceccarelli 2011; Kahn et al. 2006). Dabei sollen sich, was die Innervation und Mobilisierung oberer Extremitäten betrifft, solche Lösungsangebote als effektiv erwiesen haben, welche in einer Kombination spezifischer Trainingsaufgaben mit Lernangeboten bestehen, die ihrerseits an persönlichen Präferenzen auszurichten sind und auf diese Weise zu einem besseren >Selbstmanagement < führen (Matarić et al. 2009). Auch lässt der gegenwärtige Entwicklungsstand offenbar stärker individualisierende Strategien technischer Unterstützung des Trainings zu durch kontinuierlichen Abgleich mit aktuellen Daten von Mobilitäts- sowie willkürlichen exekutiven Funktionen nach dem Konzept des assist-as-needed (Blank et al. 2014). Hinzu kommen speziell für die Mobilisierung und Bewegungsunterstützung von Schlaganfallpatient"innen entwickelte tragbare Orthesen, die ihnen ein Gangtraining, das Steigen von Treppen und weitere rehabilitative physische Übungen ohne zusätzliche Hilfe durch weitere Personen erlauben (Dahl/Boulos 2014). Bei nicht mehr gegebenen Möglichkeiten des Rückgewinns von Mobilität bieten sich inzwischen technisch weit fortgeschrittene, mit Monitoren auch zur ständigen Datenübertragung ausgestattete Rollstühle an (Cooper et al. 2008).

Tatsächlich gibt es Hinweise dafür, dass durch den Einsatz robotischer Systeme der Erfolg rehabilitativer Maßnahmen in der Weise gesteigert werden kann, dass Patient*innen eine größere Bereitschaft und ein gesteigertes Engagement der Teilnahme an klinischen Übungsprogrammen zeigen (Chong Li et al. 2014; Winkle et al. 2018). Auch spielt der Einspareffekt von Personalkosten eine keineswegs un- 
tergeordnete Rolle (Pepito/Locsin/Constantino 2019, S. 453) angesichts der für Rehabilitationsmaßnahmen im Bereich der Mobilität erforderlichen größeren Therapieteams. Die Langzeiteffekte einer durch Roboter unterstützten Therapie werden als vergleichbar angesehen mit denen konventioneller Interventionen (Cooper et al. 2008). Über das ursprünglich klinische Setting hinaus hat die medizinische Robotik immer mehr Zugang auch zu außerklinischen Anwendungen nicht nur im Bereich der häuslichen Rehabilitation, sondern auch im Bereich der beruflichen Arbeit gefunden.

\section{Neuro-Rehabilitation im klinischen Setting}

Autonome Systeme im Bereich der Neuro-Rehabilitation sind speziell dem Berufsfeld Pflege nur eingeschränkt zuzuordnen. Allerdings werden aufgrund zunehmender Verflechtungen pflegerisch-therapeutischer Aufgaben die professionellen Grenzen zwischen Pflege respektive Care und den klassischen Therapieberufen wie etwa der Physiotherapie zusehends porös - bei weiterhin fortbestehendem Anspruch originärer Qualifikationen. Nachstehend werden Technik-Entwicklungsprojekte benannt, die auf eine verbesserte Mobilität beeinträchtigter Menschen abzielen (Hülsken-Giesler/Remmers 2020): Das im Projekt REHATHESE (2018) entwickelte robotische System ist auf orthopädische Rehabilitation der unteren Extremitäten durch aktive, sensorgesteuerte Orthesen ausgerichtet. Auf Basis einer Analyse der Bewegungs- und Belastungsmuster lassen sich Veränderungen im Gangverhalten oder kritische Situationen identifizieren und somit Therapien individualisieren. Mit dem aus dem Projekt MOPASS (2015) hervorgegangenen Roboter wurde ein individuell angepasstes Gangrehabilitationssystem entwickelt, mit dem >passives Gehen` auf Laufbändern ersetzt und Risiken von Stürzen bei Schrittübungen ausgeschlossen werden können. Das im Projekt RECUPERA Reha (2017) entwickelte selbsttragende Ganzkörper-Exoskelett dient der robotischen Oberkörperassistenz. Es soll »kinematisch annähernd den gesamten Bewegungsraum des menschlichen Körpers erfassen und energieautark agieren können.« (Ebd.) Auch der im Projekt ROREAS (2016) entwickelte Roboter gehört zur Gattung der Therapieroboter. Er dient Schlaganfallpatient*innen zur Unterstützung des Gangtrainings in Einrichtungen der Rehabilitation und gleichzeitig als Erinnerungs- und Navigationshilfe. Zu Demonstrationszwecken existiert ein knapp fünfminütiges Video, das stark auf Produktwerbung ausgerichtet ist. Den Bekundungen des Klinikleiters zufolge soll der Roboter Therapeut*innen oder Pflegefachpersonen nicht ersetzen (Remmers 2020b).

In der filmischen Demonstration von ROREAS ist keine persönliche Ansprache des Patienten erkennbar. Er wirkt durchgehend niedergeschlagen, leicht indifferent, was möglicherweise als eine Reaktion auf eine (gegebenenfalls schlaganfallbedingte) Mobilitätseinschränkung gedeutet werden könnte. Der einseitigen An- 
sprache durch den Roboter vermag der Patient lediglich durch standardisierte Antwortvorgaben in Form von Tastenbefehlen zu entsprechen. Auf weitere Anfragen des Roboters, inwieweit er Informationen verstanden habe beziehungsweise weitere Informationen wünsche, reagiert der Patient ohne jede innere Regung. Dem gestischen Ausdruck nach lässt sich das Verhalten als eher >pflichtschuldige< Reaktion auf standardisierte Informationen oder Anfragen interpretieren. Eine Kommunikation mit dem nach- beziehungsweise vorlaufenden Therapieroboter findet nicht statt.

\section{Kontrastierung: Struktureigenschaften einer therapeutisch-rehabilitativen Dyade}

Man wird konstatieren müssen, dass mit dem instrumentellen Einsatz eines Roboters ein adjuvantes Training ermöglicht und von einigen Patient"innen auch als willkommen empfunden wird. Vergleichende Untersuchungen zu den erzielten Wirkungen liegen nicht vor und hätten sich auch durch ein anspruchsvolles Studiendesign auszuweisen (Altenbuchner/Weber 2020). Freilich sind Besorgnisse nicht von der Hand zu weisen: Aufgrund einer immer dünner werdenden Personaldecke wird der substituierende Einsatz von Therapierobotern immer wahrscheinlicher. Aber nur dann wäre ihr Einsatz gerechtfertigt, wenn Isolationstendenzen bewusst entgegengewirkt werden würde (Deutscher Ethikrat 2020). Unbedingt müsste sich das gesamte therapeutische Team konzeptionell darüber im Klaren sein, dass das rehabilitative Geschehen nur dann effektiv sein kann, wenn die leiblich vermittelte Sphäre personalen Selbsterlebens der Patient"innen systematisch mit einbezogen wird, vor allem unter Gesichtspunkten der Plastizität hirnorganischer Funktionen, denen stets unter Berücksichtigung einer psychosomatischen Verarbeitungseinheit sensorischer Innervierungen ein geistig-seelisches Korrelat entspricht. Aus Gründen einer psychophysischen Einheit der Person ist daher ein als "Resonanzraum« (Rosa 2018) zu verstehender Interaktionszusammenhang rehabilitativer Maßnahmen bei allen Versuchen ihrer technischen Unterstützung beziehungsweise Substitution zu reklamieren - wobei die Einbeziehung sprachlicher und mimischer Kommunikation eine besondere Herausforderung darstellt, der schwer entsprochen werden kann (Wade et al. 2011).

Was besagt das auf phänomenaler Ebene? Patient"innen und Therapeut"innen bilden auf basalem Niveau physischer Kooperation eine rehabilitative Einheit. In dieser unmittelbaren Kooperation ist das Erlernen beispielsweise einer durch körperlich kontrollierte Bewegungen herzustellenden Rumpfstabilität unmittelbar rückgekoppelt an Wiederholungen ontogenetischer Erfahrungen; das heißt an elementare Empfindungen des Berührens und Berührt-Werdens, des Fühlens und Gefühlt-Werdens. Die motorische Rückgewinnung von Gang- und damit Gleichge- 
wichtsicherheit ist gekoppelt an die leibliche Präsenz eines, die eigenen Bewegungen spürbar mitvollziehenden Kooperationspartners. Die Wiederaneignung motorischer Fähigkeiten vollzieht sich auf dem Wege eines unverzichtbar mitschwingenden Verhaltens der anderen Person. Die auf diese Weise erzeugten sensorischen Stimulationen sind bei begleitender Instruktion/Kommunikation gekoppelt mit einem gleichsam automatisch mitlaufenden kognitiven Training. Die allein in Gestalt einer Dyade sich vollziehende und zu gewährleistende physische Balancearbeit kann ohne jenes intrapsychische Korrelat, ein sich gleichzeitig herstellendes mentales Gleichgewicht, überhaupt nicht erzielt werden. Es ist jene direkte physische/leibliche Kooperation der Patient*innen und der Therapeut*innen, welche überhaupt erst eine elementare Erfahrung von Selbstwirksamkeit ermöglicht. Genau aus diesem Grunde eines weitaus wirksameren emotionalen Erlebens dürften nicht nur wesentlich stabilere, sondern auch nachhaltigere Effekte eines interpersonal basierten Mobilitätstrainings zu erwarten sein. Auf einen solchen buchstäblich integrativen, gewiss nur mit aufwendigen Verfahren messbaren Rehabilitationserfolg sind auf rein funktionelle Parameter abstellende Studien bislang kaum ausgerichtet.

\section{Conclusio}

Unsere eingangs gestellte Frage lautete, inwieweit technische Systeme wie beispielsweise Therapieroboter jenseits rein verhaltensbezogener Funktionalitäten den Anforderungen eines als interpersonale Einheit verstandenen therapeutischrehabilitativen Geschehens zu entsprechen vermögen. Unsere vorläufige Antwort lautet: Fasst man originäre Struktureigenschaften von Care Work als Beziehungsarbeit ins Auge, so sind es einige (wenige) instrumentelle Routinetätigkeiten (vor allem mit physischem Belastungscharakter), die durch moderne Assistenztechnologien wahrscheinlich deutlich entlastet werden können. Das gilt a fortiori für das von uns als therapeutisch-rehabilitative Dyade akzentuierte, stark auf körperlichleibliche Gegenseitigkeit ausgerichtete Interaktionsgeschehen, das lediglich in funktionellen Anteilen eines Rückgewinns beispielsweise der Mobilität technisch unterstützt werden kann. Bei alledem ist daran zu erinnern, dass die Verletzlichkeit des zumeist alten, rehabilitationsbedürftigen und -fähigen Menschen in Abhängigkeit von seiner kognitiven und psychischen Verfasstheit einem vor allem persönlich nicht begleiteten Einsatz von Therapierobotern enge Grenzen setzt. Freilich ist die Frage einer persönlichen Begleitung technischer Interventionen nicht einmal die vorrangige; im Vordergrund steht vielmehr die Frage, inwieweit technische Hilfen so eingesetzt werden können, dass das elementar dyadisch strukturierte Rehabilitationsgeschehen in seinen vor allem Selbstvertrauen bildenden Effekten, den Erfahrungen seigenleiblich wiedergewonnener 
Selbstwirksamkeit und damit verbundenen emotionalen Stabilisierungen nicht gestört wird - zum Beispiel dadurch, dass Technik zu Ungunsten der personalen Einbettung die gesamte Aufmerksamkeit auf sich zieht.

\section{Literatur}

Altenbuchner, Amelie/Weber, Karsten (2020): »Geriatric trauma patients as research subjects in a technology-driven research project «, in: Haltaufderheide, Joschka/Hovemann, Johanna/Vollmann, Jochen (Hg.), Aging between participation and simulation. Ethical dimensions of social assistive technologies in elderly care, Berlin/Boston: de Gruyter, S. 87-103.

Baltes, Margaret M./Wahl, Hans-Werner (1996): »Patterns of communication in old age: The dependence-support and independence-ignore script «, in: Health Communication, 8, S. 217-231.

Baltes, Paul B./Baltes, Margaret M. (1990): »Psychological perspectives on successful aging: The model of selective optimization with compensation«, in: Baltes, Paul B./Baltes, Margaret M. (Hg.), Successful aging: Perspectives from the behavioral sciences, New York: Cambridge University Press, S. 1-34.

Barnard, Alan (2016): "Radical nursing and the emergence of techniques as healthcare technology«, in: Nursing Philosophy, 17, S. 8-18.

Beasley, Ryan A. (2012): »Medical robots: Current systems and research directions«, in: Journal of Robotics, 2012, S. 1-14.

Becker, Gabriele/Kruse, Andreas/Tronnier, Jutta/Roepke-Brandt, Birgit/Natus, Anna/Theissen, Helena/Wetzel, Andrea (2006): »Rehabilitationsverlauf und Nachhaltigkeit: Erste Ergebnisse einer Studie zur Rehabilitation von Schlaganfallpatienten«, in: Zeitschrift für Gerontologie \& Geriatrie, 39, S. 365-370.

Bengel, Jürgen/Strittmatter, Regine/Willmann, Hildegard (2001): Was erhält Menschen gesund? Antonovskys Modell der Salutogenese - Diskussionsstand und Stellenwert (Forschung und Praxis der Gesundheitsförderung, Band 6, erweiterte Neuauflage), Köln: Bundeszentrale für gesundheitliche Aufklärung.

Bertolini, Andrea/Arian, Shabahang (2020): "Do robots care? Towards an anthropocentric framework in the caring of frail individuals through assistive technology«, in: Haltaufderheide, Joschka/Hovemann, Johanna/Vollmann, Jochen. (Hg.), Aging between participation and simulation. Ethical dimensions of social assistive technologies in elderly care, Berlin/Boston: de Gruyter, S. 35-52.

Blank, Amy A./French, James A./Pehli van, Ali Utku/O'Malley, Marcia K. (2014): »Current trends in robot-assisted upper-limb stroke rehabilitation: Promotiong patient engagement in therapy«, in: Current Physical Medicine and Rehabilitation Reports, 2, S. 184-195. 
BMFSFJ. Bundesministerium für Familie, Senioren, Frauen und Jugend (2020): Achter Altersbericht. Ältere Menschen und Digitalisierung, Drucksache 19/21650, Berlin: Deutscher Bundestag.

BMG. Bundesministerium für Gesundheit (2019): Zahlen und Fakten zur Pflegeversicherung. https:/www.bundesgesundheitsministerium.de/fileadmin/Dat eien/Downloads/Statistiken/Pflegeversicherung/Zahlen_und_Fakten/Zahlenu-Fakten-zur-Pflegeversicherung_2019.pdf (zuletzt besucht am 26.09.2020).

Bröckling, Ulrich (2007): Das unternehmerische Selbst. Soziologie einer Subjektivierungsform, Frankfurt a.M.: Suhrkamp.

Cavan, Ruth Shonle/Burgess, Ernest W./Havighurst, Robert J./Goldhamer, Herbert (1949): Personal adjustment in old age, Chicago: Science Research Associates.

Ceccarelli, Marco (2011): »Problems and issues for service robots in new applications«, in: International Journal of Social Robotics, 3, S. 299-312.

Cesta, Amedeo/Coradeschi, Silvia/Cortellessa, Gabriella/Gonzales, Javier/Tiberio, Lorenza/Von Rump, Stephen (2010): Enabling social interaction through embodiment in ExCITE. https://www.researchgate.net/profile/Silvia_Corade schi/publication/228963861_Enabling_Social_Interaction_Through_Embodim ent_in_ECITE/links/ofcfd5077bb4796d50000000.pdf (zuletzt besucht am 14 . 10.2020).

Chan, Marie/Esteve, Daniel/Escriba, Christophe/Campo, Eric (2008): »A review of smart homes - Present state and future challenges«, in: Computer Methods and Programs in Biomedicine, 91, S. 55-81.

Chong, Li/Rusák, Soltán/Horváth, Imre/Linhong, Ji/Yuemin, Hou (2014): Current status of robotic stroke rehabilitation and opportunities for a cyber-physically assisted upper limb stroke rehabilitation. https://www.researchgate.net/pu blication/262764024_CURRENT_STATUS_OF_ROBOTIC_STROKE_REHABIL ITATION_AND_OPPORTUNITIES_FOR_A_CYBER-PHYSICALLY_ASSISTED _UPPER_LIMB_STROKE_REHABILITATION/download (zuletzt besucht am 20.11.2020).

Conradi, Elisabeth (2001): Take Care. Grundlagen einer Ethik der Achtsamkeit, Frankfurt/New York: Campus.

Cooper, Rory A./Dicianno, Brad E./Brewer, Bambi/LoPresti, Edmund/Ding, Dan/ Simpson, Richard/Grindle, Garret/Wang, Hongwu (2008):»A perspective on intelligent devices and environments in medical rehabilitation«, in: Medical Engineering \& Physics, 30, S. 1387-1398.

Crimmins, Eileen M./Beltrán-Sánchez, Hiram (2011): »Mortality and morbidity trends: Is there compression of morbidity?«, in: Journal of Gerontology: Series B, 66, S. 75-86.

Cumming, Elaine/Henry, William Earl (1961): Growing old: The process of disengagement, New York: Basic Books. 
Dahl, Torbjorn Semb/Boulos, Maged N. Kamel (2014): »Robots in health and social care: A complementary technology to home care and telehealthcare? «, in: Robotics, 3, S. 1-21.

Deutscher Ethikrat (2020): Robotik für gute Pflege. Stellungnahme. https://www. ethikrat.org/fileadmin/Publikationen/Stellungnahmen/deutsch/stellungnahm e-robotik-fuer-gute-pflege.pdf (zuletzt besucht am 26.09.2020).

Ehrentraut, Oliver/Hackmann, Tobias/Krämer, Lisa/Schmutz, Sabrina (2015): Zukunft der Pflegepolitik - Perspektiven, Handlungsoptionen und Politikempfehlungen. http://library.fes.de/pdf-files/wiso/12140.pdf (zuletzt besucht am 12.01.2021).

European Commission (2010): A Digital Agenda for Europe. https://www.bmvi .de/SharedDocs/EN/Documents/VerkehrUndMobilitaet/g7-eu-kommissiondigitale-agenda-fuer-europa.pdf?_blob=publicationFile (zuletzt besucht am 03.02.2018).

Fasola, Juan/Matarić, Maja J. (2012): »Using socially assistive human-robot interaction to motivate physical exercise for older adults«, in: Proceedings of the IEFE, 100, S. 2512-2526.

Fried, Linda P./Tangen, Catherine M./Walston, Jeremy/Newman, Anne B./Hirsch, Calvin/Gottdiener, John/Seeman, Teresa/Russell, Tracy/Kop, Willem J./Burke, Gregory/McBurnie, Mary Ann (2001): »Frailty in older adults: Evidence for a phenotype «, in: The Journals of Gerontology: Series A, 56, S. M146-M156.

Friedman, Avi (2002): The adaptable house: Designing homes for change, New York City: McGraw-Hill Professional.

Fries, James F. (1980): »Aging, natural death, and the compression of morbidity«, in: The New England Journal of Medicine, 303, S. 130-136.

Geyer, Siegfried (2015): »Die Morbiditätskompressionsthese und ihre Alternativen«, in: Das Gesundheitswesen, 77, S. 442-446.

Gliesche, Pascal/Seibert, Kathrin/Kowalski, Christian/Domhoff, Dominik/Pfingsthorn, Max/Wolf-Ostermann, Karin/Hein, Andreas (2020): »Robotic assistence in nursing care: Survey on challenges and scenarios«, in: International Scholarly and Scientific Research \& Innovation, 14, S. 257-262.

Goldstein, Kurt (2014 [1934]): Der Aufbau des Organismus. Einführung in die Biologie unter besonderer Berücksichtigung der Erfahrungen am kranken Menschen, Paderborn: Wilhelm Fink Verlag.

Holt-Lunstad, Jilanne/Smith, Timothy B./Layton, J. Bradley (2010): Social relationships and mortality risk: A meta-analytic review. https://journals.plos.o $\mathrm{rg} /$ plosmedicine/article?id=10.1371/journal.pmed.1000316 (zuletzt besucht am 15.11.2020).

Hülsken-Giesler, Manfred/Remmers, Hartmut (2020): Autonome Assistenzsysteme in der Pflege: Potenziale und Grenzen aus pflegewissenschaftlicher Sicht 
(Pflegewissenschaft und Pflegebildung, Band 17), Göttingen: Vandenhoeck \& Ruprecht unipress (Universitätsverlag Osnabrück).

Johansson, Karin (2013): "Have they done what they should? Moral reasoning in the context of translating older person's everyday problems into eligible needs for home modification services«, in: Medical Anthropology Quarterly, 27, S. 414-433.

Kahn, Leonard E./Lum, Peter S./Rymer, W. Zev/Reinkensmeyer, David J. (2006): »Robot-assisted movement training for the stroke-impaired arm. Does it matter what the robot does? « in: Journal of Rahabilitation Research and Development, 43, S. 619-630.

Karol, Elizabeth (2016): »Tangible and intangible elements of design for well-being in the home«, in: Gerontechnology, 15, S. 227-232.

Khaksar, Seyed Mohammad Ssadegh/Khosla, Rajiv/Chu, Mei Tai/Shahmehr, Fatemeh S. (2016): „Service innovation using social robot to reduce social vulnerability among older people in residential care facilities«, in: Technological Forecasting \& Social Change, 113, S. 438-453.

Kornwachs, Klaus (2013): Philosophie der Technik. Eine Einführung. München: C.H.Beck.

Kruse, Andreas (2011): Kreativität im Alter, Heidelberg: Universitätsverlag Winter.

Kruse, Andreas (2017): Lebensphase hohes Alter. Verletzlichkeit und Reife, Heidelberg: Springer.

Kruse, Andreas/Rentsch, Thomas/Zimmermann, Harm-Peter (Hg.) (2012): Gutes Leben im hohen Alter. Das Altern in seinen Entwicklungsmöglichkeiten und Entwicklungsgrenzen verstehen, Heidelberg: Akademische Verlagsgesellschaft.

Kuhlmey, Adelheid (2009): »Spezielle Versorgungsanforderungen bei älteren und alten Menschen. Im Spiegel des neuen Sachverständigengutachtens«, in: Zeitschrift für Gerontologie und Geriatrie, 42, S. 425-431.

Kunzler, Angela/Gilan, Donya/Kalisch, Raffael/Tüscher, Oliver/Lieb, Klaus (2018): »Aktuelle Konzepte der Resilienzforschung«, in: Der Nervenarzt, 89, S. 747-753.

Leiber, Simone/Rossow, Verena/Frerk, Timm (2020): Das Geschäft mit der Sorge. Unternehmen im Feld der sogenannten 24-Stunden-Pflege, Bonn: FriedrichEbert-Stiftung.

Lindenberger, Ulman/Smith, Jacqui/Mayer, Karl Ulrich/Baltes, Paul B. (Hg.) (2010): Die Berliner Altersstudie, 3., erweiterte Auflage, Berlin: Akademie Verlag.

Matarić, Maja J./Tapus, Adriana/Winstein, Carolee/Eriksson, Jon (2009): »Socially assistive human-robotics for stroke and mild TBI rehabilitation «, in: Studies in Health Technology and Informatics, 145, S. 249-62.

MOPASS (2015): Mobiles, dem Patienten angepasstes, robotergestütztes Gangrehabilitationssystem. MOPASS Schlussbericht. https://www.tib.eu/de/suchen/i d/TIBKAT:868304212/(zuletzt besucht am 21.10.2020). 
Nemet, Gregory F. (2009): »Demand-pull, technology-push, and government-led incentives for non-incremental technical change«, in: Research Policy, 38, S. 700-709.

Neven, Louis (2015): »By any means? Questioning the link between gerontechnological innovation and older people's wish to live at home«, in: Journal of Technological Forecasting and Social Change, 93, S. 32-43.

Niehaus, Frank (2012): »Kompressions- versus Medikalisierungsthese: Die monetären Auswirkungen«, in: BARMER GEK Gesundheitswesen aktuell, S. 46-66.

Palmer, Jasmine Samantha/Ward, Stephen Thomas (2013): The livable and adaptable house. https://www.yourhome.gov.au/housing/livable-and-adaptable-hou se (zuletzt besucht am 12.01.2021).

Pauer-Studer, Herlinde (2006): »Feministische Ethik«, in: Düwell, Marcus/Hübenthal, Christoph/Werner, Micha H. (Hg.), Handbuch Ethik (zweite, aktualisierte und erweiterte Auflage), Stuttgart/Weimar: Metzler, S. 352-358.

Pepito, Joseph Andrew/Locsin, Rozzano C./Constantino, Rose E. (2019): »Caring for older persons in a technologically advanced nursing future«, in: Health, 11, S. 439-463.

Pohl, Carsten (2011): »Demographischer Wandel und der Arbeitsmarkt für Pflege in Deutschland: Modellrechnungen bis zum Jahr 2030«, in: Pflege und Gesellschaft, 16, S. 36-52.

Reckwitz, Andreas (2017): Die Gesellschaft der Singularitäten. Zum Strukturwandel der Moderne, Berlin: Suhrkamp.

RECUPERA Reha (2017): Ganzkörper Exoskelett für die robotische OberkörperAssistenz. https://robotik.dfki-bremen.de/de/forschung/projekte/recupera-re ha.html (zuletzt besucht am 01.10.2020).

REHATHESE (2018): Individuelle und adaptive Orthese zur Gangrehabilitation. htt ps:/www.technik-zum-menschen-bringen.de/projekte/rehathese (zuletzt besucht am 01.10.2020).

Remmers, Hartmut (2019): »Pflege und Technik. Stand der Diskussion und zentrale ethische Fragen«, in: Ethik in der Medizin, 31, S. 407-430.

Remmers, Hartmut (2020a): »Gutes Leben im Alter. Verletzlichkeit und Reife älterer Menschen«, in: Frewer, Andreas/Klotz, Sabine/Herrler, Christoph/Bielefeldt, Heiner (Hg.), Gute Behandlung im Alter? Menschenrechte und Ethik zwischen Ideal und Realität, Bielefeld: transcript Verlag, S. 95-124.

Remmers, Hartmut (2020b): »Technical utopias - political illusions? What can we expect from autonomous assistance systems for older people?«, in: Haltaufderheide, Joschka/Hovemann, Johanna/Vollmann, Jochen (Hg.), Aging between participation and simulation. Ethical dimensions of social assistive technologies, Berlin/Boston: de Gruyter, S. 201-222.

Remmers, Hartmut/Renneke, Sandra (2012): »Altersbilder bei Studierenden pflegebezogener Studiengänge. Eine empirische Untersuchung«, in: Berner, Frank/ 
Rossow, Judith/Schwitzer, Klaus-Peter (Hg.), Altersbilder in der Wirtschaft, im Gesundheitswesen und in der pflegerischen Versorgung. Expertisen zum Sechsten Altenbericht der Bundesregierung, Wiesbaden: VS Verlag für Sozialwissenschaften, S. 251-287.

Remmers, Hartmut/Walter, Ulla (2012): "Der Einfluss von Altersbildern auf Behandlung und Pflege«, in: Kruse, Andreas/Rentsch, Thomas/Zimmermann, Harm-Peter (Hg.), Gutes Leben im hohen Alter: Das Altern in seinen Entwicklungsmöglichkeiten und Entwicklungsgrenzen verstehen, Heidelberg: Akademische Verlagsgesellschaft, S. 205-230.

Richter-Kornweitz, Antje (2012): »Gesundheitliche Ungleichheit im Alter - ein Armutszeugnis«, in: Butterwegge, Christoph/Bosbach, Gerd/Birkwald, Matthias W. (Hg.), Armut im Alter. Probleme und Perspektiven der sozialen Sicherung, Frankfurt a.M.: Campus, S. 144-160.

Ropohl, Günter (2009): Allgemeine Technologie. Eine Systemtheorie der Technik (3., überarbeitete Auflage), Karlsruhe: Universitätsverlag Karlsruhe.

ROREAS (2016): Robotic rehabilitation assistant for stroke patients. https://www.y outube.com/watch?v=5r11ZKCYMHQ (zuletzt besucht am 01.10.2020).

Rosa, Hartmut (2018): Resonanz. Eine Soziologie der Weltbeziehung (Wissenschaftliche Sonderausgabe), Berlin: Suhrkamp.

Schneider, Gudrun/Driesch, Georg/Kruse, Andreas/Nehen, Hans-Georg/Heuft, Gereon (2006): »Old and ill and still feeling well? Determinants of subjective well-being in $>60$ year olds: The role of the sense of coherence«, in: American Journal of Geriatric Psychiatry, 14, S. 850-859.

Siep, Ludwig (2004): Konkrete Ethik. Grundlagen der Natur- und Kulturethik, Frankfurt a.M.: Suhrkamp.

Staudinger, Ursula (2005): »Lebenserfahrung, Lebenssinn und Weisheit«, in: Staudinger, Ursula/Filipp, Sigrun-Heide (Hg.), Enzyklopädie der Psychologie. Entwicklungspsychologie des mittleren und höheren Erwachsenenalters, Göttingen: Hogrefe, S. 739-761.

Strobelberger, Michaela/Grillich, Ludwig/Gartlehner, Gerald (2012): Evidenzrecherche zur These der Kompression der Morbidität. Studie im Auftrag des Niederösterreichischen Gesundheits- und Sozialfonds. https://docplayer.org /21746577-Evidenzrecherche-zur-these-der-kompression-der-morbiditaet-de partment-fuer-evidenzbasierte-medizin-und-klinische-epidemiologie.html. (zuletzt besucht am 16.11.2020).

Tornstam, Lars (1989): „Gero-transcendence: A reformulation of the disengagement theory«, in: Aging. Clinical and Experimental Research, 1, S. 55-63.

Tronto, Joan C. (1993): Moral boundaries: A political argument for an ethic of care, New York/London: Routledge. 
von Renteln-Kruse, Wolfgang/Krause, Tom (2004): »Fall events in geriatric hospital in-patients. Results of prospective recording over a 3 year period «, in: Zeitschrift für Gerontologie und Geriatrie, 37, S. 9-14.

Wade, Eric/Dye, Jonathan/Mead, Ross/Matarić, Maja J. (2011): »Assessing the quality and quantity of social interaction in a socially assistive robot-guided therapeutic setting«, in: IEEE (Hg.), International Conference on Rehabilitation Robotics [Proceedings], S. 1-6.

Winkle, Katie/Caleb-Solly, Praminda/Turton, Ailie/Bremner, Paul (2018): »Social robots for engagement in rehabilitative therapies: Design implications from a study with therapists «, in: ACM/IEEE (Hg.), Proceedings of the 2018 ACM/IEEE International Conference on Human-Robot Interaction, S. 289-297. 



\section{Altersgerechte Assistenzsysteme und Nutzungshemmnisse}





\section{Die tatsächliche Nutzung digitaler Assistenzsysteme in der Altenpflege Ein Scoping Review}

Miriam Vetter und Laura Cerullo

\section{Alternde Gesellschaft und die Rolle digitaler Assistenztechnik}

Deutschland unterliegt einem stetigen demografischen Wandel, der trotz Zuwanderung junger Menschen verantwortlich ist für den wachsenden Anteil der älteren Bevölkerung. Im Jahr 2017 lebten in Deutschland rund 3,4 Millionen pflegebedürftige Menschen; davon waren 81 Prozent älter als 65 Jahre (Statistisches Bundesamt 2018: 8). Die Mehrheit aller Pflegebedürftigen (76\%) wird zu Hause von einem ambulanten Pflegedienst oder durch Angehörige gepflegt. Im Vergleich zu 2015 stieg die Zahl der Pflegebedürftigen in Deutschland um 19 Prozent (ebd.). Mit zunehmendem Alter erhöht sich die Wahrscheinlichkeit, pflegerische Betreuung zu benötigen. Die demografische Entwicklung zugunsten der älteren Bevölkerung stellt die pflegerische Versorgung bereits jetzt - und vor allem zukünftig - insbesondere durch den zunehmenden Fachkräftemangel in der Pflege vor große Herausforderungen (vgl. Frommeld et al. 2020; Scorna et al. 2021). In Zukunft könnte der Einsatz innovativer Technik dieses Problem mildern, denn »dabei kann gerade Technik die Pflegenden entlasten und so Freiräume schaffen für Zuwendung, Gespräche, Aktivierung und menschliche Wärme« (Hauck/Uzarewicz 2019: V).

Diskutiert wird der Themenkomplex Pflege und Technik im Alter sowohl im öffentlichen Diskurs der Massenmedien als auch in der wissenschaftlichen Fachliteratur. Herangezogen werden hierzu Begriffe und Termini wie Digitalisierung in der Altenpflege, Pflege 4.0, altersgerechte Assistenztechnik oder Ambient Assisted Living. In den Medien berichten beispielsweise die Süddeutsche Zeitung oder DIE ZEIT über den Einzug digitaler Assistenztechnik in die Häuslichkeit von und in Einrichtungen für ältere Menschen. Dazu gehören Artikel mit plakativen Überschriften wie »Omas schlaue Wohnung« (Ludwig 2011) oder "Der unsichtbare Pfleger« (Gast 2013). In der Berichterstattung werden dabei sowohl Chancen, wie die Entlastung der Pflegekräfte, als auch Hemmnisse, wie die Finanzierung und die damit verbundenen hohen Kosten, diskutiert. In der Fachliteratur zeichnet sich die Relevanz des The- 
menfeldes an der steigenden Anzahl der Publikationen zu Pflegetechnik in den letzten Jahren ab (Krick et al. 2019: 2). Diese Veröffentlichungen basieren jedoch meist auf der Erforschung einzelner Prototypen oder laborähnlicher Experimente (Fachinger 2017: 89). In einzelnen Studien wird die Verbreitung und Nutzung von Pflege- beziehungsweise Assistenztechnik zwar als gering eingestuft (Hilbert et al. 2018: 47), jedoch gibt es hierzu bislang keinen grundlegenden Erkenntnisstand (Scorna et al. 2021).

Der vorliegende Scoping Review fokussiert vor diesem Hintergrund die Frage: Welche Technik wird in der ambulanten und stationären Pflege älterer Menschen tatsächlich eingesetzt? Der Fragestellung liegt ein weites Verständnis von digitaler Pflegetechnik zugrunde, welches von assistiven Technologien über vernetzte Systeme bis hin zu Robotik reicht. Im Rahmen des Reviews wird zunächst ein Überblick zum Themenfeld digitaler Pflegetechnik gegeben. Anschließend werden mögliche Typisierungen der Technik präsentiert. Dem folgt die Darstellung des methodischen Vorgehens und die Präsentation der Ergebnisse, die sich vor allem auf die Verbreitung des Technikeinsatzes in der ambulanten und stationären Altenpflege konzentrieren.

\section{Digitale Pflegetechnik: Wissenschaftlicher Diskurs und Forschungsstand}

In diesem Abschnitt werden Reviews zum Themenkomplex digitale Pflege- und Assistenztechnik vorgestellt. Der Überblick zur vorhandenen zusammenfassenden Literatur dient als Einstieg in die Diskussion über Technikeinsatz und Akzeptanzfaktoren sowie über Diffusionshemmnisse von Pflegetechnik in der ambulanten und stationären Pflege. Dadurch soll der Blick auf unterschiedliche Perspektiven und Annäherungsversuche der Forschung an die praktische Anwendung digitaler Pflegetechnik geöffnet werden. Bei der Einordnung entsprechender Übersichtsarbeiten zeigt sich schnell, dass deutschsprachige Literatur, auf die im Folgenden zunächst eingegangen wird, im Vergleich zu internationalen Publikationen deutlich unterrepräsentiert ist.

Einen ersten allgemeinen Überblick der Publikationen zu Pflegetechnik liefern Krick et al. im Jahr 2019 ebenfalls in einem Scoping Review. Besonders Informations- und Kommunikationstechnologien (IKT) sowie Sensorik und Robotik sind Gegenstand der untersuchten Studien und werden darin entweder auf Akzeptanz, Wirksamkeit oder Effektivität geprüft. Krick et al. (2019) kommen zu dem Ergebnis, dass Wirksamkeitsstudien bestimmter Technologien bis dato kaum publiziert wurden, ebenso wenig wie Studien für das Setting der informellen beziehungsweise der häuslichen Pflege (ebd.: 11-12). In einem weiteren internationalen Review werden IKT, Robotik, Telemedizin, Sensoren, Medikationssysteme und Videospiele differenziert betrachtet und voneinander unterschieden. Während 
laut der darin analysierten Studien IKT oder Robotik bei oder zur Vorbeugung sozialer Isolation oder Depression eingesetzt werden können, sind viele Studien zur Telemedizin auf chronische Krankheiten fokussiert (Khosravi/Ghapanchi 2016: 21). Mit dem Blick speziell auf die ältere Generation gerichtet, stellen Cedillo et al. (2018: 64) fest, dass in der privaten Häuslichkeit vernetzte Systeme am häufigsten eingesetzt werden.

Mehrere Reviews belegen jedoch das experimentelle Design der Mehrzahl der Studien. Buhtz et al. (2018) kommen in ihrer Übersichtsarbeit zur Robotik für Menschen in der eigenen Häuslichkeit zu dem Fazit, dass die "Studien überwiegend explorativ oder pilotierend, ohne vergleichende Interventionen und von kurzer Interventionsdauer « (ebd.: 7) sind. Dieses Resümee wird auch in einem Review zu assistiven Technologien für Menschen mit Demenz bestätigt (Fleming/Sum 2014: 30). Deutlich wird dies ebenfalls in einem Review zum Thema Ambient Assisted Living (AAL) von Queirós et al. (2015). Von den 132 eingeschlossenen vernetzten Systemen befanden sich 83 in der Planungsphase, 43 in der Prototypenforschung und lediglich sechs in der Testphase. Dabei bezieht sich nur ein kleiner Teil von sechs Studien auf ältere Menschen, wobei hier wiederum der Fokus auf der Entwicklung der Technologie und weniger auf der Anwender*innenperspektive liegt (ebd.: 61-62). Eine stärkere Beachtung derer Bedürfnisse wird als Nutzerfreundlichkeit bezeichnet. Dieser Befund für eine nutzerfreundliche Technikkonzeption wird auch durch Calvaresi et al. (2017: 255) bestätigt.

Im Review von Yusif/Soar/Hafeez-Baig (2016) wurden Hemmnisse in der Akzeptanz assistiver Technologien älterer Menschen untersucht. Die häufigsten Begründungen gegen den Einsatz von Technologien sind das fehlende Vertrauen in deren Funktionalität sowie die Verletzung der Privatsphäre. Zudem wird der Einsatz als sinnlos, nicht für den täglichen Gebrauch geeignet oder als stigmatisierend bewertet (ebd.: 115). Peek et al. (2014) kategorisieren insgesamt 27 Faktoren, welche die Nutzung digitaler Technik im Privaten begünstigen oder behindern können. Gegliedert wurden sie in die sechs folgenden Themenbereiche: Bedenken hinsichtlich des Technikeinsatzes (z.B. hohe Kosten), erwarteter Nutzen, Bedarf an Technologie, Alternativen zur bisherigen pflegerischen Versorgung (wie z.B. die Entlastung der pflegenden Angehörigen), Einfluss durch nahestehende Personen und zuletzt persönliche Bedürfnisse (wie der Wunsch, in der Häuslichkeit zu altern). Der soziale Einfluss von Familienmitgliedern kann nach Angabe der Autor*innen förderlich sein, während hohe Kosten einen hinderlichen Faktor darstellen (ebd.: 241).

Alle vorgestellten Reviews haben gemein, dass sie die verschiedenen Arten digitaler Pflegetechnik aus unterschiedlichen Blickwinkeln betrachten und auf verschiedene Art und Weise kategorisieren. Der Grund hierfür liegt in dem bis dato nicht vorhandenen Konsens zur einheitlichen Typisierung der Technologien (Kopp/Schöchlin 2014: 7). Zusätzlich lassen sich Hinweise ableiten, dass sich die 
meisten Studien mit Prototypenforschung befassen. Ob diese Schlussfolgerung für den vorliegenden Beitrag ebenso gilt, wird in den nachfolgenden Abschnitten untersucht und erläutert.

\section{Typisierung altersgerechter Assistenzsysteme}

Um dem Problem der uneinheitlichen Typisierung und Kategorisierung assistiver Technologien zu begegnen, wird zunächst eine gängige Unterteilung präsentiert, bevor auf die im Forschungsprojekt DAAS-KIN gewählte Kategorisierung eingegangen wird. Fachinger unterscheidet zwischen Assistenztechnik der ersten, zweiten und dritten Generation. Der ersten Generation werden beispielsweise Sehhilfen oder einfache mechanische Hilfen wie Hebelifte zugerechnet. Die zweite Generation lässt sich durch die mögliche Interaktion von Pflegebedürftigen beziehungsweise (Pflege-)Personal mit dem technischen System charakterisieren, wie zum Beispiel mit einem Serviceroboter. In der dritten Generation werden diese Funktionen durch Vernetzung und künstliche Intelligenz beispielsweise zu AAL-Technik weiterentwickelt, die eigenständig mit der zu pflegenden Person kommunizieren kann. Fachinger veranschaulicht diese Weiterentwicklung von Technik, die teilweise parallel eingesetzt werden kann, am Beispiel des Hausnotrufs: Bei der ersten Generation musste der Hausnotruf durch eine Bewegung aktiv bedient werden. Automatisch wiedergegeben wird der Sturz an eine Dienstelle durch vernetzte Systeme der zweiten Generation. Ab der dritten Generation kann das System selbstständig reagieren und mit den Nutzer*innen interagieren (Fachinger 2017: 83-84).

Im Rahmen von DAAS-KIN wurden insgesamt sieben Kategorien für altersgerechte Assistenzsysteme entwickelt, die aus einer Online-Befragung von Führungskräften aus Pflegeeinrichtungen hervorgingen (vgl. Haug in diesem Band). Sie basieren auf AAL-Projekten des Bundesministeriums für Bildung und Forschung (BMBF) (2009) sowie des Verbands Deutscher Ingenieure (VDI/VDE) (Weiß et al. 2013):

- Kommunikation \& Entertainment

- Serviceroboter

- Pflegeroboter

- Sicherheitssysteme

- Monitoring

- Dokumentationssysteme

- Informationssysteme

Telepräsenzroboter, Videotelefonie, computergestützte Spiele oder sozial aktivierende Robotik wie die Robbe Paro zählen zur ersten Kategorie. Servicerobotik kann durch automatisches Ausführen von Haushaltsaufgaben oder Mobilitätsdiensten 
die Pflegenden oder das Pflegepersonal entlasten (Graf et al. 2013: 1145). Durch Pflegerobotik wird mittels intelligenter Pflegewagen beispielsweise die personenbezogene Pflege unterstützt. Der Kategorie Sicherheit werden Überwachungssysteme, Sturzsensoren oder Fußmatten zugerechnet. Monitoring betrifft vor allem das Überwachen von Vitaldaten. Elektronische Dokumentationssysteme sind im Gegensatz zur klassischen Papierversion softwarebasiert und werden meist mit mobilen Endgeräten ausgeführt. Geräte zur Informationsübermittlung können die Kommunikation von und mit sprachbehinderten Personen erleichtern.

\section{Methodik}

Um für diesen Beitrag eine systematische Übersicht zur erstellen und damit die Frage zu beantworten, ob und welche Technik in der ambulanten und stationären Pflege älterer Menschen tatsächlich zum Einsatz kommt, wurde ein Scoping Review (Arksey/O'Malley 2005) durchgeführt. Diese Art der systematischen Literaturrecherche bietet die Möglichkeit, Literatur in unterschiedlichen Datenbanken mittels angepasster Suchstrategien zu finden (Elm/Schreiber/Haupt 2019; Munn et al. 2018). Zur Orientierung und Strukturierung der Durchführung diente das PRISMA-Statement (Moher et al. 2009). Neben den Aspekten der eingangs genannten Forschungsfrage wurden weiterführende Auswertungen im Hinblick auf die Kategorisierungen von Technologien, das Setting (ambulant oder stationär) und die Herkunft des jeweiligen Beitrags durchgeführt.

\section{Suchstrategien}

Im Rahmen der Durchführung des Scoping Review wurden verschiedene Suchstrategien entwickelt, die an die jeweilige Suchumgebung angepasst wurden (siehe Tabelle 1). Allen gemein ist ihre Zusammensetzung aus den folgenden drei Elementen:

- Intervention: Einsatz assistiver Technologien oder digitaler Pflegetechnik.

- Setting: ambulante oder stationäre Altenpflege.

- Outcome: (tatsächliche) Nutzung assistiver Technologien oder Pflegetechnik. 
Tabelle 1: Suchstrategien in den für den Scoping Review verwendeten Datenbanken

\begin{tabular}{|c|c|}
\hline Datenbank & Suchstrategie \\
\hline $\begin{array}{l}\text { PubMed via } \\
\text { Medline }\end{array}$ & 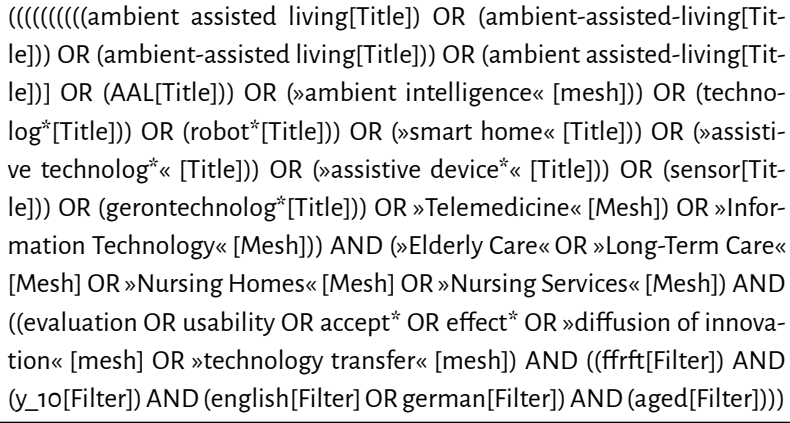 \\
\hline $\begin{array}{l}\text { CINAHL via } \\
\text { Ebsco Host }\end{array}$ & 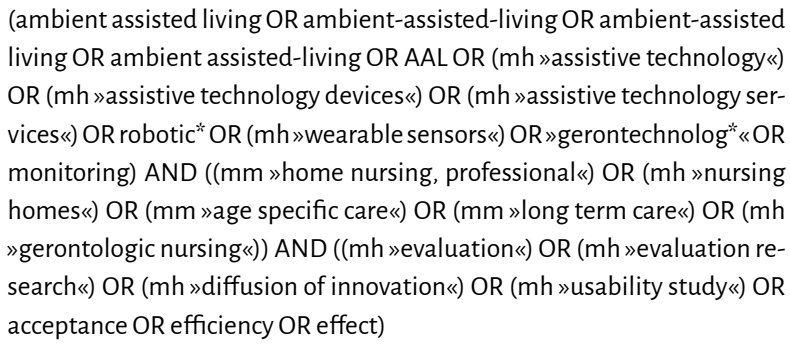 \\
\hline GeroLit & $\begin{array}{l}\text { ((Ambient Assisted Living ODER Ambient-Assisted-Living ODER } \\
\text { Ambient-Assited Living ODER Ambient Assited-Living ODER AAL) } \\
\text { ODER Techn* ODER Robot* ODER (Digital* ODER Automation) ODER } \\
\text { (Smart Home ODER Smart Living) ODER Sensor* ODER Wearable ODER } \\
\text { Assistenztechnik ODER Assist* ODER "System* ODER "Dokumentation* } \\
\text { ODER Intelligent ODER Service ODER Pflegerobot*) UND (Altenpflege } \\
\text { ODER Ambulant ODER Ambulanter Dienst ODER Altenheim ODER } \\
\text { "Pflege* ODER Pflegeheim ODER "Heim) UND (Evaluation ODER Nutz" } \\
\text { ODER Einsatz ODER "Praxis ODER Studie ODER Akzeptanz) UND jhr } \\
\text { 2010-2020 }\end{array}$ \\
\hline Google Scholar & $\begin{array}{l}\text { Altersgerechtes Assistenzsystem AND Senior* AND Altenpflege AND } \\
\text { Nutzung filetype:pdf; } \\
\text { Digital AND Technologie AND Senior* AND Altenpflege filetype:pdf }\end{array}$ \\
\hline
\end{tabular}

Quelle: Eigene Darstellung

Die einzelnen Elemente wurden durch zahlreiche Synonyme und verschlagwortete Begriffe der jeweiligen Datenbanken, das heißt der jeweiligen Suchumgebung, angepasst. Für die Recherche in der Datenbank PubMed wurden beispielsweise >Medical-Subject-Headings (MeSH-Terms) verwendet. Um die Nachvollziehbarkeit zu gewährleisten, wurde die Entwicklung der Suchstrategien, das heißt die inhaltliche Ausdifferenzierung der einzelnen Teilelemente, durch Synonyme 
und Schlagworte auf Basis der Vorlage von Hirt/Nordhausen (2020) detailliert dokumentiert. Die Recherche wurde in insgesamt vier Datenbanken durchgeführt. PubMed als medizinische Datenbank und CINAHL als Sammlung der Zeitschriftenliteratur für das Pflege- und Gesundheitswesen decken dabei den internationalen Raum ab, während GeroLit, die Datenbank des Deutschen Zentrums für Altersfragen (DZA), das deutschsprachige Pendant dazu bildet. Google Scholar wurde mittels deutscher Suchbegriffe mit zwei Suchdurchgängen vor allem nach grauer Literatur durchsucht. Die Suchbegriffe wurden hierbei lediglich in deutscher Sprache eingesetzt, da sich das Forschungsinteresse vorrangig auf den Einsatz von Pflegetechnik in Deutschland fokussiert. Zusätzlich wurden Treffer aus dem Bibliothekskatalog der Ostbayerischen Technischen Hochschule (OTH) Regensburg sowie aus Google Scholar hinzugefügt. Diese wurden bereits mittels freier Suche ermittelt, welche in Vorbereitung auf die Datenbankrecherche durchgeführt wurde.

\section{Ein- und Ausschlusskriterien}

Die mittels der Suchstrategien gefundenen Treffer wurden in drei ScreeningPhasen (Titel-, Abstract- und Volltext-Screening) anhand vorab gebildeter Kriterien (siehe Tabelle 2) ein- oder ausgeschlossen. Sämtliche Studiendesigns wurden genauso eingeschlossen wie alle Publikationsformen, wie zum Beispiel Sammelbandbeiträge oder Zeitschriftenaufsätze. Es werden Studien mit Personen ab 65 Jahren eingeschlossen, die eine pflegerische Versorgung (ambulant oder stationär) erhalten. Publikationen, in denen jüngere Erwachsene als Patient"innengruppen genannt wurden, wurden dementsprechend ausgeschlossen. Insbesondere in den Datenbanken PubMed und CINAHL verringerten sich die Trefferzahlen durch die Altersbeschränkung stark. In den Datenbanken GeroLit und Google Scholar hingegen konnte kein Altersfilter eingestellt werden. Es wurden nur Titel eingeschlossen, die sich thematisch explizit mit der Nutzung und Verbreitung digitaler Pflegetechnik oder assistiver Technologien befassen. Der Publikationszeitraum wurde aufgrund der großen Menge an Literatur zum gesamten Themenkomplex auf die letzten zehn Jahre festgelegt, wobei nur frei verfügbare Abstracts und Volltexte in deutscher oder englischer Sprache berücksichtigt wurden. 
Tabelle 2: Ein- und Ausschlusskriterien

\begin{tabular}{|c|c|c|}
\hline Domäne & Einschlusskriterien & Ausschlusskriterien \\
\hline Design & $\begin{array}{l}\text { Alle Publikationsformen (quantitative/qua- } \\
\text { litative Studien, RCTs, graue Literatur und } \\
\text { Reviews) }\end{array}$ & Keine \\
\hline $\begin{array}{l}\text { Patienten- } \\
\text { gruppe }\end{array}$ & Senior*innen ab 65 Jahren & $\begin{array}{l}\text { Jüngere Menschen unter } 64 \\
\text { Jahren }\end{array}$ \\
\hline $\begin{array}{l}\text { Interventio- } \\
\text { nen }\end{array}$ & $\begin{array}{l}\text { Digitale AAL-Technik oder assistive Techno- } \\
\text { logien }\end{array}$ & $\begin{array}{l}\text { Kein Einsatz von AAL-Technik } \\
\text { vorhanden }\end{array}$ \\
\hline Outcome & Nutzung der Interventionen & $\begin{array}{l}\text { Prototypenforschung, explora- } \\
\text { tive Studie, nur technische Ent- } \\
\text { wicklung }\end{array}$ \\
\hline Setting & Ambulante und stationäre Altenpflege & $\begin{array}{l}\text { Klinik, Arztpraxen, andere Ein- } \\
\text { richtungen }\end{array}$ \\
\hline $\begin{array}{l}\text { Publikati- } \\
\text { onsjahre }\end{array}$ & $\begin{array}{l}\text { Publikationszeitraum } \\
\text { von 01.01.2010-24.06.2020 }\end{array}$ & Publikationen vor 2010 \\
\hline $\begin{array}{l}\text { Dokumen- } \\
\text { tentyp }\end{array}$ & $\begin{array}{l}\text { Verfügbares Abstract und Volltext; } \\
\text { Sprache: englisch oder deutsch }\end{array}$ & $\begin{array}{l}\text { Kein Abstract und Volltext vor- } \\
\text { handen; andere Sprache }\end{array}$ \\
\hline
\end{tabular}

Quelle: Eigene Darstellung, angelehnt an Hirt/Nordhausen 2020

\section{Recherche}

Die Literaturrecherche erstreckte sich über vier Phasen (siehe Abbildung 1) und wurde im Juni 2020 durchgeführt. Insgesamt ergaben sich 245 Treffer, die mit einem Literaturverwaltungsprogramm gesammelt und zur weiteren Bearbeitung in Excel exportiert wurden. Nach der Entfernung der Duplikate ergab sich eine verbleibende Trefferanzahl von 238. Diese wurden anhand eines Codesystems ( $1=a k-$ zeptiert; 2 =unsicher; 3 =ausgeschlossen) von zwei Reviewerinnen in insgesamt drei Screening-Phasen voneinander unabhängig bewertet. Die als unsicher markierten Treffer wurden gemeinsam diskutiert und anschließend ein- oder ausgeschlossen. Beiträge ohne Abstract wie beispielsweise Forschungsberichte wurden in die nächste Phase aufgenommen. Am häufigsten wurden Titel aufgrund des fehlenden Bezugs zur Pflege oder des fehlenden Einsatzes von Pflegetechnik ausgeschlossen. Schlussendlich wurden elf Publikationen in die detaillierte Analyse eingeschlossen. 
Abbildung 1: PRISMA-Flussdiagramm für die Studienauswahl
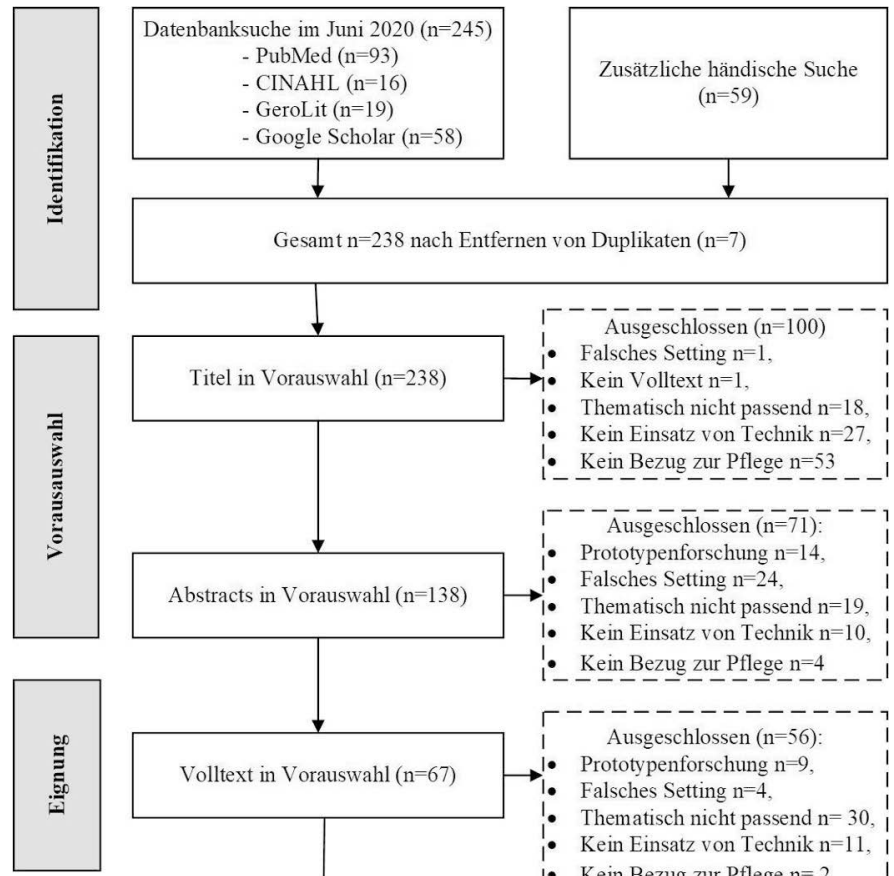

Ausgeschlossen $(\mathrm{n}=\overline{71}):--1$

- Prototypenforschung $n=14$,

- Falsches Setting $n=24$,

Thematisch nicht passend $n=19$.

1. Kein Einsatz von Technik $n=10$,

- Kein Bezug zur Pflege $n=4$

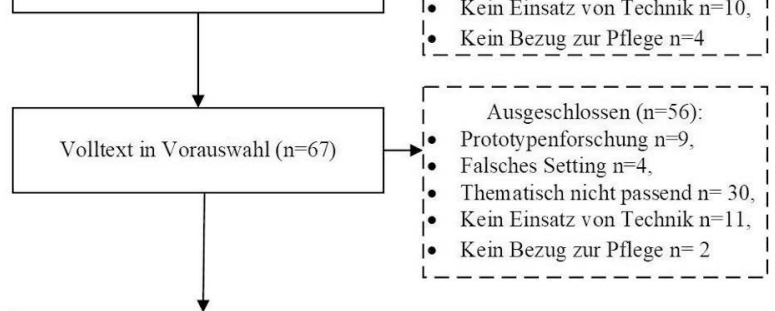

11 Artikel eingeschlossen

Quelle: Eigene Darstellung, angelehnt an Moher et al. 2009

\section{Ergebnisse}

Die zur Beantwortung der Forschungsfrage relevanten und eingeschlossenen $\mathrm{Pu}$ blikationen werden in diesem Kapitel in vier Teilabschnitten dargestellt. Zunächst stehen wesentliche Studienmerkmale im Vordergrund. Es handelt sich bei den Publikationen um unterschiedliche Studientypen: drei Zeitschriftenaufsätze, vier Projektberichte und vier Sammelbandbeiträge. Im Anschluss wird auf die in den eingeschlossenen Studien genannte Technik Bezug genommen und Faktoren vorgestellt, die gegen den Einsatz von Technik zu sprechen scheinen. Anschließend folgen Bewertungen zum Einsatz der jeweiligen Pflegetechnik. Danach werden hinderliche sowie weitere Faktoren betrachtet und im Anschluss gesondert auf die eingeschlossenen Sammelbandbeiträge eingegangen. Diese nehmen in der Ergeb- 
nisdarstellung eine besondere Rolle ein, denn es werden hier zwar Hinweise auf die Verbreitung und Nutzung digitaler Pflegetechnik gegeben, diese Bewertungen sind jedoch nicht mit statistischen Belegen gekennzeichnet. Dennoch sind sie als `hinweisgebende Quellen relevant und ein wichtiger Bestandteil der Ergebnisse.

\section{Merkmale der eingeschlossenen Studien}

Trotz der Recherche in internationalen Datenbanken stammen zehn der elf eingeschlossenen Volltexte aus Deutschland. Eine Studie wurde in der Schweiz durchgeführt (Seifert/Ackermann 2020). Letztere haben, wie einleitend geschildert, eine besondere Stellung, da sie keine klassischen Forschungsberichte darstellen, sondern als Literaturüberblick gestaltet sind (Fachinger 2017; Hilbert et al. 2018; Mielitz 2017; Wahl/Kricheldorff/Hedtke-Becker 2018). Zuletzt ist das bereits erwähnte Scoping Review zu Pflegetechnik von Krick et al. (2019) aufgenommen worden.

Bei den Projektberichten handelt es sich unter anderem um quantitative Befragungen von Mitarbeitenden aus stationären oder ambulanten Pflegeeinrichtungen (Kuhlmey et al. 2019a; Kuhlmey et al. 2019b; Merda/Schmidt/Kähler 2017; Seifert/Ackermann 2020). Ein Forschungsbericht enthält Ergebnisse der Auswertung von Beratungsprotokollen von Praxiskontakten und Workshops stationärer Altenhilfeeinrichtungen des Kuratoriums Deutschen Altenhilfe (KDA) (Sowinski/KirchenPeters/Hielscher 2013). Auch eine qualitative Befragung von Pflegepersonal zum aktuellen Technikeinsatz für ältere Menschen ist enthalten (Schlomann 2020).

Insgesamt umfassen die sechs eingeschlossenen Studien 1.731 Befragte aus der Pflege. Studienteilnehmer*innen waren bei vier der Studien Pflegemitarbeitende aus Kliniken oder Altenpflege (Kuhlmey et al. 2019a \& 2019b; Merda/Schmidt/Kähler 2017; Seifert/Ackermann 2020). Bei den Erhebungen aus dem gemischtem Setting war meist ein geringerer Anteil der Befragten Personal aus Altenpflegeeinrichtungen: Bei Merda/Schmidt/Kähler (2017: 112) sind es 41 Prozent, bei Kuhlmey et al. (2019b: 7) 14 Prozent und bei der Befragung von Seifert/Ackermann (2020: 5) 69 Prozent. Ausschließlich Personal aus der Altenpflege wurde befragt von Schlomann (2020) und Sowinski/Kirchen-Peters/Hielscher (2013). Sie richteten sich vorwiegend an die Einrichtungsleitungen (Schlomann 2020: 124; Sowinski/KirchenPeters/Hielscher 2013: 3-4).

\section{Eingesetzte Technologien}

Da über die Begrifflichkeiten der digitalen, altersgerechten Assistenztechnik kein Konsens besteht, ist von großem Interesse, welche Technik in den eingeschlossenen Publikationen eingesetzt wird. In allen Veröffentlichungen wird die Nutzung mehrerer Technologien abgefragt. Um die Darstellung in der vorliegenden Arbeit zu systematisieren, wurden die einzelnen Systeme zuerst übergeordneten Typi- 
sierungen zugewiesen, die sich an der in DAAS-KIN gebildeten und an der von Krick et al. (2019: 6) entwickelten Kategorisierung orientierten. Die zunächst sieben intern festgelegten Kategorien wurden auf fünf reduziert und zwei Rubriken (assistive Technologien und Sonstiges) aus Krick et al. stattdessen hinzugefügt. In Tabelle 3 wird deutlich, welche Typen von Technologien mit welcher Häufigkeit abgefragt wurden. In den eingeschlossenen Studien wurden durchschnittlich etwa fünf unterschiedliche Technologien aufgelistet und meist mit einer Likertskala die jeweilige Nutzung erfragt. Als Minimum mit nur vier Pflegetechniken sind hier die Studien von Merda/Schmidt/Kähler (2017) und Schlomann (2020) zu nennen. Die größtmögliche Anzahl von zehn Technologien ist bei Kuhlmey et al. (2019a, 2019b) vorzufinden.

Tabelle 3: Häufigkeiten der eingesetzten Technologien nach Publikation und Kategorie

\begin{tabular}{|l|l|l|l|l|l|}
\hline Technologie & $\begin{array}{l}\text { Kuhlmey } \\
\text { et al. } \\
2019 \\
\text { a und b }\end{array}$ & $\begin{array}{l}\text { Merda } \\
\text { et al. } \\
2017\end{array}$ & $\begin{array}{l}\text { Schlo- } \\
\text { mann } \\
\mathbf{2 0 2 0}\end{array}$ & $\begin{array}{l}\text { Seifert/ } \\
\text { Ackermann } \\
2020\end{array}$ & $\begin{array}{l}\text { Sowin- } \\
\text { ski/Kirchen- } \\
\text { Peters/Hiel- } \\
\text { scher 2013 }\end{array}$ \\
\hline Robotik & 4 & 1 & 0 & 3 & 0 \\
\hline $\begin{array}{l}\text { Sicherheits- } \\
\text { systeme }\end{array}$ & 2 & 0 & 1 & 2 & 1 \\
\hline $\begin{array}{l}\text { Monitoring } \\
\text { Assistive }\end{array}$ & 1 & 1 & 1 & 0 & 0 \\
\hline $\begin{array}{l}\text { Technologien } \\
\text { Informations- } \\
\text { und Kommu- } \\
\text { nikations-tech- } \\
\text { nologie }\end{array}$ & 1 & 1 & 1 & 0 & 1 \\
\hline $\begin{array}{l}\text { Dokumentati- } \\
\text { onssysteme }\end{array}$ & 1 & 1 & 1 & 2 & 2 \\
\hline
\end{tabular}




\begin{tabular}{|l|l|l|l|l|l|}
\hline Technologie & $\begin{array}{l}\text { Kuhlmey } \\
\text { et al. } \\
\mathbf{2 0 1 9} \\
\text { a und b }\end{array}$ & $\begin{array}{l}\text { Merda } \\
\text { et al. } \\
\mathbf{2 0 1 7}\end{array}$ & $\begin{array}{l}\text { Schlo- } \\
\text { mann } \\
\mathbf{2 0 2 0}\end{array}$ & $\begin{array}{l}\text { Seifert/ } \\
\text { Ackermann } \\
\mathbf{2 0 2 0}\end{array}$ & $\begin{array}{l}\text { Sowin- } \\
\text { ski/Kirchen- } \\
\text { Peters/Hiel- } \\
\text { scher 2013 }\end{array}$ \\
\hline Sonstiges & 0 & 0 & 0 & 0 & 1 \\
\hline Gesamt & 10 & 4 & 4 & 7 & 6 \\
\hline
\end{tabular}

Quelle: Eigene Darstellung, angelehnt an Krick et al. 2019: 9

In allen Artikeln werden assistive Technologien sowie Dokumentationssysteme berücksichtigt. Dagegen werden Robotik, Sicherheitssysteme, Informations- und Kommunikationstechnologien nicht in jeder Erhebung aufgenommen. Der Einsatz von jeglichen robotischen Systemen, Sicherheitssystemen oder Informations- und Kommunikationstechnologien wurde nicht in jeder Befragung des Pflegepersonals erfragt (siehe Tabelle 4).

Tabelle 4: Eingesetzte Systeme nach Autor*innen

\begin{tabular}{|l|l|}
\hline Publikation & Angaben zur Technologie und Nutzungshäufigkeit \\
\hline $\begin{array}{l}\text { Kuhlmey et al. 2019a } \\
\text { \& 2019b }\end{array}$ & $\begin{array}{l}\text { Hebehilfen 39\% } \\
\text { Tablets zur Dokumentation 32\% } \\
\text { Tablets zum therapeutischen Spiel und Beschäftigung 12\% } \\
\text { Sturzdetektoren 12\% } \\
\text { Erinnerungshilfen zur Medikation 5\% } \\
\text { CPS-Trackern für Demenzkranke 4\% } \\
\text { Robotik zum Materialtransport 2\% } \\
\text { Smart-Sensoren zum selbstständigen Wohnen (AAL) 2\% } \\
\text { Kuschelroboter 1\% } \\
\text { Automatische Medikamentenverteilung 1\% }\end{array}$ \\
\hline Merda/Schmidt/ & $\begin{array}{l}\text { Elektronische Dokumentation 74\% } \\
\text { Technische Assistenz 32\% }\end{array}$ \\
& $\begin{array}{l}\text { Telecare und Telemedizin 27\% } \\
\text { Robotik 21\% }\end{array}$ \\
\hline
\end{tabular}




\begin{tabular}{|c|c|}
\hline Publikation & Angaben zur Technologie und Nutzungshäufigkeit \\
\hline Schlomann 2020 & $\begin{array}{l}\text { Kaum Nutzung von digitalen Technologien wie Sensoren, Alarme, } \\
\text { Monitoring, Smartphone basierte Dokumentation und höhere Nut- } \\
\text { zung von klassischen Assistenztechnologien wie Mobilitätstechno- } \\
\text { logien, Notrufsysteme, elektronische Dokumentation, Gesundheits- } \\
\text { Monitoring }\end{array}$ \\
\hline $\begin{array}{l}\text { Seifert/Ackermann } \\
2020\end{array}$ & $\begin{array}{l}\text { Serviceroboter/Pflegeroboter/Wannenlifter } 51 \% \\
\text { Tablets } 46,9 \% \\
\text { Smartphones } 44,1 \% \\
\text { GPS-Ortung } 39,3 \% \\
\text { Videoüberwachung } 21,9 \% \\
\text { Roboter zur Aktivierung } 5 \%\end{array}$ \\
\hline $\begin{array}{l}\text { Sowinski/Kirchen- } \\
\text { Peters/Hielscher } 2013\end{array}$ & $\begin{array}{l}\text { Digitale Pflegedokumentation (sehr hoher Einsatz in der stationären } \\
\text { und ambulanten Pflege) } \\
\text { Außerklinische Intensivpflege (sehr hoch in der ambulanten Pflege, } \\
\text { mäßig im stationären Bereich) } \\
\text { Personenortungssysteme (sehr hoch im stationären Bereich, mäßig in } \\
\text { ambulanter Pflege) } \\
\text { Lifter (sehr hoch stationär, niedrig im ambulanten Bereich) } \\
\text { Bewegungsspiele wie Wii (mäßig in der stationären, niedrig in der am- } \\
\text { bulanten Pflege) } \\
\text { Animiertes Spielzeug wie Spielzeugrobbe Paro (mäßig in stationärer, } \\
\text { niedrig in der ambulanten Pflege) }\end{array}$ \\
\hline
\end{tabular}

Quelle: Eigene Darstellung

In der Befragung von Kuhlmey et al. (2019b) werden zehn Technologien in Bezug auf Kenntnis, Zugang und Nutzung, untersucht. ${ }^{1}$ Hebehilfen, die zu den assistiven Technologien gezählt werden, sind in der Alten- und Krankenpflege am häufigsten im Einsatz (39\%) (ebd.: 16). In der stationären und ambulanten Altenpflege werden sie deutlich mehr genutzt als im Krankenhaus (ebd.: 17). Auch Tablets oder Smartphones zur elektronischen Dokumentation werden mit 32 Prozent zahlreich genutzt. Diese werden in ambulanten Pflegediensten $(74 \%)$ im Vergleich $\mathrm{zu}$ Senioreneinrichtungen ( $43 \%$ ) vermehrt eingesetzt. Tablets zur sozialen Aktivierung und Sturzdetektoren erzielen eine geringe Verbreitung von je 12 Prozent. $^{2}$ Erinnerungshilfen für Medikation, GPS-Tracker für Demenzkranke, Robotik, AAL-

1 Die Prozentangaben wurden aufgrund fehlender Beschriftungen im Diagramm geschätzt.

2 Die folgenden Werte zur Verbreitung assistiver Technologien wurden von Kuhlmey et al. (2019b) nicht mehr in stationäres und ambulantes Setting unterschieden, sondern nur eine durchschnittliche Nutzung der Verwendung in Alten- und Krankenpflege dargestellt. 
Technik und die automatische Medikamentenverteilung erreichen lediglich eine Nutzung von etwa 2 bis 4 Prozent (ebd.: 16).

Die Publikationen von Merda/Schmidt/Kähler (2017) und Schlomann (2020) haben im Gegensatz dazu nur jeweils vier unterschiedliche Pflegetechniken betrachtet. Im Klinik- und Altenpflegesetting geben in der Studie von Merda/Schmidt/Kähler (2017) 74 Prozent der Befragten an, elektronische Dokumentation(-ssysteme) im Arbeitsalltag zu nutzen (ebd.: 119). Auch hier wird zwischen stationärem und ambulantem Setting unterschieden. In der stationären Altenpflege ist die Nutzung elektronischer Dokumentation etwas höher (81\%) als im ambulanten Bereich (77\%) (ebd.: 120). Technische Assistenz erreicht in dieser Befragung des Pflegepersonals eine Verbreitung von insgesamt 32 Prozent, im ambulanten Bereich ist diese mit 48 Prozent höher als im stationären Bereich mit 38 Prozent. Technische Assistenz wird im Kontext der Studie synonym verwendet $\mathrm{zu}$ Ambient Assisted Living oder zu altersgerechten Assistenzsystemen. Damit ist sensorgestützte Technik, wie beispielsweise ein intelligenter Fußboden oder Aufstehhilfen, gemeint. Die Grenze zwischen AAL-Technik und technischer Assistenz ist hier nicht trennscharf (ebd.: 21). Telecare und Telemedizin erreichen als zusammengefasste Kategorie einen Einsatz von 27 Prozent; dieser weicht in den unterschiedlichen Settings nur geringfügig voneinander ab. Der Einsatz von Robotik erreicht in dieser Studie einen Nutzungswert von 21 Prozent (Merda/Schmidt/Kähler 2017: 119-120). Wie diese Werte zustande kommen, wird nicht näher erläutert.

In den leitfadengestützten Interviews von Schlomann (2020) wird zwar der aktuelle Technikeinsatz des Pflegepersonals thematisiert, aber es können daraus keine Nutzungszahlen abgeleitet werden, sondern nur eine Tendenz. Unterteilt wird in klassische Assistenztechnologien (Mobilitätstechnologien, Notrufsysteme, elektronische Dokumentation, Gesundheits-Monitoring) und in digitale Technologien (Sensoren, Alarme, Monitoring, Smartphone-basierte Dokumentation) (ebd.: 122). Die als »klassisch« bezeichnete Pflegetechnik wird von den zehn befragten Pflegekräften häufiger genannt als die digitalen Technologien. Weitere Nutzungswerte sind aus der Studie nicht ersichtlich (ebd.: 129).

Die geschilderte Verbreitung und Nutzung wird von der Online-Studie von Seifert/Ackermann (2020) bestätigt; auch hier übertrifft die elektronische Dokumentation die Robotik in den Nutzungswerten (ebd.: 12). Es wurden 466 Beschäftigte in der Altenhilfe und weiteren sozialen Einrichtungen in der Schweiz zu ihrer Technikaffinität und Techniknutzung befragt. Die erfragte Technik beinhaltet drei robotische Systeme: Service-, Pflegerobotik sowie Robotik zur sozialen Aktivierung. Darüber hinaus wurden GPS-Ortung und Videoüberwachung, die zur Kategorie der Sicherheitssysteme gezählt wurden, den Befragten präsentiert. Es sind hohe Nutzungswerte für die Nutzung von Tablets und Smartphones zur elektronischen 
Pflegedokumentation in dieser Erhebung zu verzeichnen (ebd.: 14-18). Die prozentualen Nutzungswerte der ausgewählten Technologien sind in Tabelle 4 dargestellt.

Die Studie von Sowinski/Kirchen-Peters/Hielscher (2013) nimmt sowohl in methodischer als auch in Hinsicht auf ihre Ergebnisse eine gesonderte Stellung ein. Es handelt sich um »Praxiskontakte« zu hundert ambulanten oder stationären Altenhilfeeinrichtungen, deren Beratungsprotokolle ausgewertet wurden. Es wird angemerkt, dass es bei dieser »Praxisfeldanalyse [...] weniger um eine wissenschaftlich abgesicherte Genauigkeit und Vollständigkeit der Darstellung, sondern eher um eine Einschätzung zu den Entwicklungen und Zukunftstrends des Technikeinsatzes, die die Akteur*innen in den Einrichtungen gegenwärtig beschäftigen, « geht (ebd.: 3-4). Trotz dieser methodischen Schwierigkeiten sind die Ergebnisse prägnant. Die elektronische Pflegedokumentation wird sowohl ambulant als auch stationär sehr häufig eingesetzt und erzielt die höchsten Nutzungswerte. Die außerklinische Intensivpflege, in der pflegebedürftige Menschen engmaschig, mit pflegerischer Intensivpflege nach einem Krankenhausaufenthalt durch dauerhafte Beatmung oder künstliche Ernährung versorgt werden, weist eine geringere Verbreitung technischer Lösungen auf (ebd.: 31). Diese werden seitens spezieller ambulanter Dienste häufiger angefragt als im stationären Bereich. Auch muss angemerkt werden, dass der Technikbezug der außerklinischen Intensivpflege nicht sehr deutlich ausgeprägt erscheint. Personenortungssysteme und Lifter werden in stationären Heimen sehr häufig verwendet, ambulant dagegen weniger. Bewegungsspiele wie Wii oder animiertes Spielzeug wie die Spielzeugrobbe Paro werden stationär mäßig verwendet, ambulant dagegen kaum (ebd.: 52).

\section{Hindernisse für den Technikeinsatz in der Altenpflege}

Neben den Daten zur Nutzung werden in den sechs Studien vielfältige weitere Untersuchungen zum Stand der Digitalisierung in der Altenpflege betrieben. Die schriftliche Befragung von Kuhlmey et al. (2019a) ergibt, dass die Kenntnisse und die Nutzungsbereitschaften seitens der Pflegekräfte sehr viel höher sind als die Existenz und der Zugang zu Pflegetechnologien eine tatsächliche Nutzung erlauben. Sofern Geräte vorhanden sind, »nutzen etwa zwei Drittel der Pflegekräfte diese auch « (ebd.: 33). Die Nutzung ist dabei unabhängig von Alter, der Leitungsfunktion des Pflegepersonals oder der Art der Einrichtung. Daher kann die Nichtbereitstellung der technischen Hilfen als ein limitierender Faktor gewertet werden (ebd.).

Vor diesem Hintergrund stimmen 61 Prozent der Befragten der Aussage zu, dass ihre Einrichtung nicht angemessen auf die kommende Digitalisierung und Technisierung der Altenpflege vorbereitet sei. Der geringe Technikeinsatz wird eher als Problem auf der institutionellen, denn als Problem auf der personellen Ebene der Pflegekräfte gedeutet (Kuhlmey et al. 2019b: 19). 
Als schwerwiegendes Hindernis für den Technikeinsatz werden die fehlenden Schulungen und Unterweisungen für den Umgang mit den neuen Technologien bewertet. Die Einrichtungen müssten sich stärker auf die Etablierung und Durchführung technischer Weiterbildungsangebote konzentrieren (Kuhlmey et al. 2019a: 34; Merda/Schmidt/Kähler 2017: 148). Zudem werde in der Pflegeausbildung bislang zu wenig auf das Thema Technik eingegangen. Die Pflegekräfte werden dann im Alltag mit Technologien konfrontiert, die zum Zeitpunkt der Ausbildung noch nicht vorhanden waren. Daher werden kontinuierliche Schulungen als sehr sinnvoll erachtet (Seifert/Ackermann 2020: 31).

Neben den hohen Anschaffungskosten stehen aber unter anderem auch technische Störungen, veraltete Geräte und Verbindungsprobleme einem routinemäßigen Technikeinsatz entgegen (vgl. Haug und Scorna in diesem Band). Es wurden im Folgenden einige anschauliche, offene Nennungen angeführt, die zwar von Krankenpflegekräften stammen, aber genauso auf die Altenpflege übertragbar sind:

»Essentiell für elektronische Hilfsmittel in der Pflege sind außerdem meiner Meinung nach vor allem regelmäßige Schulungen. Auf vielen Stationen, die über elektrische Hilfsmittel verfügen, nutzen nur sehr wenige Pflegende diese, da sie oft nicht richtig eingewiesen sind und sich damit unsicher fühlen.« (Zitat 5 , Krankenpflegekraft, weiblich, 21 Jahre). »Ca. $5 \%$ des täglichen Arbeitsaufwandes ist der Umgang mit nicht ordnungsgemäß funktionierenden technischen Geräten aufgrund von Defekten, Serverproblemen, Netzproblemen, Überlastung, Veralterung usw« (Zitat 6, Krankenpflegekraft, weiblich, 56 Jahre) (Kuhlmey et al. 2019b: 15).

Nicht nur beim Pflegepersonal wird fehlende Technikeinweisung angemerkt; ebenso fehle es den älteren Menschen nach Seifert/Ackermann an Wissen zum Umgang mit den technischen Innovationen. Sie sind nicht mit der aktuellen Technik aufgewachsen und weisen somit eine andere Techniksozialisation auf. Es fehle deswegen den meisten Anwender*innen an Technikerfahrung. Durch körperliche Einschränkungen kann die Bedienung der Geräte außerdem erschwert sein. Soziale Faktoren wie begrenzte finanzielle Mittel oder fehlende geeignete Ansprechpartner*innen im sozialen Umfeld können ebenfalls hinderlich sein. Somit wird neben den Qualifizierungsmaßnahmen für das Pflegepersonal das Angebot eines technischen Supports für Anwender*innen empfohlen, die entweder ambulant oder stationär betreut werden (ebd.: 31).

\section{Bewertungen zum Einsatz von Pflegetechnik}

Im Folgenden werden einige zentrale Aussagen der analysierten Sammelbandbeiträge zur Verbreitung digitaler Assistenztechnik dargestellt, deren Ergebnisse im 
jeweiligen Beitrag jedoch nicht statistisch ausgewiesen sind. Diese Arbeiten kommen übereinstimmend zu dem Schluss, dass Pflegetechnik nicht flächendeckend im Einsatz ist (Hilbert et al. 2018: 37; Mielitz 2017: 497; Wahl/Kricheldorff/HedtkeBecker 2018: 1). Auch sie führen, wie die vorab genannten Studien, zahlreiche Hemmnisse an, die trotz der Vielzahl an Forschungsarbeiten hinderlich für eine langfristige Verbreitung altersgerechter Assistenzsysteme für ältere Menschen sind. Mielitz (2017) nennt beispielsweise die hohen Anschaffungskosten, die der geringen Nachfrage geschuldet seien. Verstärkt werde dies durch geringe Zahlungsbereitschaft oder mangelnde finanzielle Mittel der Endanwender*innen. Auch fehle es älteren Menschen an Wissen zum langfristigen Nutzen technischer Assistenzsysteme. Technische Sicherheitslücken sieht sie als weiteres Hindernis, beispielsweise in Hinblick auf den Datenschutz. Ihrer Meinung nach fehlt auch ein Ort für Beratung und Verkauf von alltagsunterstützenden Technologien (ebd.: 496). Mielitz bewertet die aktuellen Anwendungen als zu technologiezentriert, jedoch zu wenig nutzer"innenzentriert:

»Kurzum die herkömmlichen Assistenztechnologien sind im Einzelfall sehr hilfreich, doch nicht für eine Anwendung in der breiten Masse gedacht. Doch genau darum geht es bei AAL - um Assistenzlösungen, die den Menschen in unserer immer älter werdenden Cesellschaft im Alltag Unterstützung leisten können« (Mielitz 2017: 480).

Fachinger (2017) bestätigt den technischen Fokus bei der AAL-Produktentwicklung ebenfalls. Er konstatiert eine sehr große Gerätevielfalt, die aber vorwiegend von einer »Mensch und Maschine«-Ausrichtung (ebd.: 89) beherrscht werde. Die Überprüfung der Alltagstauglichkeit und deren Akzeptanz müsse noch erfolgen, damit die vernetzen Systeme, die sich noch immer in der Pilot- und Testphase befinden, routinemäßig in der ambulanten oder stationären Pflege eingesetzt werden könnten (ebd.). Neben den fehlenden Finanzierungsmöglichkeiten argumentieren Hilbert et al. (2018), dass stärker am individuellen Nutzen sowie an der breiten Akzep$\operatorname{tanz}$ angesetzt werden müsse, damit die digitale Technik eine Zukunftsfähigkeit erhalte. Denn nicht die Zahl der Forschungsaktivitäten entscheide darüber, ob die Technologie von der breiten Bevölkerung angenommen wird. Es wird für die Entwicklung standardisierter, aber noch fehlender Geschäftsmodelle für Pflegetechnik plädiert (ebd.: 41-42). Noch drastischer drücken sich Wahl/Kricheldorff/HedtkeBecker vor dem Hintergrund aus, dass seit den vergangen zwei Jahrzenten viele Forschungsarbeiten zu Alter und Technik entstanden sind, diese jedoch meist nur als Prototypen eingesetzt wurden:

»International, aber auch in Deutschland, hat sich in den zurückliegenden zwei Jahrzehnten viel in Bezug auf Forschungsarbeiten zu Alter und Technik - bisweilen auch `Gerontechnology« genannt, manchmal auch (nahezu) gleichgesetzt 
mit dem Konzept des `Ambient Assisted Living - getan. Vor unserem geistigen Auge könnte nach diesen Studien eine Reihe von Prototypen, etwa mehrere unterschiedlich smarte Rollatoren, Tracking-Systeme, Smart-Home-Lösungen, unterschiedliche Robotersysteme und mehrere robotische Tiere auftreten, zu denen unterschiedliche Intensitäten an Erprobungs- und Evaluationsergebnissen mit unterschiedlicher Robustheit vorliegen. Eines ist diesen Studien, zu denen unseres Wissens bislang kein umfassender Überblick bzw. eine entsprechende Auswertung vorliegt (aber der selektive Überblick in Jokisch/Wahl (2016)), aber doch wohl gemeinsam. Die wenigsten der generierten bzw. untersuchten Techniklösungen haben irgendeine größere Verbreitung bei älteren Menschen und/oder ihren Familienangehörigen gefunden, vielleicht mit Ausnahme der Robbe Paro, die in einer nicht unbeachtlichen Zahl von Pflegeheimen zum Einsatz kommt. Nach wie vor ist in Deutschland die am weitesten verbreitete und wirklich in die Fläche gedrungene Technologie für ältere Menschen der Notruf, was nach vielen Jahren an Forschung zu Alter und Technik doch etwas sonderbar anmutet.«(Wahl/Kricheldorff/Hedtke-Becker 2018: 1)

\section{Diskussion}

Die systematische Übersichtsarbeit zeigt, dass ...

- in jeder einbezogenen Studie eine eigene Kategorisierung mit unterschiedlichen Pflegetechniken getroffen wird,

- die enthaltenen Nutzungsanteile in den Studien zum Teil erheblich voneinander abweichen,

- die elektronische Pflegedokumentation und/oder assistive Technologien wie Hebehilfen trotz unterschiedlicher Kennzahlen aktuell am häufigsten in der Altenpflege eingesetzt werden,

- Pflegetechniken (noch) nicht flächendeckend in ambulanten oder stationären Altenhilfeeinrichtungen eingesetzt werden, sondern bislang Prototypenforschung und Pilotstudien überwiegen,

- bisher nur selektive Übersichtsarbeiten über die Verbreitung von Pflegetechnik in der nationalen Altenpflege existieren (z.B. Hilbert et al. 2018: 41; Jokisch/Wahl 2016: 6; Wahl/Kricheldorff/Hedtke-Becker 2018: 1).

Die Darstellung von Nutzungswerten von Pflegetechnologien ist ein Themengebiet, für das nur schlecht vergleichbare Zahlen vorliegen, weil jeder Studienbericht verschiedene Techniken präsentiert und diese unterschiedlich kategorisiert. Des Weiteren kann man die Technologien kaum trennscharf voneinander abgrenzen (Merda/Schmidt/Kähler 2017: 20). Trotzdem zeigt sich bei den eingeschlossenen 
Texten, dass die elektronische Pflegedokumentation und/oder assistive Technologien bei der Nutzung an erster Stelle stehen. Robotik oder vernetzte Systeme der dritten Generation werden hingegen seltener verwendet. $\mathrm{Zu}$ den Ausnahmen zählen an dieser Stelle die in der Studie von Seifert/Ackermann mit 51 Prozent am häufigsten genannten »Serviceroboter/Pflegeroboter/Wannenlifte« sowie die Ergebnisse von Merda/Schmidt/Kähler mit hohen Prozentzahlen für Robotik (21\%) und Technische Assistenz (32\%). Die vorliegenden Ergebnisse sind in der Aussage von Schlomann (2020) zusammengefasst:

»Insgesamt werden digitale Technologien im Vergleich zu den eher `klassischen< technischen Systemen von den befragten Personen als aktuell weniger relevant für den Arbeitsalltag genannt. Digitale Technologien werden in der professionellen Altenpflege bisher eher wenig genutzt, obwohl die professionell Pflegenden ein generelles Interesse und eine Offenheit äußern.«(Schlomann 2020: 129)

Pflegedokumentation und auch assistive Technologien verschaffen mehr Zeit für Pflegeaufgaben und erleichtern Arbeitsabläufe (Sowinski/Kirchen-Peters/Hielscher 2013). In Befragungen geben Pflegekräfte explizit den Wunsch nach mobilen Geräten an, um die Dokumentationszeit zu verkürzen (Schlomann 2020: 129). Damit könnte dem Zeitmangel begegnet werden, der als eine große Herausforderung im Arbeitsalltag professionell Pflegender gilt (Jenull/Brunner 2009: 6). Durch die gestiegene Verwaltungslast und Dokumentationspflicht wird die elektronische Dokumentation zukünftig zur Pflicht werden, argumentieren Sowinski/Kirchen-Peters/Hielscher (2013). Alle Pflegeeinrichtungen werden wohl in Zukunft Softwareprogramme zur Pflegeprozessbegleitung und -dokumentation nutzen (müssen) (ebd.: 53). Hebe- und Tragesysteme der ersten Generation sind vor allem in der stationären Pflege weit verbreitet, um einerseits die Gesundheit des Personals $\mathrm{zu}$ fördern und andererseits Personal einzusparen, indem zum Beispiel Waschvorgänge alleine durchgeführt werden (ebd.: 86).

Zunächst jedoch erfordert der Einsatz von Technik jeder Art Zeit und Weiterbildung. In Deutschland existieren hierzu bereits erste Qualifizierungsmaßnahmen, die aber noch nicht flächendeckend verbreitet sind und sich am Markt noch nicht etabliert haben (Merda/Schmidt/Kähler 2017: 147). Generell ist die finanzielle Frage beim Technikeinsatz in der Altenpflege noch nicht geklärt und stellt ein Hindernis für deren flächendeckende Etablierung dar (Mielitz 2017: 496).

Zusammenfassend dominiert in der Literatur aktuell die Prototypenforschung mit einem technikgetriebenen Ansatz, der als >technology push verstanden werden kann. Gefordert wird ein Perspektivenwechsel, der stärker auf die Einbindung von Anwender*innen sowie auf das Pflegepersonal eingeht (Buhtz et al. 2018: 7). Vonseiten des Pflegepersonals wird der Wunsch nach Mitgestaltung bei der Technikentwicklung geäußert (Kuhlmey et al. 2019b: 19). Dafür sollten gemeinsame For- 
mate geschaffen werden, welche die Expertisen aus Pflege und Forschung in der Entwicklung der Pflegetechnologien vereinen könnten.

\section{Limitation}

Die gewählte Methode des Scoping Reviews bietet einen Überblick über die aktuelle Literatur zur Verbreitung von Pflegetechnik in der Altenpflege. Dabei wurde die Entwicklung des Suchstrings vollständig dokumentiert und begründet. Bei den eingeschlossenen Studien handelt es sich meist um Forschungsberichte, die im Rahmen von Drittmittelprojekten initiiert wurden. Der Altersfilter kann jedoch zu einem Datenverlust in den Datenbanken PubMed und CINAHL geführt haben, da möglicherweise nicht alle Studien mit Altersgruppen kodiert werden.

\section{Schlussfolgerung}

Angesichts der wachsenden Nachfrage nach pflegerischer Versorgung in Deutschland haben Aussagen zur Verbreitung und Nutzung digitaler Pflegetechnik beziehungsweise assistiver Technologien eine hohe Priorität, da nur auf dieser Basis informierte Entscheidungen in Hinblick auf Fördermaßnamen und die Notwendigkeit weiterer technischer Entwicklungen getroffen werden können. Bislang ist die Verbreitung von Technik in ambulanten und stationären Pflegesettings wenig untersucht. Die untersuchten Studien deuten auf eine eher geringe Verbreitung hin. Dabei liegt das Hindernis wohl aber nicht aufseiten der Pflegenden, wie Kuhlmey et al. (2019a) argumentierten. Hindernisse zum Einsatz von Pflegetechnik lassen sich eher auf institutioneller Ebene verorten, da das Gesundheitswesen und die Altenhilfe noch nicht ausreichend auf die Digitalisierung vorbereitet sind (ebd.: 33). Außerdem stehen in den vorliegenden Studien meist der Technikfokus sowie die Sicht der Pflegekräfte im Vordergrund. Diese Aspekte müssten ergänzt werden, um eine stärkere Fokussierung auf die konkrete Implementierung und den tatsächlichen Einsatz der Technik zu erlangen sowie um den Fokus auf die (potenziellen) Anwender*innen selbst sowie deren Akzeptanz der Technik zu richten, damit deutlich mehr »Omas schlaue Wohnungen« (s.o.) in Deutschland geschaffen werden können. 


\section{Literatur}

Arksey, Hilary/O'Malley, Lisa (2005): »Scoping studies: towards a methodological framework«, in: International Journal of Social Research Methodology 8, S. 19-32.

Buhtz, Christian/Paulicke, Denny/Hirt, Julian/Schwarz, Karsten/Stoevesandt, Dietrich/Meyer, Gabriele/Jahn, Patrick (2018): »Robotische Systeme zur pflegerischen Versorgung im häuslichen Umfeld: Ein Scoping Review«, in: Zeitschrift für Evidenz, Fortbildung und Qualität im Gesundheitswesen 137-138, S. 1-8.

Bundesministerium für Bildung und Forschung (2009): Assistenzsysteme im Dienste des älteren Menschen, https://www.fit.fraunhofer.de/content/dam/f it/de/documents/projektportrats-aal.pdf vom 18.05.2020.

Calvaresi, Davide/Cesarini, Daniel/Sernani, Paolo/Marinoni, Mauro/Dragoni, Aldo F./Sturm, Arnon (2017): »Exploring the ambient assisted living domain: A systematic review«, in: Journal of Ambient Intelligence and Humanized Computing 8, S. 239-257.

Cedillo, Priscila/Sanchez, Cristina/Campos, Karina/Bermeo, Alexandra (2018): »A Systematic Literature Review on Devices and Systems for Ambient Assisted Living: Solutions and Trends from Different User Perspectives«, in: International Conference on eDemocracy \& eGovernment (ICEDEG), S. 59-66.

Elm, Erik/Schreiber, Gerhard/Haupt, Claudia C. (2019): »Methodische Anleitung für Scoping Reviews (JBI-Methodologie)«, in: Zeitschrift für Evidenz, Fortbildung und Qualitat im Gesundheitswesen 143, S. 1-7.

Fachinger, Uwe (2017): »Technikeinsatz bei Pflegebedürftigkeit«, in: Klaus Jacobs/Adelheid Kuhlmey/Stefan Greß/Jürgen Klauber/Antje Schwinger (Hg.), Pflege-Report 2017, Stuttgart: Schattauer, S. 83-93.

Fleming, Richard/Sum, Shima (2014): „Empirical studies on the effectiveness of assistive technology in the care of people with dementia: A systematic review«, in: Journal of Assistive Technologies 8, S. 14-34.

Frommeld, Debora/Haug, Sonja/Scorna, Ulrike/Weber, Karsten (2020): »Technik in der Pflege: Ein Allheilmittel?«, in: CAREkonkret vom 30.04.2020, S. 7.

Gast, Robert (2013): »Der unsichtbare Pfleger«, in: Die ZEIT vom 03.01.2013, S. 27-28.

Graf, Birgit/Heyer, Torsten/Klein, Barbara/Wallhoff, Frank (2013): »Servicerobotik für den demografischen Wandel. Mögliche Einsatzfelder und aktueller Entwicklungsstand «, in: Bundesgesundheitsblatt, Gesundheitsforschung, Gesundheitsschutz 56, S. 1145-1152.

Hauck, Claudia/Uzarewicz, Charlotte (Hg.) (2019): I, Robot - I, Care. Möglichkeiten und Grenzen neuer Technologien in der Pflege (Bildung - Soziale Arbeit - Gesundheit, Band 20), Berlin/München/Boston: De Gruyter; De Gruyter Oldenbourg. 
Hilbert, Josef/Becka, Denise/Cirkel, Michael/Dahlbeck, Elke (2018): »Alter und Technik: Perspektiven der Gesundheitswirtschaft«, in: Harald Künemund/Uwe Fachinger (Hg.), Alter und Technik. Sozialwissenschaftliche Befunde und Perspektiven, Wiesbaden: Springer VS, S. 33-50.

Hirt, Julian/Nordhausen, Thomas (2020): Systematische Literaturrecherche in Fachdatenbanken: Rechercheprotokoll. Eine Beilage zu RefHunter (Manual zur Literaturrecherche in Fachdatenbanken), Halle und St. Gallen, https://refhunt er.eu/rechercheprotokoll/vom 15.07.2020.

Jenull, Brigitte/Brunner, Eva (2009): »Macht Altenpflege krank?«, in: Zeitschrift für Gerontopsychologie \& -psychiatrie 22, S. 5-10.

Jokisch, Mario/Wahl, Hans-Werner (2016): Expertise zu Technik und Alter in Deutschland, Heidelberg, https://www.dggg-online.de/fileadmin/download/J okisch_Wahl_Expertise-Ueberarbeitet_v201603.pdf vom 17.07.2020.

Khosravi, Pouria/Ghapanchi, Amir H. (2016): "Investigating the effectiveness of technologies applied to assist seniors: A systematic literature review«, in: International journal of medical informatics 85, S. 17-26.

Kopp, Tobias/Schöchlin, Jürgen (2014): Der intelligente Hausschuh im blauen Ozean. Eine empirische Untersuchung zur Markteinführung eines innovativen altersgerechten Assistenzsystems (Reihe, Band 10), Lohmar: Eul.

Krick, Tobias/Huter, Kai/Domhoff, Dominik/Schmidt, Annika/Rothgang, Heinz/Wolf-Ostermann, Karin (2019): »Digital technology and nursing care: A scoping review on acceptance, effectiveness and efficiency studies of informal and formal care technologies«, in: BMC health services research 19, S. 1-15.

Kuhlmey, Adelheid/Blüher, Stefan/Nordheim, Johanna/Zöllick, Jan (2019a): »Ressource oder Risiko - Wie professionell Pflegende den Einsatz digitaler Technik in der Pflege sehen«, in: Zentrum für Qualität in der Pflege (Hg.), ZQP-Report. Pflege und digitale Technik, Berlin: ZQP, S. 31-35.

- (2019b): Technik in der Pflege - Einstellungen von professionell Pflegenden zu Chancen und Risiken neuer Technologien und technischer Assistenzsysteme. Abschlussbericht für das Zentrum für Qualität in der Pflege (ZQP), https: //www.zqp.de/wp-content/uploads/ZQP-Bericht-Technik-profPflege.pdf vom 04.05 .2020 .

Ludwig, Inga (2011): Omas schlaue Wohnung. Intelligente Technik für Senioren, https://www.sueddeutsche.de/wissen/intelligente-technik-fuer-senioren -omas-schlaue-wohnung-1.1051532-o\#seite-2 vom 27.01.2011.

Merda, Meiko/Schmidt, Kristina/Kähler, Björn (2017): Pflege 4.0 - Einsatz moderner Technologien aus der Sicht professionell Pflegender. Forschungsbericht, Hamburg, https://www.bgw-online.de/SharedDocs/Downloads/DE/Medienty pen/BGW\%20Broschueren/BGWo9-14-002-Pflege-4-0-Einsatz-moderner-Tec hnologien_Download.pdf. 
Mielitz, Stefanie (2017): »Intelligente Lösungen für den AAL-Tag!«, in: Stefan Müller-Mielitz/Thomas Lux (Hg.), E-Health-Ökonomie, Wiesbaden: Springer Gabler, S. 479-499.

Moher, David/Liberati, Alessandro/Tetzlaff, Jennifer/Altman, Douglas G. (2009): "Preferred reporting items for systematic reviews and meta-analyses: The PRISMA statement«, in: Annals of internal medicine 151, S. 264-269.

Munn, Zachary/Peters, Micah D. J./Stern, Cindy/Tufanaru, Catalin/McArthur, Alexa/Aromataris, Edoardo (2018): »Systematic review or scoping review? Guidance for authors when choosing between a systematic or scoping review approach«, in: BMC medical research methodology 18, S. 143.

Peek, Sebastiaan T. M./Wouters, Eveline J. M./van Hoof, Joost/Luijkx, Katrien G./Boeije, Hennie R./Vrijhoef, Hubertus J. M. (2014): »Factors influencing acceptance of technology for aging in place: a systematic review«, in: International journal of medical informatics 83, S. 235-248.

Queirós, Alexandra/Silva, Anabela/Alvarelhão, Joaquim/Rocha, Nelson P./Teixeira, António (2015): »Usability, accessibility and ambient-assisted living: a systematic literature review«, in: Universal Access in the Information Society 14, S. 57-66.

Schlomann, Anna (2020): »Digitale Technologien in der Altenpflege: Aktueller Technikeinsatz, Technikakzeptanz und Wünsche für digitale Unterstützung aus der Perspektive von professionell Pflegenden «, in: Pflegewissenschaft 22, S. 121-131. Scorna, Ulrike/Frommeld, Debora/Haug, Sonja/Weber, Karsten (2021): »Digitale Technik in der Pflege als Generallösung? Neue Perspektiven auf altersgerechte Assistenzsysteme«, in: Carolin Freier/Joachim König/Arne Manzeschke/Barbara Städtler-Mach (Hg.), Gegenwart und Zukunft sozialer Dienstleistungsarbeit, Wiesbaden: Springer VS, S. 299-312.

Seifert, Alexander/Ackermann, Tobias (2020): Digitalisierung und Technikeinsatz in Institutionen für Menschen im Alter, https:/www.zora.uzh.ch/id/eprint/18 5291/1/Sonderauswertung_Alter_20200131_5.pdf vom 23.06.2020.

Sowinski, Christine/Kirchen-Peters, Sabine/Hielscher, Volker (2013): Praxiserfahrungen zum Technikeinsatz in der Altenpflege, https://www.boeckler.de/p df_fof/91394.pdf vom 18.06.2020.

Statistisches Bundesamt (2018): Pflegestatistik 2017. Pflege im Rahmen der Pflegeversicherung. Deutschlandergebnisse, Wiesbaden: Destatis.

Wahl, Hans-Werner/Kricheldorff, Cornelia/Hedtke-Becker, Astrid (2018): »Technik für vulnerable ältere Menschen und ihre Angehörigen: Möglichkeiten und Grenzen«, in: Zeitschrift für Gerontologie und Geriatrie 51, S. 1-2.

Weiß, Christine/Lutze, Maxie/Compagna, Diego/Braeseke, Grit/Richter, Tobias/Merda, Meiko (2013): Unterstüzung Pflegebedürftiger durch technische Assistenzsysteme, Berlin, https://vdivde-it.de/system/files/pdfs/unterstuetzungpflegebeduerftiger-durch-technische-assistenzsysteme.pdf vom 20.05.2020. 
184 Miriam Vetter und Laura Cerullo

Yusif, Salifu/Soar, Jeffrey/Hafeez-Baig, Abdul (2016): »Older people, assistive technologies, and the barriers to adoption: A systematic review«, in: International journal of medical informatics 94, S. 112-116. 


\section{Nutzung, Planung und Bewertung digitaler Assistenzsysteme in der Pflege Ergebnisse einer Befragung von Führungskräften in ambulanten und stationären Einrichtungen}

Sonja Haug

\section{Einleitung}

Inwieweit werden digitale Assistenzsysteme in der Pflege tatsächlich angewandt? Gibt es hierbei Unterschiede zwischen ambulanten und stationären Einrichtungen? Welche möglichen Diffusionshindernisse lassen sich feststellen? Das vom Bundesministerium für Bildung und Forschung (BMBF) geförderte Projekt DAAS-KIN (»Diffusion altersgerechter Assistenzsysteme - Kennzahlenerhebung und Identifikation von Nutzungshemmnissen«) setzt an dieser Fragestellung an (vgl. Weber in diesem Band). Das Projekt geht der übergeordneten Frage nach, inwieweit die bisherige öffentliche Projektförderung (ebd.) in Bezug auf Marktentwicklung, Arbeitsplätze, Umsätze oder Verbesserung der Situation für gepflegte und pflegende Personen nachhaltig ist (vgl. Haug et al. 2020).

»Wenn man sich auf der einen Seite die sehr hohe Zahl an bislang in Deutschland (und Europa) geförderten Cero-Technologie-Forschungsprojekten (samt den Dutzenden Millionen an Fördergeldern) vor Augen führt und sich auf der anderen Seite die Frage stellt, wo zwischenzeitlich vielversprechende Techniklösungen nachhaltig Einzug in den Alltag älterer Menschen gehalten haben, dann fällt die Bilanz noch verbesserungsbedürftig aus. Was ist wirklich bislang dort sangekommen«?« (Schmidt/Wahl 2019: 546).

Potenzialstudien für den Markt der Pflegetechnik in Deutschland liegen vor (Fachinger et al. 2012; VDI/VDE-IT 2011). Fachinger (2018: 64) weist auf fehlende Geschäftsmodelle und darauf hin, dass zwar hohes ökonomisches Potenzial von assistiven Technologien gesehen wird, jedoch eine geringe Nachfrage besteht. Infolgedessen bleibt die wirtschaftliche Relevanz ungeklärt. Ein empirischer Überblick über Versorgungsdienstleistungen führt Pflegeassistenzsysteme gar nicht auf (Naumann et al. 2014). 
Seit langem wird auf fehlende Belege über Wirkungen hingewiesen. Ein Bericht des Instituts für Europäische Gesundheits- und Sozialwirtschaft GmbH (IEGUS 2013) fordert ausdrücklich Wirksamkeitsstudien. Bisher gibt es aber kein systematisches Health Technology Assessment in Bezug auf altersgerechte Assistenzsysteme. Auch für indirekte Wirkungen, zum Beispiel Kosteneinsparungen bei Versicherungsträger*innen durch vermiedene Krankenhausaufenthalte oder Arztbesuche sowie das Vermeiden eines Umzugs ins Pflegeheim, sind noch keine Nutzennachweise in der Praxis erbracht worden (Meyer 2016: 18). Meyer listet nach einer umfassenden Evaluationsstudie zwölf technische Lösungen auf, »die geeignet sein dürften, die häusliche Pflege und einen längeren Verbleib in der eigenen Häuslichkeit zu unterstützen« (Meyer 2018: 173). Sofern diese Systeme im Leistungskatalog der Sozialen Pflegeversicherung aufgenommen würden, könnte dies ihrer Ansicht nach die Marktentwicklung befördern.

Neben der Lösung unterschiedlicher technischer Herausforderungen ist »auch eine konsequente Berücksichtigung der Nutzerbedarfe, der Nutzerakzeptanz, der Einbettung in medizinische und pflegerische Versorgungsstrukturen, der Wohnumgebung und Netzwerke sowie eine Berücksichtigung ökonomischer, juristischer und ethischer Aspekte sowie nicht zuletzt eine langfristige Evaluation der Wirkungen« erforderlich (Künemund/Fachinger 2018: 10).

Ein internationales Review ergab, dass Akzeptanzstudien vor allem Roboter, Informations- und Kommunikationstechnologien oder elektronische Gesundheitsakten betreffen (Krick et al. 2019). Häusliche Pflege ist selten Untersuchungsgegenstand und Effizienz- oder Nützlichkeitsstudien sind ebenfalls nur eingeschränkt erforscht. Auch ein Literaturreview zu digitalen Assistenzsystemen im deutschsprachigen Raum bestätigt einen Schwerpunkt auf Robotik, gefolgt von Sicherheits- und Dokumentationssystemen (vgl. Vetter/Cerullo in diesem Band). Systematische Erhebungen zu den Erwartungen von Pflegenden oder Pflegeeinrichtungen bezüglich altersgerechter Assistenzsysteme oder zu Nutzungshemmnissen lagen zum Zeitpunkt der Studie ebenfalls nicht vor. ${ }^{1}$

Der vorliegende Beitrag präsentiert Ergebnisse einer Befragung im Rahmen des Projekts DAAS-KIN. Dabei werden Unterschiede in der aktuellen und geplanten Nutzung zwischen ambulanten und stationären Einrichtungen untersucht. Ausgegangen wird von den Hypothesen, dass digitale Assistenzsysteme erstens nicht sehr verbreitet sind, zweitens Unterschiede zwischen Systemen bestehen und drittens diese in der ambulanten Pflege seltener als in der stationären Pflege eingesetzt werden. Die weiteren Ziele des Projekts und des hier vorliegenden Beitrags bestehen darin, Diffusionshemmnisse bei der Verbreitung digitaler Assistenzsysteme, erwartete Auswirkungen sowie Einstellungen von Führungskräften

$1 \quad$ Inzwischen sind zwei Studien zum Einsatz von Technik in Pflegeeinrichtungen (Braeseke et al. 2020) bzw. zur Akzeptanz bei professionell Pflegenden (Zöllick et al. 2020) erschienen. 
in Pflegeeinrichtungen festzustellen. Mit dieser Erhebung der persönlichen Meinung von Führungskräften und ihrer Bewertung zum Technikeinsatz durch das Pflegepersonal wird eine Forschungslücke geschlossen.

\section{Erhebungsmethode und Stichprobe}

Zielgruppe und Untersuchungseinheit der Studie sind Pflegekräfte in leitender Position in stationären und ambulanten Pflegeeinrichtungen. Im Rahmen des Projekts DAAS-KIN wurde ein Mixed-Methods-Design gewählt, welches sowohl quantitative als auch qualitative Methoden miteinander verbindet. Eine Methoden-Triangulation »liefert eine Grundlage für die Annäherung an die Wahrheit« (Polit/Beck/Hungler 2004: 303). Die Datenerhebung und Auswertung erfolgt dabei entsprechend des jeweiligen methodischen Ansatzes; die Ergebnisse der Teilbereiche werden jedoch systematisch aufeinander bezogen (Kelle 2019). Die Methoden werden somit nicht voneinander isoliert verwendet, sondern ergänzen sich gegenseitig entsprechend dem Komplementaritätsmodell (Prein/Kelle/Kluge 1993). Die quantitative Studie wird insbesondere zur Erhebung von Häufigkeiten und zur Prüfung von Zusammenhängen angewandt.

Nach dem sequenziellen quantitativ-qualitativen Design (Kelle 2019: 169) kann der qualitative Untersuchungsteil auch vertiefende Erklärungen für quantitative Ergebnisse liefern. Gemäß dieser Sequenzabfolge sollen in der hier vorliegenden Studie mithilfe einer quantitativen Methodik Gebiete identifiziert werden, die im qualitativen Teil vertieft werden sollen (Mayer 2015: 90). Der Studie liegt somit ein »erklärend-sequenzielles Design« (Mayer 2015: 91) beziehungsweise explanatives Design (Kelle 2019: 169) mit zwei Phasen zugrunde (vgl. Scorna in diesem Band).

\section{Erhebungsmethode der Online-Befragung}

Die quantitative Befragung unter Anbieter*innen ambulanter und stationärer Pflege hat zum Ziel, die tatsächliche Verbreitung altersgerechter Assistenzsysteme, deren Investitionsumfang und ihre Auswirkungen auf Beschäftigungszahlen, Arbeitsbelastung und Pflegequalität zu erheben. Die schriftliche standardisierte Befragung wurde internetgestützt durchgeführt (Brake/Weber 2009). Vorteile von Online-Befragungen liegen unter anderem in der räumlichen und zeitlichen Unabhängigkeit, in der automatischen Filterführung und sie sind mit geringem finanziellem Aufwand verbunden. Außerdem entfallen Interviewer*innen-Effekte und Dateneingabefehler bei der manuellen Erfassung. Auch die Orientierung der Befragten an sozialen Normen fällt geringer aus (Wagner-Schelewsky/Hering 2019: 789). Allerdings setzen Online-Befragungen die Ausstattung der Zielpersonen mit Computern und Internetanschluss voraus. Online-Umfragen werden dann als 
sinnvolle Option gesehen, wenn der anvisierte Teilnehmer*innenkreis groß genug und mit Computern ausgestattet ist (ebd.).

Ein grundsätzliches Problem bei online durchgeführten Bevölkerungsbefragungen besteht darin, dass die für eine Zufallsstichprobe erforderlichen E-MailVerzeichnisse für Bevölkerungsstichproben nicht verfügbar sind (Schnell 2018). Für Bevölkerungsbefragungen sind zudem Online-Befragungen aufgrund der Alterseffekte bei der Internetnutzung und der digitalen Spaltung der Gesellschaft weniger geeignet. Bei einem Websurvey muss zudem mit dem Problem einer teilweise hohen Nonresponse-Rate gerechnet werden (Schnell 2012: 296), das heißt mit einer geringen Ausschöpfungsquote (Wagner-Schelewsky/Hering 2019: 789).

Bei den Führungskräften in der Pflege hingegen ist mit hoher Verbreitung der Internetnutzung zu rechnen. Die Befragung entspricht dem Typ 5 »Befragungen mit listenbasierten Stichproben aus Populationen mit einem hohen Abdeckungsgrad« (Baur/Florian 2009: 110). Bei einem Test dieses Typs konnte mit personalisierten Adressen eine Ausschöpfungsquote von 8,2 Prozent erzielt werden (ebd.: 119).

Besonderes Augenmerk wurde auf eine möglichst große Stichprobe und eine möglichst umfassende Abdeckung der Zielpopulation sowie einen sorgfältigen Stichprobenziehungsplan gelegt (Polit/Beck/Hungler 2012: 248). Zunächst erfolgte eine umfassende Recherche und Auflistung der Grundgesamtheit. Das Auswahlverfahren war zweistufig, wobei in einem ersten Schritt Untersuchungsregionen (Gebietseinheiten) und in einem zweiten Schritt Untersuchungseinheiten (Einrichtungen) ausgewählt wurden (Schnell/Hill/Esser 2018: 257). Die Erhebungseinheit bilden somit die Einrichtungen. Für jede Einrichtung sollte die Befragung mit einer Führungskraft durchgeführt werden.

Für die geplante Vollerhebung auf Bundesländerebene wurden vorliegende Verzeichnisse der Statistischen Ämter in Baden-Württemberg (Stand 2013), Bayern (Stand 2017), Bremen (Stand 2012, nur stationäre Einrichtungen), MecklenburgVorpommern (Stand 2017), Saarland (Stand 2017), Sachsen (Stand 2015), SchleswigHolstein (Stand 2009), Thüringen (Stand 2019) und Hamburg (Stand 2009) genutzt. Ergänzt wurden diese durch eigene Recherchen zu Einrichtungen in Berlin, Bremen (nur ambulante Einrichtungen), Hessen und Rheinland-Pfalz auf www.seniorenportal.de. ${ }^{2}$ Die Bundesländer Sachsen-Anhalt, Brandenburg, Niedersachsen und Nordrhein-Westfalen konnten in der Befragung nicht berücksichtigt werden.

Die erstellte Datenbank enthält 11.040 Einträge, wobei doppelte Einträge nicht ausgeschlossen werden können. Die vorhandenen Verzeichnisse sind nicht immer

Die Adresslisten wurden von Vanessa Mücke und Peter Wegenschimmel zusammengestellt. Fragebogen und Anschreiben wurden im Team erarbeitet (Karsten Weber, Sonja Haug, UIrike Scorna, Debora Frommeld und Peter Wegenschimmel) und von Karsten Weber verteilt. Die Online-Umfrage wurde von Ulrike Scorna in www.soscisurvey.de umgesetzt. Sonja Haug führte die Datenauswertung mit SPSS Version 24 durch. 
auf dem aktuellen Stand, fehlerhafte Adressen konnten im Vorfeld der OnlineBefragung nicht eliminiert werden. Es stellte sich heraus, dass 13,1 Prozent der Adressen nicht funktionierten. Hieraus ergibt sich eine gültige Nettostichprobe von 9.594 .

Zumeist lagen zentrale E-Mail-Adressen der Einrichtungen, erkennbar zum Beispiel durch info@, vor. Es könnte sein, dass unklar war, an wen die Umfrage weitergeleitet werden soll, und daher eine Weiterleitung meist ausblieb. Es war zu erwarten, dass die Befragung häufig bereits an dieser Stelle gestoppt wird.

Der vollstandardisierte Fragebogen umfasst 40 Fragen, die entweder dichotom sind oder in fünfstufigen Skalen Einstellungen messen. Vom 16.04.2019 bis 30.04.2019 fand ein Online-Pretest mit 64 Studierenden des berufsbegleitenden Bachelorstudienganges Pflegemanagement an der Ostbayerischen Technischen Hochschule (OTH) Regensburg und zwei Expert*innen aus Einrichtungen der ambulanten und stationären Pflege in Regensburg statt.

Der Link zur Studie wurde mit einem Anschreiben per E-Mail an den oben aufgeführten Adressverteiler versendet. Die Feldphase fand im Zeitraum vom 18.06.2019 bis 05.07.2019 statt. Es wurden insgesamt 93 Fragebögen ausgefüllt.

Es stellt sich nun die Frage, ob die Ausfälle systematisch oder zufällig erfolgten. Ein vollständig zufälliges Fehlen (MCAR - missing completely at random) muss bei der Analyse nicht berücksichtigt werden, ein zufälliges Fehlen (MAR - missing at random) kann statistisch berücksichtig werden. Das gilt nicht für ein nicht zufälliges, also systematisches Fehlen (MNAR - Missing not at random), das mit verzerrten Ergebnissen einhergeht. Ausfälle durch Verweigerung oder Nichterreichbarkeit sind meist MAR und "gut statistisch kompensierbar. Dies gilt auch bei sehr großen Anteilen von Ausfällen« (Schnell 2018: 2). Gründe für den geringen Rücklauf könnten einerseits in der Qualität der verwendeten Adresslisten und andererseits in der Thematik der Befragung liegen. Der Aktualisierungsgrad der Verzeichnisse ist bundeslandabhängig und nicht-personalisierte Adressen sind häufig; diese Problematiken werden als MAR eingestuft. Eine Verzerrung könnte durch hausinterne Regelungen zur grundsätzlichen Nicht-Teilnahme an Befragungen auftreten. Durch die Anonymisierung der Umfrage war eine gezielte E-Mail zur Erinnerung an Einrichtungen, die den Fragebogen nicht beantwortet hatten, ausgeschlossen. Nichtsdestotrotz könnte ein Grund für die Nicht-Teilnahme auch fehlendes Vertrauen in die Anonymität der Umfrage sein.

Ein inhaltsbezogener Grund könnte die fehlende Erfahrung von Einrichtungen im Bereich digitaler Assistenzsysteme sein. Es ist wahrscheinlich, dass Einrichtungen, die wenige Erfahrungen mit digitalen Assistenzsystemen aufweisen, unterdurchschnittlich bereit waren, an der Befragung teilzunehmen. Bei einem derartigen MNAR würde die Häufigkeit der Assistenzsysteme überschätzt. Da ambulante Einrichtungen gleichermaßen wie stationäre vertreten sind, beeinträchtigt dies die statistische Analyse der Unterschiede kaum. 
Die Online-Befragung diente zudem im Rahmen des Mixed-MethodsForschungsdesigns zur Ableitung von Problemkomplexen und Fragestellungen für die qualitative Befragung (vgl. Scorna in diesem Band).

\section{Beschreibung der Stichprobe}

Es beteiligten sich Einrichtungen aus Baden-Württemberg, Bayern, Berlin, Hessen, Mecklenburg-Vorpommern, Rheinland-Pfalz, Saarland, Sachsen, SchleswigHolstein und Thüringen. 58,8 Prozent der Befragten sind weiblich. Das Durchschnittsalter liegt bei 46,6 Jahren. 30,9 Prozent haben die mittlere Reife erworben, 23,5 Prozent die allgemeine oder Fachhochschulreife und 38,3 Prozent haben einen Universitäts- oder Fachhochschulabschluss. Knapp die Hälfte (46,8 \%) arbeitet im ambulanten Pflegedienst, 53 Prozent in einer stationären Einrichtung.

Die Zielgruppe der Führungskräfte wurde gut getroffen. Etwa ein Drittel der Befragten hat die Position der Pflegedienstleitung inne und knapp 60 Prozent eine andere Führungsposition innerhalb der Pflegedienstleitung. 6 Prozent sind Mitarbeiter*innen und als Pflegepersonal oder -fachkraft angestellt. Im Durchschnitt verfügen die Befragten über 23 Jahre Berufserfahrung; hierbei im ambulanten Dienst über 22,8 Jahre und im stationären Dienst über 23,4 Jahre. Etwa 40 Prozent haben eine Ausbildung als Gesundheits-, Kinder-, Heilerziehungs- oder Krankenpfleger*in, ein Viertel als Altenpfleger*in, ein Viertel auch ein Pflegestudium und knapp ein Drittel eine sonstige Berufsausbildung, beispielsweise im kaufmännisch-betriebswirtschaftlichen oder hauswirtschaftlichen Bereich.

Im Durchschnitt beschäftigen die ambulanten Pflegedienste der Stichprobe 55 Personen, die stationären Pflegeeinrichtungen hingegen 143. Die Beschäftigtenzahl der ambulanten Einrichtungen liegt im Median bei 28 und maximal bei 300. Das Maximum der untersuchten stationären Einrichtungen liegt bei 2.000, der Median bei 87 Beschäftigten. Die durchschnittliche Anzahl der Bewohner*innen beziehungsweise betreuten Personen unterscheidet sich nicht stark, wobei auch hier das Maximum mit 400 beim ambulanten und bei 1.250 beim stationären Dienst deutlich variiert. Der Anteil der kognitiv Beeinträchtigten bei den zu Pflegenden liegt nach Angaben der Befragten aus der stationären Pflege bei knapp 70 Prozent, dahingegen im ambulanten Pflegedienst nur bei 43 Prozent. Die Anzahl der Beschäftigten und der Bewohner*innen korreliert sehr stark und signifikant miteinander (Pearson's $r=0,901$ ), wohingegen keine Korrelation zwischen der Anzahl der Beschäftigten oder der Bewohner*innen mit dem Anteil der kognitiv Beeinträchtigten besteht. Es lässt sich kein Betreuungsschlüssel berechnen, da auch nichtpflegerisches Personal unter den Beschäftigten subsumiert ist. Die Befragten sind etwa gleich häufig in Einrichtungen freigemeinnütziger oder privater Träger*innen beschäftigt. 


\section{Verbreitung digitaler Assistenzsysteme}

In der Online-Befragung wurden durchgängig sieben Arten digitaler Assistenzsysteme unterschieden. Die Kategorisierung erhebt keinen Anspruch auf Vollständigkeit und ist vielleicht nicht in jedem Fall trennscharf. Als Anhaltspunkt und zur Veranschaulichung wurden jeweils Beispiele genannt. Diese wurden aus der Fachliteratur, aus Veröffentlichungen zur Projektförderung des Bundesministeriums für Bildung und Forschung (BMBF) (vgl. Weiß et al. 2013) oder aus Presseberichten übernommen (zu einer anderen Systematisierung vgl. Braeseke et al. 2020: 24).

1 Kommunikation \& Entertainment (Videotelefonie, Telepräsenzroboter, computergestützte Spiele für ältere und hochbetagte Personen, EntertainmentRoboter wie Pepper).

2 Serviceroboter (zur Übernahme von Transportaufgaben, Hol- und Bringdienste, für die Anreichung von Getränken).

3 Pflegeroboter (im Bereich der personenbezogenen Pflege wie Hebehilfen oder intelligente Pflegewagen als pflegeunterstützende Tools).

4 Sicherheitssysteme (Ortungs- und Überwachungssysteme für demenziell veränderte Personen, Sturzsensoren, Geofencing, intelligente Fußmatten, mobile Aufstehhilfen).

5 Monitoring (Vitaldatenmonitoring, Telemonitoring, Wundmanagement-Systeme).

6 Dokumentationssysteme (elektronische Patient *innenakte, elektronische Visite).

7 Informationssysteme (Tablet PCs mit Erinnerungsfunktion, Sprachassistenzsysteme, Übersetzungssysteme bei Verständigungsschwierigkeiten).

Dokumentationssysteme wurden mit Abstand am häufigsten genutzt (siehe Abbildung 1). Dies entspricht den Ergebnissen des Literaturreviews im Projekt DAASKIN (vgl. Vetter/Cerullo in diesem Band). Auch Braeseke et al. (2020: 50) dokumentieren in ihren deskriptiven Analysen ähnliche Ergebnisse. 97,5 Prozent der Befragten aus stationären Einrichtungen nutzen mindestens eine Art digitaler Assistenzsysteme. Dieser Anteil ist in ambulanten Pflegeeinrichtungen signifikant geringer (45,7\%). Insgesamt geben 54,3 Prozent der Befragten aus ambulanten Einrichtungen an, dass überhaupt keine Form digitaler Assistenzsysteme genutzt wird. Bei stationären Einrichtungen liegt dieser Anteil nur bei 2,5 Prozent; der Unterschied ist hoch signifikant.

Auch der Verbreitungsgrad der Systeme in ambulanten oder stationären Einrichtungen unterscheidet sich signifikant $\left(\mathrm{Chi}^{2}\right.$-Test $\left.\mathrm{p}=0,000\right)$. In stationären Einrichtungen werden besonders häufig Dokumentationssysteme $(85,0 \%)$ verwendet, 
gefolgt von Sicherheitssystemen (70,0\%) und seltener Kommunikations- \& Entertainmentsystemen (40,0\%). Dieses Ergebnis geht einher mit dem Befund von 2020, dass stationäre Pflegeeinrichtungen deutlich häufiger digitale Kommunikationstechnologien neu einführten oder verstärkt nutzten als ambulante Pflegeeinrichtungen (vgl. Domhoff et al. in diesem Band). In ambulanten Einrichtungen sind es vorrangig digitale Assistenzsysteme für die Dokumentation $(28,6 \%)$ und Information $(22,9 \%)$. Informationssysteme sind gleichermaßen selten und Monitoring noch seltener verbreitet. Bei diesen Arten von Systemen gibt es auch keinen signifikanten Unterschied zwischen ambulanten und stationären Einrichtungen. Serviceroboter werden in keiner Einrichtung der Stichprobe genutzt, Pflegeroboter nur in sehr wenigen stationären Einrichtungen (siehe Abbildung 1).

Abbildung 1: Nutzung digitaler Assistenzsysteme

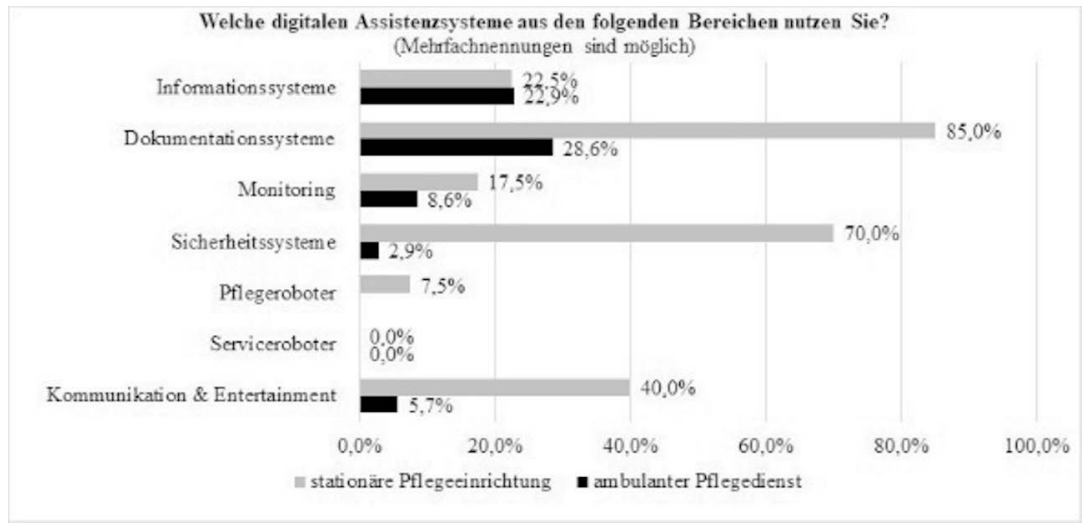

Quelle: Online-Befragung DAAS-KIN 2019

Zur Untersuchung der Faktoren, die im Zusammenhang mit einer Nutzung der Systeme stehen könnten, wurden logistische Regressionsanalysen durchgeführt. Folgende unabhängige Variablen fanden Eingang in die Analyse: Ambulante/stationäre Einrichtung, Anzahl der Beschäftigten, Anzahl der Patient*innen, Anteil kognitiv beeinträchtigter Patient*innen und Art der Träger*innen.

Insgesamt zeigt sich, dass weder die Zahl der Patient"innen noch deren kognitive Beeinträchtigung Einfluss auf den Einsatz haben. Auch die Trägerschaft der Einrichtung macht keinen Unterschied. Die Nutzung von Kommunikations- und Entertainment-, Sicherheits- und Dokumentationssystemen ist bei stationären Einrichtungen auch unter Kontrolle anderer Faktoren signifikant häufiger als bei ambulanten Einrichtungen. Kommunikations- und Entertainmentsysteme hängen daneben auch von der Anzahl der Beschäftigten ab. Monitoringsysteme 
hängen ausschließlich von der Anzahl der Beschäftigten ab. Für den Einsatz von Pflegerobotern und Informationssystemen spielen die untersuchten Faktoren keine entscheidende Rolle. Serviceroboter waren aufgrund fehlender Fälle nicht $\mathrm{zu}$ analysieren.

\section{Investitionsplanung}

Auch bei der Planung zukünftiger Nutzung unterscheiden sich die ambulanten und stationären Einrichtungen signifikant. Betrachtet man den Anteil der Einrichtungen, die eine Investition wahrscheinlich oder sehr wahrscheinlich planen, so zeigt sich, dass dies im stationären Dienst vor allem Dokumentations-, Sicherheits- und Informationssysteme betrifft sowie Kommunikation und Entertainment (siehe Abbildung 2). Die Beschaffung dieser Systeme wird signifikant seltener bei ambulanten Einrichtungen geplant $\left(\mathrm{Chi}^{2}\right.$-Test $\left.\mathrm{p} \leq 0,001\right)$. Serviceroboter und Pflegeroboter werden in stationären Einrichtungen weitaus seltener geplant, als dies angesichts der bisherigen hohen Fördersummen und der ausführlichen Fachdebatte (vgl. Weber in diesem Band) zu erwarten wäre. In den befragten ambulanten Einrichtungen sind diese Systeme nicht geplant (vgl. Braeseke et al. 2020: 67).

Abbildung 2: Planung digitaler Assistenzsysteme

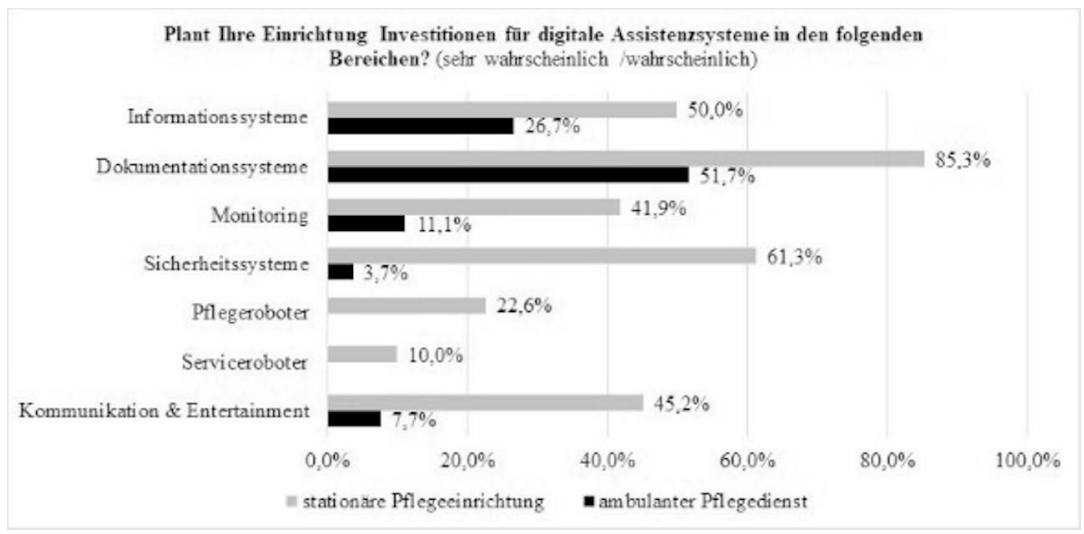

Quelle: Online-Befragung DAAS-KIN 2019

Unabhängig von der Einrichtungsart übersteigt die Häufigkeit der geplanten Investitionen, mit Ausnahme der Sicherheitssysteme, jeweils die aktuelle Nutzung (siehe Abbildung 3).

In Abbildung 4 werden zusätzliche Investitionen der Einrichtungen, die aktuell bereits ein System nutzen, mit erstmaligen Investitionserwägungen bei Einrich- 
Abbildung 3: Nutzung und Planung digitaler Assistenzsysteme

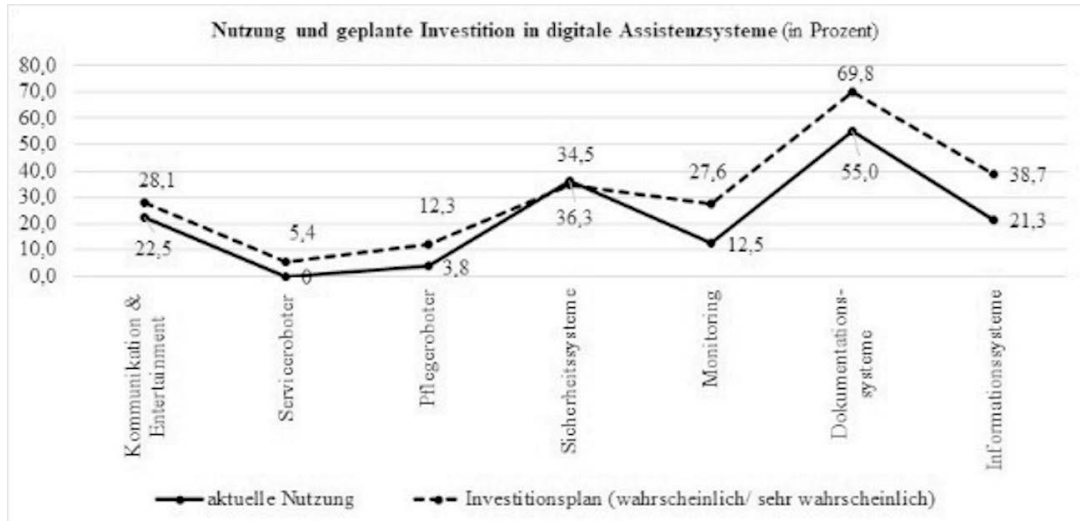

Quelle: Online-Befragung DAAS-KIN 2019

tungen, die bislang kein derartiges System nutzen, gegenübergestellt. Ist der erste Schritt gemacht, steigt die Investitionsbereitschaft der Einrichtungen.

Abbildung 4: Planung digitaler Assistenzsysteme nach Nutzung

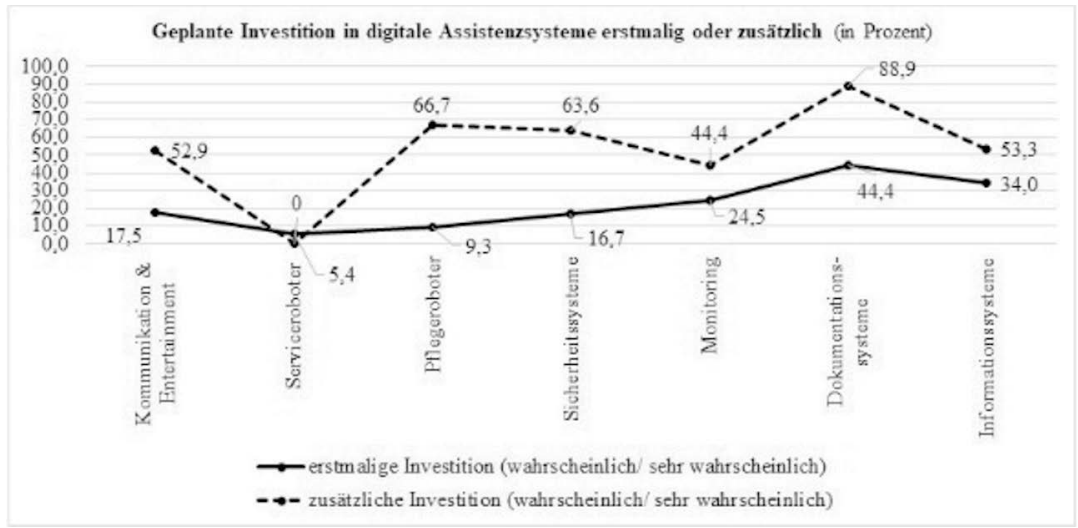

Quelle: Online-Befragung DAAS-KIN 2019

Das Maximum liegt bei zusätzlich geplanten Dokumentationssystemen $(88,9 \%)$. Der Anteil der Einrichtungen, die wahrscheinlich zusätzliche Pflegeroboter beschaffen wollen $(66,7 \%)$, darf nicht überbewertet werden, da sehr wenige Einrichtungen diese aktuell einsetzen. Da Serviceroboter in keiner Einrichtung 
genutzt werden, liegt der Anteil zusätzlicher Investitionen auch bei null. Seltener fassen Pflegeeinrichtungen, die noch nicht über entsprechende Assistenzsysteme verfügen, erstmalig Investitionen ins Auge. Auch dort ist die Beschaffung digitaler Dokumentations- und Informationssysteme am wahrscheinlichsten.

Je mehr Beschäftigte eine Einrichtung hat, umso wahrscheinlicher ist eine Investitionsplanung für jedes Assistenzsystem (Skalierung: wahrscheinlich/sehr wahrscheinlich/unentschieden/unwahrscheinlich/sehr unwahrscheinlich, Pearsons' $r, p \leq 0,01)$. Mit Ausnahme des Monitorings korreliert die Planung auch positiv mit der Anzahl der Bewohner*innen.

Multivariate Analysen der Investitionswahrscheinlichkeiten zeigen, dass diese Effekte auf den Unterschied zwischen ambulanten und stationären Einrichtungen zurückzuführen sind. Ist ein System in Planung, erhöht sich auch die Wahrscheinlichkeit für jedes andere System. Die Investitionsplanung für alle digitalen Assistenzsysteme korreliert positiv mit der Anzahl der Beschäftigten. Je höher diese ist, umso wahrscheinlicher ist die Planung der Assistenzsysteme. Mit Ausnahme des Monitorings korreliert die Planung auch positiv mit der Anzahl der Bewohner*innen: je höher die Anzahl der Bewohner"innen, umso wahrscheinlicher die Planung. Mit dem Anteil kognitiv Beeinträchtigter korreliert nur schwach die Planung von Sicherheitssystemen und von Kommunikation und Entertainment. Die Planung für digitale Assistenzsysteme der unterschiedlichsten Art korreliert wiederum miteinander. Das bedeutet, die Investitionsplanung für ein System erhöht die Wahrscheinlichkeit für jedes andere System ebenfalls. Besonders stark korrelieren die Planung für Service- und Pflegeroboter, für Sicherheitssysteme und Monitoring, Sicherheits- und Dokumentationssysteme, Sicherheits- und Kommunikationssysteme sowie Dokumentations- und Informationssysteme.

Zusätzlich wurde analysiert, ob überhaupt eine Investition geplant wird (wahrscheinlich/sehr wahrscheinlich versus unentschieden/unwahrscheinlich/sehr unwahrscheinlich). Logistische Regressionsanalysen zeigen mit einer Ausnahme keine signifikanten Effekte. Die Planung eines Sicherheitssystems ist bei stationären im Vergleich zu ambulanten Einrichtungen um den Faktor 37 erhöht. Generell hat jedoch weder die Zahl der Beschäftigten noch der Gepflegten noch der Anteil kognitiv Beeinträchtigter oder die Art der Einrichtung einen Einfluss darauf, ob Systeme geplant sind. Ambulante und stationäre Einrichtungen unterscheiden sich also wesentlich darin, wie entschieden sie bei Planungen agieren.

\section{Digitalisierungsstrategie}

Die Mehrzahl der Einrichtungen verfügt nach Aussage der Befragten über eine Digitalisierungsstrategie. Hierbei zeigen sich wiederum signifikante Unterschiede zwischen ambulanten und stationären Einrichtungen $\left(\mathrm{Chi}^{2}\right.$-Test $\left.p \leq 0,001\right)$. Der Unterschied beträgt beachtliche 29,6 Prozent (siehe Abbildung 5). Die Träger- 
schaft zeigt hier keinen signifikanten Effekt, auch wenn bei freigemeinnützigen Träger*innen Digitalisierungsstrategien stärker verbreitet sind. Eine gesonderte Betrachtung der Trägerschaft zeigt nur bei privaten Träger*innen einen signifikanten Unterschied zwischen ambulanten und stationären Einrichtungen.

Abbildung 5: Digitalisierungsstrategie

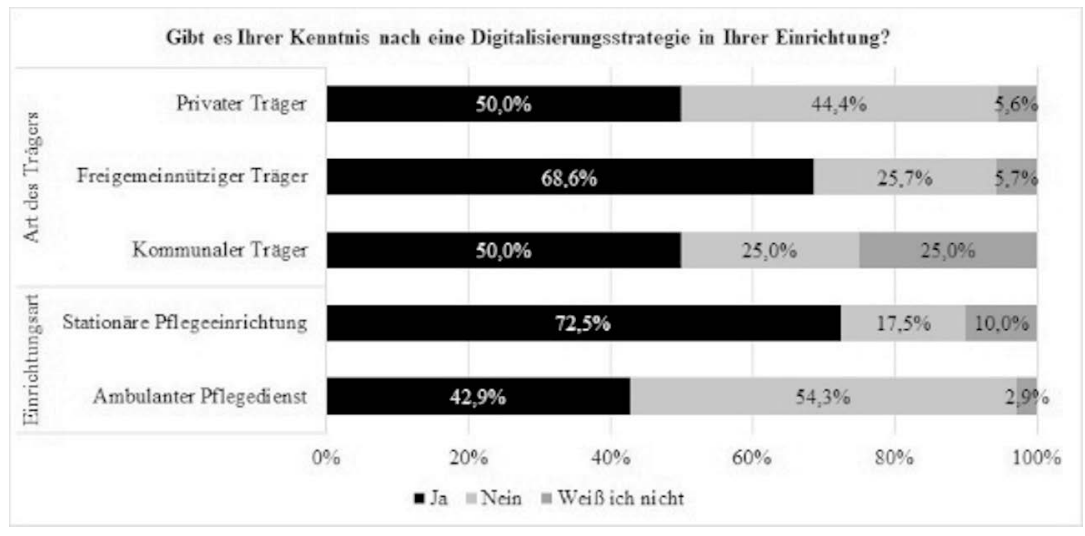

Quelle: Online-Befragung DAAS-KIN 2019

Es muss berücksichtigt werden, dass die Befragten unter einer Digitalisierungsstrategie unterschiedliches verstehen könnten. Die Studie von Braseke et al. (2020: 47) fragt konkret nach Bekanntheit und Nutzung von Fördermaßnahmen gem. $\$ 8$ Abs. 8 SGB XI und findet ebenfalls einen höheren Bekanntheits- und Nutzungsgrad bei vollstationären Einrichtungen.

\section{Diffusionshemmnisse}

Im folgenden Abschnitt werden Argumente, die für und gegen den Einsatz technischer Assistenzsysteme sprechen, einander gegenübergestellt und der jeweils erwartete Aufwand bei deren Einführung betrachtet.

\section{Gründe gegen die Beschaffung}

Die Hauptgründe, die nach Aussagen der Befragten gegen die Beschaffung digitaler Assistenzsysteme sprechen, sind im Wesentlichen finanzieller oder technischer Art. Auch Braseke et al. (2020: 68) nennen Anschaffungs- und Folgekosten/(Re-)Finanzierung als größtes Hemmnis beim weiteren Ausbau des Technikeinsatzes. Es 
werden zu hohe Kosten bei der Beschaffung (73,3\%), der Einführung $(56,7 \%)$ und im laufenden Betrieb beziehungsweise der Wartung (51,7\%) genannt (siehe Abbildung 6). Nach Ansicht von 41,7 Prozent der Befragten existieren außerdem nur unzureichende technische Lösungen oder liegt eine Inkompatibilität mit der bestehenden Infrastruktur vor (38,3\%). Ein weiteres Viertel befürchtet, dass die Technik die Erwartungen nicht erfülle (26,7\%). Relativ weit verbreitet ist auch die Befürchtung mangelnder Akzeptanz durch das Pflegepersonal (45,0\%) oder die Pflegebedürftigen (40,0\%). Diese Ergebnisse decken sich weitgehend mit den Ergebnissen der Expert*innenbefragung (vgl. Scorna in diesem Band).

\section{Abbildung 6: Faktoren gegen Beschaffung}

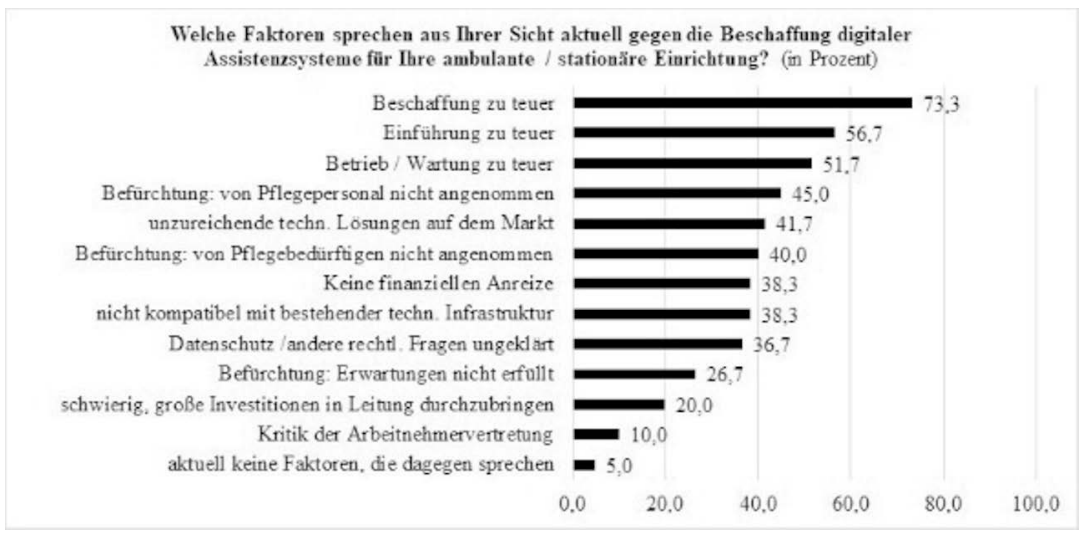

Quelle: Online-Befragung DAAS-KIN 2019

\section{Aufwand bei der Einführung}

Der Aufwand für die Wartung und Installation von Servicerobotern wird im Vergleich zu den anderen Bereichen mit Abstand am höchsten eingeschätzt (siehe Abbildung 7). Auch Pflegeroboter werden als wartungsintensiv und aufwändig bei der Installation wahrgenommen, Kommunikations- und Entertainmentsysteme hingegen vor allem bei der Installation.

Der Aufwand für die Schulung des Personals wird bei allen abgefragten Formen digitaler Assistenzsysteme im Vergleich zu Installation, Wartung und Anpassung der Arbeitsorganisation am höchsten eingeschätzt. Die Befunde entsprechen dem Literaturreview im Projekt DAAS-KIN (vgl. Vetter/Cerullo in diesem Band). Sie gehen auch einher mit der Bewertung von Klein (2019: 89) zum Wachstumsfeld der 
Abbildung 7: Aufwand Wartung und Installation

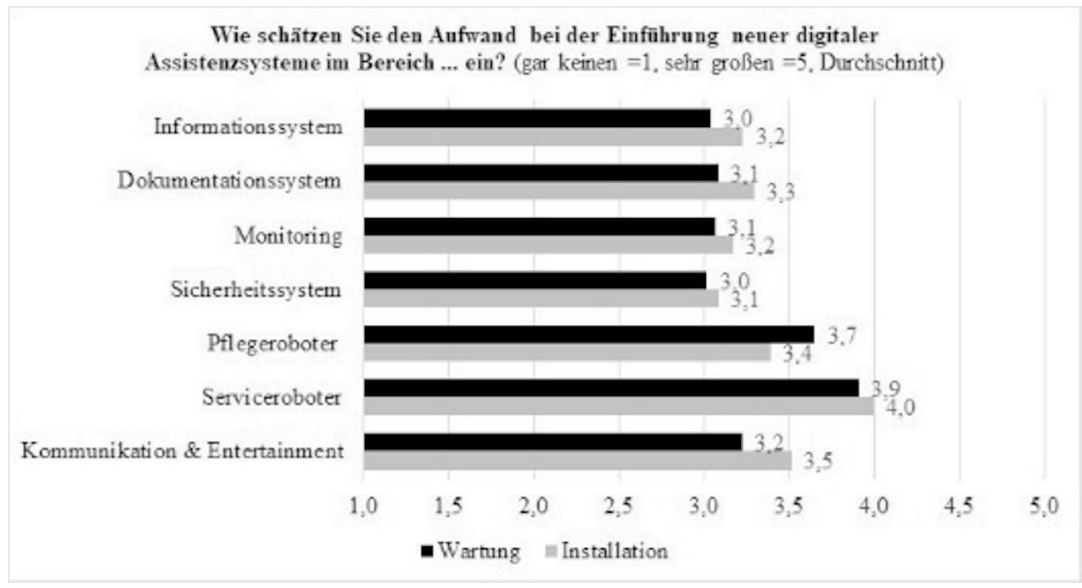

Quelle: Online-Befragung DAAS-KIN 2019

Abbildung 8: Aufwand Motivation und Schulung

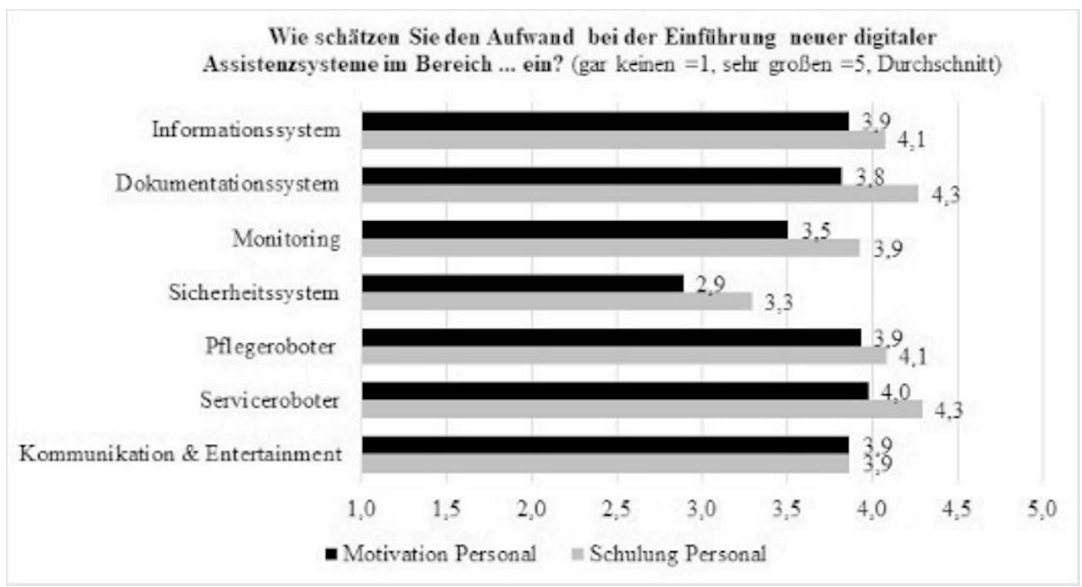

Quelle: Online-Befragung DAAS-KIN 2019

sozialen Roboter, wonach qualifizierte Kräfte nicht verdrängt und fachliches wie technisches Know-how benötigt werden.

Der Aufwand für die Motivation des Personals wird bei allen Systemen höher als der Aufwand für die Überzeugung der Pflegebedürftigen eingeschätzt (siehe 
Abbildungen 8, 9). Hoher Aufwand einer Anpassung der Arbeitsorganisation wird insbesondere bei Servicerobotern sowie Dokumentations- und Informationssystemen gesehen.

Abbildung 9: Aufwand Arbeitsorganisation und Überzeugung

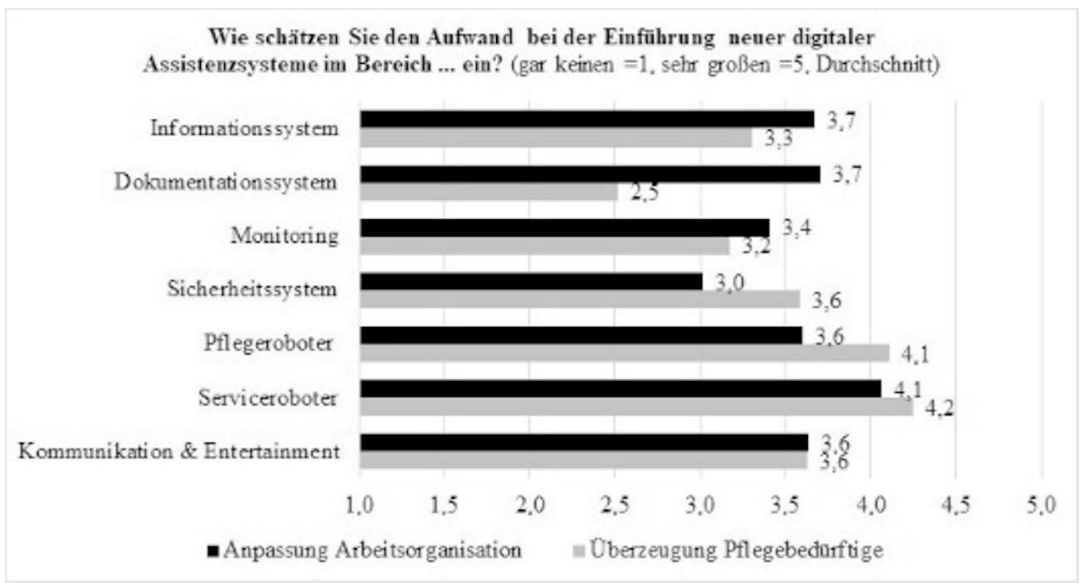

Quelle: Online-Befragung DAAS-KIN 2019

\section{Erwartete Auswirkungen}

Der folgende Abschnitt geht darauf ein, inwieweit und wie sich der Einsatz altersgerechter Assistenzsysteme auf Beschäftigungszahlen, Arbeitsbelastung und Pflegequalität ausgewirkt hat beziehungsweise welche Auswirkungen erwartet werden. Hintergrund sind Hoffnungen in Bezug auf Einsparpotenziale (s.o.).

Zudem werden jeweils die Faktoren, die nach Einschätzung der Befragten aus Sicht des Pflegepersonals für oder gegen den Einsatz digitaler Assistenzsysteme sprechen, themenbezogen diskutiert. Dadurch werden Punkte, die bei Entscheidungen über den Einsatz eine Rolle spielen, sichtbar.

Zusammenfassend werden aus Sicht des Pflegepersonals am häufigsten Probleme bei der Handhabung, Sicherheitsbedenken bei Ausfall der Technik und das Fehlen menschlicher Zuwendung vorgebracht. Fast ebenso häufig werden auch positive Faktoren gesehen, vor allem Arbeitserleichterung, eine reduzierte körperliche Belastung im Pflegealltag (z.B. rückenschonendes Arbeiten), eine reduzierte psychische Belastung im Pflegealltag und der mobile Zugriff auf Daten. Die Ge- 
wichtung von Faktoren aus der rein persönlichen Sicht der Befragten fällt leicht anders aus (siehe Abbildung 18).

\section{Ersatz menschlicher Arbeitskraft}

Die Auswertung stützt sich auf die subjektive Ansicht der Befragten im Hinblick auf den Ersatz menschlicher Arbeitskraft durch die Assistenzsysteme. Hierbei muss berücksichtigt werden, dass diejenigen, die angeben, dass kein derartiges System vorhanden ist, nicht in die Auswertung eingehen. Es zeigt sich, dass nach Aussagen der Befragten menschliche Arbeitskraft bei den meisten Systemen nicht beziehungsweise wenig ersetzt wird (siehe Abbildung 10). Lediglich bei Servicerobotern oder Sicherheitssystemen wird Einsparpotenzial an Arbeitskräften gesehen. Nach der Studie von Braeseke et al. (2020: 70) wird ein verringerter Personaleinsatz bei 12,6 Prozent der vollstationären, 26,4 Prozent der teilstationären und 19,6 Prozent der ambulanten Einrichtungen erwartet.

Abbildung 10: Ersatz menschlicher Arbeitskraft

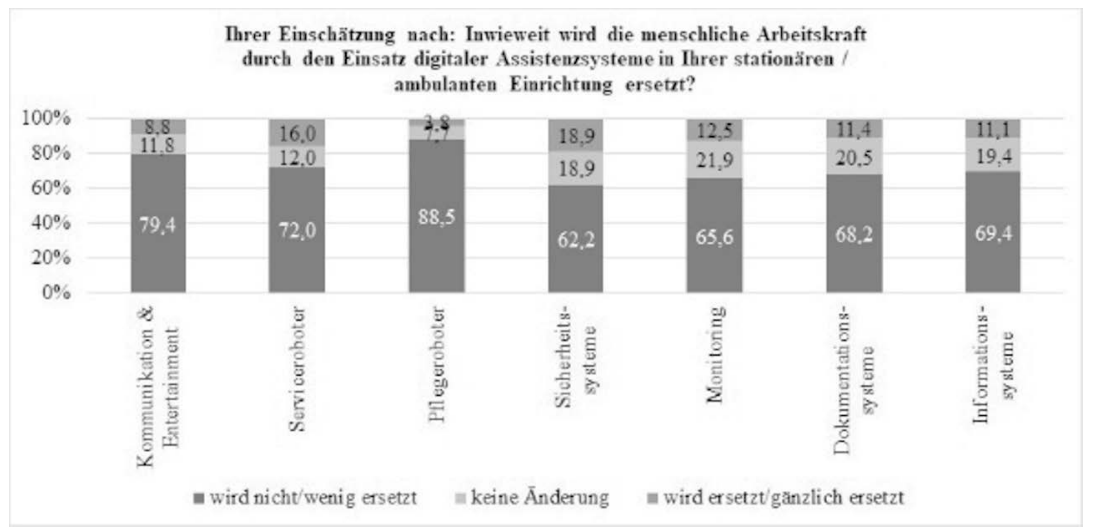

Quelle: Online-Befragung DAAS-KIN 2019

Bei den Gründen, die für oder gegen den Einsatz digitaler Assistenzsysteme aus Sicht des Pflegepersonals sprechen, spielt ein Ersatz menschlicher Arbeitskraft keine große Rolle. Von den Führungskräften wird ein Personalabbau relativ selten erwartet, wobei 26,5 Prozent einen Personalabbau als Argument gegen digitale Assistenzsysteme anführen. 5,9 Prozent sehen auch den Vorteil eines vermehrten Einsatzes gering qualifizierter Arbeitskräfte. 


\section{Arbeitsbelastung}

Eine Veränderung der Arbeitsbelastung wird bei Kommunikationssystemen sowie Servicerobotern nicht gesehen, weder nimmt diese $\mathrm{zu}$ noch ab (siehe Abbildung 11). Bei Pflegerobotern und Sicherheitssystemen sowie auch Monitoring und Dokumentationssystemen hingegen wird aus Sicht der Führungskräfte der höchste positive Effekt auf die Arbeitsbelastung erwartet.

Abbildung 11: Auswirkung aufArbeitsbelastung

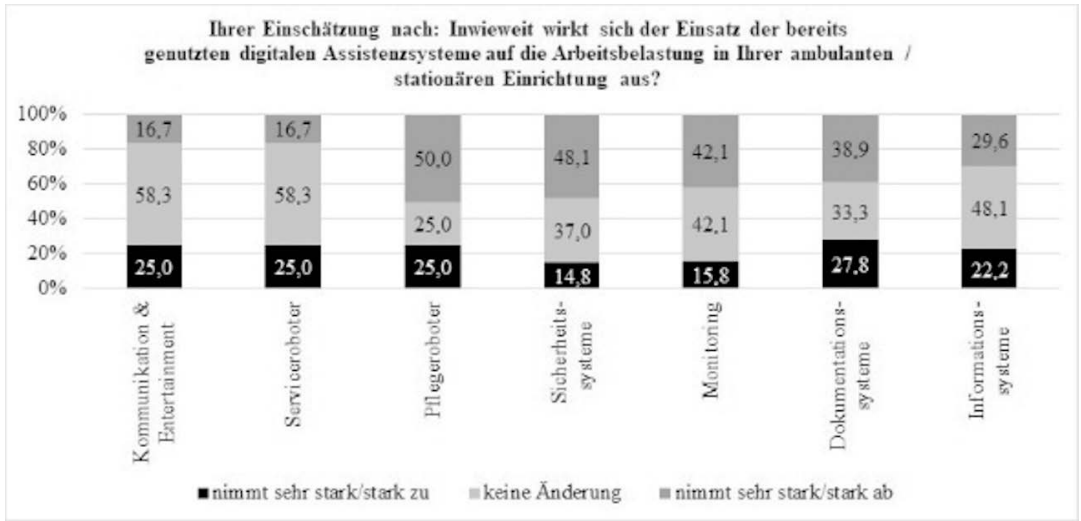

Quelle: Online-Befragung DAAS-KIN 2019

Im Zusammenhang mit der Arbeitsbelastung des Personals werden von den Führungskräften häufig Vorteile gesehen, wie Arbeitserleichterung (58,8\%), reduzierte körperliche Belastung (57,4\%), reduzierte psychische Belastung (57,4\%), während 35,3 Prozent einen komplizierteren Berufsalltag als Nachteil sehen.

\section{Pflegequalität}

Bei der Einschätzung der Auswirkungen auf die Pflegequalität zeigt sich, dass beim Einsatz von Service- und Pflegerobotern mit einer Verschlechterung gerechnet wird, wohingegen den anderen Systemen in der Tendenz Verbesserungen zugeschrieben werden. Dies gilt vor allem für Sicherheits- und Dokumentationssysteme und Monitoring. Hierbei ist zu berücksichtigen, dass sich bei den RobotikSystemen die Auswertung auf relativ wenige Fälle bezieht (siehe Abbildung 12).

Negative Auswirkungen auf die Qualität der Pflege befürchten insgesamt 44,1 Prozent. Generell würden viele Führungskräfte aus Sicht des Personals dennoch Vorteile sehen, darunter mehr Zeit für Pflegebedürftige $(47,1 \%)$, Un- 
Abbildung 12: Pflegequalität

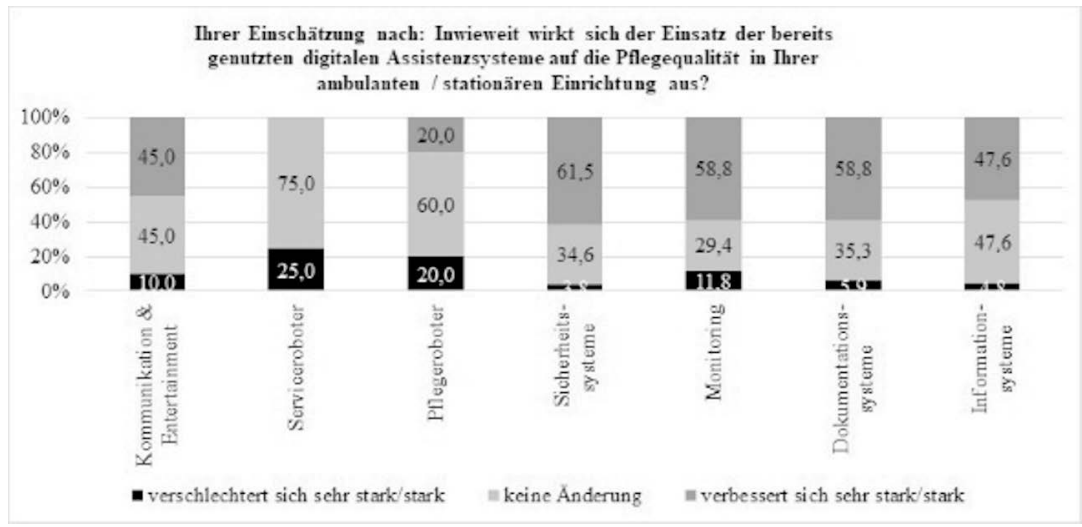

Quelle: Online-Befragung DAAS-KIN 2019

terhaltungsangebot für Pflegebedürftige $(48,5 \%)$ und individuelle Betreuung $(30,9 \%)$.

\section{Patient*innenwohl}

Die Beurteilung hinsichtlich des Patient*innenwohls fällt nach Aussagen der Führungskräfte positiv aus (siehe Abbildung 13). Besonders gut in diesem Zusammenhang werden Sicherheits- und Monitoring-Systeme beurteilt. Lediglich Pflegeroboter werden hier gleichermaßen sehr schlecht/schlecht oder sehr gut/gut bewertet.

Die Vor- und Nachteile im Hinblick auf das Wohl der Patient*innen werden ebenso ambivalent eingeschätzt. Einerseits wird eine Reduktion patient*innengefährdender Situationen (41,2\%) und eine Förderung sozialer Kontakte (17,6\%) positiv gesehen, andererseits wird ein Fehlen menschlicher Zuwendung (64,7\%) erwartet.

\section{Nützlichkeit und Bedienungsfreundlichkeit}

Effizienz, Nützlichkeit wie auch Bedienungsfreundlichkeit spielen eine große Rolle für den Einsatz digitaler Assistenzsysteme. Ein Test, nach dem Technology Acceptance Model (Davis 1993) zeigt, dass wahrgenommene Nützlichkeit den höchsten Effekt auf die Nutzung hat. Eine qualitative Befragung professionell Pflegender in der Altenhilfe bestätigt Nützlichkeit und Einfachheit als zentrale Kategorien der Ak- 
Abbildung 13: Patient*innenwohl

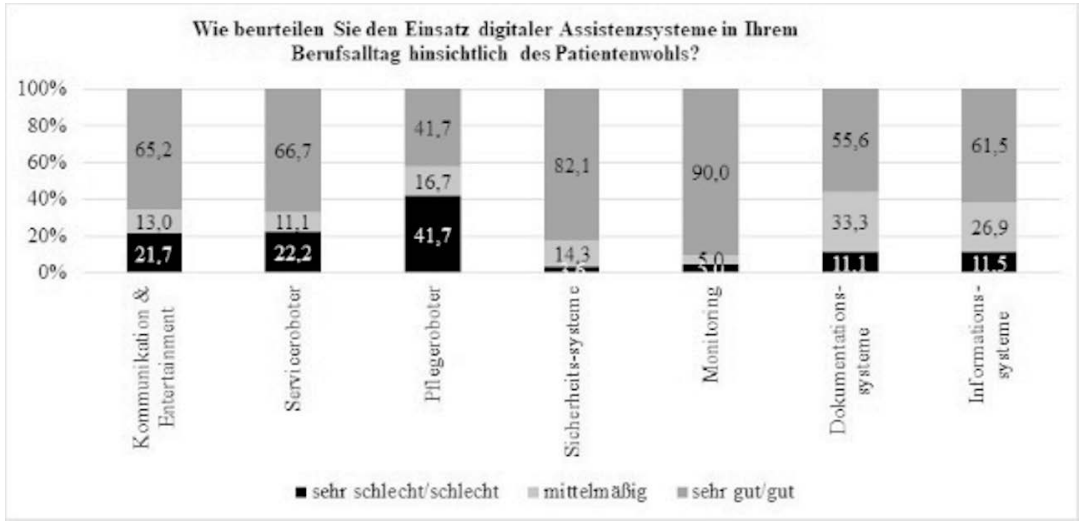

Quelle: Online-Befragung DAAS-KIN 2019

zeptanz neben anderen (Schlomann 2020). Allerdings liegen sehr wenige Studien zur Nutzung altersgerechter Assistenzsysteme vor (vgl. Vetter/Cerullo in diesem Band). Studien zur Praxistauglichkeit sind allgemein selten, da meist experimentelle Wirksamkeitsstudien mit geringer Fallzahl durchgeführt werden (Krick et al. 2019). Effizienzstudien zeigen häufig geringe Evidenz. Hohe Evidenz lässt sich vor allem für Informations- und Kommunikationstechnik oder Roboter zeigen (ebd.).

\section{Effizienz und Nützlichkeit}

Die Effizienz und Nützlichkeit der Systeme werden von den Führungskräften mit Ausnahme der Pflegeroboter als gut oder sehr gut bewertet. Monitoring- und Sicherheitssysteme werden in sehr hohem Maße als effizient und nützlich gesehen. Dies gilt nur für die Hälfte der Befragten in Bezug auf Pflegeroboter. Auch bei Kommunikations- und Entertainmentsystemen oder Servicerobotern ist die Effizienz und Nützlichkeit aus Sicht von Teilen der Führungskräfte eingeschränkt (siehe Abbildung 14).

Als vorteilhaft sehen Führungskräfte den mobilen Zugriff auf Daten (55,9\%), die Unterstützung bei der Archivierung (45,6\%), weniger Dokumentationsaufwand $(42,6 \%)$ und Zeitgewinn (41,2\%), während 13,2 Prozent als negative Auswirkung eine Standardisierung der Arbeitsschritte nennt. 
Abbildung 14: Effizienz und Nützlichkeit

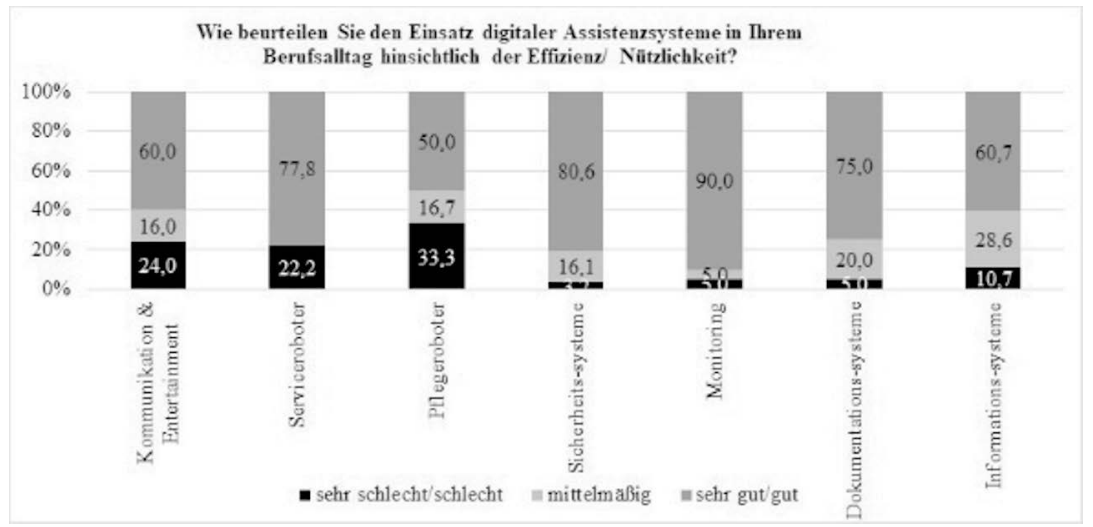

Quelle: Online-Befragung DAAS-KIN 2019

\section{Benutzungsfreundlichkeit}

Die Benutzungsfreundlichkeit wird unterschiedlich bewertet. Hierbei liegt die Bewertung der Service- und Pflegeroboter im negativen Bereich, wohingegen Sicherheitssysteme, Monitoring und auch Informationssysteme mehrheitlich als gut oder sehr gut beurteilt werden (siehe Abbildung 15).

Abbildung 15: Benutzungsfreundlichkeit

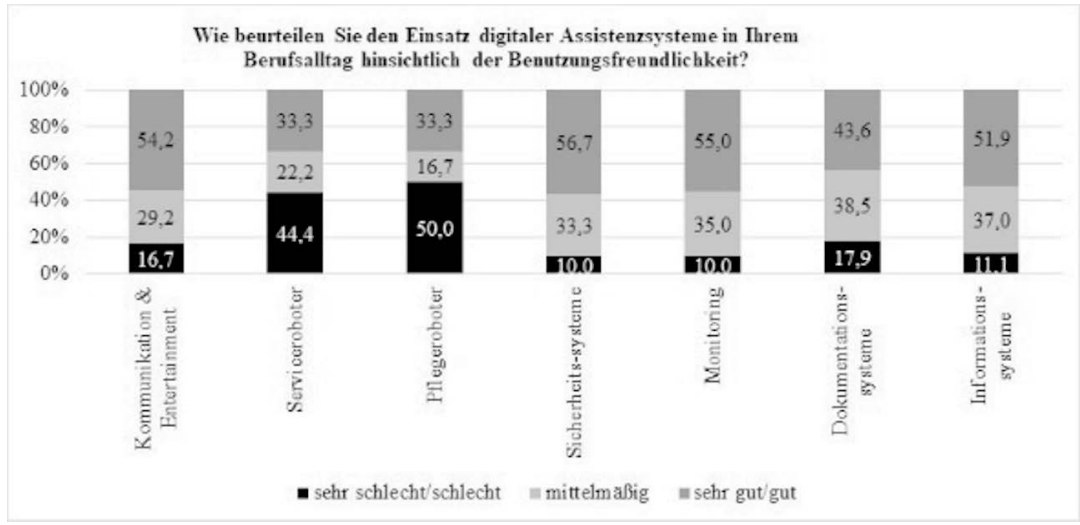

Quelle: Online-Befragung DAAS-KIN 2019 
Als Grund für den Einsatz digitaler Assistenzsysteme nennen Führungskräfte eine einfache Funktionsweise (20,6 \%) ebenso wie bessere Schulungsmöglichkeiten für das Personal (20,6\%). Allerdings sehen 69,1 Prozent beim Personal Probleme bei der Handhabung und 61,8 Prozent geben eigene fehlende Kenntnisse im Umgang mit digitalen Assistenzsystemen an.

\section{Einstellungen und Reaktionen}

Die persönliche Einstellung zur Nutzung stellt einen wichtigen Aspekt dar, denn Führungskräfte in der Pflege sind als Entscheidungsinstanzen mitverantwortlich für die Einführung digitaler Assistenzsysteme (vgl. Scorna in diesem Band). Zunächst wird auf die Einschätzung der Führungskräfte aus Sicht des Pflegepersonals und die von ihnen erwarteten Reaktionen von Pflegepersonal und Pflegebedürftigen eingegangen.

Zusammenfassend wurde nach Begründungen für und gegen den Einsatz digitaler Assistenzsysteme aus Sicht des Pflegepersonals gefragt. Fast alle Befragten nennen Gründe, die aus Sicht des Pflegepersonals für den Einsatz digitaler Assistenzsysteme sprechen. Hierbei stehen Arbeitserleichterung, reduzierte körperliche und psychische Belastung und auch der mobile Zugriff auf Daten im Vordergrund. Weiterhin wird auch das Unterhaltungsangebot für Pflegebedürftige sowie mehr Zeit beziehungsweise Zeitgewinn angemerkt. Neben der Unterstützung bei Archivierung und Dokumentation werden auch die Reduzierung gefährlicher Situationen für Patient*innen und die Erfassung von Vitalwerten als Grund genannt. Kostensenkung oder vermehrter Einsatz gering qualifizierter Arbeitskräfte werden hingegen selten angesprochen.

\section{Erwartete Reaktion}

Nach Ansicht der Befragten reagiert das Pflegepersonal durchweg positiver als die Pflegebedürftigen auf den Einsatz digitaler Assistenzsysteme (vgl. Braeseke et al. 2020: 68). Dies gilt, obgleich der Aufwand zur Motivation des Personals als höher eingeschätzt wird als der Aufwand, die Pflegebedürftigen zu motivieren (siehe Abbildungen 8 \& 9). Im Durchschnitt reagiert das Personal mittelmäßig positiv auf Kommunikationssysteme, Monitoring und Informationssysteme, tendenziell gut bei Sicherheits- und Dokumentationssystemen und schlecht auf Service- und Pflegeroboter. Die erwartete Reaktion der Pflegebedürftigen wird bei den letztgenannten Arten von Assistenzsystem als noch schlechter eingeschätzt (siehe Abbildung 16). 
Abbildung 16: Erwartete Reaktion Pflegepersonal und Pflegebedürftige

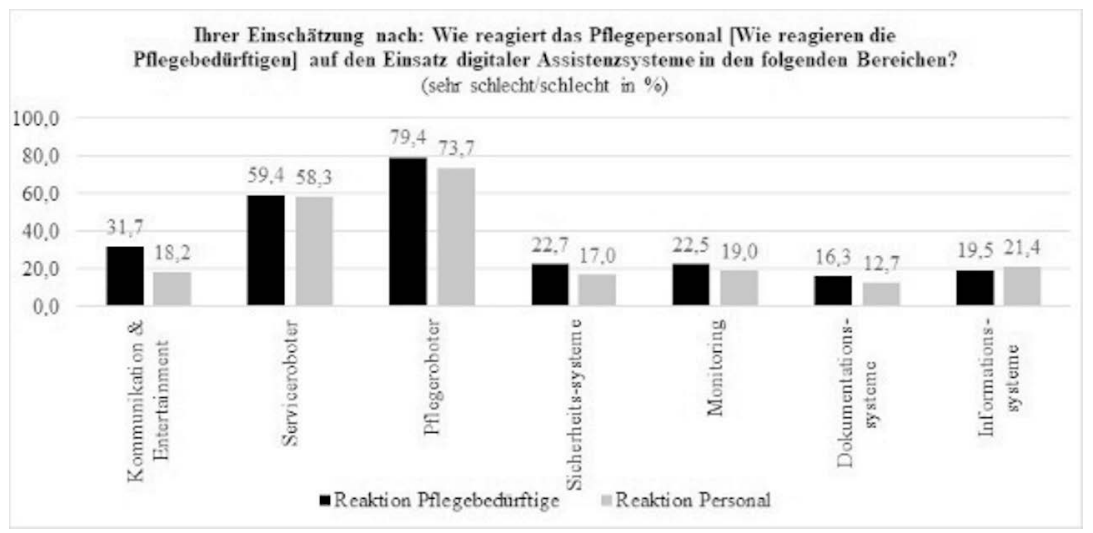

Quelle: Online-Befragung DAAS-KIN 2019

\section{Persönliche Einstellung}

Die Online-Befragung belegt eine deutliche Ablehnung von Robotern im Sinne von Pflege- $(58,5 \%)$ oder Servicerobotern $(36,0 \%)$, wohingegen alle anderen Systeme und besonders der Einsatz in den Bereichen Dokumentation (81,8\%) oder Sicherheit $(79,6 \%$ ) von den Führungskräften befürwortet wurde (siehe Abbildung 17).

Die Einstellung gegenüber den Systemen korreliert signifikant und hoch miteinander (Spearmans' Rho, 5-stufige Skala). Das heißt, wer für die Einführung eines digitalen Assistenzsystems ist, befürwortet auch alle anderen Systeme und umgekehrt, wer gegen die Einführung ist, spricht sich dann auch gegen die Einführung anderer Systeme aus. Eine Ausnahme stellen Pflegeroboter dar. Wer ihnen gegenüber negativ eingestellt ist, lehnt nicht zwangsläufig Monitoring und Sicherheitssysteme ab. Zwischen dem Alter und den Einstellungsmustern bestehen keinerlei Korrelationen. Ein $\mathrm{Chi}^{2}$-Test zeigt zudem keinen signifikanten Zusammenhang zwischen dem Geschlecht und der persönlichen Einstellung zur Einführung digitaler Assistenzsysteme. Lineare Regressionsanalysen mit der abhängigen Variable persönliche Einstellung zeigen weder Alters- noch Geschlechtseffekte.

Gemäß Theorien aus der sozialpsychologischen Einstellungs- und Kognitionsforschung wäre ein positiver Zusammenhang zwischen Nutzung und Einstellung zu erwarten. Die Theorie der kognitiven Dissonanz (Festinger 1957) geht vom Ziel der Vermeidung widersprüchlicher Kognitionen aus. Bei Nutzung eines Systems würde sich demnach eine möglicherweise zu Beginn vorhandene aversive Haltung 
Abbildung 17: Persönliche Einstellung

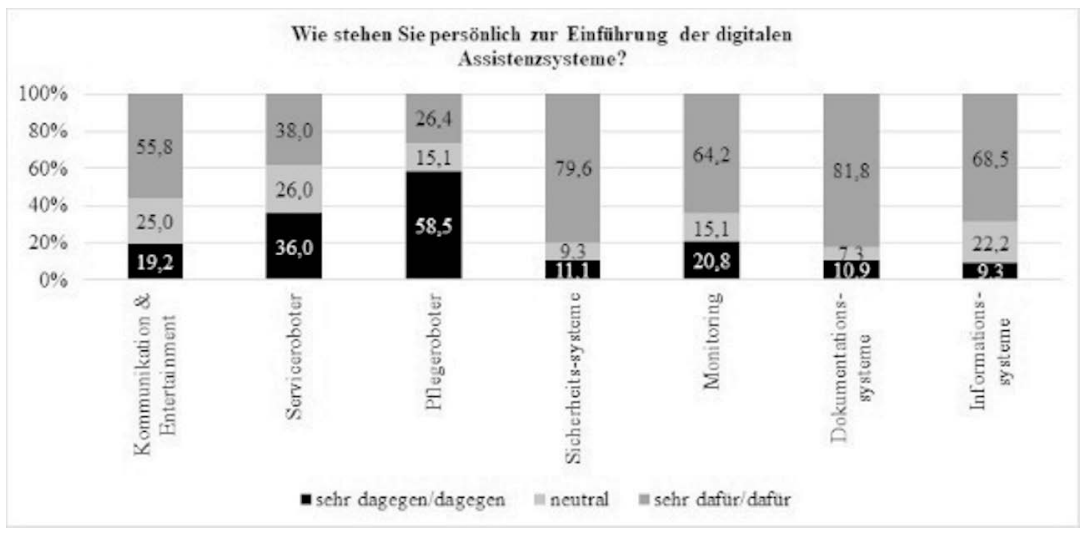

Quelle: Online-Befragung DAAS-KIN 2019

verringern, da das Bestreben besteht, dass Einstellungen konsistent zum Verhalten sind.

Die Einstellungsmuster zeigen die erwarteten Effekte. Werden Pflegeroboter, Sicherheitssystem, Monitoring oder Dokumentationssystem genutzt, sprechen sich die Befragten signifikant häufiger dafür aus, als wenn das System nicht genutzt wird ( $\mathrm{Chi}^{2}$-Test). Dies gilt mit Ausnahme von Kommunikations- oder Informationssystemen, die auch Befragte, die die jeweiligen Systeme nicht nutzen, positiv sehen. Für Serviceroboter lässt sich die Analyse nicht durchführen, da sie in keiner Einrichtung der Stichprobe genutzt werden.

Ergebnisse linearer Regressionsanalysen unter Kontrolle von Alter und Geschlecht bestätigen diesen Befund. Bei Pflegerobotern, Sicherheitssystemen, Monitoring und Dokumentationssystemen und auch bei Kommunikations- und Entertainmentsystemen erhöht die Nutzung signifikant die Akzeptanz. Bei Informationssystemen ist kein signifikanter Effekt zu finden.

Nicht untersucht werden kann, ob eine positive Einstellung zur Einführung eines Systems zu einer häufigeren Einführung führt, wie es gemäß der Theorie des geplanten Verhaltens (Ajzen 1988) und des darauf basierenden Technology Acceptance Models (Davis 1993) zu erwarten wäre. Dieser Analyseschritt wäre in einer Befragung post-facto möglich, wenn bekannt wäre, ob die befragten Führungskräfte über die Einführung entschieden haben.

Neben den Gründen gegen eine Beschaffung (siehe Abbildung 6) und Gründen, die aus Personalperspektive für oder gegen den Einsatz sprechen (siehe themenbezogene Darstellung in den Abschnitten zu Diffusionshemmnissen und erwarteten 
Auswirkungen) wurde schließlich auch nach der ganz persönlichen Sichtweise der Führungskräfte gefragt. Die Rangordnung der Gründe, die bei persönlicher Einschätzung gegen den Einsatz technischer Assistenzsysteme sprechen, (siehe Abbildung 18) unterscheidet sich zum Teil erheblich von den oben aufgeführten Faktoren aus Sicht des Pflegepersonals.

Abbildung 18: Persönliche Gründe gegen Einsatz

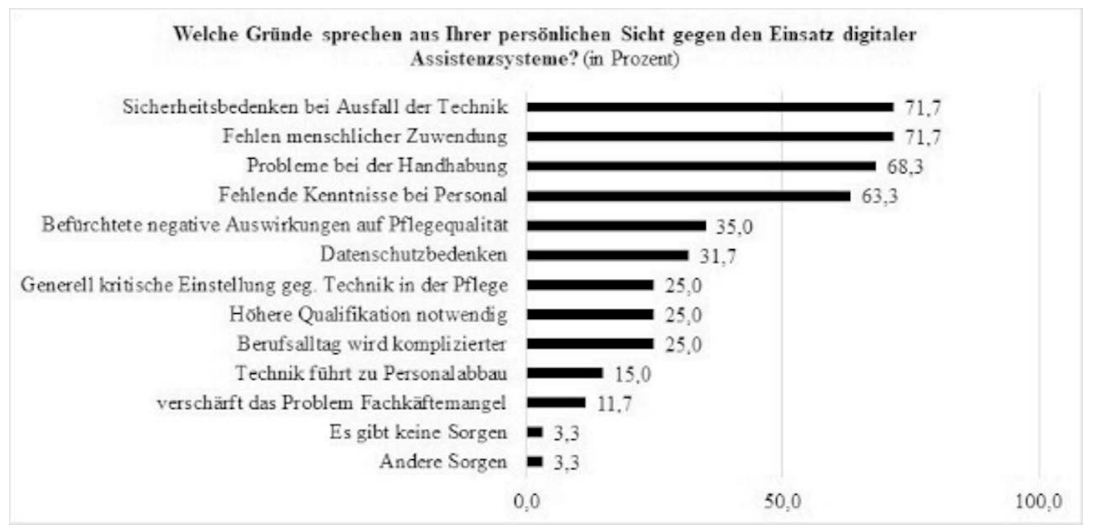

Quelle: Online-Befragung DAAS-KIN 2019

Die größten Befürchtungen aus persönlicher Sicht bestehen zu 71,1 Prozent im Fehlen menschlicher Zuwendung (vgl. Frommeld in diesem Band) und in gleichem Maße in Sicherheitsbedenken bei Ausfall der Technik. Mit geringem Abstand folgen Probleme bei der Handhabung (68,3\%) und fehlende Kenntnisse im Umgang mit digitalen Assistenzsystemen aufseiten des Personals (63,3\%). Als Gründe für einen Einsatz nennen die Befragten, gefragt nach ihrer persönlichen Meinung, die reduzierte körperliche Belastung im Pflegealltag (66,7\%), Arbeitserleichterung $(66,7 \%)$, den mobilen Zugriff auf Daten (61,7\%), weniger Dokumentationsaufwand (56,7\%) und Unterstützung bei der Archivierung (56,7\%), Zeitgewinn beziehungsweise mehr Zeit für Pflegebedürftige (53,3\%) und Unterhaltungsangebote für Pflegebedürftige $(51,7 \%)$.

\section{Zusammenfassung und Diskussion}

Digitale Systeme sind nicht isoliert zu betrachten Die Investitionsplanung eines digitalen Assistenzsystems erhöht die Wahrscheinlichkeit der Anschaffung für jedes andere System. Einrichtungen, die bereits Systeme nutzen, planen häufiger ei- 
ne zusätzliche Investition. Ein Einsatz von Assistenzsystemen geht auch mit einer positiveren Bewertung durch Führungskräfte einher. Positive Einstellungen gegenüber einem System gehen mit positiven Einstellungen gegenüber anderen Systemen einher.

Größtes Potenzial bei Dokumentationssystemen Wie erwartet bestehen große Unterschiede in der Nutzung verschiedener Arten von Assistenzsystemen und die meisten Systeme sind wenig verbreitet. Bei Dokumentations-, Informations- und Sicherheitssystemen und auch Monitoring ist das Einsatzpotenzial nach Ansicht von Führungskräften in der Pflege am höchsten und Diffusionshemmnisse sind am geringsten ausgeprägt. Diejenigen Systeme, die am stärksten in der öffentlichen Diskussion sichtbar sind, die Service- und Pflegeroboter, sind am wenigsten im Einsatz und auch sehr selten geplant. Gegenüber Robotern sind Einstellungsmuster am stärksten durch Aversion geprägt. Die Erwartungen im Hinblick auf Effizienz, Bedienungsfreundlichkeit sowie Akzeptanz durch Pflegebedürftige und Patient*innenwohl fallen am niedrigsten aus.

Häufig im Einsatz und häufig geplant sind digitale Systeme, die zur Dokumentation, aber auch Information, Sicherheit und Monitoring dienen. Die Einstellung der Pflegedienstleitungen dazu ist häufig positiv und es werden sehr viele Vorteile gesehen. Allerdings werden auch Gründe dagegen angeführt, die sich auf Pflegequalität, Qualifikation, Sicherheitsbedenken und befürchtete technische Probleme beziehen.

Geringere Verbreitung in der ambulanten Pflege Es bestätigen sich erwartete Unterschiede zwischen ambulanten und stationären Einrichtungen. Digitale Assistenzsysteme kommen in ambulanten Einrichtungen bisher wenig zum Einsatz und dementsprechend wird dort eine Investitionsplanung mit geringerer Wahrscheinlichkeit durchgeführt. Auch eine Digitalisierungsstrategie existiert in ambulanten Einrichtungen seltener. Im ambulanten Pflegebereich besteht somit der größte Nachholbedarf für die Digitalisierung.

Diffusionshemmnisse liegen in finanziellen und technischen Faktoren und der Akzeptanz von Personal und Pflegebedürftigen Gegen die Beschaffung und den Einsatz digitaler Assistenzsysteme wurde, wie oben aufgezeigt, eine Vielzahl an Gründen vorgebracht. Am häufigsten werden zu hohe Kosten genannt.

Positive Auswirkungen erwartet Aus der Perspektive des Personals sprechen aber auch viele Gründe für einen Einsatz. Es werden viele positive Auswirkungen erwartet, allerdings weniger bei Service-und Pflegerobotik als bei anderen digitalen Assistenzsystemen. 
Einsparpotenzial bei Arbeitskräften gering Beiträge zur Behebung des Fachkräftemangels werden von den befragten Führungskräften durch einen Einsatz weniger gesehen. Digitale Assistenzsysteme erfordern höher qualifiziertes und somit besser bezahltes Personal (IT und Pflege) und kosten zudem Arbeitszeit für Einführung, schulung und Wartung.

Schulungsaufwand bei der Einführung am höchsten Mit der Einführung sind neben direkten Kosten auch indirekte Kosten durch Qualifizierungsmaßnahmen verbunden. Der erwartete Schulungsaufwand übertrifft den Aufwand für alle anderen Aspekte. Hieraus ergeben sich auch Herausforderungen in der (akademischen) Pflegeausbildung.

Ablehnung von Pflegerobotik bei Pflegekräften In Bezug auf die hervorstechenden >Roboter bestehen Hoffnungen für den Abbau von Arbeitsbelastungen, während gleichzeitig starke Vorbehalte gegenüber der entlastenden Technik bestehen. Der Aufwand zur Motivierung der Pflegekräfte wird hoch eingeschätzt und die Befürchtungen richten sich darauf, die Pflegekräfte würden die Technik nicht annehmen. Dies entspricht den Befunden von Zöllick et al. (2020: 216), wonach Pflegekräfte körperliche Entlastung zu schätzen wissen, aber Technikeinsatz im Bereich sozialer oder emotionaler Zuwendung dem professionellen Selbstverständnis widerspricht.

Sicherheit und Bedienungsfreundlichkeit nicht ausreichend Digitale Assistenzsysteme werden zwar als effizient und nützlich betrachtet, jedoch nicht als bedienungsfreundlich und in der Handhabung als problematisch bewertet. Auch bestehen viele Befürchtungen, zum Beispiel Sicherheitsbedenken beim Ausfall der Technik.

Insofern ist die Technik von Assistenzsystemen im Allgemeinen, aus Sicht der Führungskräfte, noch nicht ausgereift. Für die Gestaltung, Gebrauchstauglichkeit und Akzeptanz der Pflegerobotik ist eine nutzer"innenzentrierte Entwicklung hilfreich. Diese unterliegt jedoch vielfaltigen Limitationen, sodass selten Praxistests stattfinden (Klebbe/Eicher 2020). Lösungen aus experimentellen Studien sind (noch) nicht in der Praxis angekommen (vgl. Weber in diesem Band).

Als eine Folge dieser paradoxen Befundlage mit sehr hohen Erwartungen, aber auch sehr vielschichtigen Befürchtungen in den Ergebnissen der Befragung können berechtigte Zweifel geäußert werden, ob die bisher verstärkt geförderten technischen Entwicklungen zukünftig auf die Akzeptanz bei ambulanten und stationären Pflegeeinrichtungen stoßen werden, die notwendig wäre, damit eine flächendeckende Diffusion stattfinden kann. Die hier dargestellte Perspektive der Führungskräfte wurde im Projekt DAAS-KIN ergänzt und vertieft durch qualitative Interviews (vgl. Scorna in diesem Band), wobei die Einstellungen gegenüber digi- 
talen Assistenzsystemen aus individueller, organisationaler und gesellschaftlicher Sicht untersucht wurden (vgl. Frommeld in diesem Band).

\section{Literatur}

Ajzen, Icek (1988): Attitudes, Personality, and Behavior. Milton Keynes: Open University Press.

Baur, Nina/Florian, Michael, J. (2009): "Stichprobenprobleme bei OnlineUmfragen", in: Nikolaus Jackob/Harald Schoen/Thomas Zerback, (Hg.), Sozialforschung im Internet. Methodologie und Praxis der Online-Befragung, Wiesbaden: Springer VS, S. 109-128.

Braeseke, Grit/Pflug, Claudia/Tisch, Thorsten/Wentz, Lukas/PörschmannSchreiber/Ulrike/Kulas, Heidi (2020): Umfrage zum Technikeinsatz in Pflegeeinrichtungen (UTiP): Sachbericht für das Bundesministerium, Berlin: IGES Institut.

Brake, Anna/Weber, Susanne M. (2009): »Internetbasierte Befragung«, in: Stefan Kühl/Petra Strodtholz/Andreas Taffertshofer (Hg.), Handbuch Methoden der Organisationsforschung. Quantitative und qualitative Methoden, Wiesbaden: Springer VS, S. 413-434.

Davis, Fred D. (1993): »User Acceptance of Information Technology: System Characteristics, User Perceptions and Behavioral Impacts«, in: International Journal of Man-Machine Studies 38, S. 475-487.

Fachinger, Uwe (2018): »Altern und Technik: Anmerkungen zu den ökonomischen Potentialen«, in: Harald Künemund/Uwe Fachinger (Hg.), Alter und Technik. Sozialwissenschaftliche Befunde und Perspektiven, Wiesbaden: Springer VS, S. 51-68.

Fachinger, Uwe/Koch, Hellen/Henke, Klaus-Dirk/Troppens, Sabine/Braeseke, Grit/Merda, Meiko (2012): Ökonomische Potenziale altersgerechter Assistenzsysteme. Ergebnisse der »Studie zu Ökonomischen Potenzialen und neuartigen Geschäftsmodellen im Bereich Altersgerechte Assistenzsysteme«. https://partner.vde.com/bmbf-aal/Publikationen/studien/intern/Docum ents/VDE_PP_AAL_\%C3\%96kon.\%20Potenziale_RZ_oB.pdf vom 04.05.2021.

Festinger, Leon (1957): A Theory of Cognitive Dissonance. CA: Stanford University Press.

Haug, Sonja/Frommeld, Debora/Scorna, Ulrike/Weber, Karsten (2021): Technik in der Pflege als Generallösung? Ein kritischer Blick auf altersgerechte Assistenzsysteme aus Sicht von Führungskräften in der Pflege«, in: Monitor Versorgungsforschung 14(1), S. 63-68.

IEGUS (2013): Abschlussbericht zur Studie »Unterstützung Pflegebedürftiger durch technische Assistenzsysteme«. https:/vdivde-it.de/system/files/pdfs/un 
terstuetzung-pflegebeduerftiger-durch-technische-assistenzsysteme.pdf vom 04.05.2021.

Kelle, Udo (2019): »Mixed Methods«, in: Nina Baur/Jörg Blasius (Hg.), Handbuch Methoden der empirischen Sozialforschung, Wiesbaden: Springer Fachmedien, S. 159-172.

Klebbe, Robert/Eicher Cornelia (2020):»Wer sind eigentlich diese Nutzer? Zur Rolle älterer und pflegebedürftiger Erwachsener in der Entwicklung robotischer Assistenzsysteme«, in: Jannis Hergesell/Arne Maibaum/Martin Meister (Hg.), Genese und Folgen der Pflegerobotik, Weinheim: Beltz, S. 220-252.

Klein, Barbara (2019): „Einsatz sozialer Roboter in der Pflege - Roboter als Freund und Begleiter? «, in: Zentrum für Qualität in der Pflege (Hg.), ZQP-Report Pflege und digitale Technik, Berlin: ZQP, S. 84-90.

Krick, Tobias/Huter, Kai/Domhoff, Dominik/Schmidt, Annika/Rothgang, Heinz/Wolf-Ostermann, Karin (2019): »Digital Technology and Nursing Care: A Scoping Review on Acceptance, Effectiveness and Efficiency Studies of Informal and Formal Care Technologies«, in: BMC Health Services Research $19,400$.

Künemund, Harald/Fachinger, Uwe (2018): »Einleitung", in: Harald Künemund/Uwe Fachinger, Alter und Technik. Sozialwissenschaftliche Befunde und Perspektiven, Wiesbaden: Springer VS, S. 9-14.

Mayer, Hanna (2015): Pflegeforschung anwenden: Elemente und Basiswissen für Studium und Weiterbildung, 4., vollständig überarbeitete Auflage. Wien: Facultas-WUV.

Meyer, Sibylle (2016): Technische Unterstützung im Alter - was ist möglich, was ist sinnvoll? Expertise zum Siebten Altenbericht der Bundesregierung. http://nbn -resolving.de/urn:nbn:de:0168-ssoar-49980-9 vom 04.05.2021.

Meyer, Sibylle (2018): $\gg$ Technische Assistenzsysteme zu Hause - warum nicht? Vergleichende Evaluation von 14 aktuellen Forschungs- und Anwendungsprojekten ", in: Harald Künemund/Uwe Fachinger (Hg.), Alter und Technik. Sozialwissenschaftliche Befunde und Perspektiven, Wiesbaden: Springer VS, S. 147-176.

Naumann, Dörte/Schulz, Erika/Geyer, Johannes/Korfhage, Thorben (2014): Versorgungsformen in Deutschland: Untersuchung zu Einflussfaktoren auf die Nachfrage spezifischer Versorgungsleistungen bei Pflege- und Hilfebedarf; ZQPAbschlussbericht. https:/www.zqp.de/wp-content/uploads/Abschlussbericht_ Versorgungsformen_Deutschland.pdf vom 04.05.2021.

Polit, Denise F./Beck, Cheryl T./Hungler, Bernadette P. (2004): Lehrbuch Pflegeforschung: Methodik, Beurteilung und Anwendung, Bern, Göttingen: Huber.

Prein, Gerald/Kelle, Udo/Kluge, Susann (1993): Strategien zur Integration quantitativer und qualitativer Auswertungsverfahren. https://www.sfb186.uni-brem en.de/download/paper19.pdf vom 04.05.2021. 
Schlomann, Anna (2020): »Digitale Technologien in der Altenpflege. Aktueller Technikeinsatz, Technikakzeptanz und Wünsche für digitale Unterstützung aus der Perspektive von professionell Pflegenden«, in: Pflegewissenschaft 2, 22, S. 121131.

Schmidt, Laura/Hans Werner Wahl (2019): "Alter und Technik«, in: Karsten Hank/Frank Schulz-Nieswandt/Michael Wagner/Susanne Zank (Hg.), Alternsforschung. Handbuch für Wissenschaft und Praxis, Baden-Baden: Nomos, S. 537-555.

Schnell, Rainer (2012): Survey-Interviews: Methoden Standardisierter Befragungen. Wiesbaden: Springer VS.

Schnell, Rainer/Hill, Paul. B./Esser, Elke (2018): Methoden der empirischen Sozialforschung, 11. Auflage. München: Oldenbourg.

Schnell, Rainer (2018): Warum ausschließlich >online` durchgeführte Bevölkerungsumfragen nicht >repräsentativ` sind, Working Paper 11. Duisburg: Universität Duisburg-Essen (Research Methodology Group).

VDI/VDE-IT (2011): Technologische und wirtschaftliche Perspektiven Deutschlands durch die Konvergenz der elektronischen Medien. Studie der VDI/VDE Innovation + Technik GmbH in Kooperation mit dem Institut für Gründung und Innovation der Universität Potsdam im Auftrag des Bundesministeriums für Wirtschaft und Technologie. https://www.tomsnetworking.de/uploads/media /20110630_Konvergenzstudie_Studienband.pdf vom 04.05.2021.

Wagner-Schelewsky, Pia/Hering, Linda (2019): »Online-Befragung«, in: Nina Baur/Jörg Blasius (Hg.), Handbuch Methoden der empirischen Sozialforschung, Wiesbaden: Springer VS, S. 787-800.

Weiß, Christine/Lutze, Maxie/Compagna, Diego/Braeseke, Grit/Richter, Tobias/Merda, Meiko (2013): Unterstützung Pflegebedürftiger durch technische Assistenzsysteme. Berlin: Bundesministerium für Gesundheit.

Zöllick, Jan C./Kuhlmey, Adelheid/Suhr, Ralf/Eggert, Simon/Nordheim, Johanna/Blüher, Stefan (2020): »Akzeptanz von Technikeinsatz in der Pflege. Zwischenergebnisse einer Befragung unter professionell Pflegenden«, in: Klaus Jacobs/Adelheid Kuhlmey/Stefan Greß/Jürgen Klauber/Antje Schwinger (Hg.), Pflege-Report 2019, Berlin: Springer Open, S. 201-218. 



\title{
Digitale Technik in der ambulanten und stationären Pflege
}

\author{
Eine Interviewstudie zum Einfluss des Pflegepersonals \\ auf die Einführung digitaler Assistenzsysteme
}

Ulrike Scorna

\section{Hintergrund}

Die Digitalisierung, verstanden als die verstärkte Nutzung neuer Informationsund Kommunikationstechnologien (Traum et al. 2017), durchdringt immer mehr Bereiche des täglichen Lebens, auch solche, die vorrangig auf der zwischenmenschlichen Interaktion basieren und augenscheinlich nur wenig mit Technik in Verbindung stehen, wie dem Bereich der Pflege (Bleses/Busse 2020; Friemer 2020; Hielscher 2020; Huml 2018;). Dass sich die Digitalisierung im Gesundheits- und Pflegesektor auszuweiten scheint, wird durch den Pflegenotstand forciert.

Entsprechend der Prognosen wird die demografische Entwicklung in Deutschland zu einem Wandel der Altersstruktur führen. Die Lebenserwartung steigt und mit ihr auch der Anteil der Alten und Hochbetagten, während gleichzeitig der Anteil junger, erwerbsfähiger Menschen sinkt (StBA 2019). Diese Entwicklung wird die Finanzierung der Pflege vor große Herausforderungen stellen, denn mit dem Anstieg des Anteils Alter und Hochbetagter steigt auch der Anteil der pflegebedürftigen Menschen von derzeitigen knapp 3,7 Millionen (Stand 2018) auf circa 5,9 Millionen Menschen im Jahr 2050 (BMG 2020: 17). Aufgrund der Abnahme des Anteils der erwerbsfähigen jungen Menschen wird auch die Anzahl derer, die in die Kranken- und Pflegeversicherung einzahlen, sinken, wodurch die Pflegekosten auf weniger Beitragszahler*innen umgelegt werden müssen, was zwangsläufig zu einem Anstieg der Beitragszahlungen führen wird (vgl. Bleses/Busse/Friemer 2020; Bowles/Greiner 2012; Werding 2014;).

Neben der Zunahme der Pflegebedürftigen und den Finanzierungsschwierigkeiten ist ein weiterer Aspekt des sogenannten Pflegenotstands der akute Personalund Fachkräftemangel. Schon heute fehlen in der Pflege 25.000 bis 30.000 Stellen (BMG 2018). Bis 2030 wird diese Zahl entsprechend den Prognosen auf mindestens 130.000 Stellen ansteigen (Schwinger et al. 2019: 4, 19). Der Personalmangel ergibt 
sich dabei aus zwei zum Teil aufeinander aufbauenden Problemlagen: Zum einen ist die Arbeitssituation in der Pflege äußerst prekär. Neben geringen Verdienstmöglichkeiten, Arbeiten in Schichtdiensten sowie mangelnden Aufstiegsmöglichkeiten (Golombek/Fleßa 2011) führt der Mangel an Pflegepersonal zu einem gestiegenen Arbeitsvolumen beziehungsweise einer Verdichtung der Arbeitsabläufe für die verbliebenen Mitarbeiter*innen, wodurch die Arbeitsbelastung der einzelnen Pflegekraft, speziell die psychischen und physischen Belastungen, ansteigen (Bleses/Busse 2020: 49; Boll-Westermann et al. 2019). Die Folgen sind ein Anstieg der Teilzeitquote, hohe Personalfluktuationen und krankheitsbedingte Arbeitsausfälle bis hin zu vorzeitigen Berufsaufgabe (Golombek/Fleßa 2011; Bleses/Busse/Friemer 2020). Zum anderen kann gerade auch aus diesen Gründen kein Nachwuchs generiert werden, obwohl die Nachfrage nach Pflegekräften in Anbetracht der Zunahme an Pflegebedürftigen stetig steigt (Golombek/Fleßa 2011; Bleses/Busse 2020). Der dringend benötigte Nachschub an jungen, motivierten Arbeitskräften bleibt folglich aus.

Der Pflegenotstand avanciert somit zu einem der stärksten Argumente für die digitale Transformation im Bereich der Pflege, denn mit der Einführung und Nutzung digitaler Assistenzsysteme verbindet sich nicht nur die Hoffnung auf eine Steigerung der organisatorischen Effizienz, Arbeits- und Pflegequalität sowie Senkung von Pflegekosten, sondern auch die Möglichkeit das Image des Pflegeberufes attraktiver gestalten zu können (Bleses/Busse/Friemer 2020: 1; Evans et al. 2020: 151; Hielscher 2020: 33; Huml 2018).

Derzeit erfolgt die digitale Transformation in der ambulanten und stationären Pflege zumeist in Form von Digitalisierungsprozessen im Bereich der Dokumentation und Pflegeprozessplanung (Bleses/Busse/Friemer 2020; Hielscher 2020), aber auch in den Bereichen der Sicherheits-, Informations-, Kommunikations- und Entertainmentsystemen sowie im Bereich des Monitorings, der Pflege- und Serviceroboter finden digitale Assistenzsysteme bereits vereinzelt Anwendung (Scorna et al. 2021), um einerseits die Selbständigkeit der Pflegebedürftigen zu erhalten und andererseits das Pflegepersonal bei der Arbeit am und mit Pflegebedürftigen zu unterstützen (Huml 2018).

Obwohl die Einführung digitaler Assistenzsysteme oft im Rahmen einer organisatorischen Effizienzsteigerung besonders von den leitenden Führungskräften der Pflegeeinrichtungen begrüßt und initiiert wird (Friemer 2020), ist die Rolle der Pflegekräfte dabei nicht zu vernachlässigen. Denn unabhängig davon, ob die digitalen Assistenzsysteme nur im Hintergrund zur Optimierung von Dokumentations- und Pflegeprozessen laufen oder direkt bei der Pflegearbeit mit und am Pflegebedürftigen eingesetzt werden, führt ihr Einsatz zu Veränderungen der bisherigen Arbeitsabläufe sowie Interaktionsstrukturen und erfordert besonders vom Pflegepersonal neue Handlungsstrategien und fachliche sowie überfachliche Kompetenzen, allen voran der Erwerb technischer Kompetenzen 
und die Bereitschaft zur sinnhaften Auseinandersetzung und Mitgestaltung technischer Innovationen (Bleses/Busse/Friemer 2020: 10; Friemer 2020; Hielscher 2020).

Die Digitalisierung bedeutet einen großen Wandel für die Arbeitswelt im Bereich der Pflege. Dennoch gibt es aktuell nur wenige Studien, die sich mit den Einstellungen der Pflegekräfte hinsichtlich der Einführung digitaler Assistenzsysteme in die Pflege auseinandersetzen, wie beispielsweise die Studie von Kuhlmey et al. (2019), in der die Nützlichkeit, Effektivität und Erwünschtheit des Technikeinsatzes in den vier Pflegefunktionsbereichen (körperliche Unterstützung, soziale und emotionale Unterstützung, Monitoring und Dokumentation) erhoben wurde. Welche Auswirkungen die Nutzung digitaler Assistenztechniken aber speziell auf die Pflegearbeit an sich und das Selbstverständnis der Pflegekräfte haben oder ob es Faktoren gibt, die eine erfolgreiche Technikimplantation in den Pflegealltag begünstigen können, darüber ist nur wenig bekannt (Bleses/Busse/Friemer 2020: 3, Hielscher 2020; Meyer 2016: 18).

An diesem Punkt setzt das Projekt DAAS-KIN (Diffusion altersgerechter Assistenzsysteme - Kennzahlenerhebung und Identifikation von Nutzungshemmnissen) an, um neben der Verbreitung und den Investitionskosten bisher eingesetzter digitaler Assistenzsysteme auch deren Auswirkungen auf die Pflegepraxis und das Pflegepersonal zu untersuchen. Um die Validität und damit auch die Glaubwürdigkeit der Ergebnisse zu erhöhen (Mayer 2015), wird in dem Projekt ein Mixed-Methods-Design angewandt. DAAS-KIN beinhaltet neben einer quantitativen Fragebogenerhebung (vgl. Haug in diesem Band), die vor allem die Verbreitung digitaler Assistenzsysteme, deren Investitionsumfang sowie Auswirkungen auf Beschäftigungszahlen, Arbeitsbelastung und Pflegequalität evaluiert, auch eine qualitative Studie und eine anschließende Diskurs- und modifizierte Wertbaumanalyse (vgl. Frommeld in diesem Band). In dem folgenden Beitrag sollen die Ergebnisse der qualitativen Interviewstudie vorgestellt und diskutiert werden.

\section{Methode und Fragestellung}

Die qualitative Studie erfolgte in Form leitfadengestützter Expert"inneninterviews einerseits mit Vertreter*innen der ambulanten und stationären Pflegeeinrichtungen (Führungskräfte und Pflegepersonal) und andererseits mit Vertreter*innen aus der Forschung und Wissenschaft. Die Proband"innenauswahl erfolgte nach der Methode des Theoretical Samplings durch eine bewusste Zusammenstellung der Stichprobe (Absichtsvolle Stichprobenziehung) auf Basis zuvor festgelegter und für das Forschungsvorhaben relevanter Merkmale (Schreier 2011). Voraussetzung zur Teilnahme an den Interviews war ein direkter Bezug zum Forschungsthema, das 
heißt Praxiserfahrungen mit digitalen Assistenzsystemen, bedingt durch die Ausübung eines Berufes in der ambulanten oder stationären Pflege oder der empirischen Forschung im Bereich des Pflege- und Gesundheitswesens.

Entsprechend der Methode des leitfadengestützten Interviews orientierte sich die Befragung der Proband*innen an einem zuvor erarbeiteten Fragenkatalog, welcher als grobe Orientierung gilt und entsprechend der Gesprächsdynamik sowohl in der Reihenfolge der Fragen variieren als auch um weitere Aspekte, die sich aus der Gesprächssituation ergeben, ergänzt werden kann (Helfferich 2014). Der hohe narrative Anteil ermöglicht es den Proband*innen den Gesprächsverlauf aktiv mitzugestalten und eigene, zuvor nicht angesprochene Aspekte einzubringen. Anders als bei quantitativen Verfahren kann so die inhärente Logik der Proband*innen nachvollzogen und ihr Handeln im Rahmen der Auswertung sinnhaft rekonstruiert werden (Wohlrab-Sahr/Przyborski 2008).

Ausgehend von den leitenden Forschungsfragen nach den medizinischen, pflegerischen, ökonomischen und sozialen Überlegungen sowie moralischen Werten, die die Pflege gestalten, der Frage nach grundsätzlichen Nutzungshürden sowie potenziellen organisatorischen und technischen Vorkehrungen zur Überwindung dieser, waren folgende Themenbereiche Bestandteil des Fragenkatalogs: Fragen zur Einrichtung (Lage, Größe, Trägerschaft), Fragen zur Einstellung und Motivation bezüglich der Assistenzsysteme (konkrete Nutzungserfahrung, Einfluss auf die tägliche Pflegearbeit (Chancen und Hürden), Bedingungen, unter denen eine Nutzung vorstellbar wäre) und Fragen zur Implementierung (notwendig gewordene Vorabmaßnahmen, Reaktionen des Pflegepersonals und der Pflegebedürftigen).

Insgesamt wurden zwölf Interviews im Zeitraum von Oktober 2019 bis Januar 2020 telefonisch durchgeführt und anschließend transkribiert, wovon vier Interviews mit Expert*innen aus Forschung und Wissenschaft stattfanden, drei mit Pflegekräften und fünf mit Vertreter*innen der Pflegedienstleitung. Die durchschnittliche Berufserfahrung der befragten Vertreter*innen aus dem Bereich der Pflege (Pflegepersonal, Pflegedienstleitung) beträgt 19,6 Jahre, die der Expert*innen aus Forschung und Wissenschaft 11,8 Jahre. Die Datenanalyse erfolgte mit MAXQDA nach dem Verfahren des thematischen Kodierens (Flick 2016), einer Methode basierend auf der Grounded Theory. Die einzelnen Transkripte werden zunächst offen, axial und selektiv kodiert und bilden die Grundlage für das sogenannte Kategoriensystem. In diesem Kategoriensystem werden alle relevanten Kern- und Subkategorien entsprechend verschiedener Themenbereiche geordnet. Die systematische Zuordnung ermöglicht einerseits die Veranschaulichung der spezifischen Ausprägungen einzelner Kategorien einer Gruppe und andererseits auch die vergleichende Analyse der kategorialen Ausprägungen mit unterschiedlichen Gruppen, wie beispielsweise im vorliegenden Fall von DAASKIN, ein Vergleich der gruppenspezifischen Ansichten von Pflegefachkräften und Führungskräften hinsichtlich der Kategorie aktuelle Arbeitssituation. 


\section{Ergebnisse}

\section{Ansichten zur aktuellen Situation in der Pflege}

Die Befragten berichten, dass sich die Arbeitsbedingungen in den Pflegeeinrichtungen in den letzten Jahren verschlechtert hätten. Als Ursache hierfür werden vor allem drei Gründe genannt: der Zustand der Bewohner*innen, der akute Personalmangel und das sich daraus ergebende gestiegene Arbeitspensum für das verbliebene Personal.

Bezüglich des Zustands der Bewohner*innen berichten die Befragten, dass diese heute in höherem Maße pflegebedürftig als früher seien. Der Anteil der Schwerstpflegefälle (Bewohner*innen mit einem Pflegegrad drei, vier oder fünf) hätte deutlich zugenommen und der Anteil derer, die rüstig seien und wenig oder gar keiner Pflege bedürften, hätte deutlich abgenommen (PDL_O2). Dem gestiegenen Pflegebedarf steht dabei ein immer größer werdender Mangel an Personal, insbesondere an Pflegefachkräften, gegenüber, wodurch die Versorgung der Pflegebedürftigen stark gefährdet sei (PDL_03, PP_O1, PP_03). Zum einen könnten aufgrund des personellen Engpasses kaum noch Pflegeplätze angeboten werden, zum anderen leide das Personal infolge des gestiegenen Arbeitsvolumens beziehungsweise der Verdichtung der Arbeitsprozesse zunehmend unter Zeitdruck, weshalb es hier vermehrt zu krankheitsbedingten Arbeitsausfällen und insbesondere zu Langzeiterkrankungen kommen würde. Vor allem die psychischen Erkrankungen bei dem Pflegepersonal hätten durch die permanente Überlastung zugenommen (PDL_03, PP_O1).

\section{Aktuelle Einsatzpraxis}

Die Befragten äußerten, dass derzeit in der Pflege meist noch sehr konventionell gepflegt werde und nur wenige digitale Assistenzsysteme tatsächlich eine konkrete Anwendung im Pflegealltag gefunden hätten (E_or). Ein Grund hierfür sei, dass viele Einrichtungen inhabergeführte Betriebe sind, die Digitalisierungsprozesse meist per se ablehnen, beziehungsweise darin keinen Nutzen sehen würden, da es bisher auch so funktioniert hätte (E_03). Auch wären die Unternehmen eher mit den aktuellen Problemen, also dem Fachkräftemangel und der defizitären Versorgungssituation, beschäftigt, weshalb die Einführung neuer Technologien in den Hintergrund treten würde (E_04). Die Auffassung, dass digitale Assistenzsysteme das Potenzial haben, dem Pflegenotstand effektiv zu begegnen, sei innerhalb der Geschäftsleitung der Einrichtungen nur wenig verbreitet (E_O1, PDL_04, PP_O1). Zum anderen wurde zwar in den letzten Jahren im Zuge einer technikgetriebenen Förderpolitik die Entwicklung digitaler Assistenzsysteme von der Politik gefördert, jedoch orientierte sich die Entwicklung dabei eher an den technischen 
Möglichkeiten als an dem tatsächlichen Bedarf der ambulanten und stationären Pflege. Für die pflegerische Kerntätigkeit seien derzeit zu wenig Systeme verfügbar (E_04, PDL_04). Die Befragten geben zudem an, dass es eine große Diskrepanz zwischen der öffentlichen Meinung, was digitale Assistenzsysteme können und bewirken sollen, und dem tatsächlichen Nutzen gäbe und die Resonanz gegenüber digitalen Assistenzsystemen dementsprechend zurückhaltend sei (E_04).

Die Befragung hat gezeigt, dass in den Fällen, in denen digitale Assistenzsysteme in der ambulanten oder stationären Pflege Anwendung finden, es sich vorrangig um Assistenzsysteme aus den Bereichen der Sicherheitssysteme (z.B. Sensormatten, Fluchtuhren, Hausnotrufsysteme), Dokumentation (z.B. elektronische Pflegeakte, Wunddokumentation, Medikamentenmanagementsysteme), Kommunikation und Entertainment (z.B. Alexa, Tablets mit Spielfunktion, Paro, Wii) sowie Patient*innenmonitoring (Vitaldatenmonitoring, Pulsoximeter) handelt. Digitale Assistenzsysteme, die den Bereichen der Informationssysteme oder Pflege- und Serviceroboter zugehörig sind, finden hingegen nur sehr selten beziehungsweise gar keine Anwendung.

Dass gerade Pflege- und Serviceroboter in der Pflege nur wenig Akzeptanz finden, könnte mit der Auffassung der Befragten begründet werden, dass die Pflege ein individueller und auf Empathie beruhender Prozess sei (PDL_01, PDL_O2, PP_01, PP_O2, PP_03). Der als elementar eingeschätzte persönliche Kontakt zwischen Pflegenden und Pflegebedürftigen dürfe nie ersetzt werden (PDL_02, PP_03). Digitale Assistenzsysteme, die potenziell zu einem Verlust menschlicher Zuwendung führen, beziehungsweise den zwischenmenschlichen Kontakt ersetzen könnten, oder deren Anwendung die Patient*innen irritieren, verunsichern oder gefährden würden, werden daher prinzipiell von den Befragten abgelehnt. Gleiches gilt auch für die Vorstellung, digitale Assistenzsysteme alleine im Umgang mit einem Pflegebedürftigen, das heißt ohne die zusätzliche Anwesenheit von Pflegepersonal, einzusetzen.

\section{Implementierung und erste Reaktionen}

Die Gründe, die laut den Befragten tatsächlich zu einer Implementierung digitaler Assistenzsysteme geführt haben, resultierten oft aus einer organisatorischen Notwendigkeit, um einerseits das Personal in Anbetracht des hohen Arbeitspensums zumindest punktuell zu entlasten und andererseits die Arbeitsorganisation und Arbeitsabläufe effizienter gestalten zu können. Die Aufgeschlossenheit der Geschäfts- und Pflegedienstleitung gegenüber technischen Innovationen sei dabei entscheidend, ob und in welchem Umfang digitale Assistenzsysteme implementiert beziehungsweise überhaupt in Erwägung gezogen werden (E_01, E_02, E_03).

Die Befragten berichten, dass die Implementierung oft mit Schwierigkeiten verbunden war, weshalb verschiedene Vorab- und Begleitmaßnahmen notwendig 
wurden. Die ergriffenen Maßnahmen bezogen sich dabei meist auf das Personal (Schulung und Einbindung der Mitarbeiter*innen) und die Integration der zu implementierenden digitalen Assistenzsysteme in die bestehende Informationstechnik (IT) der Einrichtung (Softwareanpassungen, Pretest, Ankauf weiterer Technik) (PDL_01, PDL_02, PDL_03, PDL_04, PDL_05, PP_02).

Wie die Befragten berichten, hätten einige Mitarbeiter*innen anfangs Skepsis gegenüber den digitalen Assistenzsystemen gehabt, welche vor allem auf Berührungsängste und Unsicherheiten im Umgang mit den Systemen zurückzuführen und besonders bei älteren, nicht technikaffinen Mitarbeiter*innen ausgeprägt gewesen sei (PDL_05, PP_02). Die Skepsis hätte aber nach einer Weile nachgelassen (PDL_04). Prinzipiell ist die Reaktion des Pflegepersonals und der Pflegebedürftigen größtenteils positiv ausgefallen. Keiner der Befragten berichtet davon, dass digitale Assistenzsysteme kategorisch abgelehnt würden.

\section{Auswirkungen durch die Nutzung}

Den Befragten zufolge habe die Nutzung der digitalen Assistenzsysteme größtenteils zu einer Arbeitserleichterung beziehungsweise zu einer Senkung der Arbeitsbelastung beigetragen, da die verwendeten Systeme zu einer psychischen und physischen Entlastung des Pflegepersonals, zu einer smarteren Arbeitsorganisation und zu Zeiteinsparungen geführt hätten. Digitale Assistenzsysteme aus dem Bereich des Monitorings hätten beispielsweise das Pflegepersonal durch die Bereitstellung zusätzlicher Patient*inneninformationen sowie den mobilen Zugriff darauf bei der situativen Entscheidungsfindung effektiv unterstützt. Dadurch konnte das Personal besonders in Notfällen schneller reagieren, was zu mehr Handlungssicherheit und zu einer psychischen Entlastung geführt habe. Auch die häufigen, vor allem nächtlichen Kontrollgänge konnten durch digitale Assistenzsysteme aus dem Bereich des Monitorings reduziert werden (PDL_02, PP_03). Bei demenziell erkrankten Pflegebedürftigen, die eine Tendenz zum Weglaufen zeigten, konnte durch die Verwendung von globalen Positionsbestimmungssystemen (GPS), zum Beispiel durch das Tragen von GPS-Armbändern, die Gefahr des Weglaufens früher erkannt und damit die zum Teil zeitlich und psychisch sehr aufwendigen Suchaktionen verhindert werden (E_O1, E_02, PDL_02, PDL_04, PDL_05). Die Befragten berichten, dass einige der Pflegefachkräfte anfangs den neuen digitalen Assistenzsystemen nicht vertraut hätten, wodurch diese zunächst zu keiner Entlastung im Arbeitsablauf geführt haben, da beispielsweise die nächtlichen, stündlichen Kontrollgänge weiterhin unternommen wurden (PP_O2). Letztendlich habe aber das Vertrauen in die Technik zugenommen, wodurch auch die nächtlichen, persönlich durchgeführten Anwesenheitskontrollen reduziert werden konnten, was von den Pflegefachkräften als allgemeine Entlastung wahrgenommen wurde. 
Besonders häufig erwähnten die Befragten, dass durch die Verwendung der digitalen Assistenzsysteme Zeit vor allem bei administrativen Tätigkeiten, wie der Dokumentation und Archivierung, eingespart werden konnte, wodurch der empfundene Stress abgenommen habe (E_03, PDL_01, PDL_03, PDL_04). Durch eine automatische Spracherkennungssoftware konnten beispielsweise Patient*innendaten schneller eingepflegt werden, da sie zeitnah diktiert und nicht mehr im Nachgang händisch eingetragen werden mussten (PDL_03). Auch habe der Einsatz der digitalen Assistenzsysteme zu einer besseren Arbeitsorganisation geführt, da beispielsweise in der elektronischen Patient*innenakte der Medikationsplan oder die Wunddokumentation sofort verfügbar war, was auch die Kommunikation und Zusammenarbeit mit dem behandelnden ärztlichen Personal verbessert habe (E_02, PDL_01, PDL_03). Dem gegenüber wurde negativ erwähnt, dass digitale Assistenzsysteme oft auch (zusätzlichen) Lärm verursachten, was als belastend empfunden wurde (PP_03).

Hinsichtlich der Pflegequalität gaben die Befragten an, dass digitale Assistenzsysteme die Patient*innenversorgung verbessert hätten, da sie für mehr Sicherheit sorgen würden (PDL_01, PDL_04, PP_02, PP_03). So würden beispielsweise durch die Anwendung der digitalen Assistenzsysteme Fehlerquellen minimiert werden, was für mehr Handlungssicherheit aufseiten des Personals gesorgt hätte (PDL_O1). Zudem konnten patient*innengefährdende Situationen, wie beispielsweise die stetig drohende Sturzgefahr aus dem Bett, durch Fallsensoren reduziert werden (PDL_04). Von den Befragten wurde in diesem Kontext negativ erwähnt, dass durch die Verwendung von digitalen Monitoringsystemen zwar die patient*innengefährdenden Situationen reduziert werden konnten, die Verwendung dieser Systeme aber gleichzeitig auch dazu geführt habe, dass weniger Kontrollgänge nötig wurden, wodurch die körperliche Ko-Präsenz und die Interaktion zwischen Pflegenden und Gepflegten abgenommen hätten (E_O1). Im Allgemeinen habe aber die Anwendung der digitalen Assistenzsysteme bei den Pflegebedürftigen zu einer Steigerung des Wohlbefindens geführt, da dem Pflegepersonal dadurch, dass Zeit bei administrativen Tätigkeiten eingespart werden konnte, nun mehr Zeit für die Pflegebedürftigen zur Verfügung stand und manche Systeme auch über ein Entertainmentangebot verfügen würden (PDL_O1, PDL_O2, PDL_04).

Obwohl theoretisch durch die Verwendung digitaler Assistenzsysteme Mitarbeiter*innen zum Beispiel im Bereich der Verwaltung eingespart werden könnten, führte in der Praxis keiner der verwendeten Systeme zu irgendeiner Art von Personaleinsparungen (E_02, E_04, PDL_04). Im Gegenteil: Dadurch, dass die Einrichtungen verstärkt digitale Assistenzsysteme eingesetzt hätten, seien sie für potenzielle Arbeitnehmer*innen attraktiver geworden, wodurch es in einigen Fällen $\mathrm{zu}$ vermehrten Initiativbewerbungen gekommen sei (PDL_05). Auch würde das in der öffentlichen Diskussion oft skizzierte Szenario der durch digitale Assistenzsysteme ersetzten Pflegekräfte keiner Realität entsprechen. Vielmehr würden die 
Systeme den Mangel an vorhandenem Personal und die daraus resultierenden Probleme (erhöhtes Arbeitspensum, Arbeiten unter Zeitdruck) abmildern, sodass sich die Arbeitsbedingungen für das verbliebene Personal verbesserten (E_03).

\section{Nutzungshemmnisse}

Digitale Assistenzsysteme haben das Potenzial, das Wohlbefinden der*des Pflegebedürftigen zu verbessern sowie im Pflegealltag zu einer Steigerung der Qualität, der Effizienz und des Sicherheitsgefühls beizutragen. Gleichwohl hat die Befragung diverse Gründe offengelegt, die eine Nutzung beziehungsweise die Anschaffung dieser Systeme erschweren.

Entsprechend der Aussagen der Befragten ist einer der meist genannten Gründe, der gegen die Nutzung von digitalen Assistenzsystemen spricht, die Zuverlässigkeit der derzeitigen Systeme beziehungsweise ihre potenzielle Störanfälligkeit sowie die damit verbundenen Sicherheitsbedenken im Falle von Fehlermeldungen oder eines gänzlichen Systemausfalls (E_04, PDL_O1, PDL_02, PDL_05, PP_02, PP_03). Sowohl die Fehlermeldung als auch das Ausbleiben einer Meldung würden zwangsläufig zu einer Fehlbehandlung der pflegebedürftigen Person führen, wodurch deren Gesundheit gefährdet werden würde. Das Vertrauen in die Systeme sei seitens der professionell Pflegenden (Pflegedienstleitung und Pflegepersonal) daher nicht hoch. Generell seien viele der aktuell erhältlichen digitalen Assistenzsysteme auch noch nicht ausgereift und daher nicht für den tatsächlichen Einsatz in der Pflege zu gebrauchen, da beispielsweise die Funktion keine Entlastung im Pflegealltag darstelle oder Akkulaufzeiten zu kurz seien (E_02, PDL_04, PDL_05, PP_03). Auch die Bedienung der digitalen Assistenzsysteme sei oft nicht intuitiv und Nutzer*innenoberflächen meist unübersichtlich, weshalb der Nutzung eine hohe Einarbeitungszeit vorausgehen müsse. Aufgrund der aktuellen Situation in der Pflege fehle es aber gerade an dieser benötigten Zeit und seitens des Personals oft auch am Interesse an einer intensiven Auseinandersetzung mit der digitalen Technik (E_O1, PP_O2, PP_03). Die Integration der digitalen Assistenzsysteme in die bestehende IT-Ausstattung der Pflegeeinrichtungen (E_O2, PDL_O1, PDL_O3) sowie die allgemeine Finanzierung dieser Systeme (E_O1, E_O2, E_O3, PDL_O1, PP_O2, PP_03) stellen ebenfalls weitere Nutzungshürden dar, da die Systeme oft nicht mit der vorhandenen Technik kompatibel sind oder deren Nutzung den Ausbau der vorhandenen digitalen Infrastruktur sowie den Ankauf zusätzlicher Technik bedarf, was mit weiteren Kosten verbunden sei, für die es kein Budget gebe.

Hinsichtlich der Zuverlässigkeit der Systeme, ihrer Handhabung und Integration in die bestehende IT der Pflegeeinrichtungen sind sich die Befragten einig, dass die Entwicklung digitaler Assistenzsysteme noch mehr Zeit benötigt, bis die Systeme in der Praxis effektiv eingesetzt werden können. Die Expert*innen aus Wissenschaft und Forschung betonen, dass derzeit zwischen den Entwickler*in- 
nen und den potenziellen Anwender*innen der digitalen Assistenzsysteme noch zu wenig kommuniziert werde. Oft herrsche seitens der Entwickler*innen ein unzureichendes Verständnis darüber vor, was die tatsächlichen Bedingungen und Anforderungen in der Pflege sind (E_02, E_04).

Wie die Befragung gezeigt hat, ist die Rolle der Anwender"innen, hier speziell des Pflegepersonals und der Pflegedienstleitung, im Kontext der Einführung digitaler Assistenzsysteme in ambulanten und stationären Pflegeeinrichtungen nicht $\mathrm{zu}$ unterschätzen, denn sie sind es letztendlich, die, unabhängig des potenziellen Nutzens der digitalen Assistenzsysteme, aufgrund ihrer Einstellung und Erfahrung mit den Systemen darüber entscheiden, ob und in welchem Umfang diese tatsächlich in der Pflege Anwendung finden. Daher ist ein sehr starkes Nutzungshemmnis die fehlende Akzeptanz des Pflegepersonals, welche vor allem auf deren Berufsethos (E_01, PDL_05, PP_02), fehlende Technikkompetenz (E_01, E_02, PDL_05) und Mitbestimmungsmöglichkeiten (E_o1, PP_o1), aber auch auf einen Mangel an Schulungsangeboten und Ansprechpartner*innen (E_02, PP_03) zurückzuführen ist. So weisen die Befragten auf die beim Pflegepersonal weit verbreitete Ansicht hin, dass die Pflege eine interaktive Arbeit ist, die auf menschlichen Beziehungen basiere, bei der jeglicher Einsatz von Technik hinderlich sei. Die Grundeinstellung gegenüber einer Technikimplementierung sei daher meist negativ, da kein Nutzen oder Vorteil darin erkannt werde (E_O1, E_02). Die Zustimmung zur Nutzung digitaler Assistenzsysteme sei bei technikaffinen Mitarbeiter*innen meist höher, da ihnen der Umgang mit Technik leichter fiele und sie die Systeme daher eher als Unterstützung empfinden würden (PDL_05). Mitarbeiter*innen, die Berührungsängste mit neuen Technologien haben oder denen der Umgang mit Technik im Allgemeinen schwerer fällt, würden sich eher gegen eine Nutzung aussprechen, da die Anwendung für sie eine zusätzliche Arbeitsbelastung darstellt, ungeachtet der potenziellen arbeitserleichternden Effekte, die durch das digitale Assistenzsystem möglich wären (E_04, PDL_01, PDL_03, PP_02). Die Motivation zur Nutzung der Systeme auch vor dem Hintergrund, dass kaum Schulungen in dem Bereich angeboten würden, sei dementsprechend gering. Bedenken hinsichtlich der Datennutzung, des Datenschutzes (PDL_03, PDL_04) sowie Unsicherheiten bei rechtlichen (E_O1, PDL_O1) und bürokratischen Vorgaben (PDL_03, PP_03) sind zusätzliche Gründe, die seitens des Pflegepersonals und der Pflegedienstleitung gegen eine Nutzung der digitalen Assistenzsysteme sprechen.

\section{Akzeptanzfaktoren}

Auf Basis der Analyse der Nutzungshemmnisse können insgesamt dreizehn Faktoren identifiziert werden, die einen entscheidenen Einfluss darauf haben, ob digitale Assistenzsysteme in der statonären und ambulanten Pflege erfolgreich implementiert und langfristig akzeptiert werden (siehe Abbildung 1). 
Abbildung 1: Akzeptanzfaktoren digitaler Assistenzsysteme in der Pflege

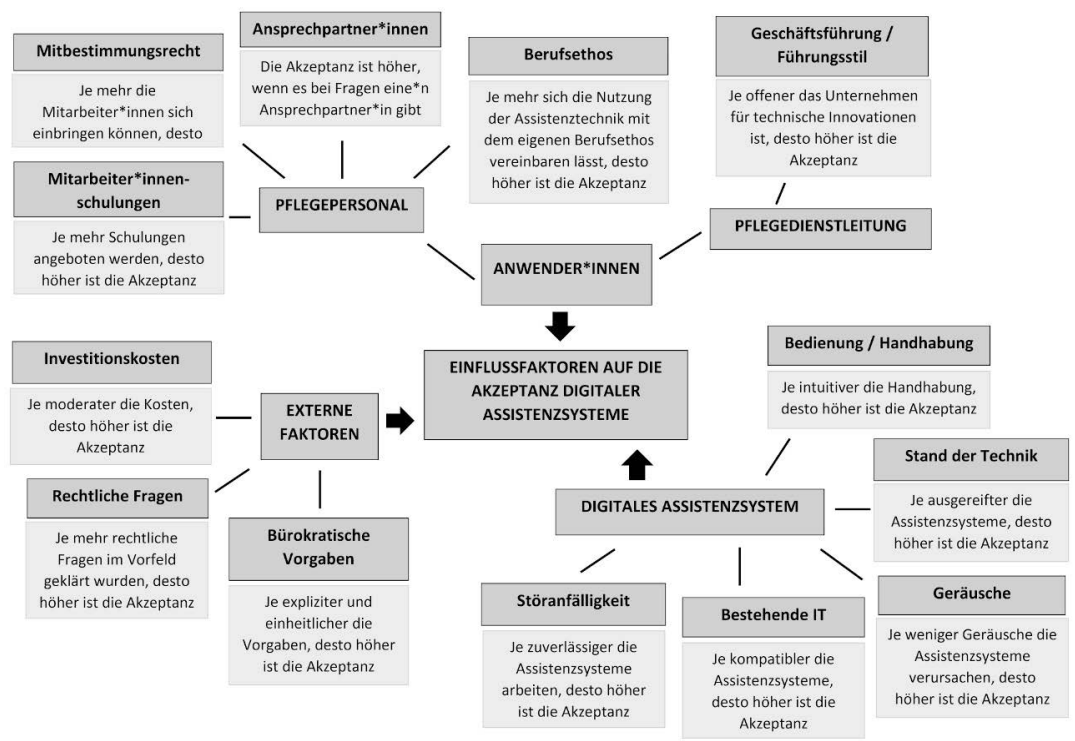

Quelle: Interviewstudie DAAS-KIN 2019

Aus Sicht der Anwender*innen (Pflegepersonal und Pflegedienstleitung) ist die Akzeptanz und damit die Wahrscheinlichkeit einer erfolgreichen Anwendung höher, je offener das Unternehmen für technische Innovationen ist und je mehr das Personal ein Mitbestimmungsrecht hinsichtlich neuer technischer Anschaffungen hat, es eine Ansprechperson bei Problemen gibt, Schulungen angeboten werden, der Nutzen des digitalen Assistenzsystems ersichtlich ist und sich dieser mit dem eigenen Berufsethos vereinbaren lässt. Die persönliche Einstellung der Pflegefachkräfte hat dabei einen entscheidenden Einfluss darauf, ob die implementierten Systeme tatsächlich im Pflegealltag Anwendung finden, um Arbeitsprozesse zu optimieren und das Personal zu entlasten. Denn findet die Implementierung unter der Belegschaft keinen Zuspruch, werden die Systeme gar nicht erst genutzt oder es kommt zu einer Parallelnutzung, das heißt, trotz des Einsatzes der digitalen Assistenzsysteme werden alte Arbeitsabläufe beibehalten, die eigentlich durch die Anwendung der technischen Systeme hätten eingespart werden sollen. Besonders oft äußerten die Befragten, wie wichtig es daher sei, die Mitarbeiter*innen von Anfang an miteinzubeziehen und sie im Umgang mit den Systemen zu schulen, denn ein nutzenorientierter Entwicklungsprozess, der die Bedürfnisse der Pflegenden berücksichtige, und regelmäßige Mitarbeiter*innenschulungen würden auch die 
Bereitschaft des Pflegepersonals erhöhen, digitale Assistenzsysteme im Pflegealltag zu integrieren und Berührungsängste sowie Unsicherheiten minimieren.

Betrachtet man das digitale Assistenzsystem selbst, so ist dessen Akzeptanz höher, je ausgereifter es ist und je zuverlässiger es funktioniert, je einfacher es in bestehende Systeme zu integrieren beziehungsweise je kompatibler es ist, je intuitiver Bedienung und Handhabung sind und je weniger (zusätzliche) Geräusche verursacht werden, da diese als Belastung empfunden werden.

Weitere Einflussfaktoren (externe Faktoren) beziehen sich auf Investitionen sowie rechtliche und bürokratische Vorgaben. Je moderater die Investitionen, je mehr rechtliche Fragen im Vorfeld geklärt werden und je expliziter und einheitlicher die bürokratischen Vorgaben zur Nutzung der Systeme sind, desto höher fällt die Akzeptanz aus und desto wahrscheinlicher ist es, dass die digitalen Assistenzsysteme eine Anwendung in der Pflege finden.

\section{Notwendige Maßnahmen seitens der Politik, Forschung und Pflegekassen}

Hinsichtlich der Maßnahmen, welche die Bedingungen der Implementierung und Nutzung digitaler Assistenzsysteme verbessern würden, sehen die Befragten vor allem Handlungsbedarf in der Politik, bei den Pflegekassen und dem Medizinischen Dienst der Krankenversicherungen (MDK) sowie im Bereich der Forschung.

Seitens der Politik erhoffen sich die Befragten eine Verpflichtung zur Digitalisierung besonders im Bereich der Dokumentation (PDL_03, PDL_04) sowie mehr finanzielle Unterstützung in Form vorgegebener Budgets und Subventionen, damit die Digitalisierung in den Pflegeheimen weiter ausgebaut werden kann, beziehungsweise die Heime sich Investitionen in diesem Bereich leisten können (PDL_04). Ein großes Manko stellen die digitalen Infrastrukturen ländlicher Einrichtungen dar, bei denen die Digitalisierung oft schon am Fehlen zuverlässiger Wireless-LAN-Systeme als Grundvoraussetzung für die Anwendung vieler digitaler Assistenzsysteme scheitere. Zwar gebe es auch schon einige gesetzliche Regelungen, aber die Umsetzung im Bereich der Pflege sei schwierig und einige der Regelungen würden den Pflegealltag verkomplizieren (PDL_O2). So werden beispielsweise einige Dokumentationen derzeit sowohl digital als auch in $\mathrm{Pa}$ pierform benötigt, was einen doppelten Arbeitsaufwand bedeute. Daher sollten bürokratische Hürden abgebaut und Vorgaben, wie die Anforderungen zum Datenschutz, überarbeitet und für den Pflegealltag angepasst werden (PDL_O3, PDL_04).

Einen starken Handlungsbedarf sehen die Befragten aber auch bei den Pflegekassen, von denen sie sich ebenfalls mehr finanzielle Unterstützung erhoffen, da es kaum Fördermittel gebe (PDL_04). Um die digitalen Assistenzsysteme prä- oder refinanzieren zu können, sollten im Pflegesatz die von der Politik vorgegebenen Budgets berücksichtigt werden, beispielsweise als Investitionspauschale oder 
monatlicher Sockelbetrag (E_03). Der derzeitige einmalige Digitalisierungszuschuss der Pflegekassen von maximal 12.000 Euro würde nach Einschätzung der Befragten nicht ausreichen (E_O2, PDL_01). Auch soll der bürokratische Aufwand, der nötig sei, um den Digitalisierungszuschuss zu beantragen, vereinfacht und die Abläufe beschleunigt werden, damit die Einrichtungen die geplanten Anschaffungen hinsichtlich der Höhe des gewährten Zuschusses besser planen können (PDL_O1). Ebenfalls sollen verstärkt Selektivverträge abgeschlossen werden, um die Patient"innenversorgung zu verbessern (E_O2).

Sowohl von der Politik als auch den Pflegekassen und vonseiten des MDKs erhoffen sich die Befragten mehr Vertrauen besonders in Bezug auf die Abrechnungssicherheit, da der digitalisierten Dokumentation oft unterstellt werde, einen $\mathrm{Ab}$ rechnungsbetrug zu begünstigen. Die Befragten betonen jedoch, dass ein solcher Abrechnungsbetrug auch in Papierform möglich sei und die Digitalisierung diese eher noch verhindern könnte (PDL_03).

Die Befragten gehen stark davon aus, dass die Kommunikations- und Informationstechnologien sich in der Pflege weiter ausbreiten werden. Vonseiten der Forschung erhoffen sie sich daher, dass bei der Entwicklung digitaler Assistenzsysteme noch mehr der Nutzen und die Anwendbarkeit in den Fokus gerückt werden (E_02, E_04) und dass es eine interdisziplinäre Zusammenarbeit zwischen Technikentwickler*innen und Anwender*innen gibt, sodass eine partizipative Technikentwicklung ermöglicht wird (E_OI). Hinsichtlich der Nutzung im Pflegealltag erhoffen sich die Befragten eine einfachere, intuitivere Handhabung der digitalen Assistenzsysteme, ohne dass lange Bedienaktivitäten entwickelt werden müssen (E_02, PP_02). Auch werden mehr Studien gefordert, die den Mehrwert der digitalen Assistenzsysteme in der Praxis evaluieren (E_04).

\section{Erwartungen und Wünsche bezüglich der zukünftigen Entwicklung digitaler Assistenzsysteme}

Auf die zukünftige Entwicklung der digitalen Assistenzsysteme im Bereich der ambulanten und stationären Pflege angesprochen, berichten die Befragten, dass sie erwarten, dass die Entwicklung mehr in Richtung Smarthome, also Sturzmatten und Tablets, gehen wird und weniger in Richtung Robotik, da diese zu kostenund wartungsintensiv sind und derzeit noch zu wenig Mehrwert generieren (E_O3, PDL_04).

Hinsichtlich des Einsatzes der digitalen Assistenzsysteme wünschen sich die Befragten, dass diese vor dem Hintergrund des Personalengpasses vor allem $\mathrm{zu}$ einer (körperlichen) Entlastung der Pflegekräfte führen wird (PDL_O1, PDL_O2, PP_01, PP_02, PP_03) und durch ihre Anwendung Zeit bei Kontrollgängen oder administrativen Tätigkeiten eingespart beziehungsweise der Aufwand vor allem bei der pflegerischen Dokumentation reduziert werden kann (PDL_04, PP_02, PP_03). 
Damit die digitalen Assistenzsysteme aber in der Pflege effektiv genutzt werden können, sei eine einfache und zuverlässige Funktionsweise ( $\left.\mathrm{PP} \_\mathrm{O} 2\right)$ sowie eine Einführung und regelmäßige Schulungen der Pflegekräfte unabdingbar (E_O2, E_O4, PDL_03, PP_03). Generell wünschen sich die Befragten, dass technologische Aspekte schon in der Ausbildung eine größere Rolle spielen, sodass Berührungsängste bei der Technikanwendung im Pflegealltag von vornherein minimiert werden können (E_04).

Die Expert*innen aus dem Bereich der Forschung und Wissenschaft wünschen sich, dass im Zuge der voranschreitenden Digitalisierung der Arbeitswelt sich auch das Selbstverständnis in der Pflege wandelt und die Verwendung digitaler Medien so selbstverständlich wird, wie beispielsweise die Nutzung eines Smartphones zum Telefonieren. Dafür sei es aber notwendig, dass digitale Assistenzsysteme nicht länger als störend oder gar als ein Eingriff in den Pflegeprozess, sondern als Werkzeuge wahrgenommen werden, welche die Pflegekräfte bei ihrer pflegerischen Tätigkeit unterstützen und dazu beitragen, die Pflegequalität im Allgemeinen zu erhöhen (E_02, E_04, PDL_01).

\section{Zusammenfassung und Diskussion}

Die digitale Transformation der Arbeitsabläufe in den ambulanten und stationären Pflegeeinrichtungen ist eine zwiespältige Angelegenheit. Zwar stellt die Nutzung digitaler Assistenzsysteme in Anbetracht der voranschreitenden Digitalisierung der Arbeitswelten und des Pflegenotstandes schon fast eine organisatorische Notwendigkeit dar, um die Versorgung der steigenden Anzahl multimorbider Pflegebedürftiger trotz fehlender Pflegekräfte und Finanzierungsschwierigkeiten der Pflegeleistungen aufgrund der sinkenden Anzahl an Beitragszahlern gewährleisten zu können; von einer euphorischen Stimmung im Zuge der Implementierung kann dennoch vor allem bei dem Pflegepersonal keine Rede sein, denn die Maxime lautet: Technik ja, aber zu welchem Zweck und in welchem Umfang?

Anders als in der öffentlichen Diskussion oft geschildert, ist der Bereich der Pflege noch weit davon entfernt, dass digitale Assistenzsysteme sukzessiv Pflegetätigkeiten übernehmen und Pflegekräfte gar ersetzt werden könnten. Sofern digitale Assistenzsysteme in ambulanten und stationären Pflegeeinrichtungen eine Anwendung finden, handelt es sich derzeit meist um Systeme aus den Bereichen der Sicherheit, Dokumentation, Kommunikation und Entertainment sowie Patient"innenmonitoring; Pflege- und Serviceroboter werden gar nicht beziehungsweise nur sehr selten und dann meist nur in Modellprojekten eingesetzt. Dies entspricht auch den Ergebnissen der Befragung von Führungskräften in der Pflege im Projekt DAAS-KIN (vgl. Haug in diesem Band). 
Dass digitale Assistenzsysteme in der Pflege so wenig Anwendung finden, dafür lassen sich diverse Gründe identifizieren. Neben der Störanfälligkeit der Technik sowie der Höhe der Investitionskosten hat besonders das Pflegepersonal einen Einfluss darauf, ob und in welchem Umfang digitale Assistenzsysteme genutzt werden. Wie die Befragung gezeigt hat, sind die Pflegemitarbeiter*innen zwar prinzipiell digitalen Assistenzsystemen gegenüber positiv eingestellt, jedoch wird dabei oft unterschieden, welche Verwendung die Systeme im Pflegealltag finden. Während digitale Systeme für administrative Tätigkeiten sehr begrüßt werden, stoßen Assistenzsysteme für den direkten Kontakt zu den Patient*innen weiterhin auf Skepsis. Grundlegend hierfür ist das Berufsethos der Pflegenden, wonach der Einsatz von Technik hinderlich für die auf zwischenmenschlichen Beziehungen und Fürsorge basierende Beziehung ist. Dieses Ergebnis entspricht auch der Studie von Kuhlmey et al. (2019:34), wonach Technik zwar in den vier untersuchten Pflegefunktionsbereichen (körperliche Unterstützung, soziale und emotionale Unterstützung, Monitoring und Dokumentation) prinzipiell positiv bewertet, beim Einsatz im Rahmen der sozialen und emotionalen Unterstützung jedoch kritischer bewertet wird. Ob digitale Assistenzsysteme seitens des Pflegepersonals akzeptiert und befürwortet werden, hängt somit vom jeweiligen Setting ab: Ob die Sinnhaftigkeit der Anwendung erschlossen wurde, die Systeme als zuverlässig gelten und sich der Einsatz mit der Berufsauffassung der Mitarbeiter*innen vereinbaren lässt beziehungsweise, ob digitale Assistenzsysteme als ein Werkzeug oder als ein sie ersetzendes Mittel angesehen werden, hat entscheidenden Einfluss.

Wie die Befragung gezeigt hat, ist daher ein wesentlicher Faktor, der zu einer erfolgreichen Nutzung digitaler Assistenzsysteme beitragen kann, die Akzeptanzschaffung innerhalb des Pflegepersonals. Seitens der Geschäfts- und Pflegedienstleitung bedeutet das eine frühzeitige Aufklärung über die Motive der Implementierung und den zu erwartenden Nutzen, denn anders als oft von den Mitarbeiter*innen vermutet sollen durch digitale Assistenzsysteme nicht Pflegekräfte eingespart, sondern die Arbeitsbedingungen für das vorhandene Personal verbessert werden.

Um die potenziellen Berührungsängste und Unsicherheiten im Umgang mit den digitalen Assistenzsystemen zu reduzieren, sind Mitarbeiter*innenschulungen unabdingbar. Auch muss es eine Möglichkeit für die Pflegenden geben, Fragen und Anmerkungen zu den verwendeten Systemen zu äußern. Wie die Befragung gezeigt hat, werden einige der Assistenzsysteme abgelehnt, da sich deren Handhabung nicht intuitiv oder unpraktisch für den Pflegealltag erweist, da beispielsweise das Interface zu komplex ist. Oft sind diese Nutzungshemmnisse leicht zu beheben, würden die Anwendungsprobleme effektiv weitergeleitet und anschließend behoben werden. Auch die Einbindung der Pflegemitarbeiter*innen hinsichtlich der Frage, ob neue digitale Assistenzsysteme angeschafft werden sollen, könnte deren Bereitschaft zur Nutzung erhöhen. 
Es bleibt festzuhalten, dass der Einsatz der digitalen Assistenzsysteme zwangsläufig auch zu einer Veränderung der bisherigen Arbeitsabläufe führt und potenziell zu einer Steigerung der Effizienz sowie der Arbeits- und Pflegequalität beitragen kann. Wenn die Systeme aber keine Akzeptanz bei den Nutzer*innen, speziell dem Pflegepersonal, finden, minimiert dies die Chance auf eine erfolgreiche Implementierung und Anwendung.

\section{Literatur}

Bleses, Peter/Busse, Britta (2020): »Digitalisierung der Pflegearbeit in der ambulanten Pflege: Herausforderungen und Gestaltungsmöglichkeiten guter Arbeitsqualität«, in: Peter Bleses/Britta Busse/Andreas Friemer (Hg.): Digitalisierung der Arbeit in der Langzeitpflege als Veränderungsprojekt. Berlin: Springer Vieweg, S. 49-64.

Bleses, Peter/Busse, Britta/Friemer, Andreas (2020): »Veränderungsprojekte Digitalisierung der Arbeit in der Langzeitpflege. Anforderungen und Gestaltungsoptionen im Rahmen umfassender Veränderungsprozesse«, in: Peter Bleses/Britta Busse/Andreas Friemer (Hg.): Digitalisierung der Arbeit in der Langzeitpflege als Veränderungsprojekt. Berlin: Springer Vieweg, S. 11-32.

Boll-Westermann, Susanne/Hein, Andreas/Heuten, Wilko/Krahn, Tobias (2019): »Pflege 2050 - Wie die technologische Zukunft der Pflege aussehen könnte«, in: Zentrum für Qualität in der Pflege (Hg.): ZQP-Report Pflege und digitale Technik. Berlin: ZQP, S. 10-17.

Bowles, David/Greiner, Wolfgang (2012). "Bevölkerungsentwicklung und Gesundheitsausgaben«, in: Gesundheit und Gesellschaft, 12 (4) (2012), S. 7-17.

Bundesministerium für Gesundheit (BMG) (2020): Zahlen und Fakten zur Pflegeversicherung, vom 17. Februar 2020. https://www.bundesgesundheitsmini sterium.de/fileadmin/Dateien/3_Downloads/Statistiken/Pflegeversicherung/ Zahlen_und_Fakten/Zahlen_und_Fakten_der_SPV_17.Februar_2020_barr.pdf (Abfrage: 05.05.2021).

Bundesministerium für Gesundheit (BMG) (2018): Beschäftigte in der Pflege, vom 20. März 2018. https://www.bundesgesundheitsministerium.de/themen/pfleg e/pflegekraefte/beschaeftigte.html (Abfrage: 05.05.2021).

Evans, Michaela/Ludwig, Christine/Gießler, Wolfram/Breuker, Gertrud/Scheda, Wolfgang (2020): »Digitalisierung für die Altenpflege«, in: Peter Bleses/Britta Busse/Andreas Friemer (Hg.): Digitalisierung der Arbeit in der Langzeitpflege als Veränderungsprojekt. Berlin: Springer Vieweg, S. 151-165.

Flick, Uwe (2016): Qualitative Sozialforschung: Eine Einführung. Reinbek bei Hamburg: Rowohlt Taschenbuch Verlag. 
Friemer, Andreas (2020): »Digitale Technik droht? Bedroht? Wirklich nur? Kompetenzentwicklung in Veränderungsprojekten«, in: Peter Bleses/Britta Busse/Andreas Friemer (Hg.): Digitalisierung der Arbeit in der Langzeitpflege als Veränderungsprojekt. Berlin: Springer Vieweg, S. 135-150.

Golombek, Josephine/Fleßa, Steffen (2011): »Einflussfaktoren auf die Verweildauer im Beruf und die Standortwahl des Arbeitsplatzes bei Gesundheits- und Krankenpflegern«, in: HBScience 2 (1) (2011), S. 3-10.

Helfferich, Cornelia (2014): »Leitfaden- und Experteninterviews«, in: Nina Baur/Jörg Blasius (Hg.): Handbuch Methoden der empirischen Sozialforschung. Wiesbaden: Springer VS, S. 559-574.

Hielscher, Volker (2020): »Digitalisierungsprozesse und Interaktionsarbeit in der Pflege«, in: Peter Bleses/Britta Busse/Andreas Friemer (Hg.): Digitalisierung der Arbeit in der Langzeitpflege als Veränderungsprojekt. Berlin: Springer Vieweg, S. 33-45.

Huml, Melanie (2018): »Digitalisierung in Gesundheit und Pflege. High-Tech für mehr Menschlichkeit«, in: Christian Bär/Thomas Grädler/Robert Mayr (Hrsg): Digitalisierung im Spannungsfeld von Politik, Wirtschaft, Wissenschaft und Recht. Berlin: Springer Gabler, S. 149-154.

Kuhlmey, Adelheid/Blüher, Stefan/Nordheim, Johanna/Zöllick, Jan (2019): »Ressource oder Risiko - Wie professionell Pflegende den Einsatz digitaler Technik in der Pflege sehen«, in: Zentrum für Qualität in der Pflege (Hg.): ZQP-Report Pflege und digitale Technik. Berlin: ZQP, S. 31-37.

Mayer, Hanna (2015): Pflegeforschung anwenden: Elemente und Basiswissen für Studium und Weiterbildung, 4. vollständig überarbeitete Auflage. Wien: Facultas-WUV.

Meyer, Sibylle (2016): Technische Unterstützung im Alter - was ist möglich, was ist sinnvoll? Expertise zum Siebten Altenbericht der Bundesregierung. https: //www.ssoar.info/ssoar/bitstream/handle/document/49980/ssoar-2016-meye r-Technische_Unterstutzung_im_Alter_-.pdf?sequence $=1 \&$ isAllowed $=y \& \ln k n$ ame=ssoar-2016-meyer-Technische_Unterstutzung_im_Alter_-.pdf (Abfrage: 04.05.2021).

Schreier, Margrit (2011): "Qualitative Stichprobenkonzepte«, in: Gabriele Naderer/Eva Balzer (Hg.): Qualitative Marktforschung in Theorie und Praxis: Grundlagen Methoden Anwendungen, 2. überarbeite Auflage. Wiesbaden: Gabler, S. 231-247.

Schwinger, Antje/Klauber, Jürgen/Tsiasiotti, Chrysanthi (2019): »Pflegepersonal heute und morgen«, in: Klaus Jacobs/Adelheid Kuhlmey/Stefan Greß/Jürgen Klauber/Antje Schwinger (Hg.): Pflege-Report 2019. Mehr Personal in der Langzeitpflege - aber woher? Berlin: Springer, S. 3-22.

Scorna, Ulrike/Frommeld, Debora/Haug, Sonja/Weber, Karsten (2021): »Digitale Technik in der Pflege als Generallösung? Neue Perspektiven auf altersgerechte 
Assistenzsysteme«, in: Carolin Freier/Joachim König/Arne Manzeschke/Barbara Städtler-Mach (Hg.): Gegenwart und Zukunft sozialer Dienstleistungsarbeit, Wiesbaden: Springer VS, S. 299-312.

Statistisches Bundesamt (StBA) (2019): Bevölkerung im Wandel. Annahmen und Ergebnisse der 14. Koordinierten Bevölkerungsvorausberechnung. Wiesbaden: Statistisches Bundesamt (Destatis).

Traum, Anne/Müller, Christoph/Hummert, Henning/Nerdinger Friedemann W. (2017): Digitalisierung. Die Perspektive des arbeitenden Individuums. White Paper Series Nr. 1. https:/www.researchgate.net/profile/Christoph-Mueller-2 5/publication/321781442_Digitalisierung_-_Die_Perspektive_des_arbeitenden_ Individuums/links/5a86a8b2a6fdcc6bra36ddco/Digitalisierung-Die-Perspekti ve-des-arbeitenden-Individuums.pdf (Abfrage: 04.05.2021).

Werding, Martin (2014): Demographischer Wandel und öffentliche Finanzen. Langfrist-Projektionen 2014-2060 unter besonderer Berücksichtigung des Rentenpakets der Bundesregierung. Arbeitspapier 01/2014. Sachverständigenrat zur Begutachtung der Gesamtwirtschaftlichen Entwicklung, https://www. sachverstaendigenrat-wirtschaft.de/fileadmin/dateiablage/download/publika tionen/arbeitspapier_01_2014.pdf (Abfrage: 05.05.2021).

Wohlrab-Sahr, Monika/Przyborski, Aglaja (Eds.) (2008): Lehr- und Handbücher der Soziologie. Qualitative Sozialforschung: Ein Arbeitsbuch. München: Oldenbourg. 


\section{Vertrauen, Wissen, Innovation und Wohltun als (neue) Herausforderungen im Kontext digitaler Assistenzsysteme}

Ergebnisse einer Diskurs- und Wertbaumanalyse

Debora Frommeld

\section{My House, My Home, My Destiny: Digitale Technologien und das Zuhause im Alter}

Die Entwicklung altersgerechter Assistenzsysteme und Ambient Assisted Living (AAL)-Technologien ist eng mit dem Ziel verbunden, die Lebensqualität älterer Menschen $\mathrm{zu}$ verbessern und ihnen möglichst lange ein unabhängiges Leben $\mathrm{zu}$ ermöglichen (vgl. Fachinger/Henke 2010; Heinze 2018; Weber in diesem Band). Einen zentralen Stellenwert nimmt das engste persönliche Lebensumfeld, das Zuhause, ein; AAL greift gerade dieses Motiv auf. Betrachtet man die Einbettung des Zuhauses im öffentlichen Diskurs, handelt es sich bei dem Wunsch älterer Menschen, so lange wie möglich selbstbestimmt und unabhängig zu leben (Heinze 2018: 19-20; Wahl/Oswald 2016: 622-628), um keine exklusive, sondern eine generelle Vorstellung eines sguten Lebens und impliziert bei allen Altersgruppen das Bedürfnis nach einem privaten Rückzugsort. So spiegeln zahlreiche Songs, die auf YouTube als Video hochgeladen und millionenfach angeklickt wurden, dieses Bild vom Zuhause bei mehreren Generationen seit Mitte des letzten Jahrhunderts wider. Hier zeigt sich eine altersunabhängige Sicht auf das Zuhause unter den ehemals beteiligten Musiker*innen, Hörer*innen sowie den internet- und musikaffinen Nutzer*innen im digitalen zweiten Jahrtausend. $\mathrm{Zu}$ den prominenten Titeln zählen sowohl ältere wie »Our House« von Crosby, Stills, Nash \& Young (1970) und Madness (1982) als auch jüngere Musikvideos wie »Home« von Michael Bublé (2005), »My House« von Flo Rida (2015) und »Lego House« von Ed Sheeran (2011).

Mit diesem Ort scheinen gewisse Vorstellungen verbunden $\mathrm{zu}$ sein, denn die Wohnung oder das Haus werden in den sozialen Medien und im TV auf Fotografien, in Werbungen und (Kurz-)Filmen mit Gemütlichkeit, Geborgenheit und einer warmen Atmosphäre porträtiert. Auch wenn in Zeiten mehrfacher Lockdowns aufgrund von COVID-19 Ausflüge und Urlaub unmöglich erscheinen, 
scheint die Wunschvorstellung eines schönen Zuhauses als Sphäre der Individualität, als Kraftort und heimelig anmutende Idylle in den Massenmedien und der Populärkultur fest verankert zu sein. Ein anstrengender Arbeitstag im Büro, im Auto oder in der Produktion lässt ein solches Zuhause zu einem Sehnsuchtsort werden. Lange vor der Ära der >Klicks` und >Likes in den sozialen Medien spiegeln die beliebten Heimatfilme, die im deutschsprachigen Raum in der Zeit nach dem Zweiten Weltkrieg produziert wurden, dieses Image des Zuhauses oder Nach-Hause-Kommens auch regional wider.

In westlichen Industriegesellschaften mit einem hohen Grad an Individualisierung, zu denen Deutschland zählt, ist es gerade die Intimsphäre und Privatheit, die Metapher des Ankommens nach einem hektischen Tag, die damit assoziiert ist. Das Zuhause hat seit dem Aufkommen von Erwerbsarbeit, geregelten Arbeitszeiten, Trennung von Arbeit und Freizeit in klassischen (männlichen) Lebensverläufen mit Ausbildung, Arbeit und Rente seit der Nachkriegszeit in den 1950er Jahren einen besonderen Stellenwert (Geißler/Meyer 2002). Kulturell eingeschrieben ist in Deutschland der Gedanke des Eigenheims und dessen Ausstattung mit Konsumgütern, die das Zuhause komfortabel und schön machen - gerade auch in Zeiten eines Lockdowns. Im Rahmen der Alters-Surveys des Bundesministeriums für Familie, Senioren, Frauen und Jugend (BMFSFJ) konnten Motel-Klingenbiel/Künemund/Bode (2005) für das Jahr 1996 zeigen, dass ein Drittel der über 65-Jährigen bereits mehr als vierzig Jahre am selben Ort wohnte, Nowossadeck/Engstler (2017) wiesen im Jahr 2014 nach, dass fast 60 Prozent der 70- bis 85-Jährigen über eine eigene Immobilie verfügten. Vor diesem Hintergrund erscheint es nachvollziehbar, dass die gewohnte persönliche Umgebung, fernab von den Blicken Dritter, bei Älteren einen hohen Stellenwert einnimmt. Privathaushalte werden neben den Kliniken und Praxen der niedergelassenen Ärzt*innen inzwischen als dritter Gesundheitsstandort bezeichnet (Heinze/Hilbert/Paulus 2009), die aus gesundheitsökonomischer Sicht unter anderem für altersgerechte Assistenzsysteme nutzbar gemacht werden könn(t)en.

Diesen Ergebnissen und Überlegungen folgend, fungiert das Zuhause im Alter als essenzieller Bezugspunkt dieses Beitrags. Ein spezifisches Gefüge - individuelle, menschliche Bedürfnisse (Mikroebene) einerseits und politische, ökonomische Forderungen (Meso- und Makroebene) nach technischen Innovationen andererseits - steht aufgrund des demografischen Wandels im Fokus des Forschungsinteresses zahlreicher Studien (vgl. den Anhang in Weber in diesem Band). Vor diesem Hintergrund werden aus verschiedenen disziplinären Perspektiven wie der Pflegewissenschaften, Gerontologie, Ethik, Soziologie und Technikfolgenabschätzung seit einigen Jahren AAL-Technologien intensiv diskutiert. Dazu zählt, wie die derzeit zur Verfügung stehende oder in Entwicklung befindliche (digitale) Pflegeund Assistenztechnik im Alltag von Pflegenden, Gepflegten und ihren Angehörigen eingeordnet werden kann oder welche Prämissen der potenzielle Einsatz erfüllen 
müsste, um unter anderem das Leben in der eigenen Wohnung oder dem Haus so lange wie möglich zuzulassen. Aktuell liegen wenige quantitative Studien vor, die auf Basis umfänglichen Datenmaterials die tatsächliche Nutzung in Deutschland aufzeigen und Hinweise geben, worin die Nutzungshemmnisse bestehen (Braeseke et al. 2020; Zöllick et al. 2020). Ihre Ergebnisse einer geringen Verbreitung von digitalen Assistenzsystemen und deren zurückhaltende Einführung in der ambulanten und stationären Altenpflege bestätigen auch Haug, Scorna sowie Vetter/Cerullo in diesem Band.

Um die skizzierte Forschungsfrage in der Tiefe $\mathrm{zu}$ untersuchen, wurde in DAAS-KIN (»Diffusion altersgerechter Assistenzsysteme-Kennzahlenerhebung und Identifikation von Nutzungshemmnissen«) ein Mixed-Methods-Design verfolgt (vgl. Haug und Scorna in diesem Band). Mit einer Wissenssoziologischen Diskursanalyse (WDA) wird auf Grundlage aktueller Studien untersucht, welche Einschätzungen und (Ideal-)Vorstellungen derzeit zum Einsatz von altersgerechten Assistenzsystemen bestehen. Der folgende Beitrag analysiert zentrale Normen und Werte und diskutiert diese zusammen mit dem Bewertungsinstrument MEESTAR - einem diskursethischen Modell zur ethischen Evaluation soziotechnischer Arrangements. Im Anschluss daran wird behandelt, von welchen diskursiven Strukturen in dem entstandenen Werteschema zentrale Impulse ausgehen, die eine künftige Nutzung unterstützen können. Die Ergebnisse werden in den Forschungstand eingeordnet und unter einem Fazit subsumiert.

\section{Zentrale Werte und Überzeugungen im Diskurs: Ein normativer Blick auf digitale Assistenzsysteme}

Die Fragestellung, nach der sich das Vorgehen dieser Teilstudie des Projekts DAASKIN richtet, resultiert aus dem Ansatz von Ortwin Renn (1999 \& 2014), das Verfahren einer Wertbaumanalyse für die Bewertung von Technikfolgen einzusetzen. Dieses Instrument kann dafür genutzt werden, Entscheidungen in einem komplexen Setting unter Zuhilfenahme eines mehrstufigen Prozesses herbeizuführen. Dabei werden unter anderem diejenigen Bewertungen, Regeln und Normen als Verhaltensvorschriften gesammelt, die in einer Gesellschaft zu einem Sachverhalt als verbindlich und legitim anerkannt sind (Renn 1999: 617-618, 2014: 165-167). Die Wertbaumanalyse wird in diesem Beitrag in modifizierter Form in Kombination mit einer WDA angewandt. Ziel der Teilstudie von DAAS-KIN ist es, ein diskursives Werteschema (siehe Abbildung 1) auf Grundlage ausgewählter Literatur zu bilden. Das Schema erfasst Werte ${ }^{1}$, die den Diskurs typischerweise kennzeichnen, und 
die Konstellation der über- und untergeordneten Werte zueinander. Dazu wurden Arbeiten fokussiert, die die Einstellung und Erfahrung zur Nutzung digitaler Assistenzsysteme evaluierten. Die Systematik und Methodik des qualitativen Teilprojekts wird im Folgenden skizziert. Danach werden die Ergebnisse vorgestellt und mithilfe von Auszügen aus dem Datenmaterial empirisch belegt.

\section{Herangehensweise und Methodik}

Die Untersuchung basiert auf einer Analyse des wissenschaftlichen Diskurses zu Technik und Alter. Dieser wird als Spezialdiskurs betrachtet, der auf PubMed prinzipiell öffentlich zugänglich ist. Es handelt sich hierbei um Wissen, das in quantitativen und qualitativen Studien zur (potenziellen) Nutzung digitaler Assistenzsysteme in der ambulanten oder stationären Pflege von Wissenschaftler*innen neu generiert, aktualisiert oder als Review zusammengestellt wurde. PubMed stellt dieses Wissen als bibliografische Datenbank zur Verfügung und deckt den gesamten Bereich der Gesundheitsforschung ab. Die gelisteten Artikel sind vorwiegend in Fachjournals publiziert worden und geben den aktuellen Forschungsstand zu (digitaler) Pflege- und Assistenztechnik wieder. Auf Grundlage einer Verknüpfung spezifischer Suchbegriffe konnten über 6.000 Artikel recherchiert werden, irrelevante Treffer wurden sukzessive ausgeschlossen und aus knapp 1.700 Treffern schließlich zwölf Fachartikel ${ }^{2}$ in die weitere Analyse einbezogen. Dieser Schritt wurde in einem zweistufigen Verfahren vollzogen, in dem zwei beteiligte Forscher*innen unabhängig voneinander, aber nach denselben Kriterien das Datenkorpus erstellten. ${ }^{3}$ Die Systematik der Suche, die Eingrenzung und Auswertung, orientiert sich an der Grounded-Theory-Methodologie (GTM) nach Strauss/Hildenbrand/Hildenbrand (1994) sowie Strauss/Corbin (1996). Dabei werden am Material Thesen generiert, die (Zwischen-)Ergebnisse laufend überprüft, abstrahiert und interpretiert. Diese iterativ-zyklische Herangehensweise leitet sich aus der qualitativen Sozialforschung $a b$ (Strübing et al. 2018). Sie ist auch typisch für das Verfahren der Wertbaumanalyse (Renn 1999: 620-621, 2014: 175) und dient zur Überprüfung der identifizierten Werte im selben inhaltlichen Kontext, aber am Datenmaterial von Interviews. Diese wurden im Rahmen eines weiteren Teilprojekts von DAAS-KIN mit Vertreter*innen der ambulanten und stationären Pflegeeinrichtungen sowie

Broadbent et al. (2016), Cresswell/Cunningham-Burley/Sheikh (2018), Johansson-Pajala/ Custafsson (2020), Klein/Schlömer (2018), Lee et al. (2018), Lehoux/Grimard (2018), Pino et al. (2015), Radic/Vosen (2020), Rantanen et al. (2018), Suwa et al. (2020), Vandemeulebroucke et al. (2019) sowie Wangmo et al. (2019).

3 Das Forscher ${ }^{\dddot{i}}$ innenteam bei diesem Schritt bestand aus der Autorin und Monika Friedl, der ich an dieser Stelle herzlich danken möchte. Nicla Kaufner unterstützte bei der Realisierung der Abbildung in diesem Beitrag. 
mit Vertreter*innen aus Forschung und Wissenschaft geführt (vgl. Scorna in diesem Band). Die Analyse der ausgewählten Texte erfolgte mit Unterstützung von MAXQDA.

Zunächst wurden infrage kommende Suchbegriffe identifiziert. Dazu zählten allgemeine Begriffe aus dem Bereich der digitalen Assistenzsysteme, die mit einem Stichwort verknüpft wurden, das eine ethische Dimension (z.B. Ethik, Moral, Norm, Wert, Standard, Erfahrung) einschließt. Teilweise wurde als zusätzliches Kriterium das Alter (z.B. alt, Alter, Ältere) und/oder die Pflege ergänzt. Dabei wurde nach Titel, Abstract und (kursorischem) Lesen des Volltexts Fachartikel in die Auswahl übernommen und die anschließende Suche je nach Treffer weiter verfeinert. Suchen mit über 1.000 Treffern wurden so lange modifiziert, bis die Trefferlisten und Texte mit dem Verfahren der GTM analysiert werden konnten. Die analysierten Werte und deren einzelne Ausprägungen werden bei einer Wertbaumanalyse in Beziehung zueinander gestellt, dabei werden Haupt- und Unterebenen gebildet. Die klassische Darstellung eines Wertbaums erfolgt in einer Baumstruktur (Renn 1999 \& 2014). Für die Belange der vorliegenden Diskurs- und Wertbaumanalyse eignet sich die Darstellung in Form eines Diagramms mit einzelnen Segmenten. Diese Struktur wurde anschließend in das Werteschema (siehe Abbildung 1) überführt.

Eingeschlossen wurden deutsch- und englischsprachige Artikel, ${ }^{4}$ die zwischen Januar 2015 und Dezember 2020 auf PubMed verfügbar waren. Kriterien für diesen Zeitraum waren möglichst kürzlich erschienene Studien, die in hinreichender Anzahl vorliegen. Im Zuge der Recherchen und Anpassungen der Suchbegriffe stellte sich heraus, dass zahlreiche Studien vorliegen, die die Einstellung gegenüber assistiven Technologien am Beispiel von Robotern in der Pflege untersuchen. Diese Variante wird in den meisten Studien genutzt, um digitale Assistenztechnologien zu beschreiben, die Pflegende und Gepflegte im Alltag unterstützen könn(t)en. ${ }^{5}$ Dieser Bestandteil der Recherche (robots) wurde zu einem zentralen Schlüsselwort und steht für eine Art von altersgerechten Assistenzsystemen.

Im Zuge der Korpuserstellung wurden einzelne Studien fokussiert, das heißt Reviews, Proceedings und graue Literatur wurden ausgeschlossen. Da Ergebnisse

PubMed ist eine englischsprachige Datenbank, das heißt deutschsprachige Artikel werden mit Titel und Abstract auf Englisch angezeigt.

Broadbent et al. (2016), Cresswell/Cunningham-Burley/Sheikh (2018), Johansson-Pajala/ Gustafsson (2020), Lee et al. (2018), Lehoux/Grimard (2018), Pino et al. (2015), Radic/Vosen (2020), Rantanen et al. (2018), Suwa et al. (2020) und Vandemeulebroucke et al. (2019) beziehen sich auf Roboter in der Pflege, die von den Befragten hinsichtlich ihres Unterstützungspotenzials bewertet wurden. Klein/Schlömer (2018) untersuchen ein robotisches Duschsystem und Wangmo et al. (2019) »intelligent assistive technology (IAT)«. Wenn im Folgenden von digitalen oder altersgerechten Assistenzsystemen bzw. AAL die Rede ist, schließen diese Begriffe die gesamte Bandbreite der Technologien ein, die in den Studien untersucht wurden (vgl. auch die Aufschlüsselung von Haug in diesem Band zu diesen Systemen). 
betrachtet werden sollen, die sich auf die Rehabilitation und Pflege älterer Menschen im Sinne altersgerechter Assistenzsysteme beziehen, wurden Artikel, die die Perspektive der ambulanten und/oder stationären Pflege nicht berücksichtigen, nicht in das Korpus aufgenommen. Ebenso wurden Studien, die ausschließlich eine Versorgung im Rahmen einer Palliativ- und Intensivpflege, dem Lebensende und in geschlossenen Stationen (z.B. Justizvollzugsanstalt, Psychiatrie) behandeln, nicht einbezogen. Genauso wurde mit Studien verfahren, die sich nicht im Kontext digitaler Assistenzsysteme oder AAL verorten lassen und die spezifische Anwendungen wie die Heimdialyse oder orthopädische Therapien des Gangbildes (z.B. mit einem Exoskelett) in einem klinischen Setting testen und die medizin(-technische) Wirksamkeit bewerten. Berücksichtigen solche Studien den Ansatz von AAL eines möglichst unabhängigen Lebens oder einer sozialen Teilhabe, wurden die Texte einbezogen.

Die modifizierte Wertbaumanalyse ist in diesem Beitrag als Diskursanalyse angelegt. Diese Kombination erlaubt, nach der »diskursiven Begründung und $\mathrm{Zu}$ sammenfassung von kollektiv vereinbarten Werten und Entscheidungskriterien « (Renn 2014: 173) zu fragen. Der Zugang untersucht wissenschaftliches Wissen und strukturiert sowie rekonstruiert im Folgenden Werte und Normen, die Pflegende, Gepflegte, Stakeholder*innen und wissenschaftliche Expert*innen im Zusammenhang mit digitalen Assistenzsystemen in den einbezogenen Studien äußern. Diese Einschätzungen der befragten Personen und Ergebnisse der Studie werden von den jeweiligen Autor*innen in einen Fachartikel eingebettet, für den Kontext eines bestimmten Journals einer speziellen Fachrichtung aufbereitet und vor diesem Hintergrund gedeutet. Die Arbeit nutzt dabei die WDA, die unter anderem eine Weiterentwicklung des diskursanalytischen Verfahrens von Michel Foucault darstellt (Keller 2011). Demzufolge werden einzelne Studienergebnisse im weiteren Verlauf zusammen analysiert, um typische Aussagen identifizieren zu können. Damit fungiert der Beitrag als wissenssoziologische Untersuchung, bei der Sprache, Aussagen und Diskurse als dynamische Komplexe betrachtet werden, die Wissen in einer Gesellschaft formulieren (Berger/Luckmann 2009: 36-43). Diese Perspektive geht davon aus, dass durch diese Aushandlungen bestimmte Deutungen entstehen, die die Alltagswelt ordnen. Auf diese Weise könnte die Einstellung gegenüber Din$g e n^{6}$ wie digitalen Assistenzsystemen nachhaltig geprägt werden. Die derzeitige potenziell zurückhaltende Nutzung oder gar Ablehnung von Assistenztechnologien (vgl. Haug und Scorna in diesem Band) könnte sich mitunter dadurch erklären lassen, wenn in den Diskurs ethische und moralische Kriterien eingelassen sind. 


\section{Ein diskursives Werteschema: Einschätzungen und Einstellungen gegenüber digitalen Assistenzsystemen}

Die Analyse eines Spezialdiskurses der Wissenschaft erscheint aufschlussreich, weil auf dieser Grundlage politische Entscheidungen getroffen werden, die ökonomisch wirksam sind und den praktischen beruflichen Alltag der Pflege betreffen können. Dies zeigt sich an den Geldern, die von den Ministerien in Deutschland für die AAL-Forschung zur Verfügung gestellt werden (vgl. den Anhang in Weber in diesem Band) und nicht zuletzt an den politischen Handlungen, die sich während der Pandemie aufgrund von COVID-19 nach den jeweiligen wissenschaftlichen Empfehlungen richten. Gerade in diesem Kontext werden grundsätzliche ethische Fragen verhandelt, zum Beispiel was den Zugang zu einer Impfung oder die gesellschaftliche (Ungleich-)Behandlung von Geimpften und Ungeimpften betrifft - also die Berücksichtigung moralischer Grundsätze in einer Gesellschaft, die auch beim Einsatz altersgerechter Assistenzsysteme regelmäßig zur Sprache kommen. Vor dem Hintergrund dieser digitalen Technologien wurde MEESTAR entwickelt.

MEESTAR stellt sieben Bewertungsdimensionen zur Verfügung (Weber 2016). Dazu zählen Gerechtigkeit, Privatheit, Selbstbestimmung/Autonomie, Fürsorge, Selbstverständnis, Sicherheit sowie Teilhabe. Diese basieren auf ethischen Vorstudien und erlauben es, eine konkrete technische Anwendung eines altersgerechten Assistenzsystems in eine von vier Stufen (von >ethisch völlig unbedenklich bis >nicht akzeptabel $)$ einzuordnen. Für die vorliegende Analyse sind die sieben Dimensionen von zentraler Bedeutung sowie die darin transportierten Beziehungen auf einer individuellen, organisationalen und gesellschaftlichen Ebene. Es bietet sich an, MEESTAR dem Werteschema gegenüberzustellen beziehungsweise MEESTAR durch das Schema evaluieren zu lassen. Alle sieben Dimensionen konnten bestätigt werden. Auf Grundlage der Diskurs- und Wertbaumanalyse wird MEESTAR um eine Dimension, nämlich Wissen \& Innovation, ergänzt und zwei Dimensionen für die Belange des Werteschemas angepasst (siehe Abbildung 1). Fürsorge \& Selbstverständnis werden in dem untersuchten Diskurs eng aufeinander bezogen, Teilhabe wird zusammen mit Wohltun diskutiert.

Die Reflexion über digitale Assistenzsysteme im wissenschaftlichen Diskurs hebt Gerechtigkeit, Privatheit, Autonomie \& Selbstbestimmung, Fürsorge \& Selbstverständnis, Wissen $\&$ Innovation, Sicherheit, Teilhabe $\&$ Wohltun als ethisch relevante Kriterien hervor. Als übergeordnetes diskursives Muster vereint Vertrauen alle Dimensionen. Das Werteschema verdeutlicht einerseits die engen, reziproken Bezüge zwischen den Werten, andererseits symbolisiert das Schema die Konstitution eines Wertegefüges als diskursive Einheit: 
Abbildung 1: Zentrale Werte im wissenschaftlichen Diskurs über digitale Assistenzsysteme

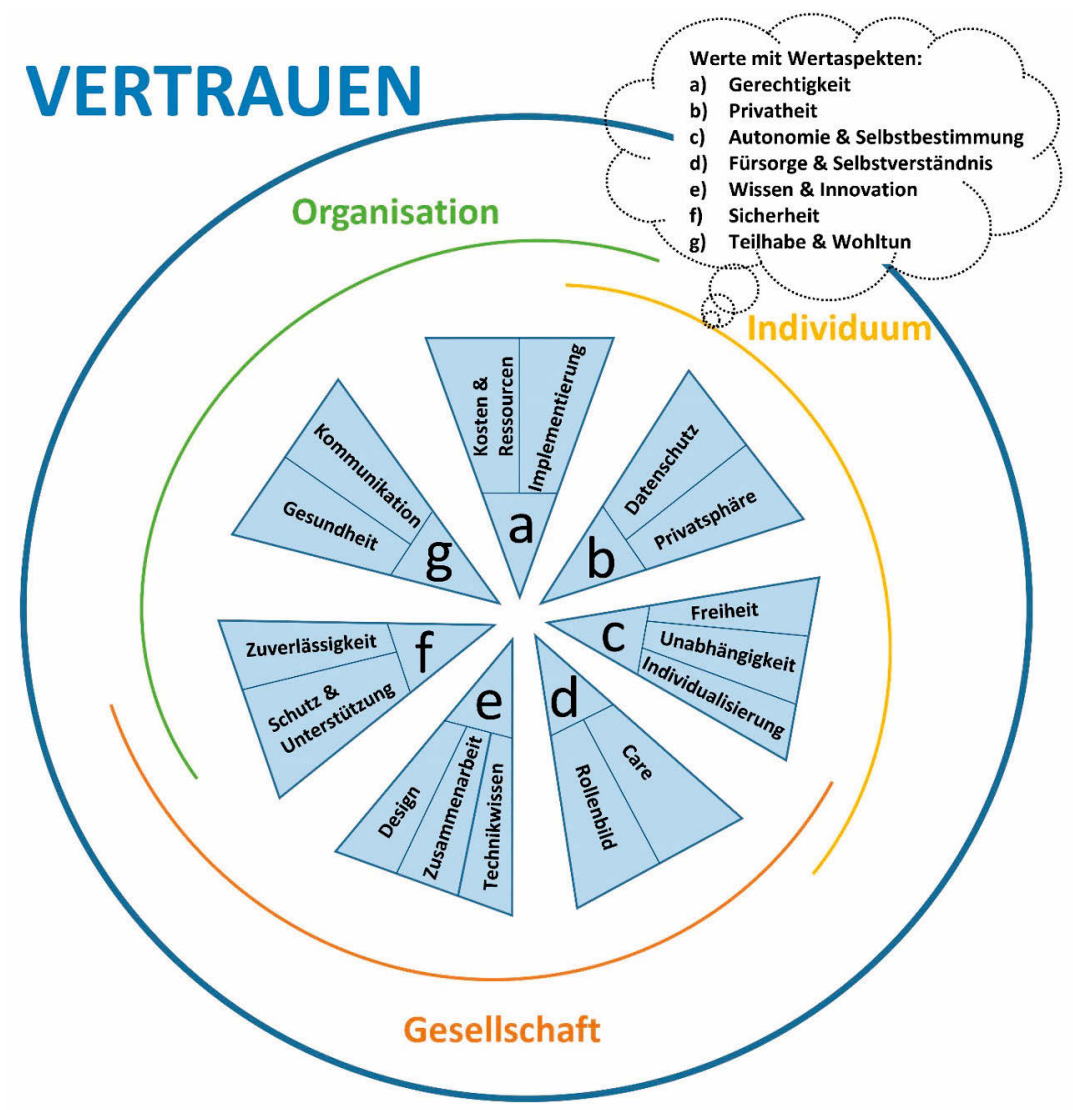

Quelle: Diskurs- und Wertbaumanalyse DAAS-KIN 2020

1 Erfährt eine oder mehrere ethische Dimension keine Berücksichtigung, wird das Gefüge in sich instabil und ethisch fragwürdig.

2 Das Verhältnis zu digitalen Assistenzsystemen wird auf drei Ebenen verhandelt: Die Bewertung auf einer individuellen Ebene, die in eine organisationale Ebene eingebettet ist. Beide beeinflussen eine gesellschaftliche Ebene beziehungsweise spiegeln diese zurück.

3 Alle drei Ebenen stehen im unmittelbaren Bezug zueinander und konstruieren im Diskurs das Verhältnis zu digitalen Assistenzsystemen beziehungsweise zur Mensch-Technik-Interaktion in Form spezifischer Dimensionen, den sieben Wertkonstellationen. 
Im Folgenden werden diese zentralen Werte mit ihren jeweiligen Ausprägungen, das heißt den untergeordneten Werten, erläutert. ${ }^{7}$

\section{a) Gerechtigkeit}

Aspekte, die mit Fragen der Gerechtigkeit verbunden sind, beziehen sich vor allem auf Kosten $\&$ Ressourcen. Dazu zählen in erster Linie die finanziellen Belastungen, die für die Endnutzer*innen anfallen können, aber kein Ausschlussprinzip bei der Anschaffung und Nutzung von digitalen Assistenzsystemen darstellen sollen (Klein/Schlömer 2018: 27, 29; Suwa et al. 2020: 9; Wangmo et al. 2019: 6): »They [professional caregivers; Erg. DF] pointed out that these technologies should be provided to anybody who needs it, and not only for those who can afford it « (Klein/Schlömer 2018: 29). In der betreffenden Akzeptanzstudie wurde eine intelligente robotische Duschhilfe von Pflegenden und Gepflegten in Fokusgruppen ethisch bewertet. In weiteren Studien wurden fehlende Gesetze und Richtlinien bemängelt, was die Absicherung von Schäden und Unfällen (Johansson-Pajala/Gustafsson 2020: 8; Suwa et al. 2020: 9) sowie die Klassifizierung von Assistenzsystemen und die Aufklärung dazu angeht, die es zum Beispiel bei frei verkäuflichen Medikamenten gibt (Johansson-Pajala/Gustafsson 2020: 5-6; Wangmo et al. 2019: 7). Bei der Implementierung von digitalen Assistenzsystemen wurde problematisiert, dass die Abläufe in den beteiligten Behörden nicht geregelt sind und eine digitale Infrastruktur in Gemeinden fehlt, in die diese wiederum nicht bereit sind zu investieren (Johansson-Pajala/Gustafsson 2020).

In Verbindung mit MEESTAR zeigen sich demnach auf der individuellen, organisationalen und gesellschaftlichen Akteur*innenebene ethische Konflikte. Bei MEESTAR werden die Vermeidung sozialer Ungleichheit und eine Berücksichtigung von Solidarität als ethisches Ziel formuliert. Konkret adressiert werden der Zugang zu altersgerechten Assistenzsystemen, die Finanzierung und eine Generationengerechtigkeit (Manzeschke et al. 2013: 17-18; Weber 2015: 258-260). Damit sind die Kosten im Zusammenhang mit den Systemen gemeint, die individuell oder von der Gemeinschaft getragen werden und zwischen den Generationen nicht $\mathrm{zu}$ ungleichen Lasten verteilt sein sollen.

\section{b) Privatheit}

Als prominente Position in ethischen Debatten gilt die Privatheit, die möglichst unantastbar und rechtlich geschützt sein sollte (Manzeschke et al. 2013: 16-17; Weber 2015: 252-255). MEESTAR ordnet den Datenschutz in diese Dimension ein, denn altersgerechte Assistenzsysteme erfordern in der Regel eine Erhebung, Verarbeitung und (kurzfristige) Speicherung von hochsensiblen, persönlichen 
(Gesundheits-)Daten. Gerade auch im privaten Umfeld soll die Technik in den Hintergrund treten beziehungsweise in gewissen Momenten inaktiv sein, die Privatsphäre also achten.

Vandemeulebroucke et al. (2019: 8) zeigen in ihren Gruppendiskussionen mit Pflegeheimbewohner*innen genau diesen Wunsch auf: »In addition, participants expressed older adults' need for personal moments, alone, with relatives or friends, without a SAR's [socially sssistive robots; Erg. DF] dominating presence. Just knowing that SARs are present and can be (de-)activated when one wishes could provide a feeling of security.«Intelligente Duschsysteme wurden in der Studie von Klein/Schlömer (2018) von älteren Pflegebedürftigen als ethisch sensibel bewertet, weil die Intimsphäre privat bleibt und die Anwesenheit von Pflegekräften nicht erforderlich ist.

Kameras, Mikrofone und Wearables, die ungewollt aktiv bleiben - weil in vielen Fällen vermutlich nicht bekannt ist, wie das Ausschalten erfolgt - stellen jedoch einen ethisch komplexen Sachverhalt dar (Johansson-Pajala/Gustafsson 2020: 6; Radic/Vosen 2020: 630, 635; Wangmo et al. 2019: 2). Eigentlich müssten die Nutzer*innen aufgeklärt und deren Einverständnis erfolgt sein, unterstützend könnten Personen im sozialen Umfeld wie Angehörige und Pflegedienstleister*innen im Umgang mit diesen Funktionen der Technik geschult sein (Wangmo et al. 2019: 5-8). Allerdings wird dieser Wissenserwerb durch die Komplexität der digitalen Assistenzsysteme erschwert und ein Einverständnis, das vorab gegeben werden sollte, müsste beim Einsatz von künstlicher Intelligenz (KI) und selbstlernenden Algorithmen stets neu eingeholt werden. In einer internationalen Vergleichsstudie konnten Suwa et al. (2020: 11-12) herausarbeiten, dass die Weitergabe und Überwachung von personenbezogenen und -beziehbaren Daten der Gepflegten und ihres sozialen Umfelds an das medizinische und pflegerische Fachpersonal unter den Befragten (ältere Menschen, Angehörige und Pflegepersonal) bekannt ist und von ihnen akzeptiert wird. Während bei den Vitalfunktionen die Zustimmung in allen Ländern (Finnland, Irland, Japan) inzwischen mit über 80 Prozent sehr hoch ist, fiel diese bei Fotos und Videos, die Pflegeroboter aufnehmen könnten, zwischen 45 und 56 Prozent am niedrigsten aus. Trotzdem weist Japan die höchste bis dato gemessene Akzeptanzrate auf. Allerdings zeigt es sich, dass ältere Menschen mit leichten kognitiven Beeinträchtigungen etwaigen Überwachungstechnologien besonders kritisch gegenüberstehen; hier gibt es einen großen Unterschied zwischen dieser Personengruppe (56\%), Pflegekräften (16\%) und der Perspektive älterer Menschen im Allgemeinen (28\%) (Pino et al. 2015: 10). 


\section{c) Autonomie \& Selbstbestimmung}

Das Recht auf Selbstbestimmung wird in den Studien unter anderem unter Prämissen einer Freiheit und Unabhängigkeit des Subjekts verhandelt. Gerade das eigenständige Leben zu Hause, in dem je nach Bedarf auf digitale Assistenzsysteme zurückgegriffen werden kann, wird unabhängig von Alter, Geschlecht und Betroffenheit beziehungsweise Pflegebedürftigkeit bevorzugt (Lehoux/Grimard 2018: 333-334). Dieser Sichtweise stimmten Pflegekräfte in einer Fragebogenstudie $(\mathrm{n}=200)$ mehrheitlich $\mathrm{zu}: » 68.5 \%$ totally or partially agreed that they would be ready to experiment with and introduce new robot technology in home care, if it could help elderly people to cope independently at home« (Rantanen et al. 2018: 1852). In verschiedenen Studien wird potenziell ein Zugewinn an Unabhängigkeit gesehen, was sich auf den Eintritt in das Pflegeheim bezieht (»it will delay my entry into a retirement home«; Pino et al. 2015: 9) oder auf die Lebensführung im Allgemeinen (»a way to empower older adults to remain autonomous«; Vandemeulebroucke et al. 2019: 7). Trotzdem wird auf Risiken hingewiesen, die eine Begrenzung der individuellen Freiheit bedeuten können. Hier werden zum Beispiel neue Abhängigkeiten befürchtet (»Being dependent upon (inhumane) humans«; Lehoux/Grimard 2018: 334) sowie neue Limitierungen der Selbstbestimmung (»I believe the use of the robot will restrict my autonomy«; Pino et al. 2015: 9). Dieses Recht auf Freiheit wird deutlich eingefordert:

»It is awful to [do; Erg. DF] that to someone who has been free and independent during all his life. Human freedom is a wonderful thing, and we must keep it during our whole life [...].« (Pino et al. 2015: 10; Herv. i. O.)

Westliche Industriegesellschaften sind von einem hohen Grad der Individualisierung gekennzeichnet. Übertragen auf den Alltag mit altersgerechten Assistenzsystemen kann davon ausgegangen werden, dass Subjekte Selbstbestimmung als etwas $>$ Normales betrachten. Die WDA zeigt auf, wie diese ethische Dimension in den Studien verhandelt wird: Entscheidungen sollen nach eigenem Ermessen und informiert getroffen werden (Klein/Schlömer 2018: 28; Wangmo et al. 2019: 4), ältere Menschen sollen die Möglichkeit haben, sich für oder gegen Technik zu entscheiden (Suwa et al. 2020: 10, 13; Vandemeulebroucke et al. 2019: 6), und die Technik sowie deren Anwendung soll an individuelle Bedürfnisse und Krankheitsbilder angepasst werden können (Lehoux/Grimard 2018: 333; Pino et al. 2015: 8). Diese Prämissen scheinen in intimen Umgebungen eine wichtige Rolle zu spielen, denn diese wurden bei der ethischen Bewertung von »I-SUPPORT«, dem System, das beim Duschen unterstützt, von Senior"innen vorgebracht (Klein/Schlömer 2018). Gerade bei Pflegerobotern spielen das Aussehen des Geräts, die individuelle Anpassung von Bestandteilen, wie dem Namen, dem Geschlecht und der Stimme sowie der Dienste, Funktionen beziehungsweise Programme des Roboters, eine zentrale 
Rolle (Klein/Schlömer 2018: 28-29; Lehoux/Grimard 2018: 336; Pino et al. 2015: 7, 11; Suwa et al. 2020: 9). Nicht zuletzt ist auch die Wohnumgebung individuell, auch daran sollte das Gerät angepasst werden können (Rantanen et al. 2018: 1856).

Weber (2016) arbeitet heraus, dass MEESTAR an Beauchamp/Childress (2019) ${ }^{8}$ anschließt, die die Autonomie als eines der vier Prinzipien begreifen, wenn es um grundsätzliche ethische Entscheidungen geht, zum Beispiel, ob der Einsatz altersgerechter Assistenzsysteme gerade auch bei älteren oder vulnerablen Personengruppen deren Autonomie berücksichtigt. Begreift man nach Weber altersgerechte Assistenzsysteme als soziotechnisches Arrangement, ist aus ethisch-moralischer Sicht auch die Selbstbestimmung der Pflegekräfte zu reflektieren, gerade bei einer möglichen Ablehnung digitaler Assistenzsysteme, auch wenn diese als sicher anerkannt ist (Cresswell/Cunningham-Burley/Sheikh 2018: 7).

\section{d) Fürsorge \& Selbstverständnis}

Die beiden MEESTAR-Dimensionen Fürsorge und Selbstverständnis drücken sich in den untersuchten Studien in einem >Berufsethos $<$ aus, das ein Altersbild antizipiert, das sich auf einen pflegebedürftigen, hochaltrigen und gebrechlichen Menschen konzentriert. Berufsethos als Begriff fasst zusammen, was im wissenschaftlichen Diskurs einerseits als professionelles Selbstverständnis der Pflegenden verhandelt wird und andererseits als Rollenbild der (professionellen) Pflege in der Gesellschaft besteht. Eng verwoben sind diese Sichtweisen mit Care, was im Diskurs die Auffassung einer >guten Pflege darstellt, wie Rudolph es in diesem Band beschreibt. Der Begriff umfasst Tätigkeiten, Zuständigkeiten und Verantwortlichkeiten im privaten Bereich (familiäre Pflege durch beispielsweise Angehörige) und im professionellen Bereich (in der ambulanten und stationären Pflege), deren Qualitäten derzeit immer noch qua Geschlecht zugeschrieben werden (vgl. Giese 2018; Porz 2018). Damit sind Sorge-Arbeiten in spezifischen Beziehungen und Interaktionen gemeint, die sozial - also aufeinander bezogen - sind. Diese Unterstützungsleistungen sollen empathisch sein und sich an den Bedürfnissen der Personen orientieren, die Hilfe empfangen, so die Idealvorstellung.

Das gesellschaftliche und berufliche Rollenbild scheint zum einen klar mit den kulturellen Vorstellungen einer >guten Pflege verbunden zu sein, woraus diffuse, aber auch konkrete Sorgen und Ängste resultieren, die sich als Narrative und Stereotype im Diskurs zeigen. Dazu zählt die Assoziation der altersgerechten Assistenzsysteme mit Robotern, die in populärkulturellen Formaten als Aggressor und

Für Beauchamp/Childress (2019) fallen Selbstbestimmung und Privatsphäre mitunter unter die Dimension der Autonomie. Der Ansatz findet vor allem im Bereich der Biomedizin und Medizinethik breite Verwendung. So stellen sich bei vererbbaren Erkrankungen und der grundsätzlichen Möglichkeit, einen Centest vorzunehmen, zahlreiche ethische Fragen, vergleiche hierzu Lenk/Frommeld (2015). 
Bedrohung dargestellt werden (Johansson-Pajala/Gustafsson 2020: 3; Pino et al. 2015: 8): »[Patients] think when you say robot ... you mean Terminator, so people are afraid « (Cresswell/Cunningham-Burley/Sheikh 2018: 4; Erg. i. O.). Diese Vorurteile gegenüber assistiven Möglichkeiten scheinen derzeit nicht aufgebrochen werden zu können und Angehörige lehnen diese bisweilen radikal ab (JohanssonPajala/Gustafsson 2020: 7; Pino et al. 2015: 8). Die Befürchtung, dass eine Technisierung der Pflege zum Personalabbau (die vor allem bei humanoiden Robotern groß ist) und zu generellen Einsparungen (und zur Verschlechterung der Pflegequalität) führen könnte, tritt im Diskurs klar hervor (Cresswell/Cunningham-Burley/Sheikh 2018: 6; Johansson-Pajala/Gustafsson 2020: 4-6; Klein/Schlömer 2018: 29; Lee et al. 2018: 2102).

Diese Deutung des Diskurses wäre jedoch zu einseitig, denn der Einsatz von altersgerechten Assistenzsystemen wird aktuell überschätzt (vgl. Haug und Scorna in diesem Band). Tatsächlich kommt eine differenzierte Perspektive auf die Systeme in zahlreichen Fragebogenstudien, Interviews und Gruppendiskussionen im Rahmen der Diskurs- und Wertbaumanalyse zum Ausdruck. Das Pflegepersonal sieht das Potenzial von digitalen Assistenzsystemen in einfachen Abläufen; dazu zählen das Messen und Überwachen von Vitaldaten, die Unterstützung bei Hebeaufgaben und zusätzliche Sicherheitsinstrumente (Johansson-Pajala/Gustafsson 2020: 3; Lee et al. 2018: 2098). So wird betont, dass bei diesen Aufgaben Ziele wie die Entlastung des pflegerischen und medizinisches (Fach-)Personals realistisch erscheinen (Cresswell/Cunningham-Burley/Sheikh 2018: 7; Klein/Schlömer 2018: 29; Lee et al. 2018: 2096, 2102; Radic/Vosen 2020: 636). Die frei gewordenen physischen und zeitlichen Ressourcen könnten dazu beitragen, dass Kernkompetenzen von Care den Patient*innen zugutekommen.

Neben dieser optimistischen Bewertung steht eine in der Pflege fest verankerte und gesellschaftlich geteilte, normative Überzeugung einer sguten Pflege`, die einfühlsam über Kommunikation und Berührung erfolgt, an die zu Pflegenden angepasst wird und nicht durch Technik ersetzt werden kann (JohanssonPajala/Gustafsson 2020: 3, 5; Klein/Schlömer 2018: 29; Lee et al. 2018: 2102; Lehoux/Grimard 2018: 334; Wangmo et al. 2019: 7). Hinzu kommt die Sichtweise, dass die berufliche Tätigkeit ohne komplexe digitale Systeme in der Vergangenheit funktioniert hat und dass ein Beruf in der Pflege gewählt wurde, weil dabei ein enger sozialer Kontakt mit Menschen im Vordergrund steht und nicht die Technik. Befürchtet wird eine Veränderung in der Beziehung zu den Patient*innen.

Eine drohende Entmenschlichung der Pflege durch Technik scheint ein generelles und dominantes Diskursmuster darzustellen, sodass gerade bei Pflegerobotern eine klare Position zu erkennen ist (Lehoux/Grimard 2018: 334; Pino et al. 2015: 9). Diese sind aktuell mit dem Selbstverständnis, das Pflegekräfte im Sinne von Care haben, nicht oder kaum vereinbar: "I'm afraid that the introduction of care robots would make the treatment of elderly people inhumane« (Rantanen et al. 
2018: 1852). Diese Auffassung scheint mit einem Altersbild zusammenzuhängen, das Alter mit Einsamkeit, sozialer Isolation, Krankheit und kognitiven Beeinträchtigungen gleichsetzt und bei dem eine Pflege durch Menschen bevorzugt wird, wie Klein/Schlömer (2018: 29), Pino et al. (2015: 9) sowie Vandemeulebroucke et al. (2019: 6) aufzeigen. Ältere Menschen, die diesem Altersbild entsprechen, sind nach Wangmo et al. (2019) als Zielgruppe für assistive Technologien vorgesehen.

Diese Ansicht von medizinischem und pflegerischem Fachpersonal sowie von Expert*innen aus dem Bereich der Gesundheits- und Humanwissenschaften, die in der Studie befragt wurden, vernachlässigt nicht nur, dass Modelle wie MEESTAR eine selbstbestimmte Entscheidung für oder gegen altersgerechte Assistenzsysteme für eine ethische Unbedenklichkeit voraussetzen, sondern geht davon aus, dass Kommunikation mit anderen Menschen und menschliche Zuwendung ein Bedürfnis aller im Alter darstellt (Manzeschke et al. 2013; Weber 2015). Dementsprechend fühlten sich die Befragten in den Studien von altersgerechten Assistenzsystemen nicht angesprochen, wenn sie sich selbst (noch) nicht in diesem Altersbild verorteten und bewerteten die Technologien aus einer zukünftigen Perspektive. Auch wird nicht zwischen Systemen mit verschiedenen Funktionen (z.B. Kommunikation, Sicherheit, Dokumentation) unterschieden, deren Auswirkung jeweils unterschiedlich bewertet werden können (vgl. Haug in diesem Band).

\section{e) Wissen \& Innovation}

Das Design von Technik, die erwünschte Zusammenarbeit auf mehreren Ebenen in Richtung partizipativer Technikgestaltung und Technikwissen werden unter Wissen \& Innovation subsumiert. Sie können als typische Aussagen im wissenschaftlichen Diskurs über digitale Assistenzsysteme und insbesondere über robotische Technologien in der Pflege identifiziert werden. MEESTAR wird dabei um eine neue Dimension ergänzt, die aus der Perspektive einer "Wissensgesellschaft « (Stehr 1994 \& 2001) gewählt wird, um die Konstellation von Pflege, Wissen und Technik auszudrücken. Hintergrund ist, dass im Zuge eines rasanten technologischen Fortschritts in Industriegesellschaften zusammen mit der Digitalisierung Technik und Technikwissen alltäglich geworden ist beziehungsweise sich im Pflegealltag aufdrängt - nicht zuletzt durch massive Investitionen von Bund und Ländern in Innovationen in diesem Anwendungsbereich (vgl. Hergesell et al. und Weber in diesem Band). Innovationen stehen in Deutschland seit Ende des 19. Jahrhunderts für ein gesellschaftliches Deutungsmuster, das Innovationen im Kontext von Ideen, Wissen, Angebot, Nachfrage und ökonomischer Macht verhandelt (vgl. Frommeld 2019: 78-84). Neu im Diskurs ist, dass unter diesem Fokus große Potenziale für das Gesundheitswesen gesehen werden, weshalb in diesem Zusammenhang der Begriff Gesundheitswirtschaft vorgeschlagen wurde (Bratan/Wyrda 2018; vgl. Hilbert et al. 2018). 
Bevor ein Produkt auf den Markt kommt, steht am Anfang die Idee und die Entwicklung hin zur Zielgruppe Senior*innen. Das Design betrifft zunächst die sinnlich wahrnehmbare Gestaltung eines Produkts. Dazu gehören Farben, Klänge und Accessoires, die als Ergänzung bei bestehenden Systemen vorgeschlagen werden, zum Beispiel bei dem oben genannten robotischen Duschsystem (Klein/Schlömer 2018: 28), sowie allgemein eine persönliche Ansprache (Radic/Vosen 2020: 636). Die Technik sollte also personalisierbar sein und damit das Subjekt in seiner Individualität und Selbstbestimmung ansprechen. In drei verschiedenen Studien wurde herausgearbeitet, dass die Roboter aus ethischen Gründen Menschen und Tieren nachempfunden sein können, aber dennoch eindeutig als Maschinen identifizierbar sein sollen (Cresswell/Cunningham-Burley/Sheikh 2018: 7; Pino et al. 2015: 6-8; Wangmo et al. 2019: 5): "I don't like a humanoid robot because it gives you the illusion of being with someone and in reality you are still alone« (Pino et al. 2015: 8). Dabei treffen unterschiedliche Erwartungen aufeinander. Ingenieur*innen bemühen sich, möglichst realistische Roboter zu entwickeln, ${ }^{9}$ aufseiten der Nutzer* innen sind diese gar nicht erwünscht: "So, we try to do our best to really make it as close as possible to the person being there « (Cresswell/Cunningham-Burley/Sheikh 2018: 6). Forschung und Entwicklung (F\&E) scheinen einen schmalen Grat zur Verfügung zu haben, denn das Design soll weder stigmatisieren, infantilisieren noch falsche Tatsachen wie die Anwesenheit eines Menschen vortäuschen - also ethisch fragwürdig sein (Cresswell/Cunningham-Burley/Sheikh 2018; Pino et al. 2015).

Als Herausforderung wird eine Zusammenarbeit diverser Akteur*innen betrachtet, zum einen im Stadium von F\&E, zum anderen auf der Ebene von Gemeinden, der öffentlichen Verwaltung und Pflege (Johansson-Pajala/Gustafsson 2020; Lee et al. 2018). Das Ziel bestünde in einem gelingenden Design und Innovationen, die sich an den Nutzer*innen orientieren. Dabei geht es um den Austausch von Wissen, also von Kompetenzen, Erfahrung und Expertise, was bis dato nicht offen erfolgt - der Diskurs aus einer wissenschaftlichen Perspektive zeigt, dass >jeder sein eigenes Süppchen kocht<. Pflege- und Innovationsdiskurs verlaufen in unterschiedlichen Mustern. So ist die Implementation mit der Beteiligung von (öffentlicher) Verwaltung, Akteur*innen im Bildungssektor und der Schulung der Nutzer*innen ein langsamer Prozess, während Innovationen ihren Erfolg gerade daraus ziehen, rasch auf den Markt gebracht zu werden.

Um die Funktionen soziotechnischer Arrangements ausschöpfen zu können, wird dem Erlernen von Technikwissen sowie den Fähigkeiten und Fertigkeiten

Das Paradox des Uncanny Valley wurde 1970 von Masahiro Mori beschrieben, der durch seine frühen Forschungsarbeiten zu Robotik und Robotern bekannt wurde, und zeigt folgenden Zusammenhang: Zunächst steigt die Akzeptanz von Robotern, je menschenähnlicher sie sind. Ab einem gewissen Punkt jedoch, dem Uncanny Valley, verkehrt sich die Akzeptanz ins Gegenteil. Vergleiche hierzu Scorna (2015: 84). 
im Umgang mit Technik ein hoher Stellenwert eingeräumt: „Such education may cover all aspects of technology, from basic computer knowledge to concrete working methods using virtual care visits or videoconferencing (JohanssonPajala/Gustafsson 2020: 5). Dabei ist die Rede von einem »shift in skill« (Cresswell/Cunningham-Burley/Sheikh 2018: 7-8) und einer neuen Generation eines Fachpersonals, das schon in der Ausbildung auf den Wandel vorbereitet wird (Johansson-Pajala/Gustafsson 2020: 3-5). Von denjenigen Pflegekräften, die bereits lange Jahre in ihrem Beruf arbeiten, werden die Motivation und Bereitschaft zum Lernen erwartet, obwohl die alten Abläufe und das traditionelle Care-Konzept für diese gut funktionieren. Erschwerend kommt hinzu, dass in solchen Konstellationen nicht nur die Pflegekräfte Angst haben, etwas kaputt zu machen, sondern auch die Gruppe der hochaltrigen Gepflegten Berührungsängste hat, da sie noch weniger an digitale Technik gewöhnt ist. Daraus wird gefolgert, dass allen Beteiligten nicht nur das Üben und Vertrautwerden mit einfacher und komplexer Technik ermöglicht, sondern insgesamt (mehr) Wissen über Technik und digitale Assistenzsysteme zur Verfügung gestellt werden sollte (Pino et al. 2015; Rantanen et al. 2018; Suwa et al. 2020; Vandemeulebroucke et al. 2019).

\section{f) Sicherheit}

Bei der Bewertung, welche ethischen und moralischen Voraussetzungen digitale Assistenzsysteme zu erfüllen haben, stellt die Sicherheit von Patient*innen einen wesentlichen Ausgangspunkt für Innovationen in diesem Bereich dar, oder anders ausgedrückt ein Maximalziel (Weber 2015). Dabei geht es im Sinne der Prinzipienethik von Beauchamp/Childress (2019) um ein physisches und psychisches Nichtschaden des Körpers und der Gesundheit, und darüber hinaus um ein Sicherheitsgefühl. Die Technologien zielen unter anderem darauf $a b$, mithilfe von Erinnerungsfunktionen und Überwachungssystemen beide Dimensionen von Sicherheit zu gewährleisten und in ihrem Effekt zu steigern.

Die untersuchten Studien greifen in ihren Ergebnissen Schutz \& Unterstützung als zentrale Wertaspekte auf (Klein/Schlömer 2018: 27-29; Lehoux/Grimard 2018: 335; Pino et al. 2015: 6-8; Rantanen et al. 2018: 1852-1853; Vandemeulebroucke et al. 2019: 7). Beides zählt zu den Facetten von Sicherheit - die Unterstützungsleistung von digitaler Technik im Alltag und deren Zuverlässigkeit. Am Beispiel der Roboter wird aufgezeigt, dass die Studienteilnehmer*innen überzeugt sind, dass damit die Sicherheit der Gepflegten durch digitale Assistenzsysteme erhöht werden kann und sie im Alltag vor Unfällen besser geschützt sind, jedoch nur unter der Prämisse, dass den Anwender*innen durch die Roboter kein Schaden zugefügt wird. Krankenschwestern und -pfleger*innen betrachten mögliche Unfälle als größten Nachteil von Robotern in der Fragebogenstudie von Lee et al. (2018: 2102). Die 
Funktionen, die als sinnvoll erachtet werden, zeigen einmal mehr die potenzielle Einbettung von Technik in den Alltag. Dabei geht es um ein vielfältiges Portfolio:

»(a) cognitive support applications to compensate cognitive impairment (e.g., locating lost items, task reminding); (b) communication services to keep an active social life (e.g., video calls, email); (c) risk prevention and healthcare applications (e.g., falls detection, management of critical situations), and (d) applications for supporting everyday tasks (e.g., online grocery shopping, journey planning, simplified Internet access)«(Pino et al. 2015: 6).

Bei der Bewertung der Zuverlässigkeit dieser Optionen kommt die Skepsis, die derzeit nicht nur in der Pflege, sondern in gesellschaftlicher und organisationaler Hinsicht gegenüber den Systemen besteht, zum Ausdruck. Die Effizienz, die Effektivität, der Nutzen und die Funktionalität werden unter Realbedingungen infrage gestellt: "What if an elderly person is moving away from a robot, can it follow the elderly person? [...] Is that person able to put the robot back on its feet again?" (Cresswell/Cunningham-Burley/Sheikh 2018: 6; Herv. i. O.). Stakeholder*innen und Entscheider*innen aus Wirtschaft, Pflege, Politik, Versicherungswesen und Medien bewerten auf Verbandsebene die aktuelle Situation zurückhaltend: »The development of robots is actually very complicated, and we are still far from having robots that are flexible and meet the - often demanding - requirements of the users« (Johansson-Pajala/Gustafsson 2020: 3).

Auf einer normativen Eben kann aus diesem Diskurs gefolgert werden, dass es zwar mannigfaltige Optionen und Potenziale für assistive Technologien gibt, die unter bestimmten Voraussetzungen angenommen werden und das Pflegepersonal entlasten könnten. So werden Systeme, die speziell der Sicherheit und dem Monitoring dienen, bei der Befragung von Führungskräften in der Pflege im Rahmen von DAAS-KIN weitgehend als effizient, nützlich und förderlich für das Patient*innenwohl bewertet (vgl. Haug in diesem Band). Vor allem aber scheitern Roboter (noch) im Alltag, weil an diese höhere Erwartungen herangetragen werden und sie stärker in eine Mensch-Technik-Interaktion eingebettet sind als zum Beispiel ein Hausnotrufknopf oder Sensormatten (Cresswell/Cunningham-Burley/Sheikh 2018: 9; Lee et al. 2018: 2100; Rantanen et al. 2018: 1856; Weber 2015: 258-259). ${ }^{10}$ Gleichzeitig lässt sich nicht bilanzieren, dass weniger Hausbesuche notwendig waren, die Kommunikation zwischen allen Beteiligten erleichtert werden konnte oder einfache Systeme weniger fehleranfällig waren.

10 Cresswell/Cunningham-Burley/Sheikh (2018: 9) unterscheiden zwischen humanoiden Robotern und Servicerobotern, wobei letztere einfache Aufgabe wie Bringdienste und Reinigungstätigkeiten in der Studie in einer kontrollierten Umgebung (z.B. im Krankenhaus) erfüllen. 


\section{g) Teilhabe \& Wohltun}

Altersgerechte Assistenzsysteme sind auch dafür vorgesehen, eine soziale Teilhabe $\mathrm{zu}$ ermöglichen und zu verbessern, sowohl zu Hause als auch in der Öffentlichkeit (Weber 2015, vgl. Weber in diesem Band). Bei MEESTAR werden bei dieser Dimension die Überlegungen von Beauchamp/Childress (2019) herangezogen, die unter Beneficience Aspekte des sogenannten Wohltuns fassen. Darunter wird die Pflicht verstanden, dass Pflege und altersgerechte Assistenzsysteme im Allgemeinen Patient*innen zugutekommen und Maßnahmen ergriffen werden, die den Patient*innen guttun. Die Analyse der zentralen Werte in den Studien verdeutlicht, dass die Dimension Teilhabe zusammen mit Wohltun verhandelt wird. Die Ergebnisse bilden unter dieser Verknüpfung die zahlreichen Herausforderungen der (professionell) Pflegenden $a b$. Aus ethisch-soziologischer Sicht steht diese Perspektive neben den Bedürfnissen der Gepflegten, während die Dimension der Fürsorge stärker die Patient*innen in den Fokus rückt. Demzufolge leiten sich aus Teilhabe $\&$ Wohltun zwei Verbindungen im Werteschema ab (siehe Abbildung 1).

Digitale Assistenzsysteme, insbesondere robotische Technologien, werden in den Befragungen und Gruppendiskussionen von Broadbent et al. (2016), Johansson-Pajala/Gustafsson (2020), Lehoux/Grimard (2018), Pino et al. (2015), Suwa et al. (2020) und Vandemeulebroucke et al. (2019) als Chance für Kommunikation betrachtet, die Geselligkeit und soziale Kontakte schafft. Ältere und isolierte Menschen gelten daher als adäquate Zielgruppe (Vandemeulebroucke et al. 2019: 5-8). Die Einsamkeit könnte durch eine Gesprächsmöglichkeit, die über robotische Systeme bereitgestellt wird, zwar nicht aufgehoben, aber abgeschwächt werden. Daneben scheint die Unterhaltung durch Medien wie Musik, eine fachliche und soziale Unterstützung aus der Ferne sowie die Bereitstellung von (Gesundheits-)Informationen eine bereichernde Option darzustellen (Pino et al. 2015; Suwa et al. 2020; Vandemeulebroucke et al. 2019). Auch bei der Befragung von Führungskräften in der Pflege werden digitale Assistenzsysteme für Kommunikation und Entertainment mehrheitlich positiv für das Patient"innenwohl oder die Pflegequalität bewertet (vgl. Haug in diesem Band). Pflegekräfte, Stakeholder*innen und ältere Menschen sehen bei all den Vorteilen trotzdem die Gefahr einer (zunehmenden) sozialen Isolation oder Entfremdung (Johansson-Pajala/Gustafsson 2020: 5; Pino et al. 2015: 10; Rantanen et al. 2018: 1852).

Die Analyse des wissenschaftlichen Diskurses zeigt auf, dass Risiken für die psychische Gesundheit, die generell im Pflegekontext für Beschäftigte bestehen (vgl. Bobbert 2019), durch digitale Assistenzsysteme (noch) nicht aufgefangen werden können. Dazu zählen das Arbeiten unter Druck und zu wenig Zeit für die Patient*innen, woraus Interrollenkonflikte mit dem Berufsbild und -ethos resultieren. Unterstützung durch Technik, die der physischen Gesundheit zum Beispiel beim Heben der Patient*innen zuträglich sein können, werden als effektiv und 
hilfreich erachtet, wie bereits erwähnt. Aus gesellschaftlicher Sicht und nach Meinung betroffener Senior* innen wäre es wünschenswert, dass digitale Assistenzsysteme diese Situation verbessern (Lehoux/Grimard 2018: 333; Wangmo et al. 2019: 7). Als zentrale und realistische Anforderung an diese Technologien erweist sich der Faktor Zeit (Lehoux/Grimard 2018: 333; Vandemeulebroucke et al. 2019: 6-7). Zeit, die durch den Einsatz von Assistenzsystemen gespart wird, könnte - so die Auffassung - nicht nur einer Fürsorge der Gepflegten zugutekommen, sondern auch im Sinne eines Prinzips des Wohltuns eine Entwicklung in Richtung einer sguten Pflege darstellen.

Pflegekräfte erklären Pflegeroboter in der Studie von Rantanen et al. (2018: 1856) trotz eines positiven Befunds von Stakeholder*innen und Senior*innen (einmal mehr) für untauglich, psychische Bedürfnisse zu erfüllen: »The results show that home care personnel do not believe in the usefulness of care robots [...] in relieving anxiety or loneliness « (ebd.). Diese Einstellung scheint die Studie von Broadbent et al. (2016: 26), bei der Roboter testweise zum Einsatz kamen, empirisch zu belegen: "There were no significant changes to quality of life or depression.« Die Zeitersparnisse könnten durch die Verwaltung und Beaufsichtigung der Systeme irrelevant werden (Lee et al. 2018: 2102). Nicht nur aus subjektiver Sicht werden demzufolge die Potenziale durch die Risiken wieder aufgehoben.

\section{Vertrauen als zentrales Konstrukt im Diskurs: Individuelle, organisationale und gesellschaftliche Perspektiven}

Die aktuelle Konstellation im Diskurs bestimmt Vertrauen als entscheidendes und dynamisches ethisch-moralisches Kriterium des Phänomens altersgerechter Assistenzsysteme (siehe Abbildung 1). Zu diesem Ergebnis kommt auch die Studie »Why addressing ethical questions in KI will benefit organizations « des Capgemini Research Institute, bei der knapp 6.000 Stakeholder*innen weltweit zu den organisationalen Herausforderungen von KI im Allgemeinen befragt wurden (Capgemini Research Institute 2019). Demnach müssen Unternehmen ihre Systeme gegenüber den Verbraucher*innen in ethischer Hinsicht rechtfertigen, wenn Nachfrage und Bindung bei den Kund"innen erreicht werden soll. Die KI-Strategie bei dieser Studie impliziert solche ethischen Anforderungen, die auf die Dimensionen Gerechtigkeit, Privatheit, Fürsorge, Wissen und Sicherheit zurückgeführt werden können. Da davon ausgegangen werden kann, dass digitale Assistenzsysteme künftig auch im privatwirtschaftlichen Bereich angeboten werden, wird gefolgert, dass Innovationen und ökonomische Prinzipien ethische Kriterien von Beginn an integrieren sollten. Auch der Literaturreview von Vandemeulebroucke/Dierckx/Gastmans (2018) auf Basis von 28 Studien bestätigt die im Werteschema (Abbildung 1) ent- 
haltenen ethischen Dimensionen Gerechtigkeit, Autonomie, Fürsorge, Sicherheit, Teilhabe und Wohltun.

Vertrauen wird im Folgenden auf Basis eines moralischen Verständnisses diskutiert und orientiert sich dabei an Pfannkuche (2012). Vertrauen auf individueller, organisationaler und gesellschaftlicher Ebene adressiert gerade im privaten Umfeld des Zuhauses folgende (normative) Anforderungen an digitale Assistenzsysteme:

Gerechtigkeit: Vertrauen der Senior*innen in die Pflegenden und die Technik »ist dort notwendig, wo eine Kontrolle ihrer Handlungen entweder nicht realisierbar ist oder es Gründe gibt, auf diese freiwillig zu verzichten « (Pfannkuche 2012: 48; vgl. auch Haug/Weber 2015). Dies trifft auf viele Einzelsituationen im Alltag älterer Menschen zu. Eine vollkommende Kontrolle in allen Interaktionen im Pflegekontext erscheint kaum realisierbar, zumal aktuell noch einige Gesetzeslücken existieren und unklar ist, ob Gesetze und Leitlinien für Organisationen und Institutionen dazu beitragen, dass digitale Assistenzsysteme künftig nach fairen Maßstäben verteilt werden (Wangmo et al. 2019: 9).

Privatheit: Die Androhung von Sanktionen bei Nichteinhaltung des Datenschutzes durch gesetzliche Regelungen, an die sich Unternehmen, Organisationen und Kommunen halten müssen, kann eine Vertrauensbasis in digitale Assistenzsysteme schaffen (Pfannkuche 2012: 51). Bei MEESTAR wird Privatheit als Voraussetzung für individuelle Freiheit und Autonomie betrachtet. So sind Aufzeichnungen, die die Technologien anfertigen, ohne erfolgte Einwilligung nach der DatenschutzGrundverordnung (DSGVO) bereits strafbar, das Abschalten oder Pausieren der Geräte muss verlässlich geschehen.

Autonomie \& Selbstbestimmung: Die Nutzer*innen haben jederzeit die Kontrolle über die Systeme, sie können das System individuell an wechselnde Bedürfnisse anpassen und fühlen sich von dem System mit den Sinnen und in ästhetischer Hinsicht angesprochen (vgl. Pino et al. 2015: 12). Diese einfach vorzunehmenden Konfigurationen kann die Akzeptanz und Bindung an das Gerät erhöhen, ähnlich wie bei Smartphones, die sich als persönliches, technisches Gadget durchgesetzt haben. Subjekte vertrauen sich dieser Technologie bereits an und trauen dieser gleichermaßen zu, Individualität zu berücksichtigen. Damit gehen sie ein Risiko ein, weil sie nicht wissen können, was mit ihren Daten geschieht, was aber nicht generell in einem Widerspruch zu Vertrauen steht (Haug/Weber 2015). MEESTAR beinhaltet, dass die Selbstbestimmung im Rahmen einer technikgestützten Fürsorge nicht begrenzt werden darf.

Fürsorge \& Selbstverständnis: Ein Pflege-Setting ist hoch interaktiv. Wird in eine 'gute Pflege vertraut, wird auf die Kompetenz und das Wollen der Pflegekräfte vertraut, genau diese Leistung zu erbringen. Diese beiden Aspekte des Vertrauens implizieren, dass sich in einer Gesellschaft die Gepflegten, Angehörigen und Pflegedienste darauf verlassen und dass sich die Pflegekräfte in moralischer Hin- 
sicht damit identifizieren, dauerhaft eine sgute Pflege umzusetzen. Ethische digitale Assistenzsysteme können als technische Maschinen Erwartungssicherheit anbieten, wenn die Pflegekräfte keine Zeit haben, erschöpft sind oder kein Vertrauen in menschliche Pflege vorhanden ist: "Some caregivers do not fulfill their job with their heart and soul« (Vandemeulebroucke et al. 2019: 6). Nicht nur die analysierten Studien, auch weitere Untersuchungen zeichnen jedoch ein anderes, klares Bild, das sich auf der individuellen und organisationalen Ebene gegenseitig verstärkt (vgl. u.a. Kristoffersson et al. 2011; Nilsen et al. 2016; Pfadenhauer/Dukat 2015; Robillard/Kabacińska 2020; Scorna in diesem Band). Diese Auffassung wirkt als machtvolles Berufsethos in der Gesellschaft: »Human contact is crucial (Wangmo et al. 2019: 7). Demnach sollen intime, private Interaktionen nicht an Roboter abgegeben werden.

Wissen \& Innovation: Zwar ist das Innovationsparadigma in Deutschland fest verankert und gilt als Garant für internationale Wettbewerbsfähigkeit (vgl. Frommeld 2019: 78-84). Nichtsdestotrotz wird ein Vertrauen in die technische Entwicklung angesichts der Pflege älterer Menschen als riskant betrachtet (JohanssonPajala/Gustafsson 2020: 3). Die Entwicklung von digitalen Assistenzsystemen und insbesondere von humanoiden Robotern wird mit Misstrauen beobachtet, einem Antagonisten von Vertrauen. In einer smarten und total vernetzten >Society 5.0<, die nach Suwa et al. (2020: 12) in Japan vorangetrieben wird, welches weltweit als Vorreiter in der Realisierung einer robotischen Pflege gilt, ist der Kontakt mit digitalen Technologien im Zuhause und im Alter alltäglicher als in Deutschland. Um Vertrauen erreichen zu können, müssen erstens die Systeme bekannter werden und zweitens die Kompetenzen im Umgang mit Technik ausgebaut werden: „Exposure is likely to be key in going forward « (Cresswell/Cunningham-Burley/Sheikh 2018: 8; vgl. Vetter/Cerullo in diesem Band). Dazu gehört neben einem Technikwissen auch eine ethische Qualifikation. Letzteres wurde $2019^{11}$ in die Pflegeausbildung integriert (Lehmeyer/Riedel 2019; Riedel 2019).

Sicherheit: Vertrauen in die Sicherheit digitaler Systeme setzt voraus, dass die Pflegenden, Angehörigen und Gepflegten davon ausgehen können, dass ihnen das System nicht schadet und dass es bei Unfällen und unter Umständen bei lebensbedrohlichen Veränderungen die zugesicherte Leistung erbringt. Johansson-Pajala/Gustafsson (2020: 6) fordern für solche Situationen eine BackupLösung. Positive Erfahrungen mit digitalen Assistenzsystemen im Alltag können diese für alle beteiligten Akteur*innen vertrauenswürdig machen. Wenn der subjektive Eindruck intersubjektiv nachvollziehbar ist, kann sich zugleich Vertrauen in der Gesellschaft einstellen (Pfannkuche 2012). Selbst wenn die Systeme in der 
Zukunft effektiv arbeiten und im Aussehen Menschen gleichen, wird es kritisch gesehen, dass zu hohe Erwartungen geweckt werden, die dann in Enttäuschung und Vertrauensverlust resultieren, wenn die Technik nicht wie ein Mensch agiert (Cresswell/Cunningham-Burley/Sheikh 2018).

Teilhabe \& Wohltun: Eine grundsätzliche Frage im Diskurs über digitale Assistenzsysteme scheint mit Teilhabe und Wohltun zusammenzuhängen. Sind digitale Systeme dazu in der Lage, existenzielle Bedürfnisse nach Nähe und Austausch zu erfüllen? Das Vertrauen in diese Fähigkeit scheint auf keinem sicheren (moralischen) Fundament zu stehen. Wie Pfannkuche (2012: 56) es umschreibt, können wir uns in diesem noch kaum vertrauten Feld der Innovationen nicht mit Wissen beruhigen. Ein Vertrauenkönnen setzt voraus, dass Subjekte sich mit dem System vertraut machen können. Die derzeitige Situation in der Pflege ist gekennzeichnet von Problematiken wie Zeitmangel, zu kurzen Patient"innenkontakten, Überlastungen und Stress (vgl. Rudolph in diesem Band; Scorna et al. 2021a \& 2021b). Aus gesellschaftlicher Sicht und nach Meinung betroffener Senior*innen wäre es wünschenswert, dass altersgerechte Assistenzsysteme das moralische Spannungsverhältnis einer Abhängigkeit von dem Kontakt zu den Pflegekräften (und deren moralische Belastung, diesen adäquat zu erfüllen) aufbrechen. Wenn die Erfahrung zeigt, dass digitale Geräte zuverlässig als Brücke dienen, emotionale Bedürfnisse zu erfüllen und sich die Gepflegten dabei wohlfühlen, könnten die Systeme unterstützen, dass die Barrieren zu einer Teilhabe am sozialen Leben im Alter niederschwelliger verlaufen. Eine solche selbstbestimmte Teilhabe könnte nach MEESTAR zu einer positiven ethischen Bewertung führen.

Der Befund einer zurückhaltenden Diffusion von digitalen Assistenzsystemen in diesem Beitrag zeigt sich nicht nur in Deutschland, sondern unter anderem auch in Schweden und den USA (Alaiad/Zhou 2014; Johansson-Pajala/Gustafsson 2020). Eine empirisch gesicherte Beurteilung der potenziell vielfältigen, in Deutschland derzeitig aber kaum verbreiteten (und wenn, dann niederschwelligen) altersgerechten Assistenzsystemen ist aufgrund der wenigen Langzeitstudien kaum möglich. Die vorliegende Untersuchung stellt mit der Berücksichtigung internationaler Studien eine Annäherung an die Einstellungen und Nutzungshemmnisse auf der individuellen, organisationalen und gesellschaftlichen Ebene dar. Aufgrund der starken normativen Überzeugung gegenüber solchen MenschTechnik-Interaktionen, die gesellschaftlich tief verankert scheinen, können die Ergebnisse auf westliche Industriegesellschaften übertragen werden.

Der Akzent, der im Diskurs aktuell auf Vertrauen und auf sieben weiteren ethischen Dimensionen liegt, ist zeitlich variabel (siehe Abbildung 1). So könnte sich mit der Zeit eine Verschiebung ergeben, bei der sich die jetzige Ordnung im Diskurs verändert, weil ein Wertaspekt aus der inneren Kreisstruktur dominant wird und den Diskurs neu konfiguriert. 


\section{Fazit: Alte Herausforderungen, neue Handlungsmöglichkeiten}

Der vorliegende Beitrag analysiert den wissenschaftlichen Diskurs zwischen 2015 und $2020 \mathrm{zu}$ digitaler Technik in der Pflege und untersucht, wie Pflegekräfte, Senior*innen und Stakeholder*innen diese Technik bewerten. Altersgerechte Assistenzsysteme scheinen die dringenden Probleme in der Pflege, vor allem aus Sicht der Pflegekräfte, allenfalls vereinzelt abschwächen zu können. Altersgerechte Assistenzsysteme funktionieren daher jetzt noch nicht (und vielleicht auch nicht künftig) als Lösung für Herausforderungen, die sich durch den demografischen Wandel stellen oder als Kompensation für personelle Engpässe in der Pflege. Dieses Ergebnis stützt sich auf ein Werteschema (siehe Abbildung 1), das auf Basis von sieben ethischen Dimensionen die gegenwärtigen Einstellungen aufzeigt, die als Hemmnisse für die Einführung der Systeme und Technologien fungieren. Durchaus realistisch erscheinende Verbesserungen wie Zeitersparnisse im Pflegealltag werden durch ein neues, spannungsreiches Verhältnis zwischen Mensch und Technik relativiert: Innovationen im Bereich von digitalen Assistenzsystemen scheinen zu den psychischen Belastungen der professionell Pflegenden beizutragen, was die Sorge um den Verlust des Arbeitsplatzes und die Befürchtung angeht, der moralischen (Selbst-)Verpflichtung einer >guten Pflege nikeinsatzes nicht nachkommen zu können. Dieser moralische Konflikt scheint auf den ersten Blick subjektiv, könnte aber sowohl die Akzeptanz des Einsatzes von assistiver Technik als auch die Attraktivität eines Arbeitsplatzes in der Pflege weiterhin negativ beeinflussen.

Die verstärkte Einbindung technischen und ethischen Wissens in die Pflegeausbildung könnte jedoch in der Zukunft ein adäquates >Handwerkszeug`für die Pflege zur Verfügung stellen, vor allem da der Kontakt mit AAL, KI und weiteren digitalen (Assistenz-)Technologien in allen Altersgruppen und Schichten der Gesellschaft zunehmen wird. Damit verbindet sich das Potenzial, einerseits die (teilweise unbegründeten) Vorbehalte gegenüber digitalen Assistenzsystemen langfristig zu wandeln, so zum Beispiel, dass eine technisierte Pflege nicht zwangsweise bedeutet, sich mit einem Roboter, der an den Terminator erinnert, konfrontiert zu sehen und andererseits die Angst oder das Misstrauen gegenüber Neuem, welche als »fear of the unknown« (Cresswell/Cunningham-Burley/Sheikh 2018: 4-6, 8; vgl. Johansson-Pajala/Gustafsson 2020: 3-5) bezeichnet wird, zu überwinden. In deutschen Haushalten hat bislang nur der Hausnotruf eine weite Verbreitung erfahren; die sspektakulären Berichte in den Medien über die Potenziale der Robotik in der Pflege basieren auf Prototypen, die mit der Realität in der privaten und professionellen Pflege nicht viel gemein haben (Wahl/Kricheldorff/Hedtke-Becker 2018). Dieses Ergebnis bestätigt sich in dem Projekt DAAS-KIN in allen Teilstudien (vgl. Haug, Scorna sowie Vetter/Cerullo in diesem Band). 
Bei Unterstützungsleistungen, die in die Intimzone der Subjekte vordringen, weichen die untersuchten Studien teilweise von der gesellschaftlichen und professionellen Auffassung einer >guten Pflege wie dem traditionellen Altersbild ab. Diesen empirischen Daten zufolge wünschen sich ältere Menschen zwar menschliche Interaktionen, sind aber nicht der Auffassung, dass die Fürsorge in diesen Situationen exklusiv in einem Setting erfüllt werden kann, bei dem eine Person die andere pflegt (vgl. u.a. Klein/Schlömer 2018). Es handelt sich gerade bei solchen Beispielen wie dem Duschen und Toilettengang vielmehr um ein individuelles Arrangement, dass zwischen Subjekten und Akteur*innen unterschiedlich - und dann eben auch einfühlsam - verhandelt werden müsste, nimmt man das Prinzip der Autonomie ernst. Es ist bei dieser Klient*innengruppe denkbar, dass die Anerkennung von Selbstbestimmung und Privatheit mithilfe von digitalen Assistenzsystemen im $\mathrm{Zu}$ hause gelingen kann, was dem Konzept eines dritten Gesundheitsstandortes und einem Wandel des Altersbildes entspräche.

Die finanziellen Mittel, die in der Vergangenheit in innovative Lösungen investiert wurden, haben noch nicht zu einer erfolgreichen Zusammenarbeit von Politik, Forschung und Wirtschaft mit den (prospektiven) Nutzer*innen geführt - den Pflegekräften, den Gepflegten und den Senior*innen im Sinne einer partizipativen Technikgestaltung. Angesichts der Herausforderungen, denen sich die Gesellschaft im Zuge des demografischen Wandels und von Digitalisierung jetzt und in der Zukunft konfrontiert sieht, erscheint es notwendig, Risiken und Chancen von altersgerechten Assistenzsystemen differenziert zu betrachten.

Schon während der pandemischen Situation im Jahr 2020 und 2021 wurde während des Lockdowns Kommunikation und soziale Teilhabe von zu Hause aus über das Internet elementar und für manche auch alltäglicher als zuvor. Jenseits der Pandemie dürfte sich der Trend der Nutzung digitaler Technologien im Alltag in Zukunft verstärken. In einer digitalen Bildergesellschaft (Frommeld 2020 \& 2021) dürfte eine Selbstinszenierung in einem Zuhause mit digitalen Assistenzsystemen, die über Fotografien in den sozialen Medien erfolgt, für künftige ältere Generationen kein unwahrscheinliches Szenario darstellen.

Der Beitrag trägt dazu bei, aktuelle normative Überzeugungen auf gesellschaftlicher, organisationaler und individueller Ebene $\mathrm{zu}$ entschlüsseln. Da sich diese nicht wesentlich unterscheiden, ist es legitim, von machtvollen Nutzungshemmnissen zu sprechen. Der Beitrag zeigt, dass nicht nur aus der subjektiven Sicht der Pflegekräfte, der Gepflegten und Stakeholder*innen in diesem Umfeld die Chancen von digitalen Assistenzsystemen durch die antizipierten Risiken aktuell wieder aufgehoben werden. Jedoch entschlüsselt die WDA mit der zentralen Position von Vertrauen im Diskurs über digitale Assistenzsysteme auch Potenziale (siehe Abbildung 1): Die ethischen Dimensionen von Vertrauen, Wissen, Innovation und Wohltun, die (neue) Handlungsmöglichkeiten eröffnen. Weitere Optionen könnten darin bestehen, dass sich die Gepflegten >ihr< Gerät je nach Bedürfnis und Geschmack 
zusammenstellen können, was in einigen der untersuchten Studien zum Ausdruck gebracht wurde. Dazu könnte auch ein ästhetisch anspruchsvolles Design altersgerechter Assistenzsysteme zählen, das eben nicht den bisherigen Stereotypen (weiß, kalt, steril, monoton) folgt.

\section{Literatur}

Alaiad, Ahmad/Zhou, Lina (2014): "The determinants of home healthcare robots adoption: An empirical investigation«, in: International Journal of Medical Informatics 83 , S. 825-840.

Beauchamp, Tom L./Childress, James F. (2019): Principles of biomedical ethics, Oxford: Oxford Univ. Press.

Berger, Peter L./Luckmann, Thomas (2009): Die gesellschaftliche Konstruktion der Wirklichkeit. Eine Theorie der Wissenssoziologie, Frankfurt a.M.: Fischer.

Blumer, Herbert (1973): »Der methodologische Standpunkt des Symbolischen Interaktionsmus«, in: Arbeitsgruppe Bielefelder Soziologen (Hg.), Alltagswissen, Interaktion und gesellschaftliche Wirklichkeit Teil: 1, Symbolischer Interaktionismus und Ethnomethodologie, Reinbek bei Hamburg: Rowohlt, S. 80-146.

Bobbert, Monika (2019): »Berufliche Pflege und soziale Gerechtigkeit: sechs sozialethische Problemanzeigen «, in: Ethik in der Medizin 31, S. 289-303.

Braeseke, Grit/Pflug, Claudia/Tisch, Thorsten/Wentz, Lukas/PörschmannSchreiber/Ulrike/Kulas, Heidi (2020): Umfrage zum Technikeinsatz in Pflegeeinrichtungen (UTiP): Sachbericht für das Bundesministerium, Berlin: IGES Institut.

Bratan, Tanja/Wyrda, Sven (2018): „Gesamtgesellschaftliche Auswirkungen des medizintechnischen Fortschritts«, in: Harald Künemund/Uwe Fachinger (Hg.), Alter und Technik. Sozialwissenschaftliche Befunde und Perspektiven, Wiesbaden: Springer VS, S. 69-89.

Broadbent, Elizabeth/Kerse, Ngaire/Peri, Kathryn/Robinson, Hayley/Jayawardena, Chandimal/Kuo, Tony/Datta, Chandan/Stafford, Rebecca/Butler, Haley/Jawalkar, Pratyusha/Amor, Maddy/Robins, Ben/MacDonald, Bruce (2016): »Benefits and problems of health-care robots in aged care settings: A comparison trial«, in: Australasian Journal on Ageing 35, S. 23-29.

Capgemini Research Institute (2019): Why addressing ethical questions in AI will benefit organizations, https://www.capgemini.com/de-de/news/ki-ethik-strat egie/vom 19.02.2021.

Cresswell, Kathrin/Cunningham-Burley, Sarah/Sheikh, Aziz (2018): "Health Care Robotics: Qualitative Exploration of Key Challenges and Future Directions«, in: Journal of Medical Internet Research 20, e10410. 
Fachinger, Uwe/Henke, Klaus-Dirk (Hg.) (2010): Der private Haushalt als Gesundheitsstandort. Theoretische und empirische Analysen, Baden-Baden: Nomos.

Frommeld, Debora (2019): Die Personenwaage. Ein Beitrag zur Geschichte und Soziologie der Selbstvermessung, Bielefeld: transcript.

— (2020): »Die riskante Quantifizierung des Selbst. Vermessung, Optimierung und Ermächtigung im Zeitalter der (digitalen) Personenwaage«, in: Markus Holzinger/Oliver Römer/Clemens Boehncke (Hg.), Soziale Welt, Sonderband 24: Soziologische Phantasie und kosmopolitisches Gemeinwesen. Perspektiven einer Weiterführung der Soziologie Ulrich Becks, Baden-Baden: Nomos, S. 366-405.

- (2021): »Digitale Neuordnung und (il)legitime Wissensregime in einer Bildergesellschaft. Von Fat Studies, Body Positivity und Transformationen im Diskurs«, in: Birgit Blättel-Mink (Hg.), Gesellschaft unter Spannung. Verhandlungen des 40. Kongresses der Deutschen Gesellschaft für Soziologie 2020, Essen: DGS, im Erscheinen.

Geißler, Rainer/Meyer, Thomas (2002): Die Sozialstruktur Deutschlands. Die gesellschaftliche Entwicklung vor und nach der Vereinigung, Bonn: Bundeszentrale für Politische Bildung.

Giese, Constanze (2018): »Professionelles Selbstverständnis und Ethik«, in: Annette Riedel/Anne-Christin Linde (Hg.), Ethische Reflexion in der Pflege. Konzepte - Werte - Phänomene, Berlin: Springer, S. 21-29.

Haug, Sonja/Weber, Karsten (2015): »Vertrauen, Kontrolle und Privatsphäre in engen sozialen Beziehungen und die Wirkungen moderner Informations- und Kommunikationstechnologie«, in: Petra Grimm/Tobias O. Keber/Oliver Zöllner (Hg.), Anonymität und Transparenz in der digitalen Gesellschaft, Stuttgart: Franz Steiner, S. 37-56.

Heinze, Rolf G. (2018): »Alter und Technik«, in: Harald Künemund/Uwe Fachinger (Hg.), Alter und Technik. Sozialwissenschaftliche Befunde und Perspektiven, Wiesbaden: Springer VS, S. 15-31.

Heinze, Rolf G./Hilbert, Josef/Paulus, Wolfgang (2009): »Der Haushalt - ein Gesundheitsstandort mit Zukunft«, in: Andreas J. W. Goldschmidt/Josef Hilbert (Hg.), Gesundheitswirtschaft in Deutschland. Die Zukunftsbranche. Beispiele über alle wichtigen Bereiche des Gesundheitswesens in Deutschland, Wegscheid: Wikom, S. 772-801.

Hilbert, Josef/Beck, Denise/Cirkel, Michael/Dahlbeck, Elke (2018): »Alter und Technik: Perspektiven der Gesundheitswirtschaft«, in: Harald Künemund/Uwe Fachinger (Hg.), Alter und Technik. Sozialwissenschaftliche Befunde und Perspektiven, Wiesbaden: Springer VS, S. 33-50.

Johansson-Pajala, Rose-Marie/Gustafsson, Christine (2020): "Significant challenges when introducing care robots in Swedish elder care«, in: Disability and Rehabilitation. Assistive Technology, S. 1-11. 
Keller, Reiner (2011): Wissenssoziologische Diskursanalyse. Grundlegung eines Forschungsprogramms, Wiesbaden: Springer VS.

Klein, Barbara/Schlömer, Inga (2018): »A robotic shower system. Acceptance and ethical issues«, in: Zeitschrift fur Gerontologie und Geriatrie 51, S. 25-31.

Kohlen, Helen (2019): »Ethische Fragen der Pflegepraxis im Krankenhaus und Möglichkeiten der Thematisierung «, in: Ethik in der Medizin 31, S. 325-343.

Kristoffersson, Annica/Coradeschi, Silvia/Loutfi, Amy/Severinson-Eklundh, Kerstin (2011): "An Exploratory Study of Health Professionals' Attitudes about Robotic Telepresence Technology«, in: Journal of Technology in Human Services 29, S. 263-283.

Lee, Jai-Yon/Song, Young A./Jung, Ji Y./Kim, Hyun J./Kim, Bo R./Do, HyunKyung/Lim, Jae-Young (2018): »Nurses' needs for care robots in integrated nursing care services«, in: Journal of Advanced Nursing 74, S. 2094-2105.

Lehmeyer, Sonja/Riedel, Annette (2019): »Ethikkompetenzerwerb im Handlungsfeld - Voraussetzungen und Impulse für die professionelle Pflegepraxis«, in: Ethik in der Medizin 31, S. 391-406.

Lehoux, P./Grimard, D. (2018): »When robots care: Public deliberations on how technology and humans may support independent living for older adults«, in: Social Science \& Medicine 211, S. 330-337.

Lenk, Christian/Frommeld, Debora (2015): »Different concepts and models of information for family-relevant genetic findings: Comparison and ethical analysis«, in: Medicine, Health Care and Philosophy 18, S. 393-408.

Manzeschke, Arne/Weber, Karsten/Rother, Elisabeth/Fangerau, Heiner (2013): Ergebnisse der Studie »Ethische Fragen im Bereich altersgerechter Assistenzsysteme«, Ludwigsfelde: VDI/VDE Innovation+Technik GmbH.

Motel-Klingenbiel, Andreas/Künemund, Harald/Bode, Christina (2005): »Wohnen und Wohnumfeld«, in: Martin Kohli/Harald Künemund (Hg.), Die zweite Lebenshälfte. Gesellschaftliche Lage und Partizipation im Spiegel des AltersSurvey, Wiesbaden: Springer VS, S. 125-175.

Nilsen, Etty R./Dugstad, Janne/Eide, Hilde/Gullslett, Monika K./Eide, Tom (2016): »Exploring resistance to implementation of welfare technology in municipal healthcare services - A longitudinal case study«, in: BMC Health Services Research 16, S. 657.

Nowossadeck, Sonja/Engstler, Heribert (2017): »Wohnung und Wohnkosten im Alter«, in: Katharina Mahne/Julia K. Wolff/Julia Simonson et al. (Hg.), Altern im Wandel: Zwei Jahrzehnte Deutscher Alterssurvey (DEAS), Wiesbaden: Springer Fachmedien, S. 287-300.

Pfadenhauer, Michaela/Dukat, Christoph (2015): »Robot Caregiver or RobotSupported Caregiving?«, in: International Journal of Social Robotics 7, S. 393-406. 
Pfannkuche, Walter (2012): »Vertrauen - Eine Frage der Moral?«, in: Heidi Möller (Hg.), Vertrauen in Organisationen. Riskante Vorleistung oder hoffnungsvolle Erwartung?, Wiesbaden: Springer VS, S. 47-62.

Pino, Maribel/Boulay, Mélodie/Jouen, François/Rigaud, Anne-Sophie (2015): »AAre we ready for robots that care for us? $<$ Attitudes and opinions of older adults toward socially assistive robots «, in: Frontiers in Aging Neuroscience 7, S. 1-15.

Porz, Rouven (2018): „Care Ethics ist nicht gleich Pflegeethik «, in: Annette Riedel/Anne-Christin Linde (Hg.), Ethische Reflexion in der Pflege. Konzepte - Werte - Phänomene, Berlin: Springer, S. 14-19.

Radic, Marija/Vosen, Agnes (2020): »Ethische, rechtliche und soziale Anforderungen an Assistenzroboter in der Pflege Sicht des Führungspersonals in Kliniken und Pflegeeinrichtungen«, in: Zeitschrift für Gerontologie und Geriatrie 53, S. 630-636.

Rantanen, Teemu/Lehto, Paula/Vuorinen, Pertti/Coco, Kirsi (2018): »The adoption of care robots in home care - A survey on the attitudes of Finnish home care personnel«, in: Journal of Clinical Nursing 27, S. 1846-1859.

Renn, Ortwin (1999): »Die Wertbaumananalyse. Band 2«, in: Stephan Bröchler/Georg Simonis/Karsten Sundermann (Hg.), Handbuch Technikfolgenabschätzung, Bonn: Ed. Sigma, S. 617-624.

- (2014): "Die Wertbaumanalyse: Ein diskursives Verfahren zur Bildung und Begründung kollektiv wirksamer Bewertungsmuster«, in: Marlen Niederberger/Sandra Wassermann (Hg.), Methoden der Experten- und Stakeholdereinbindung in der Sozialwissenschaftlichen Forschung, Wiesbaden: Springer VS, S. 165-188.

Riedel, Annette (2019): »Ethikkompetenzen vertiefen und verdichten - Welche Rolle kann die Ethik-Leitlinienentwicklung als exemplarische Methode der Ethikdidaktik in der hochschulischen Pflegeausbildung spielen?«, in: Ethik in der Medizin 31, S. 361-390.

Robillard, Julie M./Kabacińska, Katarzyna (2020): »Realizing the Potential of Robotics for Aged Care Through Co-Creation«, in: Journal of Alzheimer's Disease 76, S. 461-466.

Scorna, Ulrike (2015): "Servicerobotik in der Altenpflege. Eine empirische Untersuchung des Einsatzes der Serviceroboter in der stationären Altenpflege am Beispiel von PARO und Care-O-bot «, in: Karsten Weber/Debora Frommeld/Arne Manzeschke et al. (Hg.), Technisierung des Alltags. Beitrag für ein gutes Leben?, Stuttgart: Franz Steiner, S. 81-97.

Scorna, Ulrike/Frommeld, Debora/Haug, Sonja/Weber, Karsten (2021a): »Digitale Assistenzsysteme in der Altenpflege - Fluch oder Segen? Eine empirische Untersuchung zu Chancen, Risiken und Auswirkungen«, in: Birgit BlättelMink/Torsten Noack/Cornna Onnen et al. (Hg.), Organisationen in Zeiten der Digitalisierung, Berlin: Springer, im Erscheinen. 
— (2021b):»Digitale Technik in der Pflege als Generallösung? Neue Perspektiven auf altersgerechte Assistenzsysteme«, in: Carolin Freier/Joachim König/Arne Manzeschke et al. (Hg.), Gegenwart und Zukunft sozialer Dienstleistungsarbeit. Chancen und Grenzen der Digitalisierung in der Sozialwirtschaft, Wiesbaden: Springer VS, S. 299-312.

Stehr, Nico (1994): Arbeit, Eigentum und Wissen. Zur Theorie von Wissensgesellschaften, Frankfurt a.M.: Suhrkamp.

- (2001):»Moderne Wissensgesellschaften«, in: Aus Politik und Zeitgeschichte 36, S. 7-14.

Strauss, Anselm L./Corbin, Juliet M. (1996): Grounded Theory: Grundlagen qualitativer Sozialforschung, Weinheim: Beltz.

Strauss, Anselm L./Hildenbrand, Astrid/Hildenbrand, Bruno (1994): Grundlagen qualitativer Sozialforschung. Datenanalyse und Theoriebildung in der empirischen soziologischen Forschung, München: Fink.

Strübing, Jörg/Hirschauer, Stefan/Ayaß, Ruth/Krähnke, Uwe/Scheffer, Thomas (2018): "Gütekriterien qualitativer Sozialforschung. Ein Diskussionsanstoß«, in: Zeitschrift für Soziologie 47, S. 83-100.

Suwa, Sayuri/Tsujimura, Mayuko/Kodate, Naonori/Donnelly, Sarah/Kitinoja, Helli/Hallila, Jaakko/Toivonen, Marika/Ide, Hiroo/Bergman-Kärpijoki, Camilla/Takahashi, Erika/Ishimaru, Mina/Shimamura, Atsuko/Yu, Wenwei (2020): "Exploring perceptions toward home-care robots for older people in Finland, Ireland, and Japan: A comparative questionnaire study«, in: Archives of Gerontology and Geriatrics 91, S. 1-15.

Vandemeulebroucke, Tijs/Dierckx, Bernadette C. de/Gastmans, Chris (2018): »The use of care robots in aged care: A systematic review of argument-based ethics literature«, in: Archives of Gerontology and Geriatrics 74, S. 15-25.

Vandemeulebroucke, Tijs/Dierckx, Bernadette C. de/Welbergen, Laura/Massart, Michiel/Gastmans, Chris (2019):»The Ethics of Socially Assistive Robots in Aged Care. A Focus Group Study With Older Adults in Flanders, Belgium«, in: The Journals of Gerontology. Series B, Psychological Sciences and Social Sciences 20, S. 1-12.

Wahl, Hans-Werner/Kricheldorff, Cornelia/Hedtke-Becker, Astrid (2018): »Technik für vulnerable ältere Menschen und ihre Angehörigen. Möglichkeiten und Grenzen «, in: Zeitschrift fur Gerontologie und Geriatrie 51, S. 1-2.

Wahl, Hans-Werner/Oswald, Frank (2016):»Theories of environmental gerontology: Old and new avenues for ecological views of aging", in: Brian K. Kennedy/Jacqui Smith/Nancy Morrow-Howell et al. (Hg.), Handbook of theories of aging, New York: Springer, S. 621-641.

Wangmo, Tenzin/Lipps, Mirjam/Kressig, Reto W./Ienca, Marcello (2019): »Ethical concerns with the use of intelligent assistive technology: Findings from a qualitative study with professional stakeholders", in: BMC Medical Ethics 20, S. 1-11. 
Weber, Karsten (2015): »Meestar: Ein Modell zur ethischen Evaluierung sozio-technischer Arrangements in der Pflege- und Gesundheitsversorgung«, in: Karsten Weber/Debora Frommeld/Arne Manzeschke et al. (Hg.), Technisierung des Alltags. Beitrag für ein gutes Leben?, Stuttgart: Franz Steiner, S. 247-262.

- (2016): MEESTAR ${ }^{2}$. Ein erweitertes Modell zur ethischen Evaluierung soziotechnischer Arrangements (Zweite transdisziplinäre Konferenz zum Thema »Technische Unterstützungssysteme, die die Menschen wirklich wollen«), Hamburg: Helmut-Schmidt-Universität.

Zöllick, Jan C./Kuhlmey, Adelheid/Suhr, Ralf/Eggert, Simon/Nordheim, Johanna/Blüher, Stefan (2020): "Akzeptanz von Technikeinsatz in der Pflege«, in: Klaus Jacobs/Adelheid Kuhlmey/Stefan Greß et al. (Hg.), Pflege-Report 2019: Mehr Personal in der Langzeitpflege - aber woher?, Berlin: Springer, S. 211-218. 
Implementierung und Evaluation von (digitaler) Pflegetechnik 



\section{Möglichkeiten des Bewegungsmonitorings durch Fitnesstracker nach einer Hüftfraktur}

Amelie Altenbuchner

\section{Ein Forschungsprojekt zur Nutzbarkeit von Aktivitätstrackern in der Alterstraumatologie}

Fitnesstracker werden in gesundheitswissenschaftlichen und medizinischen Studien immer häufiger erfolgreich eingesetzt (Henriksen et al. 2018). Die Möglichkeiten für die Forschung sind immens, jedoch sollten bei einem wissenschaftlichen Einsatz der konventionellen Produkte die Aspekte Güte der Erhebung, der Ethik und des Datenschutzes sowie des Datenmanagements besonders beachtet werden (Rat für Sozial- und Wirtschaftsdaten 2020). In der Verwendung der Fitnesstracker bei sehr alten, zumeist multimorbiden und fragilen Patient*innen kommt hinzu, dass diese Lifestyle-Produkte gar nicht für diese Zielgruppe entworfen wurden. Genau in dieser Gruppe sind jedoch die Faktoren (Im-)Mobilität und körperliche (In-)Aktivität von so hoher Bedeutung für Autonomie, Lebensqualität und Lebenszeit, dass sich die Übertragung der handelsüblichen Aktivitäts-Tracking-Technologie in diesen Kontext lohnt. Zudem ist die Accelerometer-Technologie in ihrer Entwicklung bereits weit vorangeschritten (vgl. Matthews et al. 2019: 11). Als Instrument für ein Bewegungsmonitoring erfassen Fitnesstracker Bewegung und reflektieren dadurch Mobilität. Sie ergänzen das Mobilitätsassessment, das durch Fragebögen oder Tests erfolgt. Da diese Testmethoden bei sehr alten Patient*innen häufig stark begrenzt sind, zum Beispiel aufgrund kognitiver Einschränkungen (vgl. Hager 2018a), bietet das Bewegungsmonitoring eine objektivere Möglichkeit der Einschätzung von Mobilität, als es Eindrücke von Pflegenden, Angehörigen oder Patient*innen selbst erlauben (Altenbuchner/Haug/Weber 2019: 143-144).

Anhand der Erkenntnisse, die im Forschungsprojekt Nutzbarkeit von Aktivitätstrackern in der Alterstraumatologie gesammelt wurden, wird in diesem Beitrag aufgezeigt, inwieweit sich Fitnesstracker eignen, um die Mobilitätsentwicklung geriatrischer Patient*innen abzubilden. Es handelt sich um ein interdisziplinäres Forschungsprojekt, in dem in einem nach den Richtlinien der Deutschen Gesellschaft für Unfallchirurgie (DGU) zertifizierten Alterstraumazentrum (ATZ-DGU) eine Beobachtungsstudie begonnen und im häuslichen Umfeld der Patient*innen fortgesetzt 
wurde. Die Zielgruppe des Projekts sind Patient"innen, die nach der operativen Versorgung einer hüftnahen Fraktur den individuellen Rehabilitationsprozess aufnehmen.

Nach einer Einführung in die theoretische und empirische Ausgangslage für das Forschungsprojekt wird erläutert, warum sich die Fitnesstracker-Armbandlösung hervorragend für eine Beobachtung des Bewegungsverhaltens im häuslichen Umfeld eignet, auch wenn durch dieses Verfahren die tatsächlichen Schrittzahlen unterschätzt werden könnten. Hierbei stellt die Wahl der Variable der täglichen Schrittzahl eine sinnvolle Ergänzung zum regulären Mobilitätsassessment dar. Im Verlauf des Beitrags wird deutlich, dass methodische Einschränkungen aus forschungsethischen Gründen, insbesondere aufgrund der Vulnerabilität von geriatrischen Patient*innen, zum jetzigen Zeitpunkt in Kauf zu nehmen sind.

Die Nutzung des Lifestyle-Produkts Fitnesstracker in einem neuen Kontext, eine Form der »Technikgestaltung« (vgl. Banse/Hauser 2010: 18), ist gut geeignet, weil sie einem dringlichen Problem auf praxistaugliche, einfache und kostengünstige Art begegnet. Die Problembehandlung, die der Einsatz des Fitnesstrackers ermöglicht, betrifft sowohl ein Erkenntnisinteresse bezüglich des realen Bewegungsverhaltens der Zielgruppe von verunfallten über 80-jährigen Patient*innen als auch eine Möglichkeit zur Dokumentation des Mobilisierungsverlaufs im Rehabilitationsprozess.

\section{Forschungsprojekte mit geriatrischen Patient*innen}

In der ethischen, sozialen, kulturellen und technologischen Erforschung, Entwicklung und Evaluation technikgestützter Assistenzsysteme liegen hohe gesellschaftliche Erwartungen. Für ein angestrebtes >gutes Leben $<$ im Alter wird an vielen Stellen, wie etwa Forschung, Politik und Entwicklung, ein hoher Aufwand betrieben. Die Übertragung der technischen Lösungen in das Alltagsleben der alternden und sehr alten Menschen ist jedoch immer noch verbesserungsfähig (vgl. Weber 2017). Über die Verhaltensweisen der Zielgruppe liegen nur wenige gesicherte Ergebnisse vor (Haltaufderheide/Hovemann/Vollmann 2020: 5). Diese Situation betrifft nicht nur technologiegetriebene Studien. Auch für eine evidenzbasierte medizinischpflegerische Versorgung geriatrischer Patient*innen treten methodische und ethische Herausforderungen auf, die den Forschungsprozess erschweren und maßgeblich zu einer Evidenzlücke beitragen, die es noch zu schließen gilt (vgl. Deutsche Akademie der Wissenschaften 2015; Hager 2018c). Ursachen hierfür liegen unter anderem in der Teilnahmebereitschaft von Proband*innen, entsprechender Fallzahlgrößen und fehlender Daten sowie in der Einwilligungsfähigkeit (Deutsche Akademie der Wissenschaften 2015: 55). Es besteht eine Evidenzlücke und darum muss häufig auf die Daten aus jüngeren Stichprobenzusammensetzungen zu- 
rückgegriffen werden, obwohl sich physiologische und biologische Abläufe im Alter anders gestalten und lebensweltbedingte andere Versorgungsregeln gelten (Hager 2018c: 16).

Ein Beispiel ist die rasche Mobilisierung der geriatrischen Patient*innen nach der operativen Versorgung einer hüftnahen Fraktur. Obwohl bei jüngeren Personen erst nach mehreren Wochen eine Vollbelastung erlaubt ist, gilt es bei geriatrischen Patient*innen vorrangig eine Immobilität, auch vorübergehend, möglichst zu vermeiden (Hager/Krause 2018). Für diese Gruppe bedeutet eine Immobilität konkrete Risiken im akutstationären Setting zu haben, wie Thrombosen, Embolien, Pneumonien oder Dekubitus (Hagg-Grün/Nikolaus/Zeyfang 2013: 49-50) und schwerwiegende Konsequenzen in den Wochen und Monaten nach der Entlassung, wie ein erhöhtes Demenz- oder Mortalitätsrisiko (Barth/Doblhammer 2017: 223-232), aber auch ein Verlust der Selbstständigkeit und ein Umzug ins Pflegeheim, wo wiederum in der ersten Zeit ein erhöhtes Frakturrisiko besteht (Rapp et al. 2019: 12-14). Für geriatrische Traumapatient*innen ist die Mobilisierung das zentrale medizinische und pflegerische Anliegen; die Einschätzung des individuellen Ziels bei der Remobilisierung ist allerdings von starker Unsicherheit geprägt. Entscheidungen beruhen mit steigendem Lebensalter stärker auf subjektiven Einschätzungen und Erfahrungen als auf gesichertem Wissen. Das Bild, das die Beteiligten von den Patient*innen haben, beruht auch auf gesellschaftlichen Altersvorstellungen (vgl. Frommeld in diesem Band). Die Einschätzungen des medizinisch-pflegerischen Fachpersonals, der Patient*innen selbst, der Angehörigen, der Pflegekräfte, Physiotherapeut*innen und Ärzt"innen sind von gewissen verzerrten Überzeugungen geprägt, die zu unterschiedlichen Auslegungen und entsprechenden Therapieentscheidungen führen (vgl. Avers et al. 2011: 153; Hager 2018c: 16-19; Höpflinger 2014: 178). Diese Situation ist für alle Beteiligten wenig zufriedenstellend; darum fordert die Deutsche Akademie der Wissenschaften (2015) Möglichkeiten zu finden, solche Patient*innen in Forschungsdesigns einzubeziehen, die zu oft aufgrund von Alter und altersbedingten Gegebenheiten und Vorerkrankungen ausgeschlossen werden (ebd.: 55-57).

Ein Vorschlag, um dies voranzutreiben, ist die Erforschung der Möglichkeiten alltagstauglicher Geräte zum fortlaufenden Bewegungsmonitoring (Barth/Doblhammer 2017: 234-238). Ein sensorbasiertes Bewegungsmonitoring bietet die Möglichkeit einer individuellen und objektiven Beobachtung der Mobilisierung (Benzinger et al. 2014: 236), die für die Proband*innen ohne gesundheitliche Folgen, Einschränkungen oder besondere Anstrengungen gestaltet werden und pragmatisch in deren Lebenswirklichkeit zum Einsatz kommen kann (vgl. Deutsche Akademie der Wissenschaften 2015: 53). Ein Fitnesstrackerarmband erlaubt das Bewegungsverhalten nicht nur in einer laborartigen Umgebung zu erproben, wie es zum Beispiel bei Sensoren der Fall ist, die wie ein Weste am Körper getragen werden und mithilfe anderer angelegt werden müssen (vgl. Nicolai 2012: 58), son- 
dern kann alltäglich von den Proband*innen getragen werden. Dadurch wird eine Exploration des tatsächlichen Verhaltens und das Erzeugen einer zweckdienlichen Datenlage möglich (vgl. Altenbuchner/Weber/Fuchs 2019: 150-152).

Ein weiterer Grund für die Schwierigkeit in der Generierung von Evidenz liegt in den Besonderheiten der Zielgruppe selbst. Wer sind alternde Patient*innen in einem sehr hohen Alter und weshalb bedarf es bei dieser Zielgruppe einer besonderen methodischen und forschungsethischen Herangehensweise? Eine kurze Antwort auf diese Fragen lautet: Geriatrische Patient*innen haben ein altersbedingtes erhöhtes Risiko für eine Fragilität und weisen darum eine erhöhte Vulnerabilität auf. Diese Faktoren führen einerseits zu einer Forschungslücke und machen die Erforschung dieser Zielgruppe andererseits umso dringlicher.

Eine Definition des Merkmals salt gestaltet sich als schwierig, da sich abgesehen von einer Einteilung des chronologischen Lebensalters der Lebensabschnitt >Alter überaus heterogen gestalten kann (vgl. Avers et al. 2011; Weber 2017). In der Bundesrepublik Deutschland ist zurzeit knapp über ein Fünftel der Bevölkerung älter als 65 Jahre, was sich im Laufe der nächsten Jahrzehnte steigern wird (vgl. Statistisches Bundesamt 2020a). In der Geriatrie erfolgt die Altersbewertung unter Einbezug des Vorhandenseins einer altersentsprechenden Morbidität. In der Definition nach Sieber (2007) liegt der Fokus auf dem Alter, aber auch auf typischen Begleiterscheinungen des Alterns. Geriatrische Patient*innen sind solche, die ein chronologisches Alter von mindestens 65 Jahren, in aller Regel jedoch 70 Jahre, erreicht haben und zusätzlich eine »Multimorbidität« (Vorhandensein von mindestens zwei meist chronischen Krankheitsbildern oder Syndromen, vgl. Robert Koch-Institut 2021) aufweisen. Daneben sind auch all jene, die ein Lebensalter von mindestens 80 Jahren erreicht haben und eine alterstypische Vulnerabilität aufweisen, als geriatrische Patient*innen zu bezeichnen (Sieber 2007: 1190).

Das Zusammenspiel von alterstypischen Morbiditäten und der Alterskomponente kann anhand der Patient*innen mit hüftgelenksnahen Frakturen verdeutlicht werden. Die Femurfraktur ist eine der häufigsten geriatrischen Frakturen (Örgel/Petri 2018: 63). In der Bundesrepublik Deutschland kommt es jährlich pro 100.000 Einwohner*innen zu 130 »Frakturen des Femurs« (Rapp et al. 2019: 11) mit den ICD-10-GM-2021-Diagnosen S72.0- bis S72.9 (Bundesinstitut für Arzneimittel und Medizinprodukte (BfArM) 2020). Von allen Personen mit dieser Diagnose sind 86 Prozent der Patient*innen über 65 Jahre alt. Die hüftnahe Fraktur ist somit eine typische Alterserscheinung. Die Altersgruppen in Abschnitten von fünf Jahren machen mit steigendem Alter einen größer werdenden prozentualen Anteil der hüftgelenksnahen Frakturen aus. Die meisten dieser Frakturen kommen bei Personen zwischen 80 und 89 Jahren vor, danach nimmt die Zahl der Frakturen wieder ab (Statistisches Bundesamt 2020b). In den nächsten zwei bis drei Jahrzehnten wird die Anzahl der Patient*innen mit hüftnahen Frakturen um rund 70 Prozent anstei- 
gen, bei über 80-Jährigen um 150 Prozent, und diese Verunfallten werden stationär und operativ behandelt werden müssen (Kretschmer et al. 2017).

Sowohl die Ursachen für als auch die Folgen von einer solchen Fraktur sind Fragilität, Multimorbidität und Vulnerabilität. Die Beziehung dieser Begriffe werden von verschiedenen Autor*innen als Teufelskreis dargestellt oder auch als das "Fragilitätssyndrom« bezeichnet (Brecht 2018: 57; Hagg-Grün/Nikolaus/Zeyfang 2013: 49; Zeyfang/Hagg-Grün/Nikolaus 2013: 63). Ein komplexes Zusammenspiel der Ereignisabfolge der Fragilität, welche nicht linear auftritt, sondern prozesshaft und individuell-unterschiedlich sowie soziokulturell unterschiedlich bedingt verlaufen kann (vgl. Becker 2014: 24; Wahl/Heyl 2015: 24-25), lässt sich verkürzt folgendermaßen beschreiben:

Physiologisches Altern bedeutet eine Veränderung der physiologischen Abläufe der Organsysteme, wie zum Beispiel eine verringerte Nervenleitgeschwindigkeit, geringeres Herzminutenvolumen oder verringerte maximale Sauerstoffaufnahme (Denkinger/Nikolaus 2013: 8). Diese Abläufe gehen nach und nach, auch bei sehr aktiven und fitten Personen, in Erkrankungen über und führen zu einer erhöhten Fragilität (Hager 2018b: 6, 2018d: 9-10; Höpflinger 2014: 177; Sieber 2007: 1190). Frailty (engl.) und die nicht vollends passenden Entsprechungen Gebrechlichkeit und Fragilität sind bekannte Begriffe der geriatrischen Medizin. Anzeichen der Fragilität sind Schwäche, Langsamkeit und ein schlechter Allgemeinzustand (Hager/Krause 2016: 48). Frailty gilt als geriatrisches Syndrom, zu dessen Operationalisierung Messinstrumente, Skalen, Diagnosekriterien und Biomarker existieren (ebd.). Gleichzeitig ist sie ein subjektives multifaktorielles Geschehen, das schwer quantitativ zu erfassen ist und dennoch eine sinnvolle und notwendige gesundheitswissenschaftliche und medizinische Variable darstellt (Körtner 2006: 108-109). Nach Sieber ist die Therapie der Erkrankungen geriatrischer Patient*innen unter besonderer Berücksichtigung ihrer Fragilität das »archetypische Handeln« der Geriatrie (Sieber 2007: 1193).

Durch das Altern, damit einhergehende Erkrankungen und das erhöhte Fragilitätsrisiko ist früher oder später auch eine Inaktivität zu beobachten. Diese führt zu einem Verlust der Selbstständigkeit, bis letztendlich eine Immobilität eintritt. Sie stellt die Kluft zwischen der persönlichen physischen Möglichkeit beziehungsweise Fähigkeit und den Umweltanforderungen dar (Barth/Doblhammer 2017: 211). Der Mangel an körperlichen, sozialen und kognitiven Aktivitäten ist ein Gesundheitsproblem, wenn sich der Funktionsstatus der physiologischen und mentalen Abläufe zusehends verschlechtert (Hager 2018d: 10). Zunehmende Immobilität führt als Folge der Fragilität zu Frakturen, etwa durch Stürze (vgl. Brecht 2018: 59.), aber auch durch krankheitsbedingte Brüche, etwa bei Osteoporose (vgl. Örgel/Petri 2018: 63). Die Folge solcher Frakturen sind weitere Einschränkungen. Nach einer hüftnahen Fraktur steigt das Risiko einer erhöhten Sterblichkeit, einer Behinderung, des Umzugs in ein Pflegeheim und der Sekundärfrakturen. Das Risiko eines 
erneuten Bruchs in den ersten Monaten ist um das 2 bis 2,5-Fache höher als sonst (Rapp et al. 2019: 12). Der Anteil derer, die sich nach der Fraktur erholen, beträgt 40 bis 60 Prozent. Bis zu 60 Prozent der Patient*innen sind nach der Fraktur auf einen erhöhten Hilfebedarf angewiesen (ebd.: 13).

Im Zusammenspiel der Multimorbidität und Fragilität gilt für die Betroffenen eine erhöhte »alterstypische Vulnerabilität « (Sieber 2007: 1192-1193). Das bedeutet, dass eine gewisse Fragilität vorliegt, die durch den Zustand einer möglichen oder vorhandenen Schwäche und Einschränkung erzeugt wird, wobei sie nicht die Behinderung an sich bezeichnet, sondern ein erhöhtes Risiko, dass eine solche eintreten wird (ebd.). Um den methodischen und ethischen Besonderheiten gerecht zu werden, wurde eine prospektive Studie durchgeführt (Altenbuchner et al. 2018) und ein Ethikvotum eingeholt.

\section{Die Zuverlässigkeit der Fitnesstracker in der Zielgruppe}

In Anbetracht des Gangbilds der Zielgruppe muss davon ausgegangen werden, dass der Algorithmus des Fitnesstrackers bei geriatrischen Patient"innen rund 10 Prozent weniger Schritte speichert, als tatsächlich getätigt werden. Beim Gehen mit Hilfsmitteln ist ein noch stärkeres Unterschätzen möglich (vgl. Floegel et al. 2017).

In einer prospektiven Studie wurde zunächst der Fitnesstracker Fitbit AltaHR eingesetzt. Dieser wird am Handgelenk getragen und erfordert eine regelmäßige Akkuaufladung. Je nach Nutzungsintensität beträgt die maximale geschätzte Akkulaufzeit fünf bis sieben Tage (Fitbit Inc. 2019a), was für die Beobachtung auf der Station und unter Anbetracht der niedrigen Nutzungsintensität durch die Zielgruppe ausreichend ist. Nach den Erfahrungen dieser Untersuchung erfolgte in einer folgenden Längsschnittuntersuchung der Einsatz eines Fitnesstrackers der Marke Garmin vívofit 3. Dieser verfügt über eine langlebige Batterie, die ein Bewegungsmonitoring im häuslichen Umfeld ohne Akkuaufladung erlaubt. Allerdings erfordert das Gerät ein regelmäßiges Auslesen der Datenpunkte, da die Speicherkapazität für maximal vier Wochen ausreicht. Dadurch kam es zu regelmäßigen Besuchen bei den Proband"innen, anhand derer auch wichtige Erfahrungen für die Erforschung der Zielgruppe im häuslichen Umfeld gesammelt werden konnten, die an anderer Stelle veröffentlicht $\mathrm{zu}$ finden sind (vgl. Altenbuchner/Weber 2020). Im Forschungsprojekt wurde nicht primär die Genauigkeit der Fitnesstracker für die geriatrische Zielgruppe überprüft. Allerdings erfolgte im Rahmen einer Fallstudie ein Vergleich der aufgezeichneten Schrittzahlen der im Projekt eingesetzten Tracker. Die Ergebnisse werden im Folgenden vorgestellt. In Anbetracht der empirischen Datenlage wurde davon ausgegangen, dass die Geräte der Marken Fitbit und Garmin ausreichend akkurat sind. 
Wenn handelsübliche Fitnesstracker als Forschungsinstrumente eingesetzt werden, sind die Geräte dieser Marken die am häufigsten verwendeten, wie ein systematisches Review der Fitnesstracker-Verwendung aus dem Jahr 2018 zeigt (Henriksen et al. 2018). Beide Geräte beinhalten einen 3-Achsen-Beschleunigungssensor (Fitbit Inc. 2019; Garmin Ltd. 2019). Entsprechend der Funktion, Bewegungen zu erkennen, hier in Form von Schritten, werden solche Sensoren auch Accelerometer oder Motion-Sensor genannt (Bieber 2014: 16). Die Information über Schritte fließt dann in einen von den Hersteller*innen programmierten Algorithmus ein, welcher die Art der Bewegung abschätzt, klassifiziert und erkennt (ebd.). Merkmale dieses Signals sind zum Beispiel Zeitintervalle, Frequenzen und statistische Merkmale sowie eine domänen- und nutzungsabhängige Kalibrierung. Diese Merkmale können unterschiedlich kombiniert werden, sodass für die jeweilige Zielbestimmung (hier: Gehen) eine möglichst exakte Aufzeichnung möglich ist (Bieber 2014: 28-35). Das bedeutet, der 3-Achsen-Sensor erfasst in der Regel Schritte, jedoch sind Voreinstellungen und Parameter von den Hersteller*innen für ein bestimmtes Ziel, in dem Fall für eine bestimmte Kund*innengruppe und nicht für geriatrische Patient*innen, programmiert (vgl. Bieber 2014: 28-35). Dadurch kann es dazu kommen, dass geringere Schrittzahlen gespeichert werden, als vom Sensor erkannt werden. Dieser Fehler lässt sich durch das Tragen des Sensors an der Ferse (z.B. an der Socke) oder an der Hüfte (Floegel et al. 2017), mittels eines Klipps, verringern.

Jedoch hat das Tragen am Handgelenk im Längsschnitt und in der häuslichen Umgebung mehrere Vorteile: Es gestaltet sich für die Patient*innen wie das Tragen einer Armbanduhr, durch die sie sich nicht gestört fühlen. Dies darf als wichtigste Erkenntnis der prospektiven Studie verstanden werden, in der die Fragestellungen untersucht wurden, wie handelsübliche Aktivitätstracker im stationären Setting eingesetzt werden können und ob sie von der Zielgruppe akzeptiert werden. Erst durch die Bereitschaft der Patient*innen, den Fitnesstracker zu tragen, und durch die Möglichkeit, das Armband ohne Probleme selbst anlegen zu können - anders als es zum Beispiel bei einem Tragen an der Socke der Fall gewesen wäre -, war es möglich, eine Langzeitstudie sim Feld , also in der häuslichen Umgebung, durchzuführen.

In einem qualitativen Kurzinterview wurden den Proband*innen ( $n=10)$ im Rahmen der prospektiven Studie vor und nach dem Anlegen des Fitnesstrackers und einem zugehörigen Bewegungsmonitoring Fragen zu ihrer Einstellung zum Gerät und zum Tragekomfort gestellt. Das Hauptinteresse dabei galt zunächst der Bereitschaft der Zielgruppe, einen Fitnesstracker mit dem Ziel der Bewegungsmessung zu tragen; daneben sollte herausgefunden werden, ob die Durchführung der Messung aufseiten der Proband*innen so funktionieren kann, dass Messfehler kleingehalten werden. Es stellte sich heraus, dass insbesondere die Armbandlösung für die Patient*innen das entscheidende Kriterium für die Akzeptanz 
des Fitnesstrackers war. Zwar kannte vor der Aufklärung der Patient*innen und Einholung der Einwilligung nur eine Person einen Fitnesstracker und dessen Funktionen, das Tragen eines solchen lehnte aber bis auf eine Person niemand ab. Bei einem zukünftigen Tragen wollen die Patient*innen aber besonders einen gesundheitlichen Nutzen davon haben. Diesbezügliche Aussagen finden sich in vergleichbaren Akzeptanzbefragungen. Der Nutzen der Fitnesstracker wird von den alternden Befragten in einer möglichen Förderung von Gesundheitsverhalten gesehen (Preusse et al. 2017). Daneben verwiesen die Befragten der prospektiven Studie häufig auf die ihnen bekannten Notfallarmbänder, welche ebenfalls nicht als störend erlebt wurden. Nachts trugen die Patient*innen das Armband ebenfalls störungsfrei am Handgelenk. Die prospektive Studie ergab auch in einem Testlauf, dass es auf der Station möglich ist Fitnesstrackerarmbänder einzusetzen. Die dadurch beobachteten Schrittzahlen ließen bereits auf der Station eine Unterscheidung in mehrere Gruppen von Entwicklungsverläufen zu, wie im nächsten Unterkapitel beschrieben wird.

Die Tragbarkeit am Handgelenk, ergänzt durch die Wasserdichte, war das Hauptkriterium bei der Geräteauswahl, um durch den Tragekomfort und das leichte Gewicht eine Belastung zu vermeiden. Weitere relevante Faktoren waren die Art des Sensors (3-Achsen-Sensor), die Messgenauigkeit, die Akku- beziehungsweise Batterieleistung sowie der Preis und die Zugänglichkeit zu den Messgeräten (vgl. Henriksen et al. 2018). Voreinstellungen der Geräte, wie ein Vibrationsalarm bei längerer Ruhezeit, wurden zurückgesetzt oder ausgestellt, damit möglichst keine technisch-programmierten störenden oder motivierenden Faktoren auftreten konnten. Ein selbstständiges Datenauslesen war den Patient*innen nicht möglich, auch nicht durch die Unterstützung von Angehörigen, denn es lag ihnen keine Information über ihr Pseudonym und somit kein Zugang zum zugehörigen Online-Tool vor. Es wäre zwar möglich, die tägliche Schrittzahl abends vom Display abzulesen und zu notieren, jedoch darf das aufgrund des Aufwands und der Displaygröße von nur $10 \mathrm{mal} 10 \mathrm{~mm}$ (0,39 x 0,39 Zoll) (Garmin 2019) als unwahrscheinlich eingestuft werden.

Ein systematisches Review der Validität und Reliabilität handelsüblicher Fitnesstracker ergab, dass die Schrittebeobachtung mit Trackern der Marke Fitbit höchst genau und exakt sowie im Vergleich zu weiteren Funktionen wie Kalorienverbrauch oder Herzfrequenzmessung am geeignetsten ist (Evenson et al. 2015: 17), um Mobilität zu messen. Die Schritte, die von den Sensoren der Marke Fitbit gezählt werden, stimmen in sehr hohem Maße mit denen eines Forschungspedometers überein $(r=0,91)$ (Dominick et al. 2016). In einer anderen Studie wies der Sensor in der Garmin vivofit 3 unter verschiedenen Bedingungen, nämlich auf dem Laufband, auf einer Teststrecke auf dem Boden sowie im 24-Stunden-Verlauf, eine sehr hohe Übereinstimmung mit einem Forschungspedometer auf $(r=0,90$ ) (An et al. 2017: 363). Allerdings waren die beteiligten Proband*innen stets $\leq 65$ Jahre alt. 
Eine systematische Übersichtsarbeit zur Validität und Reliabilität handelsüblicher Fitnesstracker für alternde Erwachsene stellt die Ergebnisse von 290 Proband*innen im Durchschnittsalter von 70,2 Jahren $(S D=4,8)$ zusammen (Straiton et al. 2018). Die Fitnesstracker der Marke Fitbit weisen demnach eine hervorragende Intra-Klassen-Reliabilität $(0,94)$ mit einem Referenzgerät auf. Auch hier wurde deutlich, dass die Algorithmen Schritte eher unterschätzen. Tedesco et al. (2019) schlagen nach einem Vergleich verschiedener handelsüblicher Fitnesstracker und Forschungsaccelerometer (hier: ActiGraph-Modelle) im Labor vor, dass die Modelle an der Hüfte getragen werden sollten. Weder die Fußgelenk- noch die Hüftlösung ist für das vorliegende Forschungsinteresse praktikabel, denn die Patient*innen sollen trotz körperlicher Einschränkungen den Sensor selbstständig und gefahrlos anlegen und abnehmen können. Diese Möglichkeit ist durch die Armbandlösung gegeben, die von den Marken Fitbit und Garmin angeboten wird.

In Rahmen des Forschungsprojekts wurde in einer Fallstudie die Schritteanzahl verglichen, die von den beiden eingesetzten Geräten beobachtet wurde (Altenbuchner/Mücke/Haug 2020). Eine 80 Jahre alte, weibliche Probandin vollzog sechs Durchläufe auf einer Teststrecke von 50 Schritten. Es ergaben sich keine signifikanten Unterschiede zwischen dem Fitbit- und dem Garmingerät sowie der tatsächlichen Schrittzahl, die über die Beobachtungsdokumentation festgestellt wurden. Im häuslichen Umfeld, in einer 33-tägigen durchgehenden Observation, korrelierten die Schrittzahlen der beiden Geräte stark positiv und signifikant $[r=0,776$, $p \leq 0,001, B c a-K I-95 \%(0,618 ; 0,874), N=33]$. Jedoch zeichnete das Fitbitgerät durchgehend mehr Schritte auf. Es wurde von der Probandin an der dominanten Körperseite getragen, während das Garmingerät an der nicht-dominanten Körperseite getragen wurde. $\mathrm{Zu}$ den Limitationen dieser Fallstudie zählt, dass es nicht möglich war, auf der Teststrecke die Armseiten der Geräte zu wechseln. Somit konnte der Einfluss der dominanten und nicht-dominanten Seite nicht geprüft werden. Dies liegt in der Konstitution der Probandin begründet. Sie fühlte sich nach der Durchführung der sechsmaligen Teststrecke physisch und mental stark erschöpft. Darum musste an dieser Stelle unterbrochen werden. Im häuslichen Umfeld behielt die Probandin die Armbänder an der entsprechenden Seite an.

Es wird deutlich, dass kontrollierte Bedingungen (Teststrecke) genaue Ergebnisse liefern, jedoch werden im Alltag und aufgrund der Gangart der Zielgruppe Schrittzahlen eher unterschätzt. Der große Unterschied zwischen einem für Forschungszwecke entwickeltem Accelerometer und einem Fitnesstracker liegt in der Kalibrierung, nicht im Sensor (vgl. Bieber 2014: 28-35). Auch im Forschungsaccelerometer sind bestimmte Kalibrierungen enthalten, die ursprünglich nicht für alle möglichen Zielgruppen vorgesehen sind.

Bei der algorithmischen Informationsverarbeitung können systematische und unsystematische Fehler auftreten. Gründe für systematische Fehler wären in diesem Kontext inkorrekte Einstellungen für Schrittlänge und Körpergröße, die Gang- 
art und das Anbringen am falschen Arm. Dieser wäre der dominante Arm, mit dem in der Regel öfter Gegenstände getragen werden, wodurch das Mitschwingen erschwert ist. Gründe für unsystematische Fehler wären Übertragungsfehler der Daten und fehlerhaftes Auslesen, beispielsweise eines falschen Datums oder auch vieler Variationen der Schrittlängen durch unterschiedliche Geschwindigkeitsarten oder Steigungen (vgl. Fitbit Inc. 2019; Garmin Ltd. 2016; Rat für Sozial- und Wirtschaftsdaten 2020). Momentan bietet die Armbandlösung durch handelsübliche Geräte eine Lösung zur Beobachtung des Mobilitätsverhaltens der Zielgruppe, die als die zum jetzigen Zeitpunkt praktikabelste Lösung bezeichnet werden darf (vgl. Altenbuchner/Mücke/Haug 2020). In einem vorsichtigen Einsatz der Geräte, wie Tedesco et al. (2019) es ausdrücken, sollte die Möglichkeit des Auftretens systematischer Fehler in die Studienplanung einbezogen werden und im Datenmanagement und der -analyse beachtet werden. Durch eine akkurate Durchführung und Dokumentation des Datenmanagements ist es möglich unsystematische Fehler weitestgehend zu eliminieren (Altenbuchner/Mücke/Haug 2020).

\section{Schrittzahl als Mobilitätsindikator}

In diesem Abschnitt wird dargelegt, warum das sensorbasierte Bewegungsmonitoring in Form von täglichen Schrittzahlen durch das Fitnesstrackerarmband eine Erweiterung des geriatrischen Mobilitätsassesments durch Fragebatterien und Tests ermöglicht und welche neuen Erkenntnisse sich dadurch ergeben. ${ }^{1}$

Im Rahmen der explorativen Datenanalyse wurden die Fragestellungen untersucht, wie sich die Schrittzahl nach der Operation einer hüftnahen Fraktur entwickelt sowie, ob und wie viele abgegrenzte Gruppen sich bilden lassen. Zur Beantwortung der Forschungsfragen erfolgt eine deskriptive Datenanalyse und eine kmeans Clusteranalyse.

Es handelt sich um eine quantitative explorative längsschnittliche (zehn Wochen) Beobachtungsstudie im Lebensalltag der geriatrischen Patient"innen, die nach der operativen Versorgung einer hüftnahen Fraktur auf einer nach den Kriterien der DGU zertifizierten Alterstraumastation (ATZ-DGU) behandelt wurden (Deutsche Gesellschaft für Unfallchirurgie/AltersTraumaZentrum DGU 2020). Das zu beobachtende Verhalten bestand aus den täglichen Schrittzahlen der Patient*innen, die durch das technische Hilfsmittel Fitnesstracker registriert und gespeichert wurden. Für das Forschungsprojekt liegt ein positives Votum der Ethikkommission an der Universität Regensburg vor.

1 Die Veröffentlichung von Teilergebnissen des Bewegungsmonitorings erfolgte auf Tagungen (z.B. Altenbuchner/Haug/Weber 2019; Altenbuchner/Mücke/Haug 2020). Eine ausführliche Ergebnisdarlegung wird noch in einer Dissertationsschrift folgen. 
Andere Autor*innen betonen bereits die Eignung einer sensorbasierten Beobachtung der Mobilität, weil fehlende Werte, die durch das Mobilitätsassessments entstehen, ausgeglichen werden können (Benzinger et al. 2014: 240-241). Anders als das Ausfüllen von Fragebögen bietet die Beobachtung am Körper eine individualisierte Datenlage (Fleiner et al. 2016: 1687), die das Bewegungsverhalten veränderungssensitiv abbildet (Nicolai 2012: 189). Auch bei Menschen mit demenziellen Erkrankungen ist das sensorbasierte Mobilitätsmonitoring möglich, wohingegen diese Personen Anweisungen für Mobilitätstests häufig nicht nachvollziehen und durchführen können (Fleiner et al. 2016: 1691-1693). Durch die Wahl der Kennzahl Schrittzahl, die bei handelsüblichen Geräten gut geeignet ist, um die körperliche Aktivität abzubilden (Dominick et al. 2016), wird die Messung der Rehabilitationsleistungen auch für Patient*innen nachvollziehbar und relevant, wie es in der Rehabilitationsliteratur gefordert wird (vgl. Benzinger et al. 2014: 239; WeidemannWendt 2017). Die Schrittzahl bietet eine Größe, die von Patient*innen und Pflegenden sowie Angehörigen verstanden werden dürfte. Angehörige spielen in der Förderung der körperlichen Aktivität geriatrischer Patient*innen beziehungsweise alternder und sehr alter Menschen eine wichtige Rolle (Sauter et al. 2019). Entsprechend der Nachvollziehbarkeit der Schrittzahlen könnte zukünftig auch eine individuelle Handlungsanweisung, zum Beispiel durch die Hausärzt*innen, denkbar sein, wenn ein (individueller) Zielwert definiert wird (Altenbuchner/Haug/Weber 2019: 144-145).

Die sensorbasierte Beobachtung zeigte sich als geeignet, um die Mobilitätsentwicklungen abzubilden und Unterschiede in der Entwicklung aufzuzeigen. Der Vergleich der täglichen Schrittzahlen aus dem vorliegenden Projekt mit Ergebnissen ähnlicher Untersuchungen (Davenport et al. 2015; Ellingson et al. 2019; Fleig et al. 2016; Fleiner et al. 2016; Kiselev et al. 2019; O'Halloran et al. 2016) ergibt, dass die beobachteten Werte plausibel und nachvollziehbar sind. ${ }^{2}$

Fragen nach der Möglichkeit einer eindeutigen Gruppenbildung hinsichtlich der Schritte-Entwicklung sowie nach der Anzahl und den Merkmalen dieser Gruppen können folgendermaßen beantwortet werden: Es lassen sich klar abgrenzbare Gruppen bilden. Die statistischen Kennwerte im Rahmen der explorativen kmeans Clusteranalyse erlauben im Zeitverlauf von Woche zu Woche eine eindeutige Unterscheidung zwischen zwei bis vier Clustergruppen. Im Gesamtzeitverlauf, unter Einbeziehung aller Wochen, können die Patient*innen in drei heterogene Clustergruppen gruppiert werden. Der Logik der k-means Clusteranalyse folgend, unterscheiden sich die durchschnittlichen Schrittzahlen zwischen den Clustern signifikant voneinander. Die mit den höchsten täglichen Schrittzahlen ( Cluster) gehen durchschnittlich den Faktor 1,96 Mal mehr als die mit den mittelhohen/-niedrigen Schrittzahlen ( $\leftrightarrow$ - Cluster) und gehen 6,14 Mal mehr als die mit 
den niedrigen Schrittzahlen ( $k$ - Cluster), welche sich von denen mit den mittelhohen/-niedrigen Schrittzahlen um den Faktor 3,13 unterscheiden. Clusteranalysen sehen vor, die statistisch vorgefundenen Clustergruppen inhaltlich zu interpretieren und mit Namen zu versehen (Schendera 2010). Diese Interpretation erfolgte in Anbetracht der deskriptiven Merkmale der Patient"innen sowie unter Beachtung theoretischer und praktischer Versorgungs- und Mobilisierungsregeln (vgl. Denkinger/Nikolaus 2013; Hager/Krause 2018; Schlesselmann 2019; Weidemann-Wendt 2017). Dieses Vorgehen deckte die folgenden Gruppen auf: >Mobilisierte Patient"innen $<$ ( $x$ - Cluster), ,Zirkulierende Patient*innen $<(\leftrightarrow$ - Cluster) und `Gebrechliche Patient"innen $<$ ( $k$ - Cluster).

Das Cluster >Gebrechliche Patient*innen « setzt sich aus den ältesten Patient*innen zusammen. Der Altersmedian liegt über dem Median der Gesamtstichprobe. Der Altersmedian der anderen Clustergruppen liegt darunter. Zwischen den Altersmedianen der Clustergruppen besteht jedoch kein signifikanter Unterschied. Es ist keine signifikante Zugehörigkeit zu einer der drei Clustergruppen hinsichtlich einer diagnostizierten Demenz erkennbar. Die Verteilungen der geriatrischen Assessmentwerte, die den kognitiven Status, die Selbsthilfefähigkeit und Mobilität vor der Fraktur abbilden, spiegeln den Gesamtzustand vieler Patient*innen wider. Personen mit entsprechend hoher Schrittzahl sind beim Cluster >Mobilisierte Patient"innen und mit niedrigen eher beim Cluster >Gebrechliche Patient"innen $\mathrm{zu}$ finden. Jedoch konnte durch die Wahl der Kennzahl der täglichen Schrittzahl aufgedeckt werden, dass sich die Clustergruppe >Zirkulierende Patient*innen`, die sich hinsichtlich der Assessmentwerte und des Alters kaum vom Cluster >Mobilisierte Patient"innen< unterscheiden, durch eine uneindeutige Mobilitätsentwicklung von Woche $\mathrm{zu}$ Woche auszeichnen und einzelne immer wieder in die unteren Gruppen der Schrittzahlen rutschen. Diese Gruppe befindet sich in der oben beschriebenen Ereignisabfolge der Fragilität und sollte weiter hinsichtlich des Aktivitätsverhaltens beobachtet werden.

Die Verteilung der wöchentlichen Zuwachsraten unterscheidet sich in allen drei Clustergruppen nicht signifikant. Zwar starten die Gruppen mit sehr unterschiedlichen Ausgangswerten der täglichen Schrittzahlen und 'Gebrechliche Patient*innen< können erst später als die anderen einen Zuwachs erreichen, jedoch ist der durchschnittliche Zuwachs sehr ähnlich. 75 Prozent der Patient*innen, die in Alters-Trauma-Zentren behandelt werden, sind eine Woche nach der Operation "gehfähig" (Sektion Alterstraumatologie DGU 2019: 22; 33-34). Ein Großteil dieser Gruppe kann gestützt (10\%) oder mit Hilfsmitteln wie Rollatoren (26\%), Gehbock (14\%) oder Gehwagen (24\%) gehen. Nur 1 Prozent kann selbstständig gehen. 22 Prozent haben noch keine Gehfähigkeit erreicht. Durch die tägliche Schrittzahl ist es möglich, der dichotomen Variable `Gehfähigkeit< einen metrischen Wert gegenüberzustellen. 
Für Personen, die sich sehr gut erholen, darf angenommen werden, dass sich mit fortschreitender Rehabilitationszeit ihre körperliche Aktivität nicht nur in Schritten, sondern auch durch andere Aktivitäten, wie etwa Radfahren, ausgedrückt werden könnte. Darum besteht aufgrund der Wahl der Variable Schrittzahl die Möglichkeit eines Unterschätzens der Bewegung, denn Aktivitäten, die über Gehen, Laufen oder Ruhen hinausgehen, können von Fitnesstrackern nicht eindeutig erfasst werden (Garriguet/Tremblay/Colley 2015: 14). Für Personen, die eine vergleichsweise schlechte Erholung aufweisen, darf, begründet durch das Gangbild, angenommen werden, dass durch den Algorithmus des Geräts weniger Schritte gespeichert werden, als tatsächlich getätigt wurden. Der Fehler liegt in experimentellen Studien bei <10 Prozent der Schrittzahl (Floegel et al. 2017: 232). Dies trifft für die Proband*innen mit und ohne Einschränkungen der Gehfähigkeit zu. Es wäre also möglich, dass die Schritte sowohl bei hohem als auch bei geringem Rehabilitationserfolg geringfügig unterschätzt wurden. Da die Patient*innen im Laufe der Mobilisierung auf der Station, in der Rehabilitationseinrichtung und auch zu Hause mit Hilfsmitteln gehen, muss von einem Unterschätzen der tatsächlichen Schrittzahl ausgegangen werden - nicht aber von einem Überschätzen (Floegel et al. 2017; Straiton et al. 2018).

Die tägliche Schrittzahl eignete sich in einer anderen Studie sehr effektiv, um ein drohendes geriatrisches Frailty-Syndrom von Personen, die zu Hause leben, vorherzusagen (Schwenk et al. 2015). Durch eine regelmäßige pflegerische oder ärztliche Prüfung der Schrittzahl wäre vorstellbar, dass sich eine Art Frühwarnsystem für die drohende Immobilität etablieren lassen könnte.

\section{Fazit}

Sensorbasiertes Bewegungsmonitoring bietet eine Möglichkeit zum Echtzeitund Verlaufsmonitoring und somit auch eine hohe Übertragbarkeit der Beobachtungsergebnisse auf den Alltagskontext (vgl. Rat für Sozial- und Wirtschaftsdaten 2020: 9).

Beim Einsatz von Fitnesstrackern ist zu beachten, dass sie keine wissenschaftlichen Forschungsinstrumente oder gar Medizinprodukte sind. Die kommerziellen Produkte werden entsprechend der Kriterien Design und Nutzer*innenfreundlichkeit entwickelt. Für die Forschungszielgruppe bieten sie zwar einen hohen Tragekomfort, jedoch ist zurzeit eine Steuerung des Geräts und ein Ablesen beziehungsweise Auslesen der Informationen schwer vorstellbar. Es ist davon auszugehen, dass in absehbarer Zeit von den Anbietern der Fitnesstracker auch Kalibrierungen für alternde und sehr alte Menschen implementiert werden, schließlich gilt es neue Nutzer*innen und Kund"innen zu gewinnen oder auch den Bedürfnissen derer ge- 
recht zu werden, die bereits heute Fitnesstracker nutzen und mit fortschreitendem Alter weiterhin diese Technologie verwenden wollen.

Auch heute schon wäre es für Rehabilitand*innen oder pflegende Angehörige möglich, die Rehabilitationsentwicklung durch die Beobachtung der täglichen Schrittzahl selbstständig mitzuverfolgen. Andere Variablen, wie Bewegungszeit oder Geschwindigkeit oder Kalorienverbrauch und Herzfrequenz, würden hier ein weiterführendes Datenverständnis abverlangen (»Data Literacy«, vgl. Duttweiler/Passoth 2016: 16), denn die Visualisierung dieser Daten, wie sie auf den Online-Plattformen und Applikationen bereitgestellt wird, gibt ein verzerrtes Bild ab. Jedoch können sich die Beobachtenden im Rahmen eines privaten Monitorings fragen: Ist eine Steigerung, eine Verringerung oder eine Konstanz im Zeitverlauf zu beobachten? Stimmen diese Beobachtungen mit dem persönlichen, subjektiven Erleben überein? Passen diese Beobachtungen zum allgemeinen Gesundheitszustand? Wie lauten die individuellen beziehungsweise persönlichen (Rehabilitations-)Ziele? (Altenbuchner/Haug/Weber 2019: 143)

Abschließend soll ein mögliches Gefahrenpotenzial durch die Deutungsmacht der algorithmischen Datenverarbeitung beim Technikeinsatz im pflegerischen Bereich nicht unerwähnt bleiben. Höhmann/Schwarz (2017) stellen die Aussagen verschiedener kritischer Autor*innen zusammen, wie zum Beispiel Friesacher (2010: 302), der davor warnt, dass hierbei Patient*innen als "Datenträger « und Pflegende als »Vermessungstechniker« gesehen werden. Dadurch entstehen »Eigenlogiken und Sachgesetzlichkeiten technischer Lösungen «, die sich auf die Handlungen der Pflegenden und die Kommunikation mit den Patient*innen auswirken, wobei das technische Gerät zwischen den Akteur*innen steht und dadurch einen direkten persönlichen Kontakt erschwert; mitunter auch, weil die persönliche Verantwortung an die Technik abgegeben wird (Höhmann/Schwarz 2017: 155). Diesem Einwand ist entgegenzubringen, dass diese Logik auch in validierten Assessmentbatterien zu finden ist, welche wiederum oftmals gar nicht in Gänze angewendet werden können, obwohl eine Diagnostik für eine Behandlungsentscheidung erforderlich und aufgrund der Evidenzlücke in der geriatrischen Versorgung von vielen Unsicherheiten geprägt ist. Sensortechnologie erleichtert es, den Zustand der Patient*innen zu verstehen und ihren individuellen Anforderungen gerecht zu werden. Anstatt das Gefahrenpotenzial in den Gesetzlichkeiten der Technik, hier der Funktionsweise des 3-Achsen-Bewegungssensor, dem Algorithmus und der Interpretation der vorgefundenen Werte zu stark zu betonen, sollten sie als innovative Lösungsmöglichkeit gesehen werden, die zur Nachvollziehbarkeit der tatsächlichen Bewegungsfähigkeit beiträgt. Die Daten bergen auch die Forderung nach einer medizinischen und pflegerischen Interpretation und entsprechender Handlung. So konnten die täglichen Schrittzahlen des Clusters `Gebrechliche Patient*innen decken, dass diese eine vergleichbar hohe Rehabilitationsleistung wie die anderen Gruppenzugehörigen erbringen, die aber von einem weit niedrigeren Ausgangs- 
punkt startet. Auch >Zirkulierende Patient"innen sind im Zeitverlauf der Beobachtung immer wieder in der Clustergruppe mit niedriger Schrittzahl angesiedelt. Hier wird ersichtlich, dass Personen aus beiden Clustern ohne ein Bewegungsmonitoring leicht unterschätzt werden könnten, wenn sie an einem unpassenden Tag eingeschätzt werden. Obwohl >Gebrechliche Patient*innen ‘ fragil sind und anhand ihrer Assessmentwerte als weitestgehend immobil gelten, konnte die Beobachtung dieser Immobilität eine quantitative Bedeutung zuweisen, die zeigt, was für diese Patient" innen noch möglich ist. Höhmann/Schwarz (2017: 158-159) argumentieren ebenfalls, dass es kontext- und situationsbedingt sinnvoll ist, "Gestaltungspotentiale zu erkennen und nutzbar zu machen«, wenn die Implementierung innovativer technischer Lösungen erforderlich ist.

Eignen sich also konventionelle Fitnesstracker als Forschungsinstrument und als Bewegungsmonitoringsystem bei geriatrischen Patient*innen? Für zukünftige Studien an dieser Zielgruppe kann diese Frage mit einem klaren >Ja beantwortet werden. Die Nutzung von Aktivitätstrackern in der Alterstraumatologie und in weiteren geriatrischen Teilgebieten, in denen Mobilität einen wichtigen Faktor darstellt, kann der Einsatz konventioneller Fitnesstracker empfohlen werden. Diese sollten mit einem 3-Achsen-Sensor ausgestattet sein, am Handgelenk getragen werden und einen gewissen Tragekomfort beinhalten. In der Durchführung sollten Möglichkeiten des Datenschutzes, zum Beispiel durch Pseudonymisierung und Datensparsamkeit, vollumfänglich beachtet werden. Unter Beachtung dieser Punkte können die Fitnesstracker nicht nur den Bedürfnissen der Zielgruppe und den einzelnen Proband*innen gerecht werden, sondern tragen in methodischer Hinsicht dazu bei, die drängende Forschungslücke zu dieser besonderen Zielgruppe weiter zu schließen.

\section{Literatur}

Altenbuchner, Amelie/Haug, Sonja/Kretschmer, Rainer/Weber, Karsten (2018): "How to Measure Physical Motion and the Impact of Individualized Feedback in the Field of Rehabilitation of Geriatric Trauma Patients«, in: Günter Schreier/Dieter Hayn (Hg.), Health Informatics Meets eHealth: Biomedical Meets eHealth - From Sensors to Decisions. Proceedings of the 12th eHealth Conference, Amsterdam: IOS Press, S. 226-232.

Altenbuchner, Amelie/Haug, Sonja/Weber, Karsten (2019): »Exploratory Analysis of Motion Tracking Data in the Rehabilitation Process of Geriatric Trauma Patients«, in: Dieter Hayn/Alphons Eggerth/Günter Schreier (Hg.), dHealth 2019 - From eHealth to dHealth. Proceedings of the 13th Health Informatics Meets Digital Health Conference, Amsterdam: IOS Press, S. 138-145. 
— (2021): »Die ersten Schritte nach einer hüftnahen Fraktur. Sensorbasierte Bewegungsexploration bei geriatrischen Traumapatient/-innen«, in: Zeitschrift für Gerontologie und Geriatrie, S. 1-6.

Altenbuchner, Amelie/Mücke, Vanessa/Haug, Sonja (2020): »Comparing Monitoring Results of Two Motion Trackers for Geriatric Patients«, in: Günter Schreier/Dieter Hayn/Alphons Eggerth (Hg.), dHealth 2020 - Biomedical Informatics for Health and Care. Proceedings of the 14th Health Informatics Meets Digital Health Conference, Amsterdam: IOS Press, S. 77-84.

Altenbuchner, Amelie/Weber, Karsten (2020): „Geriatric trauma patients as research subjects in a technology-driven research project. A preliminary field report«, in: Joschka Haltaufderheide/Johanna Hovemann/Jochen Vollmann et al. (Hg.), Aging between Participation and Simulation, Berlin, Boston: De Gruyter, S. 87-104.

Altenbuchner, Amelie/Weber, Karsten/Fuchs, Dominik (2019): NNützlichkeit \& Nutzung - Technikakzeptanz und Technikbewertung von AAL«, in: Petra Friedrich/Dominik Fuchs (Hg.), 6. Ambient Medicine ${ }^{\circledR}$ Forum »Assistive Technik für selbstbestimmtes Wohnen« (Band 6). 19.-20. Februar 2019, Tagungsband, Göttingen: Cuvillier Verlag, S. 149-156.

An, Hyun-Sung/Jones, Gregory C./Kang, Seoung-Ki/Welk, Gregory J./Lee, JungMin (2017): »How valid are wearable physical activity trackers for measuring steps?«, in: European journal of sport science 17, S. 360-368.

Avers, Dale/Brown, Marybeth/Chui, Kevin K./Wong, Rita A./Lusardi, Michelle (2011): »Editor's message: Use of the term selderly«, in: Journal of geriatric physical therapy 34, S. 153-154.

Banse, Gerhard/Hauser, Robert (2010): »Technik und Kultur - ein Überblick«, in: Armin Grunwald/Gerhard Banse (Hg.), Technik und Kultur. Bedingungs- und Beeinflussungsverhältnisse, Karlsruhe: KIT Scientific Publishing, S. 17-40.

Barth, Alexander/Doblhammer, Gabriele (2017): »Physische Mobilität und Gesundheit im Alter«, in: Tilman Mayer (Hg.), Die transformative Macht der Demografie, Wiesbaden: Springer Fachmedien, S. 207-244.

Becker, Stefanie (2014): »Gerontologie - eine interdisziplinäre Wissenschaft «, in: Stefanie Becker/Hermann Brandenburg (Hg.), Lehrbuch Gerontologie. Gerontologisches Fachwissen für Pflege- und Sozialberufe - Eine interdisziplinäre Aufgabe, Bern: Huber, S. 21-34.

Benzinger, Petra/Lindemann, Ulrich/Becker, Clemens/Aminian, Kamiar/Jamour, Michael/Flick, Simone E. (2014): »Geriatric rehabilitation after hip fracture. Role of body-fixed sensor measurements of physical activity«, in: Zeitschrift für Gerontologie und Geriatrie 47, S. 236-242.

Bieber, Gerald (2014): Methodik zur mobilen Erfassung körperlicher Aktivität mittels Beschleunigungssensoren. Dissertation, Rostock: Universität Rostock. 
Brecht, Meiken (2018): »Stürze«, in: Klaus Hager/Olaf Krause (Hg.), ESSENTIALS Geriatrie, München: Elsevier, S. 57-61.

Bundesinstitut für Arzneimittel und Medizinprodukte (2020): ICD-10-GM Version 2021. Kapitel XIX. Verletzungen, Vergiftungen und bestimmte andere Folgen äußerer Ursachen (Soo-T98). Verletzungen der Hüfte und des Oberschenkels, https:/www.dimdi.de/static/de/klassifikationen/icd/icd-10-gm/ko de-suche/htmlgm2021/block-s70-s79.htm vom 10.03.2021.

Davenport, Sarah J./Arnold, Meaghan/Hua, Carol/Schenck, Amie/Batten, Sarah/Taylor, Nicholas F. T. (2015): »Physical Activity Levels During Acute Inpatient Admission After Hip Fracture are Very Low«, in: Physiotherapy research international 20, S. 174-181.

Denkinger, Michael/Nikolaus, Thorsten (2013): »Gesundes Altwerden«, in: Andrej Zeyfang/Ulrich Hagg-Grün/Thorsten Nikolaus (Hg.), Basiswissen Medizin des Alterns und des alten Menschen, Berlin, Heidelberg: Springer, S. 3-14.

Deutsche Akademie der Wissenschaften (2015): Medizinische Versorgung im Alter. Welche Evidenz brauchen wir? (Schriftenreihe zur wissenschaftsbasierten Politikberatung), Halle (Saale): Nationale Akademie der Wissenschaften.

Deutsche Gesellschaft für Unfallchirurgie/AltersTraumaZentrum DGU (2020): Kriterienkatalog. Version 1.3, München: AVC.

Dominick, Gregory M./Winfree, Kyle N./Pohlig, Ryan T./Papas, Mia A. (2016): »Physical Activity Assessment Between Consumer- and Research-Grade Accelerometers: A Comparative Study in Free-Living Conditions«, in: JMIR mHealth and uHealth 4, ei1o.

Duttweiler, Stefanie/Passoth, Jan-Hendrik (2016): „Self-Tracking als Optimierungsprojekt?«, in: Stefanie Duttweiler/Robert Gugutzer/Jan-Hendrik Passoth et al. (Hg.), Leben nach Zahlen. Self-Tracking als Optimierungsprojekt?, Bielefeld: transcript, S. 9-42.

Ellingson, Laura D./Lansing, Jeni E./DeShaw, Kathryn J./Peyer, Karissa L./Bai, Yang/Perez, Maria/Phillips, L. A./Welk, Gregory J. (2019): »Evaluating Motivational Interviewing and Habit Formation to Enhance the Effect of Activity Trackers on Healthy Adults' Activity Levels: Randomized Intervention«, in: JMIR mHealth and uHealth 7, e10988.

Fitbit Inc. (2019): Wie zählt mein Fitbit-Gerät Schritte?, https://help.fitbit.com/art icles/de/Help_article/1141\#steps vom 01.11.2019.

Fleig, Lena/McAllister, Megan M./Brasher, Penny/Cook, Wendy L./Guy, Pierre/Puyat, Joseph H./Khan, Karim M./McKay, Heather A./Ashe, Maureen C. (2016): »Sedentary Behavior and Physical Activity Patterns in Older Adults After Hip Fracture: A Call to Action«, in: Journal of aging and physical activity 24 , S. $79-84$.

Fleiner, Tim/Haussermann, Peter/Mellone, Sabato/Zijlstra, Wiebren (2016): »Sensor-based assessment of mobility-related behavior in dementia: Feasibility and 
relevance in a hospital context«, in: International psychogeriatrics 28 , S. 16871694.

Floegel, Theresa A./Florez-Pregonero, Alberto/Hekler, Eric B./Buman, Matthew P. (2017): »Validation of Consumer-Based Hip and Wrist Activity Monitors in Older Adults With Varied Ambulatory Abilities«, in: The journals of gerontology. Series A, Biological sciences and medical sciences 72, S. 229-236.

Friesacher, Heiner (2010): »Pflege und Technik - eine kritische Analyse«, in: Pflege \& Gesellschaft 15, S. 293-313.

Garmin Ltd. (2016): Benutzerhandbuch vívofit 3, https://www8.garmin.com/man uals/webhelp/vivofit3/DE-DE/GUID-946860A4-9C41-4B5E-9570-7413A45A3CF 6-homepage.html vom 04.05.2021.

- (2019): Mein Fitness Tracker zählt die Schritte nicht genau, https://support.gar min.com/de-DE/?faq=z1TfJCqajl8ZEZey72gg98\&productID=567813\&tab=topic s vom 01.11.2019.

Garriguet, Didier/Tremblay, Sylvain/Colley, Rachel C. (2015): Comparison of Physical Activity Adult Questionnaire results with accelerometer data, Canada, https ://www150.statcan.gc.ca/n1/pub/82-003-x/2015007/article/14205-eng.pdf vom 20.07.2020.

Hager, Klaus (2018a): »Assessment - Messen in der Geriatrie«, in: Klaus Hager/Olaf Krause (Hg.), ESSENTIALS Geriatrie, München: Elsevier, S. 21-26.

— (2018b): »Immer älter, immer kränker? «, in: Klaus Hager/Olaf Krause (Hg.), ESSENTIALS Geriatrie, München: Elsevier, S. 5-8.

- (2018c): »Therapieren oder nicht?«, in: Klaus Hager/Olaf Krause (Hg.), ESSENTIALS Geriatrie, München: Elsevier, S. 15-19.

— (2018d): »Was ist im Alter anders?«, in: Klaus Hager/Olaf Krause (Hg.), ESSENTIALS Geriatrie, München: Elsevier, S. 9-13.

Hager, Klaus/Krause, Olaf (2016): »Frailty. Neuer Begriff - Altes Problem«, in: Der Allgemeinarzt 38, S. 48-52.

Hager, Klaus/Krause, Olaf (Hg.) (2018): ESSENTIALS Geriatrie (= Elsevier Essentials), München: Elsevier.

Hagg-Grün, Ulrich/Nikolaus, Thorsten/Zeyfang, Andrej (2013): »Mobilität, Immobilität, Stürze und Folgen«, in: Andrej Zeyfang/Ulrich Hagg-Grün/Thorsten Nikolaus (Hg.), Basiswissen Medizin des Alterns und des alten Menschen, Berlin, Heidelberg: Springer, S. 39-53.

Haltaufderheide, Joschka/Hovemann, Johanna/Vollmann, Jochen (2020): »The challenge ahead«, in: Joschka Haltaufderheide/Johanna Hovemann/Jochen Vollmann et al. (Hg.), Aging between Participation and Simulation, Berlin, Boston: De Gruyter, S. 1-10.

Henriksen, André/Haugen Mikalsen, Martin/Woldaregay, Ashenafi Z./Muzny, Miroslav/Hartvigsen, Gunnar/Hopstock, Laila A./Grimsgaard, Sameline (2018): »Using Fitness Trackers and Smartwatches to Measure Physical Activity in Re- 
search: Analysis of Consumer Wrist-Worn Wearables«, in: Journal of medical Internet research 20 , e110.

Höhmann, Ulrike/Schwarz, Laura (2017): »Kompetenzanforderungen an pflegerische Führungskräfte in technikbezogenen Innovationsprozessen«, in: Mario A. Pfannstiel/Sandra Krammer/Walter Swoboda (Hg.), Digitale Transformation von Dienstleistungen im Gesundheitswesen III, Wiesbaden: Springer Gabler, S. 151-171.

Höpflinger, François (2014): »Demografisch-gesellschaftliche Wandlungen und soziale Folgen«, in: Stefanie Becker/Hermann Brandenburg (Hg.), Lehrbuch Gerontologie. Gerontologisches Fachwissen für Pflege- und Sozialberufe - Eine interdisziplinäre Aufgabe, Bern: Huber, S. 161-184.

Kiselev, Jörn/Nuritdinow, Timur/Spira, Dominik/Buchmann, Nikolaus/Steinhagen-Thiessen, Elisabeth/Lederer, Christian/Daumer, Martin/Demuth, Ilja (2019): »Long-term gait measurements in daily life: Results from the Berlin Aging Study II (BASE-II)«, in: PloS one 14, e0225026.

Körtner, Ulrich H. J. (2006): »Frailty. Medizinethische Überlegungen zur Gebrechlichkeit des alten Menschen«, in: Ethik in der Medizin 18, S. 108-119.

Kretschmer, Rainer/Loher, Martin/Apfelbacher, Christian/Nerlich, Michael (2017): »Herausforderung Fragilitätsfrakturen-aus Sicht eines Alterstraumazentrums«, in: Public Health Forum 25, S. 7146.

Matthews, Charles E./Berrigan, David/Fischer, Beate/Gomersall, Sjaan R./Hillreiner, Andrea/Kim, Youngwon/Leitzmann, Michael F./Saint-Maurice, Pedro/Olds, Timothy S./Welk, Gregory J. (2019): »Use of previous-day recalls of physical activity and sedentary behavior in epidemiologic studies: Results from four instruments«, in: BMC public health 19, S. 478.

Nicolai, Simone E. (2012): Sensorbasierte Messung und Bedeutung körperlicher Aktivität bei Patienten nach Hüftfraktur in der geriatrischen Rehabilitation. Dissertation, Stuttgart: Universität Stuttgart.

O'Halloran, Paul D./Shields, Nora/Blackstock, Felicity/Wintle, Elizabeth/Taylor, Nicholas F. (2016): "Motivational interviewing increases physical activity and self-efficacy in people living in the community after hip fracture: A randomized controlled trial«, in: Clinical rehabilitation 30, S. 1108-1119.

Örgel, Marcus/Petri, Maximilian (2018): "Alterstraumatologie«, in: Klaus Hager/Olaf Krause (Hg.), ESSENTIALS Geriatrie, München: Elsevier, S. 63-68.

Preusse, Kimberly C./Mitzner, Tracy L./Fausset, Cara B./Rogers, Wendy A. (2017): »Older Adults' Acceptance of Activity Trackers«, in: Journal of applied gerontology 36, S. 127-155.

Rapp, Kilian/Büchele, Gisela/Dreinhöfer, Karsten/Bücking, Benjamin/Becker, Clemens/Benzinger, Petra (2019): »Epidemiologie von Hüftfrakturen: Systematisches Literaturreview deutscher Daten und ein Überblick über die internationale Literatur«, in: Zeitschrift für Gerontologie und Geriatrie 52, S. 10-16. 
Rat für Sozial- und Wirtschaftsdaten (2020): Datenerhebung mit neuer Informationstechnologie - Empfehlungen zu Datenqualität und -management, Forschungsethik und Datenschutz. Berlin: RatSWD.

Robert Koch-Institut (2021): Multimorbidität, https://www.rki.de/DE/Content/Ge sundAZ/G/Gesundheit_Alter/Gesundheit_Alter_node.html;jsessionid=803ED C1885D28534F06B6FB15DDCoB4B.internet111?cms_box=2\&cms_current=Mult imorbidit\%C3\%A4t\&cms_lv2=13281366 vom 04.05.2021.

Sauter, Alexandra/Curbach, Janina/Rueter, Jana/Lindacher, Verena/Loss, Julika (2019): „German senior citizens' capabilities for physical activity: A qualitative study«, in: Health promotion international 34, S. 1117-1129.

Schlesselmann, Elke (Hg.) (2019): Bewegung und Mobilitätsförderung. Praxishandbuch für Pflege- und Gesundheitsberufe.

Sieber, Cornel C. (2007): »Der ältere Patient - wer ist das?«, in: Der Internist 48, 1190-1194.

Statistisches Bundesamt (2020a): Anteil der Bevölkerung ab 65 Jahren an der Gesamtbevölkerung in Deutschland von 1991 bis 2019, https://de.statista.com/ statistik/daten/studie/548267/umfrage/anteil-der-bevoelkerung-ab-65-jahrenund-aelter-in-deutschland/vom 04.05.2021.

- (2020b): Gesundheit. Tiefgegliederte Diagnosedaten der Krankenhauspatientinnen und -patienten 2018, https:/www.destatis.de/DE/Themen/Gesellschaf t-Umwelt/Gesundheit/Krankenhaeuser/Publikationen/Downloads-Krankenha euser/tiefgegliederte-diagnosedaten-5231301187015.xlsx?_blob=publicationFil e vom 08.03.2021.

Tedesco, Salvatore/Sica, Marco/Ancillao, Andrea/Timmons, Suzanne/Barton, John/O'Flynn, Brendan (2019): "Accuracy of consumer-level and researchgrade activity trackers in ambulatory settings in older adults«, in: PloS one 14, e0216891.

Wahl, Hans-Werner/Heyl, Vera (2015): Gerontologie, Einführung und Geschichte (Grundriss Gerontologie, Band 1), Stuttgart: Verlag W. Kohlhammer.

Weber, Karsten (2017): »Demografie, Technik, Ethik: Methoden der normativen Gestaltung technisch gestützter Pflege«, in: Pflege \& Gesellschaft 22, S. 338-352.

Weidemann-Wendt, Norma (2017): »Physiotherapeutische Arbeitsfelder in der Geriatrie«, in: Katja Richter/Christine Greiff/Norma Weidemann-Wendt (Hg.), Der ältere Mensch in der Physiotherapie, Berlin, Heidelberg: Springer, S. 157-211.

Zeyfang, Andrej/Hagg-Grün, Ulrich/Nikolaus, Thorsten (Hg.) (2013): Basiswissen Medizin des Alterns und des alten Menschen, Berlin, Heidelberg: Springer. 


\section{Digitalisierte Bewegungsentwicklung geriatrischer Traumapatient*innen Konzept und Evaluation eines Bewegungsfeedbacks}

Laura Cerullo

\section{Hintergrund}

Selbstvermessung und das Sammeln von Aktivitätsdaten zur Dokumentation des eigenen Gesundheits- und Bewegungsverhaltens ist keine neue Erscheinung der digitalen Gesellschaft (vgl. Duttweiler/Passoth 2016). Durch den Einsatz handelsüblicher Sensoren wird es zunehmend leichter, die gesammelten Daten sichtbar zu machen. Diese Daten werden algorithmisch verrechnet und visualisiert. Das Endprodukt der Visualisierung sind Bilder, Zahlen und andere Symbolsysteme, welche in Applikationen (Apps) oder auf Online-Plattformen zusammengefasst und im Vergleich mit früheren Werten oder einer Vergleichsgruppe zur Verfügung stehen (Feedback). Dabei muss das Messgerät nicht durch medizinisches oder pflegerisches Personal bedient und ausgelesen werden (vgl. ebd.).

Die Idee eines Bewegungsfeedbacks besteht im klinischen Bereich darin, mittels erfasster Bewegungsdaten beispielsweise die Bewegungsentwicklung nach einer Operation zu dokumentieren, um so Rückschlüsse bezüglich des Rehabilitationsprozesses zu ziehen. Für einen gelingenden Einsatz eines Bewegungsfeedbacks sollte daher untersucht werden, unter welchen Rahmenbedingungen digitale Sensoren in Gestalt handelsüblicher Aktivitätstracker in der geriatrischen Nachsorge sinnvoll eingesetzt werden können. Das im Folgenden beschriebene Konzept und die begleitende Evaluation eines Bewegungsfeedbacks für geriatrische Traumapatient*innen basiert auf drei Komponenten: Mittels einer umfassenden Literaturrecherche wurde ein Konzept entwickelt, welches im Rahmen der Prospektiven Studie zur Nutzung von Aktivitätstrackern in der Alterstraumatologie (vgl. Altenbuchner in diesem Band) an Patient"innen evaluiert wurde (Cerullo 2018). Für die Studie wurden neben Feldforschungsnotizen einer teilnehmenden Beobachtung auch leitfadengestützte (Kurz-)Interviews durchgeführt und qualitativ analysiert. 


\section{Konzept des Bewegungsfeedbacks}

\section{Literaturrecherche}

Die Grundlage für die Entwicklung des Bewegungsfeedbacks bildet eine Literaturrecherche, welche im Oktober 2017 in der medizinischen Fachdatenbank PubMed durchgeführt wurde. Die Recherche beinhaltete die Suchanfragen (1) exercise AND gerontology AND feedback sowie (2) exercise AND gerontology AND visual feedback. Es wurden Publikationen im Zeitraum von 2007 bis 2017 eingeschlossen. Aus den insgesamt 21 Treffern wurden sechs Studien für die Konzeption des Feedbacks herangezogen.

Um eine für die Zielgruppe älterer, zum Teil multimorbider Personen angemessenen Rückmeldung sensorbasierter (Bewegungs-)Daten geben zu können, stellte sich heraus, dass das Feedback für jede Person individuell (Forman et al. 2014), regelmäßig (Nicklas et al. 2014; Peel et al. 2016) und mit einer motivierenden Ansprache (Lauzon et al. 2008) von einer Pflegefachkraft (Nordheim et al. 2014) durchgeführt werden sollte. Dabei kann auch das soziale Umfeld beziehungsweise Angehörige mit eingebunden werden (Lauzon et al. 2008). Die dargebotenen Informationen sollten dabei eindeutig, relevant und zusammengefasst präsentiert werden (Ofli et al. 2016). Die visuelle Darstellungsweise in numerischer und grafischer Form (Lauzon et al. 2008; Ofli et al. 2016; Peel et al. 2016) sollte zur Nachvollziehbarkeit zudem durch eine verbale Unterstützung ergänzt werden (Ofli et al. 2016). Grundsätzlich ist auf eine kontrastreiche Darstellung, bevorzugt auf einem TabletGerät, $\mathrm{zu}$ achten, sodass das `Swishen`, das heißt die einfache Bedienung des Geräts mit dem Finger, und Vergrößern des Textes auf dem Gerät möglich ist (Nordheim et al. 2014). Das gesamte Feedback sollte ritualisiert ablaufen, sodass sowohl die Darstellungsform als auch die verbale Unterstützung immer dem gleichen Ablauf folgen. Dies ist insbesondere bei Menschen mit demenzieller Erkrankung von Bedeutung (ebd.).

\section{Ausgestaltung}

Das auf Basis der Literaturrecherche entworfene Bewegungsfeedback besteht aus drei Komponenten. Dieses wurde in standardisierter Form vorbereitet und dauerte pro Durchführung ungefähr fünf bis zehn Minuten:

1 Power-Point-Präsentation:

Über ein Tablet wurde die durchschnittliche Anzahl der Schritte einer Woche, der Vergleich der durchschnittlichen Schrittanzahl der ersten zur aktuellsten Woche 
sowie ein Verlaufsdiagramm dieser Daten gezeigt. Bei der Visualisierung wurde ein sehr einfaches und kontrastreiches Design in schwarz/weiß gewählt, siehe Abbildung 1.

2 Ablaufplan zur einheitlichen verbalen Unterstützung:

Für einen ritualisierten Ablauf des Feedbacks wurde ein Ablaufplan für die verbale Begleitung erstellt. Zur besseren Nachvollziehbarkeit der Informationen wurden alle visualisierten Informationen in einfachen Formulierungen deutlich ausgesprochen. ${ }^{1}$

3 Handzettel:

Um den Teilnehmenden die rückgemeldeten Informationen jederzeit zugänglich zu machen, wurde für jedes Feedback ein Ausdruck mit den rückgemeldeten Informationen ausgegeben.

\section{Untersuchungsmethodik}

Begleitend zum Rehabilitationsprozess nach einer hüftnahen Operation (vgl. Altenbuchner in diesem Band) wurde, im Rahmen der monatlich stattfindenden Besuche im häuslichen Umfeld der Patient*innen, das entwickelte Konzept des Bewegungsfeedbacks evaluiert. Die Feedback erhaltenden Personen ( $n=5)$ waren zwischen 77 und 95 Jahre alt und mehrheitlich weiblich $(n=4)$. Aufgrund des explorativen und deskriptiven Charakters des Forschungsinteresses bei der Überprüfung des Feedbacks sowie der logistisch aufwendigen Forschungsbedingungen wurde eine teilnehmende Beobachtung als Methode, ergänzt durch Leitfadeninterviews, gewählt.

\section{Teilnehmende Beobachtung}

Die vorgenommene teilnehmende Beobachtung (Schnell/Hill/Esser 2011: 381-397) sollte in Bezug auf das Bewegungsfeedback Erkenntnisse darüber liefern, inwiefern das Feedback in der vorgestellten Form angemessen für die gewählte Zielgruppe

Z.B.: »Hier können Sie den bisherigen Verlauf der Schrittzahlen von Woche 1 bis zur Woche $[X X]$ sehen. (Evtl. Frage: Können Sie das erkennen? Ich kann Ihnen die Schrittzahlen auch gerne sagen.). Im Vergleich zur durchschnittlichen Schrittzahl von Woche 1 hat sich die Anzahl vergrößert/nicht verändert/verringert. « 
Abbildung 1: Vorlage zur Power-Point-Präsentation des Bewegungsfeedbacks
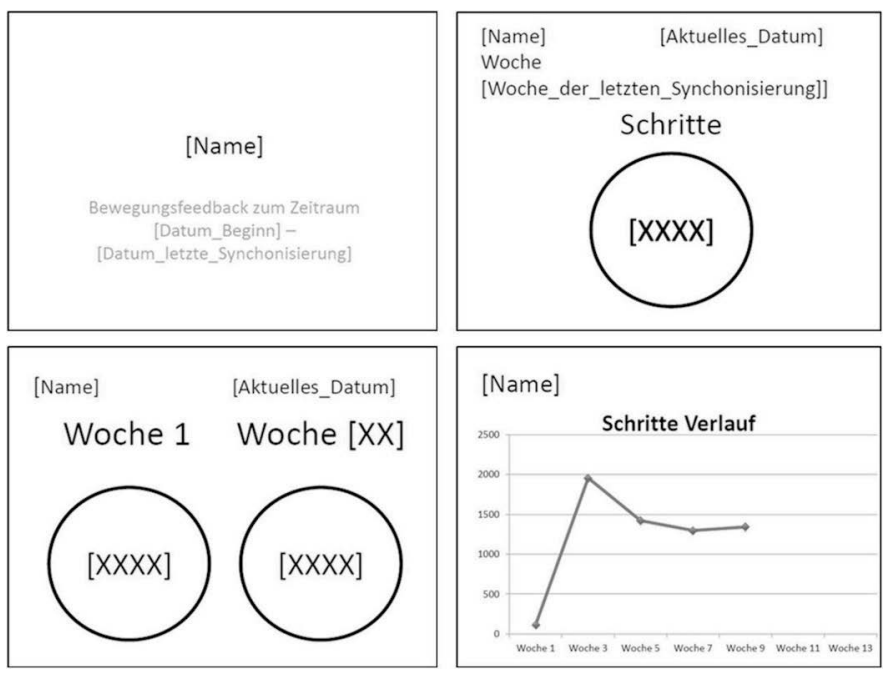

Quelle: Eigene Darstellung

ist. Hierzu wurden im Zeitraum vom 03.09.2018 bis zum 21.11.2018 elf Feldforschungsnotizen beziehungsweise Beobachtungsprotokolle (Przyborski/WohlrabSahr 2014: 49-52) für insgesamt fünf Versuchspersonen erstellt. Diese beinhalten neben dem chronologischen Ablauf des jeweiligen Besuchs auch Angaben zu Ort, Zeit, anwesende Personen während des Feedbacks, Feedback-Rezipient*innen (Patient* in oder Angehörige) und eine möglichst genaue Beschreibung des Verhaltens der Feedback erhaltenden Person während der Durchführung. Die gesammelten Feldforschungsnotizen wurden anhand der oben genannten Indikatoren aufgeschlüsselt und die beschriebenen Verhaltensweisen mittels einer einfachen qualitativen Inhaltsanalyse (Kuckartz 2018) zusammengefasst und kategorisiert.

\section{Leitfadengestützte Interviews}

Ergänzend zu den Beobachtungen wurden im Zeitraum vom 03.09.2018 bis zum 21.11.2018 leitfadengestützte (Kurz-)Interviews geführt (Przyborski/Wohlrab-Sahr 2014: 126-132), um die Beobachtungen anhand der zuvor aus den Feldforschungsnotizen gebildeten Kategorien (s.o.) zu ergänzen und gegebenenfalls zu bestätigen. Der Interviewleitfaden bestand aus sieben Fragen, die unter anderem die Nachvollziehbarkeit der rückgemeldeten Informationen sowie die motivierende oder bestä- 
tigende Wirkung des Feedbacks auf die Patient*innen thematisierten. Aufgrund logistischer Herausforderungen und organisatorischer Schwierigkeiten konnten lediglich zwei Interviews geführt werden. Ein Interview wurde mit einer pflegenden Angehörigen geführt, ein weiteres mit einer Patientin.

\section{Ergebnisse}

Das konzipierte Bewegungsfeedback war für einen Großteil der geriatrischen Patient*innen gut nachvollziehbar. Bedingungen, die dies negativ beeinflussten, waren zum einen kognitive Beeinträchtigungen im Zuge demenzieller Erkrankungen sowie die Diskrepanz zwischen der persönlichen Einschätzung der Bewegung und den dargestellten Informationen.

Es konnte gezeigt werden, dass das Interesse an der längerfristigen Bewegungsentwicklung nach einer Operation grundsätzlich besteht, gleichzeitig jedoch wenig Interesse an weiteren Informationen vorhanden ist. Während der Durchführung traten keine Probleme auf. Das lässt auf eine gute Durchführbarkeit und angemessene Darstellungsform der rückgemeldeten Informationen über ein Tablet schließen.

In Bezug auf auftretende Störfaktoren konnte im Setting der (Haus-)Besuche herausgestellt werden, dass unvorhersehbare Ereignisse wie beispielsweise ein Aufenthalt im Krankenhaus oder die Anwesenheit dritter Personen die Durchführung des Feedbacks stören können. Auch können aufgrund der charakteristischen Multimorbidität geriatrischer Patient*innen oft andere Themen eine höhere Relevanzstruktur aufweisen. Diesen Störfaktoren kann im gewählten Setting kaum vorgebeugt werden, sodass die Konsequenz eine erforderliche Flexibilität der Feedback gebenden Person ist. Dennoch konnte für die Zielgruppe festgestellt werden, dass das Feedback innerhalb der regelmäßigen Besuche einen Anreiz beziehungsweise eine Bestätigung darstellt und somit die Motivation zu mehr Bewegung gesteigert werden kann.

\section{Schlussfolgerung}

Das konzipierte Feedback für geriatrische Patient*innen zu Bewegungsdaten (Anzahl der Schritte) mittels Tablet hat das Potenzial, im Rehabilitationsprozess unterstützend zu wirken. Dabei ist besonders auf die Art und Weise der Darstellung $\mathrm{zu}$ achten. Die einfache, klare und zusammengefasste Rückmeldung von Informationen hat sich für die Zielgruppe als angemessen erwiesen - ebenso wie die Veranschaulichung der Daten über ein Tablet. Auch der monatliche Rhythmus des Feedbacks war angemessen gewählt. 
Die bestätigende und motivierende Wirkung des Feedbacks auf Patient*innen und Angehörige ist als Indikator zu sehen, dass die Implementierung eines regelmäßigen Bewegungsfeedbacks im Rehabilitationsprozess indiziert ist. Darauf aufbauend gilt es, weitere vergleichbare Studien im klinischen, ambulanten und auch im häuslichen Setting durchzuführen, um die hier vorliegenden Ergebnisse weitreichender zu untersuchen.

\section{Literatur}

Cerullo, Laura (2018): Self-Tracking im hohen Alter: Bewegungsfeedback für Patient"innen der Geriatrie. Konzept und Evaluation. Unveröffentlichte Bachelorarbeit. Regensburg: Ostbayerische Technische Hochschule Regensburg.

Duttweiler, Stefanie/Passoth, Jan-Hendrik (2016): „Self-Tracking als Optimierungsprojekt?«, in: Stefanie Duttweiler/Robert Gugutzer/Jan-Hendrik Passoth/Jörg Strübing (Hg.), Leben nach Zahlen. Self-Tracking als Optimierungsprojekt?, Bielefeld: transcript, S. 9-44.

Forman, Daniel E./LaFond, Karen/Panch, Trishan/Allsup, Kelly/Manning, Kenneth/Sattelmair, Jacob (2014): »Utility and efficacy of a smartphone application to enhance the learning and behavior goals of traditional cardiac rehabilitation: A feasibility study«, in: Journal of cardiopulmonary rehabilitation and prevention 34, S. 327-334.

Kuckartz, Udo (2018): Qualitative Inhaltsanalyse. Methoden, Praxis, Computerunterstützung, Weinheim, Basel: Beltz Juventa.

Lauzon, Nicola/Chan, Catherine B./Myers, Anita M./Tudor-Locke, Catrine (2008): »Participant experiences in a workplace pedometer-based physical activity program «, in: Journal of physical activity \& health 5, S. 675-687.

Nicklas, Barbara J./Gaukstern, Jill E./Beavers, Kristen M./Newman, Jill C./Leng, Xiaoyan/Rejeski, W. J. (2014): »Self-monitoring of spontaneous physical activity and sedentary behavior to prevent weight regain in older adults«, in: Obesity (Silver Spring, Md.) 22, S. 1406-1412.

Nordheim, Johanna/Hamm, Sabine/Kuhlmey, Adelheid/Suhr, Ralf (2014): »TabletPC und ihr Nutzen für demenzerkrankte Heimbewohner.«, in: Zeitschrift für Gerontologie und Geriatrie 48, S. 543-549.

Ofli, Ferda/Kurillo, Gregorij/Obdržálek, Štěpán/Bajcsy, Ruzena/Jimison, Holly B./Pavel, Misha (2016): »Design and Evaluation of an Interactive Exercise Coaching System for Older Adults: Lessons Learned«, in: IEEE journal of biomedical and health informatics 20, S. 201-212.

Peel, Nancye M./Paul, Sanjoy K./Cameron, Ian D./Crotty, Maria/Kurrle, Susan E./Gray, Leonard C. (2016): "Promoting Activity in Geriatric Rehabilitation: A Randomized Controlled Trial of Accelerometry«, in: PloS one 11, e0160906. 
Przyborski, Aglaja/Wohlrab-Sahr, Monika (2014): Qualitative Sozialforschung. Ein Arbeitsbuch (Lehr- und Handbücher der Soziologie), München: Oldenbourg. Schnell, Rainer/Hill, Paul B./Esser, Elke (2011): Methoden der empirischen Sozialforschung, München: Oldenbourg. 



\section{Zum Potenzial grundlagenwissenschaftlicher Technikforschung für ein "gutes Leben im Alter « Ein Plädoyer für konsequente partizipative}

Technikgestaltung

Jannis Hergesell, Arne Maibaum, Andreas Bischof, Benjamin Lipp

\section{Einleitung}

In der gegenwärtigen Debatte um digitale Technologien für ältere und pflegebedürftige Menschen ist eine augenscheinliche Diskrepanz unübersehbar. Unter dem Stichwort Digitalisierung subsumierte (assistive) Technologien werden als vielversprechender Lösungsansatz für die Folgen des demografischen Wandels, des Pflegenotstandes und Garant für ein >gutes Leben`s im Alter postuliert (Maibaum/Hergesell 2020). Dem gegenüber stehen allerdings nur einzelne marktreif entwickelte Technologien. So gingen bislang, trotz massiver Forschungsförderung, auffallend wenige praxisrelevante Entwicklungen aus den zahlreichen Forschungsprojekten hervor (Kucharski/Merkel 2018; Weinberger/Decker 2015; vgl. auch die Beiträge von Haug, Scorna und Weber in diesem Band). Bisher prägen digitale Assistenzen weder den pflegerischen Alltag noch sind sie substanzielle Konstituenten von Lebensqualität im Alter (Birken et al. 2018; Hergesell 2019a). Dies trifft besonders für avancierte, mit hochgradig digitalisierungsoptimistischen Hoffnungen belegte Technologien $\mathrm{zu}$, wie etwa der Pflegerobotik oder komplexen, sensorbasierten Assistenzsystemen.

Ein seit längerem prominenter Ansatz, den ausbleibenden (erhofften durchschlagenden) Erfolg der Digitalisierung der Pflege zu erklären und dem persistenten »Mismatch (Hergesell/Maibaum/Meister 2020: 8) zwischen digitaler Technologie und pflegebedürftigem Alter zu begegnen, ist die partizipative Technikentwicklung (oder partizipatives Design).

»Ansätze der partizipativen Technikgestaltung, wie Participatory Design, haben den Anspruch zukünftige Nutzer als gleichwertige Partner in den Cestaltungsprozess zu integrieren, um ihre Perspektiven adäquat zu erfassen und tatsächlich ein- 
zubeziehen. Technologien sollen so bedarfsgerechter gestaltet werden und folglich eine breite Akzeptanz/Nutzung erreichen.«(Kucharski/Merkel 2018: 1)

Partizipatives Design stellt damit eine notwendige Reaktion auf die seit den $2000 \mathrm{er}$ Jahren massiv geförderte und geforderte Entwicklung innovativer Assistenzen für die Pflege dar. Ohne die durch partizipative Ansätze vorangetriebene - und in den letzten Jahren immer effektivere - Integration der sogenannten sozialen Dimensionen digitaler Assistenzen in Forschungs- und Entwicklungsprojekte wäre die angestrebte Forschungsförderung nur schwer realisierbar gewesen. Partizipative Methoden sollten also durch die systematische Beachtung der sozialen Strukturen, für welche die Technologie entwickelt wird, eine gelingende(re) Technikgenese und -implementierung gewährleisten. Erst durch diese Entwicklung im Forschungsfeld gelang es, sogenannte ökonomische, soziale, rechtliche und ethische Faktoren in die Forschung und Entwicklung zu integrieren und alltagstaugliche(re) Technologien zu entwickeln.

Dabei sind die vorzufindenden Ansätze, ihr methodisches Vorgehen und ihre Zielsetzungen sehr heterogen. Sie reichen von dem eher auf Akzeptanzforschung abzielenden Technology Acceptance Model (Davis 1989) bis hin zur politisch-ethisch orientierten Technikfolgenabschätzung (Grunwald 2018) oder dem User Experience Design (Sontheimer 2016). Spätestens mit der Konjunktur der (partizipativen) Aktionsforschung (Unger 2012), welche die Teilhabe der ehemals >Beforschten als Gelingensbedingung für praxistaugliche Ergebnisse versteht, wurde auch die Nutzer*inneneinbindung bei sogenannter Gerontotechnologie immer zentraler. In der Folge entwickelten sich diverse sozialwissenschaftlich orientierte Ansätze des partizipativen Designs und der partizipativen Technikentwicklung, welche Senior*innen und Pflegende aktiv in den (Forschungs- und) Entwicklungsprozess einbeziehen (Kucharski/Merkel 2018). $\mathrm{Zu}$ nennen sind etwa das szenariobasierte Design (Cieslik et al. 2012), die kontextintegrierende, praxiszentrierte Bedarfsanalyse (Birken et al. 2018) oder MEESTAR ${ }^{2}$ zur ethischen Evaluierung soziotechnischer Arrangements (vgl. Manzeschke et al. 2013; Weber 2016).

Gerade die sozialwissenschaftlich orientierten Ansätze verstehen dabei Technologie(-genese) als genuin sozial (Rammert 2006 \& 2016) und konzipieren Technikentwicklung demnach als kontingenten Aushandlungsprozess. Folglich adressieren sie wesentlich weniger technische Machbarkeit oder vermeintliche Funktionalität im Entwicklungsprozess als viel mehr soziale Dimensionen wie den Einbezug der Lebenswelt der Nutzer*innen, unterschiedliche Machtbeziehungen der beteiligten Akteur*innen und den Ausgleich von Partikularinteressen. Solche Ansätze kritisieren dabei besonders den Entwicklungsprozess verzerrende Stereotype wie die Stigmatisierung älterer Menschen als defizitäre Nutzer*innen (Compagna/Kohlbacher 2015) oder eine angebliche Innovationsresistenz von Pflegenden gegenüber neuen Technologien (Hülsken-Giesler 2015). 
Diese Entwicklungen sind sicherlich sinnvoll, tragen gegenwärtig zu einer >gelingende(ren)< Technikentwicklung bei und fördern zudem die Teilhabe der Nutzer*innen. Allerdings gilt dies bei weitem nicht für alle im Forschungsfeld vorzufindenden Projekte. Gerade bei stark anwendungsorientierter, ingenieurswissenschaftlicher Forschungsprogrammatik bleiben häufig blinde Flecken bezüglich der gesellschaftlich hinderlichen und förderlichen Faktoren gelingender Technikentwicklung. Noch immer lässt sich bei Entwicklungs- und Forschungsprojekten teils eine epistemisch auf den Einzelfall, spezifische Technologien und isolierte soziotechnische Konstellationen oder Anwendungsszenarien bezogene Fokussierung feststellen, welche noch nicht konsequent durch neue und effizientere Ansätze partizipativen Designs durchbrochen wird.

Die Nutzung grundlagenwissenschaftlicher Arbeiten und die kritische Reflexion früherer Anwendungsprojekte zur Digitalisierung des pflegebedürftigen Alters haben dazu geführt, dass die Gründe für den Erfolg oder das Scheitern von Technologien nicht mehr nur isoliert in einzelnen Szenarios oder Akteur*innenkonstellationen gesucht werden. Vielmehr verweisen die neueren partizipativen Ansätze durchaus auf die als relevant identifizierten sozialen Dimensionen der gesellschaftlichen Rahmenbedingungen assistiver Technologien. Diese bestehen aus persistenten und wirkmächtigen Strukturen wie gesellschaftlichen Innovationsdiskursen und -imperativen (Hutter et al. 2016; Rammert et al. 2016) um sgutes Altern oder ungleiche Machtbeziehungen und Wissensbestände im Gesundheitssektor. Bei der Frage nach Erfolg oder Misserfolg von Gerontotechnologie werden die Auswirkungen sozialer Rahmenbedingungen immer stärker einbezogen und haben so mittlerweile maßgeblichen Einfluss auf deren Entwicklung. Ebenso entstehen zunehmend systematisch methodische Zugriffe auf die praktische Umsetzung dieser Erkenntnis in Forschungs- und Entwicklungsprojekten. Die analytische soziale Einbettung der Technikentwicklung - oder präziser, die untrennbare Verflechtung von Sozialität und Technik (Rammert 2006) - trägt also maßgeblich zu gelingenden Forschungs- und Entwicklungskonzepten für digitale Pflegeassistenzen bei.

Diese positive Entwicklung kann durch die noch konsequentere Nutzung grundlagenwissenschaftlicher Ergebnisse fortgesetzt werden. Ein weiterer Schritt da$\mathrm{zu}$ ist der intensivierte Austausch und die wechselseitige Wahrnehmung von anwendungsorientierten und (technik-)soziologischen Forschungsprojekten. So können bereits heute erfolgreiche Ansätze der partizipativen Technikgestaltung zukünftig noch effizienter Forschungsartefakte und scheiternde Technologieimplementierung vermeiden, wenn sie über das "So-und-nicht-anders-Gewordensein« (Weber 2002 [1904]: 103) der soziohistorisch gewachsenen »Inkommensurabilität pflegerischer und technischer Eigenlogiken« (Remmers 2015: 12) informiert sind oder um die gesundheits- und innovationspolitischen Agenden (Lipp 2020) wissen, welche Einfluss auf ihre Forschung haben. Daher plädieren wir in unserem Beitrag 
für eine verstärkte Einbindung grundlagenwissenschaftlicher Ergebnisse in die anwendungsorientierte Technikforschung im Allgemeinen und die partizipative Technikentwicklung im Speziellen. Anhand von zwei exemplarischen Feldern der Technikentwicklung zeigen wir auf, wie vielschichtig die sozialen Dimensionen von Technologien für pflegebedürftiges Alter sind (vgl. Frommeld und Rudolph in diesem Band) und wie voraussetzungsvoll das historische Gewordensein des Feldes für gegenwärtige Entwicklungsvorhaben ist. Dabei ist es gerade nicht unser Ziel, auf alle Felder und Anwendungsfälle digitaler Technologien für Senior*innen und geriatrische Bedarfe verallgemeinerbare Ergebnisse vorzustellen, sondern aufzuzeigen und zu diskutieren, welchen Mehrwert grundlagenwissenschaftliche Analysen der sogenannten sozialen Dimensionen von digitalen Assistenzen für die Konzeption hemmender und scheiternder Faktoren der Technikentwicklung in der Praxis generieren.

Zuerst stellen wir dar, wie im Rahmen europäischer Innovationspolitik Robotik und Altenpflege vor dem Hintergrund des demografischen Wandels füreinander verfügbar gemacht und auf diese Weise ein Bedarf an assistiven Technologien für ältere Menschen erzeugt wurde. Danach zeigen wir auf, wie historisch gewachsene Konflikte im Pflegesektor in Deutschland die Aushandlung darüber bestimmen, welche Eigenschaften sensorbasierten Assistenzsystemen in der Altenpflege zugeschrieben werden, um den Pflegenotstand zu lösen. Abschließend diskutieren wir, wie unsere Ergebnisse produktiv in die partizipative Technikentwicklung eingebunden und zukünftig Synergien zwischen Grundlagen- und Anwendungsforschung genutzt werden können.

\section{IGutes Altern mit Technik als Ergebnis (innovations-)politischer Verschaltung}

In europäischen Innovationsprogrammen ist die Referenz auf den demografischen Wandel und die Aussicht auf eine weiter alternde Gesellschaft ubiquitär (vgl. Weber in diesem Band). Pflegerobotik soll Pflegepersonal ersetzen oder zumindest entlasten, sensorbasierte Assistenzsysteme und Smarthomes sollen den möglichst langen Verbleib in der eigenen Häuslichkeit ermöglichen. Dafür fährt die Europäische Kommission eine Vielzahl an Initiativen, Partnerschaften, Roadmaps und Gipfeln auf. In ihnen ist sgutes Altern zu einem Fixpunkt innovationspolitischer Debatten geworden, in denen Technologien für jedes gesellschaftliche Problem eine Lösung darzustellen scheinen (Pfotenhauer/Jasanoff 2017). Zur konkreten Umsetzung dieses politischen Programms rückt nun seit einiger Zeit vermehrt die partizipative Technikgestaltung in den Blick. Ältere Menschen und Pflegende sollen dabei nicht nur als Endnutzer*innen von solchen Technologien profitieren, sondern vermehrt zu deren Produktion beitragen (Macq/Tancoigne/Strasser 2020). 
Diese Herangehensweise ist überraschend. Nicht nur, weil viele der Themen, die dort verhandelt werden - Robotik, Smarthomes, eine Silver Economy -, bis vor zwei Jahrzehnten kaum bis gar nicht im (innovations-)politischen Diskurs thematisiert wurden. Auch ist es - viel grundsätzlicher - verwunderlich, dass Altern und Innovation überhaupt zueinander gefunden haben und partizipative Technikgestaltung dabei gewissermaßen zum `Schmiermittel dieser Verbindung geworden ist. Aus diskursanalytischer Sicht ist dies keineswegs eine Selbstverständlichkeit, sondern vielmehr Ausdruck eines langen Prozesses der diskursiven und politischen Verschaltung (vgl. Lipp 2019 \& 2020). Um dieses Phänomen beschreiben und verstehen zu können, bedarf es zunächst einen Schritt zurückzugehen: Wie konnte es überhaupt dazu kommen, dass Altern und Innovation, Pflege und Assistenztechnologien kompatibel wurden? Diese leitende Frage verschiebt den Fokus von der normativen Frage, was eine rationale, sgute Antwort auf den demografischen Wandel sein könnte, hin zu der verstehenden Frage, unter welchen politischen und diskursiven Bedingungen jene Kompatibilität überhaupt plausibel werden konnte.

Die These lautet dabei: Diese Verschaltung konnte nur entstehen, weil es zu mehreren Verschiebungen innerhalb der (europäischen) innovationspolitischen Arena gekommen war. Diese betreffen insbesondere die Umdeutung (a) von Altern als wirtschaftspolitisches Thema, (b) von Informations- und Kommunikationstechnologien (IKTs) als Garanten >guten Alterns und (c) von Pflege als technisierte Assistenz. Diese drei Verschiebungsmomente fügen sich zusammen zu einem Regime, innerhalb dessen technische Innovation zur umfassenden Lösung für >gutes Altern< gemacht werden konnte. In diesem Kontext kommt der partizipativen Technikgestaltung eine entscheidende, stabilisierende Funktion zu. Durch sie werden ethische und soziale Folgeprobleme umfassender Technisierung abzufangen und teils $\mathrm{zu}$ verhindern versucht. Dies lässt jedoch die weiteren politischen Annahmen eines solchen Regimes, um die es im Folgenden gehen wird, weitgehend unberührt.

\section{Die Silver Economy: Altern als wirtschaftspolitisches Thema}

In modernen Gesellschaften stellt Altern üblicherweise ein schwieriges Thema dar. Demografischer Wandel markiert dabei immer auch eine Krise abnehmender Produktivität und steigender (öffentlicher) Kosten. Ältere Menschen werden hier als unproduktiv und abhängig von wohlfahrtsstaatlichen Maßnahmen identifiziert. Solche abwertenden Stereotype setzen einen alarmistischen Diskurs in Gang, der intergenerationale Verteilungskämpfe und repressive Alterspolitiken priorisiert (Katz 1992). Kritische Gerontolog*innen zeigen solche altersfeindlichen Stereotypen seit langem anhand verschiedenster gesellschaftlicher Praxisfelder (Brauer/Clemens 2010) und auch gerade in der Entwicklung assistiver Technologien (Neven 2011) auf. 
Betrachtet man jedoch die aktuellen Entwicklungen im Rahmen europäischer Innovationspolitik, kann man eine Verschiebung beobachten, die die aktuelle Diskussion rund um Altern und Assistenztechnologien wesentlich prägt. Die sogenannte Silver Economy (EC 2015) steht paradigmatisch dafür. Ältere Menschen geraten als Konsument*innen in den Blick, die neue Bedürfnisse und Konsummuster aufweisen. Aus der Bedrohung durch den demografischen Wandel wird die Herausforderung, diesen in ökonomische Potenziale zu übersetzen. Altern wird zum Wachstumsthema und dadurch für eine Reihe neuer Akteur"innen wie Industriepolitiker*innen, Unternehmen und Tech-Start-ups interessant.

Als Folge davon wird Altern zu einem wirtschaftspolitischen Zukunftsthema, welches darauf ausgerichtet ist, von Unternehmer*innen und innovativen Ingenieur*innen verwertet zu werden (Adam/Groves 2007). Zumindest im spezifischen Kontext der Innovationspolitik wird hohes Alter nicht mehr primär innerhalb alarmistischer Register verhandelt, sondern als Gelegenheit, um neue Verbindungen zwischen dem Alltag älterer Menschen und einer im Entstehen begriffenen, digitalen Konsum-Ökonomie herzustellen. In diesem Kontext kommt der partizipativen Technikgestaltung oftmals die Funktion zu, solche neuen Konsummuster oder Bedarfe $z u$ identifizieren, um hierdurch Produkte und Dienstleistungen für einen silbernen Markt zu optimieren (Schmidt-Ruhland/Knigge 2010).

\section{Die Informationsgesellschaft: IKT als Garant sguten Alternsı}

Ein weiteres wichtiges Puzzlestück dieses Regimes ist die Annahme, dass insbesondere assistive Informations- und Kommunikationstechnologien als sozialer Inklusionsgarant für eine wachsende Bevölkerung älterer Menschen dienen sollen. Dieser Diskurs fußt zunächst auf einem responsiven, von (gesellschaftlichen) Nützlichkeitskriterien geprägten Wissenschaftsverständnis (Kaldewey 2013), welches spätestens seit der Jahrtausendwende auch im europäischen innovationspolitischen Diskurs seinen Niederschlag gefunden hat. Einerseits markiert dies die diskursive Bedingung dafür, dass ein wirtschafts- beziehungsweise innovationspolitisches Thema wie die Silver Economy im Rahmen europäischer Forschungsprogramme überhaupt erst auftauchen konnte. Neben ökonomischen Gesichtspunkten tauchen dabei aber auch verstärkt inklusionspolitische Ziele auf, wie es am Beispiel der Informationsgesellschaft sichtbar wird, welches die Landschaft europäischer Förderpolitik innerhalb der letzten beiden Dekaden geprägt hat (vgl. Lipp 2019: 92-94).

Entscheidend ist hier, dass fortan die förderpolitische Erwartung, soziale Aspekte in die Entwicklung - insbesondere von IKT - miteinzubeziehen, ubiquitär wird. IKT wird hier als Vehikel der Inklusion von »Personen mit speziellen Bedürfnissen « (EC 1999: 13, Übersetzung B.L.) und zur Verbesserung von deren Lebensqualität gerahmt. Während die Zielgruppen zu Anfang recht allgemein bleiben 
und ältere Menschen kaum explizit erwähnt werden, ändert sich dies im Laufe der folgenden Arbeitsprogramme. Ältere Menschen werden hier, im Zusammenhang mit der wachsenden Prominenz einer alternden Gesellschaft, zu der Zielgruppe von Assistenztechnologien schlechthin. Umgekehrt macht diese Fokussierung zunehmend einen Unterschied für die Erwartungshaltung an technische Entwicklungsprojekte: >Gutes Altern und Inklusion werden hier zumindest diskursiv zum zentralen Zielwert von Forschung und Entwicklung. Sichtbar wird dies an entsprechend titulierten Ausschreibungen, zum Beispiel »ICT for Independent Living, Inclusion and Governance« (EC 2007: 50) oder »Service robotics for ageing well« (EC 2009: 73).

In diesem Kontext wird Altern $\mathrm{zu}$ einer zentralen Begründungsfolie für eine Reihe ingenieurswissenschaftlicher Projekte wie Umgebungssensorik (Smarthomes) oder robotischen Assistenten. Zugleich wird die Partizipation älterer Menschen an diesen Entwicklungen aus teilhabe- und akzeptanzpolitischen Gründen immer entscheidender (vgl. Compagna/Kohlbacher 2015). Kaum ein Forschungsantrag kommt in diesen Bereichen derzeit noch ohne partizipative Elemente aus. Die Grundlagenforschung an assistiven Technologien und die sozialen Probleme einer alternden Gesellschaft sind hier - zumindest im Kontext europäischer Innovationspolitik - unwiderruflich miteinander verschaltet worden - und dies unter der Bedingung partizipativer Technikgestaltung und trotz des bis dato ausstehenden Beweises, dass diese Technologien tatsächlich einen Unterschied machen im Alltag älterer Menschen.

\section{Selbstbestimmtes Leben: Pflege als technisierte Assistenz}

In diesem Kontext rückt auch das scheinbare >Kerngeschäft< einer vom demografischen Wandel geprägten Gesellschaft in den Blick: die Pflege älterer Menschen, entweder im Pflegeheim oder in den eigenen vier Wänden. Dabei hat sich ein weiterer wichtiger Zielwert beinahe unhinterfragt im Rahmen europäischer Innovationspolitik etabliert: der Wunsch älterer Menschen, länger alleine daheim zu wohnen (Neven 2015). Wir wollen dies kurz mit einer schon frühen, exemplarischen Aussage von Engelberger (1989) aus dem Bereich der Assistenz- beziehungsweise Pflegerobotik illustrieren.

»No, the robot will not be a practical nurse; but a robotized private abode will be so much more desirable than being in a $\$ 25,000$ to $\$ 40,000$ per year nursing home, doomed to an elephant's burial ground, smelling urine unto death, and contemplating penury before blessed surcease. (Engelberger 1989: 217)

Dieses Beispiel steht für einen Diskurs, der sich gegen die Zustände institutionalisierter (nursing home) Pflegesettings wendet. Entscheidend ist dabei die Unter- 
scheidung zwischen kollektiver, institutionalisierter und privater Altersvor- und -fürsorge (private abode vs. nursing home). Eine robotisierte Pflege stellt im Sinne Engelbergers eine Zukunft in Aussicht, in der sich Privatleute Roboter zu Hause halten, um gerade nicht das (öffentliche) Pflegesystem zu belasten. Dieses Narrativ scheint dann auch wieder in innovationspolitischen Überlegungen der EU auf, wenn es beispielsweise in Robotik-Projekten darum geht, »unnötige Krankenhauseinweisung « zu verhindern (EIP on AHA 2012: 4; Übersetzung B.L.). Studien in verwandten Projekten wie der Tele-Pflege zeigen, dass solche Zielsetzungen auch gerade aus ökonomischen Kalkülen der Rationalisierung heraus formuliert werden, in der Praxis jedoch oft gegensätzliche Effekte zeitigen (Oudshoorn 2011; Pols 2012).

Doch wie genau sieht der Zugriff der Robotik auf die Pflege in diesem Zusammenhang aus? Pflege taucht hier vor allem als Portfolio trennbarer alläglicher Aktivitäten auf. Es geht dann um Essenszubereitung, Hygiene oder Einkaufen. Diese Aktivitäten des alltäglichen Lebens werden bestimmten Fähigkeiten von Robotern zugeordnet. Pflege wird im Fall Engelbergers Service-Robotik, wie auch in neueren Publikationen der Assistenzrobotik (Robinson/MacDonald/Broadbent 2014), als Sammlung assistiver Dienste verstanden. Es geht hier nicht um Pflege in einem umfänglichen Sinne, sondern um technisierte Assistenz, die in der Ausübung einzelner tasks aufgeht, die ad hoc verfügbar ist und herkömmliche PflegeArrangements verzichtbar macht (Krings/Weinberger 2017). Assistenzsysteme wie Roboter sollen demnach Teile der Pflege übernehmen, damit ältere Menschen länger in ihren eigenen vier Wänden leben können (Neven 2015). Damit manifestiert sich auch die Tendenz, Pflege mittels Technisierung hin zu individualisierten, marktorientierten und technisierten Assistenzen umzuorganisieren (Kohlbacher/Hang 2010). Wenn aktuelle Innovationspolitik also über selbstbestimmtes Leben älterer Menschen spricht, dann wird hiermit eine bestimmte Logik von Pflege impliziert (Mol 2008), die eingebettet ist in ein Regime, dem es um die Rationalisierung von Pflege sowie um deren Verlagerung ins Private geht.

\section{Blinde Flecke einer sTechno-Politikı der Innovation}

Wie wir gezeigt haben, ist eine immer enger werdende Verschaltung von technischer Innovation und >gutem Altern « keineswegs ein `natürlicher Z Zustand, sondern vielmehr das Ergebnis einiger genuin politischer Diskursverschiebungen. In diesem Kontext wurde Altern zu einem wirtschaftspolitischen Zukunftsthema, IKT zum Garanten sguten Alterns` und Pflege zumindest teils als technisierte Assistenz umgedeutet. Dieser Prozess ist nicht unschuldig, sondern hat Konsequenzen für das, was innerhalb dieses Diskurses für möglich und wünschenswert befunden werden kann. In diesem Zusammenhang sind die gegenwärtigen Anstrengungen anwendungswendorientierter partizipativer Technikgestaltung zu verstehen, wel- 
che zum Ziel haben, eben jene Vorstellungen >guten Alterns` technisch umzusetzen. Dabei geht der derzeitige Diskurs mit der Marginalisierung alternativer Strategien und Möglichkeitsräume einher, die auch über aktuelle Formen der partizipativen Technikgestaltung nur unzureichend wieder eingeholt werden können (vgl. Compagna/Kohlbacher 2015). Die Optimierung bereits entwickelter Technologien hinsichtlich der Akzeptanz von Nutzer*innen-Bedürfnissen greift dabei zu kurz, weil sie die genuine Politizität der hierdurch legitimierten Verschaltung ausklammert. $\mathrm{Zu}$ fragen ist hier also, inwieweit partizipative Formate und Methoden die politische Ebene gegenwärtiger diskursiver Verschiebungen um Altern und Innovation adressieren können.

\section{Digitale Technologien als Lösung für einen historischen Konflikt in der Altenpflege ${ }^{1}$}

Unser zweiter Fokus liegt auf der soziohistorischen Rekonstruktion der Ursachen und Folgen der Forderung nach und Implementierung von digitalen Innovationen in die Altenpflege. Da konventionelle Lösungsstrategien, wie Reformen der Pflegeversicherung oder Qualifizierung der Pflegenden, keinen oder nur unzulänglichen Erfolg zeigen, scheint die Digitalisierung der Pflege zurzeit der erfolgversprechendste Lösungsansatz. Pointiert zusammengefasst greifen von politischen Akteur*innen vorangetriebene Diskurse um innovative digitale Technologien alle Probleme der Altenpflege wie Personalmangel oder Unterfinanzierung auf und bieten eine vielversprechende Lösung für die Zukunft der älteren Menschen (HülskenGiesler/Krings 2015; Künemund 2015; Nierling/Domínguez-Rué 2016). Demzufolge wurde in den letzten Jahren eine Vielzahl an Forschungs- und Entwicklungsprojekten angestoßen, die zum Ziel hatten, die Forderungen in der Praxis umzusetzen.

Betrachtet man jedoch die Effekte digitaler Pflegetechnologien, war - zumindest bis in jüngste Vergangenheit - nur eine Minderheit geeignet, um den Problemen der Pflege wirksam und kurzfristig begegnen zu können. Auf empirischer Basis konnte lange nicht von einer schon stattgefundenen oder zeitnah zu erwartenden Digitalisierung pflegerischer Alltage mittels marktreifer Produkte gesprochen werden (Endter 2016; Weinberger/Decker 2015). Besonders avancierte Zukunftsvisionen, wie die des autonomen humanoiden Pflegeroboters, können weder kurznoch mittelfristig die strukturellen Probleme des Pflegenotstandes auflösen. Darüber hinaus galten technische und (besonders alten-)pflegerische Eigenlogiken lange Zeit nur als begrenzt vereinbar, sodass ihre nun postulierte Kompatibilität irritiert (Hülsken-Giesler/Krings 2015; Remmers/Hülsken-Giesler 2007; kritisch dazu 
Koppenburger/Wüller 2020). Zudem sind Probleme der Finanzierung und Überlastung in der Altenpflegegeschichte nicht neu oder gar gegenwartsspezifisch. Vielmehr durchzieht der Pflegenotstand fast die gesamte Soziogenese der Altenpflege. Aus diesen beiden Status quo ergibt sich die Frage, weshalb digitale Pflegetechnologien seit einigen Jahren im Innovationsdiskurs derart erfolgreich als Lösungsstrategie wahrgenommen und postuliert werden können.

Wir argumentieren, dass die derzeitige diskursive Präsenz assistiver Pflegetechnologien - und damit zusammenhängend die Rahmenbedingungen und Ausbreitung partizipativer Technikentwicklung - nicht nur in deren faktischem Nutzen begründet liegt, sondern darüber hinaus in Zusammenhang mit einem, seit der Konstitution der Altenpflege feststellbaren, historischen Konflikt steht. Dieser Konflikt zwischen pflegerisch-fachlichen und ökonomisch-instrumentell orientierten Pflegeleitbildern wird in der Gegenwart mit und über die Digitalisierung der Pflege ausgehandelt. Durch eine soziohistorische Perspektive treten auch die gegenwärtigen Effekte des Einsatzes von Pflegetechnologien und ihre potenziellen Folgen für die historisch gewachsenen Strukturen der Pflege deutlich hervor. Sie bringt also gegenüber gegenwartsfokussierten, anwendungsnahen Analysen einen deutlichen Mehrwert hervor und ist zusätzlich aufschlussreich für die zukünftige (partizipative) Konzeption von Pflegetechnologien.

\section{Struktureller Konflikt in der Soziogenese der Altenpflege in Deutschland}

In der kaiserzeitlichen Gesellschaft Ende des 19. Jahrhunderts wurde pflegebedürftiges Alter von einem privaten zu einem durch wohlfahrtsstaatliche Fürsorge zu regulierenden >Problem $\prec$. Auf diese Weise wurden volkswirtschaftliche Überlegungen und bürokratische Verwaltungslogiken für die pflegerische Versorgung im Alter relevant und wirkmächtig (Heinzelmann 2004; Kondratowitz 1990). In diesem Prozess entstanden aus der ehemaligen Armen- und Siechenfürsorge auf die Versorgung alter, pflegebedürftiger Menschen spezialisierte Einrichtungen. Diese neuen Altenheime erforderten auch ein sich von den ehemaligen >Wärtern zendes Pflegepersonal. Für die Versorgung pflegebedürftigen >Alters $<$ wurden nun zunehmend fachliche Eigenschaften benötigt, welche typisch für eine genuin altenpflegerische Tätigkeit waren (Cappell 1996; Irmak 2002). In dieser formativen Periode der Altenpflege trafen also erstmals grundlegend unterschiedliche Pflegeverständnisse aufeinander. Den sich langsam entwickelnden, an fachlichen und ethischen Leitbildern orientierten (Berufs-)Auffassungen der Pflegenden standen jene der politischen und administrativen Akteur*innen entgegen. Diese waren wesentlich wirkmächtiger, primär an einer ökonomisch effizienten Versorgung sowie an wohlfahrtstaatlichen Verwaltungslogiken orientiert. So entstand im ausgehenden 19. Jahrhundert der strukturell anfangs von ökonomisch-instrumentellen Logiken dominierte Konflikt zwischen verschiedenen Pflegeverständnissen, der auch 
heute noch vorzufinden ist und in Zusammenhang mit der Forderung nach einer Digitalisierung der Pflege steht.

In der späten Nachkriegszeit Ende der 1950er Jahre lässt sich ein deutlicher Wandel der Altenpflege und eine Emanzipation pflegerisch-fachlicher gegenüber ökonomisch-instrumenteller Pflegeleitbilder feststellen. Auf kostengünstige Versorgung und bloße >Verwahrung< basierende Pflegekonzepte erschienen nicht mehr zeitgemäß. Gesamtgesellschaftlich vorzufindende Vorstellungen von Alter als sinnstiftende, eigenständige Lebensphase, eine wachsende Solidarität mit pflegebedürftigen alten Menschen und nicht zuletzt die verbesserte ökonomische Absicherung im Alter führten zu gestiegenen Anforderungen an die Altenpflege und der Qualifizierung der Pflegenden (Heumer/Kühn 2010; Kondratowitz 1988 \& 1990). Damit einhergehend wurde der Erhalt von Selbständigkeit, Individualität und Würde im Alter zum zentralen Kriterium der psychosozial ausgerichteten Altenpflege. Die bislang die Strukturen der Altenpflege prägenden ökonomischinstrumentellen Pflegeauffassungen politischer und administrativer Akteur*innen verloren ihre ehemalige Deutungshoheit. Folglich konnten sie ihre Interessen auch nicht mehr dominant und ohne Kompromisse durchsetzen, was die Art und Weise der Konfliktaushandlung von einer machtvollen Durchsetzung hin zu einer konsensualen Aushandlung änderte.

Diese Entwicklung setzte sich bis in die 1990er Jahre fort, bis die erneute (seit der Nachkriegszeit immer wiederkehrende) Diskussion um die Folgen des demografischen Wandels und der Finanzierung der sozialen Sicherungssysteme (Kelle 2008) eine weitere Veränderung der Konfliktaushandlung bewirkte. Besonders politische Akteur*innen standen unter Druck, probate Lösungsstrategien zu entwickeln (vgl. Birg 2011; Rothgang/Müller/Unger 2012), da konventionelle Lösungen, wie beispielsweise Reformen der Pflegeversicherung oder "Ausbildungs- und Qualifizierungsoffensiven « (BMFSFJ 2015: 10) der (Alten-)Pflege, immer augenscheinlicher unzureichende Wirkung zeigten. Spätestens ab den $2000 e r$ Jahren wurde eine (technologische) Lösung für die Zunahme an altersbedingt Pflegebedürftigen und den Mangel an qualifizierten Pflegenden immer drängender (Künemund 2015). Bei der Bewältigung des Pflegenotstandes galt es zu berücksichtigen, dass sich mittlerweile weder Vertreter*innen pflegerisch-fachlicher Pflegeleitbilder mit Forderungen nach einer substanziell besseren Ressourcenausstattung, noch Vertreter*innen ökonomisch-instrumenteller Interessen mit einer Reduzierung der Altenpflege auf grundpflegerische Versorgung machtvoll und ohne grundlegende Kompromissbereitschaft durchsetzen konnten. Die historische Konfliktaushandlung führte zu einem Patt, in dem sich die unterschiedlichen Pflegeverständnisse blockierten und politische Akteur*innen zunehmend handlungsunfähig wurden (vgl. Hergesell 2019b: 250-252). Sie standen vor der scheinbar unlösbaren Aufgabe, Lösungen $\mathrm{zu}$ entwickeln, welche die konfligierenden Pflegeverständnisse miteinander integrierten. 
Aus dieser scheinbar unauflösbaren Situation heraus ist der diskursive Erfolg innovativer Pflegetechnologien zu verstehen. Besonders vermeintlich wirkmächtigen assistiven Technologien wurde zugeschrieben, alle Interessen miteinander vereinbaren zu können sowie anschlussfähig an bisherige Reformen hin zu einer Ambulantisierung und somit anschlussfähig an den historisch gewachsenen Konflikt in der Pflege zu sein (vgl. BMBF 2011 \& 2014a \& 2014b \& 2015; IPA 2017; Weiß/Lutze/Compagna 2013). Die Digitalisierung der Pflege sollte zum einen eine Effizienzsteigerung und Kostenreduktion bewirken sowie auf diese Weise die langfristige Finanzierung der Pflege im demografischen Wandel ermöglichen. Zum anderen sollten digitale Innovationen zusätzlich die überforderten Pflegenden entlasten, mehr Zeit für psychosoziale Pflege zur Verfügung stellen und die Autonomie von Senior*innen fördern. Die Digitalisierung der Pflege bot also scheinbar eine historisch einzigartige Möglichkeit, den gewachsenen Konflikt antagonistischer Pflegeleitbilder aufzulösen, und wurde dementsprechend in letzter Konsequenz von keiner Akteur*innengruppierung als Zukunftsvision verhindert. Bis in die Gegenwart entwickelte sich das immer wirkmächtigere und gesamtgesellschaftlich verbreitete Narrativ, dass ohne digitale Pflegeinnovationen eine zukunftssichere, qualitativ hochwertige Pflege und ein selbständiges, würdevolles Alter(n) im demografischen Wandel gefährdet sei.

Der derzeitige Erfolg digitaler Technologien, ihre massive Förderung und der teils irritierende Digitalisierungseuphemismus lässt sich also im Wesentlichen auch als Folge der Integration eines historisch gewachsenen Konflikts in der (Alten-)Pflege verstehen.

\section{Historisch-gewachsene Grenzen partizipativer Technikentwicklung}

Auch wenn keineswegs von einer bereits erfolgten Digitalisierung des Pflegealltags zu sprechen ist, werden seit den 2010er Jahren vermehrt digitale Assistenzen implementiert. Dabei zeigt sich, dass - anders als im Diskurs um Pflegeinnovationen postuliert - die Eigenschaften der entwickelten Technologien keine problemlose Integration verschiedener Pflegeverständnisse bewirken. Vielmehr kann es dazu kommen, dass sich, vermittelt durch Technikentwickler*innen, vermehrt ökonomisch-instrumentale Pflegeverständnisse durchsetzen beziehungsweise pflegerisch-fachliche vernachlässigt werden (Hergesell/Maibaum 2018; Hergesell 2019a; vgl. Remmers 2015). Dies kann ein Grund für in der Praxis scheiternde Technikimplementierung oder mangelnde Akzeptanz durch die Nutzer*innen sein. Diesem Missstand sollte durch partizipative Technikentwicklung, welche eine bessere Integration der Interessen ermöglichen soll, entgegengewirkt werden. Allerdings griffen die dafür genutzten Konzepte oftmals zu kurz. Teile der anwendungsorientierten Technikentwicklung versuchten substanzielle, historisch gewachsene Konfliktfelder mittels niedrigschwelliger, punktueller Interventionen aufzulösen. So wurde 
etwa angenommen, dass es sich bei Fehlentwicklungen meist um bloße >Übersetzungsfehler zwischen Technikentwickler*innen und Nutzer*innen handelt, denen allerdings - wie oben ausgeführt - strukturelle und nur schwer auflösbare antagonistische Pflegeverständnisse zugrunde liegen (vgl. Hergesell/Maibaum 2018).

Wenn vor der Nutzung neuerer Konzepte in Bedarfsanalysen im Rahmen partizipativer Forschungsprojekte Anforderungen an die Pflegetechnologien hinsichtlich der Aufrechterhaltung von Autonomie (Selbstständigkeit) im pflegebedürftigen Alter erhoben wurden, schienen die Anforderungen aller beteiligten Akteur* innen oft übereinzustimmen. Sowohl Pflegende, Senior*innen als auch Technikentwickler*innen und Mittelgeber*innen (politische und administrative Akteur*innen) sahen digitale Assistenzen als Chance dafür, ein selbstbestimmtes Leben im Alter zu ermöglichen.

Bei genauerer Betrachtung auf Grundlage einer soziohistorischen Perspektive zeigt sich jedoch, dass Pflegende und Senior*innen, gemäß ihren historisch gewachsenen Pflegeauffassungen, den Erhalt von Autonomie als genuin pflegerische Aufgabe verstehen (Hergesell 2019a \& 2019b; vgl. Frommeld und Scorna in diesem Band), die nur mittels einer personalintensiven und fachqualifizierten Pflege möglich ist. Heuristisch lassen sich so zwei verschiedene Pflegeverständnisse gegenüberstellen, welche bis zur konsequenten Anwendung von partizipativen Methoden in der Technikgenese wirkten. Die Pflegenden erwarteten von den Assistenzen, im Sinne der Auffassung von (Alten-)Pflege als lebensweltliche Begleitung, eine Entlastung von repetitiven Kontrollaufgaben, Dokumentationspflichten oder hauswirtschaftsnahen Tätigkeiten, die ihnen Freiräume für eine intensive psychosoziale und individual-situative Pflege verschaffen sollten. Bei Technikentwickler*innen und politisch-administrativen Akteur*innen war diese Auffassung dagegen weniger vorzufinden. Diese Akteur"innen verstanden Autonomie oft auf Basis ihrer ökonomisch-instrumentellen Pflegeverständnisse, stellenweise entgegengesetzt, als eine durch Technologie ermöglichte Abwesenheit von Pflegenden (vgl. Hergesell/Maibaum 2018). Demnach fokussierten sie die Entwicklung von Technologie, die etwa über sensorbasierte Kontrolle die physische Sicherheit der Gepflegten garantieren und so die Anwesenheit von Pflegenden substituieren sollte. Solange diese verschiedenen Pflegeverständnisse nicht (etwa durch neuere Ansätze der partizipativen) Technikgenese komplexitätsangemessen reflektiert wurden beziehungsweise werden, kann es dazu kommen, dass Technologien implementiert werden, die in der Praxis inkompatibel mit den Bedürfnissen der Pflegenden und Nutzer*innen sind.

Das Beispiel der technisch assistierten Autonomie sensibilisiert dafür, dass die lexikalische Übereinstimmung zwischen Vertreter*innen verschiedener Pflegeverständnisse im Entwicklungsprozess keineswegs eine semantische ist. Wird solchen Diskrepanzen während partizipativer Entwicklung nicht vollumfänglich nachgegangen, kann es dazu kommen, dass die beteiligten Akteur*innen handlungsprak- 
tisch unterschiedliche Ziele verfolgen und sich auf grundlegend verschiedene Wissensbestände beziehen - mit negativen Folgen für die Akzeptanz der Technologien. Dieser Umstand tritt erst durch method(olog)isch elaborierte Forschungsdesigns hervor und wird in anwendungsorientierten, ressourcenlimitierten Entwicklungsund Forschungsprojekten oft nur unzureichend beachtet. Nur Werkzeugsets wie MEESTAR $^{2}$ (Weber 2016) können, wenn konsequent angewendet, diese Lücke füllen.

Zusätzlich verweist die soziohistorische Perspektive auf die ungleich verteilten Machtpositionen der an der Digitalisierung beteiligten Akteur*innen. Denn nicht alle Akteur*innen haben die gleiche Chance, ihre Pflegeverständnisse während der Technikentwicklung zu artikulieren und durchzusetzen. Die Entscheidung über Technologieziele und die Art und Weise der Umsetzung durch die Technikentwickler*innen wird von politisch-administrativen Akteur*innen dominiert, daher ist es möglich, dass deren ökonomisch-instrumentelle Interpretation auch bei externer Bedarfsanalyse im Rahmen partizipativer Technikentwicklung bestehen bleibt (Hergesell/Maibaum 2018). So können sie durch ihre machtvolle Position ihre Interessen und Vorstellungen von sguter Pflegetechnologie und einem >guten Leben im Alter<, trotz Versuchen der partizipativen Technikentwicklung, privilegiert in die Technikgenese einschreiben. Der historisch gewachsene Konflikt, dem die Pflegetechnologien ihren diskursiven Erfolg verdanken, wird somit durch die Technisierung mitnichten aufgelöst, sondern lediglich verschoben. Die Digitalisierung der Pflege verlagert die Aushandlung des ursprünglichen Konflikts in die Verwendung und Prozeduren der Technologien. Der grundlegende Konflikt ist nicht Gegenstand offener Aushandlung und geht daher sozusagen "stumm« vonstatten (Braunisch/Hergesell/Minnetian 2018: 199). Im Rahmen dieses Phänomens wird die Chance, durch partizipative Technikentwicklung alle Interessen komplexitätsangemessen gleichberechtigt $\mathrm{zu}$ berücksichtigen und so mehr Akzeptanz der beteiligten Akteur*innen zu erzeugen, gefährdet. Dadurch wird das Argument für eine konsequentere, flächendeckendere Verwendung neuer Tools zur partizipativen Technikgestaltung noch einmal gestärkt.

\section{Fazit \& Diskussion}

Wie wir in zwei Feldern der Forderung nach und Entwicklung von digitalen Pflegetechnologien gezeigt haben, erklärt sich deren diskursive Präsenz nicht vorwiegend oder gar ausschließlich durch ihren tatsächlich bereits heute vorzufindenden Nutzen im Pflegealltag. Mit unserer grundlagenwissenschaftlichen Perspektive auf das Phänomen Pflegetechnologie wollen wir stattdessen dafür sensibilisieren, welche komplexen, wechselwirkenden sozialen Prozesse die gravierende Diskrepanz zwischen der Prominenz der Technologien, ihren postulierten Eigenschaften und 
ihrem tatsächlichen Einsatz produzieren. Zuerst haben wir gezeigt, wie Pflegeroboter, als Paradebeispiel für avancierte Pflegetechnologien, im Rahmen europäischer Innovationspolitik zu einer (politischen) Realität gemacht werden. In diesem Diskurs wird auf technologischen Fortschritt als Reaktion auf den demografischen Wandel gesetzt und gleichzeitig Technologie genutzt, um sozialen Wandel zu konzipieren. Daran anschließend führten wir aus, dass diese Innovationspolitik deswegen auf so fruchtbaren Boden fällt, weil sie anscheinend einen historischen Konflikt innerhalb der Altenpflege integriert und digitale Pflegetechnologien auf diskursiver Ebene eine Möglichkeit darstellen, dringend benötigte Lösungen für den Pflegenotstand zu präsentieren. Schließlich haben wir dargestellt, wie diese Gemengelage von Technikentwickler*innen aufgegriffen wird. In der Entwicklungspraxis wird dabei meist nicht Technologie für die Pflege zur Verfügung gestellt, sondern eher die Pflege für Technologie verfügbar gemacht.

Mit unserer Betrachtung der komplexen sozialen Rahmenbedingungen, in welche >gute Technik für ein gutes Leben im Alter interveniert, lassen sich also sowohl die Ursachen der diskursiven Präsenz als auch die mangelnde Akzeptanz von Pflegetechnologien in der Praxis erklären. Diese gehen weit über isolierte Faktoren in einzelnen Anwendungsszenarien hinaus und lassen sich auch nur sehr bedingt in solchen analysieren. Aus unserer Diagnose können wir jedoch allgemeine Kritikpunkte ableiten, welche für anwendungsorientierte Forschung als Heuristik fruchtbar sind.

Erstens ist zu reflektieren, dass die politische Vision von digitalen Technologien für eine alternde Gesellschaft und die Altenpflege keineswegs eine neutrale oder alternativlose Zukunftsvorstellung ist. Vielmehr ist die massive Förderung von Pflegetechnologien als politische Lösung von Krisen im Gesundheitssystem (Pflegenotstand/demografischer Wandel) zu verstehen. In diesem Rahmen werden die technologischen Lösungen an das gegenwärtige Regime der Vermarktung, Rationalisierung und Ambulantisierung angepasst. Alternative Technologien und Anwendungsszenarios werden so ausgeschlossen und bestehende Machtverhältnisse im Diskurs um Alter, Gesundheit und demografischen Wandel reproduziert. Es findet also eben keine voraussetzungsfreie Aushandlung darüber statt, wie und welche Technologien zum Einsatz kommen sollen (oder etwa welche von den Nutzer*innen erwünscht sind), stattdessen werden als geeignet wahrgenommene technologische Pfade im Vorfeld fixiert.

Die zweite Kritik zielt darauf ab, dass Technikentwickler*innen nicht auf ein neutrales Anwendungsfeld stoßen, sondern in komplexe, historisch gewachsene Strukturen und Konflikte von Akteur*innengruppen mit heterogenen Pflegeverständnissen eingreifen. Für eine erfolgreichere Technikentwicklung und -implementierung in der Altenpflege wird es zukünftig entscheidend sein zu erkennen, welche unterschiedlichen Interessen empirisch vorzufinden sind und wie diese berücksichtigt beziehungsweise integriert werden können (vgl. Haug und Scorna in diesem Band). Dazu bedarf es einer vertieften Analyse sowohl 
der soziohistorischen Strukturen der Altenpflege als auch der konkreten Akteur"innenkonstellationen, für welche Technologie entwickelt wird, sowie deren Pflegeverständnissen. Jene benötigte Analyse stellt - trotz eindeutig progressiver Entwicklungen - immer noch eine Herausforderung für partizipative Ansätze und sogenannte ethical, legal and social impact (ELSI)-Bemühungen dar.

Und drittens wollen wir dafür sensibilisieren, dass epistemische Praktiken von Technikentwickler*innen zu einer Dekontextualisierung und Dekonstruktion der Pflegepraxis in einzelne, technisch machbare Aufgaben führen können. Die Folgen sind sowohl >mechanisierte Verständnisse von Pflege als auch Dysfunktionalität von Technologie in konkreten Pflegesituationen. Die Überwindung der epistemischen Kulturen von Technikentwickler*innen ist jedoch keineswegs durch einige wenige Interventionen oder Bedarfsanalysen in partizipativen Forschungsprojekten $\mathrm{zu}$ bewerkstelligen. Stattdessen sollten die konventionellen und bisher wenig erfolgreichen Förderungs- und Finanzierungsstrukturen von Entwicklungsprojekten durchbrochen werden. Anstelle von linearen Technikentwicklungslogiken sollten offenere Projektstrukturen geschaffen werden, in welche die Wissensbestände und Bedürfnisse aus der Pflege iterativ und interaktiv einfließen können und in gemeinsam entwickelten, verbindlich vereinbarten Evaluationskriterien über den gesamten Entwicklungs- und Implementierungsprozess berücksichtigt werden.

Grundlagenwissenschaftliche Ergebnisse können auch über eine allgemeine Kritik der derzeitigen Technikgenese hinaus in aktuellen Projekten zur partizipativen Technikentwicklung genutzt werden, indem sie auf die leitenden Prinzipien partizipativen Designs bezogen werden. Wie Kensing/Greenbaum (2013) festhalten, zielt die grundlegende Idee von Partizipation darauf ab, ungleiche Machtbalancen im Sinne demokratischer Praktiken zu nivellieren, Technologien und deren Effekte immer im Kontext ihrer konkreten Nutzung zu analysieren und so situationsbasiert Lernprozesse anzustoßen sowie Gleichberechtigung zu ermöglichen. ${ }^{2}$

Eine grundsätzliche Gleichberechtigung innerhalb von Entwicklungs- und Forschungsprojekten ist nur möglich, wenn alle beteiligten Akteur*innen von Anfang an ihre Interessen artikulieren können. In den meisten Projekten werden Nutzer*innen jedoch erst hinzugezogen, nachdem durch Förderrichtlinien, Mittelgeber*innen und Technikentwickler"innen schon richtungsweisende Entscheidungen über die Art und Weise sowie den Verwendungszweck der Technologien getroffen wurden. Um eine optimale demokratische Partizipation zu ermöglichen, sollten Nutzer*innen jedoch schon bei der Planung von Förderungen und der Formulierung von Technologiezielen involviert werden. Wie gezeigt, werden sonst

2 Kensing/Greenbaum (2013: 33-34) zählen sechs Prinzipien des partizipativen Designs auf: »equalising power relations«, »democratic practices«, »situation-based actions«, »mutual learning «, »tools and techniques« und »alternative visions about technology«. 
schon vor Beginn der konkreten Technikentwicklung Pfadabhängigkeiten geschaffen und ungleiche Machtbalancen (re-)produziert. Falls ein frühzeitiger Einbezug in die formative Periode der Technikgenese nicht möglich ist, sollten Nutzer*innen zumindest möglichst rasch und in alle weiteren Phasen des Entwicklungsprozesses eingebunden werden. Auch kann ein Vetorecht, bezüglich ohne sie getroffener Entscheidungen, eine verspätete Partizipation teilweise kompensieren.

Wie ausgeführt, sind in partizipativen Projekten durchgeführte Bedarfsanalysen nicht als simple >Mitteilungく von Anforderungen der Nutzer*innen an die Technikentwickler*innen zu verstehen. Vielmehr stecken hinter vermeintlich klar zu verstehenden Anforderungen komplexe, historisch gewachsene Semantiken, die den nicht in die jeweiligen Fachkulturen beziehungsweise Lebenswelten hineinsozialisierten Akteur*innen verschlossen bleiben. Um auch zukünftig Missverständnisse oder sogar gänzlich scheiternde Kommunikation effektiv zu vermeiden, müssen Bedarfsanalysen weiterhin als wesentlich mehr als aktiv zu leistende, aufwendige Translationsarbeit verstanden werden und in diese Richtung weisende Konzepte konsequent(er) angewandt werden. Es darf sich nicht, wie zu Beginn der Entwicklung von digitalen Assistenzen, vorschnell mit einem anscheinend geteilten Konsens über Bedarf und technische Lösung zufriedengegeben werden. Stattdessen muss in einem intensiven Austausch sichergestellt werden, dass tatsächlich wechselseitiges Sinnverstehen eintritt - wie es Werkzeuge wie das bereits genannte MEESTAR ${ }^{2}$ anstreben (zur Übersicht vieler weiterer Ansätze beispielsweise Reijers et al. 2018). Dies ist durchaus als ein hermeneutischer Prozess zwischen den beteiligten Akteur*innen zu verstehen, der sozialwissenschaftlich evoziert und moderiert werden sollte. Erstrebenswert wäre ein nicht ausschließlich sprachlicher Austausch, sondern auch ein lebensweltlich-ethnografischer Zugang. Beispielsweise könnten Technikentwickler*innen über einen längeren Zeitraum an vorher als relevant identifizierten Situationen im Pflegealltag teilnehmen, um die so gewonnene Sensibilität für die Pflegekultur in die Technikentwicklung einfließen zu lassen.

Letztendlich bedeutet gemeinsames Lernen und gleichberechtigte Entwicklung aber nicht nur Anstrengungen seitens der Technikentwickler*innen den pflegerischen Alltag zu verstehen. Sprich, nicht nur Technikentwickler*innen müssen aus den Laboren in die Pflege, sondern auch Pflegende in die Labore. Auf diese Weise könnten Pflegende und Senior*innen anstatt passive >Empfänger*innen Technologien und (oft missverstandene) >Beforschte $<\mathrm{zu}$ sein, zu aktiven Akteur* innen der Technikgenese werden. Beispielsweise könnten Pflegende in partizipativen Projekten an entscheidenden Treffen der Technikentwickler*innen oder regelmäßig standfindenden Demonstrationen der Technik teilnehmen. So könnten persistente epistemische Kulturen der Technikentwickler*innen durch (Mikro-)Interventionen der Nutzer*innen beständig irritiert und aufgebrochen werden. Es ließen sich nicht nur frühzeitig Fehlentwicklungen erkennen und beheben, Nutzer*innen 
wären darüber hinaus gezwungen sich grundsätzlicher mit Technikgenese auseinanderzusetzen und Technologiekompetenz auszubilden.

Die von uns auf Basis unserer Analyse(n) formulierten Kritiken der derzeitigen Forschungs- und Entwicklungsprojekte zeigen die Notwendigkeit auf, verstärkt die sozialen Rahmenbedingungen von sguter Technik für ein gutes Alter $\mathrm{zu}$ reflektieren. Ein intensiverer Dialog zwischen grundlagenwissenschaftlicher Forschung und anwendungsorientierten Projekten kann sicherlich zu einer gelingend(er)en Technikentwicklung beitragen, steht aber noch am Anfang.

\section{Literatur}

Adam, Barbara/Groves, Chris (2007): Future Matters. Action, Knowledge, Ethics, Leiden/Boston: Brill.

Birg, Herwig (2011): Demografischer Wandel in Deutschland. Bevölkerungsentwicklung: Soziale Auswirkungen, https://www.bpb.de/izpb/55920/sozialeausw irkungen-der-demografischen-entwicklung (zuletzt abgerufen 15.12.2020).

Birken, Thomas/Pelizäus-Hoffmeister, Helga/Schweiger, Petra/Sontheimer, Rainer (2018): »Technik für ein selbstbestimmtes Leben im Alter - eine Forschungsstrategie zur kontextintegrierenden und praxiszentrierten Bedarfsanalyse«, in: Forum Qualitative Sozialforschung/Forum: Qualitative Social Research 19(1), Art. 3. DOI: 10.17169/fqs-19.1.2871.

Brauer, Kai/Clemens, Wolfgang (2010): $\mathrm{Zu}$ alt? »Ageism« und Altersdiskriminierung auf Arbeitsmärkten, Wiesbaden: VS Verlag für Sozialwissenschaften.

Braunisch, Lilli/Hergesell, Jannis/Minnetian, Clelia (2018): »Stumme Ökonomisierung - Machteffekte in Innovationsdiskursen«, in: Zeitschrift für Diskursforschung, 2. Beiheft, S. 183-215. DOI: 10.3262/ZFDB1801183.

Bundesministerium für Bildung und Forschung (BMBF) (2011): Das Alter hat $\mathrm{Zu}$ kunft. Forschungsagenda der Bundesregierung für den demografischen Wandel, Berlin/Bonn: BMBF.

Bundesministerium für Bildung und Forschung (BMBF) (2014a): Pflegeinnovationen für Menschen mit Demenz, https://www.technik-zum-menschen-bringe n.de/foerderung/bekanntmachungen/pflegeinnovationen-fuer-menschen-mit -demenz (zuletzt abgerufen 15.12.2020).

Bundesministerium für Bildung und Forschung (BMBF) (2014b): Bekanntmachung des Bundesministeriums für Bildung und Forschung von Richtlinien zur Förderung von Forschung und Entwicklung auf dem Gebiet »Pflegeinnovationen für Menschen mit Demenz«, https://www.bmbf.de/foerderungen/bekanntma chung.php?B=922 (zuletzt abgerufen 15.12.2020). 
Bundesministerium für Bildung und Forschung (BMBF) (2015): Technik zum Menschen bringen. Forschungsprogramm zur Mensch-Technik-Interaktion, Bonn: BMBF.

Bundesministerium für Familien, Senioren, Frauen und Jugend (BMfSFJ) (2015): Zwischenbericht zur Ausbildungs- und Qualifizierungsoffensive Altenpflege (2012-2015), Berlin: BMfSFJ.

Cappell, Eckhard (1996): Von der Hilfspflege zur Profession. Entstehung und Entwicklung des Altenpflegeberufs, Köln: Kuratorium Deutsche Altenhilfe.

Cieslik, Silvana/Klein, Peter/Compagna, Diego/Shire, Karen (2012): »Das Szenariobasierte Design als Instrument für eine partizipative Technikentwicklung im Pflegedienstleistungssektor«, in: Karen A. Shire/Jan Marco Leimeister (Hg.), Technologiegestützte Dienstleistungsinnovation in der Gesundheitswirtschaft, Wiesbaden: Gabler, S. 85-110.

Compagna, Diego/Kohlbacher, Florian (2015): »The limits of participatory technology development: The case of service robots in care facilities for older people«, in: Technological Forecasting and Social Change 93, S. 19-31. DOI: 10.1016/j.techfore.2014.07.012.

Davis, Fred D. (1989): "Perceived usefulness, perceived ease of use, and user acceptance of information technology«, in: MIS Quarterly 13(3), S. 319-340. DOI: 10.2307/249008.

Endter, Cordula (2016): »Skripting Age - The Negotiations of Age an Aging in Ambient Assisted Living«, in: Emma Domínguez-Rué/Linda Nierling (Hg.), Ageing and Technology. Perspectives from the Social Sciences, Bielefeld: transcript, S. 121-140.

Engelberger, Joseph F. (1989): Robotics in service, Cambridge, Mass.: MIT Press.

European Commission (EC) (1999): Information Society Technologies. A programme of Research, Technology Development \& Demonstration under the 5th Framework Programme, 1999 Workprogramme.

European Commission (EC) (2007): ICT - Information and Communications Technologies, Work Programme 2007, https://op.europa.eu/en/publication-de tail/-/publication/18422ee1-c388-4b35-9af1-b6fdfa48e833/language-en/format$\mathrm{PDF} /$ source-search (zuletzt abgerufen 05.05.2021).

European Commission (EC) (2008): ICT - Information and Communications Technologies. Updated Work Programme 2009 and Work Programme 2010, h ttps://op.europa.eu/en/publication-detail/-/publication/4c8c226c-87ac-401a-a dc4-48930bfi8666/language-en/format-PDF/source-search (zuletzt abgerufen 05.05.2021).

European Commission (EC) (2015): Growing the European Silver Economy, Brussels, http://ec.europa.eu/research/innovation-union/pdf/active-healthy-agein g/silvereco.pdf (zuletzt abgerufen 11.01.2021). 
European Innovation Partnership on Active and Healthy Ageing (EIP on AHA) (2012): Action Plan on Development of interoperable independent living solution, including guidelines for business models, Brussels https://ec.europa .eu/eip/ageing/library/action-plan-development-interoperable-independent-1 iving-solution-including-guidelines_en.html (zuletzt abgerufen 05.05.2021).

Fraunhofer Institut für Produktionstechniken und Automatisierung (IPA) (2017): Care-O-bot ${ }^{\circledR}$ 3. Produktvision eines interaktiven Haushaltsassistenten, https:/ /www.care-o-bot.de/content/dam/careobot/de/documents/Produktblaetter/P roduktblatt_Care-O-bot_3.pdf (zuletzt abgerufen 15.12.2020).

Grunwald, Armin (2018): »Technikfolgeabschätzung und Demokratie. Notwendige oder kontingente Verbindung?«, in: TATuP 27(1), S. 40-45. DOI: 10.14512/tatup.27.1.40.

Heinzelmann, Martin (2004): Das Altenheim - immer noch eine »Totale Institution«? Eine Untersuchung des Binnenlebens zweier Altenheime. Dissertationsschrift, Universität Göttingen.

Hergesell, Jannis (2019a): Technische Assistenzen in der Altenpflege. Eine historisch-soziologische Analyse zu den Ursachen und Folgen von Pflegeinnovationen, Weinheim/Basel: Juventa.

Hergesell, Jannis (2019b): »Von der Armen- und Siechenfürsorge zur digitalisierten Altenpflege. Eine figurationssoziologische Perspektive auf Pflegeinnovationen«, in: Stefanie Ernst/Guido Becke (Hg.), Transformation der Arbeitsgesellschaft. Prozess- und figurationstheoretische Beiträge, Wiesbaden: Springer, S. 235-258.

Hergesell, Jannis/Maibaum, Arne (2018): »Interests and Side Effects in the Technicization of Geriatric Care«, in: Robert Weidner/Athanasios Karafilidis (Hg.), Developing Support Technologies - Integrating Multiple Perspectives to Create Assistance that People Really Want, Wiesbaden: VS Verlag für Sozialwissenschaften, S. 163-168.

Hergesell, Jannis/Maibaum, Arne/Meister, Martin (2020): »Forschungsfeld Pflegerobotik«, in: Jannis Hergesell/Arne Maibaum/Martin Meister (Hg.), Genese und Folgen der Pflegerobotik. Die Konstitution eines interdisziplinären Forschungsfeldes, Weinheim/Basel: Juventa, S. 7-12.

Heumer, Mechthilde/Kühn, Cornelia (2010): Die Entstehung und Entwicklung der Altenpflegeausbildung: Historische Rekonstruktion des Zeitraums 1950 bis 1994 in Nordrhein-Westfalen, Hamburg: Diplomica.

Hülsken-Giesler, Manfred (2015): »Neue Technologien in der Pflege. Wo stehen wir - was ist zu erwarten?«, in: Bundesanstalt für Arbeitsschutz und Arbeitsmedizin (BAuA) (Hg.), Intelligente Technik in der beruflichen Pflege, Dortmund/Berlin: BAuA, S. 10-13. 
Hülsken-Giesler, Manfred/Krings, Bettina-Johanna (2015): »Technik und Pflege in einer Gesellschaft des langen Lebens«, in: TATuP 4(2), S. 4-11. DOI: 10.14512/tatup.24.2.4.

Hutter, Michael/Knoblauch, Hubert/Rammert, Werner/Windeler, Arnold (2016): »Innovationsgesellschaft heute. Die reflexive Herstellung des Neuen«, in: Werner Rammert/Arnold Windeler/Hubert Knoblauch/Michael Hutter (Hg.), Innovationsgesellschaft heute. Perspektiven, Felder und Fälle, Wiesbaden: VS Verlag für Sozialwissenschaften, S. 15-35.

Irmak, Kenan (2002): Der Sieche. Alte Menschen und die stationäre Altenhilfe in Deutschland 1924-1961, Essen: Klartext Verlag.

Kaldewey, David (2013): Wahrheit und Nützlichkeit. Selbstbeschreibungen der Wissenschaft zwischen Autonomie und gesellschaftlicher Relevanz, Bielefeld: transcript.

Katz, Stephen (1992): "Alarmist demography. Power, knowledge, and the elderly population«, in: Journal of Aging Studies 6(3), S. 203-225. DOI: 10.1016/08904065(92)90001-M.

Kelle, Udo (2008): »Alter \& Altern«, in: Nina Baur/Hermann Korte/Martina Löw/ Markus Schroer (Hg.), Handbuch der Soziologie, Wiesbaden: Springer, S. 1131.

Kensing, Finn/Greenbaum, Joan (2013): »Heritage: having a say«, in: Jesper Simonsen/Toni Robertson (Hg.), Routledge International Handbook of Participatory Design, London/New York: Routledge, S. 21-36.

Kohlbacher, Florian/Hang, Chang Chieh (2010): »Leveraging Disruptive Innovations for the Silver Market«, in: Florian Kohlbacher/Cornelius Herstatt (Hg.), The Silver Market Phenomenon, 2nd ed., Dordrecht: Springer, S. 65-77.

Kondratowitz, Hans-Joachim von (1988): »Allen zur Last, niemandem zur Freude. Die institutionelle Prägung des Alterserlebens als historischer Prozeß «, in: Gerd Gockenjan/Hans-Joachim von Kondratowitz (Hg.), Alter und Alltag, Frankfurt a.M.: Suhrkamp, S. 100-136.

Kondratowitz, Hans-Joachim von (1990): »Geschichte der Altenpflege«, in: Helmut Wallrafen-Dreisow (Hg.), Ich bin Altenpflegerin. Berichte aus der Praxis, Hannover: Vincentz, S. 63-76.

Koppenburger, Anne/Wüller, Hanna (2020): „Über technologisch bedingte Erschütterungen pflegewissenschaftlicher Grundannahmen«, in: Jannis Hergesell/Arne Maibaum/Martin Meister (Hg.), Genese und Folgen der Pflegerobotik, Weinheim: Beltz Juventa, S. 158-179.

Krings, Bettina-Johanna/Weinberger, Nora (2017): »Kann es technische Assistenten in der Pflege geben? Überlegungen zum Begriff der Assistenz in Pflegekontexten«, in: Peter Biniok/Eric Lettkemann (Hg.), Assistive Gesellschaft. Multidisziplinäre Erkundungen zur Sozialform »Assistenz« (Öffentliche Wissenschaft und gesellschaftlicher Wandel), Wiesbaden: Springer, S. 183-202. 
Kucharski, Alexander/Merkel, Sebastian (2018): »Partizipative Technikentwicklung von Gerontotechnologie. Ansätze für mehr Akzeptanz in der Zielgruppe«, in: IAT Forschung Aktuell 06/2018. http://hdl.handle.net/10419/179903.

Künemund, Harald (2015). »Chancen und Herausforderungen assistiver Technik. Nutzerbedarfe und Technikakzeptanz im Alter«, in: TATuP 24(2), S. 28-35. DOI: 10.14512/tatup.24.2.28.

Lipp, Benjamin (2019): Interfacing RobotCare. On the Techno-Politics of Innovation. Dissertation, Technische Universität München, Munich Center for Technology in Society. https://mediatum.ub.tum.de/doc/1472757/1472757.pdf.

Lipp, Benjamin (2020): »Genealogie der RoboterPflege. Zur politischen Rationalität des europäischen Innovationsdispositivs«, in: Jannis Hergesell/Arne Maibaum/Martin Meister (Hg.), Genese und Folgen der Pflegerobotik, Weinheim: Beltz Juventa, S. 18-45.

Macq, Hadrien/Tancoigne, Élise/Strasser, Bruno J. (2020): »From Deliberation to Production: Public Participation in Science and Technology Policies of the European Commission (1998-2019)«, in: Minerva 13(3), S. 1-24. DOI: 10.1007/s11024020-09405-6.

Maibaum, Arne/Hergesell, Jannis (2020): „2030 - Der demografische Wandel als neue soziotechnische Deadline«, in: Paulina Dobroć/Andie Rothenhäusler (Hg.), 2000 Revisited. Visionen der Welt von morgen im Gestern und Heute, Karlsruhe: KIT Scientific Publishing, S. 189-204.

Manzeschke, Arne/Weber, Karsten/Rother, Elisabeth/Fangerau, Heiner (2013): Ethische Fragen im Bereich Altersgerechter Assistenzsysteme, Berlin: VDI/ VDE.

Mol, Annemarie (2008): The logic of care. Health and the problem of patient choice, London/New York: Routledge.

Neven, Louis (2011): Representations of the old and ageing in the design of the new and emerging. Assessing the design of ambient intelligence technologies for older people, Enschede: University of Twente.

Neven, Louis (2015): »By any means? Questioning the link between gerontechnological innovation and older people's wish to live at home«, in: Technological Forecasting and Social Change 93, S. 32-43. DOI: 10.1016/j.techfore.2014.04.016. Nierling, Linda/Domínguez-Rué, Emma (2016): "All that Glitters is not Silver-Technology for the Elderly in Context. Introduction«, in: Emma Domínguez-Rué/Linda Nierling (Hg.), Ageing and Technology. Perspectives from the Social Sciences, Bielefeld: transcript, S. 9-23.

Oudshoorn, Nelly (2011): Telecare technologies and the transformation of healthcare, Basingstoke: Palgrave Macmillan.

Pfotenhauer, Sebastian/Jasanoff, Sheila (2017): »Panacea or diagnosis? Imaginaries of innovation and the >MIT model in three political cultures «, in: Social Studies of Science 47(6), S. 783-810. DOI: 10.1177/0306312717706110. 
Pols, Jeannette (2012): Care at a Distance. On the Closeness of Technology, Amsterdam: Amsterdam University Press.

Rammert, Werner (2006): »Die technische Konstruktion der Wirklichkeit als Teil der gesellschaftlichen Konstruktion der Wirklichkeit«, in: Dirk Tanzler/Hubert Knoblauch/Hans-Georg Soeffner (Hg.), Zur Kritik der Wissensgesellschaft, Konstanz: UVK, S. 83-100.

Rammert, Werner (2016): Technik - Handeln - Wissen. Zu einer pragmatischen Technik und Sozialtheorie, Wiesbaden: Springer.

Rammert, Werner/Windeler, Arnold/Knoblauch, Hubert/Hutter, Michael (2016): »Die Ausweitung der Innovationszone«, in: Werner Rammert/Arnold Windeler/Hubert Knoblauch/Michael Hutter (Hg.), Innovationsgesellschaft heute. Perspektiven, Felder und Fälle, Wiesbaden: VS Verlag für Sozialwissenschaften, S. 3-13.

Reijers, Wessel/Wright, David/Brey, Philip/Weber, Karsten/Rodrigues, Rowena/ O'Sullivan, Declan/Gordijn, Bert (2018): »Methods for Practising Ethics in Research and Innovation: A Literature Review, Critical Analysis and Recommendations", in: Science and Engineering Ethics 24(5), S. 1437-1481. DOI: 10.1007/s11948-017-9961-8.

Remmers, Hartmut (2015): »Natürlichkeit und Künstlichkeit. Zur Analyse und Bewertung von Technik in der Pflege des Menschen«, in: TATuP Praxis 24(2), S. 1120. DOI: 10.14512/tatup.24.2.11.

Remmers, Hartmut/Hülsken-Giesler, Manfred (2007): »Zur Technisierung professioneller Pflege - Entwicklungsstand, Herausforderungen, ethische Schlussfolgerungen«, in: Dominik Gros/Eva-Marie Jakobs (Hg.), E-Health und technisierte Medizin. Neue Herausforderungen im Gesundheitswesen, Berlin: LIT, S. 193-212.

Robinson, Hayley/MacDonald, Bruce/Broadbent, Elizabeth (2014): »The Role of Healthcare Robots for Older People at Home. A Review«, in: International Journal of Social Robotics 6(4), S. 575-591. DOI: 10.1007/s12369-014-0242-2.

Rothgang, Heinz/Müller, Rolf/Unger, Rainer (2012): Themenreport »Pflege 2030«. Was ist zu erwarten - was ist zu tun? Gütersloh: Bertelsmann Stiftung.

Schmidt-Ruhland, Karin/Knigge, Matthias (2010): "Integration of the Elderly into the Design Process«, in: Florian Kohlbacher/Cornelius Herstatt (Hg.), The Silver Market Phenomenon, Dordrecht: Springer, S. 45-64.

Sontheimer, Rainer (2016). User Experience und User Experience Design - Eine Übersicht zum aktuellen Stand der User Experience Research. Arbeitspapier, München: Universität der Bundeswehr.

Unger, Hella von (2012). "Partizipative Gesundheitsforschung: Wer partizipiert woran?«, in: Forum Qualitative Sozialforschung/Forum: Qualitative Social Research 13(1), Art. 7. https://nbn-resolving.org/urn:nbn:de:o114-fqs120176. 
Weber, Karsten (2016): »MEESTAR ${ }^{2}$ - Ein erweitertes Modell zur ethischen Evaluierung soziotechnischer Arrangements«, in: Robert Weidner (Hg.), Technische Unterstützungssysteme, die die Menschen wirklich wollen, Proceedingsband, Helmut-Schmidt-Universität Hamburg, S. 317-326.

Weber, Max (2002 [1904]): »Die »Objektivität« sozialwissenschaftlicher und sozialpolitischer Erkenntnis«, in: Dirk Kaesler (Hg.), Weber. Schriften 1894-1922. Ausgewählt von Dirk Kaesler, Stuttgart: Kroner, S. 77-149.

Weinberger, Nora/Decker, Michael (2015): »Technische Unterstützung für Menschen mit Demenz? Zur Notwendigkeit einer bedarfsorientierten Technikentwicklung«, in: TATuP 24(2), S. 36-45. DOI: 10.14512/tatup.24.2.36.

Weiß, Christine/Lutze, Maxie/Compagna, Diego (2013): Abschlussbericht zur Studie: Unterstützung Pflegebedürftiger durch technische Assistenzsysteme, Berlin: VDI/VDE/IEGUS. 


\section{Anforderungen an die Evaluation von altersgerechten Assistenztechnologien aus gesundheitsökonomischer Sicht}

Mareike Mähs

\section{Einleitung}

Mit dem Einsatz von altersgerechten Assistenztechnologien sind unterschiedliche Potenziale und Nutzen, jedoch auch Aufwendungen, Kosten und Risiken verbunden. So könnten mithilfe von altersgerechten Assistenztechnologien ältere Menschen unterstützt werden, ein selbstständiges Leben im gewohnten häuslichen Umfeld trotz Beeinträchtigungen oder Erkrankungen zu führen (Broek et al. 2009: 22-25). Auch könnten die Ressourcen der gesundheitlichen sowie pflegerischen Versorgung mithilfe altersgerechter Assistenztechnologien effizienter eingesetzt und die Qualität der Versorgung potenziell verbessert werden (Bendig et al. 2017: 17; Grünendahl/Leonhardt/Teich 2017: 56; Ploch/Werkmeister 2017: 33). Dementsprechend könnten mithilfe altersgerechter Technologien ältere Menschen bei der Durchführung von Alltagsaufgaben unterstützt und auf ihre Sicherheit geachtet werden. Der Aufwand für Pflegende oder Betreuende könnte somit verringert und eine Einweisung in eine Pflegeeinrichtung verzögert oder sogar verhindert werden (Grünendahl/Leonhardt/Teich 2017: 56; Heinze 2018: 17-18; Remmers 2019: 424). Ferner wird diesen Technologien ein hohes Umsatzpotenzial nicht nur im Gesundheits- und Pflegesektor, sondern auch in der Wohnungswirtschaft und im »Lifestyle«-Bereich zugeschrieben (Fachinger 2018: 51).

Ist der Einsatz altersgerechter Assistenztechnologien geplant, sind jedoch nicht nur die eingangs beispielhaft aufgeführten Nutzen, sondern auch die mit dem Einsatz verbundenen Kosten zu berücksichtigen, da die Verwendung assistierender Technologien prinzipiell von Kosten-Nutzen-Relationen abhängig ist. So ist $\mathrm{zu}$ bedenken, dass die Informationsbeschaffung vor der Anschaffung, die Anschaffung selbst und die Installation der Technologie mit Kosten verbunden ist (Hauer 2017: 322). Diese könnten von Sozialversicherungsträger*innen ganz oder teilweise erstattet werden und damit Ausgaben für diese darstellen, während gegebenenfalls ein Teil der Kosten (zum Beispiel als Mietgebühr oder Leasingrate) 
von den Nutzer*innen selbst zu tragen wäre (Moser-Siegmeth/Hofer 2013: 61). Außerdem könnten neben Anschaffungskosten organisatorische Kosten für die Implementation der Technologie in routinierte, regulierte Abläufe anfallen oder eine Anpassung notwendig werden, zum Beispiel wenn eine entsprechende Infrastruktur für die Reaktion auf Notfälle eingerichtet werden muss, die durch die Technologie erkannt wurden (Leppert/Greiner 2016: 120).

Zudem fallen auch Instandhaltungskosten für die Wartung sowie anfallende Reparaturen an (Melkas 2013: 87; Meyer 2018: 169). Bezüglich Technologien, die motorisiert sind, eine Internetverbindung benötigen oder Daten erfassen, sind ferner Betriebskosten für die technische Infrastruktur, den Strom, die Internetverbindung sowie eventuell Servicestellen, die Daten auswerten, technischen Support anbieten oder Notrufe entgegennehmen, einzubeziehen (Fioranelli 2019: 8). Zudem sind Zeitkosten und monetäre Kosten für das Anlernen und Training der Nutzer*innen sowie in vielen Fällen auch der Pflege- und Betreuungskräfte beziehungsweise Angehörigen bezogen auf die Nutzung der Technologie zu berücksichtigen (Fioranelli 2019: 8; Hauer 2017: 322; Meyer 2018: 168).

Ferner könnten Risiken mit der Anwendung einer altersgerechten Technologie verbunden sein: Deren Nutzung könnte die Behinderung der Nutzerin*innen hervorheben und mit einer Stigmatisierung dieser einhergehen (Bertrand et al. 2017: 897). Die Erhebung von Daten, zum Beispiel die Überwachung von Vitalparametern, Bewegungsverhalten oder Alltagsaktivitäten, könnte einen Eingriff in die Privatsphäre der Nutzer*innen darstellen und Unbehagen bei diesen hervorrufen (Chung/Demiris/Thompson 2016: 168; vgl. Frommeld in diesem Band). Sind diese persönlichen Daten nicht ausreichend geschützt, könnte die Sicherheit der Daten gefährdet sein, könnten die Daten für andere Zwecke missbraucht und möglicherweise dadurch den Nutzer*innen Schaden zugefügt werden (Leppert/Greiner 2016: 120).

Die Nutzung altersgerechter Assistenztechnologien könnte außerdem die Unterstützung und Anwesenheit von formellen sowie informellen Pflege- und Betreuungspersonen verringern und damit unter Umständen zur sozialen Isolation der Nutzer*innen beitragen (Chung/Demiris/Thompson 2016: 175; Remmers 2019: 424). Zudem könnte die Technologie gegen den Willen der Nutzer*innen handeln und dadurch deren Autonomie einschränken (Sharkey/Sharkey 2012: 37). Durch den Einsatz der Technologie könnten bestehende Fähigkeiten abnehmen, wenn die Technologie Aktivitäten übernimmt, die die Nutzer*innen noch selbstständig hätten übernehmen können (Elsbernd/Lehmeyer/Schilling 2015: 723).

Inwiefern sich die vorgestellten Potenziale, Risiken und Aufwendungen realisieren, ist bislang jedoch weitestgehend unbekannt (Grootven/Achterberg 2016: 36-37; Meyer 2018: 172; Remmers 2019: 416). Bei vielen Technologien, wie beispielsweise intelligenten Rollatoren, ist bislang nicht erforscht, inwiefern diese mögliche Gefahren bereits bestehender Technologien, wie zum Beispiel das Sturzrisiko tra- 
ditioneller Rollatoren, verringern oder aber sogar verstärken könnten (Werner et al. 2018: 8). Hinzu kommt, dass zwar ein Großteil der Technologien als Prototypen in Forschungsprojekten entwickelt wurde, jedoch nur wenige dieser Geräte bislang zugänglich sind (Zöllick et al. 2020: 216) beziehungsweise vermarktet werden (Hilbert et al. 2018: 41-42; Grootven/Achterberg 2016: 36). Ein möglicher Grund hierfür könnte sein, dass vielen potenziellen Käufer*innen, aber auch den Pflegenden sowie Betreuenden und Händler*innen, die diese Technologien vertreiben, das Wissen über die Verfügbarkeit dieser Technologien sowie deren Qualität und Nutzen fehlt (Bozan/Berger 2019: 4307; Fachinger 2017: 4-5; vgl. Frommeld in diesem Band). Meyer (2018: 165) gibt dementsprechend zu bedenken: »Eine Nachfrage von Kunden ist erst zu erwarten, wenn sie wissen, >was man wollen kann ‘ >welchen Nutzen man davon erwarten kann und 'wie sich die Systeme in der Praxis bewähren««. Wissen über Nutzen und Kosten derartiger Technologien wird gegebenenfalls außerdem benötigt, wenn diese Kosten von Dritten finanziert oder erstattet werden sollen (Weiß et al. 2017: 28). Sowohl für Leistungen der gesetzlichen Krankenversicherung (GKV) als auch der sozialen Pflegeversicherung gilt das Wirtschaftlichkeitsgebot, nach dem die zu erstattenden Leistungen wirksam und wirtschaftlich sein sollen ( $\$ 29$ Abs. I SGB XI; \$ 12 Abs. I SGB V).

Um die Informationsbasis über diese Technologien zu erhöhen, wird Wissen über die Effektivität, den Nutzen, mögliche Nebenwirkungen und die Kosten dieser Technologien benötigt (Grootven/Achterberg 2016: 37; Meyer 2018: 174; Weiß et al. 2017: 95). Die Feststellung der Effektivität und Wirtschaftlichkeit dieser Technologien kann mithilfe der Ergebnisse gesundheitsökonomischer Evaluationen unterstützt werden (Schöffski 2012a: 9-10). Abgesehen von der Evaluation einiger E-Health- und Telemedizin-Produkte ${ }^{1}$ fehlen gerade für altersgerechte Technologien qualitativ hochwertige gesundheitsökonomische Evaluationsstudien (Albrecht et al. 2018: 341-342; Al-Shaqi/Mourshed/Rezgui 2016: 17; Calvaresi et al. 2016: 17).

\section{Einführung in gesundheitsökonomische Evaluationen}

Mithilfe gesundheitsökonomischer Evaluationen beziehungsweise Kosten-Nutzen-Abwägungen kann die Wirtschaftlichkeit von Technologien abgeschätzt und Entscheidungsträger*innen eine Hilfestellung für eine informierte, wissenschaftlich fundierte Entscheidung gegeben werden, ob zum Beispiel eine neue Leistung Einzug in die Regelversorgung erhalten soll (Schulenburg et al. 2012: 525). Bei 
diesen Evaluationen wird der Versuch unternommen, alle relevanten Kosten und Nutzen von zwei oder mehreren Alternativen gegeneinander abzuwägen (Schöffski 2012a: 6). Dabei handelt es sich oft um den Vergleich einer neuen mit einer standardmäßig eingesetzten Maßnahme (Drummond et al. 2015: 23-24; Schulenburg et al. 2012: 529). Die Durchführung keiner Maßnahme kann auch als Alternative herangezogen werden, wenn keine etablierte Maßnahme vorhanden ist (Drummond et al. 2015: 23-24; Schulenburg et al. 2012: 529).

Eine Schwierigkeit bezüglich der Durchführung gesundheitsökonomischer Analysen besteht grundsätzlich darin, die Kosten und Nutzen angemessen abzuschätzen und zu vergleichen. Eine Monetarisierung aller Faktoren ermöglicht dabei ein Rechnen in gleichen Einheiten. Die Bewertung aller Kosten und Nutzen in Geldeinheiten ist jedoch anspruchsvoll, da nicht-monetäre und indirekte Kosten und Nutzen oft schwierig zu quantifizieren und in Geldeinheiten auszudrücken sind. Zudem wird die monetäre Bewertung von Gesundheit und Leben in der Wissenschaft kontrovers diskutiert (Breyer/Zweifel/Kifmann 2013: 20; Schöffski 2012b: 59).

\section{Die Perspektive der gesundheitsökonomischen Evaluation}

Die Erfassung der Kosten und Nutzen ist abhängig von der gewählten Perspektive: Hinsichtlich der gesamtgesellschaftlichen (volkswirtschaftlichen) Perspektive werden alle Kosten und Nutzen erfasst, unabhängig davon, bei welchen Akteur*innen sie anfallen und wer von ihnen profitiert (Greiner/Schöffski 2012: 156; IQWiG 2017: 95). Die Kostenträger*innen- beziehungsweise Sozialversicherungsperspektive ist in gesundheitsökonomischen Evaluationen die Perspektive der Organisationen, die die Finanzierung einer Maßnahme übernehmen und die Kosten dieser erstatten. Zum Beispiel werden in der GKV-Perspektive ausschließlich die Ausgaben und Nutzen betrachtet, die für die Krankenkassen anfallen (Greiner/Schöffski 2012: 157; IQWiG 2017: 94). In der Leistungsträger*innenperspektive wird die Sicht der Gesundheits- und Pflegeprofessionen, die gesundheitliche beziehungsweise pflegerische Leistungen erbringen, eingenommen (Greiner/Schöffski 2012: 158). Eine weitere Perspektive ist die der Betroffenen oder auch ihrer Angehörigen. Relevante Größen stellen hier die Lebensqualität, die Zufriedenheit mit der Maßnahme sowie mögliche $\mathrm{Zu}$ - oder Selbstzahlungen, beispielsweise für häusliche Umbaumaßnahmen, dar, deren Kosten nicht oder teilweise von den Sozialversicherungsträger*innen erstattet werden (Hauer 2017:322; Prenzler/Reddemann/Schulenburg 2010: 30). 


\section{Erfassung der Kosten und Nutzen}

In gesundheitsökonomischen Evaluationen werden nicht nur direkte sowie tangible, sondern, je nach Perspektive, auch die indirekten und intangiblen Kosten und Nutzen einbezogen (Greiner/Damm 2012: 24-25): Bei den direkten Kosten beziehungsweise Nutzen besteht ein direkter Zusammenhang zwischen der betrachteten Maßnahme und den angefallenen Kosten oder Nutzen, während bei den indirekten dementsprechend ein indirekter Zusammenhang zwischen Effekten von außerhalb und der Maßnahme besteht. Ferner werden die direkten und indirekten Kosten und Nutzen in tangibel, das heißt in Geldeinheiten ausdrückbar, und intangibel, nicht oder nur eingeschränkt in Geldeinheiten ausdrückbar, unterschieden.

Für die Erfassung relevanter Nutzen und Kosten sind diese in einem ersten Schritt zu bestimmen. Diese Bestimmung orientiert sich an der eingenommenen Perspektive und ist abhängig von der Art der Kosten-Nutzen-Analyse (Drummond et al. 2015: 46). Bei den Kosten können Ressourcenverbräuche und Produktivitätsverluste in die Evaluation einbezogen werden (ebd.: 219; IQWiG 2017: 95-96). Dazu gehört auch der Ressourcenverbrauch, der sich nicht oder nur begrenzt in Marktpreisen abbilden lässt, wie unentgeltlich erbrachte Leistungen sowie Ressourcenbereitstellungen und die aufgewendete Freizeit der Betroffenen (Drummond et al. 2015: 222).

Zur Messung des Nutzens können vielfältige Zielgrößen verwendet werden (ebd.: 124-133). So werden in klinischen Studien oft intermediäre beziehungsweise Surrogatparameter ${ }^{2}$ eingesetzt, die Veränderungen im Krankheitszustand beziehungsweise hinsichtlich körperlicher Beeinträchtigungen anzeigen, die wiederum einen Hinweis auf die Gesundheit geben können (ebd.: 27-28). Diese Herangehensweise lässt zwar in der Regel einen Vergleich zweier Maßnahmen zu, allerdings kann auf diese Weise nicht die Stärke der Verbesserung analysiert werden, da diese nicht unbedingt mit der Stärke der Verbesserung im Gesundheitszustand korreliert (ebd.: 28). Ferner ist zu beachten, dass eine objektive Messung von Vitalparametern wie zum Beispiel des Blutzuckers es zwar zulässt, Veränderungen aus medizinischer oder therapeutischer Sicht zu erfassen, diese jedoch nicht mit einer Veränderung im subjektiven Wohlbefinden oder dem subjektiven Gesundheitsstatus des Betroffenen zusammenhängen muss (Brazier et al. 2017: 14).

Um das subjektive Erleben von Krankheit, Gesundheit und deren Auswirkungen auf das generelle Wohlbefinden $\mathrm{zu}$ erfassen, werden sogenannte Patient-Reported Outcomes (PRO) durch die direkte Befragung des Individuums erhoben (Bullinger et al. 2015: 284; Koller et al. 2009: 867). Die Messung von PRO

2 Unter Surrogatparameter werden Größen wie z.B. Blutdruck- oder Blutzuckerwerte verstanden, die relativ einfach zu messen sind und deshalb als Ersatz für die eigentlich zu messende Zielgröße erhoben werden (Drummond et al. 2015: 27-28). 
kann Auskunft über die Effekte einer Maßnahme aus Sicht der Betroffenen geben beziehungsweise darüber, wie diese die Effekte wahrnehmen und welche Einstellung sie gegenüber der Maßnahme haben (Wiklund 2004: 352). Die Zufriedenheit mit der Maßnahme kann wiederum einen Einfluss auf die Adhärenz und somit auch auf das gesundheitliche Ergebnis sowie auf die erfolgreiche Nutzung einer Technologie haben (ebd.: 353).

Als PRO erhoben werden unter anderem die Lebensqualität sowie die Zufriedenheit und Präferenzen der Betroffenen (Koller et al. 2009: 867-868; Wiklund 2004: 352). Neben den quantitativen gibt es auch qualitative Verfahren zur Erfassung von PRO, wie zum Beispiel Fokusgruppen und verschiedene Interviewarten, die bislang jedoch selten Anwendung in der Lebensqualitätsforschung und noch seltener in gesundheitsökonomischen Analysen finden (Wiklund, 2004: 353). Eines dieser generischen PRO-Messinstrumente ist die Abschätzung der Lebensqualität, wobei bei Gesundheitsinterventionen oft die gesundheitsbezogene Lebensqualität (Health-Related Quality of Life - HR-QoL) fokussiert wird. Die HR-QoL ist ein multidimensionales Konzept mit den Komponenten mögliche Beeinträchtigungen, Wohlbefinden und soziales Umfeld, die wiederum von dem Gesundheitszustand beeinflusst werden (Schöffski, 2012c: 329; Testa/Simonson 1996: 835).

$\mathrm{Zu}$ bedenken ist, dass die Identifizierung der Kosten und Nutzen bis zu einem bestimmten Grad unvollständig bleibt und es nicht möglich sein wird, alle Aspekte und Effekte einer Maßnahme zu betrachten (Drummond et al. 2015: 30). Auch wenn bestimmte Kosten- und Nutzenkomponenten nicht in die Berechnungen der Kosten-Nutzen-Verhältnisse miteinfließen, weil diese beispielsweise für die bestimmte Perspektive nur eingeschränkt relevant sein oder einen vernachlässigbaren Einfluss haben dürften, können diese bestimmt und genannt werden (ebd.: 46, 220).

Als nächster Schritt erfolgt die Quantifizierung der Kosten und Nutzen beziehungsweise die Messung der Ressourcenverbräuche und Zielgrößen (Greiner/Damm 2012: 28). Für die Berechnung der Ressourcenverbräuche wird ein Mengengerüst erstellt (ebd.). Bezüglich der Mengenerfassung und späteren Bewertung der Kosten wird zwischen dem macro-costing Ansatz, bei dem aggregierte Leistungen beziehungsweise Leistungsbündel analysiert werden, und dem microcosting Ansatz, bei dem detailliert jede Einzelleistung für sich gemessen und (hinsichtlich ihrer Stückkosten) bewertet wird, unterschieden. Beide Ansätze können auch zusammen angewendet werden (Greiner/Damm 2012: 28; Drummond et al. 2015: 240).

Im zweiten Schritt ist ebenso zu bestimmen, wie die Aspekte von Gesundheit gemessen werden können. Da Gesundheit ein multidimensionales Phänomen darstellt (Brazier et al. 2017: 14), liefert der Einbezug ausschließlich eines Indikators oft keine validen Ergebnisse (Gäfgen 1990: 307). Um unterschiedliche Dimensionen von Gesundheit zu betrachten, wurden mehrdimensionale Messinstrumente 
entwickelt. Zu beachten ist hierbei, dass jedes Kriterium unabhängig von den anderen Kriterien sein sollte, das heißt Korrelationen zwischen den einzelnen Items gering sein sollten, um Redundanzen und somit Doppelzählungen zu vermeiden sowie eine eindeutige Zuordnung der Antworten zu den Items zu gewährleisten (Drummond et al. 2015: 93).

Zuletzt erfolgt die monetäre Bewertung der Kosten und Nutzen, sofern eine monetäre Bewertung abhängig von der Wahl der Art der Analyse nötig ist (Greiner/Damm 2012: 28; Schöffski 2012d: 384-385). Um vergleichende Kosten-NutzenAbwägungen durchzuführen, müssen die Nutzen und die Kosten in einer einheitlichen Maßzahl angegeben werden. Die tangiblen Kosten sowie Nutzen (wie zum Beispiel Kosteneinsparungen) können in Geldeinheiten bemessen werden. Bezüglich der tangiblen Kosten und Nutzen bedeutet dies, dass diesen Stückkosten beziehungsweise Preise zugeordnet werden (Drummond et al. 2015: 222). Die Preise sollen laut Greiner/Schöffski (2012: 160) den Opportunitätskosten entsprechen, die wiederum den Nutzen der alternativen Maßnahme darstellen, auf den verzichtet würde, wenn die zu betrachtende Maßnahme ausgeführt wird. Da für die Berechnung von Opportunitätskosten im Gesundheitswesen in der Regel keine Marktpreise vorliegen, beziehen sich Berechnungen meistens auf die verfügbaren (Verhandlungs-)Preise, die zum Beispiel Gebührenordnungen oder Standardkostenlisten entstammen (ebd.; Drummond et al. 2015: 222-223). Eine monetäre Bewertung der intangiblen Kosten und Nutzen wird zum Beispiel mithilfe der Ermittlung der individuellen Zahlungsbereitschaft versucht vorzunehmen (Breyer/Zweifel/Kifmann 2013: 49-51; Greiner/Damm 2012: S. 36-37; Schöffski 2012d: 374-375).

Wendet man die dargestellten Prinzipien und Methodik der gesundheitsökonomischen Evaluation auf altersgerechte Assistenztechnologien an, ist eine Reihe von Anforderungen bezüglich der Durchführung von Evaluationsstudien zu beachten, die im folgenden Abschnitt vorgestellt werden.

\section{Anforderungen an die Evaluation altersgerechter Assistenztechnologien}

Laut Glasgow (2007: S123) sollen die Studienergebnisse je nach gewählter Perspektive für die darin betrachtete Bevölkerung repräsentativ, generalisierbar - unter anderem hinsichtlich der betrachteten Endnutzer*innen sowie des Anwendungssettings - und schließlich wiederholbar sein, wobei die Studie auch bei Wiederholung in anderen Kontexten zu vergleichbaren Ergebnissen führen sollte. Es wird empfohlen, dass die Evaluation von unabhängigen Stellen durchgeführt wird; andernfalls könnten Interessenskonflikte bestehen, wenn zum Beispiel die Hersteller*innen selbst die Studie durchführen oder diese bezahlen (Gruber 2011: 201). Außerdem wird gefordert, dass die Ergebnisse präzise, verständlich und zeitnah gegenüber den Entscheidungsträger*innen kommuniziert werden, um eine ange- 
messene und zeiteffiziente Unterstützung anzubieten (Glasgow 2007: S122; Nielsen/Sarriá Santamera/Vondeling 2008: 25-26). Die Einhaltung dieser generellen Anforderungen bezüglich der Evaluation komplexer altersgerechter Assistenztechnologien ist jedoch herausfordernd und es zeigen sich weitere Anforderungen, die aus den spezifischen Charakteristika der Technologien resultieren.

\section{Evaluation von komplexen Technologien}

Altersgerechte Assistenztechnologien können als komplexe Technologie aufgefasst werden. Nach Shiell/Hawe/Gold (2008: 1282) können komplexe Technologien durch die folgenden Eigenschaften charakterisiert werden: Die Technologie agiert in Bezug auf die individuellen Bedarfe und das Verhalten der Nutzer"innen und es ist schwierig, lineare sowie präzise Ursache-Wirkungs-Zusammenhänge zwischen der Technologie und ihren Effekten auf die Nutzer*innen aufgrund von externen (Umwelt-)Einflüssen und unterschiedlichen Komponenten der Technologie zu bestimmen. Besonders bei Technologien, die Auswirkungen auf bestehende Systeme haben, wird die gesundheitsökonomische Evaluation und allen voran die Abschätzung zahlreicher Effekte erschwert. Dazu zählen unter anderem die Selbstorganisation und Veränderungen des Systems, die Anzahl, Perspektiven sowie das Verhalten der im System agierenden Akteur"innen und mögliche Wechselwirkungen (Lysdahl et al. 2016: 41-42; Shiell/Hawe/Gold 2008: 1282).

Altersgerechte Assistenztechnologien können auf ein bestehendes System einwirken, wenn durch dessen Einsatz routinierte Abläufe im informellen und professionellen Pflege- oder Gesundheitssystem verändert werden, zum Beispiel, wenn der Betreuungsaufwand durch den Einsatz der Technologie reduziert werden kann. Außerdem können diese Technologien mit weiteren Technologien zu einem gesamten Smarthome-System vernetzt sein. Es wäre beispielsweise eine Vernetzung der altersgerechten Assistenztechnologie mit einem Notfallsystem denkbar, das auf Lokalisierungsdaten zugreifen kann (Krieg-Brückner et al. 2011: 165). Dementsprechend wird angenommen, dass altersgerechte Assistenztechnologien Auswirkungen auf komplexe Systeme haben und es deshalb spezifische Anforderungen an die gesundheitsökonomische Evaluation dieser komplexen Technologien gibt, beispielsweise bei der Wahl eines adäquaten Evaluationsdesigns und der Abschätzung der Effekte unter Einbezug der Anwendungsumgebung (Burchert 1998: 7; Köberlein-Neu 2017: 808). Auf diese spezifischen Anforderungen wird in den folgenden Abschnitten vertieft eingegangen. 


\section{Wahl der Alternative}

Für den Vergleich einer altersgerechten Technologie mit ein oder mehreren Alternativen kann die Standardtechnologie oder auch die Alternative, keine Maßnahme durchzuführen, gewählt werden. Führt man einen Vergleich mit der Alternative >keine Maßnahme durch, könnte die Kontrollgruppe zu heterogen sein, um sie mit der Interventionsgruppe vergleichen zu können. Zum Beispiel könnten Proband*innen in der Kontrollgruppe unabhängig von der Studie alternative Technologien wie Hilfen zur Kompensation einer Beeinträchtigung nutzen und eine Vergleichbarkeit nicht mehr gegeben sein oder die Ergebnisse verzerrt werden (Fuhrer 2007: 154). Deshalb bietet es sich wenn möglich an, als Alternative eine andere, ähnliche Technologie heranzuziehen, die möglicherweise keine innovativen oder intelligenten Funktionen besitzt, jedoch ebenso dieselben Beeinträchtigungen kompensieren kann, um eine Vergleichbarkeit zwischen Interventions- und Kontrollgruppe zu erreichen (Fuhrer 2007: 154; Ohinmaa/Hailey/Roine 2001: 191).

Herausforderungen bei der Interpretation der Ergebnisse der Vergleichsstudie können dann auftreten, wenn die Alternative spezielle Funktionen hat, die schwierig zu generalisieren sind, oder wenn die Alternative viele Funktionen hat und somit der Effekt nicht mehr auf eine bestimmte Funktion zurückzuführen ist beziehungsweise nicht mehr mit dem der Alternative verglichen werden kann (Fuhrer 2007: 154). Weist die Technologie nicht nur vergleichbare, sondern auch zusätzliche Funktionen im Vergleich zur Standardtechnologie auf, kann es möglicherweise sinnvoll sein, mehrere Vergleichsstudien mit jeweils unterschiedlichen Alternativen durchzuführen (Wade et al. 2017: 788).

Hinsichtlich des Einsatzes innovativer Technologien können außerdem Effekte aus dem neuartigen Einsatz der Technologie resultieren, die eine Vergleichbarkeit mit der Standardtechnologie erschweren, so beispielsweise, wenn Lern- und Gewöhnungseffekte auftreten oder die technische Infrastruktur nicht adäquat ist (Taylor 2005: 170). Nicht nur die altersgerechte Technologie selbst, sondern auch die Alternative ist deshalb präzise in einer Evaluationsstudie zu beschreiben und zu charakterisieren (Wade et al. 2017: 788). Dies gilt auch für die Wahl des Zeithorizontes, die im folgenden Abschnitt thematisiert wird.

\section{Zeithorizont der Evaluation}

Bezüglich der Wahl des Zeithorizontes benötigen die Entscheidungsträger*innen einerseits genügend Informationen über die Effekte der Technologie, weshalb Daten systematisch und über einen längeren Zeitraum erhoben werden müssten (Glasgow 2007: S121). Dabei kann die Durchführung von Evaluationsstudien und der Prozess zur Entscheidung einer Erstattungsfähigkeit mehrere Jahre dauern. Andererseits wird empfohlen, dass die Ergebnisse der Evaluation den 
Entscheidungsträger*innen zeitnah vorliegen, um die Entscheidung früh- und rechtzeitig unterstützen zu können. Auch sind die Kosten bei längerer Dauer der Evaluation tendenziell höher (Mühlbacher/Juhnke 2018: S82; The Lewin Group 2000: 36). $\mathrm{Zu}$ beachten ist zudem, dass altersgerechte Assistenztechnologien aufgrund der hohen Geschwindigkeit des technischen Fortschritts kürzere Innovationszyklen aufweisen können (Bartelmes et al. 2009: 17; Manych 2018: 20). Um die Planungssicherheit der Unternehmen zu erhöhen, werden daher zügig durchgeführte Evaluationen mit transparenten Entscheidungskriterien benötigt (Mühlbacher/Juhnke 2018: S82).

Um die Entscheidungsträger*innen zeitnah in ihrer Entscheidung, beispielsweise über die Erstattungsfähigkeit einer Technologie, unterstützen zu können, kann eine Evaluation mit einem kurzen Zeithorizont gewählt werden. Diese erste Evaluation mit kurzem Zeithorizont könnte im Rahmen einer bedingten regulatorischen Entscheidung über die Erstattungsfähigkeit durchgeführt werden und an die Bedingung geknüpft sein, dass weitere Evaluationen zu den langfristigen Effekten der Wirksamkeit und Wirtschaftlichkeit durchgeführt werden (Kirisits/Redekop 2013: 22; Mühlbacher/Juhnke 2018: S84). So bedarf es weiterer Evaluationen, um Lern- und Gewöhnungseffekte sowie die langfristigen Effekte (weiterentwickelter) altersgerechter Assistenztechnologien feststellen zu können und die Abschätzung weiterer sowie langfristiger Nutzen- und auch Kostenkomponenten miteinzubeziehen (Bartelmes et al. 2009: 17; Henschke et al. 2015: 324; Köberlein-Neu 2017: 808-810; Tarricone/Torbica/Drummond 2017: 146). Dementsprechend könnte eine entwicklungsbegleitende Evaluation durchgeführt werden, wie sie im folgenden Abschnitt thematisiert wird.

\section{Entwicklungsbegleitende Evaluation}

Die Evaluation komplexer Technologien kann sich an den Phasen des Produktlebenszyklus der Technologie orientieren, um deren Modifikationen sowie Weiterentwicklungen zu berücksichtigen (Agboola et al. 2014: e75; Campbell et al. 2000: 694; Catwell/Sheikh 2009: 3; Dintsios et al. 2017: 657-658; Perleth/Lühmann 2010: 830). Für die Einteilung der Phasen werden unterschiedliche Konzepte vorgeschlagen, die an die Evaluation von Medizinprodukten, Telehealth sowie Arzneimitteln und an die Abläufe der Technikentwicklung angelehnt sind (Campbell et al. 2000: 694-696; Fatehi et al. 2016: 760-762; Mühlbacher/Juhnke 2018: S82-S83). Der Prozess sowie die Entwicklung der Technologie sind abzubrechen, wenn sich schwerwiegende negative Effekte bezüglich der Sicherheit, Wirksamkeit und/oder Wirtschaftlichkeit zeigen (Bartelmes et al. 2009: 23). Diese Vorgehensweise kann potenziell die Unsicherheit über (langfristige) Effekte komplexer Technologien, die sich im Produktlebenszyklus verändern, verringern, die Transparenz der Erstattungs- 
entscheidung erhöhen und gegebenenfalls den Prozess der Entscheidungsfindung beschleunigen (Mühlbacher/Juhnke 2018: S84-S86; Perleth/Lühmann 2010: 829).

Hinsichtlich der Ziele und eingenommenen Perspektiven in der gesundheitsökonomischen Evaluation muss das Evaluationsdesign unter Umständen angepasst oder weiterentwickelt werden. Im Rahmen dieser entwicklungsbegleitenden Evaluation können Wirkungsstudien durchgeführt und diesbezüglich Wirkungsmodelle erstellt werden, die im nächsten Abschnitt betrachtet werden.

\section{Erstellung von konzeptionellen Wirkungsmodellen}

Um die Ursache-Wirkungsbeziehungen komplexer Technologien sowie die Wirkung der Technologie auf komplexe Systeme im Rahmen der Evaluation aufzudecken, können konzeptionelle Wirkungsmodelle erstellt werden (Blettner et al. 2018: 44-45). Wirkungsmodelle bieten den Vorteil, eine grafische Übersicht über die Wirkungsweise der Technologie, ihrer Komponenten und Kontextfaktoren zu geben, damit die Auswirkungen der Technologie sowie die daraus resultierenden Zusammenhänge besser verstanden werden können (Köberlein-Neu 2017: 812; Rohwer et al. 2016: 19). Somit können mögliche Zusammenhänge und Wirkungspfade identifiziert werden, die kurz-, mittel- sowie langfristig von der Technologie ausgehen und zu den intendierten Ergebnissen führen (Blettner et al. 2018: 44; Campbell et al. 2007: 457). Dabei werden oftmals lineare Ursache-Wirkungs-Zusammenhänge, sogenannte Wirkungsketten, betrachtet (Blettner et al. 2018: 44; Campbell et al. 2007: 457).

Mithilfe von Wirkungsmodellen können auch komplexe Zusammenhänge bezüglich der Technologie sowie weitere möglicherweise nicht intendierte Einflussfaktoren bedacht und analysiert werden (Campbell et al. 2007: 457). Zudem können im Rahmen der Entwicklung von Wirkungsmodellen schon vor der Evidenzgenerierung die heuristisch angenommenen oder theoriebasierten Wirkzusammenhänge aufgezeigt und dadurch die Evidenzgenerierung unterstützt werden (Rohwer et al. 2016: 15-16; Wahlster et al. 2016: 27). Mithilfe von Evidenz, die aus unterschiedlichen Studien resultiert, können die identifizierten Zusammenhänge und Wirkungspfade dann überprüft, verändert und validiert werden (Blettner et al. 2018: 43-44; Campbell et al. 2007: 457-459).

Komplexe Technologien können aus mehreren Komponenten bestehen, sodass im Rahmen derartiger Modelle die Wirkungen der einzelnen Komponenten einer Technologie separat und vertieft abgeschätzt werden können (Campbell et al. 2007: 457). Es kann ebenfalls abgeschätzt werden, wie die Einzelkomponenten insgesamt zur Wirkung der Technologie beitragen (Leppert/Greiner 2016: 120; Puskin et al. 2010: 97). Die genauere Abschätzung der Wirksamkeit beziehungsweise Effektivität ist abhängig von der Wahl des Studiendesigns. Im nächsten Abschnitt werden 
unterschiedliche Studiendesigns, die in einer Evaluationsstudie Anwendung finden können, vorgestellt.

\section{Wahl des Studiendesigns}

Welche Studiendesigns sich für die gesundheitsökonomische Evaluation komplexer Technologien eignen, wird kritisch diskutiert, in dem Sinne, dass für Gesundheitstechnologien eine gleich hohe Evidenz wie für Arzneimittel vorliegen sollte (Catwell/Sheikh 2009: e1000126; Perleth/Lühmann 2010: 827-828). Dementsprechend können, wie für Arzneimittel, zur Evaluation derartiger Technologien randomisiert-kontrollierte Studien (Randomised Controlled Trials - RCT) in strikt kontrollierten Laborumgebungen durchgeführt werden. Neben den Wirksamkeitswerden auch Wirtschaftlichkeitsstudien im Rahmen von RCT durchgeführt und neben dem klinischen Nutzen die Kosten erfasst (Köberlein-Neu 2017: 817).

$\mathrm{Zu}$ beachten ist hier allerdings, dass die Ergebnisse aus dem RCT unter bestimmten Umständen nicht für die gesundheitsökonomische Evaluation nutzbar sind, wenn die Anwendung der Technologie in der Testumgebung von Alltagsbedingungen abweicht und so die Wirksamkeit der Technologie im täglichen Leben nicht erfasst werden kann (Cook/Drummond/Heyse 2004: 161; Greiner/Damm 2012: 37-38). Daten aus internationalen multizentrischen Studien aus unterschiedlichen Gesundheitssystemen können aufgrund der fehlenden Vergleichbarkeit mit der nationalen Bevölkerung und dem nationalen Gesundheitssystem ebenfalls oftmals überhaupt nicht oder ausschließlich eingeschränkt genutzt werden (Cook/Drummond/Heyse 2004: 173-174; Greiner/Damm 2012: 38).

Andere Forscher*innen argumentieren hingegen, dass Beobachtungs- und epidemiologische Studien wie Kohorten-, Fall-Kontroll-, Querschnitts- oder VorherNachher-Studien für gesundheitsökonomische Evaluationen komplexer Technologien und für längere Zeithorizonte eingesetzt werden können. Diese bergen allerdings das Risiko von Verzerrungen bei der Interpretation der Ergebnisse, da in diesen Studien externe Faktoren häufig nicht kontrolliert werden können (Craig et al. 2008: a1655; Lindwedel-Reime et al. 2016: 63-64; Shiell/Hawe/Gold 2008: 1283). Eine weitere Möglichkeit zur Abschätzung der Wirksamkeit und Wirtschaftlichkeit besteht hinsichtlich des Einsatzes mathematischer Modelle, mit denen versucht wird, die kurz-, mittel- und langfristigen Effekte der Technologie zu extrapolieren (Köberlein-Neu 2017: 817; Shiell/Hawe/Gold 2008: 1283; Tarricone/Torbica/Drummond 2017: 147).

Ebenso kann es sinnvoll sein, qualitative Verfahren in die Evaluation miteinzubeziehen, um beispielsweise das Nutzungsverhalten potenzieller Nutzer*innen sowie Kontextfaktoren besser zu verstehen (Agboola et al. 2014: e75; Glasgow 2007: S121). Diese können im späteren Anwendungsgebiet der Technologie, zum Beispiel der Wohnumgebung älterer Menschen, durchgeführt werden, um die Wirkung und 
den Nutzen unter Alltagsbedingungen erfassen zu können (Eftring/Frennert 2016: 280). Ein solches Verfahren ist beispielsweise der aktivitätsbasierte Ansatz, mithilfe dessen in der häuslichen Umgebung der Nutzer*innen oder in realitätsnahen Settings wie Living Labs die Technologie bei der Durchführung von Alltagsaktivitäten der Nutzer*innen getestet werden (Helle/Iwarsson/Brandt 2014: 866). Liegen noch keine anwendungsfähigen Prototypen der Technologie vor, können Fallstudien, Vignetten, Use-Cases, Persona sowie Szenarien im Rahmen explorativer Studien entwickelt und eingesetzt werden, um den möglichen Einsatz der Technologie und Anwendungsszenarien über die Charakteristika, das Leben und mögliche Herausforderungen der Nutzer*innen hinsichtlich des Einsatzes der Technologie aufzuzeigen und $\mathrm{zu}$ analysieren. ${ }^{3}$

Konsens besteht bei vielen Forscher*innen dahingehend, dass ein Methodenmix aus qualitativer und quantitativer Forschung zur Anwendung kommen sollte (Agboola et al. 2014: e75; Blettner et al. 2018: 46; Colomer et al. 2014: 7300). Dieser kann dazu beitragen, dass die komplexen Wirkzusammenhänge der Technologie mit unterschiedlichen Studiendesigns abgeschätzt sowie die Ergebnisse verglichen, Verzerrungen in den Ergebnissen reduziert und unsichere Ergebnisse transparent gemacht werden können (Colomer et al. 2014: 7300). Dabei ist oft eine Balance $\mathrm{zu}$ finden zwischen aufwendigen Studiendesigns hoher Evidenzstufen, die zu Ergebnissen führen, die möglichst eindeutig interpretiert werden können, und einfacher konzipierten Studien unter Alltagsbedingungen, die potenziell zu Ergebnissen führen, die den realen Nutzungskontext widerspiegeln (Agboola et al. 2014: e75; Lindwedel-Reime et al. 2016:28). Zudem wird empfohlen, Studien in der Praxis beziehungsweise im Anwendungskontext unter Alltagsbedingungen, zum Beispiel in der gewohnten häuslichen Umgebung der Nutzer*innen, durchzuführen, um die kontextuellen Faktoren und das Verhalten der Nutzer*innen derartiger Technologien abschätzen zu können (Colomer et al. 2014: 7302; Martins et al. 2015: 926).

Ferner ist die Auswahl des Studiendesigns abhängig von den Nutzer*innen der altersgerechten Assistenztechnologie. So können die Endnutzer*innen sowie weitere Beteiligte, die mit der Technologie in Kontakt treten (z.B. Angehörige, Pflegesowie Betreuungskräfte, Therapeut"innen und Ärzt*innen), in die Evaluation einbezogen werden, sofern sich deren Einbezug nicht aufgrund von ethischen Erwägungen verbietet (Colomer et al. 2014: 7301; Lindwedel-Reime et al. 2016: 25-28.). Allerdings kann der Einbezug von Nutzer*innen mit spezifischen Charakteristika die Praktikabilität der Studie und Validität der Ergebnisse beeinflussen: Dies kann

Für nähere Informationen zu diesen Methoden und deren Vor- sowie Nachteile bezüglich deren Einbezugs in gesundheitsökonomische Evaluationen vergleiche Aldrich 2018: S65-S69 sowie Brazier et al. 2017: 236-239; zum Einsatz dieser Methoden für altersgerechte Assistenztechnologien vergleiche Becker et al. 2013: 16 sowie Greenhalgh et al. 2015: 5-6. 
zum Beispiel die Verständlichkeit sowie Durchführbarkeit der Befragung oder Nutzung der Technologie betreffen, wenn Proband"innen mit körperlichen oder kognitiven Einschränkungen einbezogen werden (Lindwedel-Reime et al. 2016: 29-30). Zudem kann beim Einbezug hochaltriger oder kranker Proband*innen das Risiko erhöht sein, dass diese aus der Studie aus gesundheitlichen Gründen oder aufgrund des Versterbens vorzeitig ausscheiden müssen (ebd.: 28).

\section{Fazit und Ausblick}

Um die Potenziale und Risiken sowie den Nutzen und die Kosten altersgerechter Assistenztechnologien abzuschätzen, sind methodisch hochwertige Evaluationsstudien nötig. Die Methodik der gesundheitsökonomischen Evaluationen kann angewendet werden, um Kosten-Nutzen-Abwägungen bezüglich der Technologie und geeigneten Alternativen durchzuführen.

Hinsichtlich der Durchführung gesundheitsökonomischer Evaluationen altersgerechter Assistenztechnologien ist es dabei sinnvoll, eine Reihe von Anforderungen zu beachten, wie

- die Erstellung konzeptioneller Wirkungsmodelle, die mit aktueller Evidenz überprüft und angepasst werden,

- den Einbezug potenzieller Nutzer*innen sowie weiterer Personen, die in Kontakt mit der Technologie treten,

- die Beachtung der speziellen Charakteristika der Proband"innen bei der Studienplanung hinsichtlich ethischer Erwägungen, der praktischen Durchführbarkeit der Studie und möglicher Verzerrungen der Ergebnisse,

- die Durchführung von prospektiven Studien, die sich am Lebenszyklus der Technologie orientieren,

- die Anwendung eines Methodenmixes aus experimentellen und Beobachtungsstudien sowie qualitativen und quantitativen Studien,

- die Evaluierung der Technologie nicht nur im klinischen, sondern auch und vor allem im Alltagssetting,

- die zeitnahe Bereitstellung der Ergebnisse der Evaluationsstudie,

- die Aktualisierung der Evaluationsstudie mit neuer Evidenz und Evidenz zu den langfristigen Folgen der Technologie sowie

- die Offenlegung der Generalisierbarkeit sowie Übertragbarkeit der Ergebnisse hinsichtlich der Anwendung der Technologie in anderen Kontexten und/oder Settings.

Ferner ist $\mathrm{zu}$ bedenken, dass die Kosten und Nutzen altersgerechter Assistenztechnologien in unterschiedlichen Bereichen liegen können und gegebenenfalls 
ergänzend zur Ökonomik interdisziplinäre Expertise aus unterschiedlichen Fachdisziplinen (z.B. Philosophie, Sozialwissenschaften, Psychologie, Gesundheits- sowie Pflegewissenschaften, Statistik, Informatik, Ingenieurswissenschaften) für die Durchführung qualitativ hochwertiger Evaluationen benötigt wird.

\section{Literatur}

Achelrod, Dmitrij/Schreyögg, Jonas/Stargardt, Tom (2017): »Health-economic evaluation of home telemonitoring for COPD in Germany: Evidence from a large population-based cohort «, in: The European Journal of Health Economics 18(7), S. 869-882.

Agboola, Stephen/Hale, Timothy M./Masters, Caitlin/Kvedar, Joseph/Jethwani, Kamal (2014): " Real-World< Practical Evaluation Strategies: A Review of Telehealth Evaluation «, in: JMIR Research Protocols 3(4), S. e75.

Akiyama, Miki/Yoo, Byung-Kwang (2016): »A Systematic Review of the Economic Evaluation of Telemedicine in Japan«, in: Journal of Preventive Medicine and Public Health 49(4), S. 183-196.

Albrecht, Urs-Vito/Kuhn, Bertolt/Land, Jörg/Amelung, Volker E./von Jan, Ute (2018): "Nutzenbewertung von digitalen Gesundheitsprodukten (Digital Health) im gesellschaftlichen Erstattungskontext«, in: Bundesgesundheitsblatt - Gesundheitsforschung - Gesundheitsschutz 61(3), S. 340-348.

Aldrich, Stephen C. (2018): »Integrating Scenario Planning and Cost-Benefit Methods«, in: Hastings Center Report 48(1), S. S65-S69.

Al-Shaqi, Riyad/Mourshed, Monjur/Rezgui, Yacine (2016): »Progress in ambient assisted systems for independent living by the elderly«, in: SpringerPlus 5(1), S. 624 .

Bartelmes, Marc/Neumann, Ulrike/Lühmann, Dagmar/Schönermark, Matthias P./Hagen, Anja (2009): Methoden zur frühen entwicklungsbegleitenden Bewertung innovativer medizinischer Technologien (HTA-Bericht 94. Schriftenreihe Health Technology Assessment (HTA) in der Bundesrepublik Deutschland), Köln: Deutsches Institut für Medizinische Dokumentation und Information (DIMDI).

Becker, Heidrun/Scheermesser, Mandy/Treusch, Yvonne/Auerbach, Holger/Meier, Flurina (2013): Robotik in Betreuung und Gesundheitsversorgung (TA-SWISS 58/2013), Zürich: vdf Hochschulverlag AG an der ETH Zürich.

Bendig, Thomas/Bleses, Peter/Breuer, Jens/Buhr, Regina/Egbert, Nicole (2017): Leitlinien Pflege 4.0. Handlungsempfehlungen für die Entwicklung und den Erwerb digitaler Kompetenzen in Pflegeberufen, Berlin: Gesellschaft für Informatik e. V. 
Bertrand, Kim/Raymond, Marie-Helene/Miller, William C./Martin Ginis, Kathleen A./Demers, Louise (2017): "Walking Aids for Enabling Activity and Participation: A Systematic Review «, in: American Journal of Physical Medicine \& Rehabilitation 96(12), S. 894-903.

Blettner, Maria/Dierks, Marie-Luise/Donner-Banzhoff, Norbert/Hertrampf, Katrin/Klusen, Norbert/Köpke, Sascha/Masanneck, Michael/Pfaff, Holger/Richter, Rainer/Sundmacher, Leonie (2018): »Überlegungen des Expertenbeirats zu Anträgen im Rahmen des Innovationsfonds«, in: Zeitschrift für Evidenz, Fortbildung und Qualität im Gesundheitswesen (ZEFQ) 130(1), S. 42-48.

Bozan, Karoly/Berger, Andrew (2019): »Revisiting the Technology Challenges and Proposing Enhancements in Ambient Assisted Living for the Elderly«, in: Proceedings of the 52nd Hawaii International Conference on System Sciences, Hawaii: HICSS, S. 4307-4316.

Brazier, John/Ratcliffe, Julie/Salomon, Joshua A./Tsuchiya, Aki (2017): Measuring and Valuing Health Benefits for Economic Evaluation, Oxford: Oxford University Press.

Breyer, Friedrich/Zweifel, Peter/Kifmann, Mathias (2013): Gesundheitsökonomik, Berlin/Heidelberg: Springer Gabler.

Broek, Ger van den/Cavallo, Filippo/Odetti, Luca/Wehrmann, Christian (2009): Ambient Assisted Living Roadmap, Berlin: VDI/VDE-IT, AALIANCE Office.

Bullinger, Monika/Blome, Christine/Sommer, R./Lohrberg, D./Augustin, M. (2015): „Gesundheitsbezogene Lebensqualität - ein zentraler patientenrelevanter Endpunkt in der Nutzenbewertung medizinischer Maßnahmen«, in: Bundesgesundheitsblatt-Gesundheitsforschung-Gesundheitsschutz 58(3), S. 283-290.

Burchert, Heiko (1998): Ökonomische Evaluation von Telematik-Anwendungen im Gesundheitswesen und Schlußfolgerungen für ihre Implementierung. Veröffentlichungen des Lehrstuhls für Allgemeine Betriebswirtschaftslehre und Betriebliche Finanzwirtschaft, insbesondere Unternehmensbewertung, Greifswald: Ernst-Moritz-Arndt-Universität Greifswald.

Calvaresi, Davide/Cesarini, Daniel/Sernani, Paolo/Marinoni, Mauro/Dragoni, Aldo Franco/Sturm, Arnon (2016): »Exploring the ambient assisted living domain: A systematic review«, in: Journal of Ambient Intelligence and Humanized Computing 8(2), S. 239-257.

Campbell, Michelle/Fitzpatrick, Ray/Haines, Andrew/Kinmonth, Ann Louise/ Sandercock, Peter/Spiegelhalter, David/Tyrer, Peter (2000): »Framework for design and evaluation of complex interventions to improve health «, in: British Medical Journal (BMJ) 321(7262), S. 694-696.

Campbell, Neil C./Murray, Elizabeth/Darbyshire, Janet/Emery, Jon/Farmer, Andrew/Griffiths, Frances/Guthrie, Bruce/Lester, Helen/Wilson, Phil/Kinmonth, 
Ann L. (2007): »Designing and evaluating complex interventions to improve health care«, in: British Medical Journal (BMJ) 334(7591), S. 455-459.

Catwell, Lorraine/Sheikh, Aziz (2009): »Evaluating eHealth Interventions: The Need for Continuous Systemic Evaluation«, in: PLOS Medicine 6(8), S. e1000126.

Chung, Jane/Demiris, George/Thompson, Hilaire J. (2016): „Ethical Considerations Regarding the Use of Smart Home Technologies for Older Adults: An Integrative Review«, in: Annual Review of Nursing Research 34(1), S. 155-81.

Colomer, Juan B. M./Salvi, Dario/Cabrera-Umpierrez, Maria F./Arredondo, Maria T./Abril, Patricia/Jimenez Mixco, Viveca/Garcia-Betances, Rebeca/Fioravanti, Alessio/Pastorino, Matteo/Cancela, Jorge/Medrano, Alejandro (2014): »Experience in evaluating AAL solutions in living labs«, in: Sensors 14(1), S. 7277-7311.

Cook, John/Drummond, Michael/Heyse, Joseph F. (2004): »Economic endpoints in clinical trials«, in: Statistical Methods in Medical Research 13(2), S. 157-176.

Craig, Peter/Dieppe, Paul/Macintyre, Sally/Michie, Susan/Nazareth, Irwin/Petticrew, Mark (2008): »Developing and evaluating complex interventions: The new Medical Research Council guidance«, in: British Medical Journal (BMJ) 337(1), S. $a 1655$.

Dintsios, Charalabos-Markos/Icks, Andrea/Gerber-Grote, Andreas/Chernyak, Nadja (2017): »Effizienzbewertung im Rahmen des GKV-Versorgungsstärkungsgesetzes: Was kann die Gesundheitsökonomie hierzu beitragen?«, in: Sozialer Fortschritt 66(10), S. 651-673.

Drummond, Michael F./Sculpher, Mark J./Claxton, Karl/Stoddart, Greg L./Torrance, George W. (2015): Methods for the Economic Evaluation of Health Care Programmes, Oxford: Oxford University Press.

Eftring, Hakan/Frennert, Susanne (2016): »Designing a social and assistive robot for seniors«, in: Zeitschrift für Gerontologie und Geriatrie 49(4), S. 274-281.

Elsbernd, Astrid/Lehmeyer, Sonja/Schilling, Ulrike (2015): »Der Traum vom aufrechten Gang: Rollatoren sind in der Gesellschaft weit verbreitet - sie haben jedoch noch immer erhebliche Schwächen«, in: Pflegezeitschrift 68(12), S. 722-725.

Fachinger, Uwe (2017): »Technische Lösungen für ein selbstbestimmtes Leben im Alter«, in: Archiv für Wissenschaft und Praxis der sozialen Arbeit 48(2), S. 2-8. Fachinger, Uwe (2018): »Altern und Technik: Anmerkungen zu den ökonomischen Potentialen«, in: Harald Künemund/Uwe Fachinger (Hg.), Alter und Technik: Sozialwissenschaftliche Befunde und Perspektiven, Wiesbaden: Springer Fachmedien, S. 51-68.

Fatehi, Farhad/Smith, Anthony C./Maeder, Anthony/Wade, Victoria/Gray, Leonard C. (2016): "How to formulate research questions and design studies for telehealth assessment and evaluation«, in: Journal of Telemedicine and Telecare 23(9), S. 759-763. 
Fioranelli, Emilio (2019): »Robotik, Pflege und Gesundheitsökonomie - Pflegeroboter: Eine Kosten-Nutzen-Analyse«, in: Gesundheitsökonomie \& Qualitätsmanagement 24(1), S. 8-10.

Fuhrer, Marcus J. (2007): "Assessing the efficacy, effectiveness, and cost-effectiveness of assistive technology interventions for enhancing mobility«, in: Disability and Rehabilitation: Assistive Technology 2(3), S. 149-158.

Gäfgen, Gérard (1990): Gesundheitsökonomie. Grundlagen und Anwendungen, Baden-Baden: Nomos.

Glasgow, Russell E. (2007): »eHealth Evaluation and Dissemination Research«, in: American Journal of Preventive Medicine 32(5S), S. 119-126.

Greenhalgh, Trisha/Procter, Rob/Wherton, Joe/Sugarhood, Paul/Hinder, Sue/ Rouncefield, Mark (2015): "What is quality in assisted living technology? The ARCHIE framework for effective telehealth and telecare services«, in: BMC Medicine 13(1), S. 91.

Greiner, Wolfgang/Damm, Oliver (2012): »Die Berechnung von Kosten und Nutzen«, in: Oliver Schöffski/Johann-Matthias Graf von der Schulenburg (Hg.), Gesundheitsökonomische Evaluationen, Berlin/Heidelberg: Springer, S. 23-42.

Greiner, Wolfgang/Schöffski, Oliver (2012): »Grundprinzipien einer Wirtschaftlichkeitsuntersuchung«, in: Oliver Schöffski/Johann-Matthias Graf von der Schulenburg (Hg.), Gesundheitsökonomische Evaluationen, Berlin/Heidelberg: Springer, S. 155-180.

Grootven, Bastiaan van/Achterberg, Theo van (2016): »The European Union's Ambient and Assisted Living Joint Programme: An evaluation of its impact on population health and well-being «, in: Health Informatics Journal 25(1), S. 27-40.

Gruber, Hans-Georg (2011): Telemonitoring-Systeme: Gesundheitsökonomische Evaluation und Innovationsbarrieren, Bayreuth: Rechts- und Wirtschaftswissenschaftliche Fakultät Universität Bayreuth.

Grünendahl, Martin/Leonhardt, Sven/Teich, Tobias (2017): »Mehrwert in der häuslichen Pflege durch vernetzte Ambient-Assisted-Living-Systeme«, in: Mario A. Pfannstiel/Sandra Krammer/Walter Swoboda (Hg.), Digitale Transformation von Dienstleistungen im Gesundheitswesen III: Impulse für die Pflegepraxis, Wiesbaden: Springer Fachmedien, S. 55-69.

Hauer, Katharina (2017): »Brauche ich das überhaupt?! - Qualität assistiver Technologien aus Sicht von älteren Personen in häuslicher Pflege«, in: Mario A. Pfannstiel/Sandra Krammer/Walter Swoboda (Hg.), Digitale Transformation von Dienstleistungen im Gesundheitswesen III: Impulse für die Pflegepraxis, Wiesbaden: Springer Fachmedien, S. 307-325.

Heinze, Rolf G. (2018): »Alter und Technik«, in: Harald Künemund/Uwe Fachinger (Hg.), Alter und Technik: Sozialwissenschaftliche Befunde und Perspektiven, Wiesbaden: Springer Fachmedien, S. 15-31. 
Helle, Tina/Iwarsson, Susanne/Brandt, Ase (2014):»Validation of housing standards addressing accessibility: Exploration of an activity-based approach«, in: Journal of Applied Gerontology 33(7), S. 848-869.

Henschke, Cornelia/Panteli, Dimitra/Perleth, Matthias/Busse, Reinhard (2015): "Taxonomy of medical devices in the logic of health technology assessment «, in: International Journal of Technology Assessment in Health Care 31(5), S. 324-330.

Hilbert, Josef/Becka, Denise/Cirkel, Michael/Dahlbeck, Elke (2018): »Alter und Technik: Perspektiven der Gesundheitswirtschaft«, in: Harald Künemund/Uwe Fachinger (Hg.), Alter und Technik: Sozialwissenschaftliche Befunde und Perspektiven, Wiesbaden: Springer Fachmedien, S. 33-50.

IQWiG (2017). Allgemeine Methoden, https://www.iqwig.de/de/methoden/metho denpapier.3020.html vom 10.07.2017.

Kirisits, Andreas/Redekop, W. Ken (2013): »The Economic Evaluation of Medical Devices«, in: Applied Health Economics and Health Policy 11(1), S. 15-26.

Köberlein-Neu, Juliane (2017): »Evaluationsmethoden«, in: Stefan Müller-Mielitz/ Thomas Lux (Hg.), E-Health-Ökonomie, Wiesbaden: Springer Fachmedien, S. 807-819.

Koller, Michael/Neugebauer, Edmund A. M./Augustin, Matthias/Büssing, Arndt/Farin, Erik/Klinkhammer-Schalke, Monika/Lorenz, Wilfried/Münch, K./Petersen-Ewert, C./von Steinbüchel, N./Wieseler, B. (2009): »Die Erfassung von Lebensqualität in der Versorgungsforschung - konzeptuelle, methodische und strukturelle Voraussetzungen«, in: Gesundheitswesen 71(12), S. 864-872.

Krieg-Brückner, Bernd/Röfer, Thomas/Shi, Hui/Gersdorf, Bernd (2011): »Mobilitätsassistenz im >Bremen Ambient Assisted Living Lab (BAALL)«, in: Nova Acta Leopoldina 104(1), S. 157-174.

Leppert, Florian/Greiner, Wolfgang (2016): »Finanzierung und Evaluation von eHealth-Anwendungen«, in: Florian Fischer/Alexander Krämer (Hg.), eHealth in Deutschland, Berlin/Heidelberg: Springer, S. 101-124.

Lindemann, Ulrich/Schwenk, Michael/Klenk, Jochen/Kessler, Max/Weyrich, Michael/Kurz, Franziska/Becker, Clemens (2016): »Problems of older persons using a wheeled walker«, in: Aging Clinical and Experimental Research 28(1), S. 215-220.

Lindwedel-Reime, Ulrike/Röll, Natalie/Lautenschläger, Sindy/Gradel, Cornelia/König, Peter/Kunze, Christophe (2016): Effekte und Nutzen altersgerechter Assistenzsysteme (ENAS). Leitfaden für die Planung und Durchführung von Studien zur Evaluation neuer technischer Assistenzsysteme in Forschungs- und Entwicklungsprojekten (FZI-Report 02/2016), Karlsruhe: FZI Forschungszentrum Informatik am Karlsruher Institut für Technologie.

Lysdahl, Kristin B./Mozygemba, Kati/Burns, Jake/Chilcott, James B./Brönneke, Jan B./Hofmann, Bjørn (2016): Guidance for assessing effectiveness, economic aspects, ethical aspects, socio-cultural aspects and legal aspects in complex tech- 
nologies, https:/www.integrate-hta.eu/wp-content/uploads/2016/08/IPP_Gui dance-INTEGRATE-HTA_Nr.3_FINAL.pdf vom 04.05.2021.

Manych, Matthias (2018): »4. Plenumsveranstaltung der B. Braun-Stiftung. Von der Innovation zur Regelversorgung «, in: Monitor Versorgungsforschung 11(4), S. 20.

Martins, Maria M./Santos, Cristina P./Frizera, Anselmo/Ceres, Ramón (2015): »A review of the functionalities of smart walkers«, in: Medical Engineering \& Physics 37(10), S. 917-928.

Melkas, Helinä (2013): »Innovative assistive technology in Finnish public elderlycare services: A focus on productivity«, in: Work 46(1), S. 77-91.

Meyer, Sibylle (2018): »Technische Assistenzsysteme zu Hause - warum nicht? Vergleichende Evaluation von 14 aktuellen Forschungs- und Anwendungsprojekten«, in: Harald Künemund/Uwe Fachinger (Hg.), Alter und Technik: Sozialwissenschaftliche Befunde und Perspektiven, Wiesbaden: Springer Fachmedien, S. 147-176.

Moser-Siegmeth, Verena/Hofer, Kathrin (2013): »Assistive Technologien für ältere Menschen: Nutzen für EndanwenderInnen und Herausforderungen im Einsatz«, in: SWS Rundschau 53(1), S. 57-72.

Mühlbacher, Axel C./Juhnke, Christin (2018): »Nutzenbewertung für Untersuchungs- und Behandlungsmethoden mit Medizinprodukten hoher Klassen: Die Abwägung von Patientennutzen, Evidenz und Zugang«, in: Gesundheitswesen 80(2), S. S80-S87.

Nielsen, Camilla P./Sarriá Santamera, Antonio/Vondeling, Hindrik (2008): »Policy processes and health technology assessment «, in: Marcial V. Garrido/Finn B. Kristensen/Camilla P. Nielsen/Reinhard Busse (Hg.), Health Technology Assessment and Health Policy-Making in Europe. Current status, challenges and potential, Copenhagen: WHO Regional Office for Europe, S. 19-30.

Ohinmaa, Arto/Hailey, David/Roine, Risto (2001): »Elements for assessment of telemedicine applications «, in: International Journal of Technology Assessment in Health Care 17(2), S. 190-202.

Perleth, Matthias/Lühmann, Dagmar (2010): »Nutzen- und Wirtschaftlichkeitsbewertung der biomedizinischen Technik«, in: Bundesgesundheitsblatt - Gesundheitsforschung - Gesundheitsschutz 53(8), S. 825-830.

Ploch, Uwe/Werkmeister, Till (2017): Digitalisierung und Technisierung der Pflege in Deutschland. Aktuelle Trends und ihre Folgewirkungen auf Arbeitsorganisation, Beschäftigung und Qualifizierung, Hamburg: DAA-Stiftung Bildung und Beruf.

Prenzler, Anne/Reddemann, Sebastian/Schulenburg, Johann-Matthias von der (2010): »Die Wahl der Perspektive bei gesundheitsökonomischen Evaluationen - eine interdisziplinäre Analyse«, in: G\&S Gesundheits- und Sozialpolitik 64(2), S. 30-37. 
Puskin, Dena S./Cohen, Zohara/Ferguson, A. Stewart/Krupinski, Elizabeth/ Spaulding, Ryan (2010): "Implementation and Evaluation of Telehealth Tools and Technologies«, in: Telemedicine and e-Health 16(1), S. 96-102.

Remmers, Hartmut (2019): »Pflege und Technik. Stand der Diskussion und zentrale ethische Fragen«, in: Ethik in der Medizin 31(4), S. 407-430.

Rohwer, Anke/Booth, Andrew/Pfadenhauer, Lisa/Brereton, L./Gerhardus, Ansgar/Mozygemba, Kati/Oortwijn, Wija/Tummers, Marcia/Wilt, G. J. van der/Rehfuess, Eva (2016): »Guidance on the use of logic models in health technology assessments of complex interventions «, https:/www.integrate-hta .eu/wp-content/uploads/2016/02/Guidance-on-the-use-of-logic-models-in-he alth-technology-assessments-of-complex-interventions.pdf vom 05.05.2021.

Sanyal, Chiranjeev/Stolee, Paul/Juzwishin, Don/Husereau, Don (2018): »Economic evaluations of eHealth technologies: A systematic review«, in: PLOS ONE 13(6), S. 1-11.

Schöffski, Oliver (2012a): »Einführung«, in: Oliver Schöffski/Johann-Matthias Graf von der Schulenburg (Hg.), Gesundheitsökonomische Evaluationen, Berlin/Heidelberg: Springer, S. 3-12.

Schöffski, Oliver (2012b). "Grundformen gesundheitsökonomischer Evaluationen«, in: Oliver Schöffski/Johann-Matthias Graf von der Schulenburg (Hg.), Gesundheitsökonomische Evaluationen, Berlin/Heidelberg: Springer, S. 43-70.

Schöffski, Oliver (2012c). »Lebensqualität als Ergebnisparameter in gesundheitsökonomischen Studien«, in: Oliver Schöffski/Johann-Matthias Graf von der Schulenburg (Hg.), Gesundheitsökonomische Evaluationen, Berlin/Heidelberg: Springer, S. 327-340.

Schöffski, Oliver (2012d). »Nutzentheoretische Lebensqualitätsmessung«, in: Oliver Schöffski/Johann-Matthias Graf von der Schulenburg (Hg.), Gesundheitsökonomische Evaluationen, Berlin/Heidelberg: Springer, S. 341-391.

Schulenburg, Johann-Matthias von der/Greiner, Wolfgang/Jost, F./Klusen, N./Kubin, M./Leidl, Reiner/Mittendorf, T./Rebscher, Herbert/Schöffski, Oliver/Vauth, C./Volmer, T./Wahler, S./Wasem, Jürgen/Weber, C. (2012): »Anhang«, in: Oliver Schöffski/Johann-Matthias Graf von der Schulenburg (Hg.), Gesundheitsökonomische Evaluationen, Berlin/Heidelberg: Springer, S. 525-534.

SGB V-Das Fünfte Buch Sozialgesetzbuch-Gesetzliche Krankenversicherung - (Artikel 1 des Gesetzes vom 20. Dezember 1988, BGBl I, S. 2477, 2482), das zuletzt durch Artikel 5 des Gesetzes vom 27. März 2020 (BGBl I, S. 587) geändert worden ist.

SGB XI - Das Elfte Buch Sozialgesetzbuch - Soziale Pflegeversicherung - (Artikel 1 des Gesetzes vom 26. Mai 1994, BGBl I, S. 1014, 1015), das zuletzt durch Artikel 4 des Gesetzes vom 27. März 2020 (BGBl I, S. 580) geändert worden ist. 
Sharkey, Amanda/Sharkey, Noel (2012): »Granny and the robots: Ethical issues in robot care for the elderly«, in: Ethics and Information Technology 14(1), S. 27-40. Shiell, Alan/Hawe, Penelope/Gold, Lisa (2008): „Complex interventions or complex systems? Implications for health economic evaluation«, in: British Medical Journal (BMJ) 336(7656), S. 1281-1283.

Tarricone, Rosanna/Torbica, Aleksandra/Drummond, Michael (2017): »Key Recommendations from the MedtecHTA Project«, in: Health Economics 26(S1), S. 145-152.

Taylor, Paul (2005): »Evaluating telemedicine systems and services«, in: Journal of Telemedicine and Telecare 11(4), S. 167-177.

Testa, Marcia A./Simonson, Donald C. (1996): »Assessment of Quality-of-Life Outcomes«, in: Current Concepts 334(13), S. 835-840.

The Lewin Group (2000): Assessment of Approaches to Evaluating Telemedicine, Virginia: Office of the Assistant Secretary for Planning and Evaluation, Department of Health and Human Services.

Wade, Victoria/Barnett, Adrian G./Martin-Khan, Melinda G./Russell, Trevor (2017): »Designing quantitative telemedicine research«, in: Journal of Telemedicine and Telecare 23(9), S. 786-791.

Wahlster, Philip/Brereton, Louise/Burns, Jake/Hofmann, Björn/Mozygemba, Kati/Oortwijn, Wija/Pfadenhauer, Lisa/Polus, Stephanie/Rehfuess, Eva/ Schilling, Imke/Hoorn, Ralph van/Wilt, Gert J. van der/Baltussen, Rob/Gerhardus, Ansgar (2016): Guidance on the integrated assessment of complex health technologies - The INTEGRATE-HTA Model, https://www.integrate -hta.eu/wp-content/uploads/2016/02/Guidance-on-the-integrated-assessm ent-of-complex-health-technologies-the-INTEGRATE-HTA-model.pdf vom 05.05.2021.

Weiß, Christine/Lutze, Maxie/Stock Gissendanner, Scott/Peters, Verena (2017): Abschlussbericht. Nutzen und Finanzierung technischer Assistenzsysteme aus Sicht der Pflegeversicherung und weiterer Akteure der Verantwortungsgemeinschaft am Beispiel der Quartiersvernetzung, Berlin: IEGUS, VDI/VDE-IT. Werner, Christian/Ullrich, Phoebe/Geravand, Milad/Peer, Angelika/Bauer, Jürgen M./Hauer, Klaus (2018): »A systematic review of study results reported for the evaluation of robotic rollators from the perspective of users«, in: Disability and Rehabilitation Assistive Technology 13(1), S. 31-39.

Wiklund, Ingela (2004): »Assessment of patient-reported outcomes in clinical trials: The example of health-related quality of life«, in: Fundamental \& Clinical Pharmacology 18(3), S. 351-363.

Zöllick, Jan C./Kuhlmey, Adelheid/Suhr, Ralf/Eggert, Simon/Nordheim, Johanna/Blüher, Stefan (2020): "Akzeptanz von Technikeinsatz in der Pflege«, in: Klaus Jacobs/Adelheid Kuhlmey/Stefan Greß/Jürgen Klauber/Antje Schwinger 
(Hg.), Pflege-Report 2019: Mehr Personal in der Langzeitpflege - aber woher?, Berlin/Heidelberg: Springer, S. 211-218. 



\section{Technikimplementierung in der Pflege: die Bedeutung der soziotechnischen Innovationsbewertung aus gesundheitsökonomischer Sicht}

Jürgen Zerth

\section{Pflegetechnologien und Anwendung: Eingangsstreiflichter}

Pflege und die Bedeutung der Technik für diese werden in vielfältiger Hinsicht im pflegepolitischen Diskurs thematisiert, mit staatlichen Mitteln unterstützt und politische Initiativen lassen den Eindruck entstehen, Pflegetechnologien könnten einen wesentlichen Beitrag zur Unterstützung, Förderung von Pflege und Pflegenden und zur langfristigen Steigerung von Qualität, Effektivität und Effizienz insbesondere in der Langzeitpflege beitragen (Daum/Ploch/Werkmeister 2017; Fachinger 2017). Ein Blick auf die Frage, welche Technologien für die Pflege entwickelt worden sind und welche letztendlich Eingang in die Pflegepraxis gefunden haben, zeigt mit Referenz auf die dazu bereits veröffentlichte Literatur ein eher ernüchterndes Ergebnis (Hülsken-Giesler/Zelt/Weidner 2017; Weiß et al. 2017).

Die Implementierungsbarrieren für die Umsetzung von Pflegetechnologien haben sowohl institutionelle, organisatorische und letztendlich akteur"innenbezogene Gründe (Schneider/Besser/Geithner 2020) und nach der sogenannten e-PflegeStudie sind insbesondere unklare Umsetzungsmodelle (auf der Anwendungsseite) beziehungsweise Geschäftsmodelle auf der Angebotsseite sowie mangelndes pflegepraktisches Wissen über den zielgenauen Einsatz der Technologien innerhalb des Pflegeprozesses für eine nachhaltige Implementierung abträglich (HülskenGiesler/Zelt/Weidner 2017: 33).

Die Autor*innen des Pflegethermometers 2018 (Isfort et al. 2018) haben beispielsweise Entscheidungsträger*innen aus der stationären Pflege ( $N=1067)$ gefragt, welche Technologiekategorien eher und welche weniger in der Praxis umgesetzt werden, und es lässt sich (nach dieser Querschnittsbefragung) festhalten, dass Technologien im Sinne der grundlegenden Infrastruktur, etwa einfache elektronisch verstellbare Betten oder insbesondere Dienstplansoftware, die höchste Nutzung aufweisen. Je stärker Technologien jedoch in die direkten 
Abbildung 1: Einsatz unterstützender Technologien, die eher genutzt werden ( $N=1067)$;

Abbildung 2: Einsatz unterstützender Technologien, weniger genutzt wer$\operatorname{den}(N=1067)$
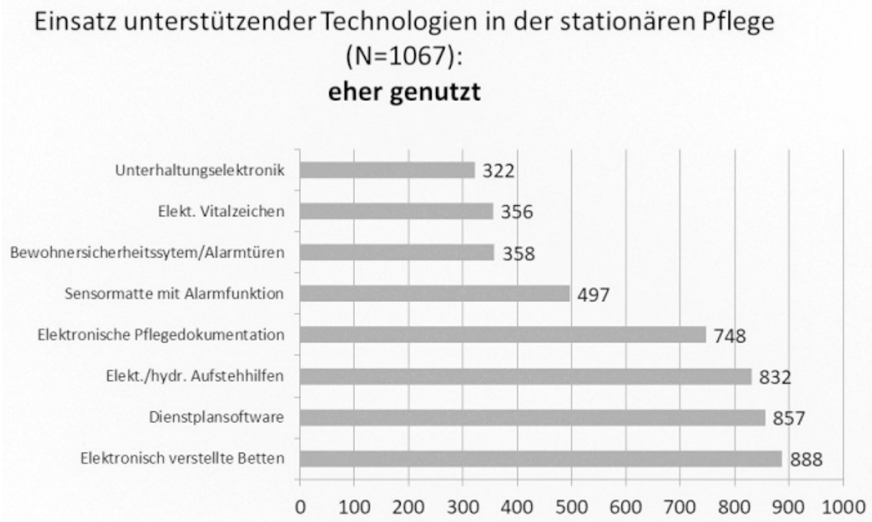

Einsatz unterstützender Technologien in der stationären Pflege

$(\mathrm{N}=1067)$ :

weniger genutzt

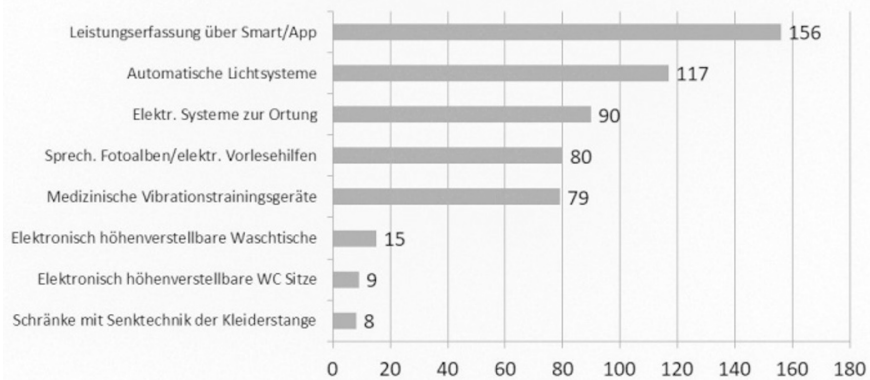

Quelle: Isfort et al. 2018: 62

Pflegebeziehungen eingreifen oder durch Personalisierungsmöglichkeiten gar Pflegebeziehungen zwischen Pflegenden und Gepflegten ändern können, etwa 
weil eine Monitoringfunktion durch eine Sensorik übernommen wird, desto weniger sind derartige Technologien vorhanden beziehungsweise werden genutzt (siehe Abbildung 1 und 2).

Gerade der Blick auf die häufig genutzten Technologien in der Befragung weist auf die Bedeutung infrastruktureller Technologien, wie höhenverstellbare Betten oder auch Dokumentationssysteme, hin. Aber auch die Umsetzung von derartigen Technologien lässt sich mit Bezug auf Ergebnisse etwa in der Pflege-4.0-Studie mit hoher Plausibilität als Aufholprozess klassifizieren (Merda/Schmidt/Kähler 2017). So können etwa die Ergebnisse dieser Studie deutliche Hinweise dazu geben, dass die Verbreitung und Umsetzung elektronischer Dokumentationssysteme im Bereich der Langzeitpflege im Vergleich zum Akutkrankenhaus mit einem deutlichen Time-Lag versehen ist und insbesondere die ambulante Pflege wesentliche Umsetzungslücken aufweist (ebd.: 46-48). Hier ist gerade im Vergleich zu den Entwicklungen in der stationären Akutversorgung ein deutlicher zeitlicher Rückstand festzuhalten, insbesondere mit Blick auf den europäischen Vergleich.

Einen erweiterten Blick auf empirisch dokumentierte Umsetzungsproblematiken zeigen Zöllick et al. (2019) in ihrer Studie auf, die den Blick auf die geäußerte Wahrnehmung von Pflegekundigen ( $\mathrm{N}=127$ Teilnehmende) im ambulanten Setting legt, inwiefern Technologien (1) grundsätzlich bekannt sind, es (2) einen Zugang zu den Technologien gibt und (3) diese auch eingesetzt werden.

In dieser Studie lassen sich grob drei Kategorien der technischen Transformation identifizieren, die nach der Kombination der Bekanntheit, des Zugangs und des Einsatzes geordnet werden können:

- Kategorie 1: Technologien wie Hebehilfen, Smartphones oder Tablets, letztgenannte insbesondere $\mathrm{zu}$ dokumentarischen Zwecken. Hier zeigt die Untersuchung eine überwiegende Kenntnis bei den Pflegekundigen (über $80 \%$ der Antworten), jedoch ist bei diesen Technologien ein deutlicher Abstand zum Zugang und ein noch deutlicherer Abstand zur Nutzung festzuhalten.

- Kategorie 2: Grob lassen sich in diese Kategorie Technologien wie Erinnerungshilfen für die Medikation, Tablets zum (therapeutischen) Spielen und zur Beschäftigung, Sturz-Dektektoren und GPS-Tracker für Demenzkranke einordnen. Die Kenntnis bei den Pflegekräften ist hier schon deutlich niedriger (im Durchschnitt $50 \%$ ), die Differenz zum wahrgenommenen Zugang erscheint noch größer (20\%) und die Nutzung pendelt sich zwischen 10 und 15 Prozent ein.

- Kategorie 3: Der Blick auf die Ergebnisse von Zöllick et al. ordnet in einer dritten Kategorie etwa Roboter zum Materialtransport, Smart-Sensors zum selbständigen Wohnen, Kuschelroboter sowie auch automatische Medikamentenverteilung zu. Hier ist allein schon die Kenntnis der Pflegekundigen deutlich niedriger, zwischen 20 und 30 Prozent, wohingegen Zugang und Nutzung in 
der Wahrnehmung auf sehr niedrigem Niveau zusammenfallen, niedriger als 10 Prozent.

Als grobe Zusammenfassung der empirischen Fallbeispiele scheinen sowohl die Aspekte des Umsetzungswissens, wie bereits in der e-Pflege-Studie adressiert, relevant zu sein als auch die Frage nach dem adäquaten Blick auf die von den Hersteller*innen gewählten Markteintritts- und Implementierungsstrategien.

Beide Aspekte sind im Hinblick auf das Gesundheits- und das Pflegesystem zu berücksichtigen, vor allem wenn es gilt, dass Technologien im Gesundheits- wie im Pflegesystem in den meisten Fällen als Teil personenorientierter Dienstleistungen charakterisiert werden müssen.

So kommen Autor*innen wie etwa Omachonu/Einspruch (2010:13) zum Ergebnis, den Technologie- und Innovationsimplementierungsprozess als Teil eines Innovationsmodells im Kontext von Dienstleistungsorganisationen zu beschreiben. Rahmenmodelle zur Technologiebegleitung und -implementierung, exemplarisch etwa das NASSS-Framework, sehen den Zusammenhang der Technologieimplementierung im Kontext der Setting-Bedingungen, der konkreten darauf passenden Technologie, der Akteur*innenreaktionen und der zusammenhängenden organisatorischen Rahmenbedingungen, die letztendlich in einer losen Wechselbeziehung zwischen angebots- und bedarfsbezogenen Faktoren eingeordnet werden könnten (Abimbola et al. 2019: 2; Greenhalgh et al. 2018).

Für die nachfolgende Betrachtung soll daher die Frage in den Vordergrund gestellt werden, inwiefern Akteur"innen-, Technologie- und Organisationsbetrachtungen der Technologieimplementierung in der Pflege in einer taxonomischen Herangehensweise dargestellt werden können, um damit einen Zusammenhang $\mathrm{zu}$ möglichen Hinweisen zu innovations- und implementierungsförderlichen Impulsen abzuleiten. In diesem Sinne ist die Bedeutung des Technologiebegriffs im pflegerischen Kontext nochmals aufzugreifen.

\section{Pflegetechnologien als Teil eines soziotechnischen Systems}

\section{Die Bedeutung der Sorge- und Interaktionsarbeit Pflege}

Die Beschreibung von Omachonu/Einspruch (2010), Technologien im Gesundheitswesen als Teil des organisatorischen und prozessualen Kontextes zu deuten, lenkt den Blick auf die Verortung von Technik als Teil einer konkreten Pflegehandlung und eines übergeordneten, phänotypischen Pflegeprozesses (Schneider/Besser/Geithner 2020: 622-623). Mit einer ersten Bezugnahme auf sozial- und insbesondere pflegewissenschaftliche Perspektiven lässt sich Pflege zunächst als Form der Sorgearbeit und der Sorgebeziehung illustrieren. Diese impliziert vor 
allem die Beziehung zwischen einer*einem Sorgenden und einer*einem, die*der Sorge erhält (Becka/Borchers/Evans 2016).

Diese Sorgebeziehung ist in einem weiteren Sinne immer eingebettet in einen Anlass- und Kontextbezug der Sorge (Nitsche 2017). So sind pflegerische Handlungen im Kontext einer Operation stärker auf medizinische Bedarfe ausgelegt, wohingegen Pflegebeziehungen zur Aufrechterhaltung der Aktivitäten des täglichen Lebens eher soziale Bedarfslagen in Augenschein nehmen werden (zur Verknüpfung von Cure und Care bspw. Hoberg/Klie/Künzel 2013: 13).

Sickau/Thiele (2017) unterteilen in Anlehnung an Böhle (2011) die pflegerische Arbeit in die analytischen Komponenten der Interaktionsarbeit, nämlich Kooperationsarbeit, Emotions- und Gefühlsarbeit sowie das subjektiv empfundene Arbeitshandeln (Böhle 2011; Sickau/Thiele 2017: 40-44). Hier greifen je nach Setting dann unterschiedliche Notwendigkeiten, die pflegerische Dienstleistung im Mix professioneller Kompetenzen und Kompetenzen der Familien- und/oder Selbstpflege zu organisieren (Schallermair 1999: 19).

Diese akteur*innenbezogenen Beschreibungen sind dann Teil der normativen Pflegearbeit, die auf einer organisatorischen Ebene durch die räumlichen sowie sächlichen Strukturen als auch durch die als notwendig vorgegebenen Prozesshandlungen - etwa durch Expert*innenstandards - eingeordnet werden (Sickau/Thiele 2017: 40). Die Akteur*innen- und Organisationsstrukturen sind in einem weiteren Schritt wieder Teil des institutionellen Kontextes von Pflege innerhalb einer (national) definierten Umsetzung des Begriffs der sozialen Sicherung. Letztgenannte könnte wirtschaftspolitisch als Ausdruck der Umsetzung der Interpretation einer Wohlfahrtsüberlegung ausgelegt werden (Zerth 2020a: 121-124).

Hier lässt sich etwa eine weiterführende Diskussion führen, inwiefern die institutionelle Gestaltung von Langzeitpflege als Teil einer Philosophie sozialer Sicherung primär komplementär zu einer Politik der Wohlfahrtssteigerung eingeordnet werden kann oder stärker im Sinne einer Befähigung zur Gestaltung derselben interpretiert werden sollte. ${ }^{1}$ Unabhängig von dieser grundlegenden Diskussion, jedoch innerhalb eines gezeichneten Rahmens institutioneller Ordnung, ist die Interaktionsarbeit Pflege aus organisationsökonomischer Sicht aus dem Wechselspiel der personalen Ressourcen im Care-Mix zwischen professionell und informell Pflegenden und den dazugehörigen settingabhängigen Ressourcen zu erläutern (ebd.: 128-129).

Technologie als Teil eines soziotechnischen Systems verändert dabei sowohl die Verhaltens-als auch die Verhältnisstruktur des Pflegehandelns, daneben greifen als verbindende Klammer die Kategorien der Akteur*innen- und Organisationskultur 
als moderierende Variablen zwischen Struktur- und Prozessbetrachtung einer Interaktionsarbeit ein.

\section{Technologien als Veränderung operanter und operander Bedingungen}

Aus dienstleistungsökonomischer Sicht erfolgt ein Austausch unterschiedlicher Teamproduktionsphänomene zwischen personalen, operanten Ressourcen und den organisationsbezogenen, operanden Bedingungen, wie es beispielsweise bei Zerth (2017: 248-249) beziehungsweise grundsätzlich bei Bornewasser/Frenzel/Tombeil (2016: 16-17) gezeigt wird. ${ }^{2}$

Technik kann somit sowohl die operanten wie die operanden Ressourcen beeinflussen, indem beispielsweise standardisierte, sensorgestützte Monitoringsysteme pflegerelevante Informationen an die Pflegekraft zurückspielen, somit exemplarisch das Monitoring eines pflegerelevanten Risikos (etwa Dekubitus) nicht mehr von einem routinemäßigen Kontrollprozess der Pflegekraft (und daher der verfügbaren Kapazität) abhängig ist, sondern durch die Sensorik anlassbezogen erhoben wird (Schneider/Besser/Geithner 2020: 622-624).

In dieser Hinsicht sind Monitoringprozesse verstetigbar und somit - die technische Prädiktionsgüte sei unterstellt - die Monitoringinformation als Prozessinformation weniger unsicher, was ökonomisch als Verbesserung von Qualitätsinformationen interpretierbar ist. Somit wird die kapazitive Wirkung derartiger prozessveränderte Technologien unmittelbar ableitbar, da sowohl die Eigenschaften des Leistungsgegenstandes, des Leistungsprozesses sowie am Ende auch des Leistungsergebnisses im Sinne einer sich weiterentwickelten Aufbau- und Ablauforganisation sich verändern (Schneider/Besser/Zerth 2017: 210-213).

Im günstigsten Fall wird im Vergleich zum Status quo die gleiche Pflegekapazität mit einem geringeren Einsatz für die pflegepraktische Tätigkeit ausgestattet sein. Der dahinter liegende ökonomische Wirkungskanal liegt darin begründet, dass personenorientierte Dienstleistungen als Interaktionsarbeit von (1) unterschiedlichen Formen der Zusammenarbeit zwischen Dienstleistungsnehmer*innen und Dienstleistungsgeber*innen gekennzeichnet sind (vorbereitend, gemeinsam durchführend, nachbereitend), dabei (2) diese Zusammenarbeit mit Unsicherheiten über die Mitwirkung der Dienstleistungsnehmer"innen verknüpft ist, somit die damit verbundenen Prozessinformationen unterschiedlicher Akteur*innen beeinflusst und häufig nur unzulänglich interpretiert werden können, die (3) Wertschöpfung beziehungsweise der pflegerelevante Outcome sich schwierig messen

Die Bezugnahme auf operante und operande Ressourcen stammt direkt aus der Diskussion einer »Value-Co-Creation«, die weniger die Nutzung von Dienstleistungen durch verschiedene Akteur*innen, sondern eher die Interaktions- und Kommunikationsbeziehungen im wertschöpfenden Sinn in den Vordergrund stellt (Lusch/Nambisan 2015). 
lassen und letztendlich das (4) organisatorische Setting das Zusammenspiel der Pflegearbeit wesentlich beeinflusst. Es liegt hier eine Wertschöpfungsstruktur einer personenbezogenen Dienstleistung vor (Picot et al. 2012: 274-275).

Technik ist im Sinne einer aufgabenbezogenen Interpretation somit Teil dieser Interaktionsbeziehung und lässt sich so in die methodischen Kategorien aufgabenbezogener Technologiebegriffe (Schreyögg/Geiger 2016: 227) beziehungsweise in weiterer Interpretation als Teil eines soziotechnischen Systems beschreiben (siehe Abbildung 3).

Abbildung 3: Soziotechnisches System Pflege

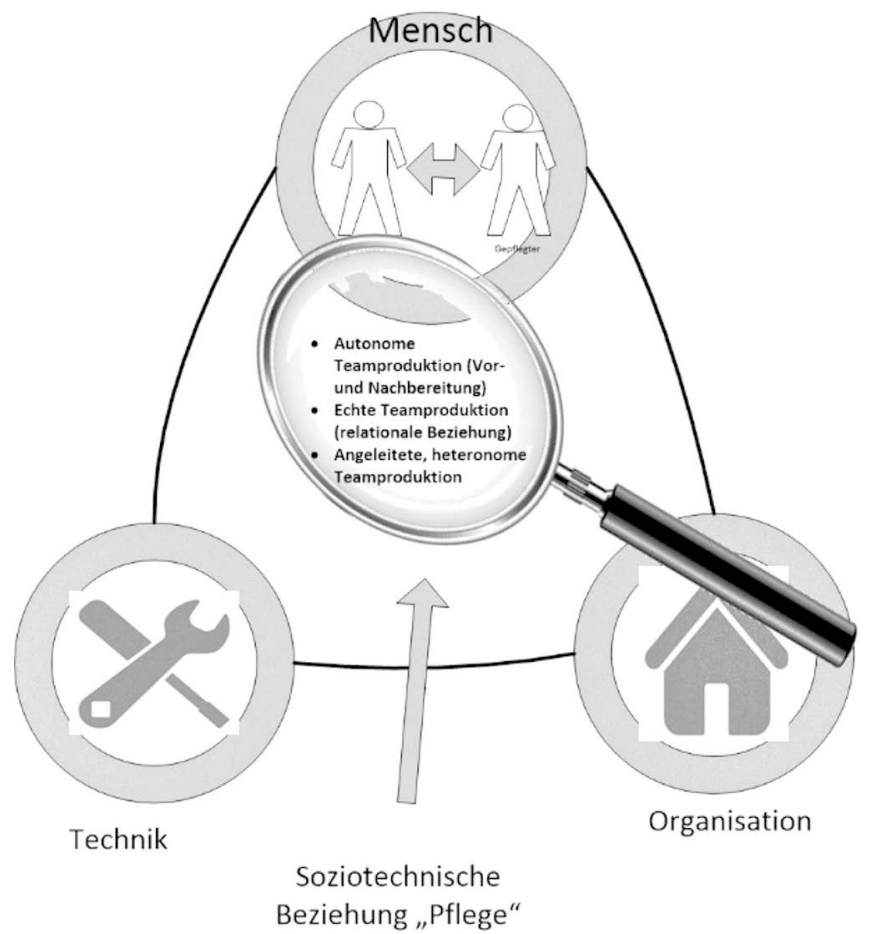

Quelle: Eigene Darstellung

Die möglichen Implikationen von Pflegetechnologien lassen sich in dieser Hinsicht und bei Berücksichtigung der Interaktionsarbeit Pflege unterscheiden, insofern Technologien direkt in der Pflegebeziehung (primäre Interaktion, etwa ein sensorgestütztes Monitor oder ein robotisches System) oder als sekundäre Interaktion (Wirkkanal beim Pflegenden, etwa Pflegedokumentation) eingeordnet werden können und dabei gerade die Prozessinformationen und somit die Qualitätsunsi- 
cherheiten bei unzureichender Kontrolle der Prozessunsicherheiten durch technologischen Einsatz reduziert werden würden (Zerth 2020c: 127-129).

Die Bezugnahme auf den - zumindest im organisierten, professionellen Pflegekontext - relevanten Pflegeprozess aus (1) strukturierter Informationserhebung, (2) klient*innenbezogener Maßnahmenplanung, (3) darauf folgender Durchführung der Pflegemaßnahmen und letztendlich (4) Bewertung des pflegepraktischen Erfolgs gibt Anknüpfungspunkte für die oben geschilderte kapazitätswirksame Veränderung durch einen prozessveränderten Charakter von Pflegetechnologien als Teil des soziotechnischen Systems (Schneider/Besser/Geithner 2020: 622623). Dabei darf jedoch nicht unterstellt werden, dass alle Pflegetechnologien ausschließlich prozessveränderte Effekte nach sich ziehen und viele Anknüpfungspunkte von Technologien - gerade mit Blick auf Hilfsmittel - direkt in der singulären Assistenz an den Pflegebedürftigen ausgelegt sind (Zerth 2017: 244).

Gleichwohl gilt festzuhalten, dass Pflegetechnologien, verstanden als Teil des soziotechnischen Systems der Pflege, die Frage nach einer Veränderung des Careund Skill-Mixes mit Blick auf zu erwartende Effekte bezüglich unterschiedlicher Case-Mix-Szenarien der Pflege implizieren. Technologien werden somit als ein Instrument rationalen Personaleinsatzes interpretiert (Görres/Böttcher/Schumski 2019: 139). Dies gilt insbesondere bei unzureichender Entwicklung pflegerischer Kapazitäten, sei es in der formalen Pflege als auch in der Familienpflege.

Eine Untersuchung von Curtis/Brooks (2020) zu Implementierungsfaktoren digitaler Pflegetechnologien in stationären Pflegeeinrichtungen, bei der sowohl Pflegekräfte als auch Einrichtungsleiter*innen, Gepflegte und deren Angehörige befragt wurden, zeigt die Wahrnehmung der Befragten, indem Technologien durch den zu erwartenden Wirkungseffekt auf die Pflegezeit und die daraus ableitbaren Qualitätseffekte für die Pflege bewertet wurden, was wiederum unmittelbar mit der Attraktivität des Pflegeberufes und der Bereitschaft, in diesem $\mathrm{zu}$ verbleiben, korrespondiert. Auch wenn die Ergebnisse dieser Studie rein auf professionelle Settings rekurrieren, lässt sich die Bedeutung von Technologien als integraler Teil einer Interaktionsbeziehung auch im Sinne des SOK-Modells nach Baltes/Baltes (1990) widerspiegeln (Schmidt/Wahl 2016: 9-10). Dabei weisen Schmidt/Wahl (2016) jedoch nicht auf die unmittelbare Beziehungsebene zwischen Gepflegten und Pflegenden hin, sondern rekurrieren auf die gesellschaftliche Einbettung. Aus einem eher ökonomischen Blickwinkel, etwa in Analogie zum oben zitierten NASSS-Ansatz, lässt sich somit unmittelbar die Frage nach den Nutzenoder Wertversprechen nicht nur auf der individuellen Ebene, sondern letztendlich auf der gesellschaftlichen Ebene ableiten (Greenhalgh et al. 2018: 3). 


\section{Einschub: Effektivität und Effizienz von Pflegetechnologien}

Ein Blick auf die (internationale) Literatur zur Effektivität und letztendlich auch zu Effizienzergebnissen von Pflegetechnologien weist darauf hin, dass es einerseits eine Vielzahl von technologischen Entwicklungen gibt, andererseits eine systematische Erfassung von Effektivitäts- oder gar Effizienzeffekten, insbesondere verknüpft mit nachhaltigen an Evidenzermittlung orientierten Designs, eher spärlich vorhanden ist (Ienca et al. 2017; Krick et al.2019).

Zwar können die Scoping-Reviews deutlich machen, dass es eine wachsende Zahl von Studien gibt, die akteur*innenbezogene Informationen zur handlungsbezogenen Akzeptanz, hier unterschieden in divergente Pflegesettings und auch Technologieklassen, beschreiben. Übertragbare, belastbare Ergebnisse zur Effektivität und insbesondere zur Effizienz sind jedoch noch eher spärlich zu finden. Eine wesentliche Ursache dafür mag auch in der häufig noch unzureichenden empirischen Datenbasis liegen. Die Zahl der Studien, die einen kontrollierten Wirkungsvergleich mit ausreichender Fallzahl und hinreichender Untersuchungsdauer vorweisen können, ist somit noch eher klein (Krick et al. 2019). Folglich sind Aussagen zu versorgungsrelevanten Effekten nur sehr eingeschränkt möglich.

An dieser Stelle lässt sich auch der Blick auf die Bedeutung des Bildes einer soziotechnischen Innovation nochmals aufgreifen. In dieser Fokussierung wird letztendlich die Mehrdimensionalität sichtbar, Technologien in einer gegebenen Interaktions- und Organisationsbeziehung $\mathrm{zu}$ betrachten. Gleichwohl lassen sich mit diesen gesetzten Rahmenbedingungen jedoch für die Ermittlung eines Prädiktions- oder Übertragungswertes, ob eine Technologie auch in strukturund prozessähnlichen Settings ebenfalls einsetzbar ist, nur sehr eingeschränkte Ableitungen treffen. La Rocca (2018) untersucht beispielsweise anhand einer systematischen Literaturanalyse die Bedeutung des Zusammenspiels von Technologien ohne und mit fokussierter Passung in den organisatorischen und institutionellen Kontext. Gesundheits- und Pflegetechnologien haben nach diesem Stand eine höhere Implementierungswahrscheinlichkeit, falls es $\mathrm{zu}$ einem systematischen Dialog und Anpassungsprozess mit den verschiedenen Stakeholder*innen einer Produkt-Dienstleistungskombination kommt.

So ist das soziotechnische Arrangement im Blick einer Technologieimplementierung mit verschiedenen Ebenen der Anwendbarkeit beziehungsweise Übertragbarkeit verbunden, hier in enger Anlehnung an das NASSS-Framework (Greenhalgh et al. 2018:3). Pflegetechnologien als Teil einer komplexen Intervention können zunächst innerhalb eines gegebenen, spezifischen Settings auf die Anwendungstauglichkeit überprüft werden. Abbildung 4 greift das Bild der Passung einer Alltagstauglichkeit der Technologie im Wechselspiel zwischen Mensch, Technik und Organisation auf. Mit dieser Alltagstauglichkeit ist jedoch noch nicht zwangs- 
läufig die Prädiktion für die Übertragbarkeit für weitere rähnlicheく Settings (’was lässt sich für andere Settings ableiten?)) abzuleiten.

Abbildung 4: Soziotechnisches System Pflege-mit veränderten Settings

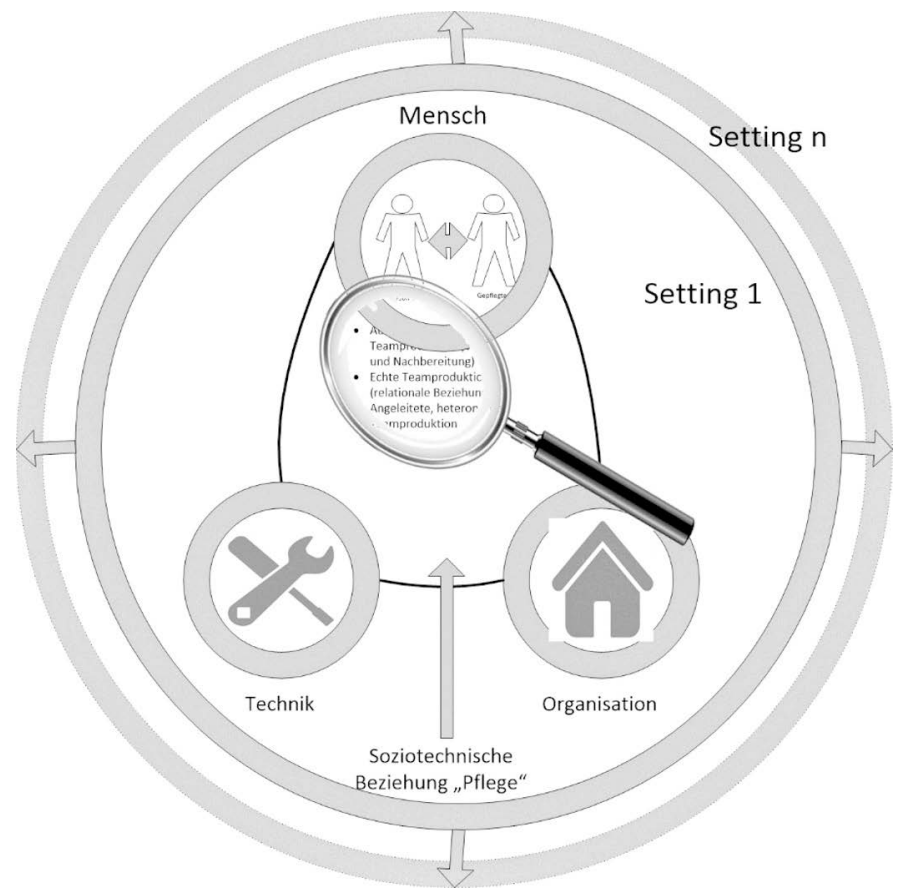

Quelle: Eigene Darstellung

Bezugnehmend auf die Rolle der handlungsrelevanten Stakeholder*innen in pflegerischen Settings (vgl. Studie von Curtis/Brooks 2020) gilt es, die Bedeutung der Pflegetechnologien in enger Verknüpfung mit dem Ablauf und der Organisation des pflegerischen Arbeitsprozesses (insbesondere in professionellen Pflegekontexten) zu betrachten. In der Folge sind projektierte Erwartungen zu Änderungen des Care- und Skill-Mixes bei zunehmender Implementierung von Technologien plausibel. Hier kann beispielsweise der Blick auf die Arbeit von Lu/Rui/Seidmann (2019) hilfreich sein, welche in Bezug auf den US-amerikanischen Kontext die Auswirkungen einer Implementierung eines digitalen Medikationsmanagementsystems in stationären Pflegeeinrichtungen untersucht hat.

Die Autor*innen konnten zeigen - hier hilft die Bedeutung eines erweiterten soziotechnischen Systems Pflege gemäß Abbildung 4 -, dass Wirkeffekte auf den Care-Mix einerseits erst über einen Zeitraum mehrerer Jahre wirksam wurden und 
andererseits die Bedeutung der Rahmensetzung, insbesondere die initiale Struktur des Care-Mixes sowie die Budgetsituation, einen wesentlichen Einfluss hatte. Gerade diejenigen Einrichtungen konnten im Sinne eines Komplementäreffektes stärker profitieren, die mit einem niedrigeren Skill-Mix begonnen haben, das heißt mit einem höheren Anteil gering qualifizierter Pflegekräfte. Somit lässt sich wiederum ein Bezug auf die empirischen Erfahrungen von Digitalisierungstechnologien im Gesundheitswesen allgemein nehmen.

Eine Arbeit von Dranove et al. (2014) zur Analyse von Electronic Health Records (EHR) in akutstationären Einrichtungen betont etwa den komplementären Wirkhebel von Technologien, wie oben eingangs im ersten Abschnitt beschrieben. Diese, zum Beispiel Dokumentationssysteme, werden nicht in der unmittelbaren Pflegebeziehung wirksam, sondern sind durch die komplementäre, systematische Informationsaufarbeitung als Hebel für einen langfristig höheren Wirkeffekt zu interpretieren. Exemplarisch können Dranove et al. etwa zeigen, dass sich Wirkeffekte einer Einführung eines Electronic Health Record weniger für die durchschnittlichen Patient*innen deutlich machen, jedoch bei Patient*innen mit höherer Fallschwere und Komplexität unmittelbar greifen.

Mit Blick auf die genannten Beispiele aus der Literatur lässt sich als Zwischenfazit festhalten, dass gerade die Verknüpfung der akteur*innenbezogenen als auch organisatorischen Bezüge eines gegebenen soziotechnischen Systems und die Veränderung(en) der hierfür relevanten Randbedingungen offensichtlich erklärende Hinweise zur Frage von Implementierungserfahrungen und Implementierungsprädiktionen für Pflegetechnologien notwendig machen.

\section{Was befördert Adoption und Implementierung? Methodische Annäherungen}

\section{Die Wechselbedeutung zwischen Adoption, Implementierung und Technikauswahl}

Wenn Pflegetechnologien sich gut im Bild eines soziotechnischen Systems abbilden lassen, ohne dabei die Bedeutung alleinstehender Technologien, die etwa als Hilfsmittel direkt und weitgehend isoliert den Pflegebedürftigen helfen können, in Abrede zu stellen ${ }^{3}$, dann gilt es die Interaktionsebenen von Pflegearrangements, 
mit anderen Worten den Innovations- und Diffusionsbedingungen von Pflegetechnologien, eine größere Beachtung zu schenken. Dies gilt insbesondere dann, wenn Aussagen zur Implementierungsfähigkeit über ein gegebenes Setting hinaus für andere Pflegearrangements getroffen werden sollen (siehe Abbildung 4).

Pflegetechnologien werden, wenn diese im engeren Sinne für den Versorgungsbereich der Langzeitpflege eingeführt werden sollen, in einem gemischten institutionellen Setting implementiert, in dem sowohl regulierte Vertrags- oder Ausschreibungskontexte marktähnliche Strukturen wiedergeben und gleichzeitig auch die Bedeutung der Familienpflege einen wichtigen Blick auf eine ehrenamtliche, weitgehend informelle Versorgungsstruktur freigibt (Nies/Leichsenring/Mak 2013; Rothgang/Larisch 2014: 227). In diesem Sinne sind einfache Analogien einer Innovationslogik per se schwierig auf den Pflegekontext anzuwenden; vor allem gilt es festzuhalten, dass es insbesondere, wie im Gesundheitswesen auch, ein eher systematisches Auseinanderfallen der Stakeholder*innen-Rollen gibt: (1) Anwender*innen einer Technologie, (2) Nutznießer*innen, (3) Nachfrager*innen und unter Umständen noch (4) Kostenträger*innen (Oberender/Zerth/Engelmann 2017: 134-135).

Die im ersten Kapitel zitierte Studie von Zöllick et al. (2019) weist nicht nur auf das Auseinanderfallen von Kenntnis, Zugang und Nutzung von Pflegetechnologien hin, sondern gibt auch implizit den Hinweis auf die Bedeutung der verschiedenen institutionellen und organisatorischen Arrangements, die letztendlich bei einer Implementierungsentscheidung zu berücksichtigen sind.

So sind exemplarisch in der stationären Pflege die Pflegekräfte zwar die hauptsächlichen Anwender*innen und unter Umständen bei pflegeentlastenden Technologien auch Nutznießer*innen der Technologie, aber nicht die marktwirksamen Nachfrager*innen und letztendlich auch nicht, bei Berücksichtigung verschiedener Refinanzierungsbedingungen, die Kostenträger*innen. In dieser Hinsicht bemühen einige Ansätze in der Literatur das Bild einer auch durch Technik induzierten Organisationsinnovation ${ }^{4}$, die somit die Marktseite einer Investitionsentscheidung (zum Beispiel Einrichtungsleiter*in fungiert als Nachfrager*in) von der Implementierungsseite (Pflegekräfte sind als Fachpromotor*innen innerhalb der Einrichtung für die Nutzung zuständig) trennen können.

diesem Fall die Gehfähigkeit - durch den Gehroller kompensiert würde (vgl. zum Überblick über unterschiedliche Technologieklassen Klein 2020: 21-29).

4 So unterscheiden Omachonu/Einspruch (2010: 14) in einer taxonomischen Form Innovationsprozesse im Cesundheitswesen in die direkt am Versorgungsprozess wirksamen operativen Dimensionen, die wiederum eingebettet sind in Umgebungsdimensionen auf der direkten organisatorischen Ebene, die in einem weiteren Schritt Teil eines institutionellen Settings sind. Auf der grundlegenden industrieökonomischen Ebene kann hier der Bezug zu Roberts (2007) hergestellt werden, der die Bedeutung der Organisations- und Managementimplikationen technologischer Innovationen beschreibt. 
In dieser Hinsicht kann der Implementierungsprozess in verschiedene Teilaspekte zerlegt werden, die etwa zunächst die Auswahlentscheidung für eine Technologie mit den daran anschließenden Implementierungsnotwendigkeiten verknüpfen. In Anlehnung an ein akteur*innenbezogenes Modell nach Behkami/Daim (2016) lässt sich eine Summe der Adoptionsentscheidungen der Akteur*innen aus den Kategorien (A) Wissen und Haltung, (B) befördernde, prozessuale und strukturelle Bedingungen sowie $(C)$ unterstützende persönliche Fähigkeiten zusammenfügen (vgl. Behkami/Daim 2016: 38-39). Somit kann sowohl der Schluss zur Idee der operanden und operanten Ressourcen gezogen, als auch die Hypothese einer Adoptionsneigungsfunktionalität eines definierten pflegerischen Settings fokussiert werden (Zerth 2020: 602). Die Adoptionsneigung eines definierten Settings $i$ lautet folglich:

$$
\text { Adoptionsneigung }\left(\text { Setting }_{\mathrm{i}}\right)=\mathrm{f}_{\mathrm{i}}\left(\mathrm{A}_{\mathrm{i}}+\mathrm{B}_{\mathrm{i}}+\mathrm{C}_{\mathrm{i}}\right)
$$

Die Adoptionsneigung könnte in einer Makrobetrachtung als ein Parameter für die Beförderung und/oder Verzögerung einer Innovationsimplementierung bei bestehender Innovationsneigung einer Organisation oder eines (Sozial-)Unternehmens interpretiert werden (Zerth 2020b: 604). Mit Blick auf die Implementierung im Pflegemarkt und zur Beschreibung einer Inventions-, Innovations- und Imitationslogik könnte die Summe aller Innovations- und Adoptionsneigungen eine Annäherung an die Diffusionsgeschwindigkeit im Markt widerspiegeln, wie es in analoger Weise in ökonomischen Diffusionsmodellen dargelegt wird. ${ }^{5}$

Beim nochmaligen Blick auf die Mikrostrukturen der Implementierung von Pflegetechnologien, insbesondere der Bedeutung der Akteur*innenrollen im Kontext soziotechnischer Systeme, lässt sich im Sinne von Peek et al. (2014) die Unterscheidung zwischen einer Vor-Implementierungs- und Nach-ImplementierungsPrädiktion einwerfen und in freier Analogie auf pflegerische Investitionsentscheidungen anpassen.

Wenn etwa Einrichtungsleiter*innen im Bild des regulierten Marktes die Frage zu berücksichtigen haben, wie diese bei einer Investitionsentscheidung die ReFinanzierung auch mit Blick auf mögliche Wettbewerber*innen im regionalen regulierten Markt berücksichtigen müssen, gilt es bei gegebener Entscheidung dann

$5 \quad$ Hier vergleichend auch exemplarisch die Zusammenfassung von Diffusionsmodellen durch Adler/Kowalczuk (2018). 
die Wahrscheinlichkeit einer erfolgreichen Umsetzung durch die handelnden (Pflege-)Akteur*innen im pflegerischen Setting abzuschätzen, um eine möglichst gute `Ex ante-wie Ex-post-Effizienz`zu gewährleisten. Somit kann im Sinne einer Rückwärtsinduktion eine Abschätzung der Adoptionsneigung eine wesentliche Information für die Optimierung der Ex ante-Investitionsentscheidung werden. Dies erlaubt den Blick auf das soziotechnische System Pflege nochmals anhand eines Fallbeispiels deutlicher zu umreißen.

\section{Adoptionsneigung als Spiegel der Technikbereitschaft und -erwartung: ein Fallbeispiel}

Im Rahmen des BMBF-geförderten Projekts »Pflegepraxiszentrum Nürnberg « (FKZ: 16SV7898) wurden verschiedene Untersuchungen zur grundlegenden Technikbereitschaft von Pflegekräften vorgenommen (Prescher et al. 2020 \& 2021). Ein Beispiel knüpft an der potenziellen Implementierung einer sensorgestützten Technologie zur Dekubitusprophylaxe an. Im Rahmen der Stichprobe wurden 408 pflegekundige Personen zu Aspekten einer ungerichteten Technikbereitschaft sowie im Sinne eines Vignettenbeispiels zur Dekubitusprophylaxe befragt.

Die Vignetten umfassten kurz gesprochen folgende Fallbeispiele, die (hypothetisch) an Pflegekräfte gerichtet waren und neben sozioökonomischen Aspekten, den Kategorien der ungerichteten Technikbereitschaft im Sinne von Neyer/Felber/Gebhardt (2012), nämlich Technikaufgeschlossenheit, Technikkompetenzüberzeugung und Technikkontrollüberzeugung sowie noch je neun Items zu technikbezogenen Kategorien (in Anlehnung an das Technology Usage Inventory nach Kothgassner et al. 2012) integriert haben, wie Nützlichkeit (für Gepflegte und Pflegende), Skepsis (bezüglich der Anwendung) und einem isolierten Item, das ethische Bedenken formuliert hat (Prescher et al. 2021):

- Fall 1: Stellen Sie sich vor, in Zukunft misst ein Sensor in einem intelligenten Pflaster, wann Patient*innen, die sich nicht ausreichend eigenbewegen, umgelagert werden müssen. Das Pflaster wird von den Patient*innen beziehungsweise Bewohner*innen am Körper getragen. Sie erhalten eine Nachricht auf Ihr Endgerät (z.B. Tablet, Computer oder Smartphone): »Bitte Herrn M. umlagern«. Sie gehen zu Herrn M. und lagern ihn um. Das Pflaster dokumentiert das Umlagern automatisch. Sie müssen selbst nicht mehr erkennen, dass umgelagert werden muss, sondern nur noch aktiv umlagern.

- Fall 2: Stellen Sie sich vor, der Sensor aus Fall 1 ist mit der intelligenten Matratze von Herrn M. verbunden. Sobald der Sensor die Notwendigkeit zum Umlagern erkennt, lagert die intelligente Pflegematratze durch einen Wechsel des Druckes Herrn M. selbständig um und dokumentiert das Umlagern. Sie müssen 
also weder erkennen, dass umgelagert werden muss, noch müssen Sie selbständig umlagern.

- Fall 3: Stellen Sie sich vor, dass der Sensor aus Fall 1 mit einem Roboter verbunden ist. Sobald der Sensor die Notwendigkeit zum Umlagern erkennt, lagert der Roboter Herrn M. um und dokumentiert das Umlagern. Sie müssen also weder erkennen, dass umgelagert werden muss, noch müssen Sie selbständig umlagern.

Die Fälle unterscheiden sich aus einer technologischen Sicht insbesondere zwischen den Fällen 1 sowie 2 und 3. Im Fall 1 liegt eine Art shalb-autonomes‘ System vor, da die pflegerelevante Monitoring-Information zwar über den Sensor unabhängig von der Anwesenheit einer Pflegekraft erfolgt und auch ein Algorithmus aufgrund der autonom erhobenen Daten eine Bedarfsmeldung für pflegerische Aktionen an die Pflegekraft weiterleitet, die Handlung selbst muss aber durch die Pflegekraft erfolgen. Bei den Fällen 2 und 3 liegt eine Art sautonomes` System vor, da hier die Handlung auch unabhängig vom unmittelbaren Eingreifen beziehungsweise auch ohne jegliches Eingreifen der Pflegekraft erfolgt - nur die technische Realisierung des Umlagerns unterscheidet sich.

Die Mittelwertvergleiche der abgefragten Konstrukte (1) Nützlichkeit, (2) Skepsis und (3) Ethische Bedenken hat nun gezeigt, dass mit wachsender Entfernung der erwarteten Technologiewirkung aus dem unmittelbaren Interaktionszusammenhang Pflegender zu Gepflegter nicht nur die Nützlichkeit abnimmt, sondern vor allem die Skepsis und die ethischen Bedenken deutlich ansteigen und zwar nicht nur im Vergleich des Falls 1 zu den Fällen 2 und 3, sondern auch isoliert von Fall 2 zu Fall 3, auch wenn von Fall 2 zu Fall 3 technisch mit Blick auf die Handlungsrolle der Pflegekraft im Vergleich zu Szenario 1 keine entscheidende Änderung mehr zu konstatieren ist (siehe Tabelle 1).

Tabelle 1: Vignettenvergleich zur Technikbereitschaft

\begin{tabular}{|l|l|l|l|}
\hline Fallkonstellation & Nützlichkeit (1-5) & Skepsis (1-5) & $\begin{array}{l}\text { Ethische Bedenken } \\
(1-5)\end{array}$ \\
\hline Fall 1 (Pflaster) & 3,70 & 2,54 & 2,38 \\
\hline $\begin{array}{l}\text { Fall 2 (Intelligente } \\
\text { Matratze) }\end{array}$ & 3,47 & 2,94 & 2,93 \\
\hline Fall 3 (Roboter) & 2,79 & 3,64 & 3,94 \\
\hline
\end{tabular}

Quelle: Eigene Darstellung nach Prescher et al. (2021) 
Eine Möglichkeit zur Interpretation der Ergebnisse könnte in der Tatsache begründet sein, dass Pflegekräfte sowohl ihr Handeln als auch das Ergebnis ihres pflegerischen Tuns in einer professionellen Verantwortung halten wollen. Je schwieriger eine Technik angesichts ihrer Eigenschaften aus Sicht der Pflegekräfte $\mathrm{zu}$ kontrollieren beziehungsweise gegenüber den Pflegebedürftigen (und gegebenenfalls auch gegenüber anderen Stakeholder*innen wie den Sorgeberechtigten) $\mathrm{zu}$ garantieren ist, desto geringer scheint die perspektivische Technikbereitschaft ausgeprägt $\mathrm{zu}$ sein. Im Vergleich $\mathrm{zu}$ Fall 2 könnten im Fall 3 deutlich mehr potenzielle Handlungen durch den Pflegeroboter denkbar sein.

Mit Bezug auf das obige Bild der Adoptionsneigung könnte somit die Bedeutung des Wechselspiels zwischen der individuellen pflegerischen Kompetenz der Pflegekundigen, der erwarteten Eigenschaften (in der Nutzung und der Wirkung) der zu implementierenden Technologie sowie den damit korrespondierenden Informationen $\mathrm{zu}$ beabsichtigten Einsatzszenarien im konkreten Pflegesetting für die Adoptionsneigung eine hohe Relevanz besitzen. Auch wenn der Fall 2 unmittelbar die Pflegekraft in eine zunächst lediglich kontrollierende und nicht eingreifende Handlungsrolle versetzt, lässt sich der Möglichkeitsspielraum für die Veränderung der Interaktionsbeziehung Pflege aus dem Fall 2 deutlicher abzeichnen als im Fall 3. Welche Rückkoppelungen zum Bild eines Adoptions- und Implementierungsszenarios lassen sich nun ableiten?

\section{Grobe Implikationen für ein (gesundheitsökonomisches) Implementierungsmuster}

Die bisherigen Ausführungen machen deutlich, dass mit dem Bild des soziotechnischen Systems, gerade mit Blick auf sich veränderte und erweiterte Settingeinordnungen (siehe Abbildung 4), Technologien sowohl in Hinblick auf die Handlungsals auch Interaktionsrolle $\mathrm{zu}$ beschreiben sind. Die Scopinguntersuchungen etwa von Krick et al. (2019) oder auch Ienca et al. (2017) weisen jedoch auf die geringe empirische Belastungsfähigkeit der meisten Studien hin, zumindest wenn es um die Implementierungstauglichkeit in realen, alltagstauglichen Settings geht. Somit lässt sich mit gewisser Plausibilität der Notwendigkeit der Förderung umsetzungsrelevanter Organisationsexperimente das Wort reden, also Formen des kontrollierten Experimentierens in realweltlichen Umgebungen (Zerth 2020). Ausgehend von einer derartigen Betrachtungsidee bleiben aber noch wesentliche Fragen zur Gestaltung solcher kontrollierten Experimentiermodelle übrig.

Wenn zur Beschreibung einer Technikimplementierung als Teil eines soziotechnischen Systems zunächst die Handlungsrollen der Investitionsentscheider*innen (etwa Einrichtungsleiter*in als >Nachfrager*in`) und der Anwender*innen und Nutznießer*innen (Pflegekräfte als hauptsächliche Anwender*innen) zweckmäßig zu differenzieren sind und diese dann im Kontext pflegepraktischer 
Bedarfe in den Bedingungen des Pflegesettings interpretiert werden müssen, dann lässt sich ein Bild einer phasenorientierten Adoptions- und Implementierungsstrategie denken. Dieses Bild, in weiter Anlehnung an Schäfer/Keppler (2013: 39) sowie Behkami/Daim (2016), greift die Idee von Rogers (1995) auf, Adoption und Diffusion als Wissens- und Lernprozess zu beschreiben (Zerth 2020b: 608-609). Mit Bezug auf das soziotechnische System Pflege wäre es dann denkbar, exemplarisch drei Stufen des Auswahlphänomens zu nutzen, gefolgt von zwei Stufen der Implementierung:

- Die Auswahlentscheidung würde zunächst das (1) Wissen über ein ausgewähltes pflegepraktisches Problem und den dazu passenden Technologien mit einer (2) wissensbasierten Prüfung verknüpfen, um zu testen, ob eine potenzielle Pflegetechnologie angesichts ihrer pflegerelevanten Eigenschaften und der induzierten Finanzierungsaspekte einen Zusatznutzen gegenüber einer Status quo-Lösung erzeugt. Nach Abschluss dieser Phase erfolgt dann die (3) wissensbasierte Entscheidung zur Implementierung oder Nicht-Implementierung. Diese Entscheidung würde in einem Idealmodell schon die

- (4) Implementierungsphase mitdenken, die im Fall einer Umsetzung einer Investitionsentscheidung die Adoption der Technologie im Sinne einer Organisationsinnovation zur Folge hätte und die oben bereits skizzierten Aspekte der Adoptionsbereitschaft(en) gerade von Pflegekräften in den Blick nehmen müsste. Die Begleitung und ein organisatorisches Lernen während des Adoptionsprozesses sind die Folge. Im (5a) Falle, dass eine projektierte Technologie nicht angeschafft würde, wäre auch zu überlegen, ob das identifizierte pflegepraktische Problem durch Veränderungen im Status quo organisiert werden könnte. Im (5b) Falle der Anschaffung wäre perspektivisch zu kontrollieren, ob sich die Implementierbarkeit letztendlich auch erfolgreich umsetzen lässt.

Folgt man dem Narrativ dieses Phasenmodells, liegt ein möglicher ordnender Handlungsblick auf weitgehend relevante Stufen notwendiger Adoptions- und Diffusionsaspekte vor, die sich hinsichtlich zu wählender Dimensionen etwa mit entsprechenden Rahmenmodellen zur Technikbewertung und -auswahl, etwa dem NASSS-Framework oder auch dem kürzlich veröffentlichen Framework eines »digital nursing technology outcome framework « von Krick et al. (2020) verknüpfen lassen. Im letztgenannten Framework werden explizit, immer gewichtet nach outcome-relevanten Perspektiven, Handlungszuordnungen an eine akteur*innenbezogene Effektivität, einer interaktionsbezogenen Effektivität sowie einer organisationsbezogenen Effektivität genutzt (ebd.: 6).

Es gilt jedoch festzuhalten, dass in den bisher herangezogenen Überlegungen Technologien als gegebenes Sujet interpretiert wurden, mit denen Veränderungen auf der Akteur*innen-, Interaktions- und Organisationsbeziehung im gegebenen so- 
ziotechnischen System betrachtet werden müssen. Die Auseinandersetzung mit einer weiteren Rolle von Hersteller*innen und/oder Technologieanbieter*innen in Kontext des soziotechnischen Systems wurde ausgeblendet beziehungsweise nicht näher erörtert. Es wäre aber zu überlegen, ob nicht gerade in weiterer Analogie an das industrieökonomische Bild des Wechselspiels eines anbieter*innenseitigen stechnology push < und eines bedarfs- beziehungsweise nachfrageseitigen >demand-pulk (Godin/Lane 2013) noch eine nicht nur interessante, sondern auch notwendige weitere Interaktionsbeziehung in einer erweiterten Schicht des soziotechnischen Bildes integriert werden könnte.

Shaw et al. (2018) versuchen das Wechselspiel zwischen Pull- und PushFaktoren in einer Heuristik aus den Merkmalen >Team+Routine`vor Technikeinführung und >Team+Tool+Routine nach Technikeinführung zu betrachten (ebd. 2018: 4), was Ähnlichkeiten zum oben formulierten Bild der soziotechnischen Innovation hat, jedoch in letztgenannter Form grundsätzlich unterstellt wird, dass auch im Status quo Technologien (tools) bereits vorhanden waren. Hier in dezidierterer Form phänomenologisch den Charakter von Pflegetechnologien weiterzuentwickeln, bietet Raum für (anwendungsorientierte) zukünftige Forschung.

Gleichwohl gilt festzuhalten, dass der groben Skizzierung des obigen Phasenmodells, betrachtet in Kombination mit einem mehrstufigen Bild des soziotechnischen Systems, Orientierungspunkte für umsetzungsrelevante Implementierungsinformationen, unter Umständen gar als eine Art Annäherung an eine Implementierungsprädiktion, hinzugefügt werden können. So könnte in einer ersten Stufe der Implementierungsprognose (Technologie wird in einem gegebenen Setting implementiert) sowohl die Adoptionsneigungen der Umsetzungspromotor*innen entwickelt (etwa Pflegekräfte in der stationären Altenpflege) als auch die Bezugnahme auf die Investitionsentscheider*innen hergestellt werden. Mit der Differenzierung der Rollenzuschreibungen Nutzer*in (Anwender*in), Nutznießer*in, Nachfrager*in und Kostenträger*in sind dann Bezugnahmen auf eine mögliche Übertragbarkeit auf ein struktur- und prozessähnliches Setting in einer zweiten Stufe plausibel möglich. Im Idealfall gilt es dann die Adoptionsneigung innerhalb eines gegebenen Settings daraufhin zu kontrollieren, wie isoliert oder übertragbar diese für ähnliche Settings sind.

Notwendige Kriterien der Abbildung von Implementierbarkeit liegen im gemeinsamen Betrachten akteur*innen- und handlungsbezogener Kriterien und Dimensionen, insbesondere auch auf die Wirkung- und Outcome-Orientierung fokussiert, wie etwa Krick et al. (2020) es formulieren. Darüber hinaus gilt es das Zusammenwirken der unmittelbar handelnden Stakeholder*innen (innerhalb eines definierenden Settings) mit den Stakeholder*innen in den Blick zu nehmen, etwa Nachfrager*innenrollen oder Kostenträger*innen, die für die langfristige Entscheidung über die Implementierung einer Technologie verantwortlich zeichnen. 
In gewisser Weise liegt hier ein Forschungsprogramm im Sinne der Schaffung pflegerelevanten Adoptions- und Implementierungswissens zum Abbau von Markteintrittsschranken in den Pflegemarkt vor.

\section{Schlussimpuls}

Nicht nur plausible Hinweise und anekdotische Evidenzen, sondern auch wachsende empirische Belege machen deutlich, dass technische Innovationen in den seltensten Fällen an der Technik im engeren Sinne scheitern, sondern unzureichende Beachtung oder Kenntnis beziehungsweise auch Handlungsanleitungen zu akteur*innenbezogenen, organisatorischen, organisationskulturellen und letztendlich systematischem Implementierungswissen fehlen. Auch wenn Pflegetechnologien in einer hohen Variabilität verfügbar und einsetzbar sind, gilt es doch strenger den Blick auf ihr Wirk- und Nutzungsversprechen zunächst in der Sorgebeziehung Pflege, im Kontext des adäquaten Settings und letztendlich in der institutionellen Zuordnung zu legen.

Das Narrativ des Phasenmodells in Kombination mit den Schichten soziotechnischer Systeme greift Ideen von Frameworks, exemplarisch dem NASSSFramework, auf und könnte aber noch stärker beitragen, zwischen akteur*innen-, interaktions- und organisationsrelevanten Fragestellungen $\mathrm{zu}$ unterscheiden. Somit tragen unterschiedliche Forschungs- und insbesondere stärker zu fördernde Anwendungskontexte dazu bei, pflegerelevantes Implementierungswissen systematisch zu erheben, zu sammeln und daraus zu lernen.

Letztendlich sind Fragen der Implementierung von Pflegetechnologien nicht von den übergeordneten Fragen zur Weiterentwicklung des regulierten Pflegemarktes und der institutionellen Strukturen des Sorgesystems Pflege zu trennen. So sind Ansätze erfolgreicher Technologieimplementierungen als Teil einer Infrastruktur Pflege zu betrachten, die etwa veränderte Informations-, Finanzierungs-, Zulassungs- und Bewertungsbedingungen umfassen. Inwiefern beispielsweise Entwicklungen von Plattformen als hilfreiche Anknüpfungspunkte auch für ein kontrolliertes Experimentieren mit pflegerischen Sorgeangeboten und mit Technik im Kontext dieser Plattformen verstanden werden können (Greschke 2017), bleibt zu diskutieren. 


\section{Literatur}

Abimbola, Seye/Patel, Bindu/Peiris, David/Patel, Anushka/Harris, Mark/Usherwood, Tim/Greenhalgh, Trisha (2019): The NASS framework for ex post theorisation of technology-supported change in healthcare: Worked example of the TORPEDO programme, in: BMC Medicine 17, S. 1-17.

Adler, Jost/Kowalczuk, Pascal (2018): Abbildung der Diffusion von Innovationen anhand des Bass-Modells: Möglichkeiten der Parameterschätzung, in: WiST 9, S. 10-17.

Baltes, Paul/Baltes, Margret (1990): Psychological perspectives on successful aging: The model of selective optimization with compensation, in: Paul Baltes/Margret Baltes (Hg.): Successful aging: Perspectives from the behavioral sciences. New York: Cambridge University Press, S. 1-34.

Becka, Denise/Borchers, Uwe/Evans, Michaela (2016): Pflegen, Kümmern und Begleiten: Berufspolitische Entwicklungspfade zur Sorgearbeit vor Ort, in: Klaus Jacobs/Adelheid Kuhlmey/Stefan Greß/Jürgen Klauber/Antje Schwinger (Hg.): Pflege-Report 2016. Schwerpunkt: Die Pflegenden im Fokus. Stuttgart: Schattauer, S. 151-167.

Behkami, Nima/Daim, Tugrul (2016): Methods and Models, in: Tugrul Daim/Nima Behkami/Nuri Basoglu/Orhun Kök/Liliya Hogaboam (Hg.): Healthcare Technology Innovation Adoption. Electronic Health Records and Other Emerging Health Information Technology Innovations. Heidelberg, New York, Dordrecht, London: Springer, S. 37-81.

Böhle, Fritz (2011): Interaktionsarbeit als wichtige Arbeitstätigkeit im Dienstleistungssektor, in: WSI Mitteilungen 9, S. 456-461.

Bornewasser, Manfred/Frenzel, Stefan/Tombeil, Anne-Sophie (2016): Ansatzpunkte zur Bestimmung der Produktivität von Dienstleistungen im Krankenhauskontext, in: Mario Pfannstiel/Christoph Rasche/Harald Mehlich (Hg.): Dienstleistungsmanagement im Krankenhaus. Nachhaltige Wertgenerierung jenseits der operativen Exzellenz. Wiesbaden: Springer Gabler, S. 47-70.

Curtis, Katherine/Brooks, Sheila (2020): Digital health technology: Factors affecting implementation in nursing homes, in: Nursing Older People 32, S. 14-21.

Daum; Mario/Ploch, Uwe/Werkmeister, Till (2017): Digitalisierung und Technisierung der Pflege in Deutschland. Aktuelle Trends und ihre Folgewirkungen auf Arbeitsorganisation, Beschäftigung und Qualifizierung. Hg. v. DAA-Stiftung Bildung und Beruf. Hamburg. Online verfügbar unter https://www.daa-stiftung.de/fileadmin/user_upload/digitalisierung_und_ technisierung_der_pflege_2.pdf vom, zuletzt geprüft am 27.09.2020.

Dranove, David/Forman, Chris/Goldfarb, Avi/Greenstein, Shane (2014): The Trillion Dollar Conundrum: Complementarities and Health Information Technology, in: American Economic Journal: Economic Policy 6, S. 239-270. 
Fachinger, Uwe (2017): Technikeinsatz bei Pflegebedürftigkeit, in: Klaus Jacobs/Adelheid Kuhlmey/Stefan Greß/Jürgen Klauber/Antje Schwinger (Hg.): Pflege-Report 2017. Schwerpunkt: Die Versorgung der Pflegenden. Stuttgart: Schattauer, S. 83-93.

Godin, Benoît/Lane, Joseph (2013): Pushes and Pulls: Hi(S)tory of Demand Pull Model of Innovation, in: Science, Technology, \& Human Values 38, S. 621-654.

Görres, Stefan/Böttcher, Silke/Schumski Lisa (2019): Rationaler Personaleinsatz in der Alten- und Langzeitpflege, in: Klaus Jacobs/Adelheid Kuhlmey/Stefan Greß/Jürgen Klauber/Antje Schwinger (Hg.): Pflege-Report 2019. Mehr Personal in der Langzeitpflege - aber woher? Berlin: Springer, S. 137-145.

Greenhalgh, Trisha/Wherton, Joe/Papoutsi, Chrysanthi/Lynch, Jenni/Hughes, Gemma/A'Court, Christine/Hinder, Sue/Procter, Rob/Shaw, Sara (2018): Analysing the role of complexity in explaining the fortunes of technology programmes: Empirical application of the NASSS framework, in: BMC Medicine 16:66.

Greschke, Maximilian (2017): Marktplätze in der Pflege - Konsolidierung und Effizienzsteigerung durch Digitalisierung, in: David Matusiewicz/Christian Pittelkau/Arno Elmer (Hg.): Die Digitale Transformation im Gesundheitswesen. Berlin: Medizinisch Wissenschaftliche Verlagsgesellschaft, S. 183-187.

Hecker, Christian (2013): "Soziale Gerechtigkeit« als Befähigungsgerechtigkeit-Subsidiarität, Verantwortungsfähigkeit und Eigenverantwortung im Rahmen liberaler Ordnungspolitik und christlicher Gesellschaftskritik, in: ORDO 64, S. 99-133.

Hoberg, Rolf/Klie, Thomas/Künzel, Gerd (2013): Strukturreform Pflege und Teilhabe. Politikentwurf für eine nachhaltige Sicherung von Pflege und Teilhabe. Freiburg. Online verfügbar unter https://agp-freiburg.de/downloads/pflege-teilha be/Reformpaket_Strukturreform_PFLEGE_TEILHABE_Langfassung.pdf, zuletzt geprüft am 10.03.2018.

Hülsken-Giesler, Manfred/Zelt, Thilo/Weidner, Frank (Hg.) (2017): ePflege. Informations- und Kommunikationstechnologie für die Pflege. Studie im Auftrag des Bundesministeriums für Gesundheit. Berlin, Vallendar, Köln: Roland Berger $\mathrm{GmbH}$, Deutsches Institut für angewandte Pflegeforschung e. V., Philosophisch-Theologische Hochschule Vallendar.

Ienca, Marcello/Fabrice, Jotterand/Elger, Bernice/Caon, Maurizio/Pappagallo, Alessandro/Kressig, Reto/Wangmo, Tenzin (2017): Intelligent Assitive Technology for Alzheimer's Disease and Other Dementias: A Systematic Review, in: Journal of Alzheimer's Disease 56, S. 1301-1340.

Isfort, Michael/Rottländer, Ruth/Weidner, Frank/Gehlen, Danny/Hylla, Jonas/Tucman, Daniel (2018): Pflege-Thermomether 2018. Eine bundesweite Befragung von Leitungskräften zur Situation der Pflege und Patientenversorgung in der stationären Langzeitpflege in Deutschland. Hg. v. Deutsches 
Institut für angewandte Pflegeforschung e. V. Köln. Online verfügbar unter ht tps://www.dip.de/fileadmin/data/pdf/projekte/Pflege_Thermometer_2018.pd f, zuletzt geprüft am 26.09.2020.

Klein, Barbara (2020): Hilfsmittel, Assistive Technologien und Robotik. Selbstständigkeit und Lebensqualität im Alter erhalten. Stuttgart: Kohlhammer.

Kothgassner, Oswald D./Felmhofer, Anna/Hauk, Nathalie/Kastenhoer, Elisabeth/Gomm, Jasmine/Kryspin-Exner, Ilse (2012): TUI. Technology Usage Inventory. Hg. v. ICARUS Research Team 2012. Wien. Online verfügbar unter https://www.ffg.at/sites/default/files/allgemeine_downloads/thematisc he\%2oprogramme/programmdokumente/tui_manual.pdf, zuletzt geprüft am 29.01.2021.

Krick, Tobias/Huter, Karin/Domhoff, Dominik/Schmidt, Annika/Rothgang, Heinz/Wolf-Ostermann, Karin (2019): Digital technology and nursing care: A scoping review on acceptance, effectiveness and efficiency studies of informal and formal care technologies, in: BMC Health Service Research 19, S. 1-15.

Krick, Tobias/Huter, Karin/Seibert, Kathrin/Domhoff, Dominik/Wolf-Ostermann, Karin (2020): Measuring the effectiveness of digital nursing technologies: Development of a comprehensive digital nursing technology outcome framework based on a scoping review, in: BMC Health Service Research 20:243.

La Rocca, Antonella (2018): Networked Innovation in Healthcare: Literature Review and Research on the Interplay of Inner and Outer Contexts of Innovation, in: Thomas Hoholm/Antonella La Rocca/Margunn Aanestad (Hg.): Controversies in Healthcare Innovation. Basingstoke: Palgrave Macmillan, S. 247-277.

Lu, Susan/Rui, Huaxia/Seidmann, Abraham (2019): Does Technology Substitute for Nurses? Staffing Decisions in Nursing Homes, in: Management Sciences 64, S. $1842-1859$.

Lusch, Robert/Nambisan, Satish (2015): Service Innovation: A Service-Dominant Logic Perspective, in: MIS Quaterly 39, S. 155-175.

Merda, Meiko/Schmidt, Kristina/Kähler, Bjørn (2017): Pflege 4.0 - Einsatz moderner Technologien aus Sicht professionell Pflegender. Forschungsbericht. Hg. v. Berufsgenossenschaft für Gesundheitsdienst und Wohlfahrtspflege (BGW). Hamburg. Online verfügbar unter https://www.bgw-online.de/SharedDocs/ Downloads/DE/Medientypen/BGW\%20Broschueren/BGWo9-14-002-Pflege4-o-Einsatz-moderner-Technologien_Download.pdf?_blob=publicationFile, zuletzt geprüft am 05.05.2021.

Neyer, Franz/Felber, Juliane/Gebhardt, Claudia (2012): Entwicklung und Validierung einer Kurzskala zur Erfassung der Technikbereitschaft, in: Diagnostica 58, S. 123-134.

Nies, Henk/Leichsenring, Kai/Mak, Sabrina (2013): The Emerging Identity of Long-Term Care and the Role of Informal Carers, in: Kai Leichsenring/Jenny 
Billings/Henk Nies (Hg.): Long-Term Care in Europe. Improving Policies and Practice. Houndsmill: Palgrave Macmillan, S. 19-41.

Nitsche, Sabine (2017): Gesundheitskompetenz im Unternehmen: Der richtige »Fit « zwischen Verhalten und Verhältnis, in: Uwe Bettig/Mona Frommelt/Martina Roes/Roland Schmidt/Günter Thiele (Hg.): Pflegeberufe der Zukunft: Akademisierung, Qualifizierung und Kompetenzentwicklung. Jahrbuch Pflegemanagement. Heidelberg: medhochzwei, S. 51-62.

Oberender, Peter/Zerth, Jürgen/Engelmann, Anja (2017): Wachstumsmarkt Gesundheit. 4. Auflage. Konstanz, München: UVK Verlagsgesellschaft.

Omachonu, Vincent/Einspruch, Norman (2010): Innovation in Healthcare Delivery Systems: A Conceptual Framework, in: The Innovation Journal: The Public Sector Innovation Journal 15, S. 1-20.

Peek, Sebastian/Wouters, Eveline/Van Hoof, Joost/Luijkx, Katrien/Boeije, Hennie/Vrijhoef, Hubertus (2014): Factors influencing acceptance of technology for aging in place: A systematic review, in: International Journal of Medical Informatics 83, S. 235-248.

Picot, Arnold/Dietl, Helmut/Franck, Egon/Fiedler, Marina/Royer, Susanne (2012): Organisation: Theorie und Praxis aus ökonomischer Sicht. Stuttgart: Schäffer Poeschel.

Prescher, Thomas/Müller, Sebastian/Jaensch, Peter/Schneider, Michael/Zerth, Jürgen (2021): Technik, Pflege und prozessorientierte Organisationsentwicklung: Nachhaltige Implementierung durch gezielte Bildungsmaßnahmen, in: Carolin Freier/Joachim König/Arne Manzeschke/Barbara Städtler-Mach (Hg.): Gegenwart und Zukunft sozialer Dienstleistungsarbeit. Springer VS, im Erscheinen.

Prescher, Thomas/Schneider, Michael/Müller, Sebastian/Jaensch, Peter/Zerth, Jürgen (2020): Technik, Pflege und prozessorientierte Lernkulturentwicklung. Konstitution und Konstruktion der Technikimplementierung in Pflegeeinrichtungen am Beispiel der Dekubitusprophylaxe, in: Pädagogik der Gesundheitsberufe 7, S. 115-121.

Roberts, Edward (2007): Managing Invention and Innovation, in: Research Technology Management 50, S. 35-54.

Rogers, Everett (1995): Diffusion of Innovations. $4^{\text {th }}$ edition. New York: The Free Press.

Rothgang, Heinz/Larisch, Joachim (2014): Pflegeökonomie - eine neue Subdisziplin der Gesundheitsökonomie, in: David Matusiewicz/Jürgen Wasem (Hg.): Gesundheitsökonomie. Bestandsaufnahme und Entwicklungsperspektive. Berlin: Duncker \& Humblot, S. 211-240.

Schäfer, Martina/Keppler, Dorothee (2013): Modelle der technikorientierten Akzeptanzforschung. Überblick und Reflexion am Beispiel eines Forschungsprojekts zur Implementierung innovativer technischer Energieeffizienz-Maßnahmen. Berlin: TU Berlin, Zentrum Technik und Gesellschaft. 
Schallermair, Christian (1999): Ökonomische Merkmale sozialer Dienstleistungen und deren Beschäftigungspotentiale am Beispiel der stationären Altenpflege. Bayreuth: P.C.O.Verlag.

Schmidt, Laura/Wahl, Hans-Werner (2016): Wie verändert Technik das Alter(n) und die Gerontologie?, in: Angewandte Gerontologie Appliquée 1, S. 7-10.

Schneider, Michael/Besser, Jürgen/Geithner, Silke (2020): Technologische Innovationen in der Pflege: Von der routinebasierten zur anlassinduzierten Pflege, in: Mario Pfannstiel/Kristin Kassel/Christoph Rasche (Hg.): Innovation und Innovationsmanagement im Gesundheitswesen. Technologien, Produkte und Dienstleistungen voranbringen. Wiesbaden: Springer Gabler, S. 615-632.

Schneider, Michael/Besser, Jürgen/Zerth, Jürgen (2017): Individualisierung durch Digitalisierung am Beispiel der stationären Pflegeversorgung - Organisationsund informationsökonomische Aspekte, in: Mario Pfannstiel/Patrick DaCruz/Harald Mehlich (Hg.): Digitale Transformation von Dienstleistungen im Gesundheitswesen II. Impulse für das Management. Wiesbaden: Springer Gabler, S. 205-226.

Schreyögg, Georg/Geiger, Daniel (2016): Organisation. Grundlagen moderner Organisationsgestaltung. Mit Fallstudien. 6. Auflage. Wiesbaden: Springer Gabler.

Shaw, James/Agarwal, Payal/Desveaux, Laura/Palma, Daniel/Stamenova, Vess/Jamieson, Trevor/Yang, Rebecca/Bhatia, Sacha/Bhattacharyya, Onil (2018): Beyond »implementation«: Digital health innovation and service design, in: npj Digital Medicine 1:48.

Sickau, Sabine/Thiele, Günter (2017): Die pflegerische Arbeit und der Umgang mit deren Anforderungen, in: Uwe Bettig/Mona Frommelt/Martina Roes/Roland Schmidt/Günter Thiele (Hg.): Pflegeberufe der Zukunft: Akademisierung, Qualifizierung und Kompetenzentwicklung. Jahrbuch Pflegemanagement. Heidelberg: medhochzwei, S. 37-50.

Weiß, Christine/Lutze, Maxie/Gissendanner, Scott/Peters, Verena (2017): Nutzen und Finanzierung technischer Assistenzsysteme aus Sicht der Pflegeversicherung und weiterer Akteure der Verantwortungsgemeinschaft am Beispiel der Quartiersvernetzung - Abschlussbericht. Gefördert vom Bundesministerium für Gesundheit (BMG). Berlin, Online verfügbar unter https://www.iit-berlin. $\mathrm{de} / \mathrm{de} /$ publikationen/nutzen-und-finanzierung-technischer-assistenzsysteme -aus-sicht-der-pflegeversicherung-und-weiterer-akteure-der-verantwortung sgemeinschaft-am-beispiel-der-quartiersvernetzung/at_download/download, zuletzt geprüft am 27.09.2020.

Zerth, Jürgen (2017): Assistenzsysteme als Teil einer Organisationsinnovation in der häuslichen Pflege, in: Herbert Rebscher/Stefan Kaufmann (Hg.): Digitalisierungsmanagement in Gesundheitssystemen. Heidelberg: medhochzwei, S. 239-257. 
Zerth, Jürgen (2020): Effektivität und Effizienz von Pflegetechnologien - was lässt sich sagen? Eine erste Annäherung. Hg. v. MedtecOnline. Online verfügbar unter https://medteconline.de/impulse/blog/detail/effektivitat-und-effizienz-vo n-pflegetechnologien-was-lasst-sich-sagen-eine-erste-annaherung-1, zuletzt geprüft am 08.09.2020.

Zerth, Jürgen (2020a): Aktuelle Herausforderungen der Sozialen Marktwirtschaft - Wohlfahrtsstaat, das Beispiel »Langzeitpflege«: Neuformulierung zwischen befördernden Strukturen und organisierter Pflegeinfrastruktur, in: Christian Müller/Elmar Nass/Johannes Zabel (Hg.): Soziale Marktwirtschaft - Ordnung der Zukunft. Münster: Aschendorff-Verlag, S. 121-141.

Zerth, Jürgen (2020b): Innovation und Imitation - zur Diskussion einer nachhaltigen Implementierung. Eine Betrachtung am Beispiel von technischen Innovationen im Pflegemarkt, in: Mario Pfannstiel/Kristin Kassel/Christoph Rasche (Hg.): Innovation und Innovationsmanagement im Gesundheitswesen. Technologien, Produkte und Dienstleistungen voranbringen. Wiesbaden: Springer Gabler, S. 597-614.

Zerth, Jürgen (2020c): Digitalisierung und Pflege - anlassbezogene Pflege, Plattformen und Implikationen für Effektivität, Effizienz und mögliche Ordnungsregeln, in: Jürgen Zerth/Hedwig Francois-Kettner (Hg.): Pflege-Perspektiven: ordnungspolitische Aspekte. Erkenntnisse aus der Versorgungsforschung und Implikationen für eine "gute Praxis« der Pflege. Heidelberg: medhochzwei, S. 119-136.

Zöllick, Jan/Kuhlmey, Adelheid/Suhr, Ralf/Eggert, Simon/Nordheim, Johanna/Blüher, Stefan (2019): Akzeptanz von Technikeinsatz in der Pflege. Zwischenergebnisse einer Befragung unter professionell Pflegenden, in: Klaus Jacobs/Adelheid Kuhlmey/Stefan Greß/Jürgen Klauber/Antje Schwinger (Hg.): Pflege-Report 2019. Mehr Personal in der Langzeitpflege - aber woher? Berlin: Springer, S. 211-218. 



\section{Autor*innen}

Amelie Altenbuchner, M. A., ist Erziehungswissenschaftlerin und wissenschaftliche Mitarbeiterin am Institut für Sozialforschung und Technikfolgenabschätzung (IST) an der OTH Regensburg. Ihre Hauptaufgabe liegt in der Ausgestaltung verschiedener Aspekte der quantitativen Forschungsmethodik. Im Rahmen interdisziplinärer und multi-professioneller sozial- und gesundheitswissenschaftlicher Projekte, wie dem Forschungsprojekt zur Nutzbarkeit von Aktivitätstrackern in der Alterstraumatologie, gehören auch Forschungsethik und Technikfolgenabschätzung zu ihren Aufgaben.

Andreas Bischof, Dr. phil., ist Nachwuchsgruppenleiter an der Technischen Universität Chemnitz. Er wurde dort 2016 mit einer ethnografischen Überblicksstudie $\mathrm{zu}$ epistemischen Praktiken der Sozialrobotik promoviert. $\mathrm{Zu}$ seinen Forschungsschwerpunkten zählen Digitale Kulturen, Technik für Alter(n), Mensch-Roboter-Interaktion sowie inter- und transdisziplinäre Methodologien. Er engagiert sich besonders für integrierte Projektkonstellationen in der MenschTechnik-Interaktion, in denen sowohl Perspektiven Betroffener als auch sozialund geisteswissenschaftlicher Disziplinen frühzeitig und entscheidungsmächtig eingebunden sind. Ergebnisse dieser Arbeit sind unter anderem das »Netzwerk Integrierte Forschung « (BMBF) und das internationale Socio-Gerontechnology Network.

Elmar Buchner, Priv.-Doz. Dr. rer. nat., ist Geowissenschaftler, arbeitet aber auch an den Schnittstellen der Geowissenschaften zur Sozial- und Gesundheitsforschung. In diesem Zusammenhang ist er stellvertretender Leiter des DigiHealth Instituts der Hochschule Neu-Ulm (HNU). Neben seinen Aufgaben in der Hochschulleitung der HNU beschäftigt er sich mit Themen wie dem Monitoring von potenziellen epidemiologischen Risikofaktoren (z.B. im Projekt SWAMMP) oder mit ethischen Gesichtspunkten und der damit einhergehenden gesellschaftlichen Akzeptanz beim Einsatz erneuerbarer Energien wie der Geothermie. 
Laura Cerullo, M.A., war von 2019 bis 2021 wissenschaftliche Mitarbeiterin am Institut für Sozialforschung und Technikfolgenabschätzung (IST) an der Ostbayerischen Technischen Hochschule (OTH) Regensburg. Sie untersuchte ein digitalisiertes Bewegungsfeedback im Rahmen der Prospektiven Studie zur Nutzung von Aktivitätstrackern in der Alterstraumatologie. Innerhalb des Projekts DAAS-KIN (Diffusion altersgerechter Assistenzsysteme - Kennzahlenerhebung und Identifikation von Nutzungshemmnissen) bearbeitete sie das Scoping Review zur Verbreitung altersgerechter Assistenztechnik in der Altenpflege.

Dominik Domhoff, M.A., ist Gesundheitswissenschaftler am Institut für Public Health und Pflegeforschung (IPP) der Universität Bremen. Neben Forschung $\mathrm{zu}$ Versorgungsstrukturen pflegebedürftiger Menschen beschäftigt er sich mit innovativen digitalen Technologien wie künstlicher Intelligenz in der Pflege. In der Studie zur Situation der Langzeitpflege in Deutschland während der CoronaPandemie war er für die Online-Befragung und Auswertung quantitativer Daten für diesen Beitrag zuständig.

Marina Fotteler, M.Sc., ist wissenschaftliche Mitarbeiterin am Institut DigiHealth der Hochschule Neu-Ulm und Doktorandin an der Universität Ulm. In ihrer Forschung beschäftigt sie sich mit altersgerechten Assistenzsystemen sowie den Möglichkeiten der digitalen Biopsie für Neurochirurgie und -pathologie. Sie studierte Public Health an der University of Southern Denmark und der University of North Carolina at Greensboro, USA und schloss mit einem M.Sc. ab. Sie hat einen B.Sc. in Informationsmanagement im Gesundheitswesen der Hochschulen Ulm und Neu-Ulm.

Debora Frommeld, Dr. phil., ist Soziologin und wissenschaftliche Mitarbeiterin am Institut für Sozialforschung und Technikfolgenabschätzung (IST) der OTH Regensburg. Sie promovierte mit einer Arbeit zur Selbstvermessung und untersuchte die Geschichte und Soziologie der Personenwaage. In ihrer Forschung und Lehre beschäftigt sie sich mit (ästhetischen) Alltagspraktiken an der Schnittstelle von Gesundheit, Körper, Technik und Digitalisierung. Im Rahmen des Projekts DAAS-KIN (Diffusion altersgerechter Assistenzsysteme - Kennzahlenerhebung und Identifikation von Nutzungshemmnissen) war sie zuständig für die Diskurs- und Wertbaumanalyse.

Sonja Haug, Prof. Dr. phil. habil., ist Professorin für Empirische Sozialforschung an der Ostbayerischen Technischen Hochschule Regensburg und Ko-Leiterin des Instituts für Sozialforschung und Technikfolgenabschätzung (IST). In ihrer Forschung beschäftigt sie sich mit demografischem Wandel, Migration sowie sozialen Aspekten von Technik im Bereich Gesundheit und Wohnen. Im Rahmen des 
Projekts DAAS-KIN (Diffusion altersgerechter Assistenzsysteme - Kennzahlenerhebung und Identifikation von Nutzungshemmnissen) war sie zuständig für die quantitative Online-Befragung und stellvertretende Projektleitung.

Jannis Hergesell, Dr. phil., studierte Altertumswissenschaften an der FU Berlin und Soziologie technikwissenschaftlicher Richtung an der TU Berlin. Er leitet das soziologische Teilprojekt eines interdisziplinären Projektverbundes zu Chancen sensorbasierter Pflegetechnologien bei der Unterstützung von stationären Pflegekräften am Arbeitsbereich Soziologie der Digitalisierung am Institut für Soziologie der Leibniz Universität Hannover. Zudem ist er Projektleiter eines Forschungsprojekts zur Arbeitsmarktintegration am Fachgebiet Methoden der empirischen Sozialforschung am Institut für Soziologie der TU Berlin. Seine Forschungsschwerpunkte sind Technik- und Gesundheitssoziologie, qualitative Methodologie sowie Digitalisierungsforschung.

Felix Holl, M.Sc., ist Medizininformatiker und als wissenschaftlicher Mitarbeiter im Institut DigiHealth der Hochschule Neu-Ulm tätig. Er promoviert über die Entwicklung von Evaluationsmethoden für mHealth-Anwendungen an der LudwigMaximilians-Universität München. Als Fulbright-Stipendiat hat er einen M.Sc. in Global Health Sciences an der University of California, San Francisco erworben. Seine Forschungsschwerpunkte sind Evaluationsmethoden in der Medizininformatik sowie die Anwendung der Medizininformatik in der Entwicklungshilfe.

Benjamin Lipp, Dr. rer. soc., studierte Soziologie, Volkswirtschaftslehre und Kriminologie an der Ludwig-Maximilans-Universität in München. Er ist wissenschaftlicher Mitarbeiter am Munich Center for Technology in Society (MCTS) der Technischen Universität München. Dort promovierte er zur Pflegerobotik im Kontext europäischer Innovationspolitik. Derzeit forscht er im Rahmen des EU-Projekts SCALINGS zu ko-kreativen Praktiken in der Pflegerobotik. Seine Forschungsschwerpunkte liegen im Bereich der Technik- und Wissenschaftssoziologie, Science and Technology Studies und Innovationsforschung mit speziellem Fokus auf die technik-politischen Rahmenbedingungen von Entwicklung und Innovation im Bereich von Informations- und Kommunikationstechnologien.

Mareike Mähs, M.Sc., arbeitet als Gesundheitswissenschaftlerin am Institut für Gerontologie an der Universität Vechta. Zurzeit ist sie im Verbundprojekt ActiVAtE_Prevention (»Activity Tracking Data to Understand Volition, Attrition and Engagement towards Healthy Behaviors in Diabetic Patients and Controls«) beschäftigt und promoviert zur gesundheitsökonomischen Evaluation von AAL-Technologien für ältere Menschen in der häuslichen Versorgung. Ihre Forschung fokussiert die 
sozialpolitischen und gesundheitsökonomischen Aspekte sowie Evaluation von gesundheitlichen und pflegerischen Innovationen.

Arne Maibaum, Dipl.-Soz.-Wiss., studierte Sozialwissenschaften und Psychologie an der Universität Duisburg-Essen. Derzeit ist er wissenschaftlicher Mitarbeiter an der TU Berlin und promoviert dort zu Robot-Competitions und zur Robotik als Wissenschaft. Seine Schwerpunkte sind die Technik- und Wissenschaftssoziologie/Science and Technology Studies und die Innovationsforschung, besonders in Bezug auf Mensch-Roboter-Interaktion, Robotik als Wissenschaft und die sogenannte Pflegerobotik.

Michael Örtl, B.Sc., ist wissenschaftlicher Mitarbeiter im Institut DigiHealth und für das Verbundprojekt CARE REGIO tätig. Seinen Bachelor in Medizinischer Informatik absolvierte er an der Universität zu Lübeck und studiert aktuell Internetund Webbasierte Systeme an der Universität Darmstadt. Sein Interesse gilt vor allem der Verarbeitung, dem digitalen Austausch und der Auswertung medizinischer und pflegerischer Daten und der Verbreitung der erarbeiteten Methoden in die Praxis für Mediziner*innen und Pfleger*innen.

Hartmut Remmers, Prof. Dr. phil. habil., ist seit 2019 Seniorprofessor im Institut für Gerontologie der Universität Heidelberg. Vor seiner Pensionierung 2018 war er Leiter der Abteilung Pflegewissenschaft im Institut für Gesundheitsforschung und Bildung an der Universität Osnabrück. Seine Arbeitsschwerpunkte waren dort unter anderem: Krankheitsbelastung und Krankheitsbewältigung im Kontext der Biografie-Forschung, Interventionskonzepte insbesondere bei Körperbild-/Selbstbild-Störungen. Ethik im Gesundheitswesen, Medizin- und Pflegeethik. Zahlreiche Forschungsprojekte waren thematisch in den Bereichen onkologische Pflege, Palliative Care, Alter und Technik angesiedelt.

Heinz Rothgang, Prof. Dr. , leitet die Abteilung Gesundheit, Pflege und Alterssicherung am Forschungszentrum Ungleichheit und Sozialpolitik (SOCIUM) der Universität Bremen. Er forscht zu Fragen des Gesundheitssystems, der Langzeitpflege und der Alterssicherung auf lokaler, nationaler und international vergleichender Ebene und ist Projektleiter im Pflegeinnovationszentrum im BMBF Cluster $\mathrm{Zu}$ kunft der Pflege. In der Studie zur Situation der Langzeitpflege in Deutschland während der Corona-Pandemie war er Teilprojektleiter.

Clarissa Rudolph, Prof. Dr. habil., ist Professorin für Politikwissenschaft und Soziologie an der OTH Regensburg und hat seit WiSe 2020/21 eine Forschungsprofessur für Geschlechterforschung und Gender Studies inne. Ihre Forschungsschwerpunkte sind Analysen der Geschlechterverhältnisse in unterschiedlichen 
gesellschaftlichen Bereichen, Arbeitsmarkt- und Sozialpolitik sowie Rechtsextremismus. Projektleitungen unter anderem zur Interessenvertretung von Pflegekräften und zu Förderprojekten für Geschlechtergerechtigkeit in MINTStudiengängen.

Martin Schmieder, Dr. rer. nat., ist Geowissenschaftler und arbeitet derzeit am Institut DigiHealth der Hochschule Neu-Ulm (HNU) am Tripelpunkt der Erdwissenschaften, der Biologie und des Gesundheitswesens. Ein besonderes Augenmerk liegt dabei auf der multispektralen Satelliten-Fernerkundung stehender Gewässer als potenzielle Brutstätten krankheitsübertragender Stechmückenarten. Des Weiteren betreibt er die Geschäftsstelle der Gemeinsamen Ethikkommission der Hochschulen Bayerns (GEHBa).

Ulrike Scorna, M.A., ist Sozialwissenschaftlerin am Institut für Sozialforschung und Technikfolgenabschätzung (IST) an der OTH Regensburg. Ihre Forschungsschwerpunkte liegen im Bereich Digitaler Assistenztechnik und Medizinsoziologie sowie der ethischen, rechtlichen und sozialen Aspekte dieser. Im Rahmen des Projekts DAAS-KIN (Diffusion altersgerechter Assistenzsysteme - Kennzahlenerhebung und Identifikation von Nutzungshemmnissen) war sie zuständig für die qualitativen Interviews.

Kathrin Seibert, M.Sc., ist Pflegewissenschaftlerin am Institut für Public Health und Pflegeforschung (IPP) der Universität Bremen. Ihre Forschungsschwerpunkte liegen in den Bereichen innovativer digitaler Technologien in der Pflege und Versorgungsqualität pflegebedürftiger Menschen. In der Studie zur Situation der Langzeitpflege in Deutschland während der Corona-Pandemie war sie für die Online-Befragung und die Auswertung qualitativer Daten für diesen Beitrag zuständig.

Walter Swoboda, Prof. Dr. med., ist Leiter des Instituts DigiHealth und Forschungsprofessor der Fakultät Gesundheitsmanagement an der Hochschule Neu-Ulm. Seine Interessen liegen in der Digitalisierung von Medizin und Pflege, eHealth, der Versorgungsforschung und dem medizinischen Informationsmanagement. Er ist einer der Leiter der gemeinsamen Ethikkommission der Hochschulen Bayerns (GEHBa) und Beirat der Stiftskliniken Weißenhorn, außerdem Mitglied der Deutschen Gesellschaft für medizinische Informatik, Biometrie und Epidemiologie (GMDS) und der Deutschen Gesellschaft für Informatik (GI).

Miriam Vetter, M.A., hat Soziale Arbeit an der OTH Regensburg studiert. Im Anschluss absolvierte sie den Master der Sozialen Arbeit mit dem Schwerpunkt Inklusion und Exklusion. Von 2019 bis 2020 war sie wissenschaftliche Mitarbeiterin 
im Institut für Sozialforschung und Technikfolgenabschätzung (IST) an der OTH Regensburg. Sie bearbeitete im Projekt DAAS-KIN das Scoping Review zur Verbreitung altersgerechter Assistenzsysteme.

Karsten Weber, Prof. Dr. phil. habil., ist Ko-Leiter des Instituts für Sozialforschung und Technikfolgenabschätzung (IST) sowie einer der drei Direktoren des Regensburg Center of Health Sciences and Technology (RCHST) der Ostbayerischen Technischen Hochschule Regensburg. Er hält außerdem eine Honorarprofessur für Kultur und Technik an der BTU Cottbus-Senftenberg. Der Schwerpunkt seiner Forschung liegt im Bereich der sozialen Auswirkungen von Informationstechnik im Gesundheitswesen sowie im Bereich der gesellschaftlichen Konsequenzen von KI. Im Rahmen des Projekts DAAS-KIN (Diffusion altersgerechter Assistenzsysteme - Kennzahlenerhebung und Identifikation von Nutzungshemmnissen) war er Projektleiter.

Karin Wolf-Ostermann, Prof. Dr., leitet die Abteilung Pflegewissenschaftliche Versorgungsforschung am Institut für Public Health und Pflegeforschung (IPP) der Universität Bremen. Sie forscht zu Strukturen, Prozessen und Ergebnissen der Gesundheitsversorgung von Menschen mit Hilfe- und Pflegebedarf sowie zur Wirkung von bestehenden und innovativen Versorgungskonzepten und Technologien und ist Projektleitung im Pflegeinnovationszentrum im BMBF Cluster Zukunft der Pflege. In der Studie zur Situation der Langzeitpflege in Deutschland während der Corona-Pandemie war sie Projektleiterin.

Jürgen Zerth, Prof. Dr. rer. pol. habil., ist Professor für Wirtschaftswissenschaften, insbesondere Gesundheitsökonomie, an der Wilhelm Löhe Hochschule in Fürth. Gleichzeitig leitet er dort das Forschungsinstitut IDC. Der Schwerpunkt der Arbeit von Jürgen Zerth bewegt sich im Kontext von gesundheits- und institutionenökonomischen Analysen von Technologie- und Organisationsmodellen im Gesundheits- und im Pflegebereich. Als Institutsleiter des IDC ist Jürgen Zerth Teil des Pflegepraxiszentrums Nürnberg (PPZ), das als Teil des BMBF-Clusters "Zukunft der Pflege« die Frage der Implementierung und Implementierbarkeit von Pflegetechnologien untersucht. 


\section{Soziologie}

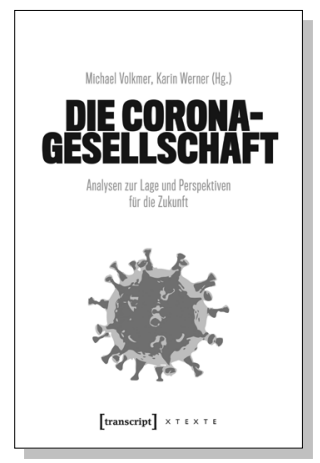

Michael Volkmer, Karin Werner (Hg.)

Die Corona-Gesellschaft

Analysen zur Lage und Perspektiven für die Zukunft

2020, 432 S., kart., Dispersionsbindung, 2 SW-Abbildungen 24,50€ (DE), 978-3-8376-5432-5

E-Book:

PDF: $21,99 €$ (DE), ISBN 978-3-8394-5432-9

EPUB: $21,99 €$ (DE), ISBN 978-3-7328-5432-5

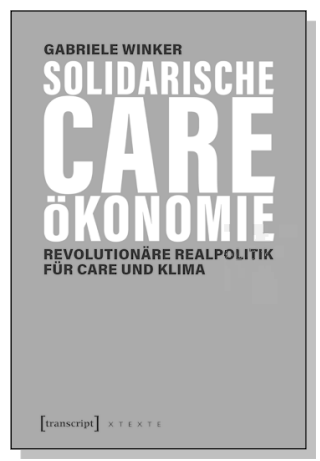

Gabriele Winker

\section{Solidarische Care-Ökonomie}

Revolutionäre Realpolitik für Care und Klima

März 2021, 216 S., kart.

$15,00 €(D E), 978-3-8376-5463-9$

E-Book:

PDF: $12,99 €$ (DE), ISBN 978-3-8394-5463-3

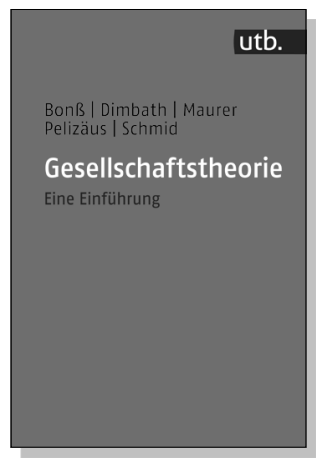

Wolfgang Bonß, Oliver Dimbath,

Andrea Maurer, Helga Pelizäus, Michael Schmid

Gesellschaftstheorie

Eine Einführung

Januar 2021, 344 S., kart.

25,00€ (DE), 978-3-8376-4028-1

E-Book:

PDF: 21,99 € (DE), ISBN 978-3-8394-4028-5 


\section{Soziologie}

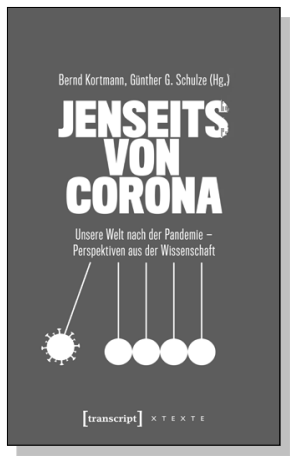

Bernd Kortmann, Günther G. Schulze (Hg.)

Jenseits von Corona

Unsere Welt nach der Pandemie -

Perspektiven aus der Wissenschaft

2020, 320 S., Klappbroschur, Dispersionsbindung, 1 SW-Abbildung

22,50€ (DE), 978-3-8376-5517-9

E-Book:

PDF: 19,99€ (DE), ISBN 978-3-8394-5517-3

EPUB: $19,99 €$ (DE), ISBN 978-3-7328-5517-9

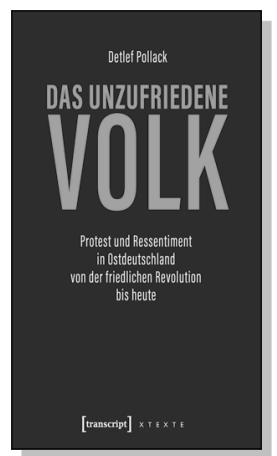

Detlef Pollack

\section{Das unzufriedene Volk}

Protest und Ressentiment in Ostdeutschland von der friedlichen Revolution bis heute

2020, 232 S., Klappbroschur, Dispersionsbindung, 6 SW-Abbildungen

20,00€ (DE), 978-3-8376-5238-3

E-Book:

PDF: $17,99 €$ (DE), ISBN 978-3-8394-5238-7

EPUB: $17,99 €(D E)$, ISBN 978-3-7328-5238-3

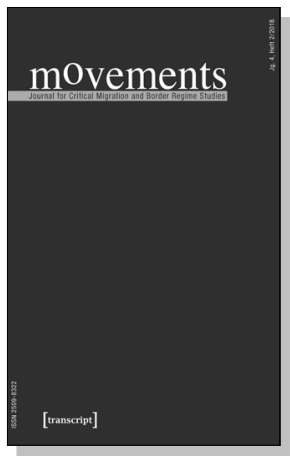

Juliane Karakayali, Bernd Kasparek (Hg.)

movements.

Journal for Critical Migration and Border Regime Studies

Jg. 4, Heft 2/2018

2019, 246 S., kart.

24,99€ (DE), 978-3-8376-4474-6 